This electronic thesis or dissertation has been downloaded from the King's Research Portal at https://kclpure.kcl.ac.uk/portal/

\title{
Sulforaphane-mediated inhibition of SHP2 as a potential pharmacotherapy for Noonan syndrome
}

Smith, Joy Lynne

Awarding institution:

King's College London

The copyright of this thesis rests with the author and no quotation from it or information derived from it may be published without proper acknowledgement.

\section{END USER LICENCE AGREEMENT}

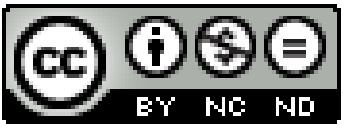

Unless another licence is stated on the immediately following page this work is licensed

under a Creative Commons Attribution-NonCommercial-NoDerivatives 4.0 International

licence. https://creativecommons.org/licenses/by-nc-nd/4.0/

You are free to copy, distribute and transmit the work

Under the following conditions:

- $\quad$ Attribution: You must attribute the work in the manner specified by the author (but not in any way that suggests that they endorse you or your use of the work).

- $\quad$ Non Commercial: You may not use this work for commercial purposes.

- $\quad$ No Derivative Works - You may not alter, transform, or build upon this work.

Any of these conditions can be waived if you receive permission from the author. Your fair dealings and other rights are in no way affected by the above.

Take down policy

If you believe that this document breaches copyright please contact librarypure@kcl.ac.uk providing details, and we will remove access to the work immediately and investigate your claim. 


\title{
Sulforaphane-mediated inhibition of SHP2 as a potential pharmacotherapy for Noonan syndrome
}

\author{
Joy Smith \\ King's College London \\ The School of Cardiovascular Science and Medicine \\ The Rayne Institute \\ $4^{\text {th }}$ Floor Lambeth Wing \\ St Thomas' Hospital \\ London, SE1 7EH
}

Thesis undertaken for the degree of Doctor of Philosophy

\section{KING'S




\section{Acknowledgements}

Firstly, l'd like to say an enormous thank you to Professor Philip Eaton. I truly believe there would have been no better mentor for me through this process and I will always appreciate the support, guidance and vast opportunities you have given me.

To everyone in the Eaton Lab and the windowless pit we call the mezzanine office, words aren't quite enough. Not only have you endured my (often questionable) choice in music, but my frequent cries for science help have never gone unanswered, and I hope you all know how grateful I am. An extra special thank you must be given to Becky, Mariana and Hyunju. You've each at some point pulled me back from the brink of insanity, even if you haven't realised it.

Scientists aside, I owe a lot of this to my parents, Lynne and lan. Your constant encouragement over the last 10 years has helped keep me going, and I will always be grateful that you think I'm a genius.

To my three wonderful best friends, Laura, Leah and Louise...thank you for listening. Your nods pretending to understand when I'm dragging on about science haven't gone unnoticed.

Finally, it wouldn't be right to not acknowledge the main man in my life. Your ability to listen, understand, help and support me has been inspirational. So, to my dog, Chase, I can't thank you enough. Jokes aside, Dom, you've been there through it all and although I'll never understand how you put up with it, I will be forever grateful for everything you've done. 


\section{Abstract}

Sulforaphane (SFN) is an electrophilic isothiocyanate which can adduct cysteine thiols within proteins. Protein targets of SFN were immunoprecipitated from cardiac tissue of wildtype (WT) mice following in vivo treatment with the electrophile using a validated polyclonal antibody developed in-house to panspecifically detect SFN adducted to cysteines. Combined with quantitative proteomics, this confirmed the non-receptor protein-tyrosine phosphatase, SHP2, as a target of SFN. SFN is intrinsically unstable at room temperature, therefore, a chemically stabilised variant developed by Evgen Pharmaceuticals (UK) known as Sulforadex (SFX-01), was used in subsequent experiments.

Using a commercially available phosphatase activity assay, SFX-01 was shown to inhibit recombinant SHP2 in vitro, as well as that in cardiac tissue of mice administered SFX-01 in their drinking water for 4 days. We speculated that SFX01 may be therapeutic in diseases where SHP2 is hyperactive, such as Noonan syndrome (NS). Indeed, using an NS mouse model, Ptpn11 ${ }^{\mathrm{D} 61 \mathrm{G} /+}$, a mutation resulting in hyperactivity of the phosphatase, SFX-01 time-dependently inhibited cardiac SHP2 activity.

$100 \%$ of homozygous and $\sim 50 \%$ of heterozygous $\operatorname{Ptpn} 11^{\mathrm{D} 61 \mathrm{G} /+}$ mice die midgestation due to severe skeletal or cardiac defects, with the remaining 50\% surviving to adulthood where they show non-cardiac features of NS. To assess if SFN-induced inhibition of SHP2 in the homozygous or heterozygous foetus could improve embryonic development, breeding pairs consisting of WT only or NS only parents were administered SFX-01 before conception and continued during pregnancy. SFX-01 treatment induced SFN-protein labelling of foetal tissue but also reduced litter sizes born from NS breeding pairs and genotyping showed only WT mice were born. This adverse effect may be due to SFN increasing the phospho-activation of ERK, which is deleterious in embryonic development of NS foeti. However, SFX-01 had no adverse impact on the pregnancies of WT mice.

Adult NS mice develop splenomegaly and myeloproliferative disease which can further develop into leukaemia. With this in mind, adult WT or NS mice were administered SFX-01 for 10 weeks to assess if prolonged treatment with the drug 
would inhibit SHP2 activity and reduce the incidence of myeloproliferative disease in the NS mouse model. Using whole blood cell staining, ultrasound and flow cytometry, lower total white blood cell count, spleen size and myeloid cell count in the blood, bone marrow and spleen of NS mice by SFX-01 was seen compared to water only controls. SHP2 activity was also attenuated in the spleen of both WT and NS mice, strongly suggesting this therapeutic action of SFX-01 was mediated by inhibition of SHP2 phosphatase activity.

Unexpectedly, even though phosphatase activity was inhibited following 4-day or 10-week treatment with SFX-01, this occurred without evidence of an SHP2-SFN adduct in the tissue of WT or NS mice. Data from biochemical analyses involving biotinylated iodoacetamide (BIAM) labelling, the polyethylene glycol (PEG)switch method or phenylarsinic acid (PAA)-binding, showed that SFN induced an inhibitory modification within SHP2 between two vicinal thiols within the active domain of the phosphatase, which to reiterate was not stable SFN adducts. Data from complementary studies using site-directed mutagenesis of cysteines supported the concept that SFN adducts to SHP2 and inhibits it, which is followed by a proximal cysteine thiol mediating its removal or truncation, with the resulting modification maintaining inhibition of the phosphatase. This SFN-induced inhibitory modification may be the formation of an intramolecular disulfide bond or perhaps the chemical modification of the SFN adduct to a dithiolethione.

Additional data has also shown that an SFN adduct can transfer from one thiol to another, so-called 'trans-thiolation'. Using bovine serum albumin with an SFN adduct, which had been purified by large-format gel filtration on a protein chromatograph, transfer of the adduct to other cysteine-containing molecules such as haemoglobin or glutathione was observed.

SFX-01, a stabilised SFN variant in phase 2 clinical trials, inhibits WT SHP2 as well as a hyperactive Ptpn $11^{\mathrm{D} 61 \mathrm{G} /+}$ mutant form expressed in many patients with NS. Consistent with this, SFX-01 significantly corrects the myeloproliferative disease found in Ptpn $11^{\mathrm{D} 61 \mathrm{G} /+} \mathrm{NS}$ mice. Thus, in conclusion, SFX-01 has potential as a new therapy for the treatment of NS. 


\section{Table of contents}

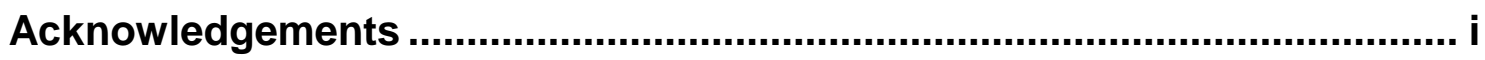

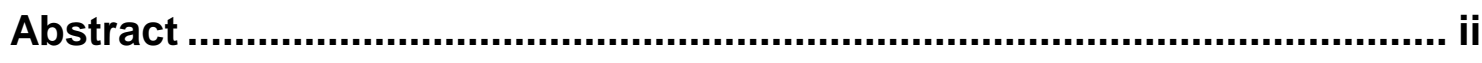

Table of contents

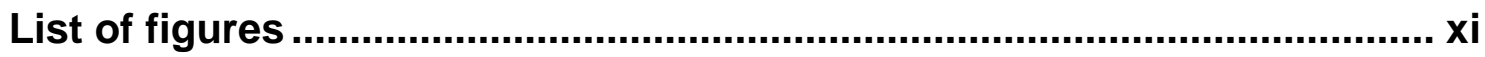

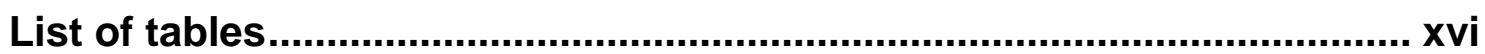

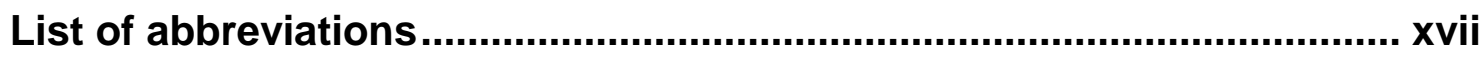

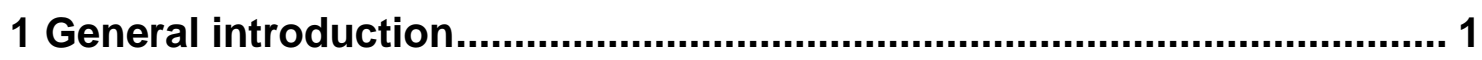

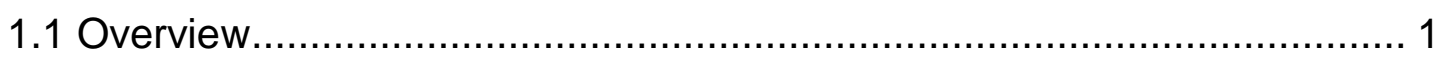

1.2 Biochemistry of cysteine thiol oxidation .............................................. 1

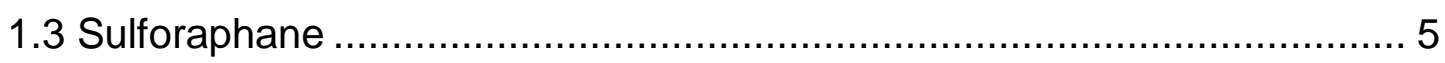

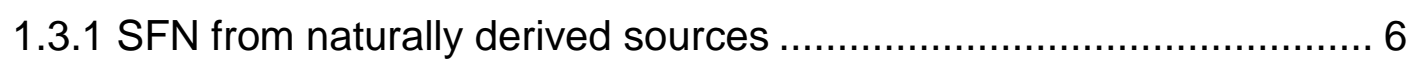

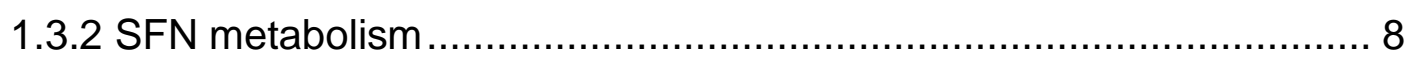

1.3.3 Modulation of signalling pathways by SFN ..................................... 11

1.3.3.1 Modulation of the NF-KB signalling pathway by SFN................ 11

1.3.3.2 Modulation of the KEAP1/Nrf2 signalling pathway by SFN ........ 11

1.3.4 Protein targets of SFN............................................................ 14

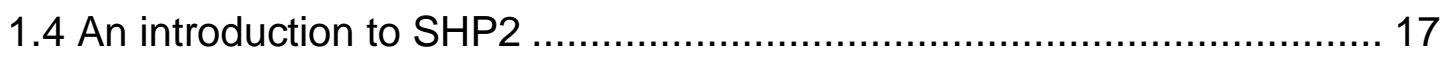

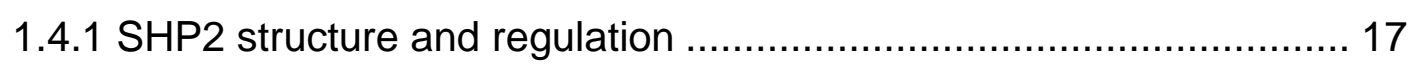

1.4.2 Cell signalling pathways regulated by SHP2 …............................ 19

1.4.2.1 Regulation of integrin signalling by SHP2 ……..................... 20

1.4.2.2 Regulation of insulin signalling by SHP2 …........................... 21

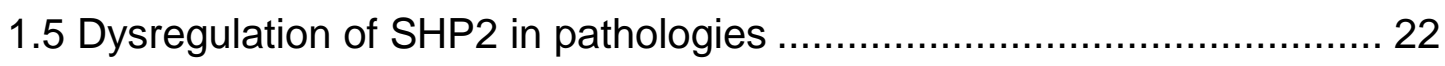

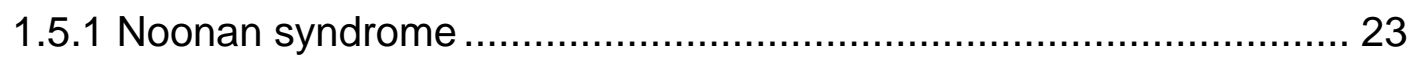

1.5.1.1 Ptpn11 mutations associated with NS ..................................... 24

1.5.1.2 NS mouse model with a Ptpn11 ${ }^{\mathrm{D} 61 \mathrm{G} /+}$ mutation .......................... 26

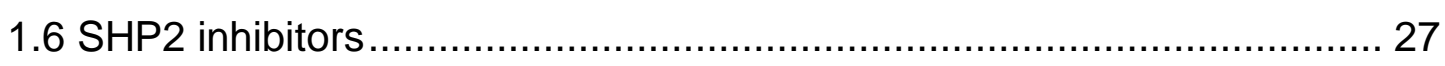

1.6.1 Sulforaphane as an inhibitor or SHP2 ………............................ 29 
1.7 Research aims

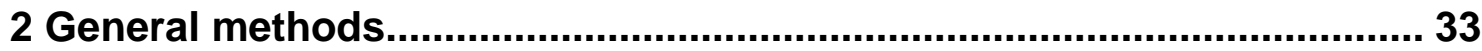

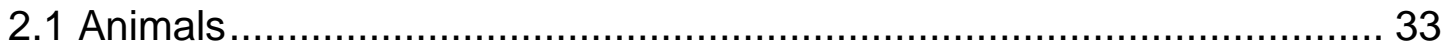

2.2 Tissue isolation and preparation for western immunoblotting or protein immunoprecipitation ............................................................ 33

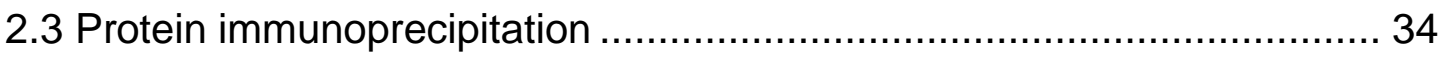

2.4 Phosphatase activity assay ....................................................... 35

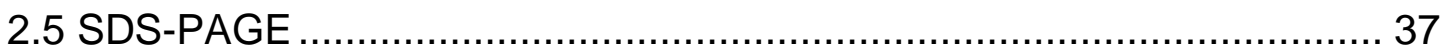

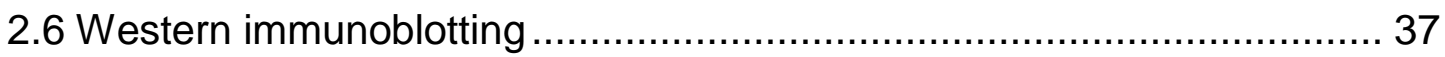

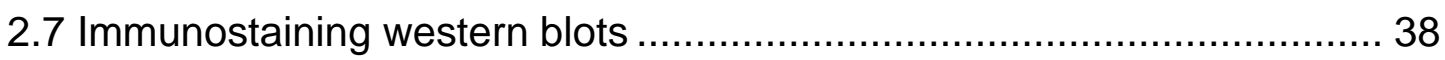

2.8 Enhanced chemiluminescence and quantification ............................ 39

2.9 Colloidal Coomassie staining ............................................... 40

2.10 Statistical analysis .................................................................. 40

2.11 Affinity purification of SFN antibody .......................................... 40

2.12 High-performance liquid chromatography (HPLC) ......................... 42

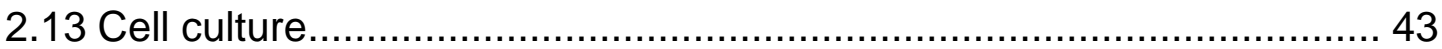

3 Sulforaphane inhibits SHP2 activity in vitro and in vivo with effects on

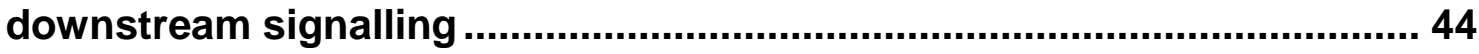

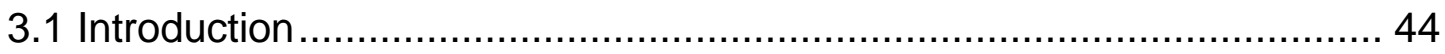

3.1.1 SHP2 regulation of the Ras/ERK signalling pathway ................... 44

3.1.2 SHP2 regulation of the JAK/STAT GH signalling pathway .............. 48

3.1.3 GH treatment of NS patients ................................................. 50

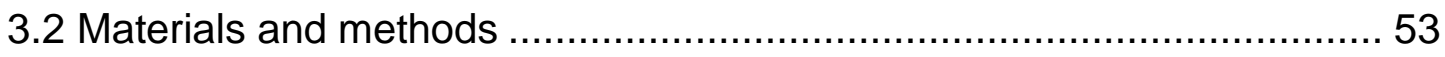

3.2.1 SDS-PAGE and western blotting ......................................... 53

3.2.2 Immunoprecipitation ......................................................... 53

3.2.3 Preparation of recombinant SHP2 for in vitro experiments.............. 54

3.2.4 Optimisation of the SHP2 phosphatase activity assay .................. 54

3.2.5 Preparation of SFN stock for use in in vitro experiments................. 55 
3.2.6 Treatment of SHP2 with SFX-01 and determination of IC50 of inhibition by SFN 55

3.2.7 Determining the stability of SFN in water over time ...................... 55

3.2.8 Acute or chronic treatment with SFN in vivo................................ 56

3.2.9 Growth hormone treatment in vivo ........................................ 56

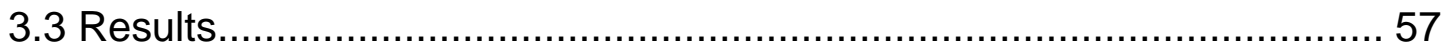

3.3.1 Optimisation of the fluorescence-based SHP2 phosphatase activity assay $\quad 57$

3.3.2 SFN inhibits SHP2 phosphatase activity in vitro.......................... 59

3.3.3 SFN adducts recombinant SHP2 ......................................... 61

3.3.4 Characterisation of the NS mouse model ................................6 63

3.3.5 SFN adducts protein targets in a concentration-dependent manner in vivo.

3.3.6 Acute treatment with SFX-01 does not inhibit SHP2 phosphatase activity in vivo

3.3.7 SFX-01 is stable in water for 6 days 69

3.3.8 SFN adducts protein targets in a time-dependent manner in vivo .... 71 3.3.9 Chronic SFX-01 treatment inhibits SHP2 phosphatase activity in vivo.

3.3.10 Treatment with clinically-relevant amounts of SFX-01 inhibits SHP2 phosphatase activity

3.3.11 Chronic SFX-01 treatment increases global tyrosine

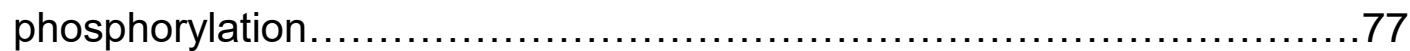

3.3.12 Chronic SFX-01 treatment increases ERK phosphorylation .......... 77

3.3.13 SFX-01 or rmGH treatment increases STAT5 phosphorylation...... 78

3.3.14 STAT5 phosphorylation by $40 \mu \mathrm{g} r \mathrm{mGH}$ is potentiated by SFX-01. 80 3.4 Discussion ......................................................................... 81

4 Sulforaphane can trans-thiolate between proteins............................. 90

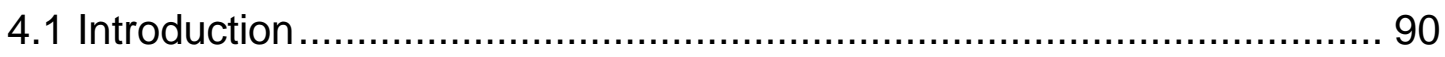

4.1.1 SFN adducts are reversible ................................................. 90

4.2 Materials and methods ............................................................ 92

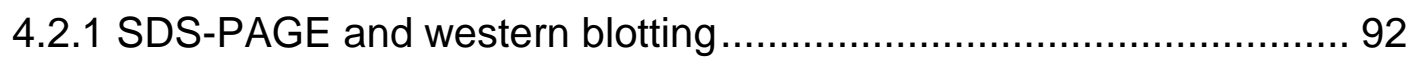


4.2.2 Immunoprecipitation 92

4.2.3 Preparation and administration of broccoli sprouts......................... 93

4.2.4 Proteomic analysis following SFX-01 treatment ............................. 94

4.2.5 Protein separation by size-exclusion chromatography ..... 94

4.2.6 Treatment of purified BSA-SFN with cysteine containing compounds. 97

4.2.7 Using HPLC to assess transfer of an SFN adduct from SHP2 to GSH. .98

4.2.8 Isolation of adult mouse ventricular myocytes 99

4.2.9 Treatment of HEK293 cells. 100

4.3 Results 101

4.3.1 SFN adducts a $\sim 15 \mathrm{kDa}$ protein in cardiac tissue. 101

4.3.2 Protein-SFN adducts are detected after consumption of broccoli sprouts. 102

4.3.3 SFX-01 treatment increases Trx and GRX1 protein expression..... 103 4.3.4 Trx or GRX1 do not comigrate with the $\sim 15 \mathrm{kDa}$ protein adducted by SFN 104

4.3.5 Immunoprecipitation of the $\sim 15 \mathrm{kDa}$ protein adducted by SFN was unsuccessful... 104

4.3.6 The $\sim 15 \mathrm{kDa}$ protein adducted by SFN was identified by mass spectrometry. 105

4.3.7 SFN can adduct $\mathrm{Hgb} \beta$ 108

4.3.8 The $\sim 15 \mathrm{kDa}$ protein adducted by SFN comigrates with $\mathrm{Hgb} \beta \ldots . . .109$

4.3.9 Immunoprecipitation of $\mathrm{Hgb} \beta$-SFN was unsuccessful 109

4.3.10 SFX-01 treatment did not increase $\mathrm{Hgb} \beta$ expression 110

4.3.11 $\mathrm{Hgb} \beta$ was not detected in isolated cardiomyocytes. 111

4.3.12 Detection of cardiac Hgb-SFN is attenuated by coronary perfusion 112

4.3.13 BSA-SFN was purified using size-exclusion chromatography 113

4.3.14 The interaction between BSA and SFN is stable in a PBS buffer. 117

4.3.15 SFN can transfer from GSH to cellular proteins 118

4.3.16 L-cysteine can remove the SFN adduct from BSA 119

4.3.17 SFN can transfer from BSA to $\mathrm{Hgb} \beta$ 
4.3.18 SFN can transfer from SHP2 to GSH ...................................... 122

4.3.19 SFN can transfer from BSA to a protein present in the blood....... 124

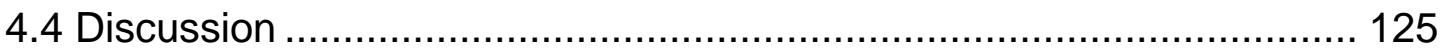

5 Therapeutic potential of sulforaphane in a NS mouse model............... 139

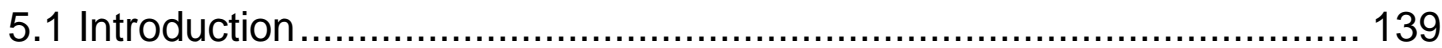

5.1.1 The role of SHP2 in cardiac development .................................. 139

5.1.2 White blood cell production ........................................................ 142

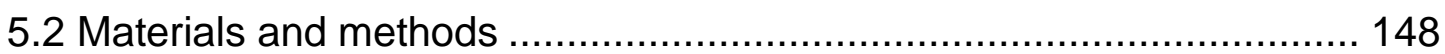

5.2.1 SDS-PAGE and western blotting .............................................. 148

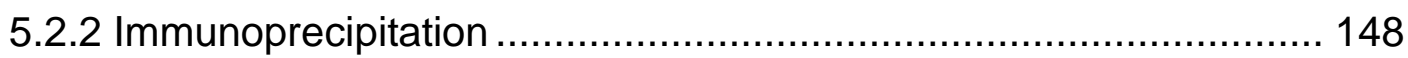

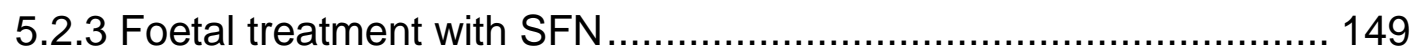

5.2.4 Blood sampling via the tail vein ................................................. 149

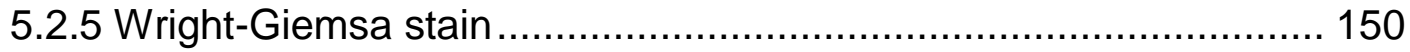

5.2.6 Tissue preparation for flow cytometry ......................................... 150

5.2.7 Cell staining for flow cytometry .............................................. 151

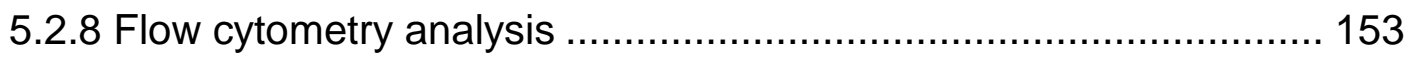

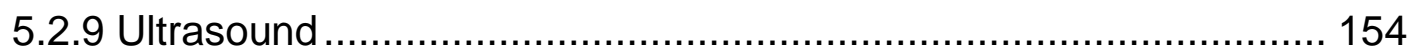

5.2.10 Macrophage inflammatory protein 1 alpha (MIP1 $\alpha$ ) measurement 154

5.2.11 Macrophage inflammatory protein 2 (MIP2) measurement........... 155

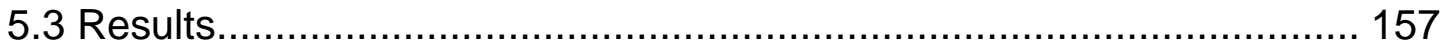

5.3.1 High amounts of SFX-01 is embryonic lethal in pregnancies carrying NS foeti.

5.3.2 Only WT neonates are born following foetal treatment with low amounts of SFX-01

5.3.3 Foetal treatment with SFX-01 only partially reduces neonatal SHP2 phosphatase activity

5.3.4 Protein-SFN adducts are detected in neonates following foetal

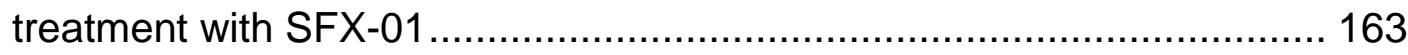

5.3.5 Foetal treatment with SFX-01 increases neonatal ERK

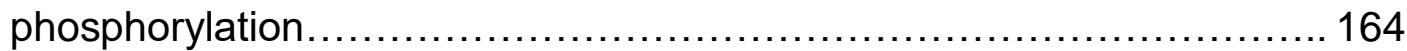

5.3.6 SFX-01 treatment lowers total white blood cell count in WT and NS mice. 
5.3.7 SFX-01 treatment lowers myeloid cell count in the blood and spleen of NS mice.

5.3.8 SFX-01 treatment reduces the plasma concentration of MIP2 but not MIP1a in NS mice

5.3.9 SFX-01 treatment reduces the spleen growth of NS mice 173

5.3.10 SFN adducts proteins in the spleen.

5.3.11 SFX-01 treatment reduces SHP2 phosphatase activity in the spleen of NS mice.

5.4 Discussion

6 The mechanism of sulforaphane-induced inhibition of SHP2 189

6.1 Introduction 189

6.1.1 Catalytic mechanism of SHP2 189

6.2 Materials and methods 192

6.2.1 SDS-PAGE and western blotting..... 192

6.2.2 Immunoprecipitation 192

6.2.3 $\mathrm{H}_{2} \mathrm{O}_{2}$ treatment of recombinant SHP2 192

6.2.4 Polyethylene glycol (PEG)-switch method. 192

6.2.5 Biotinylated iodoacetamide (BIAM) labelling method 193

6.2.6 Phenylarsinic acid (PAA) labelling method 194

6.2.7 Amplification and purification of WT and Cys ${ }^{459}$ Ser SHP2 plasmid 194 6.2.8 Site-directed mutagenesis of SHP2 plasmid 196

6.2.9 Transformation of Escherichia coli and plasmid purification following mutagenesis 198

6.2.10 Transfection of HEK293 cells with SHP2 plasmids 198

6.2.11 Cell treatment with SFX-01. 199

6.2.12 Generation of 5-thio-2-nitrobenzoic acid (TNB) 199

6.2.13 Treatment of TNB and analysis by HPLC 200

6.2.14 Liquid chromatography-mass spectrometry analysis of GSH following

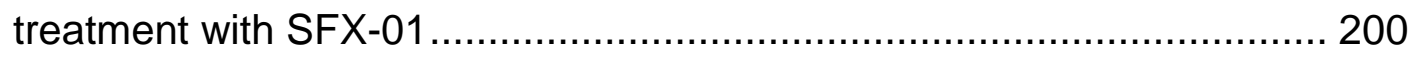

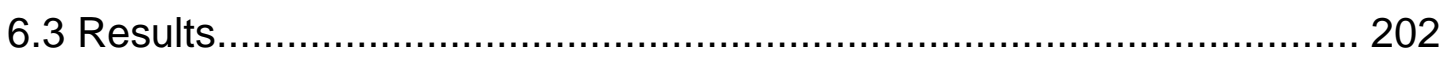

6.3.1 An SFN adduct is not detected upon inhibited cardiac SHP2 ........ 202 
6.3.2 Recombinant SHP2 migrates faster by SDS-PAGE following $\mathrm{H}_{2} \mathrm{O}_{2}$ treatment.

6.3.3 SFN treatment induces a non-reducible molecular weight shift of SHP2 in vivo.

6.3.4 SFN-induced inhibition of cardiac SHP2 is not reversed by treatment with DTT 204

6.3.5 SFN treatment induces a small mobility shift of cardiac SHP2 following the PEG-switch assay 205

6.3.6 In vivo treatment with SFX-01 reduces BIAM labelling SHP2........ 206

6.3.7 In vivo SFX-01 treatment reduces PAA labelling of SHP2 ............ 207

6.3.8 Cysteine to serine SHP2 mutants were successfully generated..... 208 6.3.9 SFN adducts proteins in a concentration-dependent manner in HEK293 cells

6.3.10 Cellular transfection with SHP2 plasmid increases protein expression in an amount-dependent manner

6.3.11 Cys $^{459}$ Ser SHP2 mutation does not affect plasmid transfection efficiency or protein expression 216

6.3.12 The phosphatase activity of SHP2 is lost following mutation of the protein's catalytic cysteine 216

6.3.13 Mutation of any cysteine pair within the active site of SHP2 results in retention of the SFN adduct 219

6.3.14 dTNB is formed following incubation of TNB with SFX-01 221

6.3.15 The identification of a dithiolethione was achieved by mass spectrometry following incubation of GSH with SFN 224

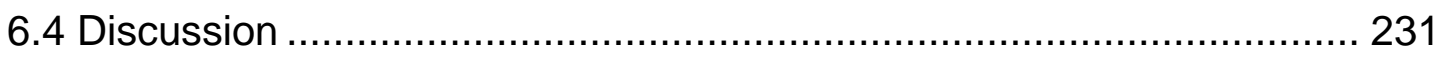

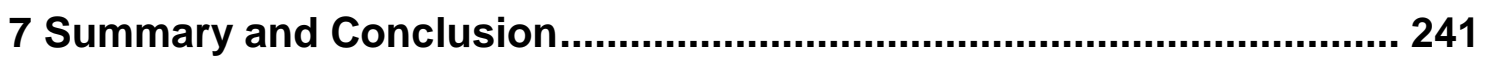

7.1 Summary 241

7.2 Conclusion 252

7.3 Future work 253

8 Bibliography 257 


\section{List of figures}

Figure 1.1 Summary of common oxidative modifications of cysteine thiols.

Figure 1.2. Summary of possible disulfide bond formation following thiol oxidation. 5

Figure 1.3. SFN can adduct protein cysteine thiolates.

Figure 1.4. A diagram showing hydrolysis of glucosinolate and subsequent formation of bioactive compounds.

Figure 1.5. Generation of SFN by enzymatic hydrolysis of glucoraphanin. ............ 8

Figure 1.6. Pathway for SFN metabolism.

Figure 1.7. Modulation of antioxidant and xenobiotic detoxification enzymes by the KEAP1/Nrf2 signalling pathway.

Figure 1.8. SHP2 structure and mechanism of activation by docking phosphotyrosine residues. 19

Figure 1.9. Regulation of integrin and insulin signalling by SHP2. 22

Figure 1.10. Location of 12 known NS-associated SHP2 mutations. 25

Figure 3.1. Diagram of the Ras/ERK signalling pathway 46

Figure 3.2. SHP2 positively regulates Ras/ERK signalling at the intracellular membrane of the endoplasmic reticulum. 48

Figure 3.3. Diagram of the JAK/STAT signalling pathway 50 Figure 3.4. Optimisation of protein and DTT concentrations for a fluorescence-based phosphatase activity assay. 58

Figure 3.5. SFX-01 inhibits SHP2 phosphatase activity in vitro........................60

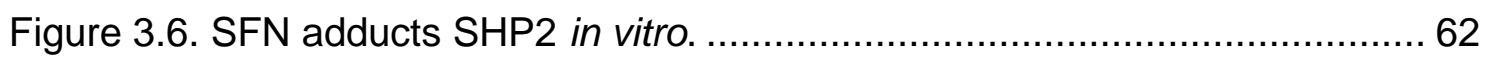

Figure 3.7. Characterisation of the NS mouse model..................................... 64

Figure 3.8. SFN adducts proteins in vivo in a concentration-dependent manner. . 66 Figure 3.9. Acute exposure to SFX-01 does not inhibit cardiac SHP2 phosphatase activity. 68

Figure 3.10. HPLC chromatograms showing SFX-01 is stable in water for 6 days.

Figure 3.11. Protein-SFN adducts accumulate in vivo in a time-dependent manner.

Figure 3.12. 4-day treatment with SFX-01 inhibits cardiac SHP2 phosphatase activity.

Figure 3.13. Treatment with SFX-01 at clinically-relevant concentrations inhibits SHP2 activity. 76 
Figure 3.14. SFX-01 treatment increases global tyrosine phosphorylation. 77

Figure 3.15. SFX-01 treatment increases ERK phosphorylation over time. 78

Figure 3.16. SFX-01 and rmGH increase STAT5 phosphorylation. 79

Figure 3.17. SFX-01 potentiates phosphorylation of STAT5 by $40 \mu \mathrm{g} \mathrm{rmGH}$. 80

Figure 3.18. Protein-disulfide reduction by DTT. 82

Figure 4.1. A diagram illustrating the size-exclusion chromatography system. 97

Figure 4.2. SFN adducts a $\sim 15 \mathrm{kDa}$ protein in cardiac tissue of WT mice. 101

Figure 4.3. Ingestion of naturally-derived sources of SFN results in protein adduct formation. 102

Figure 4.4. SFN treatment increases protein expression of Trx and GRX1 103

Figure 4.5. Neither Trx nor GRX1 comigrate with a $\sim 15 \mathrm{kDa}$ protein target of SFN. 104

Figure 4.6. A $\sim 15 \mathrm{kDa}$ protein adducted by SFN was not successfully immunoprecipitated from cardiac tissue. 105

Figure 4.7. The $\sim 15 \mathrm{kDa}$ protein adducted by SFN can be visualised following electrophoresis. 106

Figure 4.8. Mass spectra of the Hgb $\beta 1$ peptide sequence GTFASLSELHCDK which

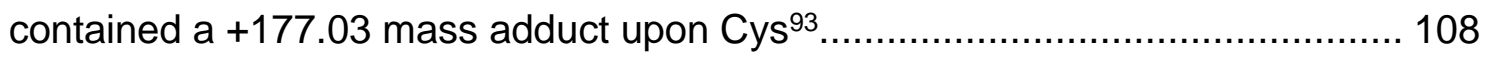

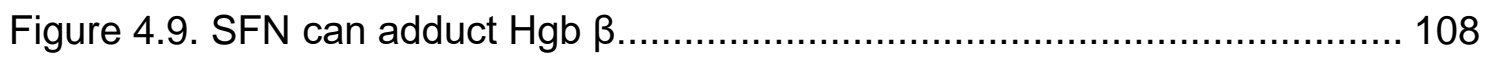

Figure 4.10. Hgb $\beta$ comigrates with the SFN adducted $\sim 15 \mathrm{kDa}$ protein............ 109

Figure 4.11. Hgb $\beta$-SFN was not successfully immunoprecipitated from cardiac tissue.

Figure 4.12. Spectral counts of $\mathrm{Hgb} \beta$ in cardiac tissue of WT mice following treatment with SFX-01 for 10 days in their drinking water.

Figure 4.13. A representative immunoblot showing $\mathrm{Hgb}$ was not detected in isolated adult mouse ventricular myocytes. 112

Figure 4.14. Hgb $\beta$-SFN present in cardiac tissue was perhaps from residual blood. 113

Figure 4.15. Chromatograms showing retention times for BSA treated with SFN.

Figure 4.16. BSA-SFN is present in eluates following size-exclusion chromatography. 116

Figure 4.17. The SFN adduct is stable upon BSA within a PBS buffer............... 117

Figure 4.18. SFN can transfer from GSH to cellular proteins. .......................... 118

Figure 4.19. L-cysteine can remove the SFN adduct from BSA....................... 119 
Figure 4.20. SFN can transfer from BSA to $\mathrm{Hgb} \beta$.

Figure 4.21. HPLC chromatograms showing SFN moving from SHP2 to GSH... 123

Figure 4.22. SFN can transfer from BSA to blood proteins.

Figure 4.23. A diagram showing the enzymatic reactions involved in a two-step dithiol mechanism for catalytic reduction of protein disulfides by Trx. 128

Figure 4.24. A diagram showing enzymatic reactions of GRX1. 128

Figure 4.25. Proposed mechanisms for transfer of the SFN adduct to Trx 130

Figure 4.26. Proposed mechanisms for transfer of the SFN adduct to GRX1 .... 130 Figure 4.27. Proposed mechanism for trans-thiolation of SFN from BSA to Hgb $\beta$ involving directed nucleophilic attack of the unbound thiol towards to adduct. .... 137 Figure 5.1. White blood cell production in the bone marrow by haematopoiesis. 145 Figure 5.2. Foetal treatment with high amounts of SFX-01 pre-gastrulation is embryonic lethal in pregnancies carrying NS foeti. 158

Figure 5.3. Foetal treatment with high amounts of SFX-01 post-gastrulation under these conditions is embryonic lethal in pregnancies carrying NS foeti. 159

Figure 5.4. Foetal treatment with low amounts of SFX-01 results in the birth of only WT neonates in pregnancies carrying NS foeti. 160

Figure 5.5. Foetal treatment with SFX-01 partially reduces SHP2 phosphatase activity in HET neonates.

Figure 5.6. SFN adducts proteins in WT or HET neonates following foetal treatment with SFX-01. 163

Figure 5.7. Foetal treatment with SFX-01 increases ERK phosphorylation in WT neonates. 164

Figure 5.8. SFX-01 treatment reduces white blood cell count in WT and NS mice. 166

Figure 5.9. Representative flow cytometry analyses of blood from 22-week-old NS mice. 168

Figure 5.10. SFX-01 treatment reduces neutrophil count in the blood of NS mice. 169

Figure 5.11. SFX-01 treatment causes a small reduction in neutrophil cell count in the bone marrow of NS mice. 170

Figure 5.12. SFX-01 treatment reduces total myeloid cell count in the spleen of NS mice.

Figure 5.13. The concentration of MIP2 but not MIP1a is reduced in the plasma of NS mice following SFX-01 treatment. 
Figure 5.14. Adult NS mice have splenomegaly.

Figure 5.15. The growth of the spleen of NS mice is reduced when they receive a 10-week treatment of SFX-01.

Figure 5.16. Protein-SFN adducts are detected in the spleen following SFX-01 treatment.

Figure 5.17. SFX-01 treatment reduces the phosphatase activity of SHP2 in the spleen of NS mice.

Figure 6.1. Catalytic mechanism of cysteine-based PTPs, including SHP2.

Figure 6.2. WT SHP2 plasmid used for site-directed mutagenesis.

Figure 6.3. An SFN adduct is not detected upon SHP2 following chronic in vivo treatment with SFX-01.

Figure 6.4. $\mathrm{H}_{2} \mathrm{O}_{2}$-treated recombinant SHP2 migrates faster on a polyacrylamide gel.

Figure 6.5. A small portion of cardiac SHP2 migrates faster a polyacrylamide gel following SFX-01 treatment. 204

Figure 6.6. SFX-01 treatment inhibits SHP2 phosphatase activity in vivo, which is not recovered by DTT. 205

Figure 6.7. Analysis of the oxidative modification of cardiac SHP2 following in vivo treatment with SFX-01 using the PEG-switch method. 206

Figure 6.8. BIAM labelling of cardiac SHP2 is reduced following SFN-induced inhibition of the protein. 207

Figure 6.9. PAA labelling of cardiac SHP2 is reduced following SFN-induced inhibition of the protein. 208

Figure 6.10. Nucleotide sequence alignment of Cys ${ }^{333}$ Ser SHP2 mutant with WT plasmid.

Figure 6.11. Nucleotide sequence alignment of Cys ${ }^{367}$ Ser SHP2 mutant with WT plasmid.

Figure 6.12. Nucleotide sequence alignment of $\mathrm{Cys}^{333 / 367}$ Ser SHP2 mutant with WT plasmid.

Figure 6.13. Nucleotide sequence alignment of $\mathrm{Cys}^{333 / 459}$ Ser SHP2 mutant with WT plasmid. 213

Figure 6.14. SFN adducts proteins in HEK293 cells in a concentration-dependent manner. 214

Figure 6.15. Cellular transfection with higher amounts of SHP2 plasmid generates increased protein expression. 215 
Figure 6.16. SHP2 protein expression is unaffected by a Cys ${ }^{459}$ Ser mutation. ... 216

Figure 6.17. Cys ${ }^{459}$ Ser SHP2 protein has no phosphatase activity

Figure 6.18. SFN adduct upon SHP2 is stabilised following mutation of two active site cysteines. 220

Figure 6.19. HPLC chromatograms of standards used for analysis. 222

Figure 6.20. HPLC chromatograms showing SFN incubation with TNB inducing dTNB formation. 223

Figure 6.21. Representative chromatograms from LC performed prior to linear ion trap MS. 225

Figure 6.22. Representative mass spectrums from linear ion trap MS performed following the incubation of GSH with L-SFN. 226

Figure 6.23. Full MS performed following the incubation of GSH with L-SFN. .... 227

Figure 6.24. $\mathrm{MS}^{2}$ performed following the incubation of GSH with L-SFN. 228

Figure 6.25. $\mathrm{MS}^{3}$ performed following the incubation of GSH with L-SFN. 229

Figure 6.26. The amount GSH-SFN formed following incubation of GSH with L-SFN increases in a time-dependent manner 230

Figure 6.27. The amount of GSSG formed following the incubation of GSH at room temperature increases in a time-dependent manner. 230

Figure 6.28. A PyMol image of the active site of SHP2 ............................... 235

Figure 6.29. A proposed scheme of SFN-induced intra-disulfide formation. 238

Figure 6.30. A proposed scheme for SFN-induced dithiolethione formation between two vicinal thiols within the active site of SHP2. 240

Figure 6.31. A possible cyclocondensation reaction of an SFN adduct with vicinal thiols within the active site of SHP2. 240 


\section{List of tables}

Table 1.1. Protein targets of SFN in cardiac tissue of WT mice identified by quantitative mass spectrometry following oral gavage with SFN....................... 16

Table 2.1. HPLC protocol for analysing the stability of SFX-01 in water ............... 43

Table 3.1. List of primary antibodies used for western blotting in chapter 3......... 53

Table 4.1. List of primary antibodies used for western immunoblotting in chapter 4.

Table 4.2. List of antibodies used for immunoprecipitation in chapter 4

Table 4.3. Chromatography protocol for purifying BSA-SFN from unbound electrophile.

Table 4.4. Solutions used for the isolation of adult mouse ventricular myocytes. 100

Table 4.5. Proteins identified by LC-MS/MS from cardiac tissue of WT mice...... 107

Table 5.1. List of primary antibodies used for western immunoblotting in chapter 5. 148

Table 5.2. List of antibodies used for immunoprecipitation in chapter 5 148

Table 6.1. List of primary antibodies used for western immunoblotting in chapter 6. 192

Table 6.2. List of primers used to generate cysteine to serine SHP2 mutants. ... 197

Table 6.3. PCR protocol used for site-directed mutagenesis. 197 


\section{List of abbreviations}

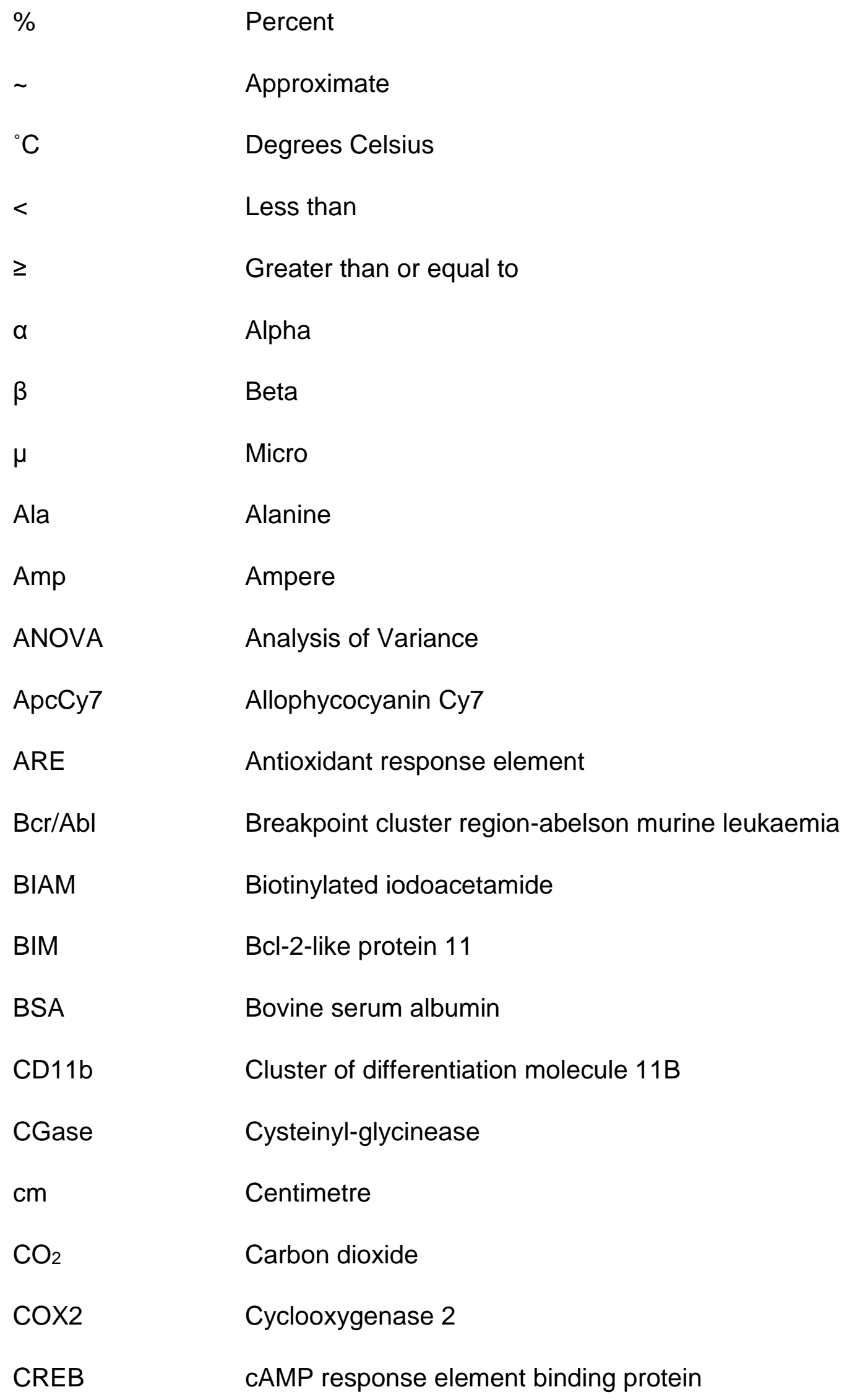

$\mathrm{cm}$

$\mathrm{CO}_{2}$

$\operatorname{cox} 2$

CREB

Percent

Approximate

Degrees Celsius

Less than

Greater than or equal to

Alpha

Beta

Micro

Alanine

Ampere

Analysis of Variance

Allophycocyanin Cy7

Antioxidant response element

Breakpoint cluster region-abelson murine leukaemia

Biotinylated iodoacetamide

Bcl-2-like protein 11

Bovine serum albumin

Cluster of differentiation molecule 11B

Cysteinyl-glycinease

Centimetre

Carbon dioxide

Cyclooxygenase 2

cAMP response element binding protein 


\begin{tabular}{|c|c|}
\hline CUL3 & Cullin 3 \\
\hline Cys & Cysteine \\
\hline $\mathrm{D}$ & Aspartic acid \\
\hline DiFMUP & 6,8-Difluoro-4-Methylumbelliferyl Phosphate \\
\hline DMEM & Roberto Dulbecco modified eagle medium \\
\hline DMSO & Dimethyl sulfoxide \\
\hline DNA & Deoxyribonucleic acid \\
\hline DPBS & Dulbecco's phosphate buffered saline \\
\hline DTT & Dithiothreitol \\
\hline ECL & Enhanced chemiluminescence \\
\hline EDTA & Ethylenediaminetetraacetic acid \\
\hline EGTA & Egtazic acid \\
\hline ELISA & Enzyme-linked immunosorbent assay \\
\hline EphR & Ephrin receptor \\
\hline ERK & Extracellular signal-regulated kinase \\
\hline FAK & Focal adhesion kinase \\
\hline FBS & Foetal bovine serum \\
\hline FITC & Fluorescein isothiocyanate \\
\hline FSC & Forward scatter \\
\hline$G$ & Glycine \\
\hline g & Gram \\
\hline GAB1 & GRB2-associated-binding protein 1 \\
\hline GAP & GTPase activating protein \\
\hline GAPDH & Glyceraldehyde 3-phosphate dehydrogen \\
\hline
\end{tabular}




\begin{tabular}{|c|c|}
\hline GCS & Glutamate cysteine ligase \\
\hline G-CSF & Granulocyte-colony stimulating factor \\
\hline GDP & Guanine diphosphate \\
\hline GF & Growth factor \\
\hline GFR & Growth factor receptor \\
\hline $\mathrm{GH}$ & Growth hormone \\
\hline GHR & Growth hormone receptor \\
\hline GM-CSF & Granulocyte-macrophage-colony stimulating factor \\
\hline GRB2 & Growth factor receptor-bound protein 2 \\
\hline GRX1 & Glutaredoxin 1 \\
\hline GRXR & Glutaredoxin reductase \\
\hline GSH & Glutathione \\
\hline GST & Glutathione S-transferase \\
\hline GTP & Guanine triphosphate or $y$-glutamyl-transpeptidase \\
\hline $\mathrm{H}$ & Hydrogen \\
\hline $\mathrm{H}_{2} \mathrm{O}_{2}$ & Hydrogen peroxide \\
\hline $\mathrm{H}_{2} \mathrm{~S}$ & Hydrogen sulfide \\
\hline $\mathrm{H}_{2} \mathrm{SO}_{4}$ & Sulfuric acid \\
\hline $\mathrm{HCl}$ & Hydrogen chloride \\
\hline HEK293 & Human embryonic kidney 293 cells \\
\hline HET & Heterozygous \\
\hline $\mathrm{Hgb}$ & Haemoglobin \\
\hline HNO & Nitroxyl \\
\hline $\mathrm{HO} 1$ & Heme oxygenase 1 \\
\hline
\end{tabular}




\begin{tabular}{|c|c|}
\hline HOM & Homozygous \\
\hline HPLC & High-performance liquid chromatography \\
\hline HRP & Horse radish peroxidase \\
\hline HSC & Haematopoietic stem cells \\
\hline HSD & Honestly significant difference \\
\hline $\mathrm{IC}_{50}$ & Half maximal inhibitory concentration \\
\hline IGF1 & Insulin-like growth factor 1 \\
\hline $\lg G$ & Immunoglobin G \\
\hline $\mathrm{IL}$ & Interleukin \\
\hline iNOS & Inducible nitric oxide synthase \\
\hline IRS1 & Insulin receptor substrate 1 \\
\hline ITC & Isothiocyanate \\
\hline JAK2 & Janus kinase 2 \\
\hline JMML & Juvenile myelomonocytic leukaemia \\
\hline $\mathrm{kDa}$ & Kilodaltons \\
\hline KEAP1 & Kelch Like ECH Associated Protein 1 \\
\hline $\mathrm{kHz}$ & Kilohertz \\
\hline KSR & Kinase suppressor of Ras \\
\hline L & Litre \\
\hline LB & Luria-Bertani \\
\hline LC-MS/MS & Liquid chromatography-tandem mass spectrometry \\
\hline L-SFN & L-sulforaphane \\
\hline Ly6C & Lymphocyte antigen $6 \mathrm{C}$ \\
\hline Ly6G & Lymphocyte antigen 6G \\
\hline
\end{tabular}




\begin{tabular}{|c|c|}
\hline Lys & Lysine \\
\hline$m / z$ & Mass-to-charge ration \\
\hline MAP2K1 & Mitogen-activated protein kinase kinase 1 \\
\hline MAPK & Mitogen-activated protein kinase \\
\hline M-CSF & Macrophage-colony stimulating factor \\
\hline MEK & MAPK/ERK kinase \\
\hline $\mathrm{mg}$ & Milligram \\
\hline $\mathrm{MHz}$ & Megahertz \\
\hline MIP1a & Macrophage inflammatory protein 1 alpha \\
\hline MIP2 & Macrophage inflammatory protein 2 \\
\hline $\mathrm{ml}$ & Millilitre \\
\hline MPP & Multipotent progenitor \\
\hline mRNA & Messenger ribonucleic acid \\
\hline MRP1 & Multidrug resistance-associated protein 1 \\
\hline $\mathrm{N}_{2} \mathrm{O}_{3}$ & Dinitrogen trioxide \\
\hline NAC & $\mathrm{N}$-acetylcysteine \\
\hline $\mathrm{NaCl}$ & Sodium chloride \\
\hline $\mathrm{NAD}(\mathrm{P}) \mathrm{H}$ & Nicotinamide adenine dinucleotide phosphate \\
\hline $\mathrm{NaHCO}_{3}$ & Sodium bicarbonate \\
\hline NAT & $\mathrm{N}$-acetyltransferase \\
\hline$N F-k B$ & Nuclear factor-kappa B \\
\hline $\mathrm{nm}$ & Nanometre \\
\hline nmol & Nanomoles \\
\hline NO & Nitric oxide \\
\hline
\end{tabular}




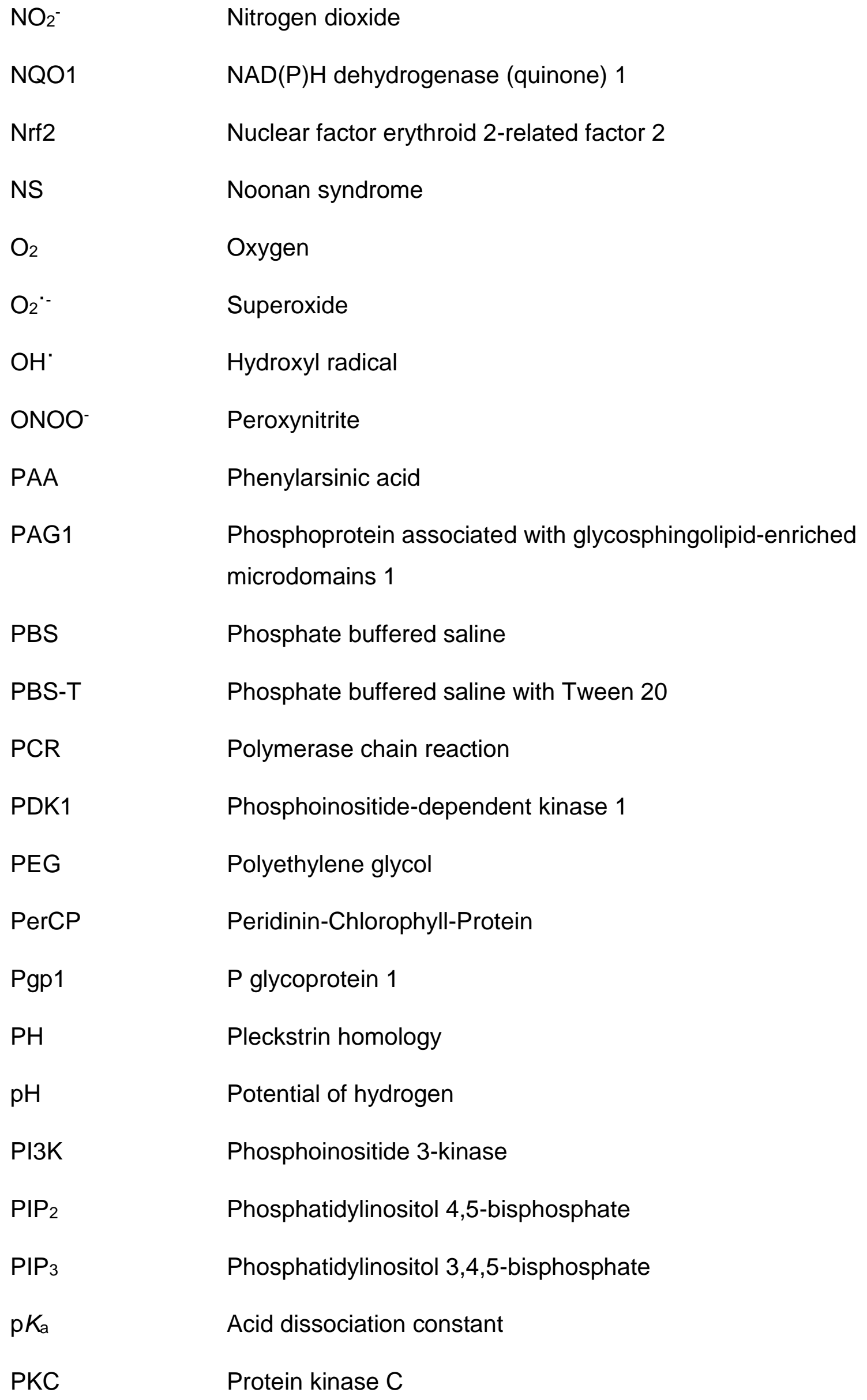




$\begin{array}{ll}\text { Plcy1 } & \text { Phospholipase C } \mathbf{1} \\ \text { PMT } & \text { Photomultiper tubes } \\ \text { Pro } & \text { Proline } \\ \text { PTP } & \text { Protein-tyrosine phosphatase } \\ \text { Ptpn11 } & \text { Protein-tyrosine phosphatase non-receptor type 11 } \\ \text { PVDF } & \text { Polyvinylidene difluoride } \\ \text { Raf1 } & \text { Rapidly accelerated fibrosarcoma 1 } \\ \text { RBX1 } & \text { RING-box protein 1 } \\ \text { Redox } & \text { Reduction and oxidation } \\ \text { rhGH } & \text { Recombinant human growth hormone } \\ \text { RhoA } & \text { Ras homolog gene family A } \\ \text { rmGH } & \text { Recombinant mouse growth hormone } \\ \text { RNS } & \text { Reactive nitrogen species } \\ \text { ROCK } & \text { Rho-associated protein kinase } \\ \text { ROS } & \text { Reactive oxygen species } \\ \text { Rpm } & \text { Rotations per minute } \\ \text { RTK } & \text { Receptor tyrosine kinase } \\ \text { S } & \text { Sulfur } \\ \text {-S } & \text { Thiolate } \\ \text { S6K1 } & \text { Sibosomal s6 kinase 1 } \\ \text { SCF } & \text { SDS-PAGE }\end{array}$




\begin{tabular}{|c|c|}
\hline SFN & Sulforaphane \\
\hline SFX-01 & Sulforadex \\
\hline$-\mathrm{SH}$ & Thiol \\
\hline $\mathrm{SH} 2$ & Src homology 2 \\
\hline SHP2 & Src homology 2-domain containing tyrosine phosphatase 2 \\
\hline$-\mathrm{SO}_{2} \mathrm{H}$ & Sulfinic acid \\
\hline$-\mathrm{SO}_{3} \mathrm{H}$ & Sulfonic acid \\
\hline soc & Super optimal broth with catabolite repression \\
\hline socs & Suppressors of cytokine signalling \\
\hline$-\mathrm{SOH}$ & Sulfenic acid \\
\hline SOS1 & Son of sevenless 1 \\
\hline SSC & Side scatter \\
\hline STAT & Signal transducer and activator of transcription \\
\hline TBS-T & Tris buffered saline with Tween 20 \\
\hline TCEP & Tris(2-carboxyethyl)phosphine \\
\hline TNB & 5-thio-2-nitrobenzoic acid \\
\hline TPO & Thrombopoietin \\
\hline $\operatorname{Trx}$ & Thioredoxin \\
\hline Tyr & Tyrosine \\
\hline UGT & UDP glucuronosyltransferase family 1 member $A 1$ \\
\hline UV & Ultraviolet \\
\hline WT & Wildtype \\
\hline wt/vol & Weight to volume ration \\
\hline ZAP70 & Zeta chain of $T$ cell receptor associated protein kinase \\
\hline
\end{tabular}




\section{General introduction}

\subsection{Overview}

The following introduction provides background information to the studies conducted in this research project, which explored the potential for the electrophilic isothiocyanate (ITC) sulforaphane (SFN) to adduct and inhibit src homology 2 (SH2)-domain containing tyrosine phosphatase 2 (SHP2). First, an overview of how changes in the oxidation-reduction (redox) state of cells can induce a variety of oxidative post-translational modifications of protein cysteine thiols. How such modifications, as well as adduction of the protein by SFN, may impact on the structure and function of SHP2, which is also known as proteintyrosine phosphatase (PTP) non-receptor type 11 (Ptpn11), is then considered in detail. SHP2 is hyperactivated in humans with Noonan syndrome (NS), a disorder that presents with an array of debilitating symptoms for which there is an unmet therapeutic need. A transgenic mouse model of NS that was engineered to express a single gain-of-function mutation in SHP2, Ptpn11 ${ }^{\mathrm{D} 61 \mathrm{G} /+}$, was studied in the context that SFN may be therapeutic by attenuating the activity of this phosphatase.

\subsection{Biochemistry of cysteine thiol oxidation}

Post-translational modifications, such as phosphorylation and methylation, can alter protein structure and function and are fundamental processes in regulating cellular homeostasis or in response to internal or external stimuli. More recently,

oxidation and reduction of proteins by cellular oxidants and reductants respectively has been recognised as a mediator of cellular communications. Amino acids most susceptible to oxidation are those containing sulfur or aromatic 
side chain groups ${ }^{1}$. Their oxidation by reactive oxygen species (ROS), reactive nitrogen species (RNS) or hydrogen sulphide $\left(\mathrm{H}_{2} \mathrm{~S}\right)$ allows changes in cellular oxidant status to be relayed into regulatory responses, which has led to these species being classified as signalling mediators in their own right. ROS form following the addition of electrons to $\mathrm{O}_{2}$. This can occur either spontaneously or be mediated by enzymes such as myeloperoxidase, xanthine oxidase, nicotinamide adenine dinucleotide phosphate $(\mathrm{NAD}(\mathrm{P}) \mathrm{H})$ oxidase, cytochrome p450, uncoupled nitric oxide (NO) synthase, as well as electrons formed by the mitochondria ${ }^{23}$. The addition of a single electron to $\mathrm{O}_{2}$ generates a superoxide anion $\left(\mathrm{O}_{2}{ }^{-}\right)$. Following the addition of a second electron, the functionally efficient secondary messenger $\mathrm{H}_{2} \mathrm{O}_{2}$ is produced. If a third electron is accepted, a highly reactive hydroxyl radical is formed $\left(\mathrm{OH}^{-}\right)$. The production of $\mathrm{NO}$ leads to the subsequent formation of RNS, including nitroxyl ( $\mathrm{HNO})$, nitrite $\left(\mathrm{NO}_{2}{ }^{-}\right)$, dinitrogen trioxide $\left(\mathrm{N}_{2} \mathrm{O}_{3}\right)$ and peroxynitrite (OONO-).

Thiol groups $(-\mathrm{SH})$ within cysteines are considered the amino acid side chain most vulnerable to oxidative modification. However, reaction with oxidants predominantly occurs when this thiol group resides in a deprotonated (loss of $\mathrm{H}^{+}$) thiolate state $\left(-S^{-}\right)$. The ability for a thiol to reside as a thiolate depends on its acid disassociation constant $\left(\mathrm{p} K_{\mathrm{a}}\right)$, which is defined as the $\mathrm{pH}$ at which the thiolate and thiol form of a certain cysteine is equal ${ }^{4}$. A lower $p K_{a}$ value corresponds to increased susceptibility for the thiol to become deprotonated at cellular $\mathrm{pH}$ and therefore is considered more reactive towards oxidants. Most cellular thiols hold a $p K_{a}$ of $\sim 8.3$ and are therefore mainly in their protonated, unreactive state at a typical cellular $\mathrm{pH}$ of 7.0-7.4. The microenvironment of proteins can, however, alter the $\mathrm{p} K_{\mathrm{a}}$ of $\mathrm{a}$ thiol ${ }^{5}$. For example, if the cysteine resides near positively 
charged side chains of an arginine residue then electrostatic interactions between these amino acids will stabilise the negatively charged thiolate and help maintain a low $p K_{\mathrm{a}}$. Negatively charged side chains of aspartate and glutamate, on the other hand, will increase the $\mathrm{p} K_{\mathrm{a}}$ by destabilising the negatively-charged cysteine side chain ${ }^{6}$.

Thiolates can undergo a range of oxidative modifications as shown in figure 1.1. Whilst RNS can induce protein nitrosylation or nitrothiol formation, $\mathrm{H}_{2} \mathrm{O}_{2}$ can modify a thiolate to form a sulfenic acid intermediate $(-\mathrm{SOH})$. If this sulfenic acid intermediate is not rapidly reduced, hyperoxidation can occur to generate a sulfinic $\left(-\mathrm{SO}_{2} \mathrm{H}\right)$ and then a sulfonic acid $\left(-\mathrm{SO}_{3} \mathrm{H}\right)$, each with increasing irreversibility. The modifications induced by oxidants can either evoke a signalling event on their own or act as an intermediate step leading to further, perhaps more stable alterations that mediate any associated functional alteration. For example, the generation of a sulfenic acid intermediate in close proximity to a vicinal thiol may facilitate intramolecular disulfide bond formation with the generation of $\mathrm{H}_{2} \mathrm{O}$ as a condensation product as shown in figure 1.2. All these considerations contribute to the intricate and selective signalling that can be orchestrated by alterations in the abundance of cellular oxidants. Oxidation of protein thiolates may also induce homo- or heterodimers via intermolecular disulfide bond formation between proteins of the same or different species respectively (figure 1.2). Disulfide bonds can then be reduced by cellular antioxidants such as disulfide reductases including thioredoxin ( $\operatorname{Tr} x)$ and glutaredoxin 1 (GRX1), whose mechanisms of action are outlined in chapter $4{ }^{7}$. Dysregulation of cellular oxidant and antioxidant levels can result in altered protein oxidation states, which has been identified in a range of pathological scenarios ${ }^{8-15}$. Understanding more 
about these mechanisms of protein oxidation and why their alterations lead to pathology may offer therapeutic opportunities.

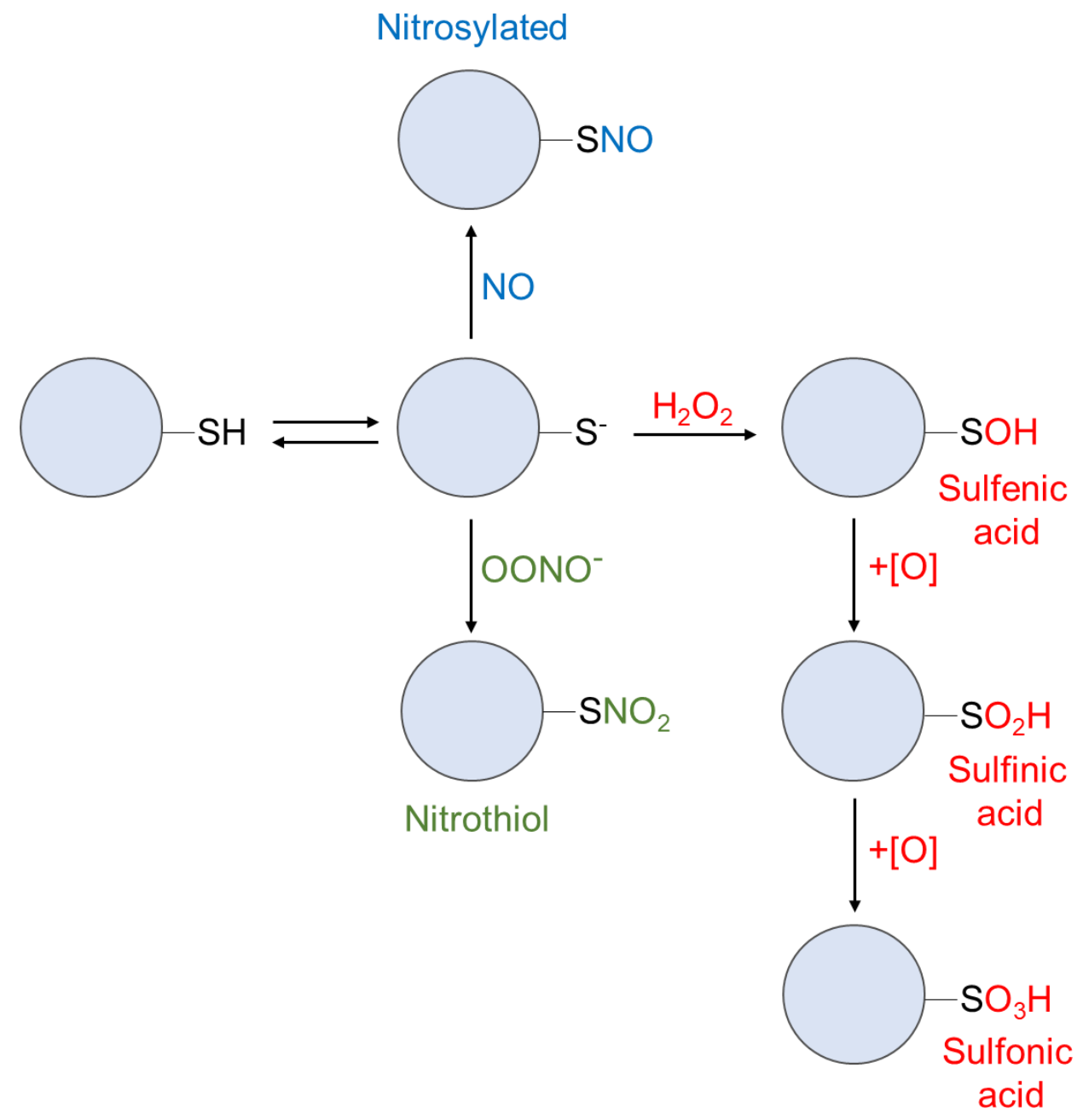

Figure 1.1 Summary of common oxidative modifications of cysteine thiols. 

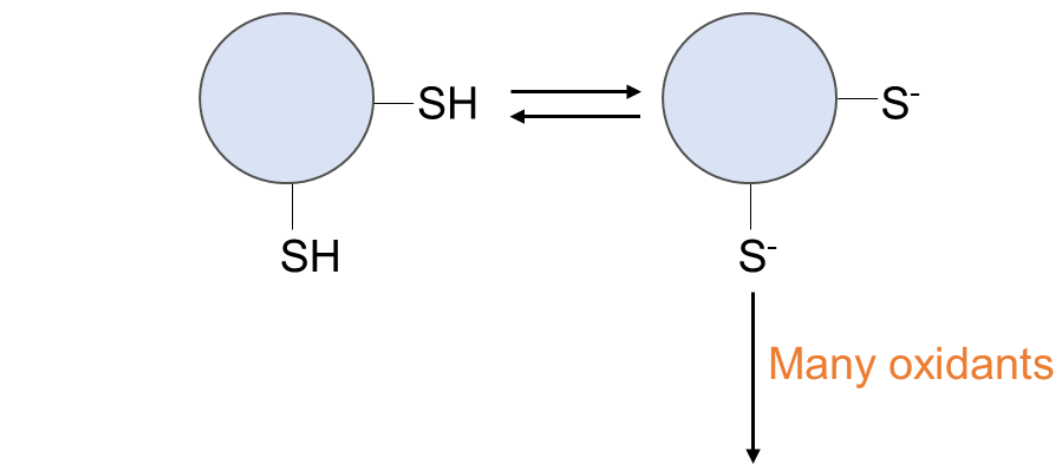

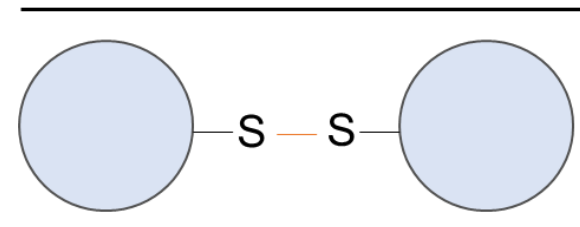

Inter-disulfide

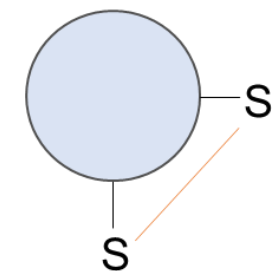

Intra-disulfide

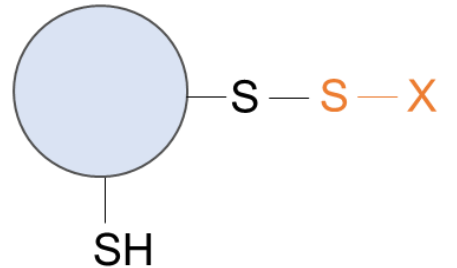

Thiolated

Figure 1.2. Summary of possible disulfide bond formation following thiol oxidation.

\subsection{Sulforaphane}

As well as oxidants, negatively charged moieties such as cysteine thiolates can also react and so adduct with electrophilic compounds such as SFN, as shown in figure 1.3. SFN is an ITC, which are characterised by an $\mathrm{N}=\mathrm{C}=\mathrm{S}$ functional group. Donation of electrons by the carbon atom across the double bonds results in a delta positive charge of the atom, which underlies its electrophilic properties. 


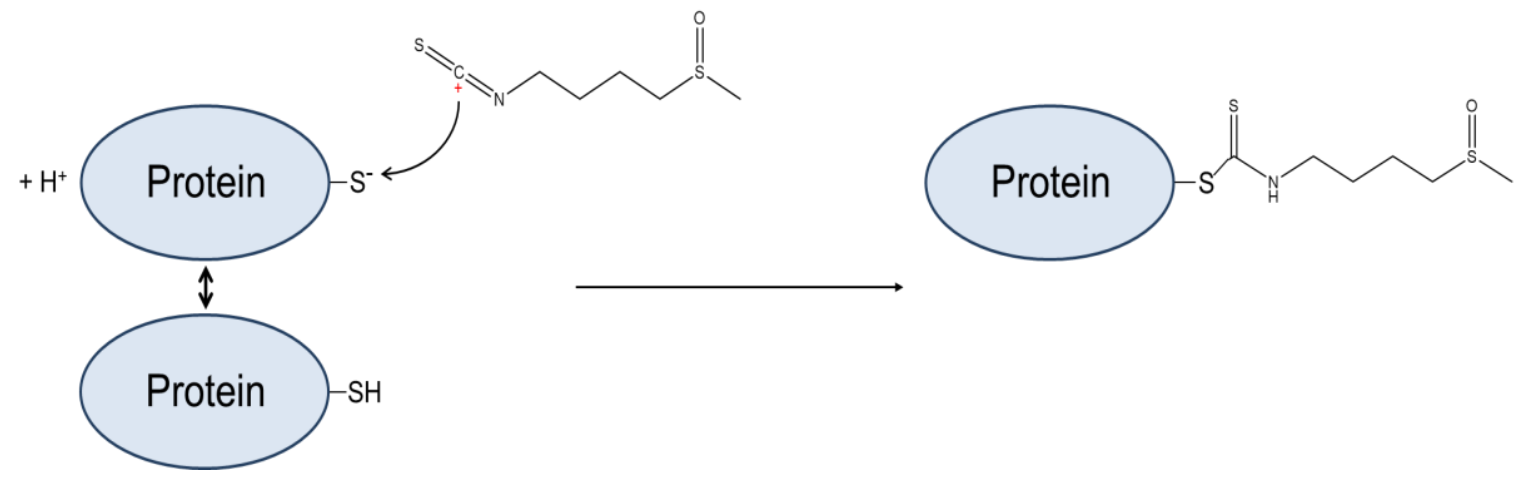

Figure 1.3. SFN can adduct protein cysteine thiolates.

Following electrophilic attack of the positively charged carbon within its ITC functional group, SFN can adduct nucleophilic protein thiolates.

\subsubsection{SFN from naturally derived sources}

Whilst this research project used chemically synthesised derivatives of SFN, SFX-01 or L-SFN, naturally, ITCs are derived from cruciferous vegetables, such as broccoli, cabbage, kale and Brussels sprouts ${ }^{16}$. ITCs themselves are not present within these plants but form following enzymatic cleavage of parent phytochemicals named glucosinolates. Chewing releases $\beta$-thioglucosidase myrosinase enzymes from extracellular vesicles, which are then free to hydrolyse the thioglucosidic bond of glucosinolates yielding glucose and thiohydroxamateO-sulfonate, an unstable aglycone ${ }^{17}$. The latter then undergoes spontaneous rearrangement into different bioactive molecules including ITCs, thiocyanates and nitriles (figure 1.4) ${ }^{17}$. 


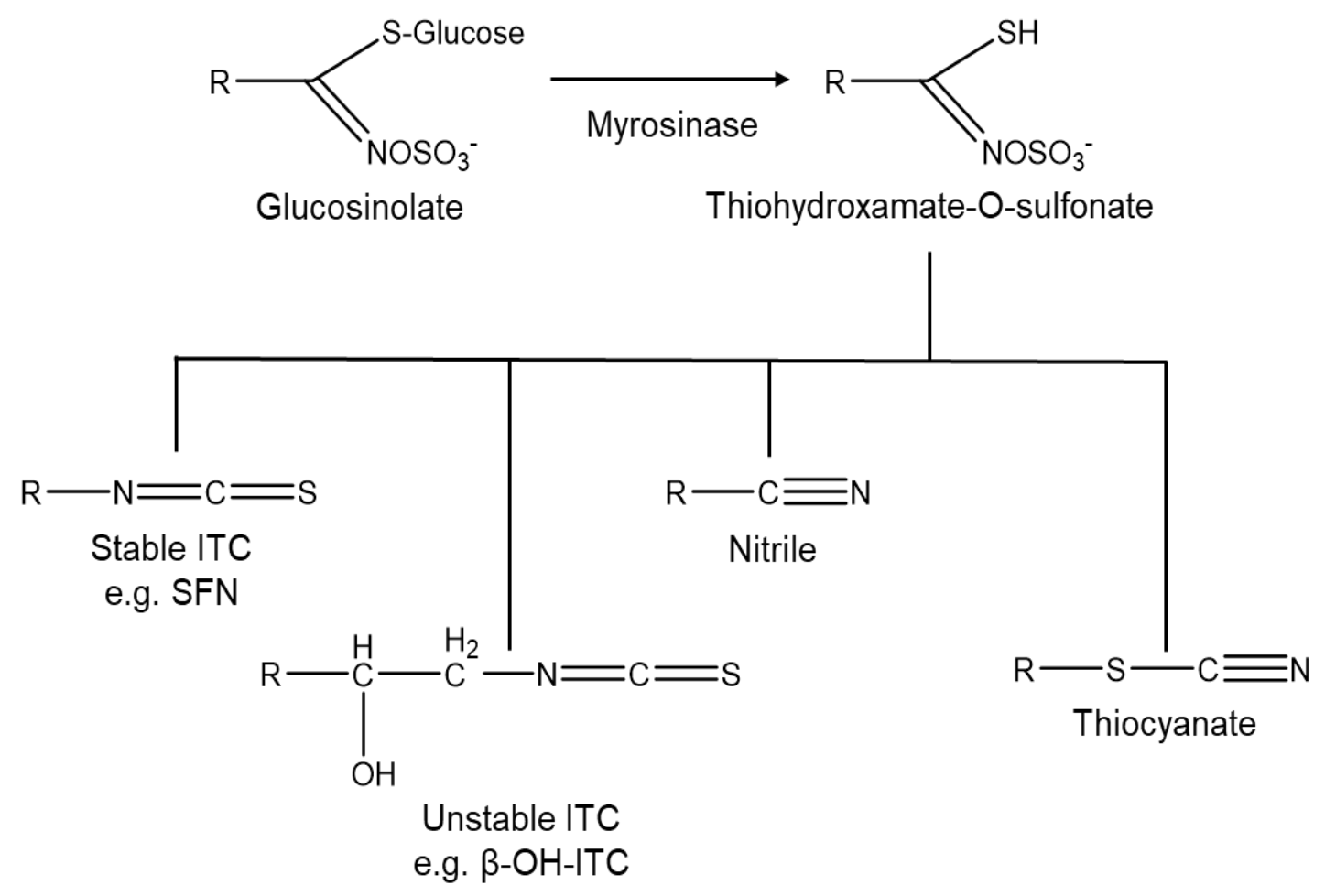

Figure 1.4. A diagram showing hydrolysis of glucosinolate and subsequent formation of bioactive compounds.

Hydrolysis of glucosinolate not only occurs in the mouth cavity but also by $\beta$ thioglucosidase found in gut microflora ${ }^{18}$. The abundance and occurrence of different glucosinolates vary between members of the cruciferous vegetable family and their hydrolysis products therefore also differ ${ }^{19}$. The predominant glucosinolate within broccoli is glucoraphanin whose hydrolysis produces SFN (figure 1.5) ${ }^{20}$. Naive broccoli sprouts are a particularly good source of glucoraphanin, found at concentrations 20-50-fold higher than the mature form of the plant ${ }^{21}$. 
<smiles>CS(=O)CCCC/C(=N/OS(=O)(=O)[O-])SC1OC(CO)C(O)C(O)C1O</smiles>

Glucoraphanin

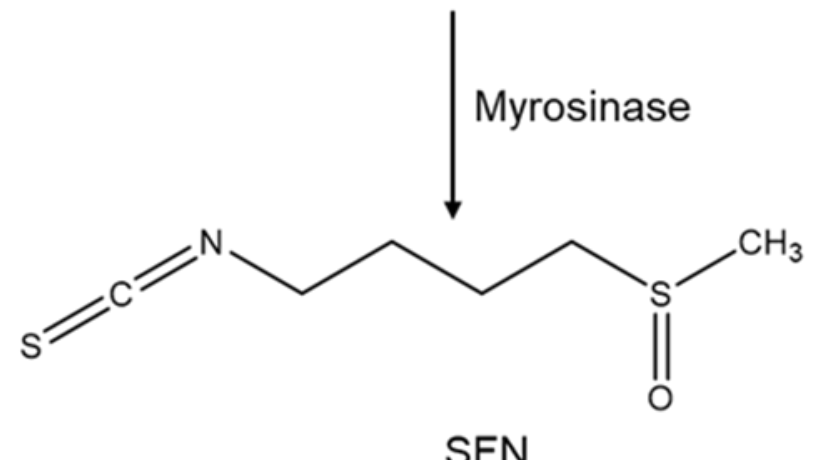

SFN

Figure 1.5. Generation of SFN by enzymatic hydrolysis of glucoraphanin.

\subsubsection{SFN metabolism}

The antioxidant tripeptide glutathione (GSH) can conjugate with free electrophiles such as SFN, with this adduction often the first stage of metabolism for such xenobiotic compounds ${ }^{22}$. Most ingested SFN passively enters small intestine epithelial cells where it is adducted by GSH, which is shown in figure 1.6. GSH-SFN then effluxes back into the lumen through the unidirectional efflux transporters multidrug resistance-associated protein 1 (MRP1) or P glycoprotein 1 (Pgp1). Both function as multispecific organic anion transporters and are expressed and distributed ubiquitously or in the intestinal epithelium respectively 23. A proportion of unconjugated SFN bypasses the tripeptide and passes into 
the systemic circulation, where it may become conjugated to GSH or alternatively passively enter tissue cells ${ }^{23}$. Once inside the cell, SFN can adduct nucleophilic moieties and regulate protein function, or again is liable to encounter and adduct with GSH that is abundant in the cytosol ${ }^{23}$. Conjugation of SFN to GSH may even occur following trans-thiolation from a cysteine-containing protein, a concept discussed in greater detail in chapter 4. GSH-SFN is transported from the cell into the systemic circulation where it is metabolised sequentially by $y$ glutamyl-transpeptidase (GTP), cysteinyl-glycinease (CGase) and Nacetyltransferase (NAT) to form SFN-cysteine-glycine and SFN-N-acetylcysteine respectively (SFN-NAC) as shown in figure 1.6, which undergo urinary excretion 24. 


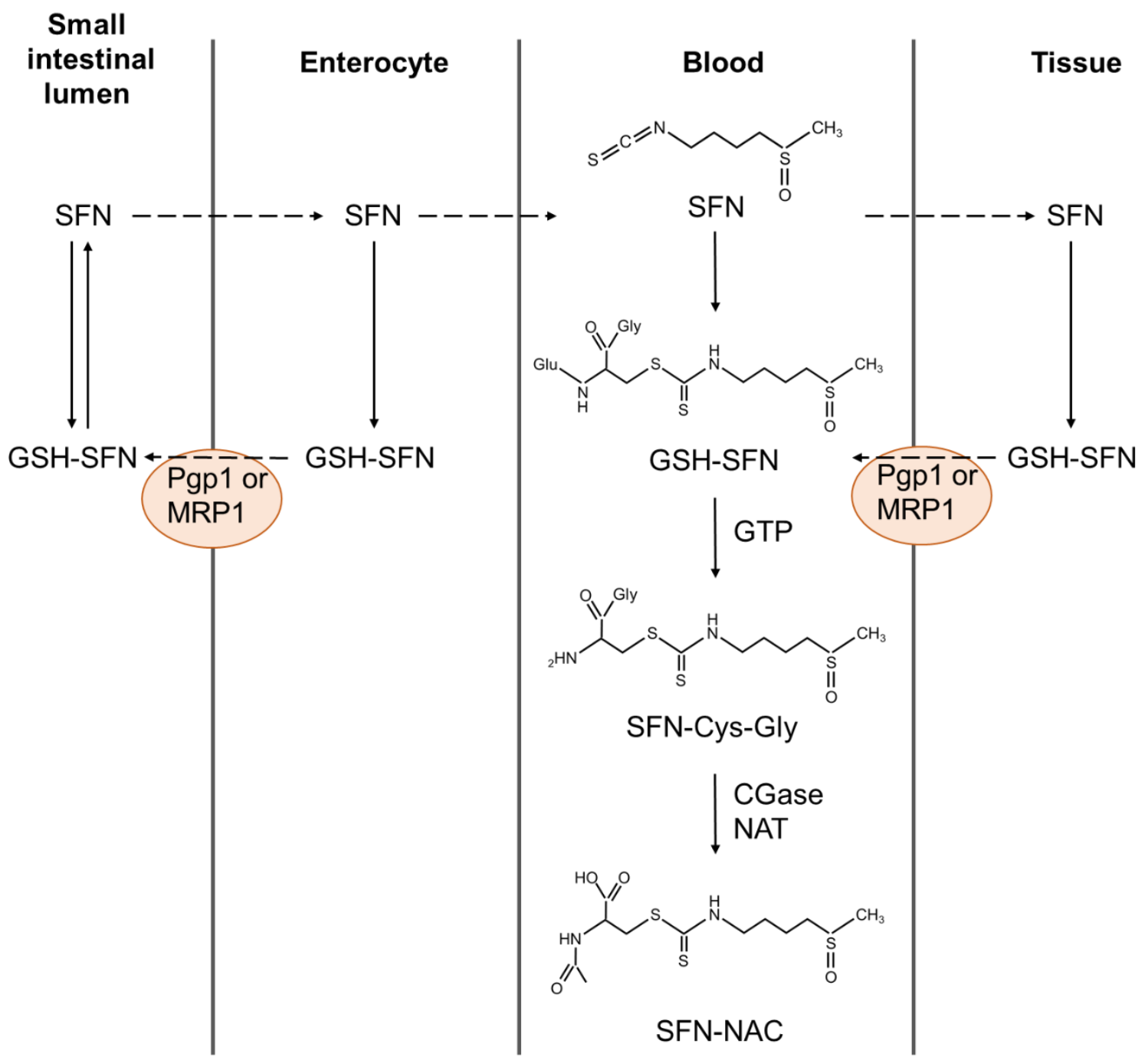

Figure 1.6. Pathway for SFN metabolism.

Following absorption from the small intestines into enterocytes, SFN may rapidly conjugate GSH which passes back to the small intestinal lumen through Pgp1 or MRP1 transporters. Alternatively, passive movement of free SFN into the blood may conjugate free GSH or continue into tissue cells. This simplified schematic shows conjugation to cellular GSH, which is discussed in greater detail in chapter 4, which is transported back to the blood through Pgp1 or MRP1 transporters. Sequential metabolism of GSH-SFN to SFN-cysteineglycine and SFN-NAC occurs enzymatically by GTP and CGase NAT respectively with SFN-NAC undergoing urinary excretion. 


\subsubsection{Modulation of signalling pathways by SFN}

This introduction has outlined that SFN can adduct nucleophilic cysteine thiols. This interaction may alter the biological function of a protein and potentially regulate downstream signalling pathways. The two most studied and characterised cellular events which can be altered by protein adduction of SFN are nuclear factor-kB (NF-kB) and KEAP1/nuclear factor erythroid 2-related factor 2 (Nrf2) signalling pathways which are detailed below.

\subsubsection{Modulation of the NF-KB signalling pathway by SFN}

$\mathrm{NF}-\mathrm{kB}$ is a transcription factor whose adduction by lipopolysaccharides during chronic inflammation causes translocation into the nucleus and upregulation of proinflammatory enzymes including inducible NO synthase (iNOS) and cyclooxygenase 2 (COX2). Subsequent elevation of cellular levels of NO and prostaglandins respectively can lead to DNA damage through nitrosative deamination of DNA bases, facilitating cancer initiation and progression ${ }^{25}$ and enhance proliferation and invasiveness of cancer cells as well as inhibit apoptosis 26.

SFN is a negative regulator of this signalling event by adducting cysteine thiols within NF-kB which impairs DNA binding of the transcription factor ${ }^{27}$. Further to this, SFN adducts Trx and GSH which are also required for NF-kB function ${ }^{27}$. Such anti-inflammatory effects of SFN can, therefore, prevent an inflammatory response from occurring which may prevent the progression of certain cancers.

\subsubsection{Modulation of the KEAP1/Nrf2 signalling pathway by SFN}

Under homeostatic conditions, KEAP1 forms a homo-dimer within the cytosol and is part of a protein complex with the E3 ubiquitin ligase RING-box protein 1 
(RBX1) as well as the RBX1 scaffold protein Cullin 3 (CUL3), as shown in figure 1.7. The function of this complex is to retain the transcription factor Nrf2 in the cytosol and target it for degradation, so acting as a negative regulator to prevent its translocation into the nucleus. Nrf2 initially interacts with KEAP1 at the proteins $\mathrm{N}$-terminal ETGE motifs ${ }^{28}$. Secondary interactions with a KEAP1 DLG motif position the transcription factor for ubiquitination by RBX1, targeting Nrf2 for proteasomal degradation. The reaction of an oxidant at one or multiple cysteines within KEAP1 induces structural changes with the resultant release of Nrf2 from the complex 2930 . The transcription factor then stabilises and accumulates within the cytosol with subsequent translocation to the nucleus. Nrf2 then binds the antioxidant response element (ARE) DNA-promoter binding region found in the 5 '-flanking region of phase 2 and antioxidant genes, upregulating multiple antioxidant enzymes which act to attenuate pathological damage caused by ROS, RNS and electrophiles as outlined in figure 1.7. Examples of these enzymes include the ubiquitous disulfide reductases Trx and GRX1 whose mechanisms of action are outlined in chapter 4. Gamma-glutamylcysteine synthetase (GCS) expression is also increased, an enzyme which catalyzes the rate-limiting step in GSH synthesis ${ }^{31}$. This results in increased production of GSH, a key player in GRX1-mediated disulfide reduction.

It is now well established SFN adducts KEAP1 at Cys ${ }^{151}$, and modification of this cysteine by oxidants is shown to induce the structural change which leads to the release of Nrf2 from the cytosolic KEAP1/RBX1/CUL3 complex ${ }^{32}$. Adduction of SFN at this cysteine induces the same structural modification (figure 1.7) and the electrophile is the most potent naturally occurring inducer of phase 2 enzymes ${ }^{33}$ 3435. 


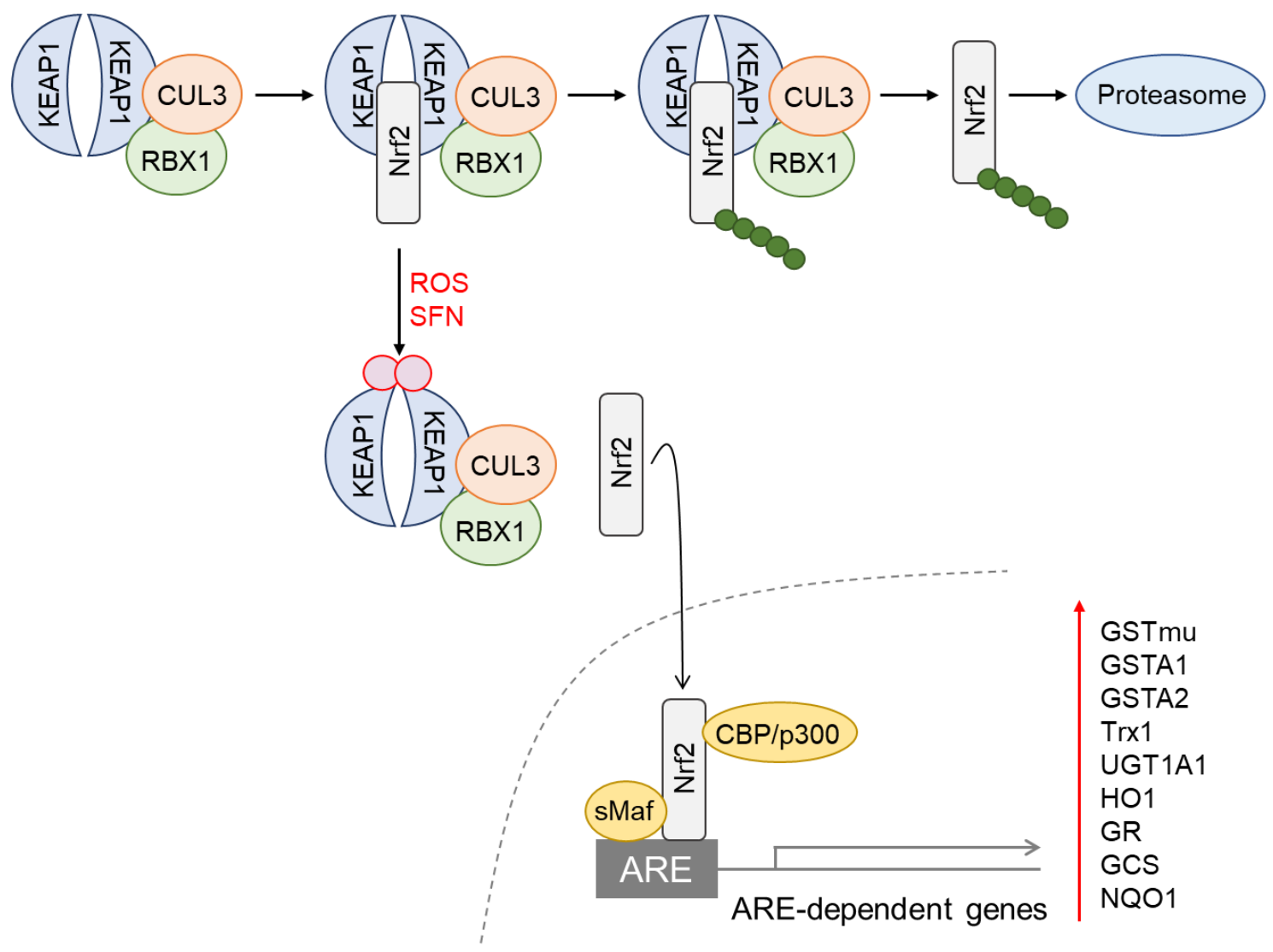

Figure 1.7. Modulation of antioxidant and xenobiotic detoxification enzymes by the KEAP1/Nrf2 signalling pathway.

Nrf2 is sequestered in the cytosol via interaction with a KEAP1/RBX1/CUL3 complex. Upon ubiquitination by RBX1, Nrf2 is degraded following targeting to proteasomes. Adduction of ROS or SFN to essential cysteines upon KEAP1 drive a conformational change allowing release of Nrf2. Following its translocation into the nucleus, the transcription factor adducts $\mathrm{ARE}$ along with small musculoaponeurotic fibrosarcoma (sMAF) and CREB-binding protein/p300 (CBP/p300). This drives upregulation of antioxidant and xenobiotic detoxification enzymes. GST = glutathione Stransferase. Trx1 = thioredoxin 1. UGT = UDP glucuronosyltransferase Family 1 Member A1. $\mathrm{HO} 1$ = heme oxygenase $1 . \mathrm{GR}=$ glucocorticoid receptor. $\mathrm{GCS}=$ glutamate-cysteine ligase. $\mathrm{NQO} 1=\mathrm{NAD}(\mathrm{P}) \mathrm{H}$ quinone dehydrogenase 1. 


\subsubsection{Protein targets of SFN}

As outlined above, the most studied protein-SFN interactions are its adduction to KEAP1 or NF-KB. However, a wide range of proteomic studies have identified hundreds of protein targets of SFN in a variety of cell lines exposed to this electrophile ${ }^{36-41}$. The reactive nature of SFN, together with is relatively simple structure, underlies a lack of selectively in the targets it interacts with and potentially covalently adducts to. Whilst Angeloni et al have documented cardiac protein targets of SFN following treatment of isolated neonatal Wistar rat cardiomyocytes in vitro ${ }^{42}$, in vivo targets after oral consumption of SFN are largely unknown.

This issue was addressed using quantitative mass spectrometry (MS) conducted by Dr Ewald Schroder from our research group. This proteomic analysis was carried out following immunoprecipitation of SFN-adducted proteins from cardiac tissue of mice using a polyclonal rabbit antibody developed in our laboratory which detects SFN adducted to protein cysteines. This methodology, therefore, allowed identification of proteins containing a reactive cysteine which may be altered or functionally important in adaptive mechanisms that occur during pathological conditions, whereby adduction by SFN regulates their activity. The analysis of biological replicates of wildtype (WT) mice orally gavaged with SFN, which were statistically compared to untreated controls, showed 40 significant cardiac protein targets of the electrophile under the conditions tested, which are shown in table 1.1. Notably, a high proportion of the targets identified were mitochondrial proteins. If SFN were to adduct and inhibit these proteins, perhaps the transport of pyruvate into the mitochondria or its subsequent conversion inside the organelle may be altered. Investigated changes in metabolites and 
ROS production following treatment with SFN may therefore warrant further investigation.

Nevertheless, another significant protein target of SFN in cardiac tissue was Ptpn11, commonly named SHP2, which is highlighted in red in table 1.1. The interaction between SFN and SHP2 has been the main focus of this research project. 


\begin{tabular}{|c|c|}
\hline Protein name & Abbreviation \\
\hline 2-oxoglutarate dehydrogenase, mitochondrial & ODO1_MOUSE \\
\hline 3-ketoacyl-CoA thiolase, mitochondrial & THIM_MOUSE \\
\hline Acetyl-CoA acetyltransferase, mitochondrial & THIL_MOUSE \\
\hline ADP/ ATP translocase 2 & ADT2_MOUSE \\
\hline ATP synthase subunit alpha, mitochondrial & ATPA_MOUSE \\
\hline ATP synthase subunit gamma, mitochondrial & ATPG_MOUSE \\
\hline Carnitine O-palmitoyltransferase 1 , muscle isoform & CPT1B_MOUSE \\
\hline Cytochrome b-c1 complex subunit 1 , mitochondrial & QCR1_MOUSE \\
\hline Cytochrome b-c1 complex subunit 2, mitochondrial & QCR2_MOUSE \\
\hline Dihydrolipoyl dehydrogenase, mitochondrial & DLDH_MOUSE \\
\hline E3 ubiquitin-protein ligase & NEDD4_MOUSE \\
\hline Electron transfer flavoprotein oxidoreductase, mitochondrial & ETFD_MOUSE \\
\hline Electron transfer flavoprotein subunit alpha, mitochondrial & ETFA_MOUSE \\
\hline Elongation factor TU, mitochondrial & EFTU_MOUSE \\
\hline Enoyl-CoA delta isomerase 1 , mitochondrial & ECl1_MOUSE \\
\hline Fructose-bisphosphate aldolase A & ALDŌA_MOUSE \\
\hline Fumarate hydratase, mitochondrial & FUMH MOUSE \\
\hline Isocitrate dehydrogenase (NADP), mitochondrial & IDHP_MOUSE \\
\hline Isovaleryl CoA dehydrogenase, mitochondrial & IVD MOUSE \\
\hline LIM domain-binding protein 3 & LDB3_MOUSE \\
\hline L-lactate dehydrogenase $\mathrm{B}$ chain & LDHB_MOUSE \\
\hline Malate dehydrogenase, mitochondrial & MDHM_MOUSE \\
\hline Medium-chain specific acyl-CoA dehydrogenase, mitochondrial & ACADM_MOUSE \\
\hline Myosin-6 & MYH6_MOUSE \\
\hline Myosin-7 & MYH7 MOUSE \\
\hline Myosin-8 & MYH8 MOUSE \\
\hline Myosin-binding protein C, cardiac-type & MYPC3_MOUSE \\
\hline $\begin{array}{l}\mathrm{NADH} \text {-ubiquinone oxidoreductase } 75 \mathrm{kDa} \text { subunit, } \\
\text { mitochondrial }\end{array}$ & NDUS1_MOUSE \\
\hline Perilipin-4 & PLIN4_MOUSE \\
\hline Phosphate carrier protein, mitochondrial & MPCP MOUSE \\
\hline Phosphoglycerate kinase 1 & PGK1_MOUSE \\
\hline Protein-tyrosine phosphatase non-receptor type 11 & PTPN11_MOUSE \\
\hline $\begin{array}{l}\text { Pyruvate dehydrogenase E1 component subunit alpha, somatic } \\
\text { form, mitochondrial }\end{array}$ & ODPA_MOUSE \\
\hline Pyruvate kinase PKM & KPYM_MOUSE \\
\hline Sarcoplasmic/endoplasmic reticulum calcium ATPase 2 & AT2A2_MOUSE \\
\hline Short-chain specific acyl-CoA dehydrogenase, mitochondrial & ACADS_MOUSE \\
\hline Succinate dehydrogenase flavoprotein subunit, mitochondrial & DHSA_MOUSE \\
\hline Trifunctional enzyme subunit alpha, mitochondrial & ECHA_MOUSE \\
\hline Trifunctional enzyme subunit beta, mitochondrial & ECHB MOUSE \\
\hline Voltage-dependent anion-selective channel protein 2 & VDAC2_MOUSE \\
\hline
\end{tabular}

Table 1.1. Protein targets of SFN in cardiac tissue of WT mice identified by quantitative mass spectrometry following oral gavage with SFN.

These studies were conducted by Dr Ewald Schroder at King's College London. 


\subsection{An introduction to SHP2}

SHP2 is a ubiquitously expressed non-receptor protein-tyrosine phosphatase. Like oxidative modifications, phosphorylation of proteins can influence their activity, complex association and/or localisation and protein phosphorylation status is orchestrated by the balanced actions of kinases and phosphatases. To analyse the chemical adduction of SFN to SHP2 and understand the biological consequences of this interaction it is important to consider how the phosphatase is regulated as well as the signalling events that this protein controls.

\subsubsection{SHP2 structure and regulation}

SHP2 is a $68 \mathrm{kDa}$ protein with two tandem SH2 domains, a central PTP catalytic domain and a C-terminal domain containing two tyrosine phosphorylation sites at residues 542 and 580, as well as a proline-rich motif. Biochemical analysis and crystallographic studies showed when SHP2 is in an inactive state the backside loops of the N-terminal SH2 domains of SHP2 fold across its PTP catalytic site where it is maintained by $\mathrm{H}$ bonding as shown in figure $1.8{ }^{43}$. The phosphatase domain is therefore physically inaccessible and SHP2 is biologically inactive due to phosphorylated substrates having limited access to the catalytic site. Binding of these $\mathrm{N}$-terminal $\mathrm{SH} 2$ domains to phospho-tyrosine residues upon receptor tyrosine kinases (RTKs) (e.g. growth factor receptor (GFR) and ephrin receptors (EphR)), cytokine receptors or scaffold proteins (e.g. insulin receptor substrate 1 (IRS1), focal adhesion kinase (FAK), GFR-bound protein 2-associated-binding protein 1 (GAB1)) induces a conformational change that relieves auto-inhibition of the catalytic domain as depicted in figure $1.8{ }^{44} 45$. The same can be achieved following docking of the $\mathrm{N}$-terminal $\mathrm{SH} 2$ domains to phospho-tyrosine residues 542 and 580 in its own C-terminal domain following phosphorylation by cytosolic 
kinases protein kinase $\mathrm{C}(\mathrm{PKC})$ or zeta chain of $\mathrm{T}$-cell receptor-associated protein kinase 70 (ZAP70), or by membrane-bound RTKs such as EphA2, for example 464748 . This unfolding relieves autoinhibition and allows access of phosphorylated substrates to the active domain containing a catalytic cysteine, Cys ${ }^{459}$, which is fundamental to the phosphatase activity of SHP2. Incorporated within Cys $^{459}$ is a low $\mathrm{p} K_{\mathrm{a}}$ thiol which means that at physiological $\mathrm{pH}$ it predominantly exists as an ionised thiolate, which enables nucleophilic attack of the sulfur towards a phosphate group, which is crucial for dephosphorylation of target proteins. As well as structural inhibition by $\mathrm{N}$-terminal $\mathrm{SH} 2$ domains, further modulation of the phosphatase's activity can occur via oxidative modification of Cys ${ }^{459}$. The catalytic domain of the phosphatase and mechanism of substrate dephosphorylation by SHP2 are reviewed in detail in chapter 6 . 


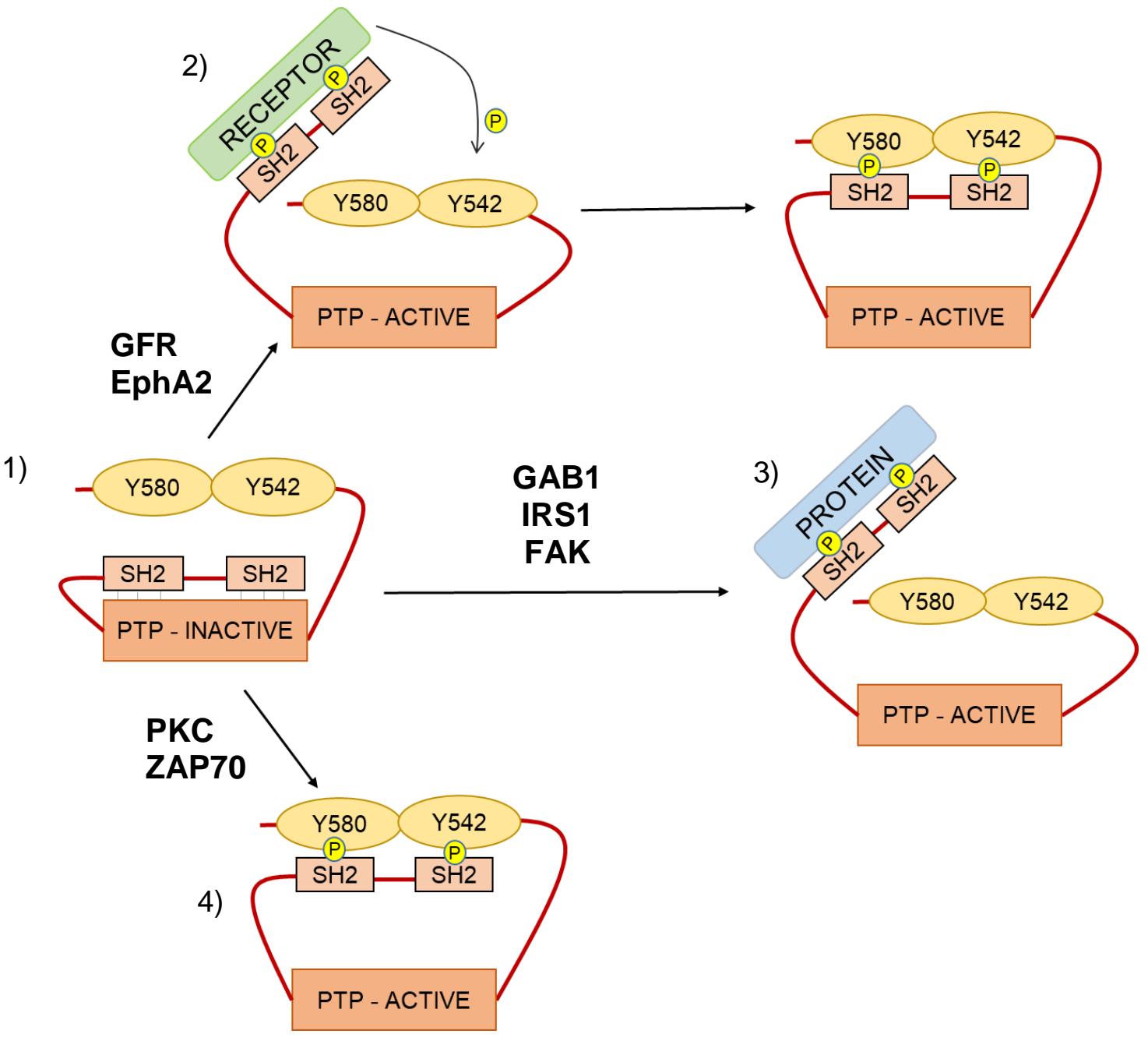

Figure 1.8. SHP2 structure and mechanism of activation by docking phosphotyrosine residues.

In the inactive state, N-terminal SH2 domains of SHP2 sterically hinder access of phospho-tyrosine substrates to the catalytic domain and blocks phosphatase activity (1). Binding of N-terminal $\mathrm{SH} 2$ domains by specific phospho-tyrosine-containing motifs releases this inhibition. These phospho-tyrosine residues may be an activated receptor (2), a protein which forms part of a scaffold complex (3) or tyrosine-phosphorylated Cterminal tail of SHP2 (4).

\subsubsection{Cell signalling pathways regulated by SHP2}

The dephosphorylation of RTKs, cytokine receptors, associated scaffold proteins and other cytosolic substrates by SHP2 regulates a wide variety of downstream signalling events which control cell proliferation, differentiation, migration, cell 
cycle progression and apoptosis. Regulation of such signalling pathways often has significant biological consequences including in embryonic development and white blood cell production, which have been a focus of these studies. Ras/ERK and janus kinase (JAK)/signal transducer and activator of transcription (STAT) are the most studied signalling pathways regulated by SHP2 and are outlined in detail in chapter 3. Nevertheless, a simple overview of SHP2's involvement in other key signalling events illustrates its importance in multiple biological functions.

\subsubsection{Regulation of integrin signalling by SHP2}

Heterodimerization of $\alpha$ and $\beta$ integrin receptor subunits occurs following binding of their extracellular domains to extracellular-matrix proteins. This dimerization increases the affinity of the receptors intracellular domains towards ligands including protein-tyrosine kinases focal adhesion kinase (FAK) and Src family kinase (SFK) ${ }^{49}$. FAK firstly docks to the cytoplasmic tail of the integrin $\beta$ subunit along with focal adhesion proteins talin and paxillin. Upon activation, FAK autophosphorylates itself, creating a docking site for SFK, which in turn becomes phosphorylated by FAK. Phospho-tyrosines within SFK provide docking sites for the N-terminal SH2 domains of SHP2, as shown in figure 1.9. Upon binding, SHP2 positively regulates integrin signalling by dephosphorylating inhibitory phospho-tyrosines within FAK, stimulating downstream events which are essential to embryonic development, tissue maintenance and repair, host defence and haemostasis ${ }^{50}$. Downstream, SHP2 also dephosphorylates p190 Ras homolog gene family member A (RhoA) guanine triphosphate (GTP)aseactivating protein (p190RhoAGAP). This facilitates the exchange of RhoA 
guanine diphosphate (GDP) for GTP, activating Rho-associated protein kinase (ROCK) to further stimulate cell proliferation and migration ${ }^{51}$.

\subsubsection{Regulation of insulin signalling by SHP2}

Binding of insulin to extracellular a subunits of insulin homodimer receptors induces a conformational change which facilitates transphosphorylation of tyrosine residues within intracellular $\beta$ subunits ${ }^{52}$. Pleckstrin homology $(\mathrm{PH})$ domains of insulin receptor substrate 1 (IRS1) subsequently dock these phospho-tyrosine residues and the substrate itself becomes phosphorylated by the activated receptor. This allows association of IRS1 with the regulatory subunit of phosphoinositide 3-kinase (PI3K), p85, through the substrates $\mathrm{SH} 2$ domains which brings the kinase in proximity to the cell membrane as shown in figure 1.9. The catalytic subunit of PI3K, p110, then phosphorylates phosphatidylinositol4,5-bisphosphate $\left(\mathrm{PIP}_{2}\right)$ leading to the formation of $\mathrm{PIP}_{3}$. A key downstream effector, Akt (otherwise known as protein kinase B) is recruited and phosphorylated by PI3K-dependent protein kinase 1 (PDK1), which stimulates downstream glycogen synthesis, promoting cell survival and growth ${ }^{53}$. As well as binding p85, phospho-tyrosine residues of IRS1 also bind N-terminal SH2 domains of SHP2, and the phosphatase is a negative regulator of insulin signalling by dephosphorylating this substrate 5455 . 


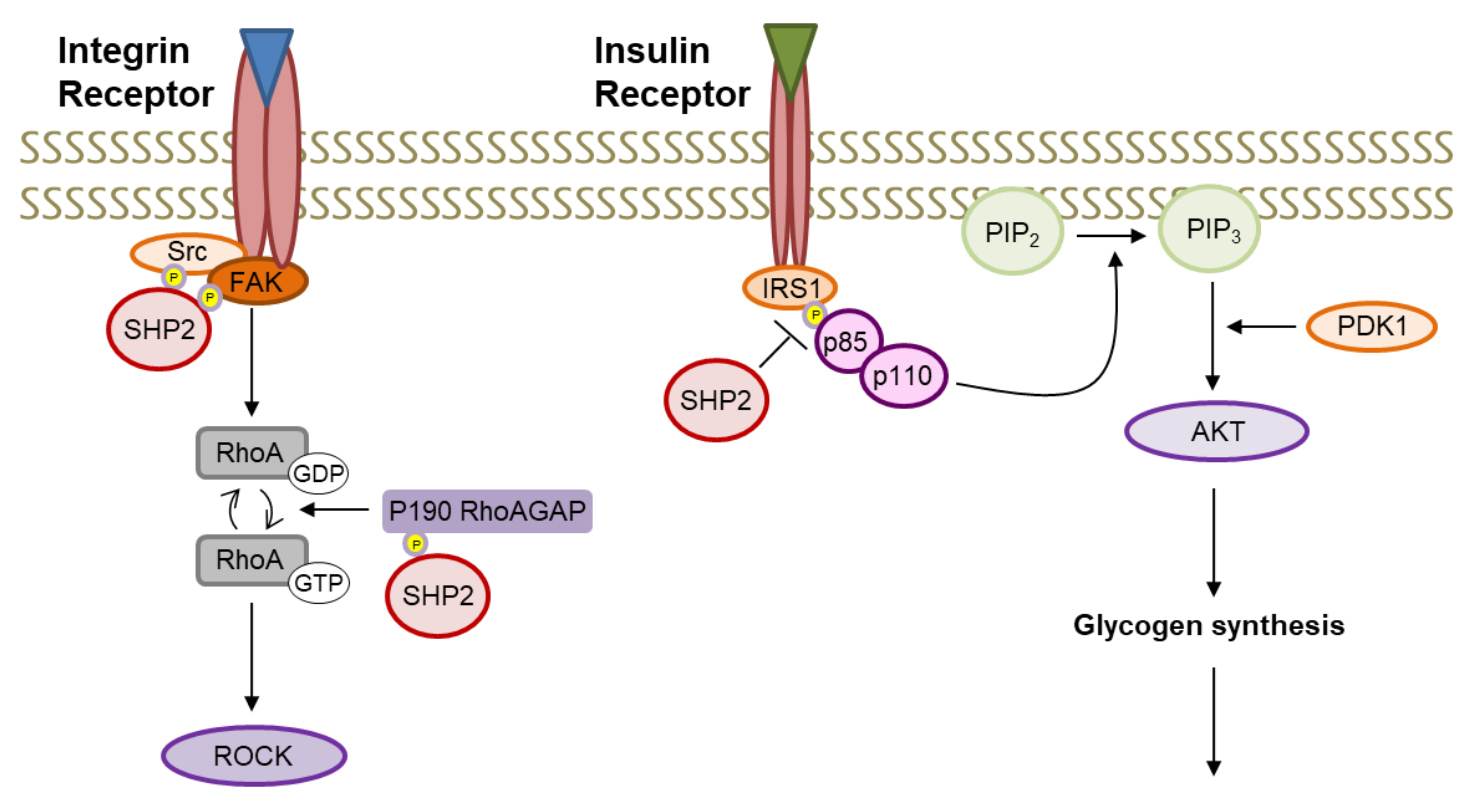

Proliferation, migration

Survival, growth

\section{Figure 1.9. Regulation of integrin and insulin signalling by SHP2.}

Following activation of integrin receptors, SHP2 dephosphorylates inhibitory phosphotyrosines of FAK and p190RhoAGAP, leading to activation of RhoA/ROCK signalling and promoting cell proliferation and migration (left pathway). Dephosphorylation of IRS by SHP2 negatively regulates insulin signalling following insulin receptor activation, providing a negative feedback mechanism for PI3K/Akt signalling and controlling cell survival and growth (right pathway).

\subsection{Dysregulation of SHP2 in pathologies}

As SHP2 is a regulator of multiple signalling events which modulate a number of cellular processes, it is unsurprising that dysregulation of its phosphatase activity due to somatic or germline mutations can initiate and/or maintain a variety of pathogenic outcomes.

SHP2 acts upstream as well as downstream of different proto-oncogenes. A detailed review of how the phosphatase functions in certain cancer cell lines is not in the scope of this thesis, but an essential and relevant message in the 
context of this project is that somatic mutations of Ptpn11, the gene encoding SHP2, drive initiation and progression of hematologic malignancies. These include juvenile myelomonocytic leukaemia (JMML) and solid tumours associated with breast, colon, lung, brain and thyroid cancer, among others 5657 58 59. Germline mutations in Ptpn11 have also been identified in two multisymptomatic developmental disorders named NS, which is the main focus of this thesis, and NS with multiple lentigines (formerly termed LEOPARD syndrome). Both are characterised by multiple, almost identical, congenital defects. However, remarkably $50 \%$ of NS patients are genetically characterised by gain-of-function mutations of SHP2 6061 , whilst NS with multiple lentigines is commonly driven by loss-of-function 6263 , highlighting the complexity that results from dysregulation of this phosphatase.

\subsubsection{Noonan syndrome}

NS is an autosomal dominant and genetically heterogeneous condition with an estimated prevalence of 1 in 1000-2500 live births. The clinical diagnosis of NS is established on the basis of distinctive features, including facial dysmorphism (high forehead, hypertelorism, down-slanting palpebral fissures, epicanthal folds, ptosis, low-set ears), reduced growth, spinal abnormalities, intellectual impairment, feeding difficulties in infancy, bleeding tendencies and hearing loss 6465 . Other common phenotypic characteristics include a broad or webbed neck, unusual chest shape with increased coagulation defects, lymphatic dysplasia, cryptorchidism and ocular abnormalities. With the exception of Down's syndrome, NS is the most common syndromic cause of congenital heart defects, occurring in around $50-80 \%$ of patients 66 67. Pulmonary valve stenosis, often with dysplasia, is the most common cardiac manifestation, resulting from 
dysplastic valve leaflets. Other cardiac structural deformities include atrial and ventricular septal defects, mitral valve stenosis, branch pulmonary artery stenosis and hypertrophic cardiomyopathy. Infants with NS are also predisposed to developing myeloproliferative disease, which may regress without the need for treatment, but has been shown to escalate to JMML in a cohort of these patients 6869

In $\sim 50 \%$ of NS patients, these manifestations occur due to gain-of-function heterozygous germline mutations in Ptpn $11^{6061}$. The remaining $50 \%$ of patients have mutations in one of the 6 following genes; Son of Sevenless 1 (SOS1), Rapidly accelerated fibrosarcoma 1 (Raf1), BRaf, KRas, NRas or mitogenactivated protein kinase kinase 1 (MAP2K1) 7071 . All 7 genes encode components or regulators of the Ras/mitogen-activated protein kinase (MAPK) pathway and therefore places NS in a group of developmental disorders termed RASopathies.

\subsubsection{Ptpn11 mutations associated with NS}

Currently, 12 germline, missense mutations in Ptpn11 have been identified in NS patients. Energetic-based structural and biochemical analysis has identified that all of these are gain-of-function alterations, causing hyperactivation of SHP2. As shown in figure 1.10 the majority of these mutations occur in or around regions of SHP2 that form interactions between the proteins N-SH2 and PTP domains ${ }^{72}$ 7374 . As discussed previously, interactions between these two domains form an autoinhibitory mechanism, preventing access of phosphorylated proteins to the catalytic cysteine of the phosphatase. Mutations in these areas, therefore, disrupt the intermolecular interactions, relieving the autoinhibited conformation which results in constitutive SHP2 activation and enhanced downstream signalling. In 
rare cases, mutations occur within the $\mathrm{C}-\mathrm{SH} 2$ domain or within the peptide linking the $\mathrm{N}-\mathrm{SH} 2$ and $\mathrm{C}-\mathrm{SH} 2$ domains, although with the same effect of relieving the autoinhibited structure conformation of the protein ${ }^{75}$. In contrast, SHP2 mutations identified in NS with multiple lentigines typically only effect residues within the PTP domain of the protein, resulting in decreased catalytic activity, highlighting that positioning of the mutation within the Ptpn11 gene drives the subsequent effect on SHP2 activity ${ }^{76}$.

SHP2 is a regulator of multiple stages of white blood cell production as well as embryonic development, including of the heart, which I outline in detail in the introduction of chapter 5 . With this in mind, it is perhaps unsurprising that 10 of the 12 missense, gain-of-function mutations in Ptpn11 identified in NS patients are correlated with one or multiple congenital heart and skeletal defects as well as a myeloproliferative phenotype.

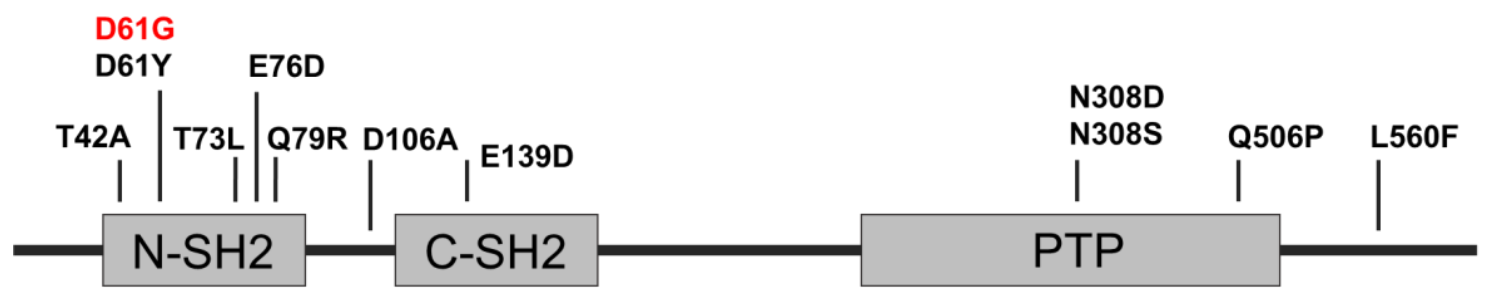

\section{Figure 1.10. Location of 12 known NS-associated SHP2 mutations.}

The majority of germline, missense mutations identified in NS patients occur in or around the N- or C-SH2 domains of SHP2, preventing autoinhibitory interactions with the PTP domain of the phosphatase. The mutation highlighted in red, D61G, causes the most hyperactive form of SHP2. 


\subsubsection{NS mouse model with a Ptpn11 ${ }^{\mathrm{D61G} /+}$ mutation}

The D61 residue within the N-SH2 domain of SHP2, which is highlighted in red in figure 1.10, participates in an $\mathrm{H}$ bond network between the $\mathrm{N}-\mathrm{SH} 2$ domain and water molecules within the PTP domain of the phosphatase, forming a major component in maintaining the proteins autoinhibited conformation. Replacement of this negatively charged aspartic acid for an uncharged glycine residue (D61G) is a commonly occurring NS-associated mutation. This charge change prevents the formation of the inhibitory N-SH2/PTP domain interface. This opens up the structure of the protein, allowing access of phosphorylated substrates to the catalytic cysteine of SHP2 and increases the basal activity of the phosphatase 77. Using a gene-targeting vector, the Neel group generated a heterozygous knock-in Ptpn11 ${ }^{\text {D61G/+ }}$ mouse model with a C57BL/6J/129S4/SvEv hybrid genetic background ${ }^{78}$. To do so a D61G mutation was introduced into J1 embryonic stem cells of $129 S 4 / S v E v$ background. Correctly mutated embryonic stem cells were transfected with a plasmid expressing Cre recombinase to excise the neo cassette and two properly excised clones were injected into C57BL/6J blastocysts. Characterisation showed these mice exhibit many of the phenotypes that characterise NS patients such as short stature, facial dysmorphia, severe cardiac defects, myeloproliferative disease and splenomegaly.

As with human NS patients who have a Ptpn11 mutation, not all offspring of the $\mathrm{D} 61 \mathrm{G} /+$ mouse model exhibit the same phenotypes. Phenotypes associated with NS are therefore defined as variably penetrant i.e. they may be present in some patients and not others. Further to this, two mice who have the same phenotype may present them with different severity. Whilst haematopoietic, craniofacial and growth defects are completely penetrant, occurring in all heterozygous mice, the 
severity of these phenotypes differ between mice. On the other hand, cardiac defects are incompletely penetrant, with $50 \%$ of heterozygous embryos dying prematurely with a spectrum of cardiac defects, whilst the rest exhibit mild valvular hyperplasia during embryogenesis which resolves before birth. As homozygous Ptpn11 $11^{\mathrm{D} 61 \mathrm{G}+++}$ is embryonic lethal it is evident gene dosage and expressivity of the mutant correlate with the severity of the defects. Cell- and pathway-specific differences in sensitivity to D61G also likely account for exacerbation of some phenotypes over others. The Neel group conclude incomplete penetrance is reflected by the amount of strain-specific genetic modifiers present within each mouse which is reflected by their $129 \mathrm{~S} 4 / \mathrm{SvEv}$ and/or C57BL/6J background, and therefore the degree to which they affect certain modifier genes ${ }^{73}$. However genomic scans using single nucleotide polymorphism panels have failed to identify strong modifier loci ${ }^{73}$.

Regardless of severity and penetrance, this single gain-of-function mutation of D61 to G61 results in the greatest activation of SHP2 associated with NS and evokes all major phenotypes seen in human patients. The Ptpn11 $11^{\mathrm{D} 61 \mathrm{G} /+}$ mouse is, therefore, an optimal animal model for delineating the potential therapeutic benefit of SHP2 inhibitors.

\subsection{SHP2 inhibitors}

As hyperactivation of SHP2 is causative of clinical manifestations present in 50 $\%$ of NS patients ${ }^{6061}$ as well as the initiation and progression of many cancers 56575859 , it is rational to assume that inhibition of the phosphatase's activity would be beneficial in these pathologies. The identification of inhibitors of its phosphatase activity has therefore been a major research goal. However, structural homology between many PTPs, as well as the charged active site of 
the phosphatase have made the development of an inhibitor selective of SHP2 challenging.

Studies have identified compounds which are potent inhibitors of SHP2 phosphatase activity in cultured cell lines as well as recombinant protein. For example, the commercially available phenylhydrazonopyrazolone sulfonate (PHPS1) is a phospho-tyrosine mimetic with a half maximal inhibitory concentration $\left(\mathrm{IC}_{50}\right)$ value for SHP2 of $2.1 \mu \mathrm{M}$. At these low concentrations, PHPS1 is selective for SHP2 in cultured epithelial cells, however, adducts other PTPs at higher concentrations including SHP1 and PTP1B ${ }^{79}$. The compound has low cell-membrane permeability which reduces the bioavailability of the drug in vivo. The Zhang group demonstrated naphthyl and polyaromatic salicylic acidbased compounds exhibit enhanced affinity for PTPs ${ }^{80} 81$. Using a library of salicylic acid-based compounds, they identified II-BO8 as an inhibitor of SHP2 which has an $\mathrm{IC}_{50}$ towards the phosphatase that is at least 3 times lower than for 11 other PTPs that were tested ${ }^{82}$. Even so, multiple other protein targets of the compound have been identified and the biological consequences of these interactions are currently unknown. Through virtual screening and experimental assays Zhang et al identified the natural compound cryptotanshinone as an inhibitor of SHP2; an active ingredient of the herbal plant Salvia miltiorrhiza, commonly used in Asian countries to treat coronary heart disease, acute ischemic stroke and cancer ${ }^{83}$. However, again, lack of selectivity of cryptotanshinone for SHP2 was a concern and further studies utilising it as a treatment are yet to emerge.

Structural studies showed all three inhibitors dock at the periphery of the protein's catalytic domain via polar and non-polar interactions. Such interactions either 
facilitate the closed autoinhibitory conformation of SHP2 or prevent electrostatic interactions otherwise essential to the catalytic activity of the phosphatase. Notably, they do not cause inhibition of SHP2 by adduction to the protein's catalytic cysteine.

\subsubsection{Sulforaphane as an inhibitor of SHP2}

The catalytic cysteine of SHP2, Cys ${ }^{459}$, facilitates successful dephosphorylation of target substrates by nucleophilic attack towards the phosphorus ion. The phosphatase activity of SHP2 can be inhibited by oxidative modification of the negatively charged thiolate group of this cysteine. As SFN adducts nucleophilic moieties such as thiolates, it was anticipated this catalytic cysteine was where the electrophile was adducting SHP2. If so, adduction of SFN may inhibit the phosphatases activity. Indeed, preliminary in vitro studies carried out by Lewis et al demonstrated dietary ITCs such as SFN inhibit the phosphatase activity of recombinant SHP2.

Due to the covalent nature of the interaction between SFN and target proteins, adduction of the electrophile is often perceived as permanent ${ }^{84}$. The permanent adduction of chemical compounds has been shown to induce haptenization and drive an immune response towards proteins which are now rendered as foreign 85. Drugs which form noncovalent electrostatic interactions such as $\mathrm{H}$ bonds, ionic bonds, van der Waals forces or hydrophobic bonds can overcome haptenization as they allow for transient association and disassociation with target proteins, which generates an equilibrium between a protein-bound and -unbound state. However, this also leads to reduced potency of the drug due to the discontinuous effect on protein function. Continual disassociation of the drug also increases the 
time in which is it vulnerable to metabolism and excretion 8687 . Typically, higher drug amount and more frequent dosage are therefore needed.

The high electrophilicity of SFN together with is relatively simple structure underlies a lack of selectively in the targets it interacts with and potentially covalently adducts to. Such electrophilic compounds are therefore typically unfavoured due to unknown and perhaps undesired biological effects that occur as a result of adduction to off-target proteins. However, off-target effects are not always a concern. For example, the thiol-reactive reagent ethacrynic acid inhibits protein phosphatase $2 \mathrm{a}$ and lymphoid tyrosine phosphatase achieved through chemical modification of their catalytic cysteines, with its adduction to additional off-target proteins having no undesired biological consequences 888990 . Indeed, published study results from human clinal trials using SFN have shown no severe side effects and minimal non-severe effects following chronic long-term use 9192 939495 .

The increased biochemical efficiency of compounds which form covalent interactions is beneficial in terms of therapeutic margins, as low amounts of the drug are required along with fewer doses, and engineering drugs which encompass covalent properties, as well as reversibility, are now considered by some to be highly desirable. Such drugs are currently being achieved through incorporation of a nitrile group into electron-deficient alkenes ${ }^{96} 97$. This allows for initial covalent interaction but also increases the acidity of the adduct, favouring the reverse reaction. Incorporation of this electrophile into a noncovalent proteinrecognition scaffold further aids the dissociation of the drug ${ }^{96}$. The covalent interaction of SFN with protein thiols is historically perceived as permanent ${ }^{98}$, however, recently published observations show thiol-SFN adducts are in fact 
reversible, with transfer between thiol groups being possible ${ }^{99}$. Although such trans-thiolation of SFN may lower protein selectivity, therapeutic desirability is increased by the covalent but reversible nature of the interaction.

The development of SFN as a drug has been perturbed by its unstable nature, requiring the electrophile to be stored in a frozen buffer containing dimethyl sulfoxide (DMSO) 100101 102. To overcome this, Evgen Pharmaceuticals developed Sulforadex (SFX-01), a chemically stabilised powder whereby SFN is encapsulated in an a-cyclodextrin complex ${ }^{103}$. This encapsulation prevents thermal degradation of SFN above $-20{ }^{\circ} \mathrm{C}$, allowing storage of the drug at room temperature. Therapeutic potential of SFX-01 is currently being trialled in patients with prostate and breast cancer which to our knowledge also has little if any, toxicity concerns at the therapeutic concentrations used ${ }^{104}{ }^{105}$. The drug has been kindly donated for use throughout this research project.

\subsection{Research aims}

The data presented herein has overall furthered our understanding of SFNmediated inhibition of SHP2 and the benefits this may have in a scenario where hyperactivation of the phosphatase is pathological, such as NS. This thesis has four chapters of results from experiments carried out with the following research aims:

- Chapter 3: determine if SFN inhibits SHP2 phosphatase activity in vitro as well as in WT mice and the Ptpn11 $11^{\mathrm{D} 1 \mathrm{G} /+} \mathrm{NS}$ mouse model.

- Chapter 4: identify protein targets of SFN in cardiac tissue of mice following chronic treatment with SFX-01 and secondly explore if the electrophile can trans-thiolate between two thiol-containing proteins. 
- Chapter 5: determine the effects of SFN on embryonic development and the progression of myeloproliferative disease in the Ptpn11 $1061 \mathrm{G} /+\mathrm{NS}$ mouse model.

- Chapter 6: analyse the mechanism by which SFN inhibits SHP2 phosphatase activity. 


\section{General methods}

All chemicals were purchased from Merck, formerly Sigma-Aldrich, unless stated otherwise.

\subsection{Animals}

Male and female WT C57BL/6J and heterozygous Ptpn11 $1^{\mathrm{D} 61 \mathrm{G} /+}$ mice (strain B6;129S4-Ptpn11tm1Bgn/Mmjax), referred to from here on as NS mice, were purchased from the Mutant Mouse Regional Research Centre and maintained in the animal facilities at King's College London. Mice aged between 12 and 15 weeks were used for experiments unless otherwise stated and treatment protocols are outlined in corresponding specific methods sections. Breeding and storerooms were maintained at $25{ }^{\circ} \mathrm{C}$ with a 12-hour light/dark cycle. Studies were performed in accordance with the Home Office guidance in the Operation of the Animals Scientific Procedures Act 1986, published by Her Majesty's Stationary Office.

\subsection{Tissue isolation and preparation for western immunoblotting or protein immunoprecipitation}

At the end of in vivo experiments, mice were sacrificed using a single intraperitoneal injection comprised of $70 \%$ sodium pentobarbitone and $30 \%$ sodium heparin. Once the mouse had lost consciousness, tested by ensuring the mouse's foot pain reflex was absent, tissue was rapidly isolated, flushed with saline (unless stated otherwise), placed immediately into liquid nitrogen and stored at $-80{ }^{\circ} \mathrm{C}$. When required, tissue was weighed and added to $10 \% \mathrm{wt} / \mathrm{vol}$ ice-cold homogenisation buffer (100 mM Tris- $\mathrm{HCl}, 150 \mathrm{mM} \mathrm{NaCl,} 1 \mathrm{mM}$ egtazic acid (EGTA), 1\% triton, $\mathrm{pH}$ 7.4) supplemented with metal-chelator-free protease 
inhibitor (Roche). On ice, tissue was homogenised using a mechanical tissue homogeniser. If sodium dodecyl sulphate polyacrylamide gel electrophoresis (SDS-PAGE) was desired, equal volumes of homogenate and 2X SDS-PAGE sample buffer (100 mM Tris- $\mathrm{HCl}$ pH 6.8, $4 \%$ SDS, $20 \%$ glycerol, $0.01 \%$ bromophenol blue) were mixed together and stored at $-20{ }^{\circ} \mathrm{C}$ until required. If protein immunoprecipitation was desired, samples were maintained on ice and immunoprecipitation protocol was carried out immediately following homogenisation.

\subsection{Protein immunoprecipitation}

$1 \mathrm{ml}$ of control group tissue lysate or $500 \mu \mathrm{l}$ of drug-treated group tissue lysate was transferred into separate microcentrifuge tubes and centrifuged at $15000 \mathrm{x}$ g for 10 minutes at $4{ }^{\circ} \mathrm{C} .50 \mu$ l of supernatant from each sample was added to 50 $\mu$ I 2X SDS-PAGE sample buffer in separate tubes to be used as 'input', whilst the remaining supernatant was transferred into fresh microcentrifuge tubes and placed on ice. Primary antibodies were bought either unconjugated or preconjugated to agarose beads which were prepared using the following protocols:

Primary antibody pre-conjugated to an agarose bead: $100 \mu \mathrm{l}$ of the agarose beadconjugated primary antibody and $50 \mu \mathrm{l}$ of unconjugated agarose bead alone (if phosphatase activity assay was required) were washed $3 \mathrm{X}$ via centrifugation at $15000 \times \mathrm{g}$ at $4{ }^{\circ} \mathrm{C}$ for 3 minutes in $1 \mathrm{ml}$ phosphate buffered saline (PBS). After the final wash, beads were re-suspended in either $100 \mu \mathrm{l}$ or $50 \mu \mathrm{l}$ PBS supplemented with metal chelator free protease inhibitor ( 1 tablet per $50 \mathrm{ml}$, Roche), depending on starting volume. $50 \mu \mathrm{l}$ of agarose bead-conjugated primary antibody was added to $400 \mu \mathrm{l}$ of tissue lysate supernatant from control or drugtreated mice. The remaining $50 \mu \mathrm{l}$ of agarose bead-conjugated primary antibody 
was added to $400 \mu \mathrm{l}$ PBS to be used as a negative control. $50 \mu \mathrm{l}$ of unconjugated agarose bead was added to $400 \mu \mathrm{l}$ of tissue lysate supernatant from the control group, which was also to be used as negative controls. Samples were rotated at 20 rotations per minute (rpm) for 3 hours at $4{ }^{\circ} \mathrm{C}$.

Primary antibody not conjugated to an agarose bead: $10 \mu \mathrm{l}$ of primary antibody was added to $400 \mu$ lissue lysate supernatant and rotated at $20 \mathrm{rpm}$ overnight at $4{ }^{\circ} \mathrm{C}$. The following morning, $20 \mu \mathrm{l}$ protein A/G plus agarose beads (Santa Cruz \#sc-2003) were added and rotated at $20 \mathrm{rpm}$ for a further 3 hours at $4{ }^{\circ} \mathrm{C}$.

Following incubation of tissue homogenate supernatants with pre-conjugated or unconjugated primary antibodies, samples were centrifuged at $15000 \times \mathrm{g}$ at $4{ }^{\circ} \mathrm{C}$ for 5 minutes and the supernatant was collected as 'output'. Samples were again washed $3 \mathrm{X}$ via centrifugation at $15000 \times \mathrm{g}$ at $4{ }^{\circ} \mathrm{C}$ for 3 minutes in PBS and resuspended in $125 \mu$ l PBS following the final wash. For western immunoblotting, proteins were eluted from the beads by boiling for 5 minutes at $95{ }^{\circ} \mathrm{C}$ followed by centrifugation at $15000 \mathrm{xg}$ at $4{ }^{\circ} \mathrm{C}$ for 3 minutes. The supernatant was added to an equal volume of 2 X SDS-PAGE sample buffer, labelled as 'capture' and stored at $-20{ }^{\circ} \mathrm{C}$ until required. For phosphatase activity assay, the protein was left conjugated to the bead and the activity assay protocol was performed immediately after immunoprecipitation.

\subsection{Phosphatase activity assay}

SHP2 phosphatase activity was measured using an EnzChek Phosphatase Kit (Thermo Fisher). This kit contained a fluorogenic substrate named 6,8-difluoro4-methylumbelliferyl phosphate (DiFMUP). Cleavage of its phosphate group generates the fluorescent reaction product 6,8-difluoro-4-methylumbelliferone. 
Therefore, a higher fluorescent readout correlated to higher phosphatase activity. Continuous assessment of activity could be achieved using this assay, which allowed for endpoint experiments as well as the determination of the initial rate of reaction. Immunoprecipitation of protein from tissue lysate was carried out as described above and preparation of recombinant protein is outlined in the corresponding specific methods section of chapter 3. All reagents were brought to room temperature before use. In an area of reduced lighting, a $10 \mathrm{mM}$ stock solution of DiFMUP was prepared by dissolving one vial of DiFMUP in $200 \mu \mathrm{l}$ of $\mathrm{N}, \mathrm{N}$-dimethylformamide and a working $200 \mu \mathrm{M}$ solution was subsequently prepared by diluting $1 \mu \mathrm{l}$ of the stock in $49 \mu \mathrm{l}$ PBS. $50 \mu \mathrm{l}$ of protein-containing sample was loaded into a black 96 -well flat-bottomed plate (Thermo Fisher). All samples were run in triplicate. The reaction was initiated by rapidly adding $50 \mu \mathrm{l}$ of the DiFMUP working solution into each protein-containing well. DiFMUP alone and SFX-01 alone were used as negative controls for in vitro studies. Tissue lysate incubated with unconjugated agarose bead and antibody/bead or antibody alone, as described previously, were used as a negative control for in vivo studies. Fluorescence was measured with a microplate reader (Gemini XPS) at an excitation of $360 \mathrm{~nm}$ and an emission of $460 \mathrm{~nm}$ at room temperature. Readings were taken every 1 -minute over a 10 -minute time period and analysed using SoftMax Pro software. Unless otherwise stated, the 10-minute time point was taken for analysis. Values were calculated by using the average of the triplicate samples and subtracting the average negative control value from each sample. 0-100 $\mu \mathrm{M}$ DiFMU standards were also used in each experiment and a line of best fit was generated. The amount of product formed from experimental 
samples was calculated by extrapolating the corresponding $Y$ value from the line of best fit.

\subsection{SDS-PAGE}

Protein-containing samples were subjected to SDS-PAGE using a Bio-Rad miniprotean tetra cell system. Precast polyacrylamide protein gels were purchased from Bio-Rad and consisted of a stacking gel and a 4-20 \% gradient resolving gel. Gels were held in place using gel chambers which were then inserted into a buffer tank and submerged in reservoir buffer. A 1X working solution of reservoir buffer (25 mM Tris, 192 mM glycine, $0.1 \%$ SDS, $\mathrm{pH}$ 8.3) was made by diluting $100 \mathrm{ml}$ of a $10 \mathrm{X}$ stock in $900 \mathrm{ml}$ of deionised water. $50 \mu \mathrm{l}$ of the protein sample was mixed via vortex with $50 \mu \mathrm{L} 2 \mathrm{X}$ SDS-PAGE sample buffer before being loaded into the gel. When using a fifteen-well gel, $10 \mu \mathrm{l}$ of the sample was loaded into each well and $15 \mu \mathrm{l}$ was loaded if a ten-well gel was used. $3 \mu \mathrm{l}$ of a molecular marker (Precision Plus protein dual colour standards, Bio-Rad) was loaded in a neighbouring lane, which contained proteins of various molecular weights, providing a visual aid to determine transfer efficiency as well as identifying the molecular weight of proteins. Electrophoresis was performed at $50 \mathrm{~V}$ for $\sim 15$ minutes to allow proteins to migrate through the stacking gel followed by $180 \mathrm{~V}$ until the dye front had reached the bottom of the resolving gel.

\subsection{Western immunoblotting}

Immunoblot polyvinylidene fluoride (PVDF) transfer membranes (Bio-Rad), were soaked in $100 \%$ methanol for 2 minutes followed by submersion in transfer buffer (25 mM Tris, 192 mM glycine, $20 \%$ vol/vol ethanol, pH 8.3). Once electrophoresis was complete, the polyacrylamide gel was removed from the plastic cassette 
using a metal opening lever and carefully placed on top of the PVDF membrane. The gel and membrane were then sandwiched between two stacks of electrode paper which had been wetted with transfer buffer. The sandwich was placed inside the bottom half of a Trans-Blot semi-dry rapid transfer system cassette (Bio-Rad). Air bubbles and excess transfer buffer were removed by rolling a long plastic tube over the top of the sandwich. The top of the transfer system cassette was then added and the whole cassette placed inside the semi-dry transfer machine. If one membrane was transferred at a time, $1.3 \mathrm{amps}$ and a voltage of 25 were applied for 7 minutes and if two membranes were transferred, 2.5 amps and a voltage of 25 was applied for the same amount of time. Once complete, the stacks of electrode paper and gel were discarded, and the membrane was then ready for immunostaining.

\subsection{Immunostaining western blots}

Membranes were blocked on a shaker for 1-hour at room temperature in a solution containing either of the following buffers: PBS containing $0.1 \%$ Tween 20 (PBS-T) plus $10 \% \mathrm{wt} / \mathrm{vol}$ non-fat milk powder (Marvel) for non-phosphorylated proteins of interest or Tris buffer solution containing $0.1 \%$ Tween (TBS-T) plus $10 \% \mathrm{wt} / \mathrm{vol}$ bovine serum albumin (BSA) for phosphorylated protein targets. The blocking solution was then discarded and desired primary antibody, diluted in either PBS-T plus $5 \% \mathrm{wt} / \mathrm{vol}$ milk or TBS-T plus $5 \% \mathrm{wt} / \mathrm{vol}$ BSA, was added to the membrane and incubated at room temperature for 2 hours on a shaker. Primary antibody dilution was determined either from the manufacturer's recommendations or from previous studies. The membrane was then washed for 1-hour at room temperature in either PBS-T or TBS-T, with the wash solution changed every 20 minutes. A solution containing horseradish peroxidase (HRP) 
linked secondary antibodies (Cell Signalling) diluted 1:1000 in either PBS-T plus $5 \% \mathrm{wt} / \mathrm{vol}$ milk or TBT-T plus $5 \% \mathrm{wt} / \mathrm{vol}$ BSA were then added to the membrane and incubated at room temperature for 1 -hour on a shaker. The membrane was again washed for 1 -hour at room temperature in either PBS-T or TBS-T, with the wash solution changed every 20 minutes and were then ready for enhanced chemiluminescence $(E C L)$ and quantification to be performed.

\subsection{Enhanced chemiluminescence and quantification}

ECL was performed using Pierce ECL western immunoblotting substrate (Thermo Fischer). $500 \mu \mathrm{l}$ of solution A was mixed with $500 \mu \mathrm{l}$ solution B and then pipetted onto the membrane ensuring even and total coverage. After 1-minute membranes were placed inside a clear plastic wallet and air bubbles were removed. The plastic wallet was fixed in place inside a film cassette and chemiluminescence was detected using photographic film (GE Healthcare) which was subsequently developed using an automated machine (Fuji RG II). After development was complete, membranes were stained using Coomassie Blue (0.2\% Coomassie Blue R, $7.5 \%$ acetic acid, $50 \%$ ethanol) for 30 minutes followed by $3 \times 10$-minute washes in destaining solution (5\% acetic acid, $50 \%$ methanol). This was carried out to identify the efficiency of the transfer process and to aid in quantification of protein loading. For cellular experiments the analysis of GAPDH protein was used as quantification of protein loading. Developed film and Coomassie Blue stained membranes were digitally scanned and quantified by densitometry using Image Studio Lite (LI-COR Biosciences). 


\subsection{Colloidal Coomassie staining}

Following protein separation by SDS-PAGE, it was sometimes desirable to visualise total protein within samples. Once electrophoresis was complete, gels were stained overnight at $4{ }^{\circ} \mathrm{C}$ with Colloidal Coomassie Blue, which was made of two solutions: solution A ( $85 \%$ orthophosphoric acid; $0.6 \mathrm{M}$ ammonium sulphate) and solution B (5 \% Coomassie G 250). $20 \%$ methanol is added to the solution directly before use. The following morning, Colloidal Coomassie stain was removed and the gel was destained in deionised water at room temperature, on a shaker, until desired background staining was achieved.

\subsection{Statistical analysis}

All data sets with an $n \geq 3$ are displayed as mean values \pm the standard error of the mean. Data sets containing only two groups were analysed for statistically significant differences using independent samples $t$-test. Data sets of three or more were first analysed using one-way analysis of variance (ANOVA) to identify the presence of statistically significant differences between any of the groups. If a statistical difference were identified, a follow-up Tukey's honest significant difference (HSD) test was performed to identify which groups within the data set held the significant difference. Statistical analysis was carried out using Microsoft Excel. Differences were considered significant at the $95 \%$ confidence level $(p<0.05)$.

\subsection{Affinity purification of SFN antibody}

The anti-SFN primary antibody was purified from rabbit serum which had been stored at $-80{ }^{\circ} \mathrm{C}$ since harvesting. To do this, an affinity column using $\mathrm{N}$ hydroxysuccinimide) NHS-activated Sepharose was used (GE Healthcare) which 
was made via the batch method. $5 \mathrm{ml}$ of activated Sepharose was added to a 10 $\mathrm{ml}$ plastic column and washed in $10-15$ column volumes of cold $1 \mathrm{mM} \mathrm{HCl}$ to remove the storage solvent. NHS-activated Sepharose was then incubated with $50 \mathrm{mM}$ L-cysteine in $8 \mathrm{ml}$ coupling solution (0.2 $\left.\mathrm{M} \mathrm{NaHCO}_{3}, 0.5 \mathrm{M} \mathrm{NaCl}, \mathrm{pH} 8.3\right)$. The matrix mixture was left rotating overnight at $4{ }^{\circ} \mathrm{C}$ to allow coupling of cysteine to the NHS-activated Sepharose via its amino terminus. The following morning the column was centrifuged at $1000 \times \mathrm{g}$ for 1 -minute to pellet the Sepharose beads and the supernatant was discarded. To minimise hyperoxidation and block any free NHS groups that had not been coupled to cysteine, beads were resuspended in $8 \mathrm{ml} 0.1 \mathrm{M}$ Tris $-\mathrm{HCl} \mathrm{pH} 7.5$ and incubated for 2 hours at room temperature. Rapid alternate washes were then carried out; firstly, using $0.1 \mathrm{M}$ Tris- $\mathrm{HCl} \mathrm{pH} 8.5$ followed by $0.1 \mathrm{M}$ acetate buffer $\mathrm{pH} 4.0$ plus $0.5 \mathrm{M} \mathrm{NaCl}$ and this process was repeated three times. The column was then washed in 5 column volumes $10 \mathrm{mM}$ dithiothreitol (DTT) in PBS pH 8.0, to reduce any disulfide bonds that may have formed, which was followed by 10 column volumes PBS. After the final wash, $5 \mathrm{ml} 50 \mathrm{mM}$ L-SFN was added to the beads and incubated overnight at $4{ }^{\circ} \mathrm{C}$ to enable coupling of SFN to the free-thiol group of cysteine to occur. The following morning, the column was washed with 20 column volumes PBS supplemented with $20 \%$ DMSO followed by 5 column volumes PBS to remove any unbound SFN. $10 \mathrm{ml}$ of rabbit antiserum was added to the column and rotated overnight at $4{ }^{\circ} \mathrm{C}$. The following morning, beads were poured into a disposable PD10 column (GE Healthcare), the antiserum was allowed to drip through the column and the eluate was repassed through the beads to optimise binding. The column was then washed $3 \mathrm{X}$ with $10 \mathrm{ml}$ PBS plus $500 \mathrm{mM} \mathrm{NaCl}$ followed by $10 \mathrm{ml}$ PBS. $17 \mathrm{ml}$ of acid-elution buffer (100 mM glycine, pH 2.5) was 
added to the column to elute acid-labile antibodies which were collected in a 15 $\mathrm{ml}$ falcon tube containing $3 \mathrm{ml} 1 \mathrm{M}$ Tris, $\mathrm{pH}$ 8.0. The column was then neutralised by washing with $9 \mathrm{ml}$ PBS. $17 \mathrm{ml}$ of basic-elution buffer (100 mM triethylamine, $\mathrm{pH}$ 11.5) was added to the column to elute alkali-labile antibodies which were collected in a $15 \mathrm{ml}$ falcon tube containing $3 \mathrm{ml} 1 \mathrm{M}$ acetate $\mathrm{pH}$ 5.0. The column was subsequently washed with $9 \mathrm{ml}$ PBS and stored in PBS plus $0.05 \%$ azide at $4{ }^{\circ} \mathrm{C}$. The two pools of antibody were then concentrated using spin columns with a $50 \mathrm{~K}$ cut-off (Milipore) via centrifugation at $3000 \mathrm{xg}$ at $4{ }^{\circ} \mathrm{C}$ into storage buffer (PBS, $0.1 \%$ Tween-20, $1 \%$ trehalose, $0.01 \%$ azide). Pools were concentrated until optical density at $280 \mathrm{~nm}$ exceeded 1 (where $1 \mathrm{OD}$ at $280 \mathrm{~nm}$ $=0.7 \mathrm{mg} / \mathrm{ml} \operatorname{lgG}$ ). The purified antibody was finally aliquoted into $25 \mu \mathrm{l}$ aliquots and stored at $-20{ }^{\circ} \mathrm{C}$ until required for western immunoblotting or immunoprecipitation.

\subsection{High-performance liquid chromatography (HPLC)}

The HPLC system (LC-10 AD liquid chromatograph, Shimadzu) consisted of a gradient pump used at a flow rate of $1 \mathrm{ml} /$ minute, an injection valve programmed to inject $20 \mu \mathrm{l}$ of sample, a UV detector set to $205 \mathrm{~nm}$ and a Supelco reverse phase Titan C-18 column (Sigma-Aldrich) held in a column oven set to $37^{\circ} \mathrm{C}$. The mobile phase consisted of buffer A (100\% deionised water) and buffer B (90 $\%$ acetonitrile:10 \% deionised water) and the binary gradient programme is outlined in table 2.1. 


\begin{tabular}{|l|l|l|}
\hline Time point, minutes & Function & Percentage \\
\hline 0 & Buffer A & 100 \\
& Buffer B & 0 \\
\hline 5 & Buffer A & 100 \\
& Buffer B & 0 \\
\hline 15 & Buffer A & 0 \\
& Buffer B & 100 \\
\hline 20 & Buffer A & 0 \\
& Buffer B & 100 \\
\hline 25 & Buffer A & 100 \\
& Buffer B & 0 \\
\hline 25 & Stop & \\
\hline
\end{tabular}

Table 2.1. HPLC protocol for analysing the stability of SFX-01 in water

\subsection{Cell culture}

HEK293 cells (a cell line derived from human embryonic kidney) were maintained in Dulbecco's modified Eagles media (DMEM) plus GlutaMAX-I (Thermo Fischer), supplemented with $10 \%$ foetal bovine serum (FBS) and penicillin/streptomycin ( $1 \mathrm{unit} / \mathrm{ml} ; 1 \mathrm{\mu g} / \mathrm{ml}$ ) in a $95 \% \mathrm{O}_{2} / 5 \% \mathrm{CO}_{2}$ incubator at 37 ${ }^{\circ} \mathrm{C}$. Once cells reached $70-80 \%$ confluency, media was removed and cells were washed in $10 \mathrm{ml}$ of warmed Dulbecco's PBS. Cells were detached from the flask by incubating with $1.5 \mathrm{ml}$ warmed trypsin- EDTA (Invitrogen Life Technologies) for 5 minutes. The detachment of cells was confirmed using light microscopy. Warmed media was added to reach the desired dilution and cells were seeded into either a fresh T75 flask (10 ml total volume) for maintaining the cell line or into 6-well plates $(2 \mathrm{ml} /$ well) for experiments (Thermo Fisher). Transfection and treatment protocols are outlined in the corresponding specific methods sections. 


\section{Sulforaphane inhibits SHP2 activity in vitro and}

\section{in vivo with effects on downstream signalling}

\subsection{Introduction}

\subsubsection{SHP2 regulation of the Ras/ERK signalling pathway}

SHP2 is a positive regulator of the Ras/ERK signalling pathway. The Ras/ERK cascade is the most extensively studied MAPK signalling pathway and couples signals from tyrosine kinase GFRs to a broad complement of effector proteins, including transcription factors. Binding of growth factors (GF) such as plateletderived GF, epidermal GF and fibroblast GF drives dimerization and transphosphorylation of the receptors. Stimulation causes recruitment of SHP2 to phosphorylated receptors whereby it likely positively regulates GF signalling via both phosphatase-dependent and -independent mechanisms by acting as a scaffold protein (figure 3.1) ${ }^{59}$. SHP2 then becomes phosphorylated at its Cterminal domain, firstly on Tyr ${ }^{542}$ followed by $\mathrm{Tyr}^{580}$, with the former being the major docking site for GFR-bound protein 2 (GRB2) ${ }^{106}$. This interaction forms the basis of a larger complex comprising GAB1, the PH domains of which likely bind the SH2 domains of SHP2 ${ }^{107}{ }^{108}$. SOS, a guanine nucleotide exchange factor is then recruited to this complex, catalysing the exchange of GDP from the small GTPase Ras for GTP, a key step in Ras reactivation ${ }^{109}$. The protooncogene serine/threonine kinase RAF is next recruited, where it is activated by a complex multistage process likely involving the kinase suppressor of Ras (KSR), although this remains unconfirmed 110111 112. RAF drives phosphorylation and activation of the dual-specificity mitogen-activated protein/extracellular signal-related kinase kinases (MEK) 1 and 2 which 
subsequently phosphorylate a tyrosine and then a threonine residue in the threonine-glutamic acid-tyrosine motif within the activation loop of ERK 1 (p44) and 2 (p42), resulting in their activation 113 114. ERK then directly phosphorylates multiple proteins and transcription factors, including c-Myc, Ets 1, the oncogene proteins c-Jun and c-Fos which form activator protein (AP) 1, as well as activating NF-KB and cAMP response element binding protein (CREB) via phosphorylation of their upstream kinases, ІкB kinase and ribosomal s6 kinase 1 (S6K1) respectively 115116117 . The expression of proteins involved in cell cycle progression and apoptosis prevention, including cyclins and cyclin-dependent kinases, GFs, cytokines and B-cell lymphoma 2 are subsequently upregulated. Non-transcriptional events are also triggered, including S6K1 regulation of autocrine signalling in pancreatic $\beta$ cells as well as interleukin 2 driven T-cell proliferation ${ }^{118} 119$.

Phospho-tyrosines on both Ras and GFRs provide docking sites for the GTPase-activating protein RasGAP, which negatively regulates the ERK pathway by hydrolysing RasGTP to GDP ${ }^{120}$. SHP2, on the other hand, stimulates signalling and is strongly suggested to act upstream of Ras, as dominant SHP2 negative fibroblasts show defective Ras activation ${ }^{121}$. SHP2 preferentially dephosphorylates tyrosine residues upon both GFRs and Ras, hindering RasGAP binding and promoting Ras activation 76 122. SHP2 also dephosphorylates Sprouty, a Ras/ERK pathway inhibitor. Sprouty becomes tyrosine phosphorylated in response to GFR activation and binds to $\mathrm{SH} 2$ domains of GRB2, which prevents docking of SOS (figure 3.1) ${ }^{123124 .}$ 


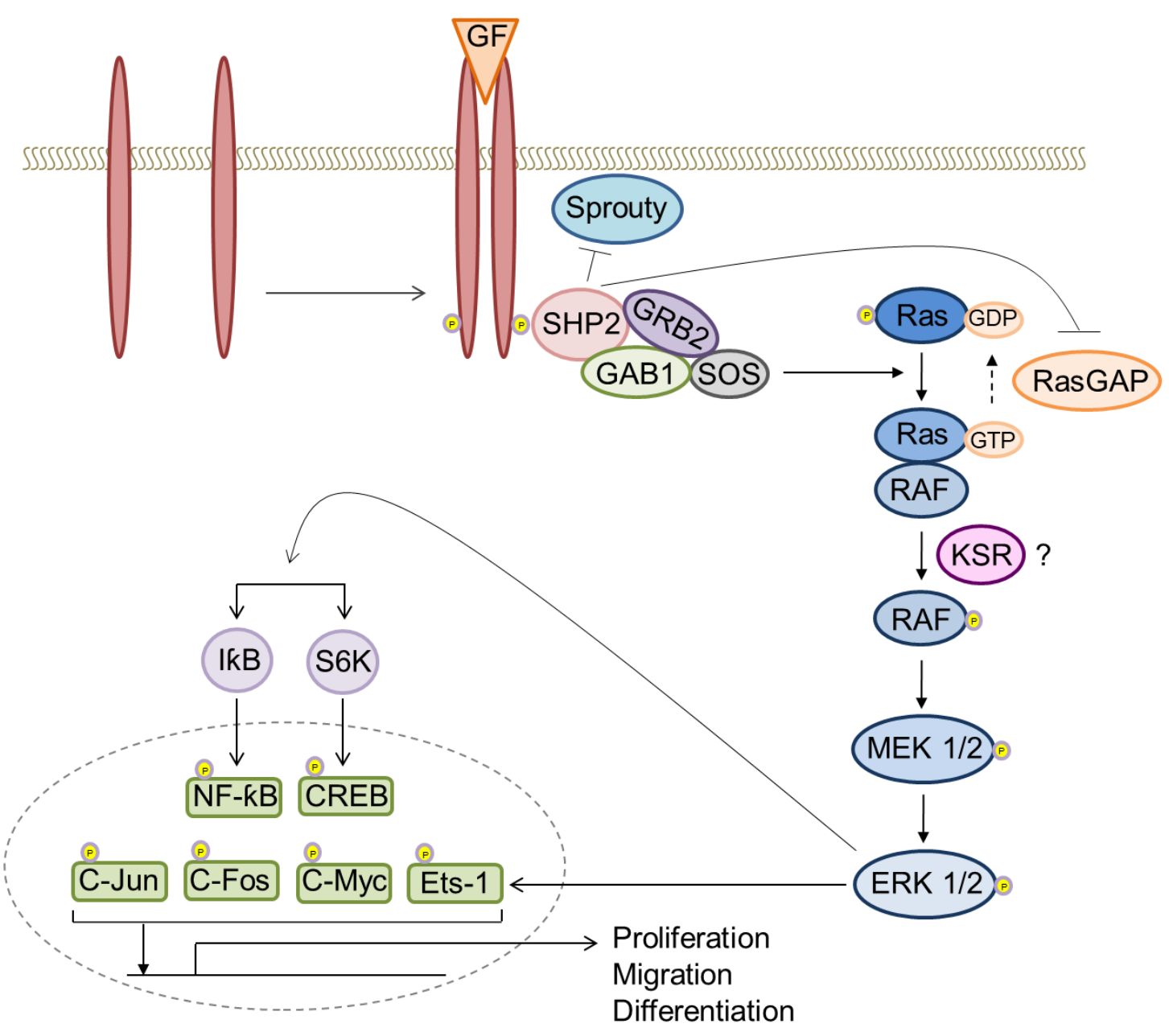

Figure 3.1. Diagram of the Ras/ERK signalling pathway.

GF binding drives receptor dimerization and trans-phosphorylation, providing docking sites for a protein complex involving SHP2 and SOS. Downstream ERK 1/2 activation occurs via RasGTP formation by SOS and subsequent RAF and MEK 1/2 phosphorylation. ERK phosphorylates a range of transcription factors, some via upstream kinases, resulting in upregulation of proteins required for proliferation, migration and apoptosis. SHP2 is a positive regulator of Ras/ERK signalling by dephosphorylating phospho-tyrosines on the negative regulator Sprouty, as well as Ras which prevents RasGAP binding and GTP hydrolysis.

ERK signalling, which can be stimulated independently of GFRs, is also initiated at the intracellular membrane of the endoplasmic reticulum in an SFK-dependent manner, which is negatively regulated by C-terminal Src kinase (Csk). Following 
GF stimulation Csk is recruited to areas where SFKs localise via interaction of its SH2 domains with phospho-tyrosines on phosphoprotein associated with glycosphingolipid-enriched microdomains 1 (PAG1) 125126 (figure 3.2). Csk phosphorylates tyrosine residues within the C terminal of SFKs which inhibits their activity. SHP2, on the other hand, can positively regulate SFK by docking GAB1 where it dephosphorylates PAG1 and blocks Csk recruitment ${ }^{126}$. Once activated, SFK phosphorylates downstream targets including phospholipase $\mathrm{C} Y$ 1 (Plcy1), which catalyses phospholipid hydrolysis and the generation of second messengers such as diacylglycerol (DAG) or inositol triphosphate (IP3) that can activate the Ras/ERK signalling pathway ${ }^{126}$ (figure 3.2). 


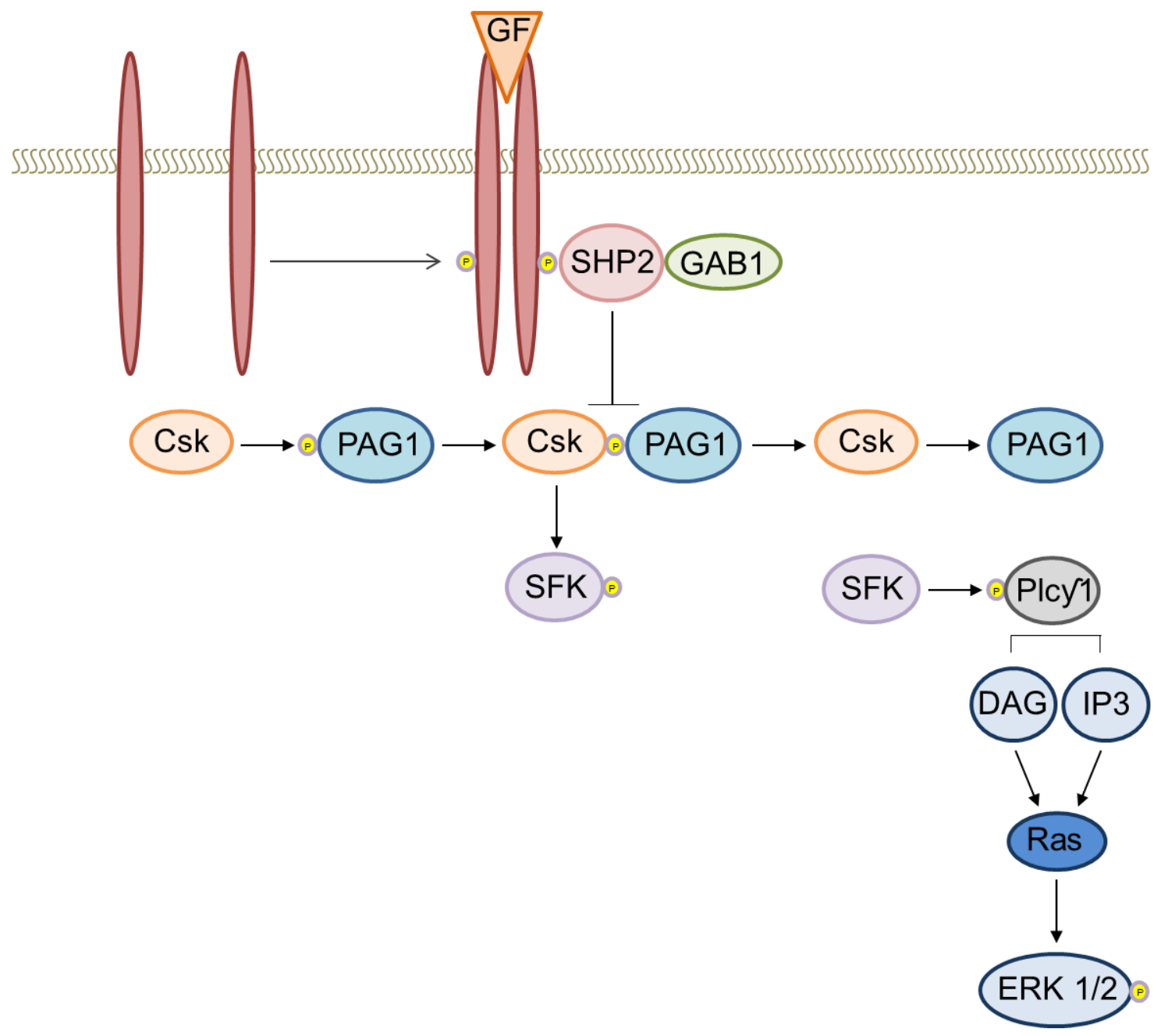

Figure 3.2. SHP2 positively regulates Ras/ERK signalling at the intracellular membrane of the endoplasmic reticulum.

Following GFR activation, Csk docks phospho-tyrosines on PAG1, bringing it into proximity with SFK, which it inactivates by phosphorylation. SHP2 docking to phosphotyrosines on the receptor allows localisation to Csk/PAG1 where it dephosphorylates PAG1 and prevents Csk binding, allowing stimulation of the Ras/ERK pathway by phosphorylation of PIcy1 by SFK.

\subsubsection{SHP2 regulation of the JAK/STAT GH signalling pathway}

The JAK/STAT GH signalling pathway promotes cell cycle progression and proliferation as well as apoptosis following binding of $\mathrm{GH}$ to $\mathrm{GH}$ receptors (GHR), 
and SHP2 is a negative regulator of this pathway ${ }^{127}$. GHs are secreted from the lateral wing of the anterior pituitary gland following synthesis by somatotropic cells. Binding of $\mathrm{GH}$ to GHRs initiates homodimerization of two monomeric receptor subunits ${ }^{128}$. The cytoplasmic domain of each receptor monomer is associated with JAK2, bound via an amino-terminal FERM (Band-4.1, ezrin, radixin, moesin) domain ${ }^{129}$. JAK2 belongs to a family of non-RTKs and is the only member involved in hormone-like cytokine signalling ${ }^{130}$. Dimerization of the receptor allows trans-phosphorylation and subsequent activation of the kinase domain of each JAK2 ${ }^{131}$. Activated JAK2 then auto-phosphorylates itself on additional tyrosine residues as well as others in the cytoplasmic domain of the GHR ${ }^{132}$. These phospho-tyrosine residues provide docking sites for a family of transcription factors named STAT, including STAT5 ${ }^{133}$. JAK2 subsequently phosphorylates conserved carboxy-terminal tyrosine residues within STAT5 proteins, which facilitates their dimerization and translocation into the nucleus where they bind regulatory sequences to activate or repress the transcription of target genes ${ }^{127} 134$. Phospho-tyrosine residues within the kinase domain of JAK2 also provide docking sites for PTPs including SHP2 ${ }^{135}$. Through western blotting and knock-out models, the Carter-Su group demonstrate C-terminal $\mathrm{SH} 2$ domains of SHP2 directly dock phospho-Tyr ${ }^{595}$ within GHRs, activating the phosphatase ${ }^{135}$. SHP2 then acts as a negative regulator of GH signalling by dephosphorylating phospho-tyrosine residues on GHRs and/or JAK2 which removes binding sites for downstream signalling proteins including STAT5 (figure 3.3) ${ }^{135} 136$. 


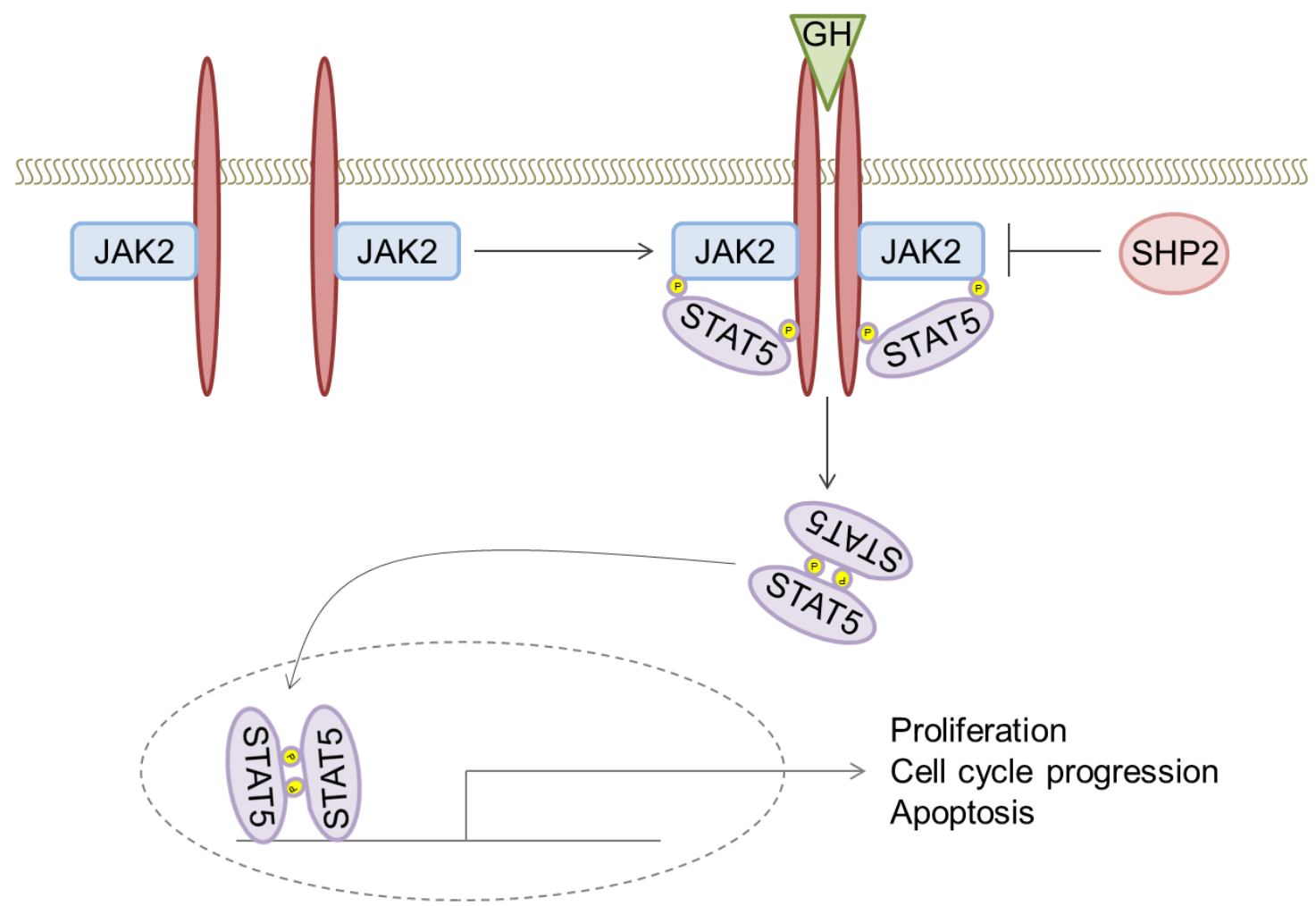

Figure 3.3. Diagram of the JAK/STAT signalling pathway.

$\mathrm{GH}$ binding to GHRs causes dimerization of these RTKs. JAK2 proteins subsequently become trans-phosphorylated providing docking sites for STAT5 followed by their dimerization and translocation to the nucleus. STAT5 binding to DNA drives upregulation of proteins involved in proliferation, cell cycle progression and apoptosis. SHP2 is a negative regulator of this pathway by dephosphorylating JAK2 proteins.

\subsubsection{GH treatment of NS patients}

As mentioned previously, a distinctive feature of NS is short stature, occurring in almost $80 \%$ of patients ${ }^{137}$. Currently, treatment of attenuated growth in NS patients is limited to recombinant human (rh) $\mathrm{GH}$ therapy. Initial clinical trials in children showed promising results - increasing growth by roughly $5 \mathrm{~cm}$. However, ongoing improvements in adult height are still inconclusive ${ }^{138}$. Safety reports on the use of rhGH in children raise concerns about potential off-target effects on bone development due to intracranial pressure, scoliosis, and muscle and joint 
discomfort in some patients ${ }^{139}$. Further to this, information about the potential impact of GH therapy on ventricular development in patients with cardiac defects and/or abnormal cardiac function is also unavailable. As well as GHs regulating JAK/STAT signalling, there is also cross talk with the ERK pathway. Hyperactivation of ERK or upregulation of the kinase is seen in multiple cancers and its activation aids the growth and proliferation of these cells. Therefore, the prospect of a potentiated incidence of cancer following GH therapy warrants consideration. Although GH therapy may provide short term benefits to children with NS, the concerns highlighted above make it evident that further studies into utilising $\mathrm{GH}$ as novel therapies are needed.

Short stature is most prevalent in NS patients with mutations in Ptpn11, the gene which encodes SHP2 ${ }^{140}$. In vivo studies identified reduced sensitivity to GHs in NS mice which are genetically engineered with a gain-of-function SHP2 mutation, although exactly how hyperactivation of the phosphatase induces growth retardation is still under investigation. The Yart group showed an association between early postnatal growth delay and low levels of insulin-like growth factor 1 (IGF1) in a mouse model expressing a gain-of-function SHP2 mutation, Ptpn11 1 D61G/+ 141. They subsequently found inhibition of SHP2 in GH responsive cell lines resulted in increased IGF1 levels following GH stimulation ${ }^{141}$. Inhibitors of SHP2, or combined treatment with GH, may offer benefits over current therapies.

Within the phosphatase domain of SHP2 lays a catalytic cysteine, Cys ${ }^{459}$, which must be in its negatively charged thiolate state to successfully carry out nucleophilic attack towards the phosphorus ion of phosphorylated substrates and dephosphorylate them, and the structure of the protein's active domain and its 
catalytic mechanism are outlined in chapter 6 . Due to the electrophilic nature of SFN, it was logical to assume it would adduct at this nucleophilic catalytic cysteine and here I sought to determine if treatment of SHP2 with SFX-01 would inhibit the proteins phosphatase activity both in vitro and in vivo. Complementary experiments investigating alterations to downstream signalling pathways were also performed. 


\subsection{Materials and methods}

\subsubsection{SDS-PAGE and western immunoblotting}

SDS-PAGE and western immunoblotting were performed as outlined in the general methods. For this chapter the following primary antibodies were used:

\begin{tabular}{|l|l|l|}
\hline Primary antibody & \multicolumn{1}{|c|}{ Company } & Species \\
\hline Sulforaphane & In-house & Rabbit \\
\hline SHP2 (for immunocapture) & Santa Cruz \#sC-280 & Rabbit \\
\hline Total ERK 1/2 & Cell signalling \#9102 & Rabbit \\
\hline Phosphorylated ERK 1/2 & Cell signalling \#9101 & Rabbit \\
\hline SHP2 (for immunodetection) & R\&D Systems \#AF1894 & Goat \\
\hline Phospho-tyrosine PY20 & Santa Cruz \#sC-508 & Mouse \\
\hline Phosphorylated STAT5 & Cell signalling \#9351 & Rabbit \\
\hline
\end{tabular}

Table 3.1. List of primary antibodies used for western blotting in chapter 3.

\subsubsection{Immunoprecipitation}

Immunoprecipitation of proteins from tissue was performed as outlined in the general methods. For this chapter, the capture antibody was agarose-conjugated anti-SHP2 (Rabbit, Santa Cruz, \#sc-7384 AC). Subsequent immunoblotting was carried out using an anti-SHP2 primary antibody (Goat, R\&D Systems \#AF1894) and an anti-goat secondary antibody. 


\subsubsection{Preparation of recombinant SHP2 for in vitro experiments}

Recombinant human SHP2 protein (Abcam) bought at $0.73 \mathrm{mg} / \mathrm{ml}$ in storage buffer (50\% glycerol, $0.05 \%$ Tween 20, $75 \mathrm{mM} \mathrm{NaCl}, 25 \mathrm{mM}$ Tris HCl, $2 \mathrm{mM}$ EDTA, $10 \mathrm{mM} \mathrm{GSH}, 1 \mathrm{mM} \mathrm{DTT}, \mathrm{pH}$ 8.0) was stored at $-80{ }^{\circ} \mathrm{C}$. When required, SHP2 was placed onto ice where it was held until experiments commenced. Prior to use in experiments, varying amounts of SHP2 were added to PBS, on ice, to make a total volume of $40 \mu \mathrm{l}$, which was routinely desalted to remove undesirable components of the storage solution, and in doing so swap for a suitable buffer. Desalting was carried out using a $7 \mathrm{kDa}$ cut-off $0.5 \mathrm{ml}$ Zeba Spin desalting column (Thermo Fisher) which was first centrifuged at $1500 \times \mathrm{g}$ at $4{ }^{\circ} \mathrm{C}$ for 1 -minute to remove the storage buffer. The spin column was then washed three times to remove any remaining sodium azide from the resin by adding $100 \mu \mathrm{l}$ of experimental buffer $(25 \mathrm{mM}$ Tris $\mathrm{HCl}, 75 \mathrm{mM} \mathrm{NaCl}, 0.05 \%$ Tween 20, $2 \mathrm{mM}$ EDTA, varying DTT concentration as described below, $\mathrm{pH}$ 7.2) to the top of the resin and centrifuging as before. Following the final wash step, the spin column was moved to a microcentrifuge tube containing $10 \mu$ of the experimental buffer at reagent concentrations which generated a final concentration of $25 \mathrm{mM}$ Tris $\mathrm{HCl}, 75 \mathrm{mM} \mathrm{NaCl}, 0.05 \%$ Tween 20, $2 \mathrm{mM}$ EDTA with varying DTT concentrations, $\mathrm{pH}$ 7.2. $40 \mu \mathrm{l}$ of SHP2 in PBS was then added to the top of the resin and centrifuged at $1500 \times \mathrm{g}$ at $4{ }^{\circ} \mathrm{C}$ for 2 minutes. SHP2 was then placed back on ice until the experiment began.

\subsubsection{Optimisation of the SHP2 phosphatase activity assay}

A $50 \mu \mathrm{l}$ sample of varying amounts of recombinant SHP2, prepared as described above, containing varying concentrations of DTT, was incubated at room temperature for 6 hours prior to loaded into a 96-well flat-bottomed plate and 
phosphatase activity was measured using the fluorescence-based assay as described in detail in the general methods.

\subsubsection{Preparation of SFN stock for use in in vitro experiments}

A $50 \mathrm{mM}$ SFN stock was prepared by dissolving $58 \mathrm{mg}$ SFX-01 in $1 \mathrm{ml}$ of deionised water. The sample was mixed by vortexing until all SFX-01 was dissolved. Working solutions were made by diluting the stock in deionised water. SFN stock was freeze-thawed no more than 4 times before being replaced with a new batch.

\subsubsection{Treatment of SHP2 with SFX-01 and determination of IC $\mathrm{C}_{50}$ of inhibition by SFN}

Nine concentrations of SFX-01 from 0.007-1.75 $\mu \mathrm{M}$ were incubated with 0.011 $\mu \mathrm{g} / \mathrm{ml}$ recombinant SHP2 for varying lengths of time in PBS containing $0.016 \mathrm{mM}$ DTT. Each experiment was performed in triplicate and the activity of the protein was assessed using the fluorescence-based phosphatase activity assay as outlined in the general methods. The $\mathrm{IC}_{50}$ for SFN-dependent inhibition of recombinant SHP2 phosphatase activity in vitro was determined using the curvefitting programme GraphPad Prism (GraphPad Software Inc.).

\subsubsection{Determining the stability of SFN in water over time}

$12.5 \mathrm{mg}$ SFX-01 was dissolved by vortexing it in $5 \mathrm{ml}$ deionised water to make a final solution of $2.5 \mathrm{mg} / \mathrm{ml}$ SFX-01 $(0.385 \mathrm{mg} / \mathrm{ml} \mathrm{SFN})$. Dissolved SFX-01 was stored in the dark at room temperature. Each morning the solutions were analysed by HPLC using the protocol outlined in the general methods. A deionised water only sample was used as a negative control followed by a 0.385 $\mathrm{mg} / \mathrm{ml}$ SFN standard to determine its retention time. 


\subsubsection{Acute or chronic treatment with SFN in vivo}

For acute SFN treatment, SFX-01 $(0-10 \mathrm{mg} / \mathrm{kg}$, which is equivalent to $0-1.54$ $\mathrm{mg} / \mathrm{kg}$ SFN) was dissolved in $175 \mu \mathrm{l}$ deionised water and vortexed thoroughly. WT or NS mice then received a single intraperitoneal injection of a specified concentration and were sacrificed after 30 minutes. For chronic SFN treatment, the requisite amount of SFX-01 was dissolved in deionised water to make a final amount of $2.5 \mathrm{mg} / \mathrm{ml} \mathrm{SFX-01.} \mathrm{WT} \mathrm{or} \mathrm{NS} \mathrm{mice} \mathrm{then} \mathrm{received} \mathrm{this} \mathrm{as} \mathrm{a} \mathrm{substitute}$ for their drinking water over a 10-day period and were sacrificed at varying time points. The water containing the SFX-01 was replaced every 4 days. Following acute and chronic treatment, cardiac and liver tissue was harvested for further analyses.

\subsubsection{Growth hormone treatment in vivo}

WT mice were treated with either SFX-01 alone, recombinant mouse $\mathrm{GH}(\mathrm{rmGH})$ alone or a combination of both. 40 or $70 \mu \mathrm{g} \mathrm{rmGH}$, purchased from the HarbourUCLA Research and Education Institute, was prepared by diluting 40 or $70 \mu \mathrm{g}$ in $175 \mu \mathrm{l}$ saline supplemented with $10 \mathrm{mM} \mathrm{NaOH}$ and injected subcutaneously. 2.5 mg SFX-01 was prepared by diluting $2.5 \mathrm{mg}$ in $175 \mu \mathrm{l}$ deionised water and injected intraperitoneally. Vehicle-treated control mice received an equivalent volume of saline by intraperitoneal injection. When combined treatment was required, rmGH was first injected subcutaneously followed immediately by an intraperitoneal injection of SFX-01. Mice were sacrificed at time points $0,30,60$ or 120 minutes after treatment and cardiac tissue was harvested. 


\subsection{Results}

\subsubsection{Optimisation of the fluorescence-based SHP2 phosphatase activity}

assay

A commercial fluorescence-based phosphatase activity assay kit, which required optimisation prior to use in the in vitro experiments, was used to assess the catalytic activity of recombinant SHP2 as well as that found in tissues in vivo. As expected, increasing amounts of recombinant SHP2 $(0.011-0.73 \mu \mathrm{g} / \mathrm{ml})$ prepared as described previously, showed proportionately greater phosphatase activity (figure 3.4 A). For this first experiment, an experimental buffer containing $1 \mathrm{mM}$ DTT was used. Fluorescence of sufficient intensity above baseline was generated with $0.011 \mu \mathrm{g} / \mathrm{ml} \mathrm{SHP2}$ and was the concentration used in subsequent experiments. Desalting $0.011 \mu \mathrm{g} / \mathrm{ml}$ recombinant SHP2 into an experimental buffer containing increasing concentrations of DTT, from $0-1 \mathrm{mM}$, was found to increase phosphatase activity in a concentration-dependent manner (figure 3.4 B). As minimal DTT was desired, as it would likely also react with SFN, the lowest concentration of $0.016 \mathrm{mM}$ was chosen for subsequent experiments. 
A)

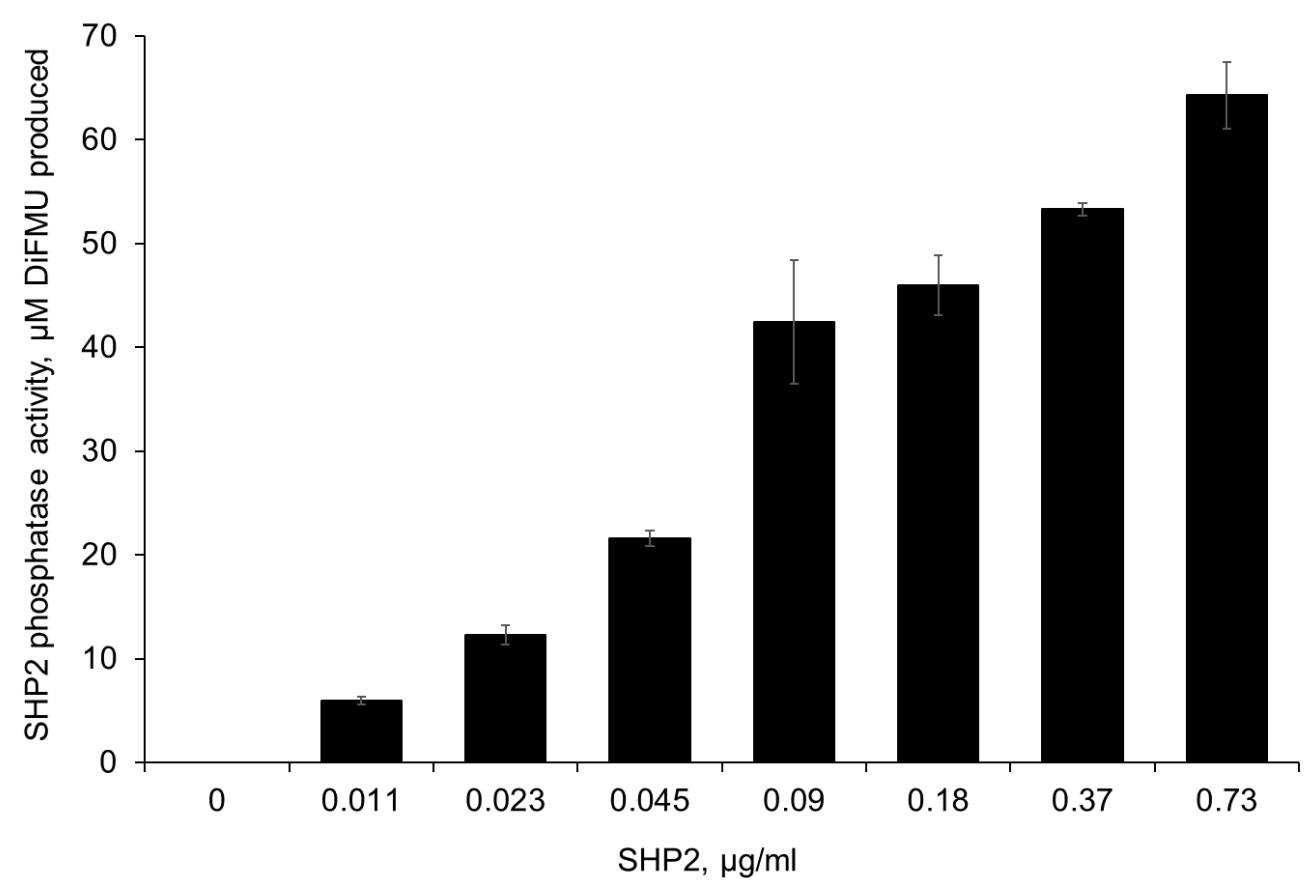

B)

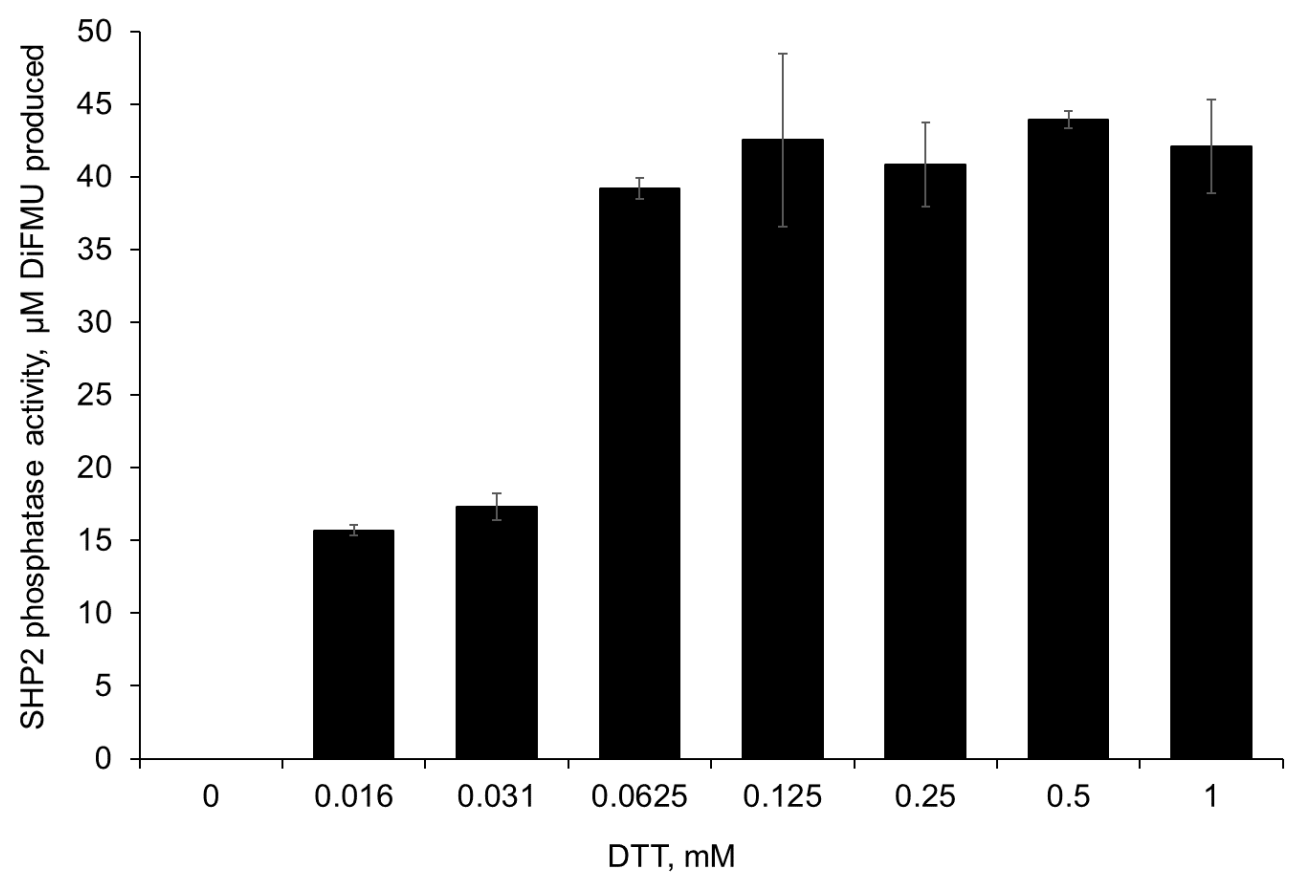

Figure 3.4. Optimisation of protein and DTT concentrations for a fluorescencebased phosphatase activity assay.

A) A fluorescence-based phosphatase activity assay showing an increase in SHP2 activity when increasing amounts of recombinant protein were used in the assay. The experimental buffer used in this experiment contained $1 \mathrm{mM}$ DTT. B) A fluorescencebased phosphatase activity assay showing an increase in SHP2 phosphatase activity when the protein was incubated in an experimental buffer which contained increasing concentrations of DTT. 


\subsubsection{SFN inhibits SHP2 phosphatase activity in vitro}

Concentration- and time-dependent inhibition of SHP2 phosphatase activity was seen using the fluorescence-based activity assay following incubation of 0.011 $\mu \mathrm{g} / \mathrm{ml} \mathrm{SHP2}$ in the presence of $0.016 \mathrm{mM}$ DTT with $0-1.75 \mu \mathrm{M}$ SFX-01 for 0-6 hours (figure $3.5 \mathrm{~A}$ ). Using the initial rate of reaction, calculated between the first and third minute of the assay, the $\mathrm{IC}_{50}$ for SFN-dependent inhibition of SHP2 was calculated for each time point. These values were time-dependent, varying from 47.28-0.49 $\mu \mathrm{M}$ respectively (figure $3.5 \mathrm{~B}$ ). 
A)

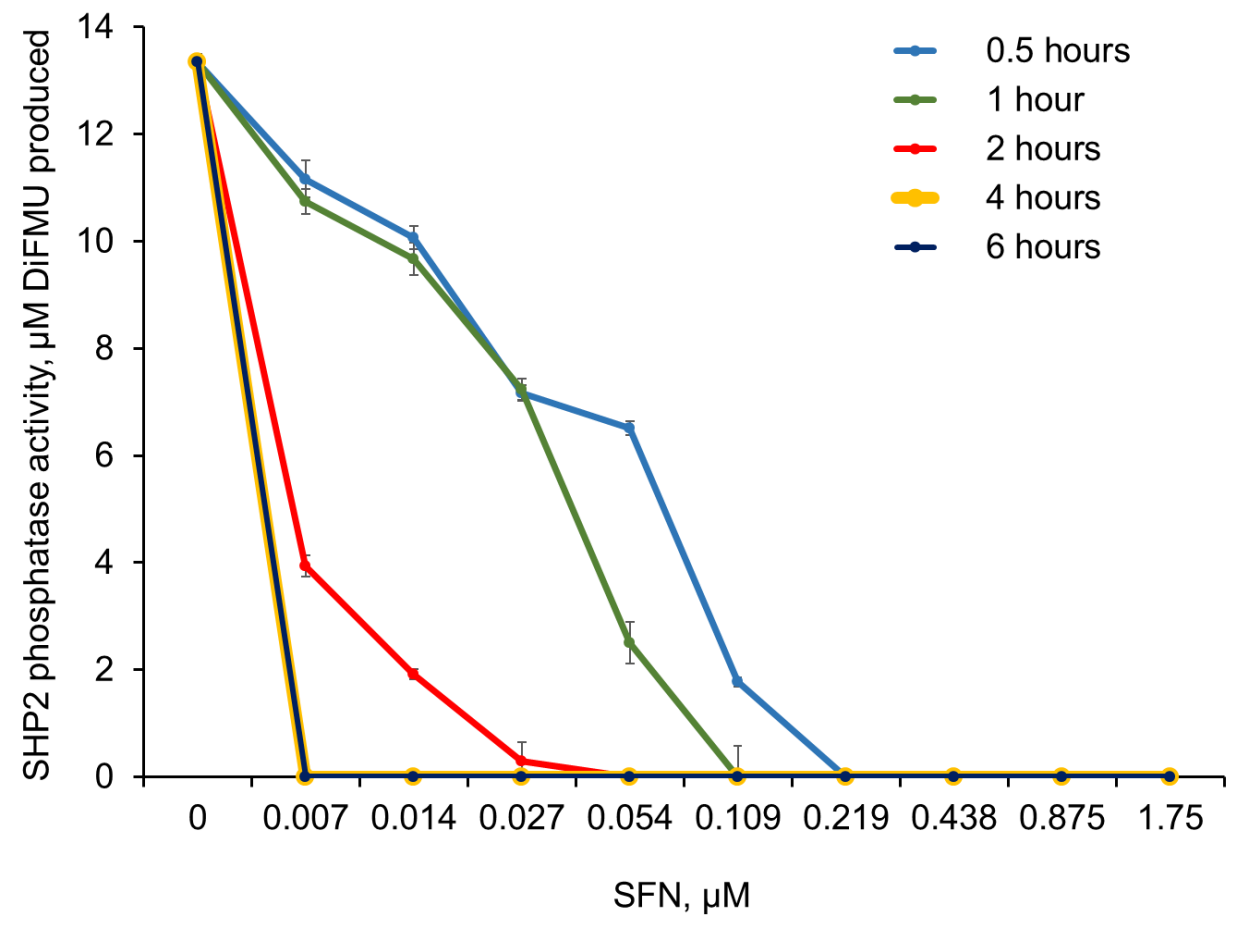

B)

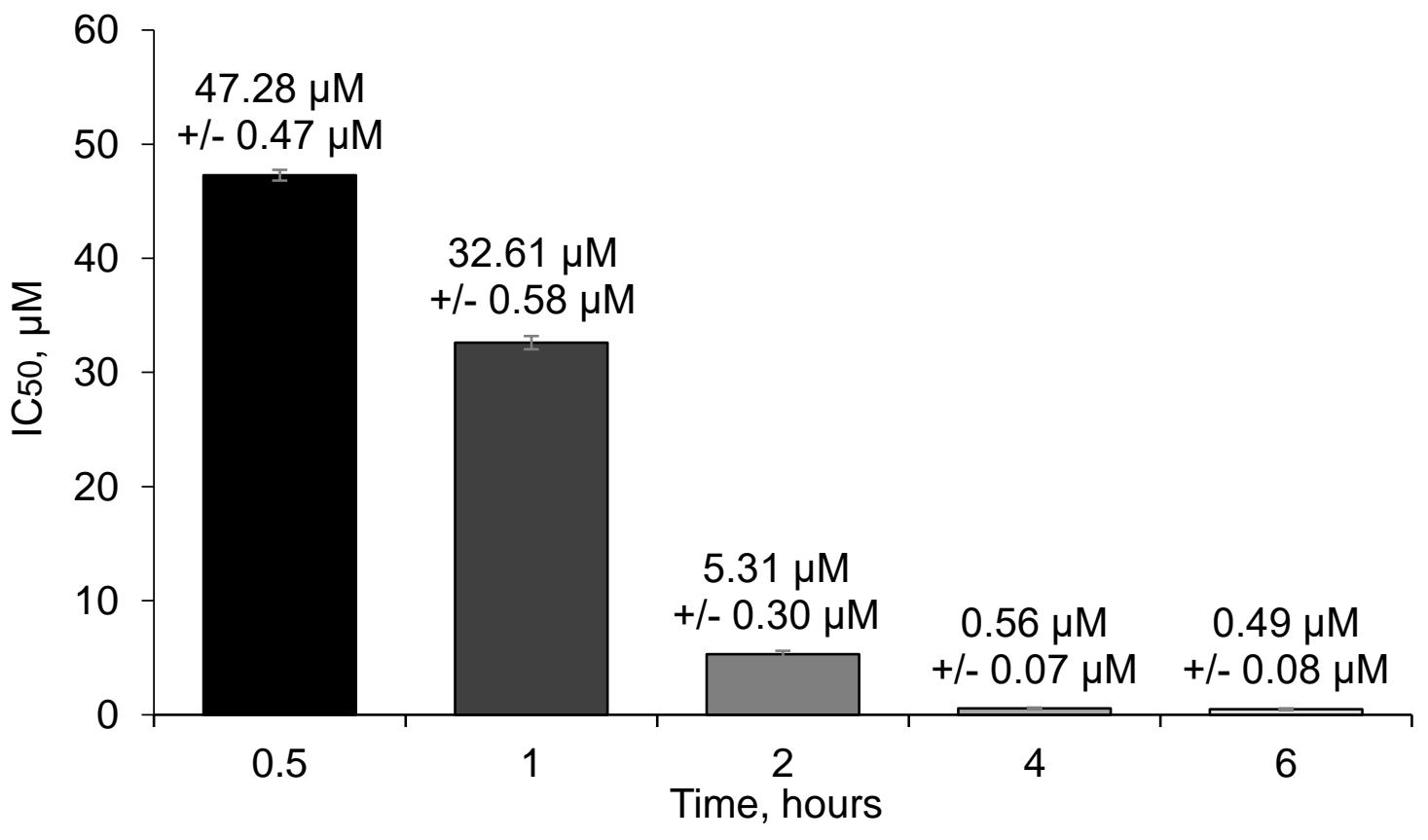

Figure 3.5. SFX-01 inhibits SHP2 phosphatase activity in vitro.

A) A fluorescence-based phosphatase activity assay carried out using $0.011 \mathrm{mg} / \mathrm{ml}$ recombinant SHP2 in a buffer containing $0.016 \mathrm{mM}$ DTT incubated with varying concentrations of SFX-01 for varying lengths of time. SFX-01 inhibits SHP2 phosphatase activity in a concentration- and time-dependent manner. B) $\mathrm{IC}_{50}$ values of SFN inhibition of SHP2 in different conditions. ( $n=5,{ }^{*} p<0.05$ versus $0 \mu M$ SFN control). 


\subsubsection{SFN adducts recombinant SHP2}

Western immunoblotting showed SFN adduction to recombinant SHP2 following incubation with $1.75 \mu \mathrm{M}$ SFX-01 for 30 minutes which was stable for a further 6 hours (figure 3.6 A). SHP2-SFN was also detected following incubation of the protein with $0.109 \mu \mathrm{M}$ SFX-01 for $0.5-6$ hours although a loss of the adduct was detected at the 2-6-hour time points compared to $0.5-1$ hours (figure $3.6 \mathrm{~B}$ ). Adduction of the protein by the electrophile was only detected following 2 hours of incubation with $0.007 \mu \mathrm{M}$ SFX-01 which again decreased at later time points (figure $3.6 \mathrm{C}$ ). 
A)

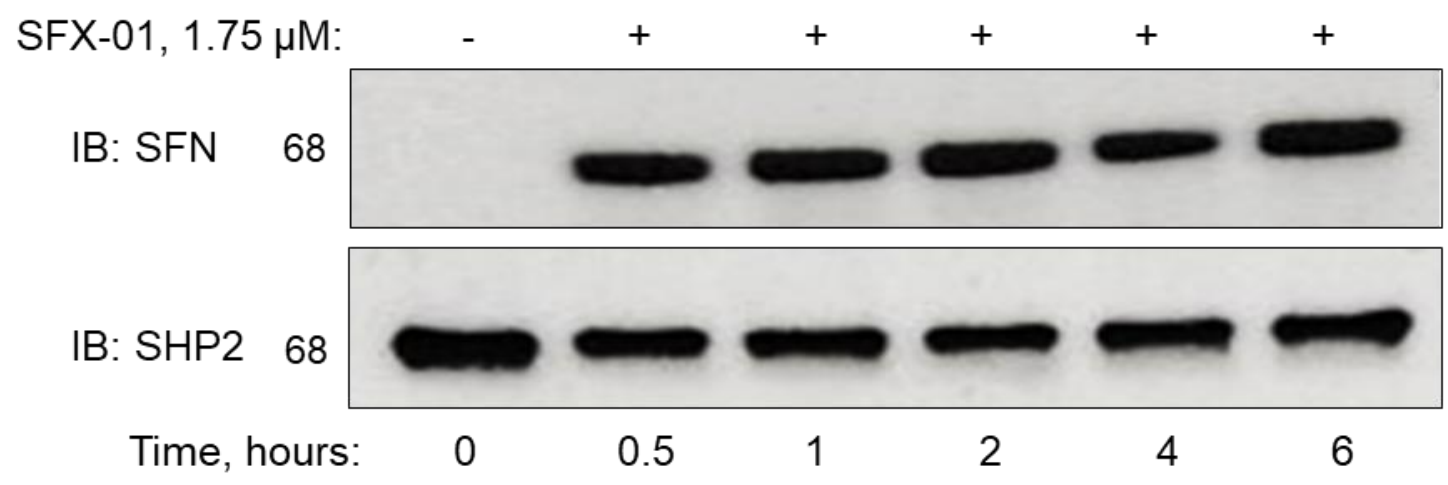

B)

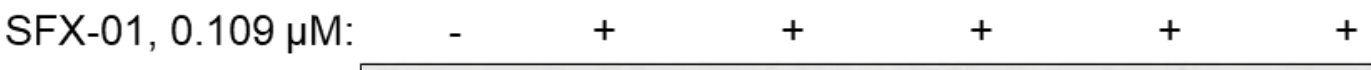

IB: SFN 68

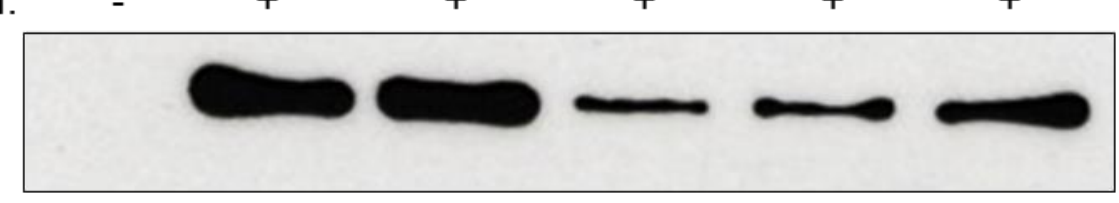

IB: SHP2 68

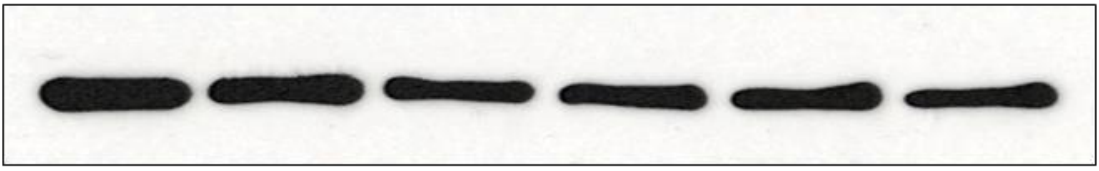

$\begin{array}{lllllll}\text { Time, hours: } & 0 & 0.5 & 1 & 2 & 4 & 6\end{array}$

C)

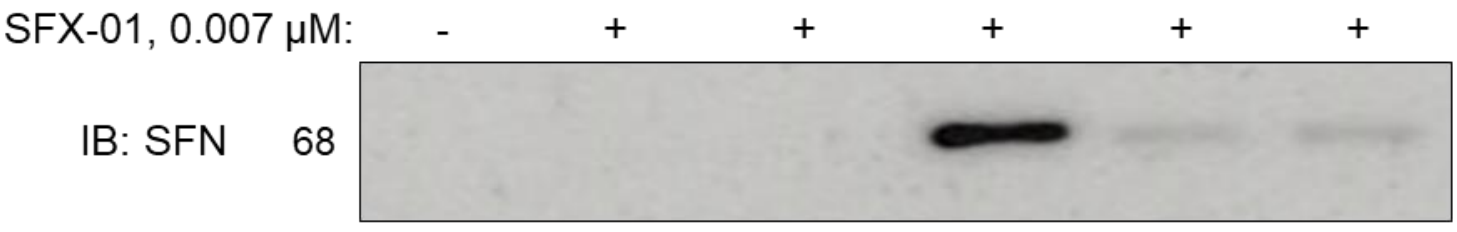

IB: SHP2 68

Time, hours: 0

0.5

1

2

4

6

Figure 3.6. SFN adducts SHP2 in vitro.

A) A representative immunoblot showing SFN adduction upon SHP2 following 30-minute incubation with $1.75 \mu \mathrm{M}$ SFX-01 which was stable for 6 hours. B) A representative immunoblot showing SHP2-SFN following 30-minute incubation with $1.09 \mu \mathrm{M}$ SFX-01. Reduction of the adduct was seen following 2 hours of incubation. C) A representative immunoblot showing SFN adduction upon SHP2 following 2 hours incubation with 0.007 $\mu \mathrm{M}$ SFX-01. Almost complete loss of the adduct was seen 4 hours post-incubation. 


\subsubsection{Characterisation of the NS mouse model}

Daily weighing from 3-22 weeks of age showed both male and female NS mice weigh less than their WT counterparts (figure 3.7 A). Visual inspection showed that NS mice were shorter in height and length, had a flatter skull and a webbed neck compared to their WT littermates (figure 3.7 B). Immunoblotting of SHP2 protein immunoprecipitated from cardiac tissue of WT or NS mice revealed comparable protein expression. However, a subsequent activity assay identified 3.5-fold hyperactivity of SHP2 in the mutants compared to WT (figure 3.7 C). Immunoblotting also revealed elevated ERK phosphorylation in the cardiac tissue of NS mice, with total ERK protein remaining comparable to WT littermates (figure $3.7 \mathrm{D}$ ). 
A)

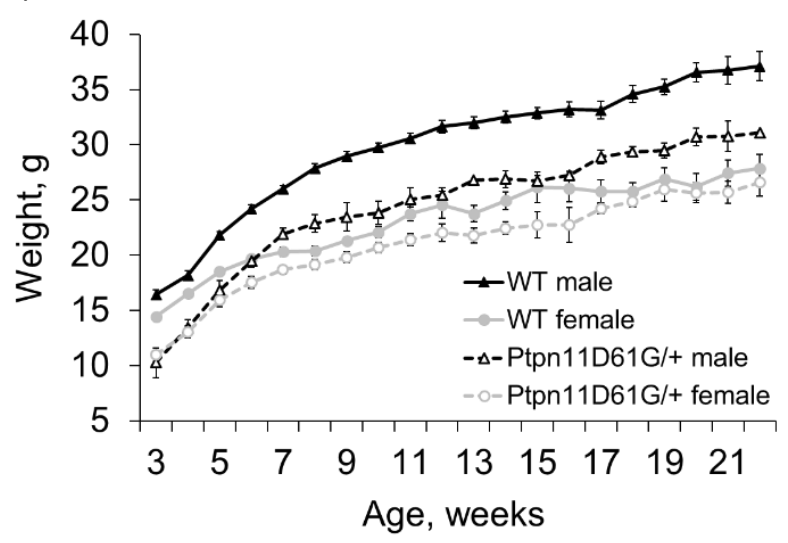

B)

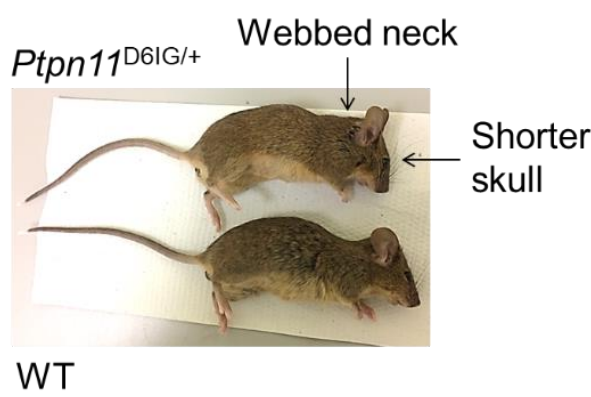

C)

D)

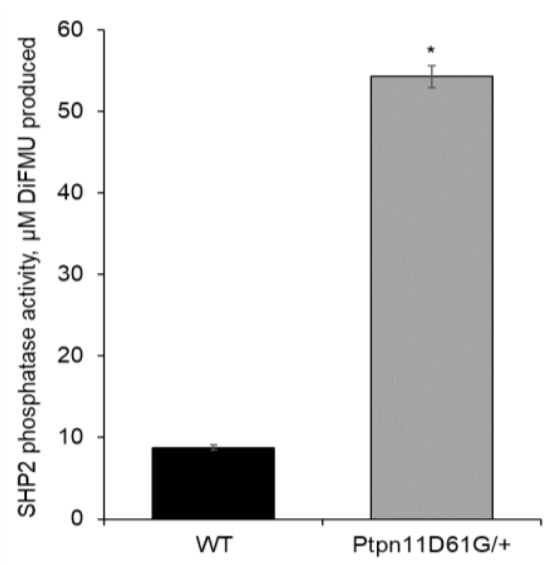

E)
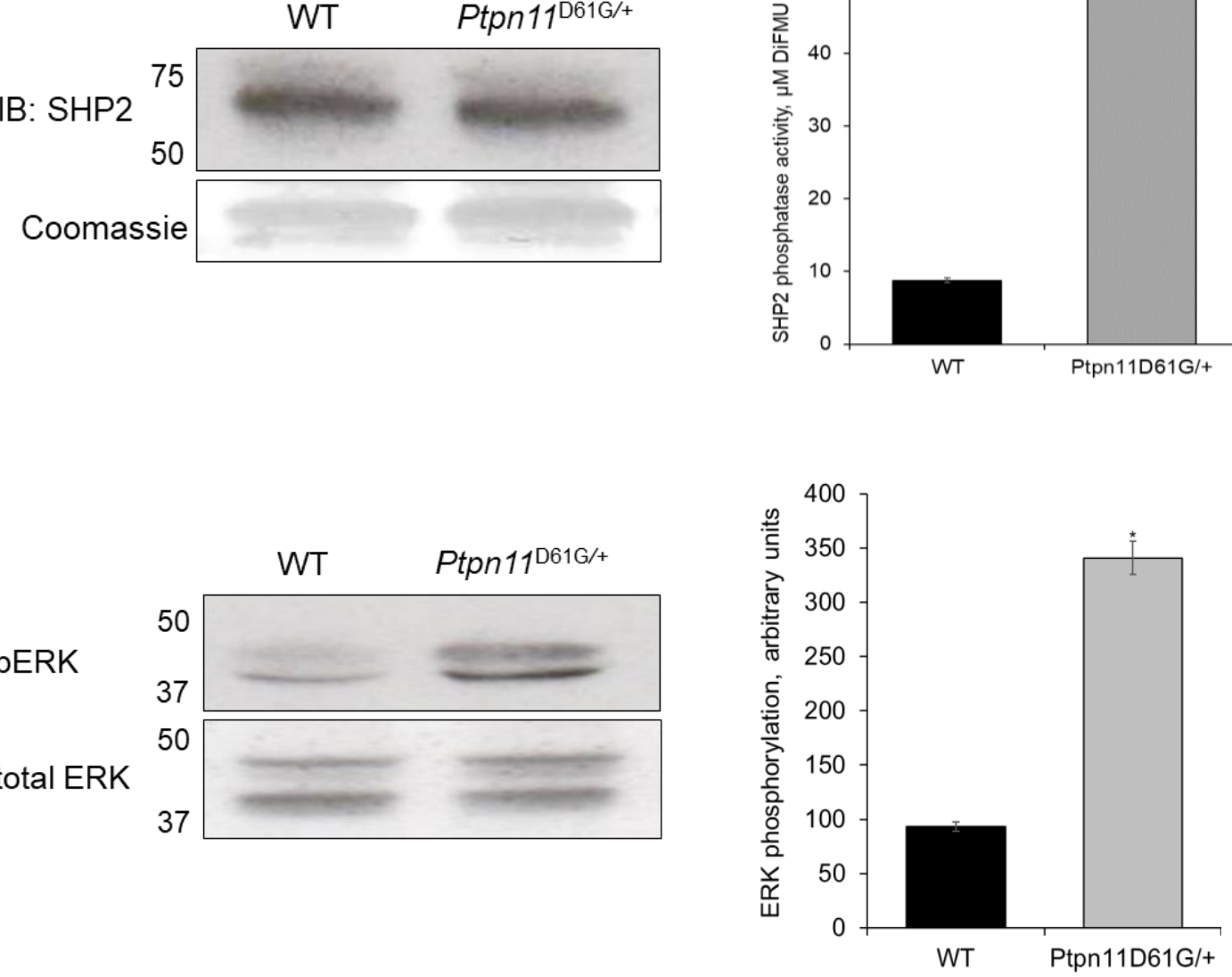

Figure 3.7. Characterisation of the NS mouse model.

A, B) NS mice present with a webbed neck, shorter skull and weigh less than their WT littermates. C, D) An immunoblot and fluorescence-based phosphatase activity assay showing comparable protein levels but increased phosphatase activity in NS mice using SHP2 immunoprecipitated from WT or NS cardiac tissue. E) An immunoblot showing increased ERK phosphorylation in cardiac tissue of NS mice. $\left(n=10,{ }^{*} p<0.05\right.$ versus WT). 


\subsubsection{SFN adducts protein targets in a concentration-dependent manner in}

vivo

To establish if SFN could adduct protein targets following in vivo treatment with SFX-01 instead of L-SFN, which was used in preliminary experiments to identify protein targets of the electrophile in cardiac tissue, WT or NS littermate mice were intraperitoneally injected with varying concentrations of SFX-01 acutely for 30 minutes. An increase in SFX-01 concentration $(0-10 \mathrm{mg} / \mathrm{kg})$ correlated with the increased abundance of protein-SFN adducts in cardiac and liver tissue of WT mice (figure 3.8 A, B). SFX-01 treatment of NS mice also resulted in protein-SFN adducts in both cardiac and liver tissue, however, only one concentration (10 $\mathrm{mg} / \mathrm{kg}$ ) was examined in these mice (figure 3.8 C, D). 
A) WT heart
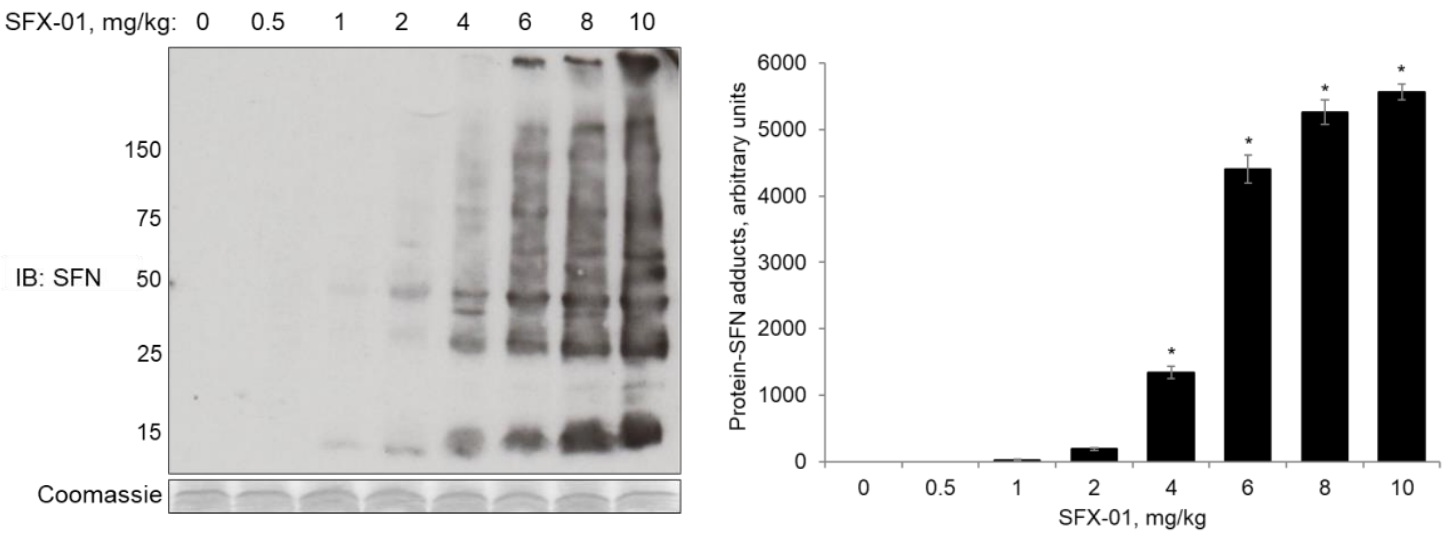

B)

WT liver
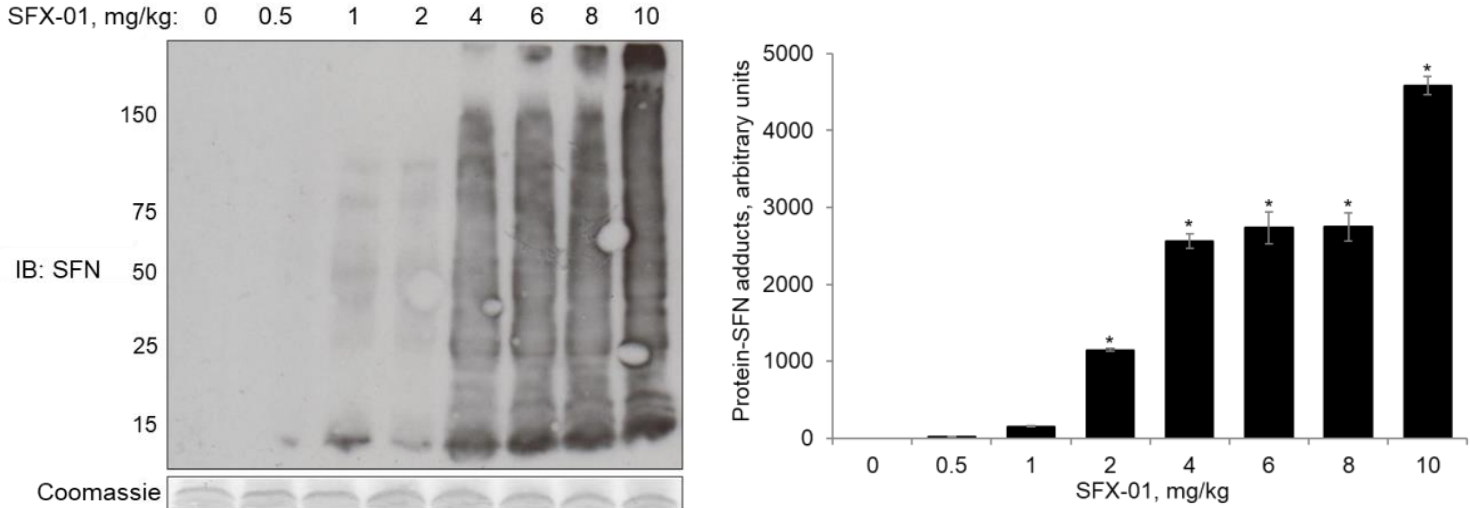

C)

D)

Ptpn11 $10616 /+$ heart
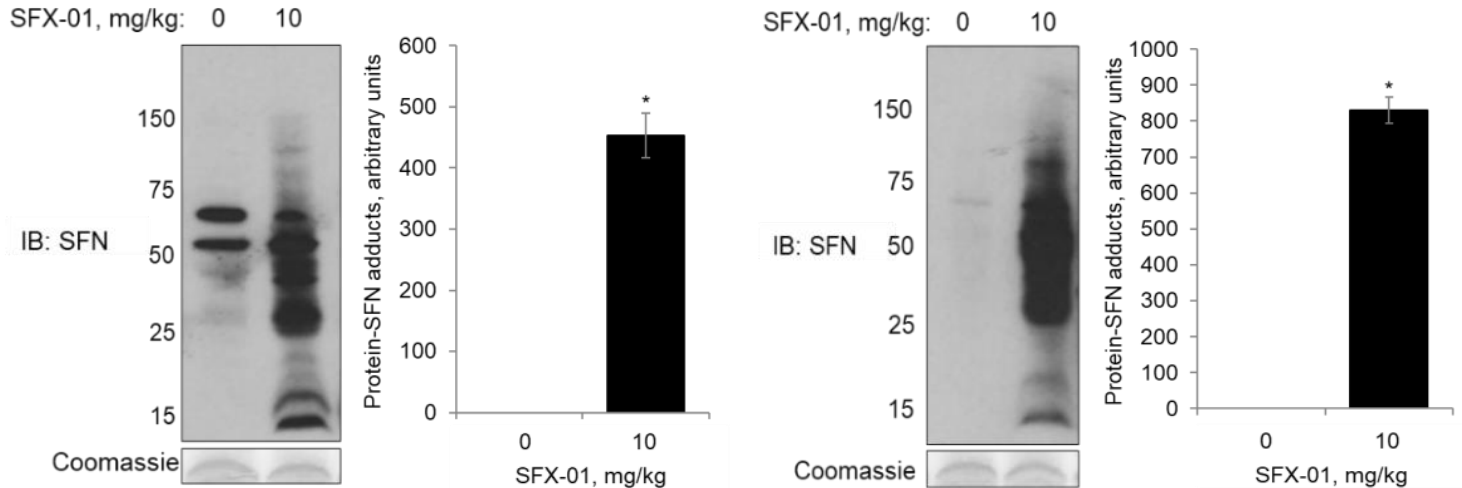

Figure 3.8. SFN adducts proteins in vivo in a concentration-dependent manner.

A-D) Immunoblots showing concentration-dependent increase in protein-SFN adducts in the cardiac and liver tissue of WT or NS mice 30 minutes after intraperitoneal injection of varying concentrations of SFX-01. Note: only one concentration of SFX-01 was assessed in NS mice as the number of these mice was limited. ( $n=6,{ }^{*} p<0.05$ versus water only control). 


\subsubsection{Acute treatment with SFX-01 does not inhibit SHP2 phosphatase activity in vivo}

To assess inhibition of SHP2 phosphatase activity following acute treatment with SFX-01, SHP2 was immunoprecipitated from the cardiac tissue of WT or NS mice following intraperitoneal injection with $10 \mathrm{mg} / \mathrm{kg}$ SFX-01. Immunoblotting of the input and immunocaptured SHP2 identified comparable protein expression between WT and NS mice (figure 3.9 A). SHP2 phosphatase activity was basally higher in the NS mice compared to WT, consistent with their activating mutation (figure 3.9 B). However, no inhibition in activity was observed following the 30 minute treatment of SFX-01 in either WT or NS mice (figure 3.9 B). 
A)

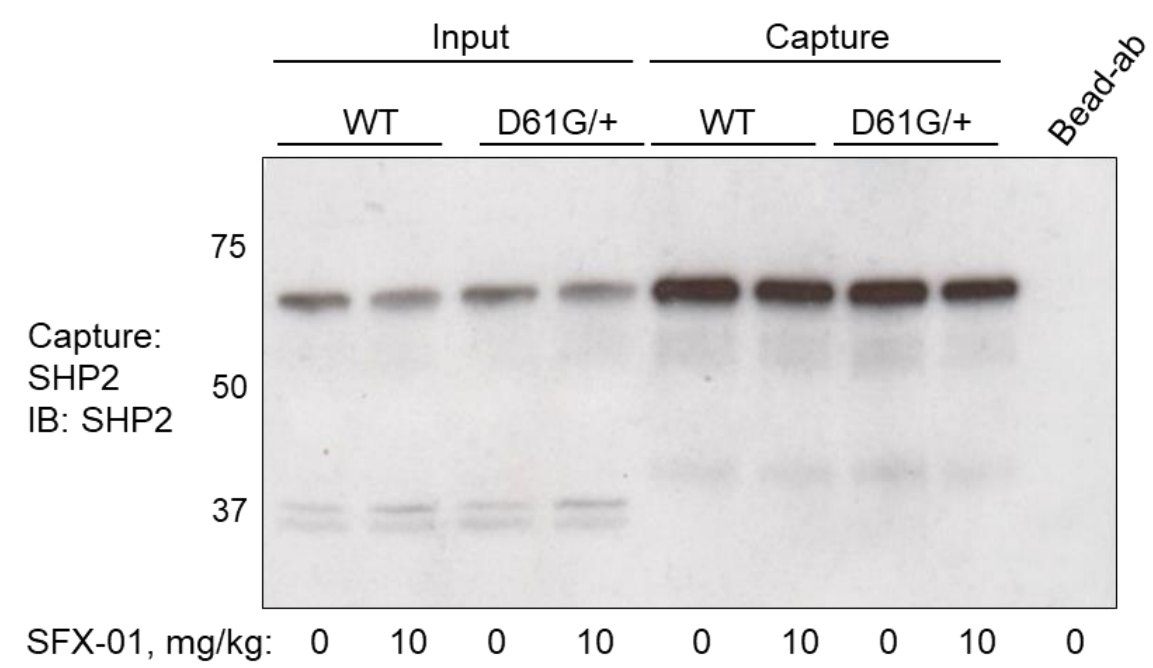

B)

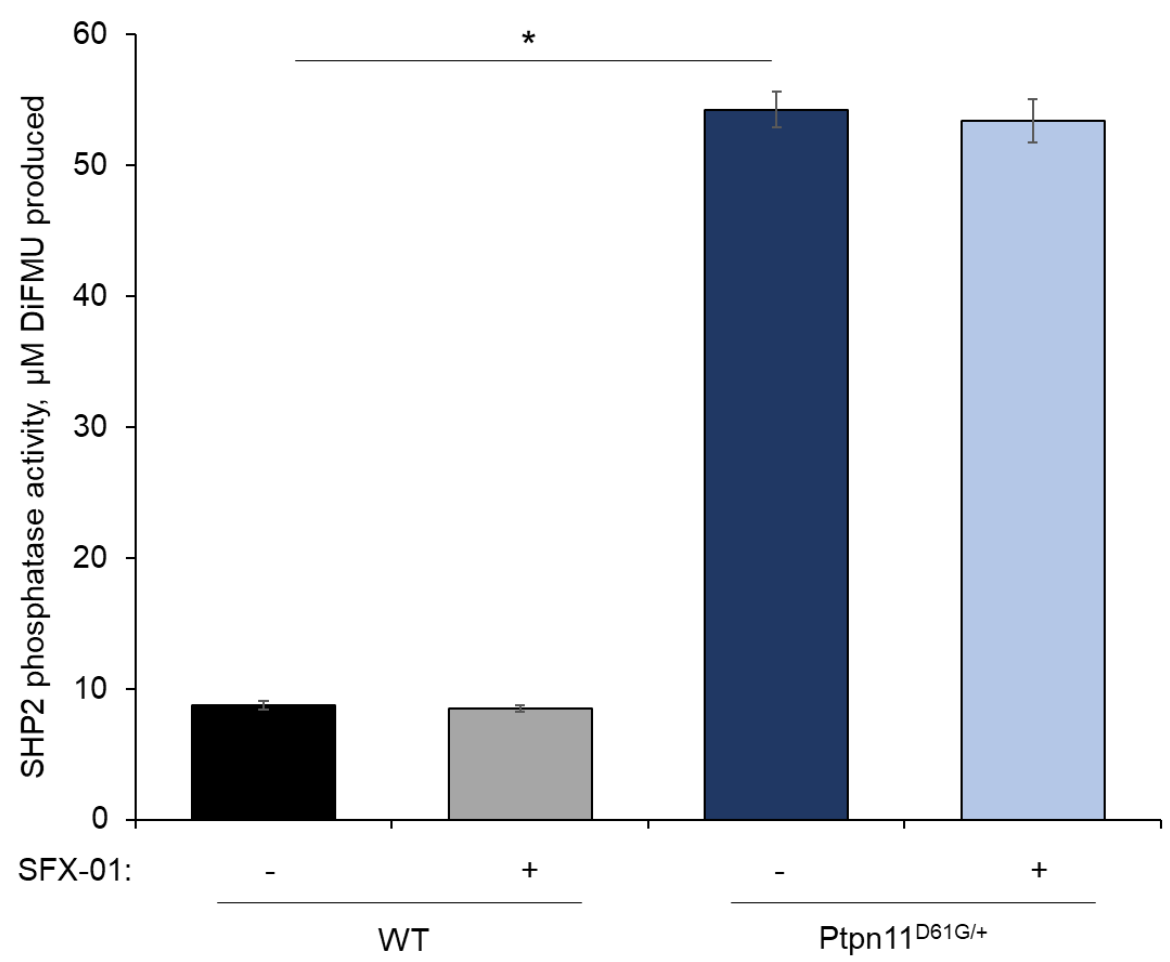

Figure 3.9. Acute exposure to SFX-01 does not inhibit cardiac SHP2 phosphatase activity.

A) An immunoblot of input and capture samples following immunoprecipitation of SHP2 from cardiac tissue of WT or NS mice 30 minutes after intraperitoneal injection of 10 $\mathrm{mg} / \mathrm{kg}$ SFX-01. B) A fluorescence-based phosphatase activity assay using immunoprecipitated SHP2 shows no inhibition of SHP2 phosphatase activity following 30-minute treatment with $10 \mathrm{mg} / \mathrm{kg} \mathrm{SFX-01.}(\mathrm{n}=6)$. 


\subsubsection{SFX-01 is stable in water for 6 days}

SFX-01 was dissolved in drinking water as a method of administration in some in vivo experiments. To establish the stability of SFX-01 in water over time, the drug was dissolved in water and analysed by HPLC at varying time points. A chromatogram of L-SFN, which was used as a positive control, revealed its retention time as $\sim 9.56$ minutes under the analytical conditions used (figure 3.10 B). The peak intensity of SFN following the addition of SFX-01 to water did not change over 0-6 days and it was therefore assumed the drug was stable for this duration (figure 3.10 C-E). A small peak with a retention time of $\sim 8.70$ minutes appears after 4 days, which may correspond to a degraded form of SFN, however, this has not been confirmed and was a very minimal component. 
A)

B)

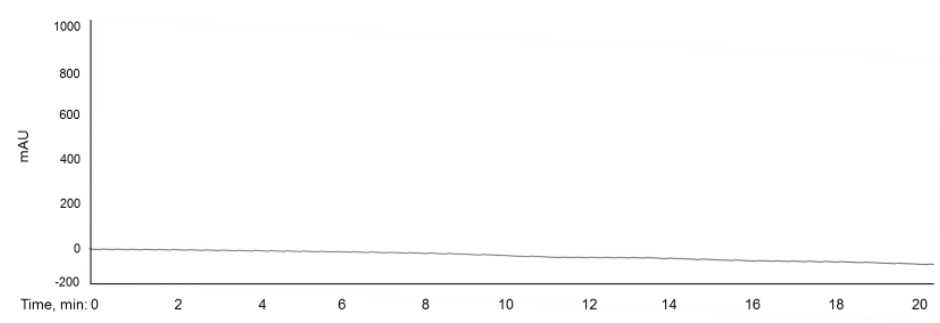

C)

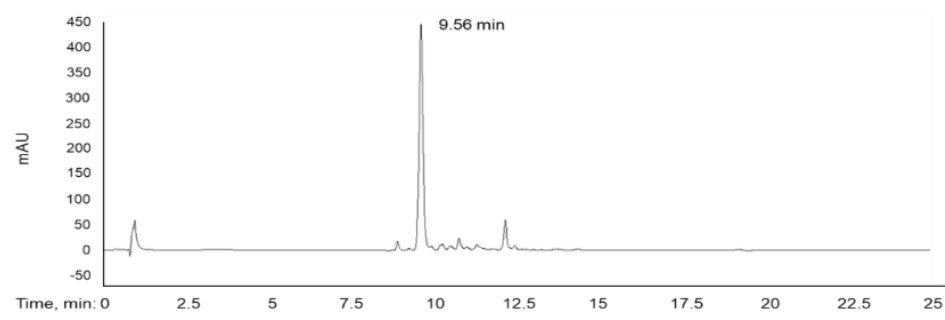

D)

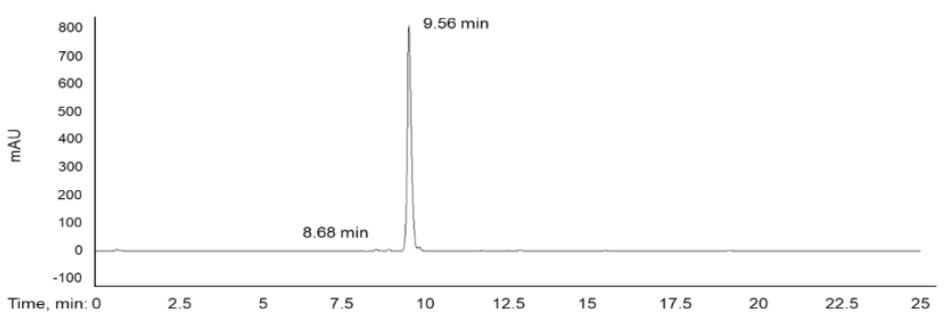

E)
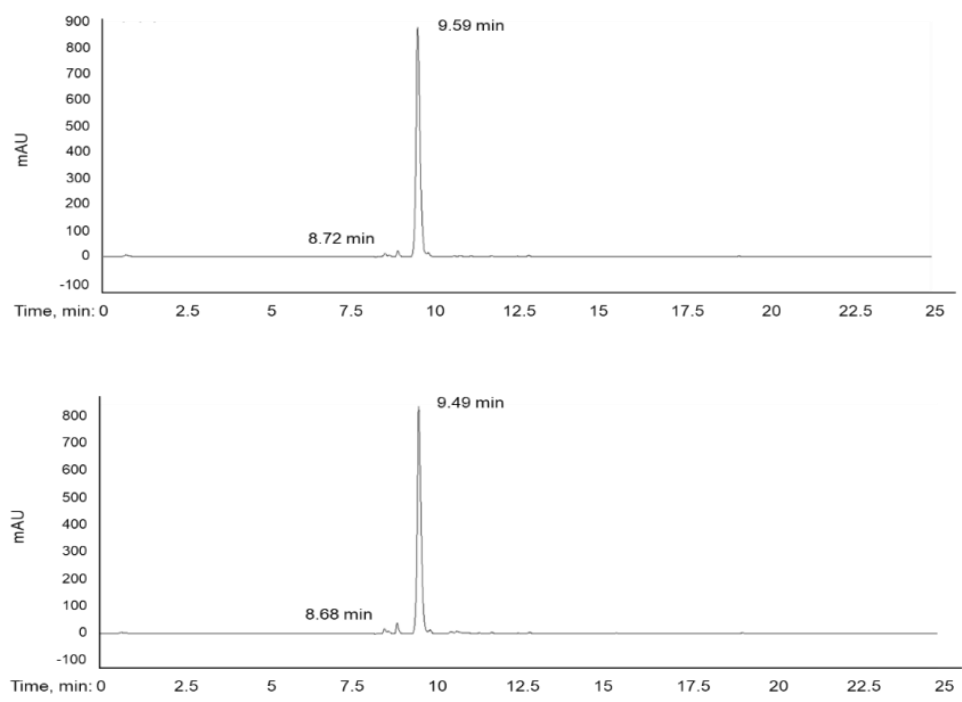

Figure 3.10. HPLC chromatograms showing SFX-01 is stable in water for 6 days.

A) A water only negative control. B) $0.385 \mathrm{mg} / \mathrm{ml} \mathrm{L-SFN}$ in water positive control. C-E) $2.5 \mathrm{mg} / \mathrm{ml} \mathrm{SFX}-01$ dissolved in water, stored at room temperature and analysed at time points of 0,4 and 6 days respectively. A small peak with a retention time of $\sim 8.70$ minutes, which likely represents a minimal amount of degradation, became apparent after 4 days. mAU represents absorbance using a UV detector set at a wavelength of 205 nm. 


\subsubsection{SFN adducts protein targets in a time-dependent manner in vivo}

As 30 -minute treatment with $10 \mathrm{mg} / \mathrm{kg}$ SFX-01 was not successful in inhibiting SHP2 phosphatase activity, WT or NS mice were subsequently treated with 2.5 $\mathrm{mg} / \mathrm{ml} \mathrm{SFX}-01$ in their drinking water for up to 10 days. Protein-SFN adducts were observed following 4 days of treatment in the cardiac tissue of WT or NS mice, which increased in a time-dependent manner (figure 3.11 A, B). 
A)

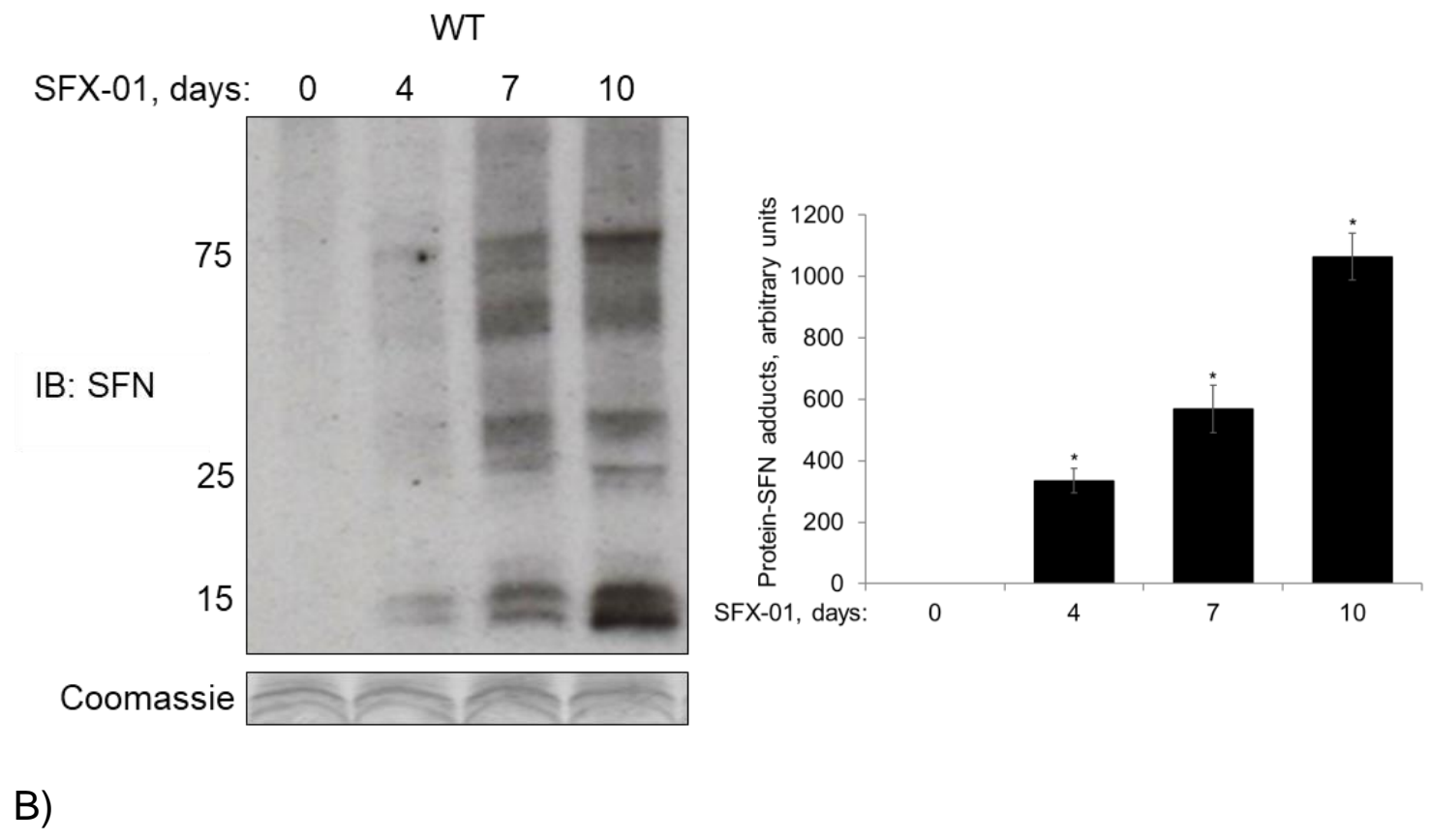

Ptpn11061G/+
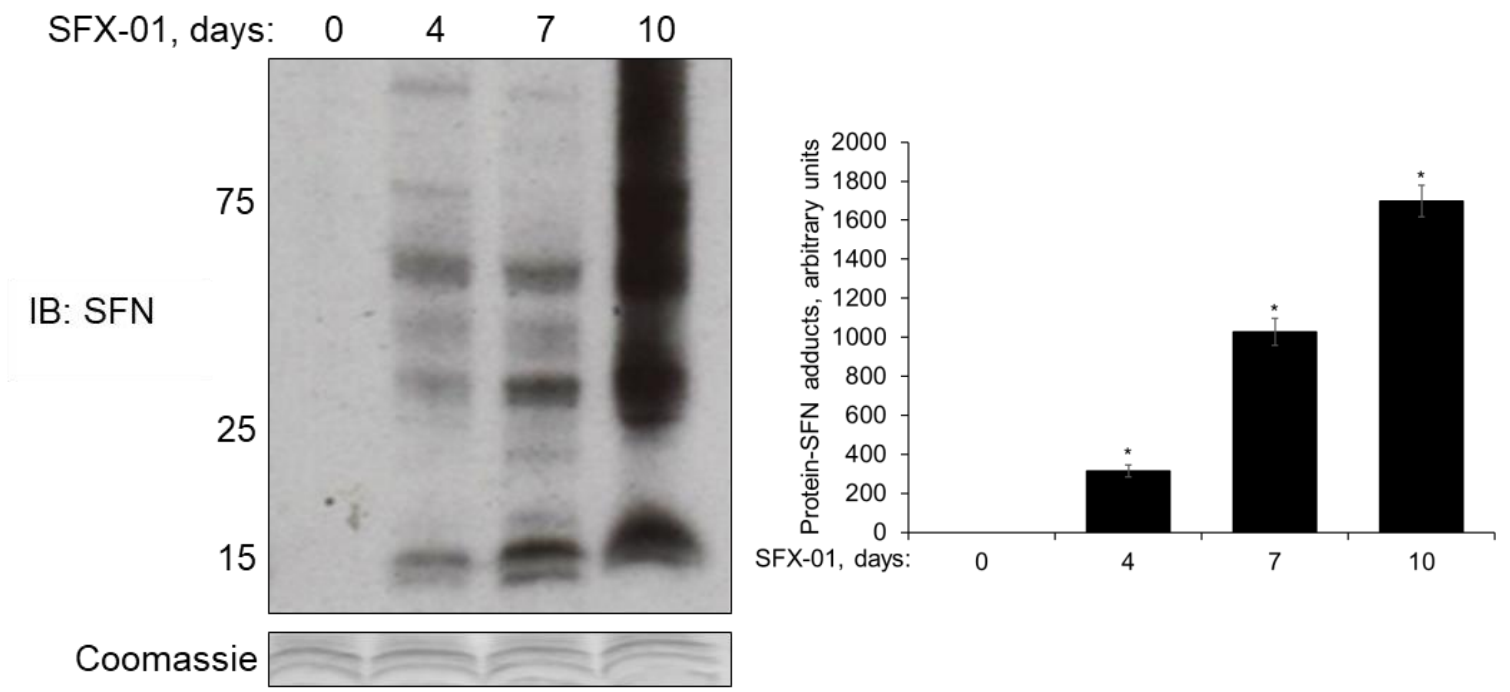

Figure 3.11. Protein-SFN adducts accumulate in vivo in a time-dependent manner.

A, B) Immunoblots showing time-dependent increase in protein-SFN adducts in the cardiac tissue of WT or NS mice after receiving $2.5 \mathrm{mg} / \mathrm{ml} \mathrm{SFX-01} \mathrm{chronically} \mathrm{for} \mathrm{up} \mathrm{to} 10$ days in their drinking water. $\left(n=10,{ }^{*} p<0.05\right.$ versus water only control). 


\subsubsection{Chronic SFX-01 treatment inhibits SHP2 phosphatase activity in vivo}

SHP2 was immunoprecipitated from the cardiac tissue of WT or NS mice following 4 days treatment with $2.5 \mathrm{mg} / \mathrm{ml} \mathrm{SFX-01} \mathrm{in} \mathrm{their} \mathrm{drinking} \mathrm{water.} \mathrm{As} \mathrm{with}$ the previous study in which the drug was administered for 30 minutes, immunoblotting of the input and immunocaptured SHP2 identified comparable protein expression between WT and NS mice (figure 3.12 A) with SHP2 phosphatase activity basally greater in NS mice compared to WT (figure 3.12 B). However, unlike the 30-minute treatment, inhibition of SHP2 phosphatase activity was observed following treatment with $2.5 \mathrm{mg} / \mathrm{ml} \mathrm{SFX-01} \mathrm{for} 4$ days in WT or NS mice (figure $3.12 \mathrm{~B}$ ). Indeed, 4 days of SFX-01 treatment nearly normalised the hyperactive SHP2 in the NS mice to WT levels. 
A)

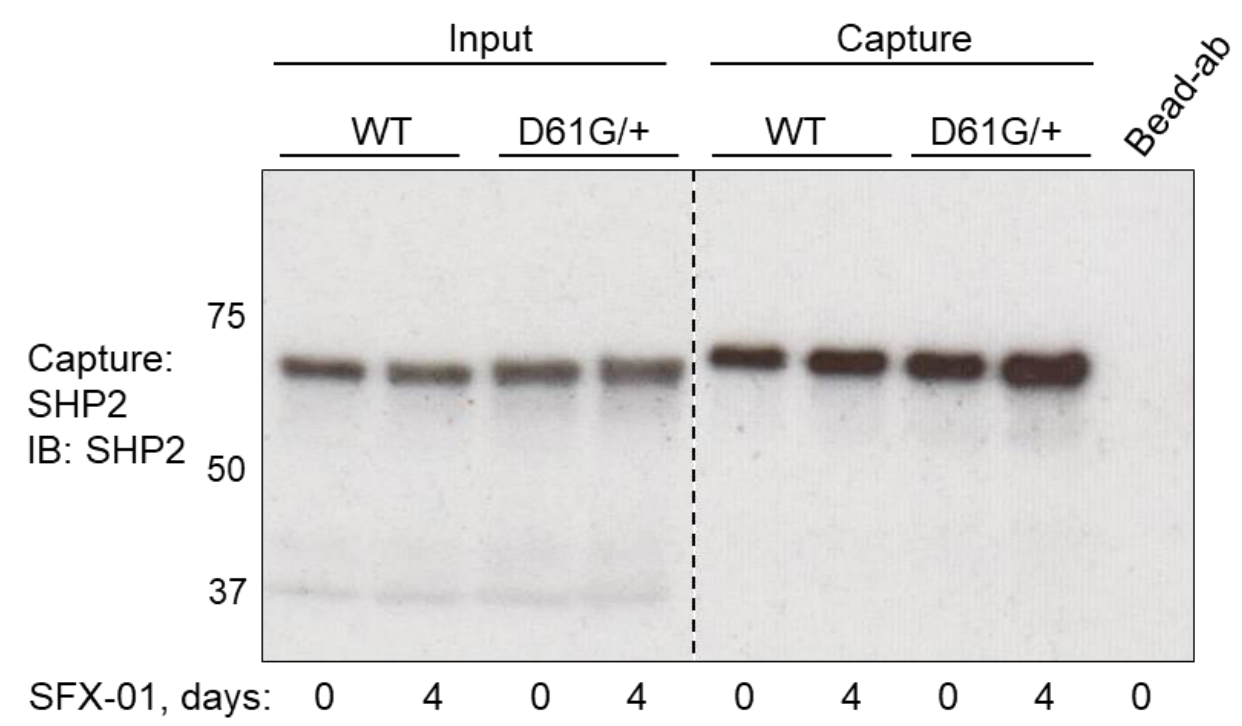

B)

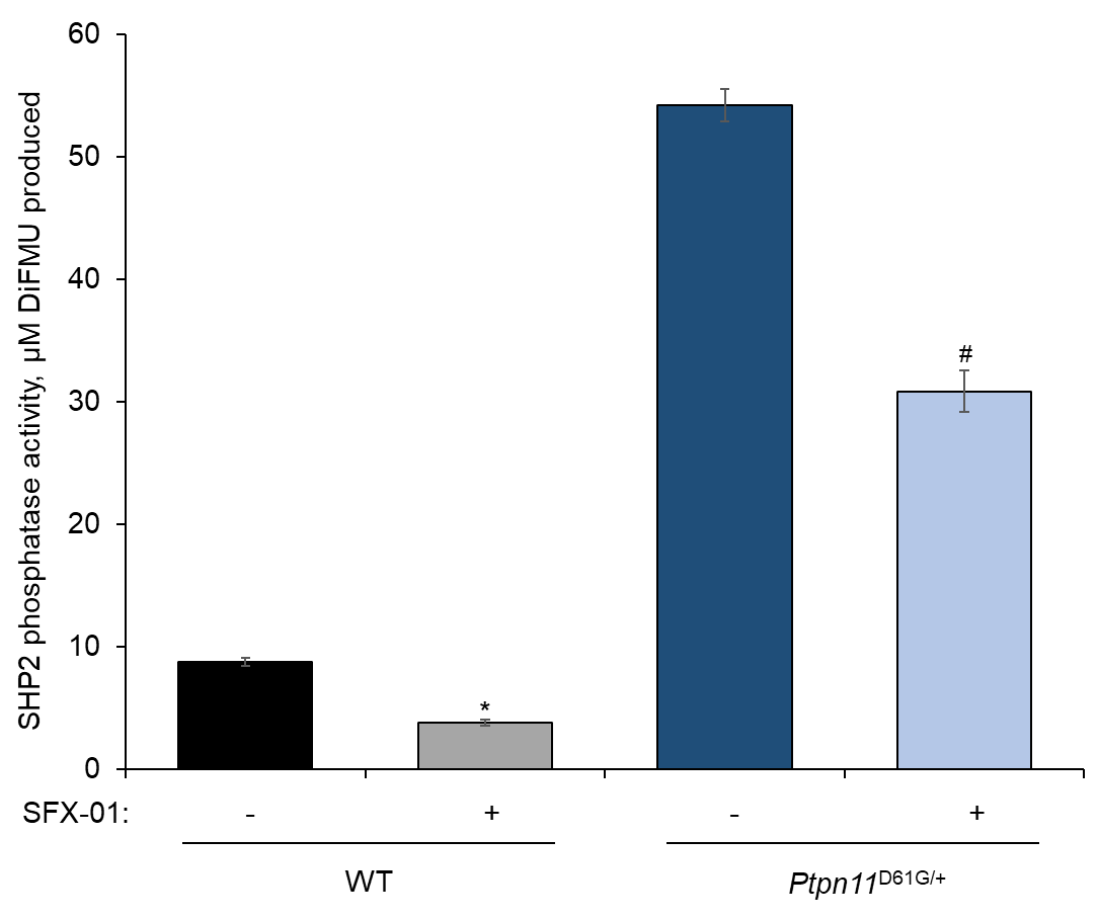

Figure 3.12. 4-day treatment with SFX-01 inhibits cardiac SHP2 phosphatase activity.

A) An immunoblot of input and capture samples following immunoprecipitation of SHP2 from cardiac tissue of WT or NS mice after receiving $2.5 \mathrm{mg} / \mathrm{ml} \mathrm{SFX-01} \mathrm{for} 4$ days in their drinking water. B) A fluorescence-based phosphatase activity assay using immunoprecipitated SHP2 from cardiac tissue shows inhibition of SHP2 phosphatase

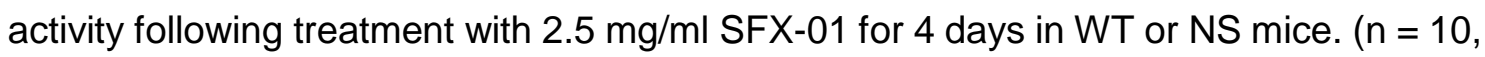
${ }^{*}$ and $\# p<0.05$ versus water only control of the same genotype). 


\subsubsection{Treatment with clinically-relevant amounts of SFX-01 inhibits SHP2}

phosphatase activity

The amount of SFX-01 used in previously described in vivo experiments where mice received the drug for 4-10 days was higher than those currently used by Evgen Pharmaceuticals in their clinical trials in humans. Thus, WT mice were orally gavaged with varying concentrations $(0-500 \mathrm{mg} / \mathrm{kg})$ of SFX-01 and 3 hours later cardiac tissue was harvested for analysis. SFX-01 caused inhibition of SHP2 activity only at $500 \mathrm{mg} / \mathrm{kg}$ (figure $3.13 \mathrm{~A}$ ). Subsequent studies in which varying amounts of the drug, which was approximately $0-500 \mathrm{mg} / \mathrm{kg}$, was provided for 7 days in the drinking water resulted in inhibition of the phosphatase as low as approximately $5 \mathrm{mg} / \mathrm{kg}$ (figure $3.13 \mathrm{~B}$ ). SHP2 immunoprecipitated from mice administered $2.5 \mathrm{mg} / \mathrm{ml}$ SFX-01 for 7 days in their drinking water served as a positive control. 
A)

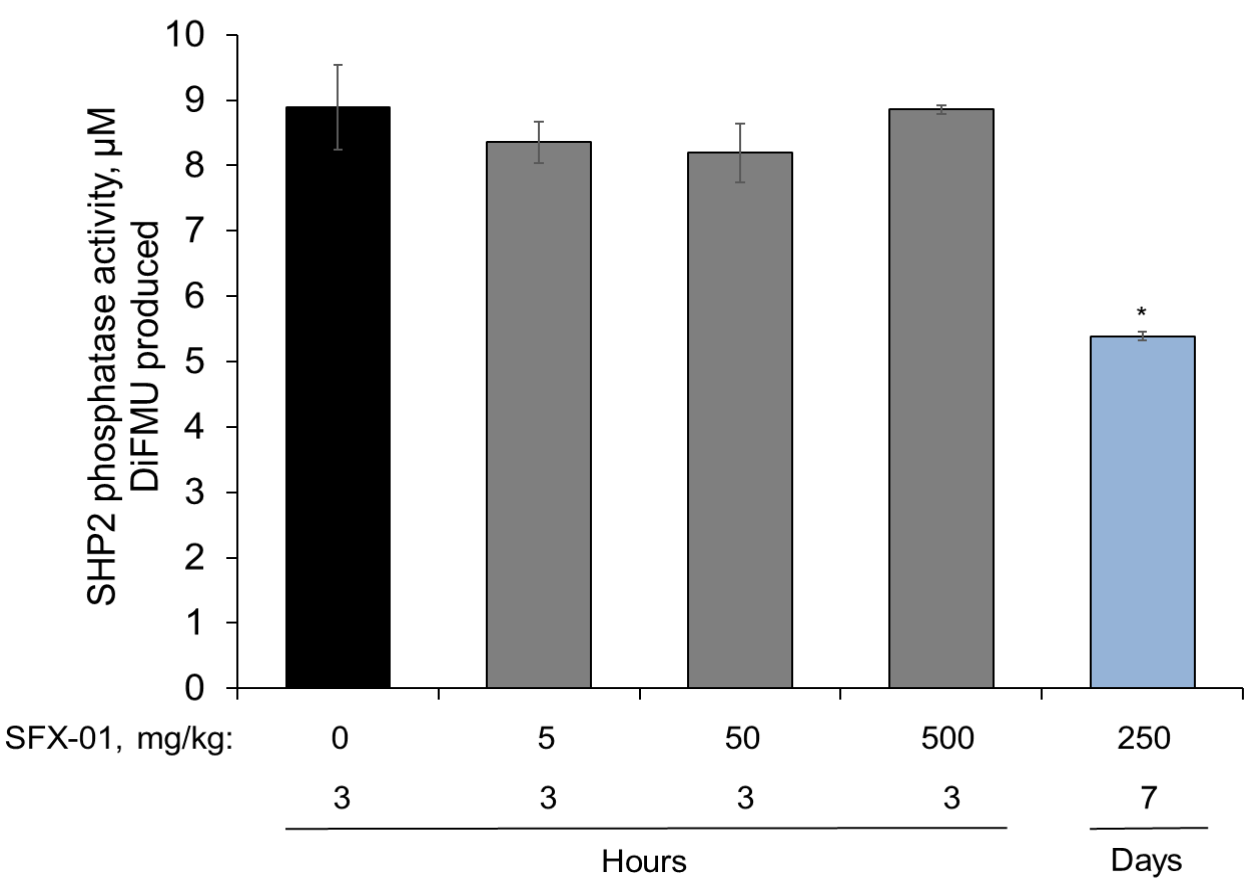

B)

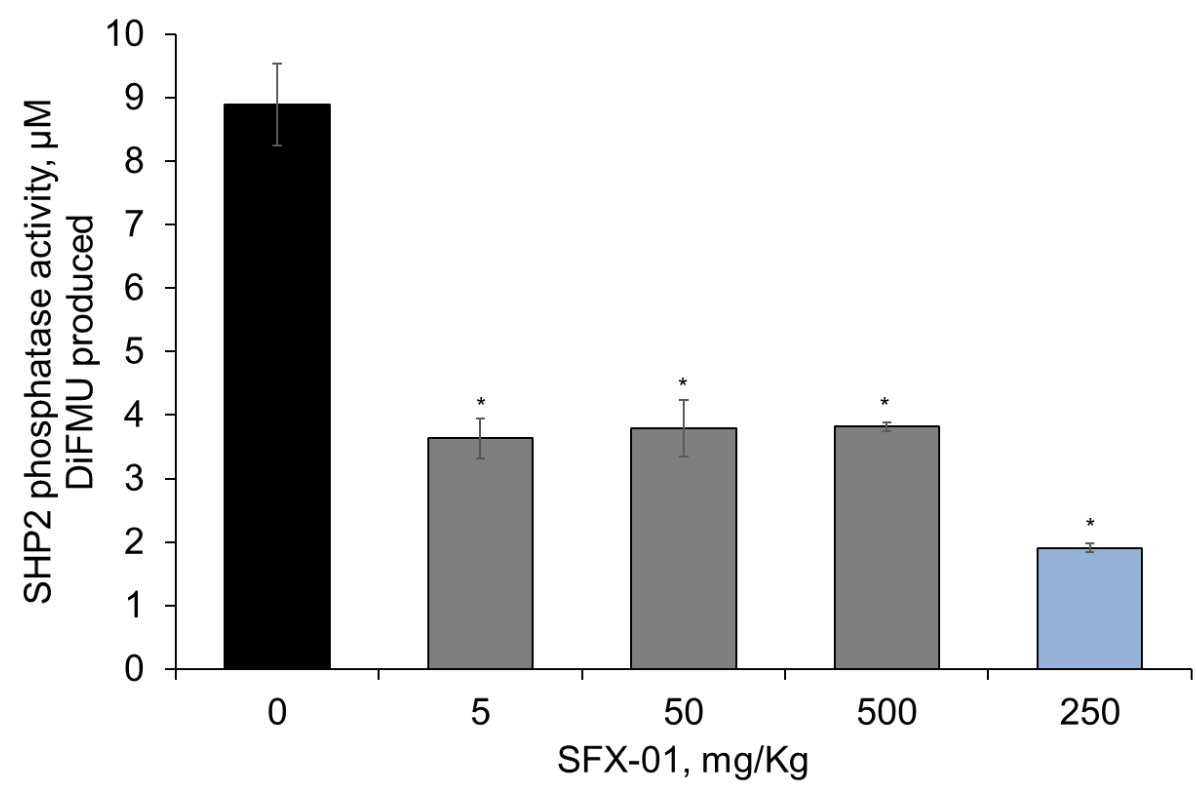

Figure 3.13. Treatment with SFX-01 at clinically-relevant concentrations inhibits SHP2 activity.

A, B) Fluorescence-based phosphatase activity assays using SHP2 immunoprecipitated from cardiac tissue of WT mice showing inhibition in activity either 3 hours post-oral gavage with $500 \mathrm{mg} / \mathrm{kg}$ SFX-01 (A) or approximately 5-500 mg/kg SFX-01 when received in their drinking water for 7 days (B). Positive control was derived from mice

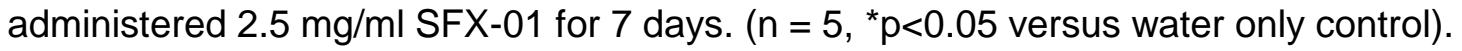




\subsubsection{Chronic SFX-01 treatment increases global tyrosine phosphorylation}

Immunoblotting with a pan-specific anti-phospho-tyrosine antibody revealed an increase in tyrosine phosphorylation of multiple proteins in cardiac tissue of WT mice following 4,7 or 10 days SFX-01 treatment at $2.5 \mathrm{mg} / \mathrm{ml}$ in their drinking water (figure 3.14).

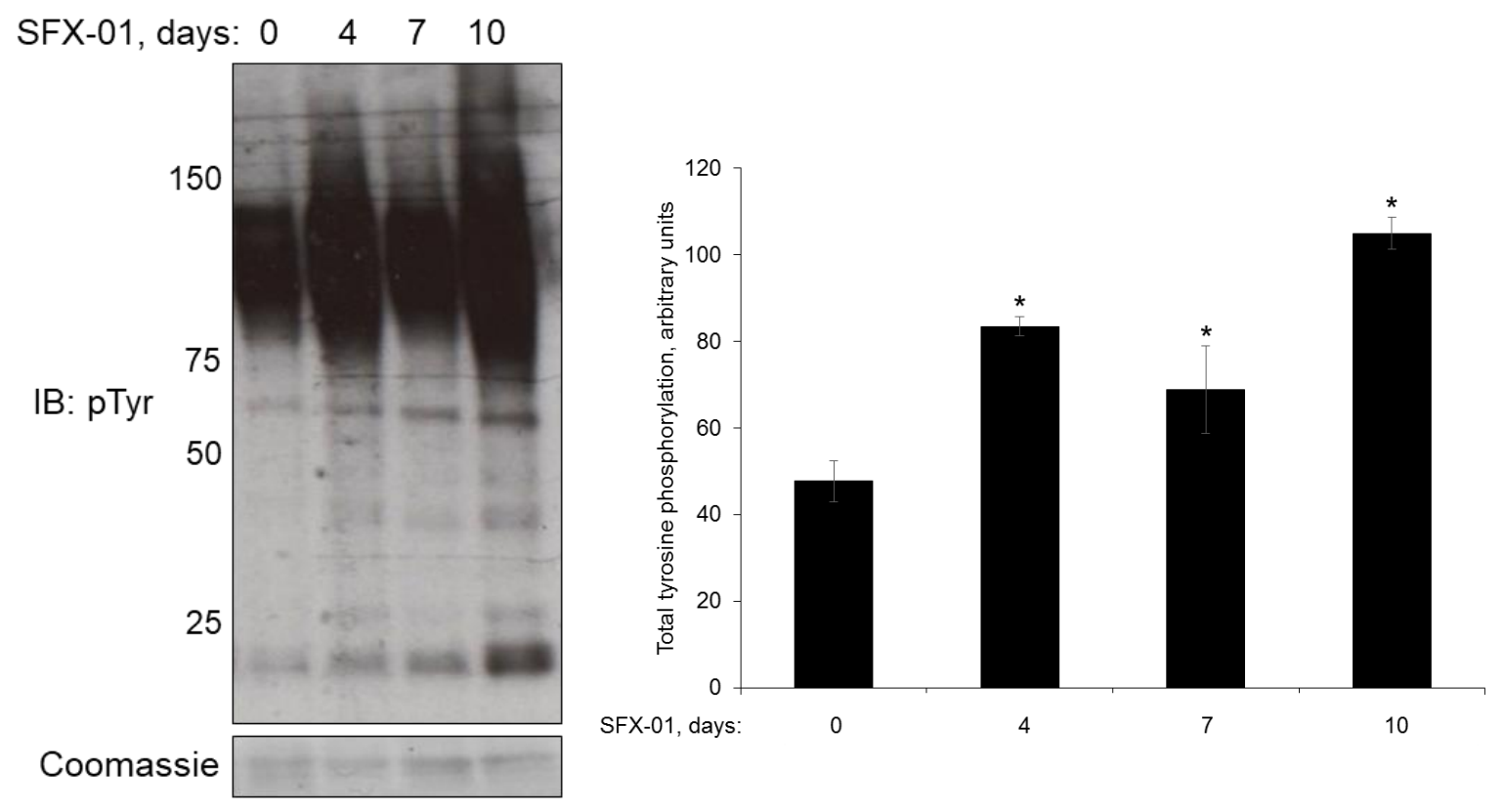

Figure 3.14. SFX-01 treatment increases global tyrosine phosphorylation.

An immunoblot showing an increase in global tyrosine phosphorylation in cardiac tissue of WT mice administered $2.5 \mathrm{mg} / \mathrm{ml} \mathrm{SFX-01} \mathrm{in} \mathrm{their} \mathrm{drinking} \mathrm{water} \mathrm{for} \mathrm{up} \mathrm{to} 10$ days. (n $=6,{ }^{*} \mathrm{p}<0.05$ versus water only control).

\subsubsection{Chronic SFX-01 treatment increases ERK phosphorylation}

Immunoblotting showed ERK phosphorylation was increased in the cardiac tissue of WT mice that received $2.5 \mathrm{mg} / \mathrm{ml} \mathrm{SFX-01} \mathrm{in} \mathrm{their} \mathrm{drinking} \mathrm{water,} \mathrm{but}$ only at the 10-day time point. A trend towards an increase was also notable after 7 days of treatment, however, this did not reach statistical significance. Over the 
duration of the chronic treatment, ERK protein expression did not change (figure 3.15).
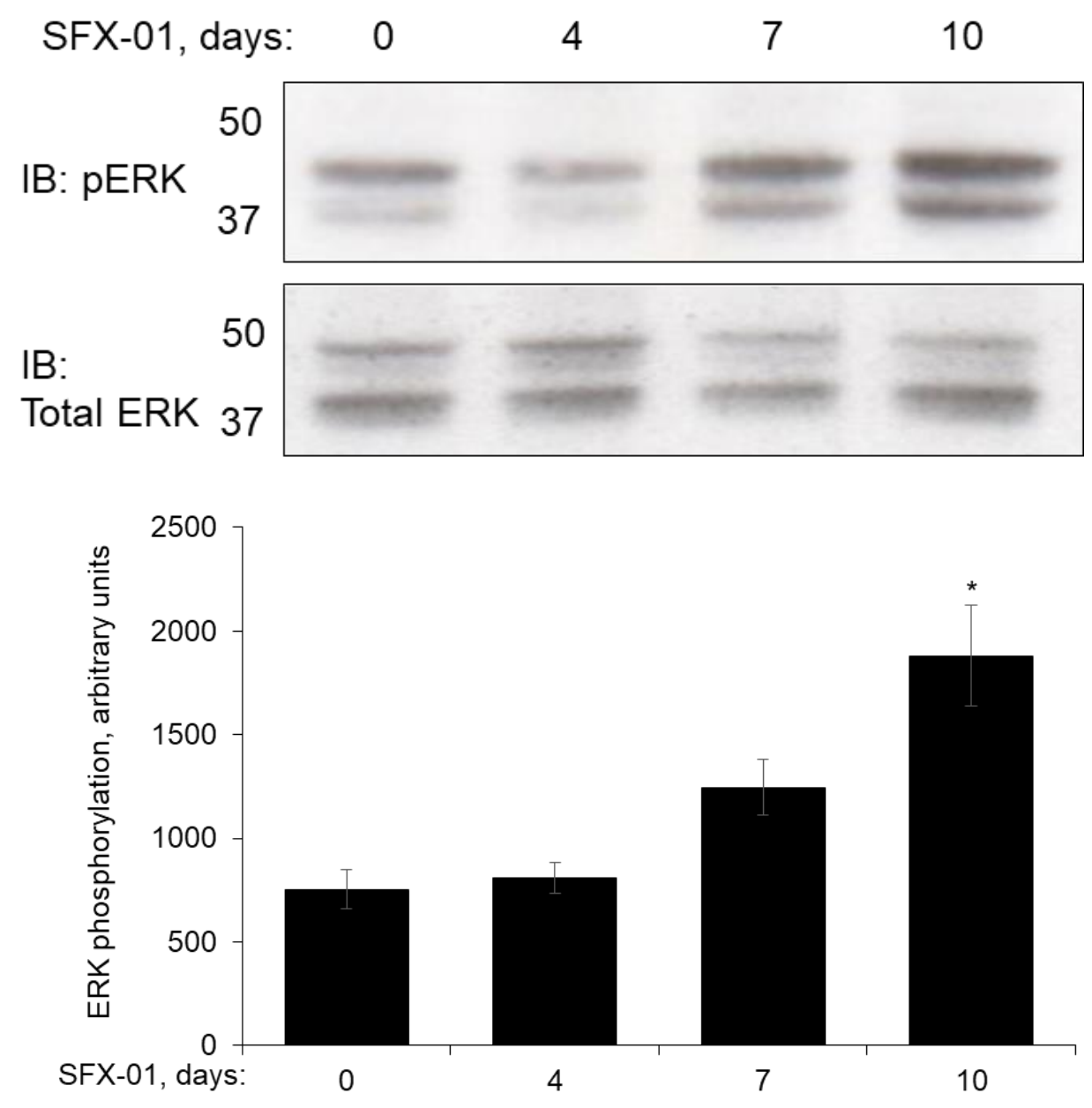

Figure 3.15. SFX-01 treatment increases ERK phosphorylation over time.

An Immunoblot showing increased ERK phosphorylation in cardiac tissue of WT mice following treatment with $2.5 \mathrm{mg} / \mathrm{ml} \mathrm{SFX-01}$ for 10 days in their drinking water. $(n=5$, ${ }^{*} p<0.05$ versus water only control).

\subsubsection{SFX-01 or rmGH treatment increases STAT5 phosphorylation}

As expected, phosphorylation of STAT5 was increased in the cardiac tissue of WT mice 30 minutes after subcutaneous injection of $70 \mu \mathrm{g} \mathrm{rmGH}$. STAT5 phosphorylation was also increased 30 minutes after an intraperitoneal injection 
of $2.5 \mathrm{mg} / \mathrm{ml} \mathrm{SFX-01,} \mathrm{although} \mathrm{to} \mathrm{a} \mathrm{lesser} \mathrm{extent} \mathrm{than} \mathrm{rhGH}$. Combination treatment with $70 \mu \mathrm{g} \mathrm{rmGH}$ and $2.5 \mathrm{mg} / \mathrm{ml} \mathrm{SFX-01} \mathrm{did} \mathrm{not} \mathrm{potentiate} \mathrm{STAT5}$ phosphorylation compared to $\mathrm{rmGH}$ alone at any time point examined. Phosphorylation decreased over time in all treatment groups, although it remained elevated compared to control 120 minutes after treatment with $\mathrm{rmGH}$ with or without SFX-01 (figure 3.16).
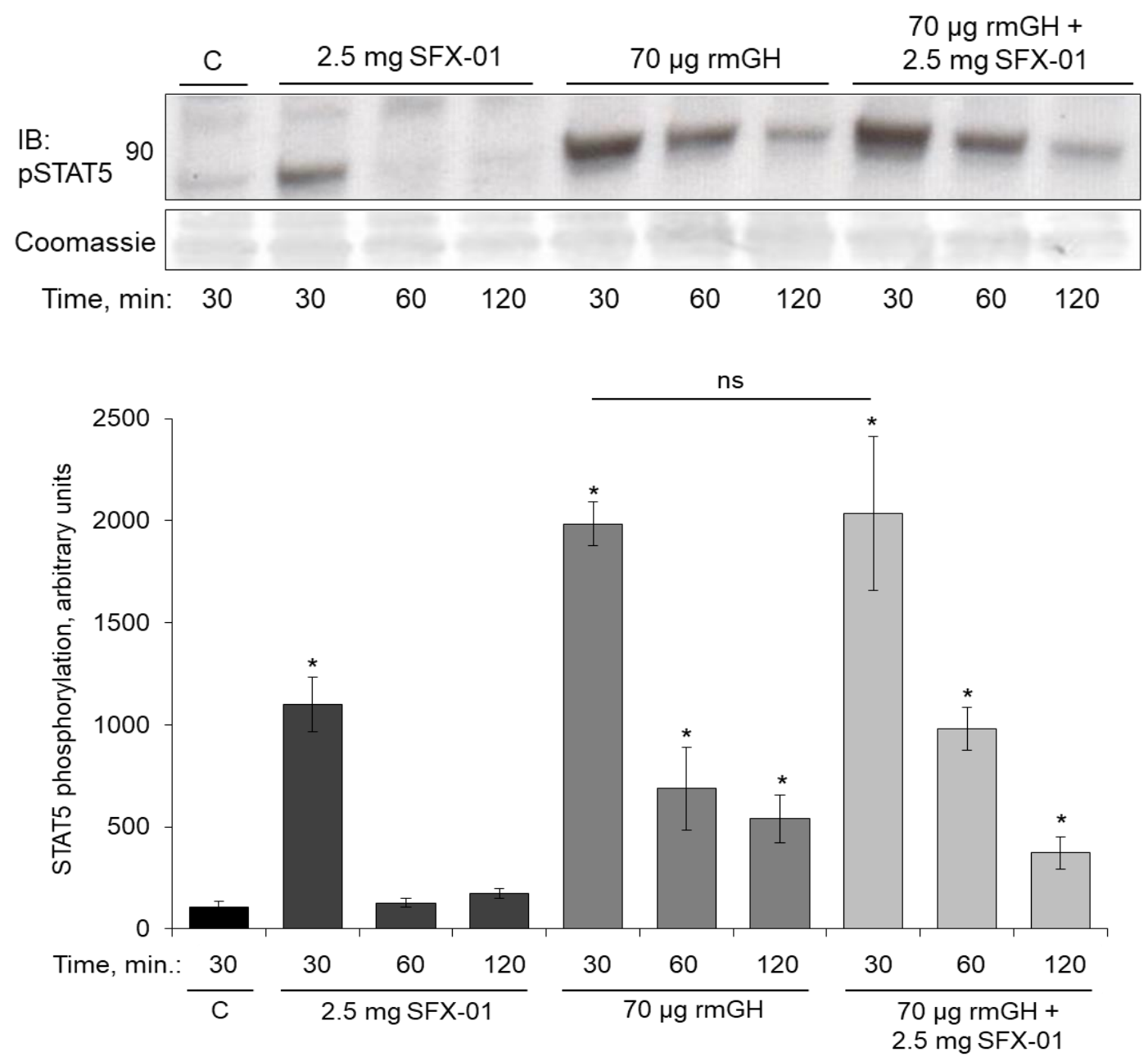

Figure 3.16. SFX-01 and rmGH increase STAT5 phosphorylation.

An immunoblot showing increased cardiac STAT5 phosphorylation 30 minutes following a single intraperitoneal injection of $2.5 \mathrm{mg} \mathrm{SFX-01} \mathrm{or} \mathrm{a} \mathrm{single} \mathrm{subcutaneous} \mathrm{injection} \mathrm{of}$ $70 \mu \mathrm{g} \mathrm{rmGH}$ to WT mice. STAT5 phosphorylation was not potentiated when SFX-01 was co-administered with $70 \mu \mathrm{gmGH}$. In all treatment groups, STAT5 phosphorylation decreased over time. $\left(n=3,{ }^{*} p<0.05\right.$ versus water control). 


\subsubsection{STAT5 phosphorylation by $40 \mu \mathrm{g} \mathrm{rmGH}$ is potentiated by SFX-01}

Previous in vivo studies showed SFX-01 did not potentiate STAT5 phosphorylation induced by $70 \mu \mathrm{g} \mathrm{rmGH}$. This experiment was repeated, but a lower $40 \mu \mathrm{g}$ dose of subcutaneously injected $\mathrm{rmGH}$ was used, as the $70 \mu \mathrm{g}$ may have been maximally stimulating STAT5 phosphorylation. As with the previous study, immunoblotting revealed an increase in STAT5 phosphorylation in cardiac tissue of WT mice 30 minutes following intraperitoneal injection of $2.5 \mathrm{mg} \mathrm{SFX-}$ 01. $40 \mu \mathrm{g} \mathrm{rmGH}$ alone increased STAT5 phosphorylation, and this was potentiated when SFX-01 was concomitantly administered (figure 3.17).
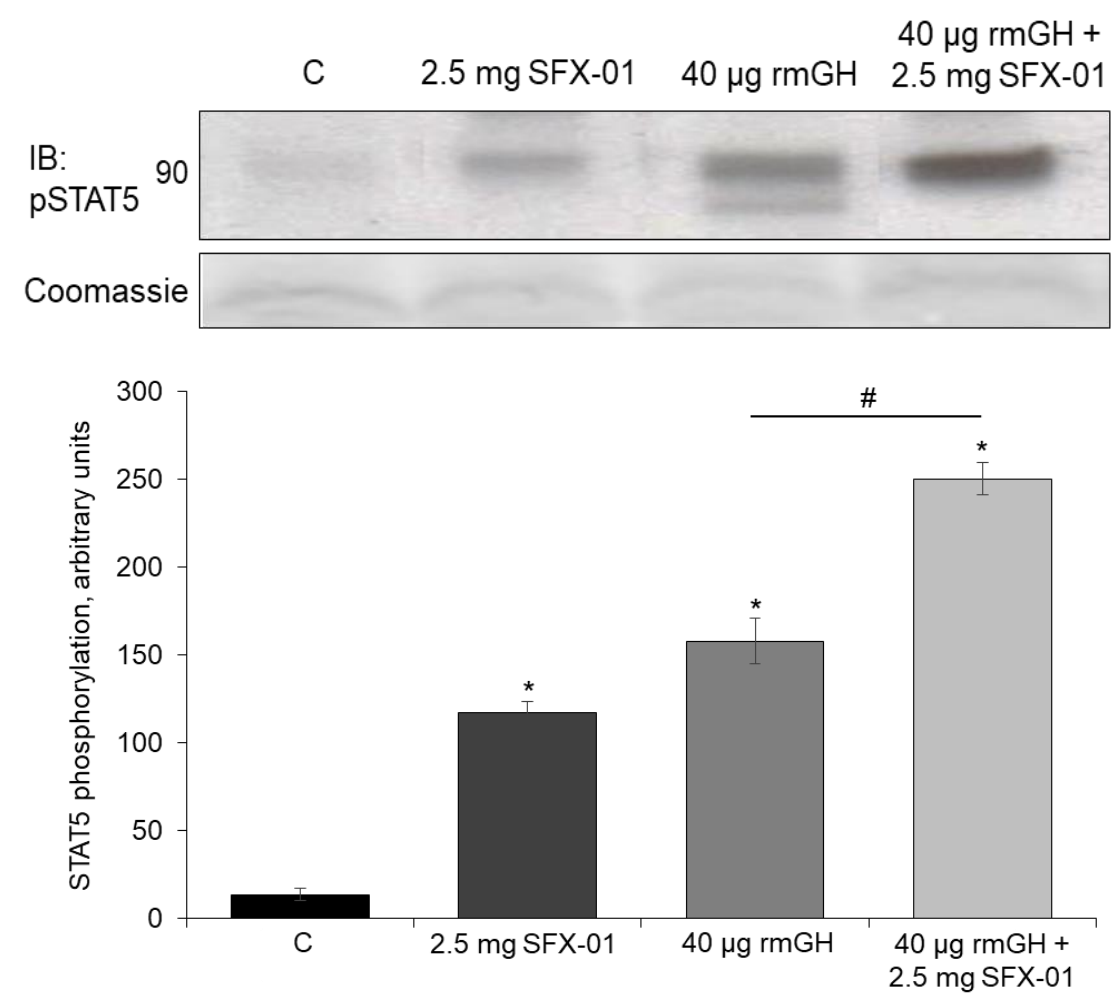

Figure 3.17. SFX-01 potentiates phosphorylation of STAT5 by $40 \mu \mathrm{g} \mathrm{rmGH}$.

An immunoblot showing increased cardiac STAT5 phosphorylation 30 minutes after a single intraperitoneal injection of $2.5 \mathrm{mg} \mathrm{SFX-01}$ or a single subcutaneous injection of $40 \mu \mathrm{g} \mathrm{rmGH}$ to WT mice. STAT5 phosphorylation was potentiated when a combined treatment of SFX-01 and rmGH was used at these amounts. $\left(n=3,{ }^{*} p<0.05\right.$ versus water control). 


\subsection{Discussion}

Studies are underway by multiple research groups to identify efficacious inhibitors of SHP2. Here, I investigated whether SHP2 was inhibited by SFN, which was rationally anticipated for the reasons outlined in the introduction of this chapter. Such inhibition could offer the prospect of a therapy for NS by normalising hyperactive SHP2 to WT levels.

Assessing the effect of SFN on SHP2 phosphatase activity required utilisation of a commercial, fluorescence-based phosphatase activity assay. Although the assay was established by the company, optimisation was required to determine the minimum amount of recombinant SHP2 protein to generate a consistently detectable signal from the fluorometer without the assay substrate becoming saturated. The experimental buffer for these experiments contained DTT, a reducing agent which resolves disulfide bonds via a disulfide-exchange mechanism (figure 3.18). DTT is essential within the storage and experimental buffer of recombinant SHP2 to maintain the catalytic and surrounding cysteines in a reduced state ${ }^{142}$, and as expected, desalting SHP2 into a solution with no DTT caused the protein to become air oxidised and inactive. As discussed in greater detail in chapter 4, SFN can 'trans-thiolate' and so move between thiolcontaining compounds and/or proteins ${ }^{99}$. As DTT is a dithiol-containing compound, I next optimised a minimal concentration in order to keep the protein reduced and active prior to the experiment, but also minimise the amount of excess thiol that SFN could potentially react directly with or trans-thiolate to or from. 

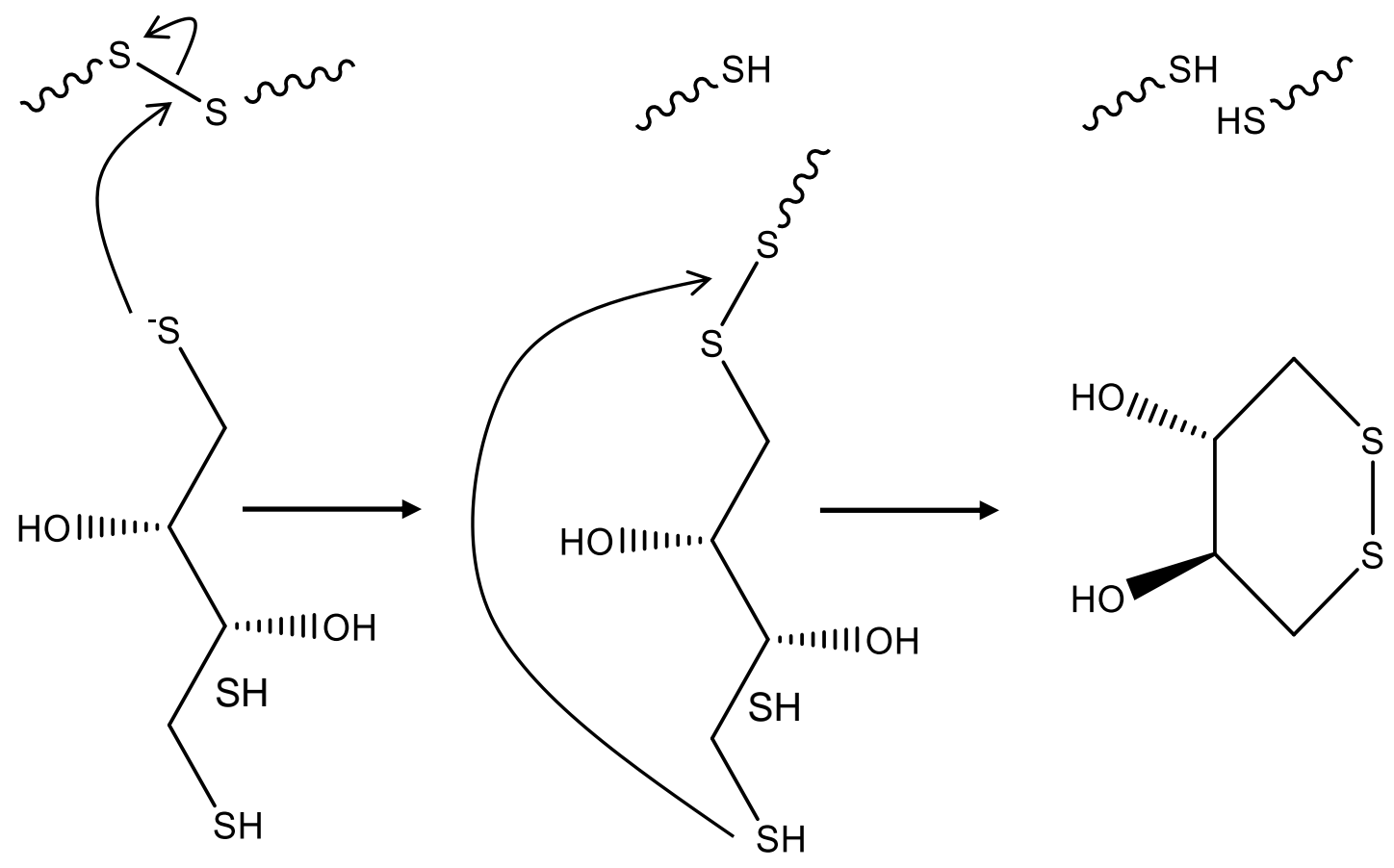

Figure 3.18. Protein-disulfide reduction by DTT.

DTT reduces disulfides via two sequential thiol-disulfide exchange reactions.

In line with the rationale presented in the introduction of this chapter, I observed the inhibition of SHP2 phosphatase activity by SFX-01 in vitro. At shorter time points inhibition was achieved in a concentration-dependent manner, likely due to more SFN being readily available to adduct the catalytic cysteine of SHP2 as the abundance of the electrophile increased. Indeed, I observed no adduction of SHP2 by SFN after a 30-minute incubation with the lowest concentration of the electrophile, whereas a high abundance of SHP2-SFN was detected at the two higher concentrations investigated. This correlates with previous observations from Lewis et al, who demonstrated that dietary ITCs, including allyl ITC and SFN, are concentration-dependent inhibitors of SHP2 phosphatase activity ${ }^{143}$. As well as this, they observed reduced inhibition of SHP2 in the presence of 
phosphate containing compounds. This is likely due to these phosphate groups being cleaved at the active site, occupying the catalytic cysteine and preventing inhibitory adduction by SFN. They therefore also hypothesised that ITC inhibition of SHP2 is active site-directed.

SFN also inhibited SHP2 in a time-dependent manner in vitro. I anticipate this was due to the covalent nature of the SHP2-SFN interaction. Covalent versus non-covalent drug-protein interactions are considered in detail in the general introduction. Non-covalent drug-protein interactions are reversible, and this generates an equilibrium between a protein-bound and -unbound state. Therefore, this class of drug typically results in an eventual plateau in protein inhibition. Compounds with high electrophilicity such as SFN, on the other hand, form covalent interactions with proteins which are typically stable and permanent, or only slowly reversed. Extended periods of time increase the opportunity for SFN to adduct the catalytic cysteine of SHP2, and if this interaction is indeed permanent this may explain the time-dependent accumulation in inhibition of the phosphatase. Indeed, incubation with low amounts of SFX-01 for longer durations resulted in the same level of inhibition of SHP2 phosphatase activity as when incubated with higher amounts for a shorter time period. This was also visualised by immunoblotting as adduction of SHP2 by SFN required 2-hour incubation with the lowest concentrations of the electrophile. This cumulative effect also results in differences in $\mathrm{IC}_{50}$ values, which range from 47.28 to 0.49 $\mu \mathrm{M}$ depending on the duration that the phosphatase was exposed to SFN. Similar observations are also documented by the Darley-Usmar group, whereby prolonged treatment (days) with the electrophile 15-deoxy- $\Delta^{12,14}$-prostaglandin $\mathrm{J}_{2}$ resulted in accumulative adduction of the signalling protein KEAP1 ${ }^{144}$. However, 
if accumulative adduction of a stable SFN adduct upon SHP2 were the sole mechanism for the time-dependent increase in inhibition of the phosphatase then I would have expected to see cumulative protein-adduct formation as measured using immunoblotting. In fact, the formation of SHP2-SFN was followed by a timedependent loss of the adduct even though the proteins phosphatase activity remained inhibited. A detailed hypothesis and proposed mechanism for SFNinduced inhibition of SHP2 are outlined in chapter 6.

I next wanted to establish if inhibition of the phosphatase by SFN could be achieved in vivo using a mouse model for NS with hyperactive SHP2 activity. Since clinical drugs are typically administered in a single, daily bolus dose I used this delivery approach of SFX-01 for initial experiments. However, even though multiple protein-SFN adducts were identified, adduction or inhibition of SHP2 by SFN was not observed. Due to the findings of the preceding in vitro experiments I speculated that a lower amount of SFX-01 administrated continually over a longer duration may provide the time needed for SFN to adduct and inhibit low abundance proteins such as SHP2; consequently, my experimental design was altered accordingly. The method of continual drug administration was by addition to the drinking water, which unlike repeated oral gavage offered a stress-free and non-invasive delivery approach. SFX-01 is orally bioavailable, and it was not rational to administer using osmotic minipumps, as this was considered a backward step in terms of developing the compound for clinical use. Also, the amount of SFX-01 that we delivered over a sustained period cannot be accommodated in a single minipump. Cardiac SHP2 phosphatase activity was inhibited in WT or NS mice following administration of lower amounts of SFX-01 in this chronic manner. Again, this provides evidence that prolonged treatment 
with low doses of SFN allows time for the electrophile to adduct and inhibit SHP2. This inhibition was maintained up to 10 days of treatment, which was perhaps due to the covalent nature of the interaction. Although, this mechanism of inhibition is challenged and reviewed in detail in chapter 6. As drug metabolism is notably higher in mice than in humans ${ }^{145} 146$ the amount of SFX-01 given throughout the chronic in vivo study was $\sim 5$-fold that used in clinical trials; $~ 250$ $\mathrm{mg} / \mathrm{kg}$ compared to $50 \mathrm{mg} / \mathrm{kg}$. However, importantly, I also established that SHP2 phosphatase activity was significantly inhibited in cardiac tissue of WT mice following 7 days treatment of SFX-01 in drinking water at a concentration as low as $\sim 5 \mathrm{mg} / \mathrm{kg}$. Again, these data provide evidence for electrophiles achieving biological effects when administered at low amounts over prolonged treatment times. Importantly, due to its requirement in essential signalling pathways, levels of SHP2 phosphatase activity were not completely reduced under any of these experimental conditions and in fact, the activity of the phosphatase in NS mice was reduced to levels comparable to their untreated WT littermates.

After establishing SFN induced inhibition of SHP2 activity I next assessed changes in signalling events downstream of the phosphatase. As SHP2 is a tyrosine phosphatase, it is logical to assume that increased global tyrosine phosphorylation following SFX-01 treatment was due, at least in part, to its inhibition. The adduction of SFN to proteins other than SHP2 also likely contributes to this biological effect and perhaps the electrophile could adduct and inhibit other PTPs with essential catalytic cysteines.

Oxidative modification of the catalytic cysteines of PTPs by ROS can also inhibit their activity, as outlined in chapter 6 . Several studies have identified SFN can increase cellular ROS 147148 149, which I speculate may involve the antioxidant 
GSH. Due to its high cytoplasmic abundance, GSH is readily available to adduct free-SFN before the electrophile can interact with other target proteins. GSH plays a major role in the removal of ROS. Therefore, although SFN induces production of this antioxidant via activation of the KEAP1/Nrf2 signalling pathway, the pool of reactive GSH is overall reduced due to adduction by the electrophile. This resulting SFN-induced increase in cellular ROS may be contributing to increased global tyrosine phosphorylation by inhibiting an array of PTPs.

SFN-induced upregulation in gene expression of many kinases via the KEAP1/Nrf2 signalling pathway, such as phosphatidylinositol 3 and 4 , protein kinase $\mathrm{C}$ and MAP kinase kinase kinase 4 and 5 has also been documented and may be another explanation for the increase in protein phosphorylation ${ }^{150}$.

SHP2 is a positive regulator of the Ras/ERK signalling pathway through mechanisms outlined in the introduction of this chapter and I rationally anticipated that SFN-induced inhibition of the phosphatase's activity would result in decreased phosphorylation and activation of ERK. The reverse effect was observed, with SFX-01 treatment inducing an increase in ERK phosphorylation in cardiac tissue. These data correlate to an accumulating body of evidence showing SFN-induced increase in ERK phosphorylation in multiple cell lines, including liver carcinoma, cortical neuronal, intestinal epithelial and glioblastoma 151152153154 . Perhaps SFN-induced inhibition of SHP2 does result in a decrease in SHP2-stimulated ERK phosphorylation due to a resulting increase in RasGAP, Sprouty and Csk activity, but these effects may be outweighed by regulation of other SHP2-dependent and -independent mechanisms which induce phosphorylation of the kinase, as described below. 
SHP2 positively regulates IGF1-induced activation of Akt 15572 156. It has been shown that following its activation, Akt can phosphorylate RAF at Ser ${ }^{259}$ in its amino-terminal regulatory domain, which inhibits the protein 157 and prevents RAF from subsequently phosphorylating ERK. Perhaps SFN-induced inhibition of SHP2 reduces Akt activation and prevents its negative regulation of ERK phosphorylation.

SFN may also increase ERK phosphorylation independent of SHP2 by increasing levels of cellular ROS. I have outlined a proposed mechanism for SFN-induced increase in ROS in detail above. ROS produced in an IGF1 dependent manner activate GFRs resulting in downstream stimulation of ERK signalling as well as other MAPKs ${ }^{158}{ }^{159}$. A common feature of GFRs is cysteine-rich motifs that are susceptible to modification by ROS, which may be responsible for driving receptor activation. Perhaps another mechanism by which oxidative stress increases ERK phosphorylation is altering the activity of MAPK phosphatases. MAPK phosphatase 1 is inhibited by $\mathrm{H}_{2} \mathrm{O}_{2}$-induced sulfenic acid intermediates upon its catalytic cysteine which results in increased phosphorylation of ERK, its primary substrate ${ }^{160}$. As well as this, activation of protein kinase $\mathrm{C} \delta$ by glutamate-induced ROS stimulates degradation of MAPK phosphatase 1 via a ubiquitin-proteasome pathway ${ }^{161}$. Finally, although the mechanism remains unclear, ROS can also induce phosphorylation of MEK 1/2 and increase downstream ERK phosphorylation 162. SFN itself also carries oxidant-like properties as it can directly adduct thiolates and inhibit protein function, much like $\mathrm{H}_{2} \mathrm{O}_{2}$. Therefore, perhaps the ROS-induced mechanism of ERK phosphoactivation considered above can also be achieved through direct adduction of mentioned protein cysteines by SFN. 
SHP2 is a negative regulator of the JAK2/STAT5 signalling pathway and finally, I assessed the impact of SFX-01 treatment on this cascade. As GHs directly bind GHRs and stimulate signalling, it was as expected that rmGH treatment alone increased STAT5 phosphorylation in cardiac tissue. One observation is a peak in STAT5 phosphorylation 30 minutes post-rmGH treatment followed by a relatively rapid decline. Studies in both rats and primary hepatocytes have also shown that high levels of circulating GH result in a peak in signalling followed by fast induction of down-regulation ${ }^{163}{ }^{164}$. Including dephosphorylation of JAK2 by SHP2, GH signalling has multiple negative feedback mechanisms, such as ligand-induced endocytosis of the GHR ${ }^{165}$, and upregulation of a family of cytokine-inducible suppressors of cytokine signalling (SOCS) ${ }^{166}$. SOCS proteins dock phospho-tyrosine residues on both JAK2 and GHRs which interferes with STAT5 binding and induces degradation of the receptor complex. Either of these negative feedback mechanism may explain the rapid decline in STAT5 phosphorylation ${ }^{167}$.

An increase in STAT5 phosphorylation followed by a time-dependent decline was also observed following treatment with SFX-01. I speculate that SFN-induced inhibition of SHP2 activity may be responsible for this increase in STAT5 phosphorylation, as the phosphatase could no longer dephosphorylate JAK2.

As explained in the results section of this chapter, two in vivo experiments were conducted using different amounts of rmGH. At higher amounts, no potentiation in STAT5 phosphorylation induced by rmGH was observed following combined treatment with SFX-01. I speculated that when mice were given high amounts of hormone, even though SFX-01 may be inhibiting SHP2 activity, JAK2 phosphorylation cannot be increased as it is already saturated by high levels of 
GH stimulation. Indeed, combined treatment with SFX-01 using less rmGH resulted in potentiated STAT5 phosphorylation compared to rmGH alone.

Interestingly, studies in rats showed that low levels of circulating GH result in maximal activation of the JAK/STAT signalling pathway ${ }^{168}$. This low level of stimulation may avoid a rapid increase in negative regulation by the mechanisms explained above. Perhaps the lower levels of GH utilised in my latter experiment prevented induction of such negative feedback loops and heightened potentiation of the combined treatment. With this in mind, it may have been beneficial to examine longer time points to determine whether sustained activation of the pathway can be detected.

These findings are, however, contradictory to previous studies which found decreased STAT3 or STAT5 phosphorylation in human nasopharyngeal, prostate and lymphocyte cancer cell lines following treatment with SFN 169170171. Although notably, these studies were all conducted using human cancer cell lines, which may have basally altered signalling mechanisms and activity and are not comparable to the murine cardiac tissue analysed in my experiments which predominantly consists of non-proliferative cardiomyocytes. For example, mRNA expression of GHR is elevated in human prostate cancer cell lines where rapid induction of JAK2/STAT5 phosphorylation by GH is detected ${ }^{172}$. Secondly, in proliferating cells, including a range of cancer cell lines, GHs induce a high level of nuclear localisation of GHRs where they can bind to transcriptional regulators themselves and upregulate target genes without driving STAT5 phosphorylation 173174 


\section{Sulforaphane can trans-thiolate between}

\section{proteins}

\subsection{Introduction}

\subsubsection{SFN adducts are reversible}

Adduction of ITCs to intracellular GSH is the first step in their metabolism ${ }^{175}$. In most cases, this adduction is permanent and results in urinary excretion of subsequent metabolites. However, the resultant dithiocarbamate can also sporadically reverse i.e. the resolution of the adduct to regenerate reactive forms of the thiol and SFN, with no catalysis by another protein or chemical ${ }^{176} 177$. Bruggeman et al found that treatment of rat liver cells with certain ITCs was cytotoxic and these same detrimental effects were seen when treated with GSHITC, where there was the apparent release of the ITC at the plasma membrane 178. They also showed that 2 hours post addition of L-cysteine to GSH-ITC, $80 \%$ of total conjugates were L-cysteine-ITC and vice versa. This process is widely regarded as non-enzymatic, although increased catalysis has been observed with the presence of GSH transferases 179180 . It is therefore recognised that adduction to GSH does not always result in detoxification of the ITC. This is not to say that reversal of the adduct occurs only intracellularly. GSH-ITC can be rapidly excreted from the cell via MRP1 and/or Pgp1, as shown in the general introduction and reversal may, therefore, occur within the bloodstream or following transportation across cellular membranes into other tissues. Irrespective of location, regenerated electrophile is again available to react with free thiols ${ }^{181}$. The Uchida group have also shown using MS analysis that as well as the spontaneous reversal of a GSH-SFN interaction, the electrophile can 
actively transfer directly from a thiol-containing protein to $\mathrm{GSH}$ without the generation of free-SFN ${ }^{182}$. This mechanism, termed 'trans-thiolation' in this document, involves direct nucleophilic attack of the reactive thiolate of GSH to the protein-SFN interaction. These data, therefore, suggest the transient movement of an SFN adduct between protein thiols, which may or may not require the flow of the electrophile to and from GSH to aid transport.

In chapter 3 I reported a time-dependent increase in protein-SFN adducts in the cardiac tissue of WT or NS mice following treatment with SFX-01 for 4-10 days. Here liquid chromatography (LC) with tandem (MS/MS) analysis was utilised to identify SFN-adducted proteins of interest following treatment with SFX-01 or broccoli sprouts, a rich source of naturally occurring SFN. Subsequent biochemical analysis was used to investigate if the transient movement of the SFN adduct was responsible for such accumulation upon these proteins, which serve as a sink for the electrophile. 


\subsection{Materials and methods}

\subsubsection{SDS-PAGE and western immunoblotting}

SDS-PAGE and western immunoblotting were performed as outlined in the general methods. For this chapter the following primary antibodies were used:

\begin{tabular}{|l|l|l|}
\hline Primary antibody & Company & Species \\
\hline Sulforaphane & In-house & Rabbit \\
\hline Thioredoxin & Abcam \#ab26320 & Rabbit \\
\hline Glutaredoxin 1 & Abcam \#ab45953 & Rabbit \\
\hline Haemoglobin & Abcam \#ab77125 & Mouse \\
\hline Haemoglobin subunit $\beta$ & Santa Cruz \#sc-31116 & Mouse \\
\hline
\end{tabular}

Table 4.1. List of primary antibodies used for western immunoblotting in chapter 4.

\subsubsection{Immunoprecipitation}

Immunoprecipitation of proteins from tissue was performed as outlined in the general methods. For this chapter the following antibodies were used: 


\begin{tabular}{|l|l|l|l|}
\hline Antibody & Company & Species & Capture beads \\
\hline Sulforaphane & In-house & Rabbit & $\begin{array}{l}\text { Protein A/G PLUS-Agarose } \\
\text { Santa Cruz \#sc-2003 }\end{array}$ \\
\hline Thioredoxin & $\begin{array}{l}\text { Abcam } \\
\text { \#ab26320 }\end{array}$ & Rabbit & $\begin{array}{l}\text { Protein A/G PLUS-Agarose } \\
\text { Santa Cruz \#sc-2003 }\end{array}$ \\
\hline Glutaredoxin 1 & $\begin{array}{l}\text { Abcam } \\
\text { \#ab45953 }\end{array}$ & Rabbit & $\begin{array}{l}\text { Protein A/G PLUS-Agarose } \\
\text { Santa Cruz \#sc-2003 }\end{array}$ \\
\hline Haemoglobin & $\begin{array}{l}\text { Abcam } \\
\text { \#ab77125 }\end{array}$ & Mouse & $\begin{array}{l}\text { Protein A/G PLUS-Agarose } \\
\text { Santa Cruz \#sc-2003 }\end{array}$ \\
\hline $\begin{array}{l}\text { Haemoglobin } \\
\text { subunit } \beta\end{array}$ & $\begin{array}{l}\text { Santa Cruz } \\
\text { \#sc-31116 }\end{array}$ & Mouse & $\begin{array}{l}\text { Protein A/G PLUS-Agarose } \\
\text { Santa Cruz \#sc-2003 }\end{array}$ \\
\hline
\end{tabular}

Table 4.2. List of antibodies used for immunoprecipitation in chapter 4.

\subsubsection{Preparation and administration of broccoli sprouts}

Individually housed male or female WT or NS mice were used in this study. Control group mice continued their regular diet of pelleted food. $15 \mathrm{mg}$ of pesticide-free broccoli sprouts (Planet Organic) were weighed and placed in a glass jar. $1 \mathrm{~g}$ of their regular pelleted food was crushed and distributed over the sprouts to encourage eating. A plastic screw lid was placed on top which had a $3 \mathrm{~cm}$ diameter access hole cut out from the middle. Each morning broccoli sprouts were replenished. Remaining broccoli sprouts were weighed to monitor consumption. Mice were sacrificed after 7 days and cardiac and liver tissue was harvested. 


\subsubsection{Proteomic analysis following SFX-01 treatment}

LC-MS/MS was carried out by Dr Xiaoke Yin and Professor Manuel Mayr (The James Black Centre, King's College London). Proteins from heart homogenates of WT mice which had received $2.5 \mathrm{mg} / \mathrm{ml} \mathrm{SFX-01} \mathrm{in} \mathrm{their} \mathrm{drinking} \mathrm{water} \mathrm{for} 7$ days were separated by SDS-PAGE. Following electrophoresis, a yellow band appeared on the polyacrylamide gel which corresponded to a $\sim 15 \mathrm{kDa}$ protein in lanes containing SFX-01-treated samples. This gel band and equivalent controls were carefully excised under sterile conditions and subjected to in-gel tryptic digestion using a ProGest (DigiLab Inc.) robotic digestion system. Peptides were subsequently separated by nanoflow LC using a reverse-phase column (PepMap C18, $3 \mu \mathrm{M}, 100 \AA ̊, 25 \mathrm{~cm} \times 75 \mu \mathrm{m}$ inner diameter, Thermo Fisher). Separated peptides were then applied to an interfacing linear ion trap mass spectrometer (LTQ Orbitrap XL, Thermo Fisher) and collected from the analyser using full ion scan mode over an $\mathrm{m} / \mathrm{z}$ range of $300-2000$. MS/MS was performed to analyse the top six ions using dynamic exclusion. Generated spectra were analysed against the mouse protein database using the Mascot search engine (Matrix Science). Two missed cleavages per peptide were allowed. Protein identifications were verified using the proteomics computer program Scaffold (Version 4, Proteome Software Inc.). Peptides with above $95 \%$ probability of identification were accepted as specified by the Scaffold software. A modification of $177.03 \mathrm{Da}$ was searched for using the proteomic software PEAKS (Bioinformatics Solutions Inc.).

\subsubsection{Protein separation by size-exclusion chromatography}

In order to explore the transfer of the electrophile between proteins, a thiolcontaining protein with SFN adducted was required and for these experiments 
BSA was used. A $200 \mathrm{mM}$ BSA stock was made by dissolving $13.30 \mathrm{mg}$ BSA in $1 \mathrm{ml}$ of either $10 \mathrm{mM}$ Tris $\mathrm{pH} 7.4$ or PBS pH 7.4. A $50 \mathrm{mM}$ SFN solution was then made by dissolving $115.23 \mathrm{mg}$ SFX-01 in $2 \mathrm{ml}$ of either $10 \mathrm{mM}$ Tris buffer or PBS. $5 \mu \mathrm{l}$ of the $200 \mathrm{mM}$ BSA stock and $100 \mu \mathrm{l}$ of the $50 \mathrm{mM}$ SFN stock were added to $895 \mu \mathrm{l}$ Tris or PBS, making a working solution containing $1 \mathrm{mM} \mathrm{BSA}$ and $5 \mathrm{mM}$ SFN. The solution was then incubated at $37^{\circ} \mathrm{C}$ for 30 minutes.

BSA-SFN was separated from free-SFN by size-exclusion LC (Bio-Rad BioLogic Duoflow liquid chromatograph), which comprised a pump operated at a flow rate of $1 \mathrm{ml} /$ minute, an injection valve fitted with a $500 \mu \mathrm{l}$ sample loop, a UV detector set to $280 \mathrm{~nm}$ and a Hi-Load 16/600 Superdex 200 prep grade column (Bio-Rad). The mobile phase consisted of either $10 \mathrm{mM}$ Tris $\mathrm{pH} 7.4$ or PBS pH 7.4. The programme is outlined in table 4.3. A $10 \mathrm{mM}$ Tris or PBS buffer sample was used as a negative control followed by a $1 \mathrm{mM} \mathrm{BSA}$ standard to determine its retention time.

\begin{tabular}{|l|l|l|l|l|}
\hline $\begin{array}{l}\text { Volume, } \\
\text { ml }\end{array}$ & Function & Buffer, \% & $\begin{array}{l}\text { Volume, } \\
\text { ml }\end{array}$ & $\begin{array}{l}\text { Flow, } \\
\mathbf{m l} / \mathbf{m i n}\end{array}$ \\
\hline 0 & Isocratic Flow & 100 & 1.60 & 1.60 \\
\hline 1.60 & Load/Inject & 100 & 1.60 & 1.60 \\
\hline 3.20 & Isocratic Flow & 100 & 420 & 420 \\
\hline 423 & Stop & & & \\
\hline
\end{tabular}

Table 4.3. Chromatography protocol for purifying BSA-SFN from unbound electrophile.

When absorption at $280 \mathrm{~nm}$ was detected by visual inspection of the real-time chromatogram, the eluate was continuously collected in serial $5 \mathrm{ml}$ fractions. 
Following analysis of all samples, the pumps and column were flushed with $70 \%$ methanol for 10 minutes and left stored in $70 \%$ methanol until next required. Following the chromatography procedure, $50 \mu \mathrm{l}$ of each eluted fraction was added to $50 \mu \mathrm{l}$ of $2 X$ SDS-PAGE sample buffer and the remaining eluate divided into $100 \mu \mathrm{l}$ aliquots and stored frozen at $-20^{\circ} \mathrm{C}$ until required. Samples were then subjected to SDS-PAGE followed by either Colloidal Coomassie staining or western immunoblotting as described in the general methods.

As the methods name suggests, separation is achieved by differences in protein or compound size, more specifically their Stokes radius, which considers not only size of the solute but also its mobility within a solution ${ }^{183}$. Separation of the analytes greatly depends on the size of the pores within gel beads that form the stationary phase of the apparatus as visualised in figure 4.1. 


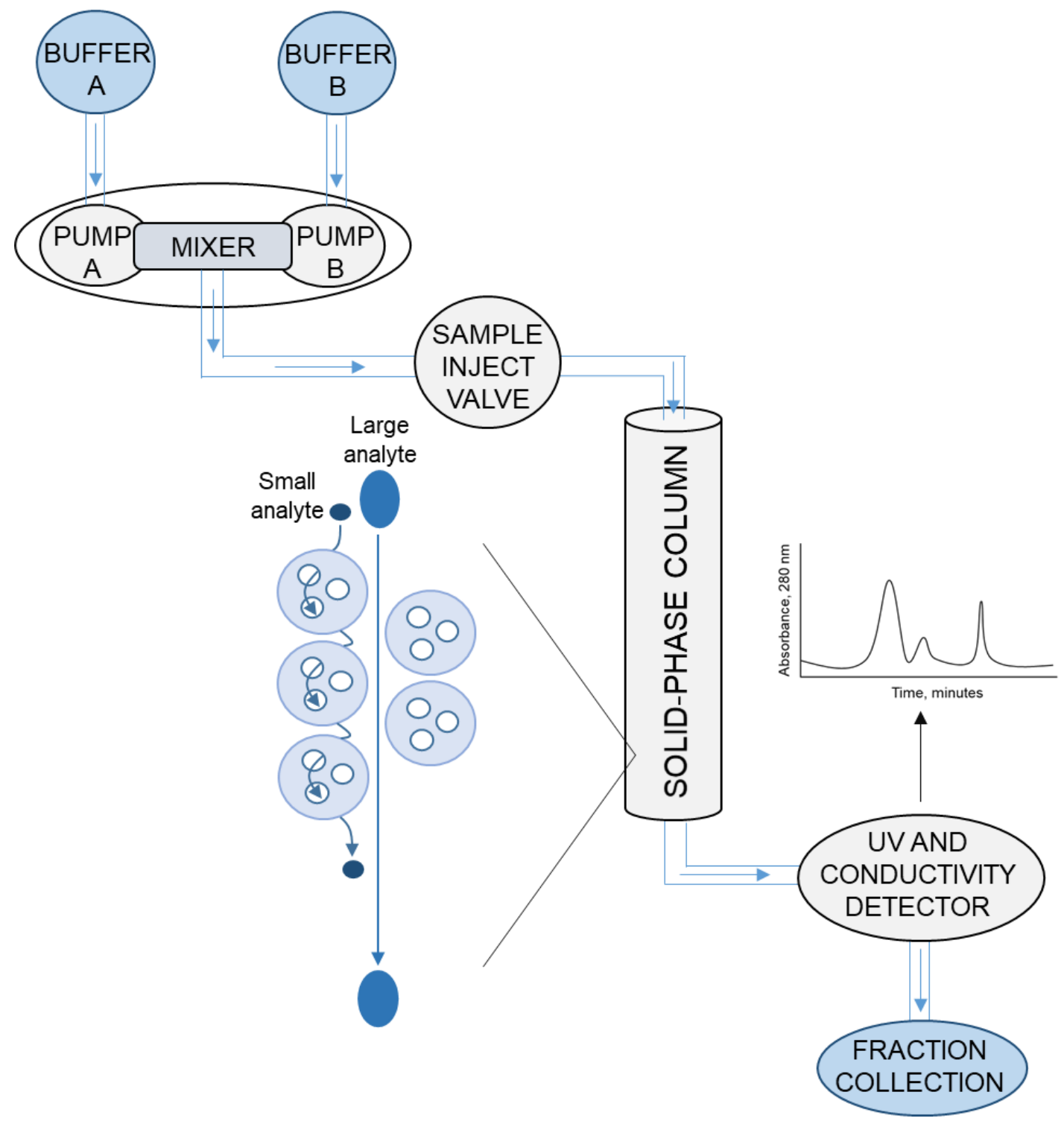

Figure 4.1. A diagram illustrating the size-exclusion chromatography system.

\subsubsection{Treatment of purified BSA-SFN with cysteine containing compounds}

To investigate the ability of SFN to trans-thiolate, BSA-SFN purified using the gel filtration outlined above was incubated with other cysteine-containing compounds, including; haemoglobin subunit $\beta(\mathrm{Hgb} \beta)$, haemoglobin $(\mathrm{Hgb})$, GSH, glyceraldehyde 3-phosphate dehydrogenase (GAPDH), L-cysteine or tissue homogenate from WT mice. $100 \mu \mathrm{l}$ of $25 \mu \mathrm{M} \mathrm{Hgb} \beta, \mathrm{Hgb}, \mathrm{GSH}, \mathrm{GAPDH}$ 
or L-cysteine in PBS pH 7.4 or 9.0 were incubated with $50 \mathrm{mM}$ Bond-Breaker Tris (2-carboxyethyl) phosphine (TCEP) solution (Thermo Fisher) for 30 minutes to reduce any oxidative modifications. Following reduction with TCEP, solutions were desalted using $0.5 \mathrm{ml}$ Zeba Spin desalting columns with a $7 \mathrm{kDa}$ molecular weight cut-off (Thermo Fisher). $100 \mu$ of cardiac, liver, kidney or blood homogenates from untreated WT mice were also prepared, using the protocol described in the general methods. $100 \mu \mathrm{l}$ of $25 \mu \mathrm{M}$ BSA-SFN, purified by sizeexclusion chromatography, was added to each solution, generating a final BSASFN concentration of $12.5 \mu \mathrm{M}$. Samples were then incubated at $37{ }^{\circ} \mathrm{C}$ for $0-60$ minutes followed by addition of an equal volume of 2X SDS-PAGE sample buffer. Negative controls were used containing either BSA, Hgb, GSH, GAPDH, Lcysteine or tissue homogenate alone. A time-matched control was also made by incubating $12.5 \mu \mathrm{M}$ BSA-SFN alone for 1 -hour at $37^{\circ} \mathrm{C}$. Western immunoblotting was carried out following the protocol described previously in the general methods.

\subsubsection{Using HPLC to assess transfer of an SFN adduct from SHP2 to GSH}

Incubation of $65 \mu \mathrm{M}$ SFX-01 with $10 \mu \mathrm{M}$ SHP2 for 30 minutes at room temperature was followed by desalting to remove any unbound electrophile using a $7 \mathrm{kDa}$ cut-off $0.5 \mathrm{ml}$ Zeba Spin desalting column (Thermo Fisher). The solution was then analysed by HPLC using the protocol outlined in the general methods and the UV detector set at a wavelength of $205 \mathrm{~nm}$. The solution was subsequently incubated with $10 \mathrm{mM} \mathrm{GSH}$ for 30 minutes at room temperature and again analysed using the HPLC method. Finally, the sample was incubated with $10 \mathrm{mM}$ TCEP and incubated at room temperature for 30 minutes and again analysed using the HPLC method. 
A deionised water-only sample was used as a negative control followed by a GSH-SFN standard to determine its retention time.

\subsubsection{Isolation of adult mouse ventricular myocytes}

Adult mouse ventricular myocytes were isolated from WT C57BL/6 mice using a collagenase-based enzymatic digestion procedure. Isolation was carried out by Dr Shiney Reji (The Rayne Institute, King's College London) using the following protocol. Hearts were excised, cannulated and sequentially perfused in Langendorff mode at $37{ }^{\circ} \mathrm{C}$ with solutions outlined in table 4.4. Perfusion was conducted using modified Tyrode's solution 1 for 2 minutes followed by calciumfree modified Tyrode's solution for 5 minutes. This was followed by subsequent perfusion with modified Tyrode's solution containing collagenase (250 mg/L) for $\sim 12$ minutes. The ventricles were then removed from the apparatus and excised into smaller pieces to allow extra surface area for the collagenase digestion to occur. The homogenate was then bubbled with $\mathrm{O}_{2}$ for 4 minutes at $37{ }^{\circ} \mathrm{C}$. Following incubation, the tissue was gently triturated until a uniform suspension was obtained and the cell suspension was subsequently filtrated through a nylon mesh. The cells were allowed to settle for 5 minutes which was followed by washing in modified Tyrode's solution 2 . The cells were again allowed to settle for 5 minutes which was followed by resuspension in modified Tyrode's solution 2. After a 2-hour recovery period, the supernatant was removed, and the cells washed in PBS prior to lysis and addition to an equal volume of 2 X SDS-PAGE sample buffer. 


\begin{tabular}{|c|c|c|c|c|}
\hline & $\begin{array}{l}\text { Modified } \\
\text { Tyrode's } \\
\text { solution 1, } \\
\text { nmol/L }\end{array}$ & $\begin{array}{l}\text { Calcium } \\
\text { Free, nmol/L }\end{array}$ & $\begin{array}{l}\text { Enzyme } \\
\text { solution, } \\
\mathrm{nmol} / \mathrm{L}\end{array}$ & $\begin{array}{l}\text { Modified } \\
\text { Tyrode's } \\
\text { solution, } \\
\text { nmol/L }\end{array}$ \\
\hline $\mathrm{NaCl}$ & 130 & 130 & 130 & 130 \\
\hline $\mathrm{KCl}$ & 5.4 & 5.4 & 5.4 & 5.4 \\
\hline $\mathrm{MgCl}_{2}$ & 1.4 & 1.4 & 1.4 & 1.4 \\
\hline $\mathrm{NaH}_{2} \mathrm{PO}_{4}$ & 0.4 & 0.4 & 0.4 & 0.4 \\
\hline HEPES & 4.2 & 4.2 & 4.2 & 4.2 \\
\hline Glucose & 10 & 10 & 10 & 10 \\
\hline Taurine & 20 & 20 & 20 & 20 \\
\hline Creatine & 10 & 10 & 10 & 10 \\
\hline EGTA & - & 0.1 & - & - \\
\hline $\mathrm{CaCl}_{2}$ & 0.75 & - & 0.5 & - \\
\hline Type-1 & - & - & $342 \mathrm{U} / \mathrm{mg}$ & - \\
\hline $\begin{array}{l}\text { Collagenase } \\
\mathrm{pH} \text { at } 37^{\circ} \mathrm{C}\end{array}$ & 7.3 & 7.3 & 7.3 & 7.3 \\
\hline
\end{tabular}

Table 4.4. Solutions used for the isolation of adult mouse ventricular myocytes.

\subsubsection{Treatment of HEK293 cells}

Just prior to treatment, growth media was removed from HEK293 cells and replaced with fresh media containing no FBS. Cells were then treated for 0-60 minutes with $10 \mu \mathrm{M}$ of either GSH-SFN (Santa Cruz \#sc-207496) or GSH.

Following treatment, $200 \mu \mathrm{l}$ of $2 X$ SDS-PAGE sample buffer was added to each well. Cells were then detached from the well using a cell scraper, moved into a microcentrifuge tube and lysed via sonication for 7 seconds at $30 \mathrm{kHz}$ and $40 \%$ amplitude. Western immunoblotting was then carried out using the protocol outlined in the general methods. 


\subsection{Results}

\subsubsection{SFN adducts a $\sim 15 \mathrm{kDa}$ protein in cardiac tissue}

As detailed in chapter 3 , treatment of WT mice with $2.5 \mathrm{mg} / \mathrm{ml} \mathrm{SFX-01} \mathrm{for} \mathrm{4-10}$ days in their drinking water identified a time-dependent increase in protein-SFN adducts in cardiac tissue. These samples were reanalysed by immunoblotting which included collecting shorter detection film exposure times, which illustrated that SFN predominantly adducts a protein with a mass of $\sim 15 \mathrm{kDa}$. This SFN adduct accumulated in a time-dependent manner (figure 4.2).

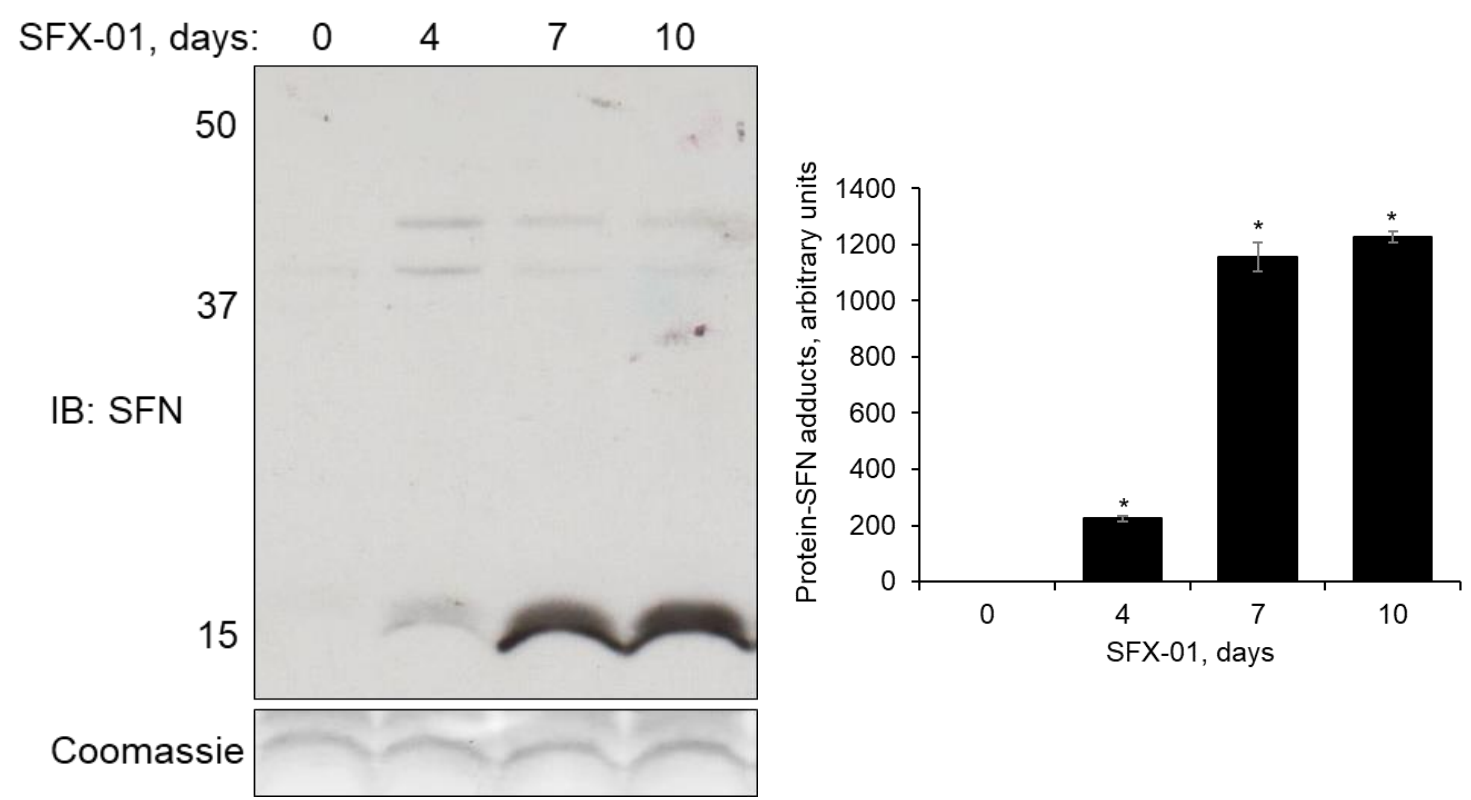

Figure 4.2. SFN adducts a $\sim 15 \mathrm{kDa}$ protein in cardiac tissue of WT mice.

An immunoblot showing a time-dependent increase in SFN adduction to a $\sim 15 \mathrm{kDa}$ protein in cardiac tissue of WT mice following treatment with $2.5 \mathrm{mg} / \mathrm{ml} \mathrm{SFX-01} \mathrm{in} \mathrm{their}$ drinking water for $4-10$ days. $\left(n=10,{ }^{*} p<0.05\right.$ versus water-only control). 


\subsubsection{Protein-SFN adducts are detected after consumption of broccoli sprouts}

I next wanted to assess if protein-SFN adducts could be detected in vivo following ingestion of a naturally occurring source of the electrophile. WT or NS mice were therefore given broccoli sprouts for 7 days. SFN adduction of a $\sim 15 \mathrm{kDa}$ protein was seen in cardiac tissue of WT or NS mice after consumption of the sprouts (figure 4.3).

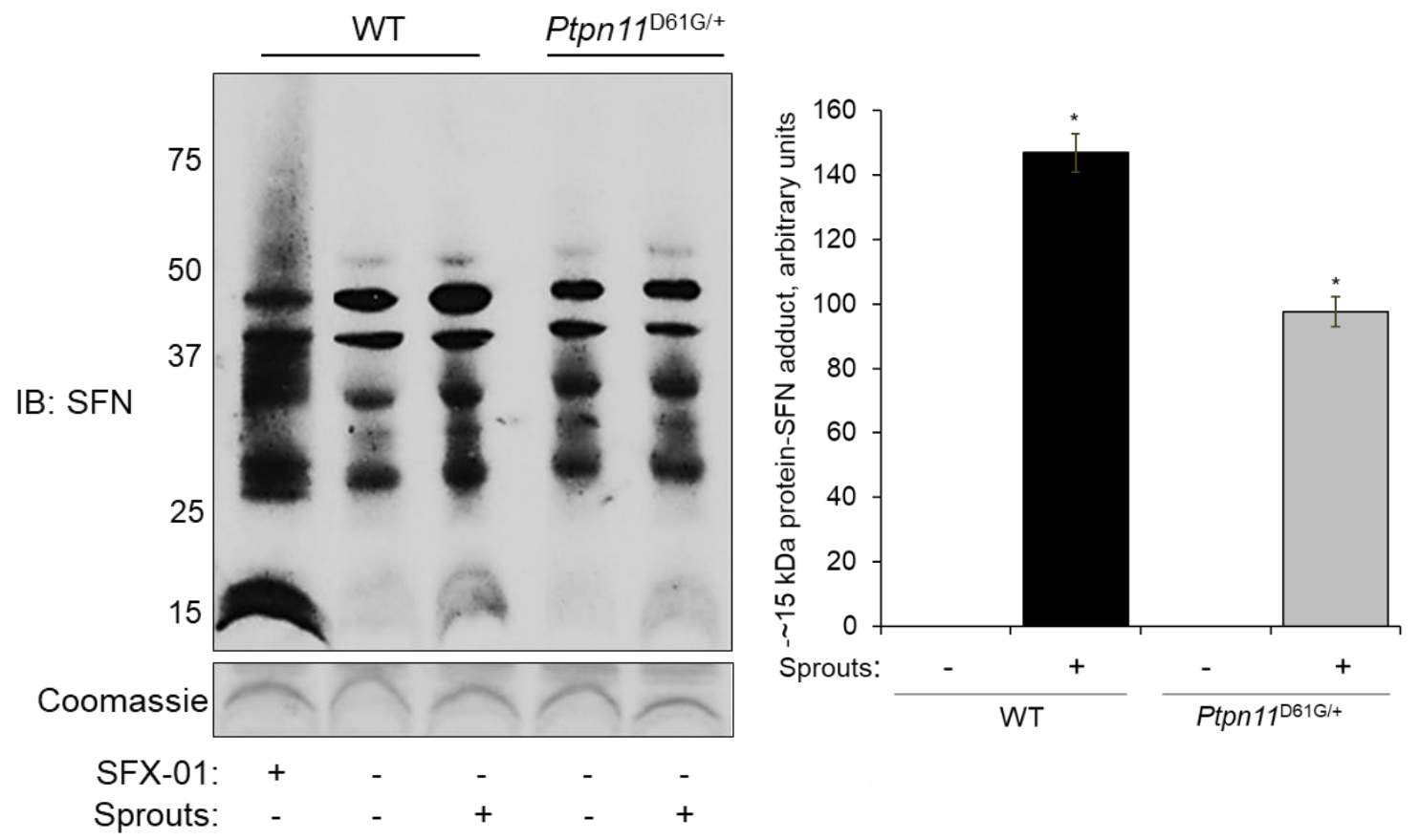

Figure 4.3. Ingestion of a naturally-derived source of SFN results in protein adduct formation.

An immunoblot showing SFN adduction of a $\sim 15 \mathrm{kDa}$ protein in cardiac tissue of WT or NS mice after eating broccoli sprouts for 7 days. $\left(n=4,{ }^{*} p<0.05\right.$ versus pelleted food only control). 


\subsubsection{SFX-01 treatment increases TrX and GRX1 protein expression}

I next began to investigate if the $\sim 15 \mathrm{kDa}$ protein adducted by SFN following treatment with SFX-01 for 4-10 days in their drinking water was Trx or GRX1, which were considered rational because they are thiol-dependent enzymes of approximately this molecular weight. Immunoblotting under reducing conditions identified an increase in protein expression of Trx or GRX1 in cardiac tissue of WT mice following 10-day treatment with $2.5 \mathrm{mg} / \mathrm{ml} \mathrm{SFX-01} \mathrm{(figure} 4.4 \mathrm{~A}, \mathrm{~B}$ ).

A)

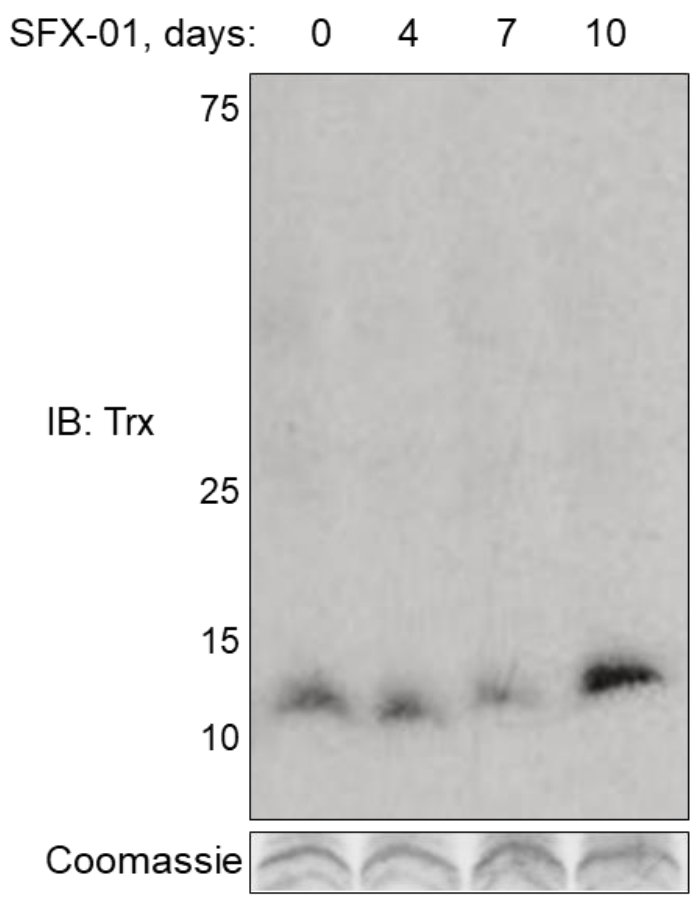

B)

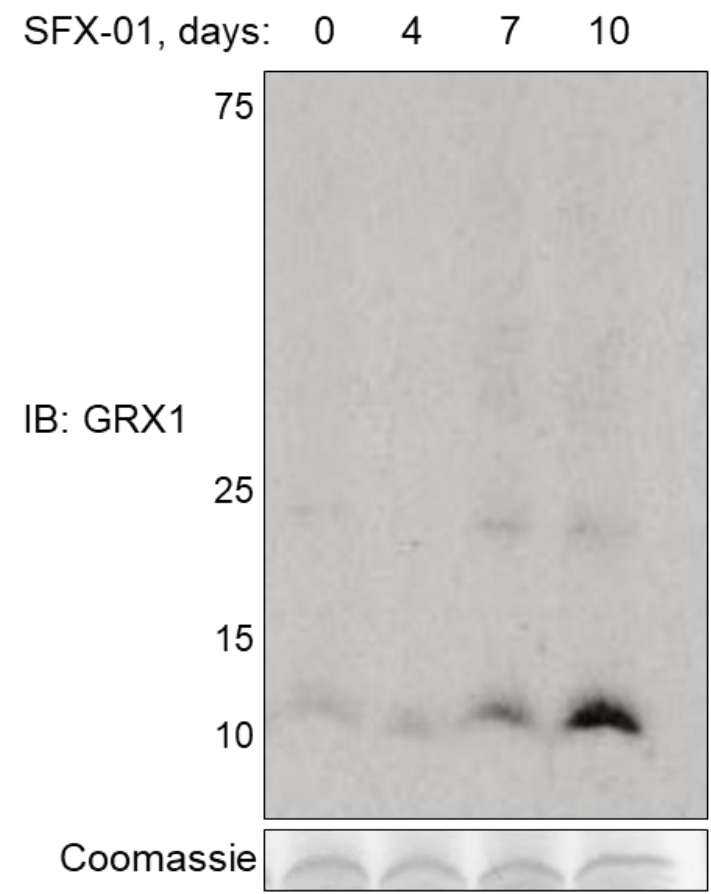

Figure 4.4. SFN treatment increases protein expression of TrX and GRX1.

A, B) Representative immunoblots showing an increase in protein expression of Trx and GRX1 in cardiac tissue of WT mice following treatment with $2.5 \mathrm{mg} / \mathrm{ml}$ SFX-01 in their drinking water for 10 days. 


\subsubsection{Trx or GRX1 do not comigrate with the $\sim 15 \mathrm{kDa}$ protein adducted by}

\section{SFN}

Proteins from cardiac tissue of WT mice who received $2.5 \mathrm{mg} / \mathrm{ml} \mathrm{SFX-01} \mathrm{in} \mathrm{their}$ drinking water were resolved in triplicate by SDS-PAGE. Immunoblotting revealed Trx nor GRX1 resolved at the same molecular weight as the $\sim 15 \mathrm{kDa}$ protein adducted by SFN (figure 4.5).

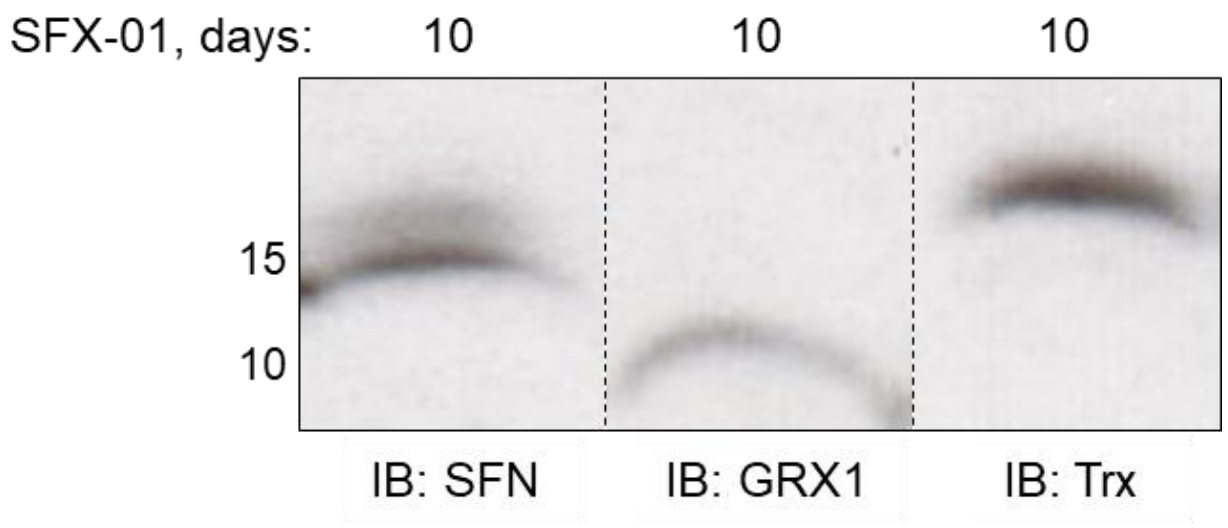

Figure 4.5. Neither Trx nor GRX1 comigrate with a $\sim 15 \mathrm{kDa}$ protein target of SFN.

A representative immunoblot showing the $\sim 15 \mathrm{kDa}$ protein adducted by SFN from the cardiac tissue of WT mice following 10-day treatment with $2.5 \mathrm{mg} / \mathrm{ml} \mathrm{SFX-01} \mathrm{in} \mathrm{their}$ drinking water did not comigrate on a polyacrylamide gel with either Trx or GRX1.

\subsubsection{Immunoprecipitation of the $\sim 15 \mathrm{kDa}$ protein adducted by SFN was unsuccessful}

I next attempted to immunoprecipitate the $\sim 15 \mathrm{kDa}$ protein adducted by SFN from cardiac tissue of WT or NS mice following 10-day treatment with $2.5 \mathrm{mg} / \mathrm{ml} \mathrm{SFX-}$ 01 in their drinking water. Immunoprecipitation was conducted using our in-house anti-SFN primary antibody, however, the protein of interest was not present in the immunocaptured sample (figure 4.6). 


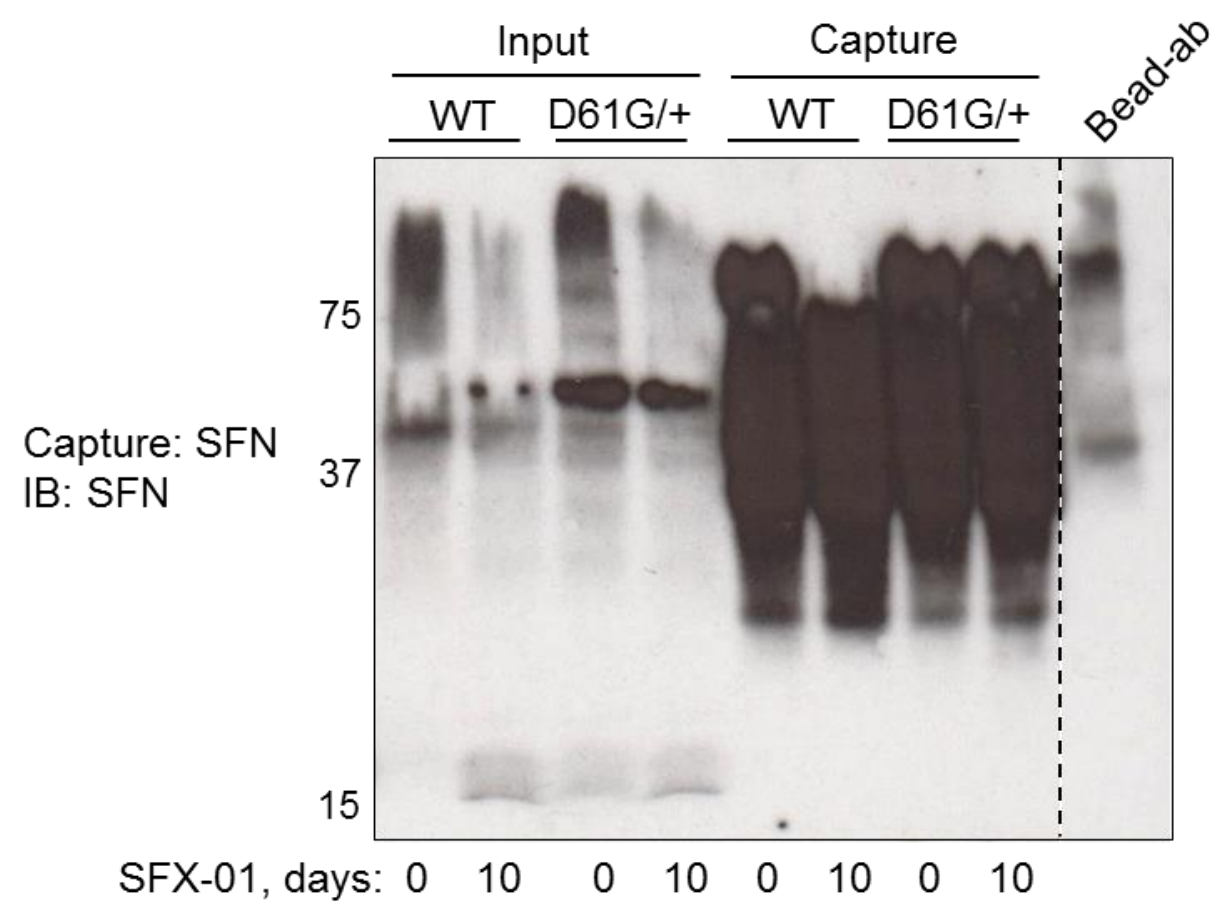

Figure 4.6. A $\sim 15 \mathrm{kDa}$ protein adducted by SFN was not successfully immunoprecipitated from cardiac tissue.

A representative immunoblot of input and immunocaptured cardiac tissue of WT or NS mice treated with $2.5 \mathrm{mg} / \mathrm{ml} \mathrm{SFX}-01$ for 10 days in their drinking water. Precipitation was conducted using the anti-SFN primary antobody although no enrichment of the $\sim 15 \mathrm{kDa}$ protein adducted by SFN was achieved.

\subsubsection{The $\sim 15 \mathrm{kDa}$ protein adducted by SFN was identified by mass spectrometry}

Unexpectedly, the $\sim 15 \mathrm{kDa}$ protein adducted by SFN following 10-day treatment with SFX-01 could be visualised on a polyacrylamide gel following electrophoresis by a yellow colouring (figure 4.7). Table 4.5 documents the proteins identified by LC-MS/MS within gel pieces excised from both SFX-01 treated and untreated samples, which was conducted using biological repreats. $\mathrm{Hgb}$ subunits $\alpha$ and $\beta 1$ ( $\mathrm{Hgb} \alpha$ and $\mathrm{Hgb} \beta 1$ respectively) were the most abundant. Subsequent analysis using the computer program Mascot identified an addition 
of $+177.03 \mathrm{Da}$, the mass of SFN, upon cysteine within the peptide sequence 'GTFASLSELHCDK', which corresponds to Cys ${ }^{93}$ of $\mathrm{Hgb} \beta 1$ (figure 4.8).

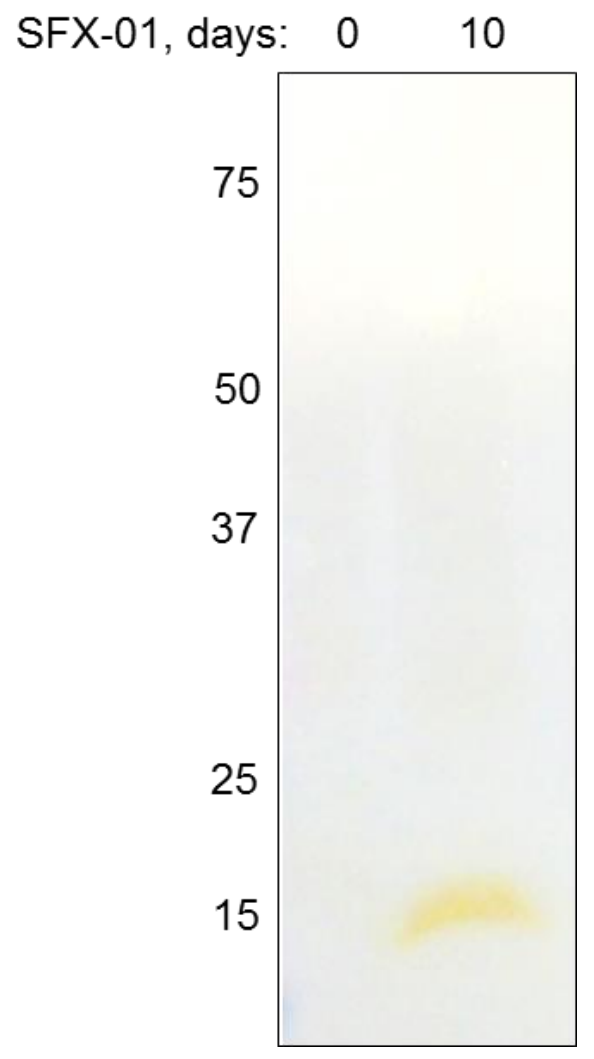

Figure 4.7. The $\sim 15 \mathrm{kDa}$ protein adducted by SFN can be visualised following electrophoresis.

A representative polyacrylamide gel showing the yellow appearance of the $\sim 15 \mathrm{kDa}$ protein adducted by SFN in cardiac tissue of WT mice who received $2.5 \mathrm{mg} / \mathrm{ml} \mathrm{SFX-01}$ in their drinking water for 10 days. 


\begin{tabular}{|l|l|l|}
\hline Protein name & Abbreviation & Mol. Wt \\
\hline Hgb subunit a & Hgb a & $15 \mathrm{kDa}$ \\
\hline Hgb subunit $\beta 1$ & $\mathrm{Hgb} \beta 1$ & $16 \mathrm{kDa}$ \\
\hline Histone H4 & $\mathrm{H} 4$ & $11 \mathrm{kDa}$ \\
\hline Cytochrome c oxidase subunit 6c & COX6C & $8 \mathrm{kDa}$ \\
\hline ATP synthase subunit e, mitochondrial & ATP51 & $8 \mathrm{kDa}$ \\
\hline Fatty acid-binding protein, heart & FABPH & $15 \mathrm{kDa}$ \\
\hline Cytochrome c, somatic & CYC & $12 \mathrm{kDa}$ \\
\hline Cytochrome c oxidase subunit 5A & COX5A & $16 \mathrm{kDa}$ \\
\hline Fatty acid-binding protein, adipocyte & FABP4 & $15 \mathrm{kDa}$ \\
\hline Cytochrome c oxidase subunit NDUFA4 & NDUA4 & $9 \mathrm{kDa}$ \\
\hline Cytochrome b-c 1 complex subunit 8 & QCR8 & $10 \mathrm{kDa}$ \\
\hline ATP synthase subunit g, mitochondrial & ATP5L & $11 \mathrm{kDa}$ \\
\hline Cytochrome c oxidase subunit 4 isoform 1 & COX41 & $20 \mathrm{kDa}$ \\
\hline Cardiac phospholamban & PPLA & $6 \mathrm{kDa}$ \\
\hline $\begin{array}{l}\text { NADH dehydrogenase subunit 1 beta } \\
\text { subunit 3 }\end{array}$ & NDUB3 & $12 \mathrm{kDa}$ \\
\hline $\begin{array}{l}\text { NADH dehydrogenase subunit 1 alpha } \\
\text { subunit 6 }\end{array}$ & NDUA6 & $15 \mathrm{kDa}$ \\
\hline Cytochrome b-c1 subunit 7 & QCR7 & $14 \mathrm{kDa}$ \\
\hline Cytochrome c oxidase subunit 5b & COX5B & $14 \mathrm{kDa}$ \\
\hline ATP synthase subunit f, mitochondrial & ATPK & $10 \mathrm{kDa}$ \\
\hline Mitochondrial pyruvate carrier 2 & MPC2 & $14 \mathrm{kDa}$ \\
\hline ADP/ATP translocase 1 & ADT1 & $33 \mathrm{kDa}$ \\
\hline NADH dehydrogenase 1 subunit C2 & NDUC2 & $14 \mathrm{kDa}$ \\
\hline NADH dehydrogenase 1 $\beta$ subunit 4 & NDUß4 & $15 \mathrm{kDa}$ \\
\hline Cytochrome c oxidase subunit 6B1 & CX6B1 & $10 \mathrm{kDa}$ \\
\hline ATP synthase protein 8 & ATP8 & $8 \mathrm{kDa}$ \\
\hline Ubiquitin-60S ribosomal protein L40 & RL40 & $15 \mathrm{kDa}$ \\
\hline Histone H2A type 2 & H2A2C & $14 \mathrm{kDa}$ \\
\hline Myoglobin & MYG & $17 \mathrm{kDa}$ \\
\hline $\begin{array}{l}\text { CDGSH iron-sulphur domain-containing } \\
\text { protein 1 }\end{array}$ & CISD1 & $12 \mathrm{kDa}$ \\
\hline NADH dehydrogenase 1 alpha subunit 7 & NDUA7 & $13 \mathrm{kDa}$ \\
\hline
\end{tabular}

Table 4.5. Proteins identified by LC-MS/MS from cardiac tissue of WT mice. 


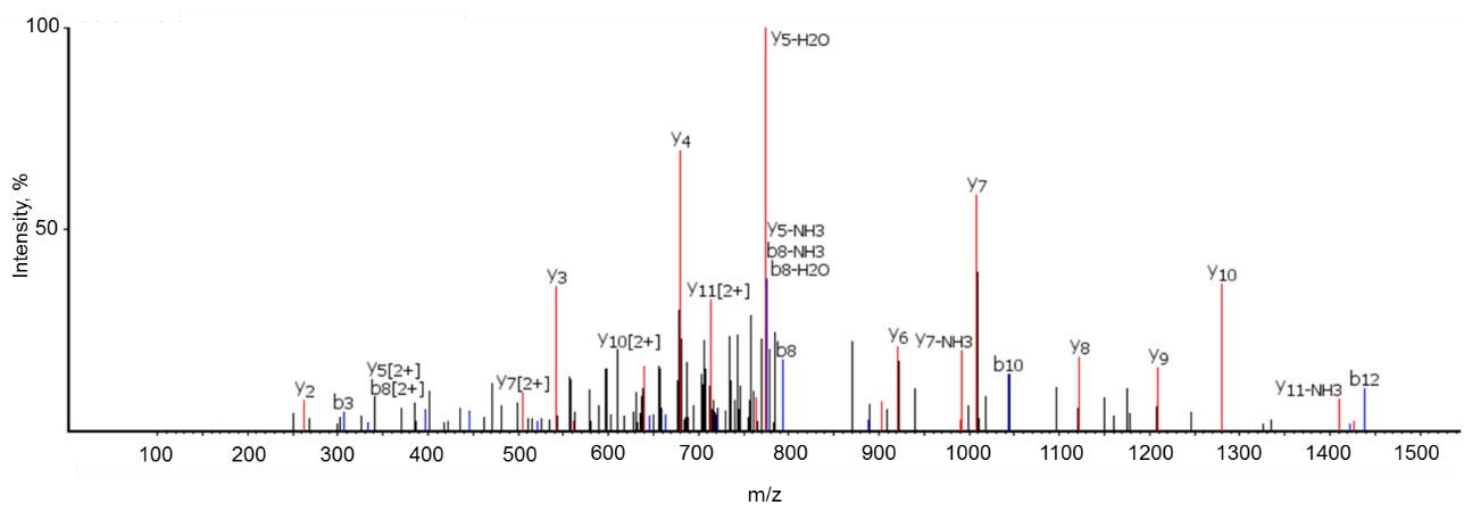

Figure 4.8. Mass spectra of the Hgb $\beta 1$ peptide sequence GTFASLSELHCDK which contained $\mathrm{a}+\mathbf{1 7 7 . 0 3}$ mass adduct upon $\mathrm{Cys}^{93}$.

\subsubsection{SFN can adduct $\mathrm{Hgb} \beta$}

Immunoblotting revealed SFN can adduct recombinant $\mathrm{Hgb} \beta$ following 30minute treatment with SFX-01 (figure 4.9). Hgb $\beta$ has a monomeric weight of 16 $\mathrm{kDa}$. SFN adducts are identified at a molecular weight of both 16 and $32 \mathrm{kDa}$, likely corresponding to both monomeric and dimerized forms of $\mathrm{Hgb} \beta$.
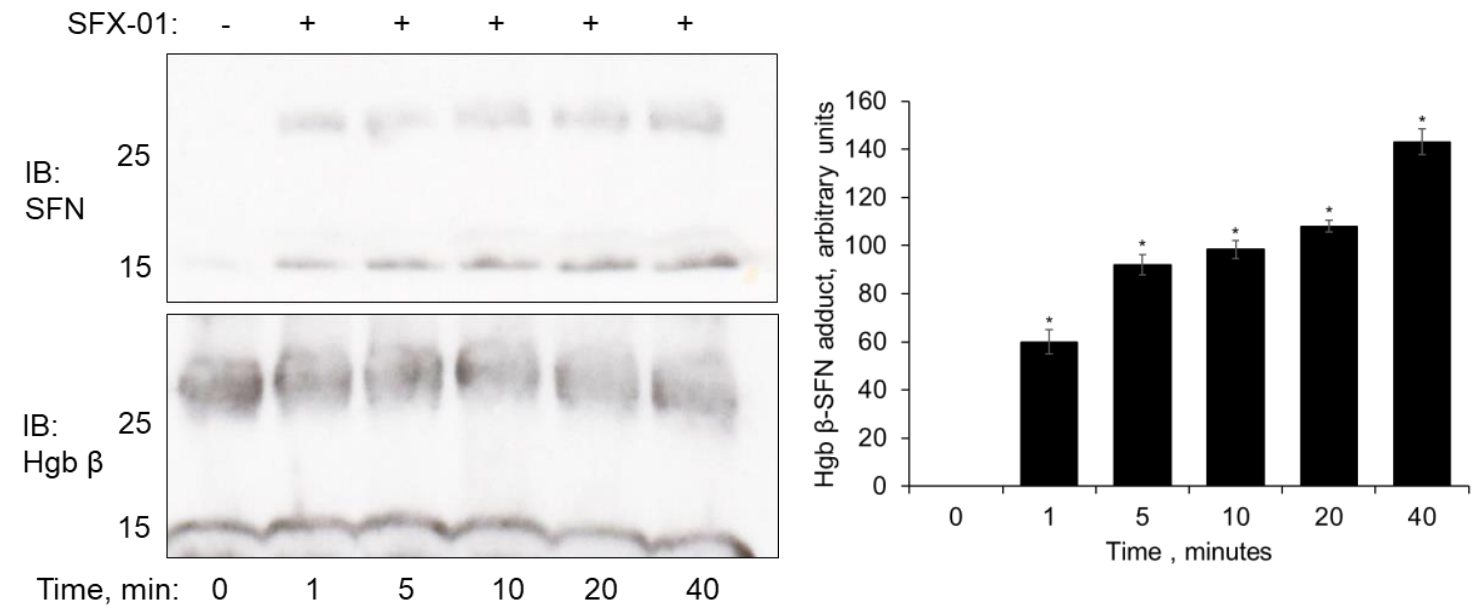

Figure 4.9. SFN can adduct $\mathrm{Hgb} \beta$.

An immunoblot showing SFN adducted to monomeric and dimeric $\mathrm{Hgb} \beta$ following incubation of recombinant protein with SFX-01 for 30 minutes. $\left(n=5,{ }^{*} p<0.05\right.$ versus untreated control). 


\subsubsection{The $\sim 15 \mathrm{kDa}$ protein adducted by SFN comigrates with $\mathrm{Hgb} \beta$}

Immunoblotting revealed the $\sim 15 \mathrm{kDa}$ protein adducted by SFN in cardiac tissue of WT mice following treatment with $2.5 \mathrm{mg} / \mathrm{ml} \mathrm{SFX-01} \mathrm{in} \mathrm{their} \mathrm{drinking} \mathrm{water} \mathrm{for}$ 4-10 days resolved at the same molecular weight as recombinant $\mathrm{Hgb} \beta$ following electrophoresis (figure 4.10).

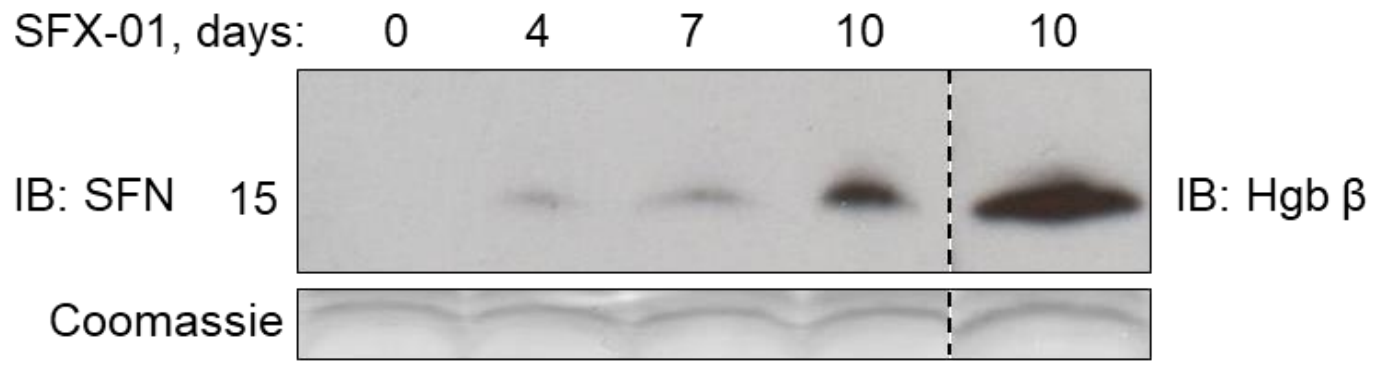

Figure 4.10. Hgb $\beta$ comigrates with the SFN adducted $\sim 15 \mathrm{kDa}$ protein.

A representative immunoblot showing comigration of $\mathrm{Hgb} \beta$ with the $\sim 15 \mathrm{kDa}$ protein adducted by SFN in cardiac tissue of WT mice following 4-10-day treatment with SFX01 in their drinking water.

\subsubsection{Immunoprecipitation of Hgb $\beta-S F N$ was unsuccessful}

I attempted to immunoprecipitate Hgb $\beta$-SFN from cardiac tissue of WT mice following 10-day treatment with $2.5 \mathrm{mg} / \mathrm{ml} \mathrm{SFX-01} \mathrm{in} \mathrm{their} \mathrm{drinking} \mathrm{water.}$ Immunoprecipitation was conducted using an anti-Hgb $\beta$ primary antibody however no presence of $\mathrm{Hgb} \beta$-SFN was detected in the immunocaptured sample (figure 4.11). 


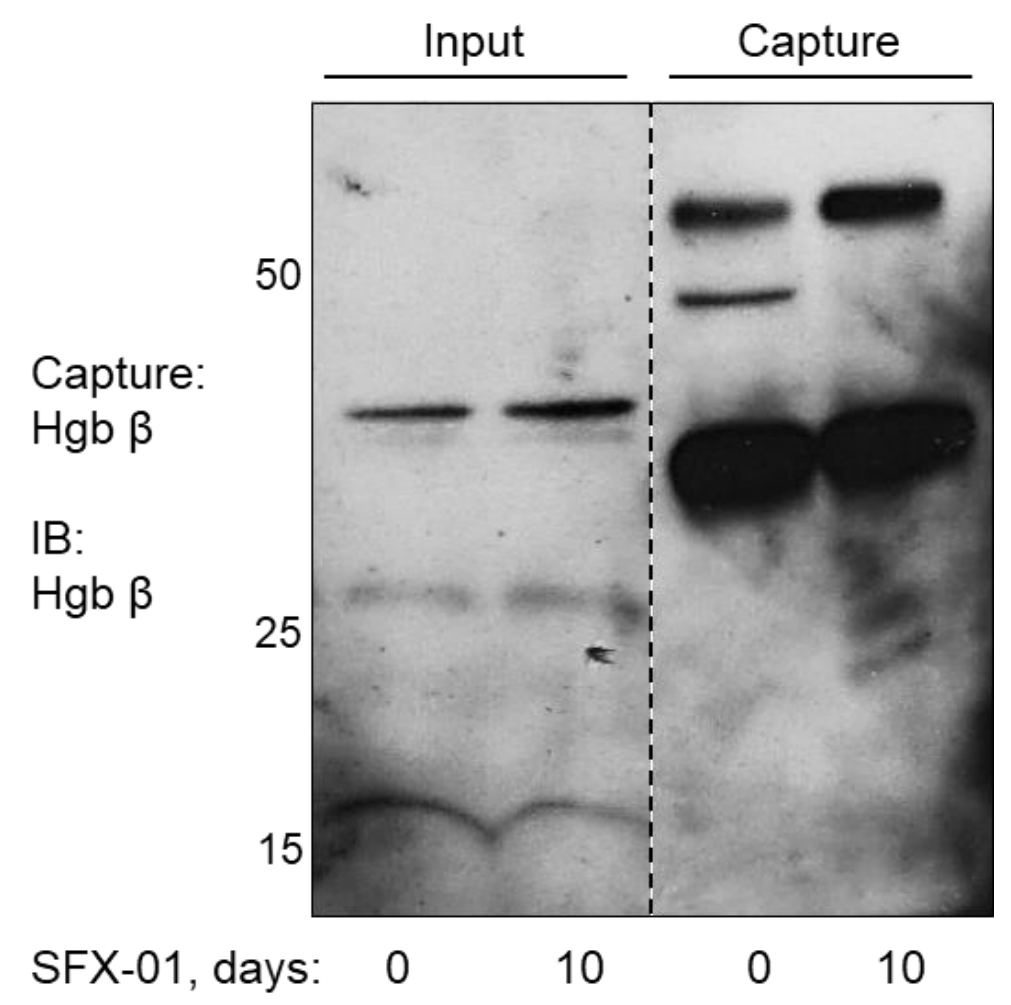

Figure 4.11. Hgb $\beta$-SFN was not successfully immunoprecipitated from cardiac tissue.

A representative immunoblot of input and immunocaptured cardiac tissue of WT mice

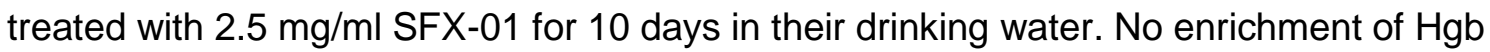
$\beta-S F N$ was achieved.

\subsubsection{SFX-01 treatment did not increase Hgb $\beta$ expression}

As the $\sim 15 \mathrm{kDa}$ protein accumulatively adducted by SFN following continuous SFX-01 treatment was identified as Hgb $\beta$ by LC-MS/MS, I assessed if the expression of the protein was increased. Spectral counts for $\mathrm{Hgb} \beta$ revealed there was no change in the abundance of the protein in cardiac tissue from WT mice

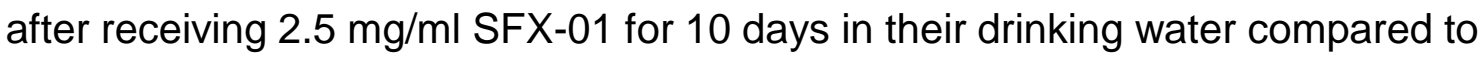
control (figure 4.12). 


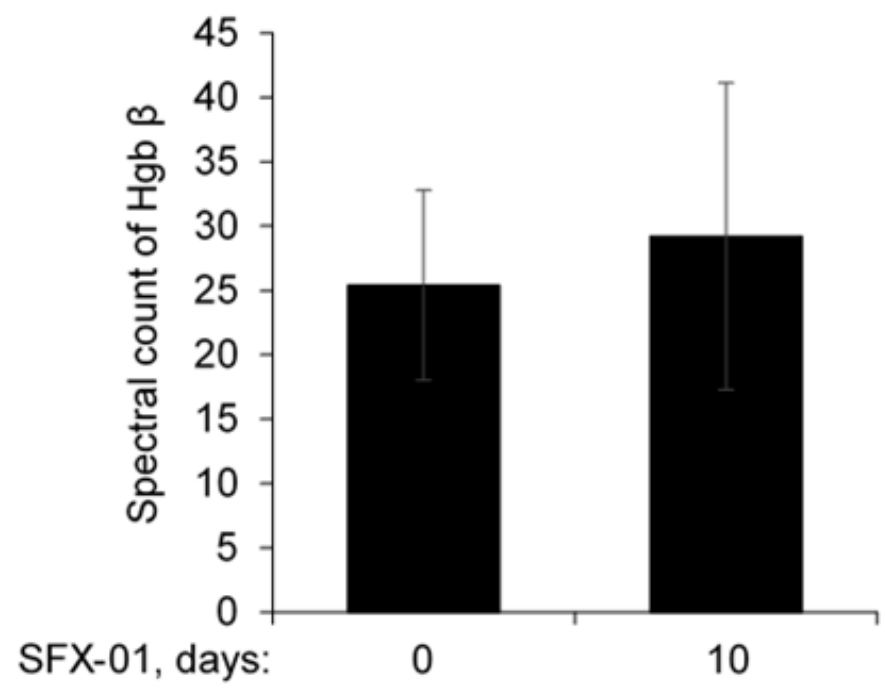

Figure 4.12. Spectral counts of $\mathrm{Hgb} \beta$ in cardiac tissue of WT mice following treatment with SFX-01 for 10 days in their drinking water.

\subsubsection{Hgb $\beta$ was not detected in isolated cardiomyocytes}

I next investigated if $\mathrm{Hgb} \beta$-SFN present in cardiac tissue was a result of this protein being present in cardiomyocytes, as it is found in tissues other than red blood cells which is discussed in detail in the discussion of this chapter. Immunoblotting analysis, with a blood sample as a positive control, did not find $\mathrm{Hgb}$ to be present in isolated adult mouse ventricular cardiomyocytes (figure 4.13). 


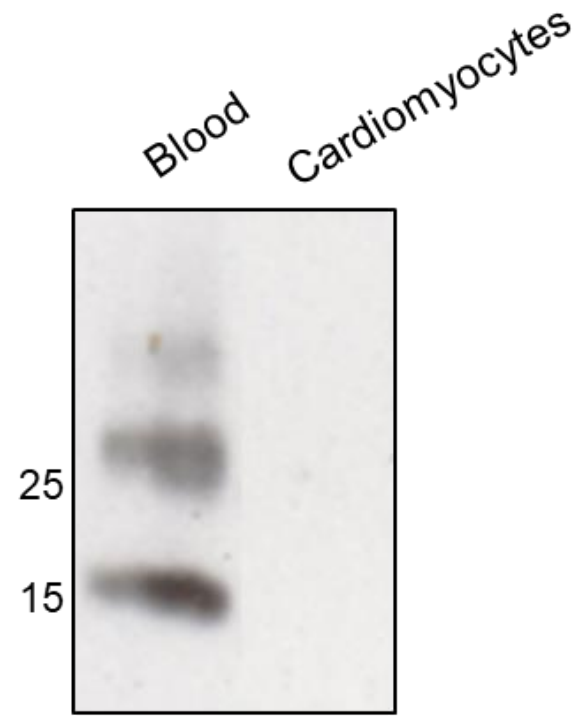

IB: Hgb

Figure 4.13. A representative immunoblot showing $\mathrm{Hgb}$ was not detected in isolated adult mouse ventricular myocytes.

\subsubsection{Detection of cardiac Hgb-SFN is attenuated by coronary perfusion} Hearts were isolated from WT mice treated with SFX-01 for 10 days and subjected to different stringencies of coronary vascular perfusion to ascertain whether the adduct observed was due to residual blood. Immunoblotting showed that flushing blood from the heart reduced the Hgb-SFN signal, consistent with the adduct being the result of modified red blood cell Hgb (figure 4.14). 


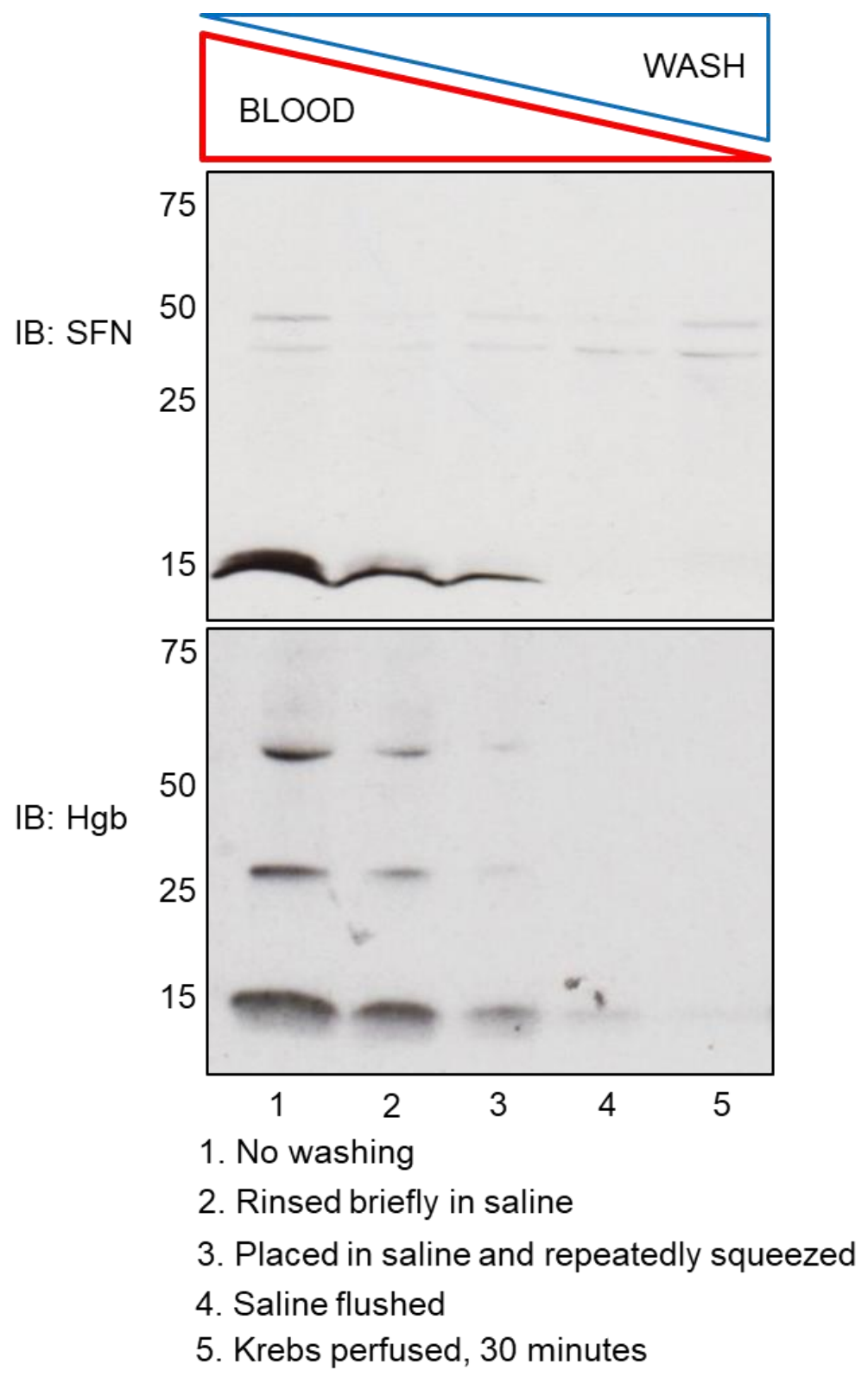

Figure 4.14. Hgb $\beta$-SFN present in cardiac tissue was perhaps from residual blood.

Representative immunoblots showing a decrease in cardiac Hgb-SFN and Hgb following perfusion of coronary vessels of hearts isolated from mice treated with SFX-01 for 10 days.

\subsubsection{BSA-SFN was purified using size-exclusion chromatography}

Following incubation of $1 \mathrm{mM}$ BSA with $5 \mathrm{mM}$ SFN for 30 minutes in either 10 $\mathrm{mM}$ Tris $\mathrm{pH} 7.4$ or PBS $\mathrm{pH} 7.4$, free-SFN was removed from either solution using 
gel filtration chromatography. Regardless of the buffer used, the chromatogram showed similar profiles with two broad peaks appeared during the 120-minute run time (figure $4.15 \mathrm{~A}, \mathrm{~B}$ ). To determine if these peaks contained BSA-SFN and assess the purity of the samples, fractions were collected and subjected to SDSPAGE followed by Colloidal Coomassie staining or western immunoblotting. Immunoblotting identified which samples contained SFN adducted to monomeric BSA and the fractions to be used in subsequent experiments are indicated by an arrow in figure 4.16 A, B. Colloidal Coomassie staining of the fractions after separation on a polyacrylamide gel together with BSA standards $(1.25-5 \mathrm{nmol})$ allowed the concentration of BSA present in the samples following chromatography to be measured. This was $\sim 50 \mu \mathrm{M}$ for the Tris-containing sample and $\sim 25 \mu \mathrm{M}$ for the PBS-containing sample (figure 4.16 C). 
A)

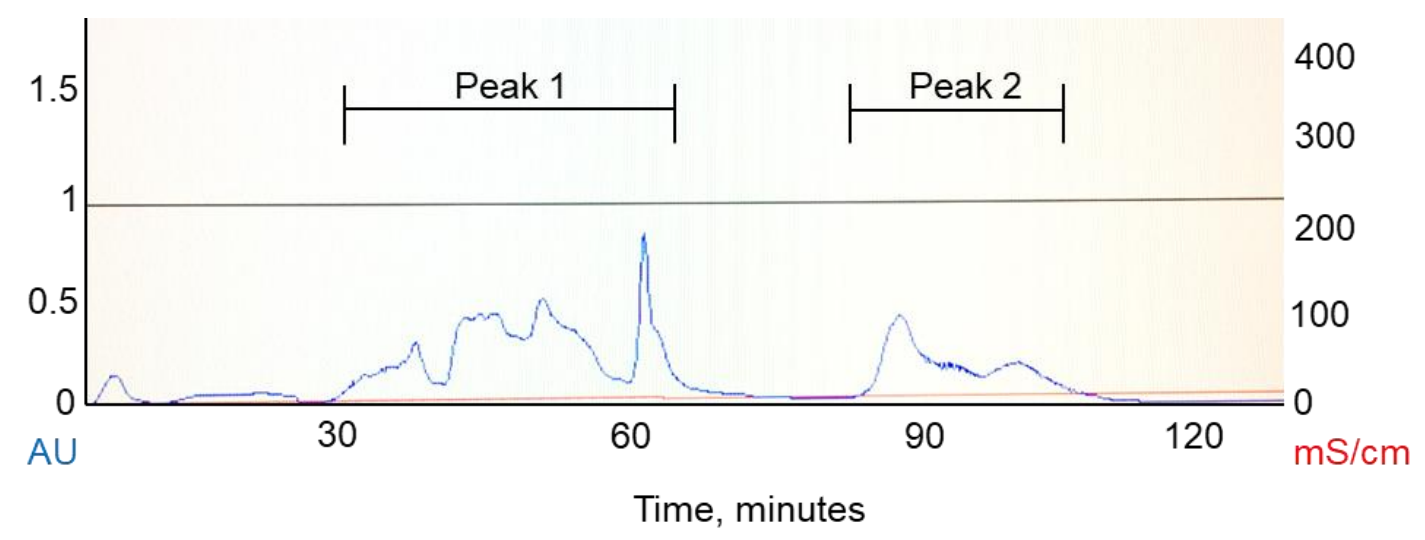

B)

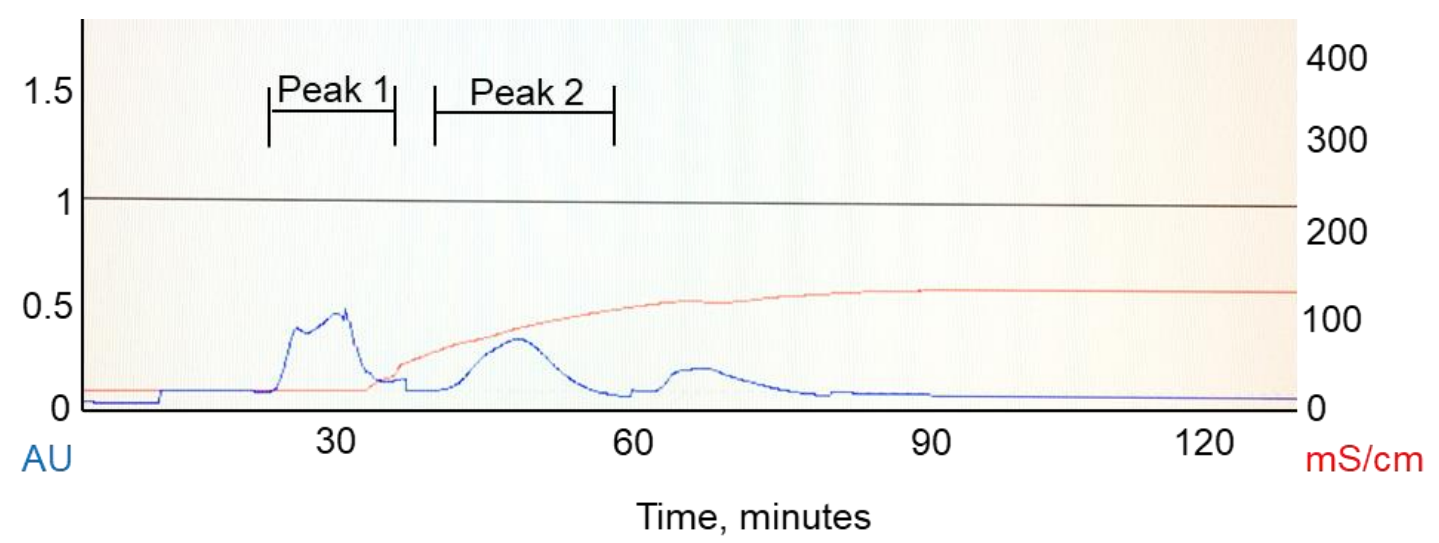

Figure 4.15. Chromatograms showing retention times for BSA treated with SFN.

A) $1 \mathrm{mM}$ BSA incubated with $5 \mathrm{mM}$ SFN in $10 \mathrm{mM}$ Tris $\mathrm{pH} 7.4 \mathrm{~B}) 1 \mathrm{mM}$ BSA incubated with $5 \mathrm{mM}$ SFN in PBS pH 7.4. AU represents absorbance using a UV detector set at a wavelength of $280 \mathrm{~nm}$.

A) 


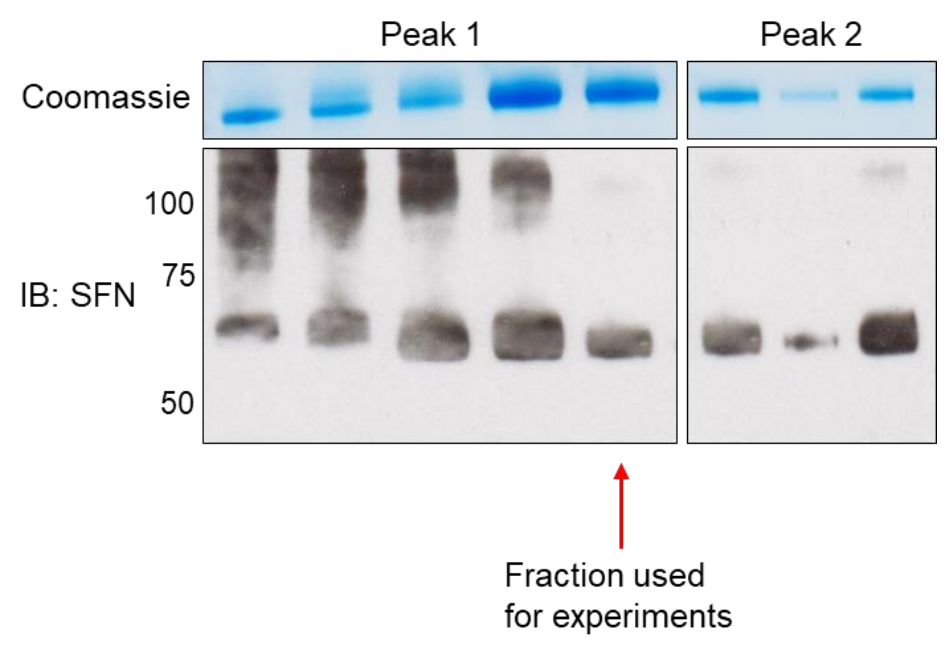

B)

C)
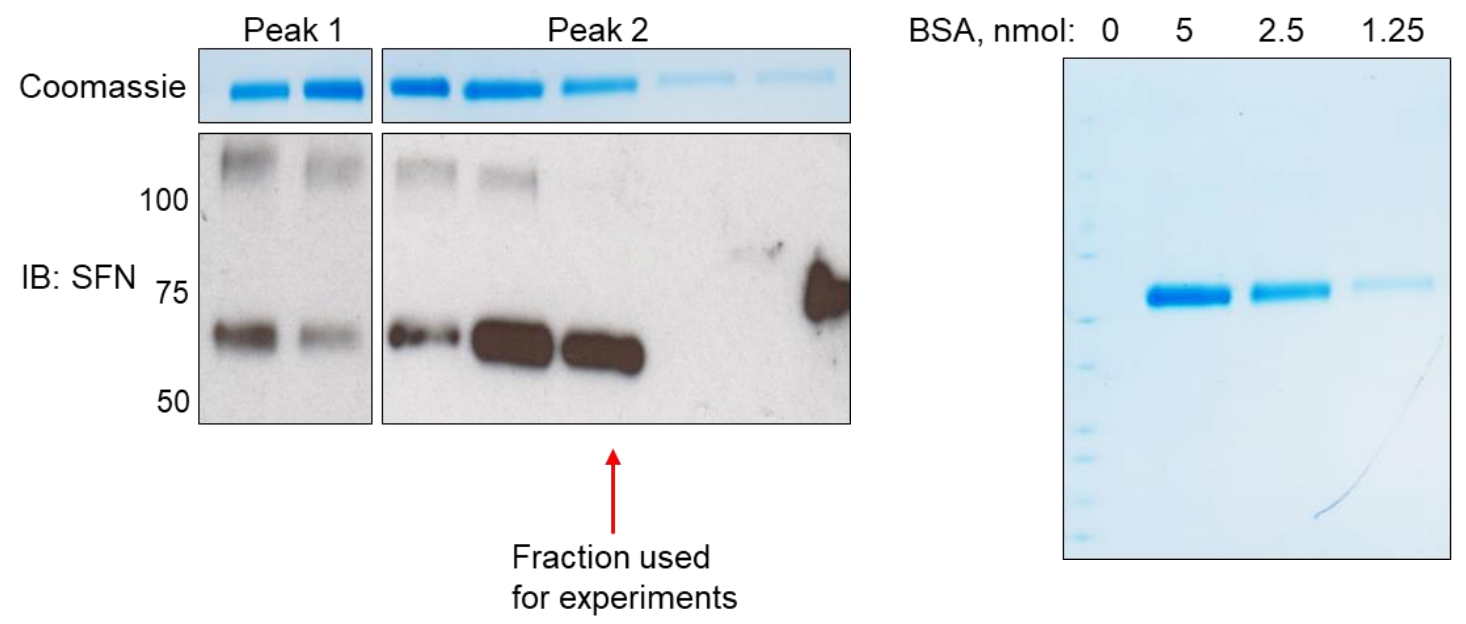

Figure 4.16. BSA-SFN is present in eluates following size-exclusion chromatography.

A, B) Immunoblots and Colloidal Coomassie stained polyacrylamide gels of fractions collected following gel filtration of BSA treated with SFN in either a $10 \mathrm{mM}$ Tris or PBS buffer respectively. Immunoblotting revealed SFN had adducted BSA and indicated which fractions contained predominantly monomeric BSA which was desirable for subsequent experiments. C) A Colloidal Coomassie stained polyacrylamide gel following electrophoresis using varying amounts of BSA to use as standards. Colloidal Coomassie stained polyacrylamide gels of gel filtrated BSA-SFN samples were compared to these standards to estimate the amount of protein present. 


\subsubsection{The interaction between BSA and SFN is stable in a PBS buffer}

To assess the stability of the SFN adduct over time, chromatographically-purified BSA-SFN was incubated at room temperature for 0-60 minutes and resolved using SDS-PAGE. Immunoblotting identified a time-dependent loss of the SFN adduct from BSA when in a Tris-containing buffer. When using a PBS buffer, the SFN adduct was stable upon BSA over time (figure 4.17 A, B).

A)
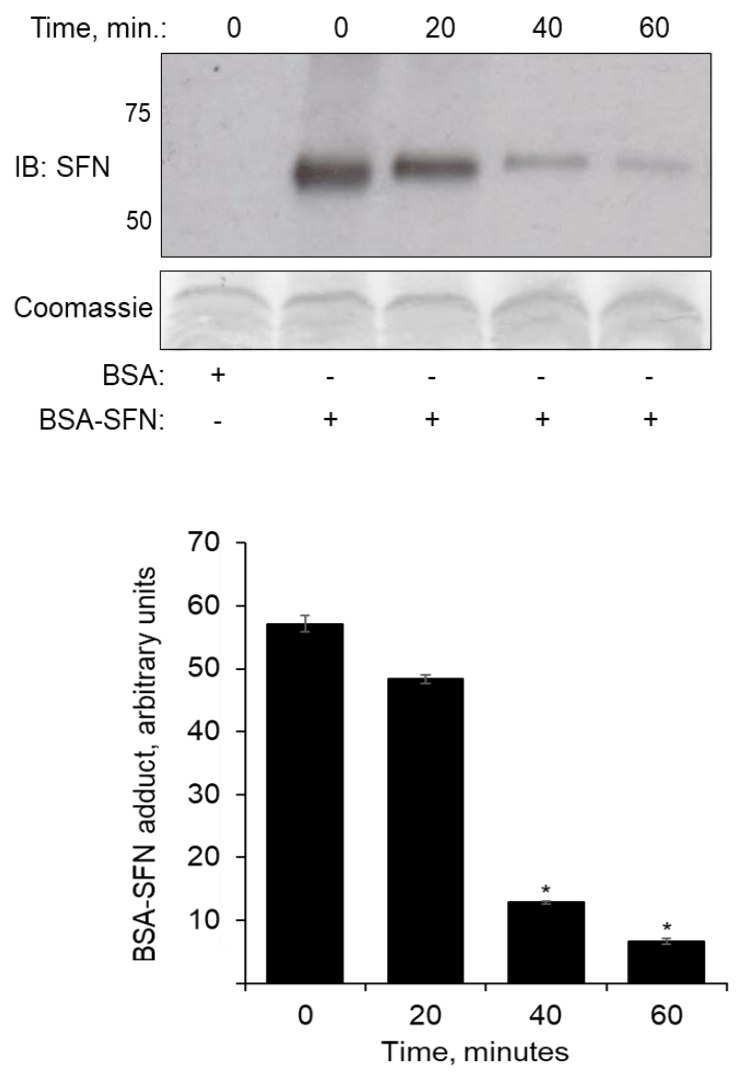

B)
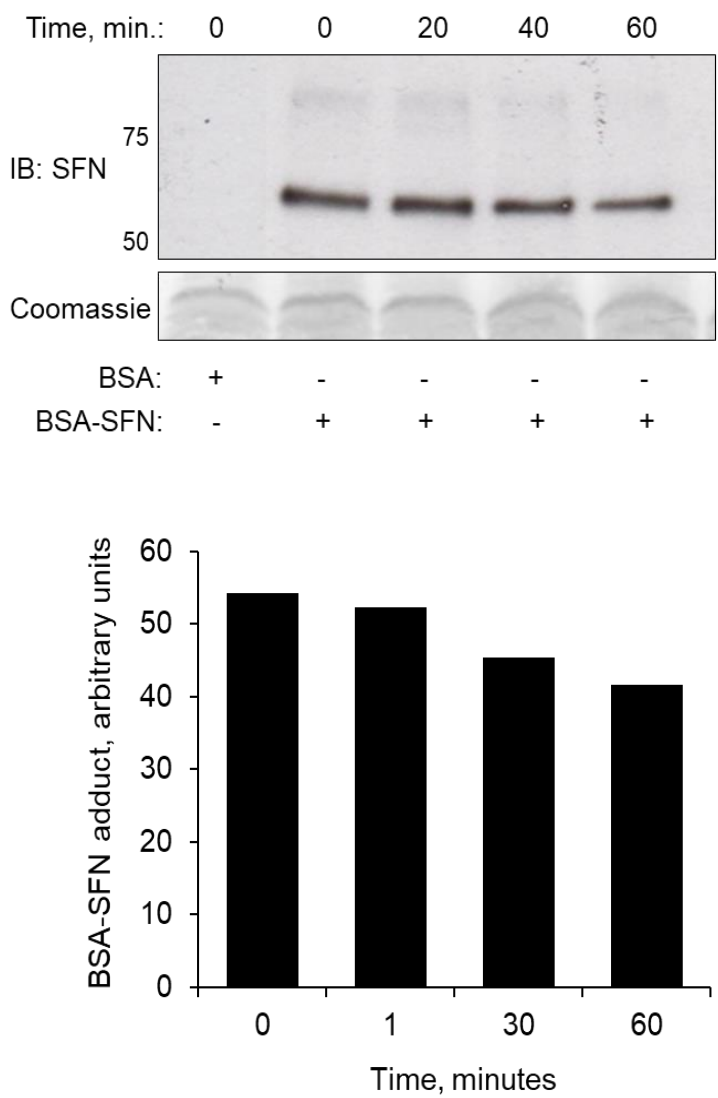

Figure 4.17. The SFN adduct is stable upon BSA within a PBS buffer.

A) An immunoblot showing removal of the SFN adduct from BSA when BSA-SFN in a $10 \mathrm{mM}$ Tris buffer purified by size-exclusion chromatography was incubated at room temperature for 0-60 minutes. B) An immunoblot showing stable adduction of SFN to BSA following incubation of chromatographically-purified BSA-SFN in a PBS buffer at room temperature for $0-60$ minutes. $\left(n=3,{ }^{*} p<0.05\right.$ versus 0 -minute control). 


\subsubsection{SFN can transfer from GSH to cellular proteins}

To investigate if SFN could transfer from GSH to cellular proteins, HEK293 cells were incubated for 30 or 60 minutes with commercially available GSH-SFN. Immunoblotting identified multiple proteins adducted by SFN following incubation for 30 or 60 minutes. Immunoblotting also revealed there was no increase in protein glutathionylation at either time point, suggesting SFN had directly transferred (trans-thiolated) from the tripeptide to cellular proteins (figure 4.18).

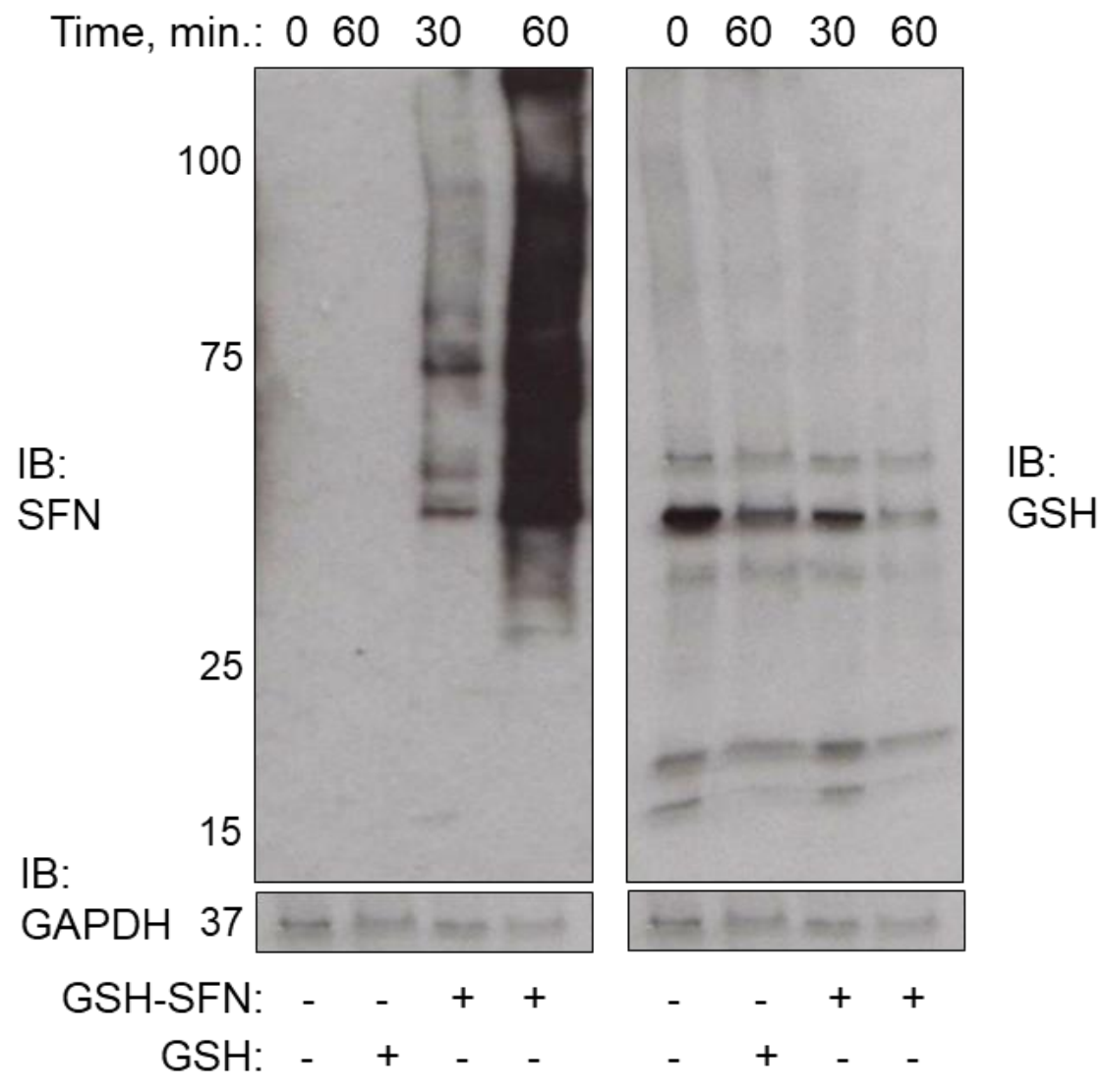

Figure 4.18. SFN can transfer from GSH to cellular proteins.

A representative immunoblot showing an increase in protein-SFN adducts following incubation of HEK293 cells with GSH-SFN for 30 or 60 minutes. No increase in glutathionylation of proteins was detected. 


\subsubsection{L-cysteine can remove the SFN adduct from BSA}

Immunoblot analysis showed there was a loss of the SFN adduct over time when BSA-SFN was incubated with L-cysteine for 5-40 minutes (figure 4.19). A timematched untreated BSA-SFN control showed no loss of the adduct over time in the absence of L-cysteine.

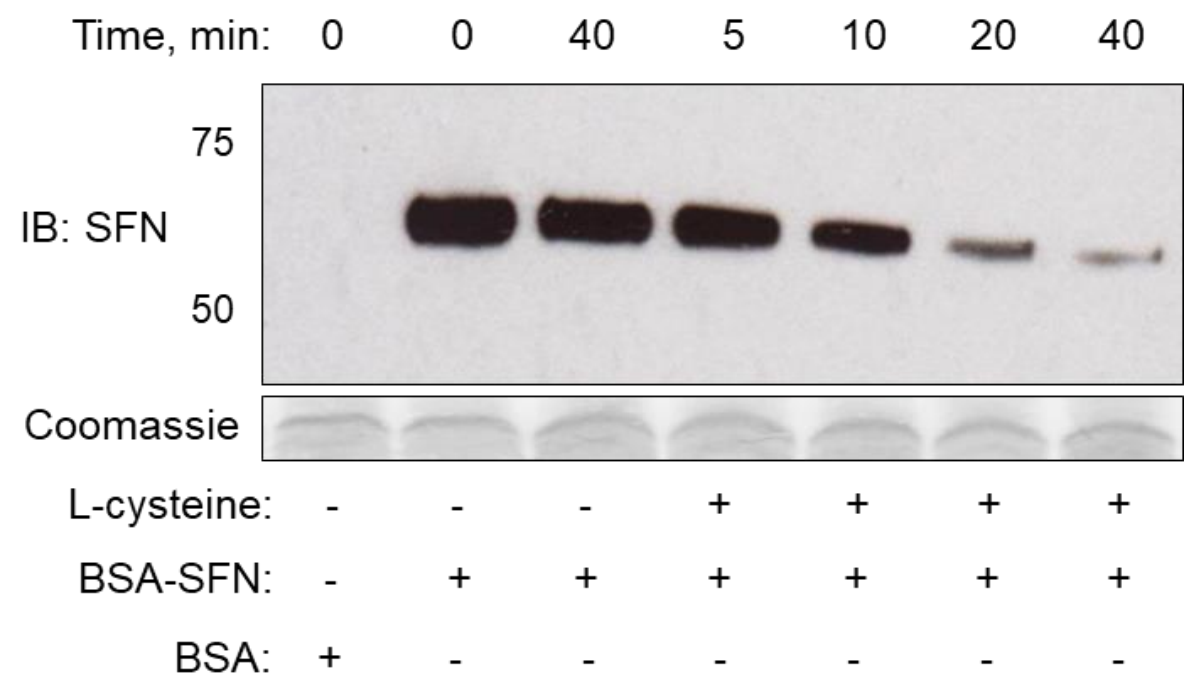

Figure 4.19. L-cysteine can remove the SFN adduct from BSA.

A representative immunoblot showing loss of the SFN adduct from BSA following incubation of BSA-SFN with L-cysteine for 5-40 minutes.

\subsubsection{SFN can transfer from BSA to $\mathrm{Hgb} \beta$}

To investigate if the SFN adduct could move between thiol-containing biomolecules, BSA-SFN purified by size-exclusion chromatography was incubated with either $\mathrm{Hgb} \beta$ or GSH for 1-60 minutes at either $\mathrm{pH} 7.4$ or $\mathrm{pH} 9.0$. Following 1-minute incubation at $\mathrm{pH} 7.4 \mathrm{Hgb} \beta$-SFN was identified by immunoblotting, consistent with rapid trans-thiolation of the electrophile from BSA. Although the amount of Hgb $\beta$-SFN did not increase, adduction of the electrophile to BSA significantly decreased over time and to a greater extent 
when incubated with GSH (figure 4.20 A). These observations were replicated at $\mathrm{pH} 9.0$, although a greater amount of $\mathrm{Hgb} \beta-\mathrm{SFN}$ was observed at all incubation times examined (figure 4.20 B). SFN adduction upon GSH was not detectable by the immunoblotting analysis due to the small size of this tripeptide. 
A)
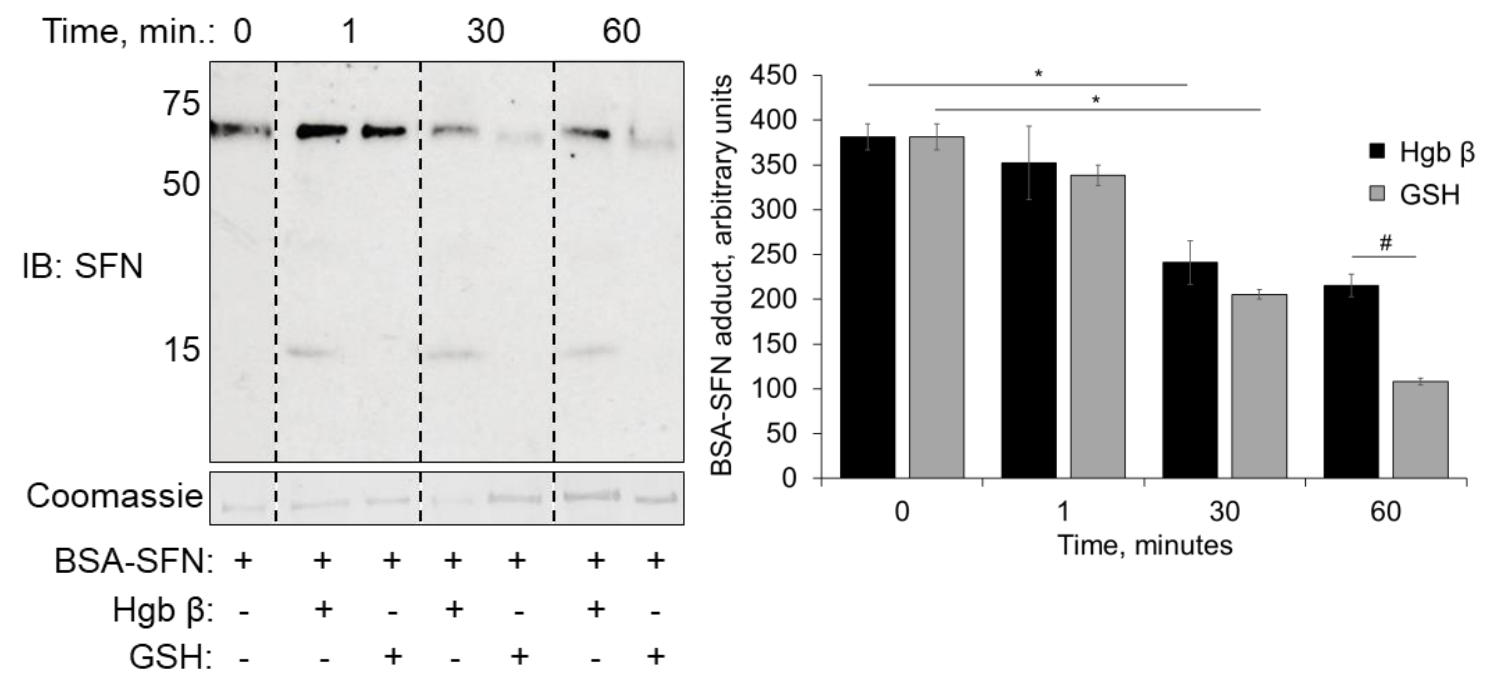

B)
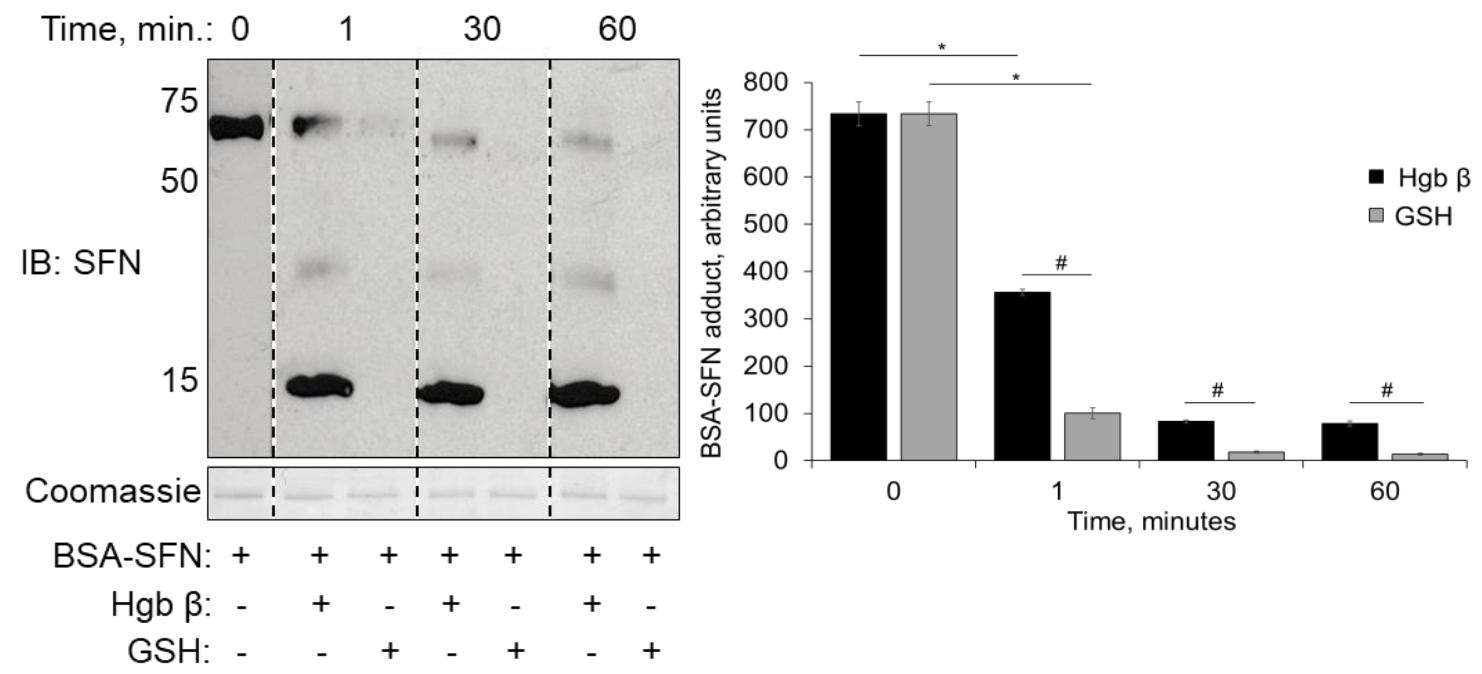

Figure 4.20. SFN can transfer from BSA to Hgb $\beta$.

A, B) Immunoblots showing time-dependent loss of SFN from BSA following incubation of BSA-SFN with either $\mathrm{Hgb} \beta$ or $\mathrm{GSH}$ for 1-60 minutes at $\mathrm{pH} 7.4(\mathrm{~A})$ or $\mathrm{pH} 9.0(\mathrm{~B})$. $\mathrm{Hgb}$ $\beta$-SFN is detected following 1-minute incubation and to a greater extent at $\mathrm{pH} 9.0(\mathrm{~B})$ than $\mathrm{pH} 7.4$ (A). GSH-SFN was not detected due to the small size of this tripeptide. $(n=3$, ${ }^{*} p<0.05$ versus 0 -minute control. $\# p<0.05$ versus treatment with $\mathrm{Hgb} \beta$ ). 


\subsubsection{SFN can transfer from SHP2 to GSH}

HPLC analysis following incubation of SFX-01 with SHP2 identified a doublet peak upon the chromatogram at 14.89 and 15.19 minutes (figure $4.21 \mathrm{~B}$ ). I hypothesised that the latter peak corresponded to SHP2 alone, whilst the peak with a shorter retention time was perhaps the protein adducted with SFN. Following incubation of the solution with GSH, HPLC analysis identified a peak at 15.19 minutes not associated with a doublet, likely unbound SHP2 and a broad peak at 9.12 minutes which correspond to a GSH-SFN standard (figure $4.21 \mathrm{C}$ ). The GSH-containing solution was then incubated with TCEP, to resolve any disulphide bonds. HPLC analysis identified two new peaks upon the chromatogram at 1.27 and 9.35 minutes. These two products were likely GSH and SFN respectively (figure $4.21 \mathrm{D}$ ), as the retention time is similar to that produced from an SFN standard shown in chapter 3 figure 3.10, and GSH is not retained on this HPLC column and will therefore pass through the column and be detected quickly. 
A)

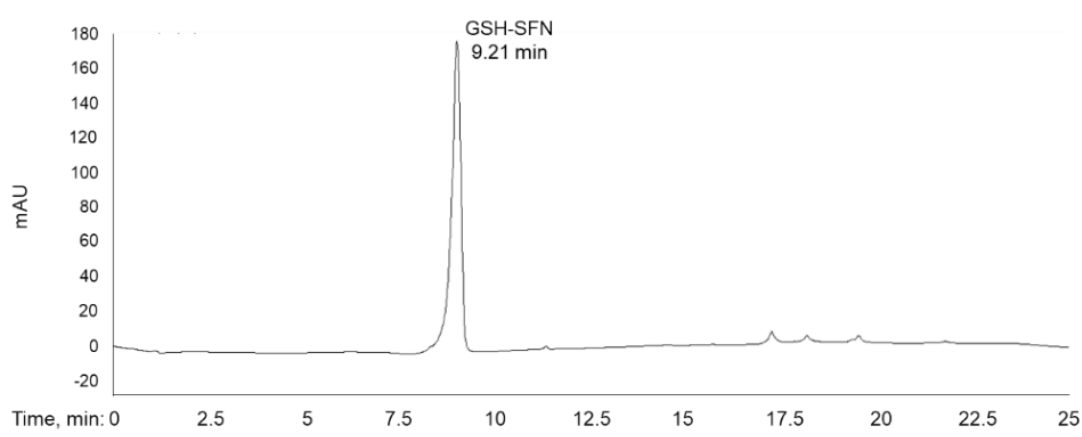

B)

C)

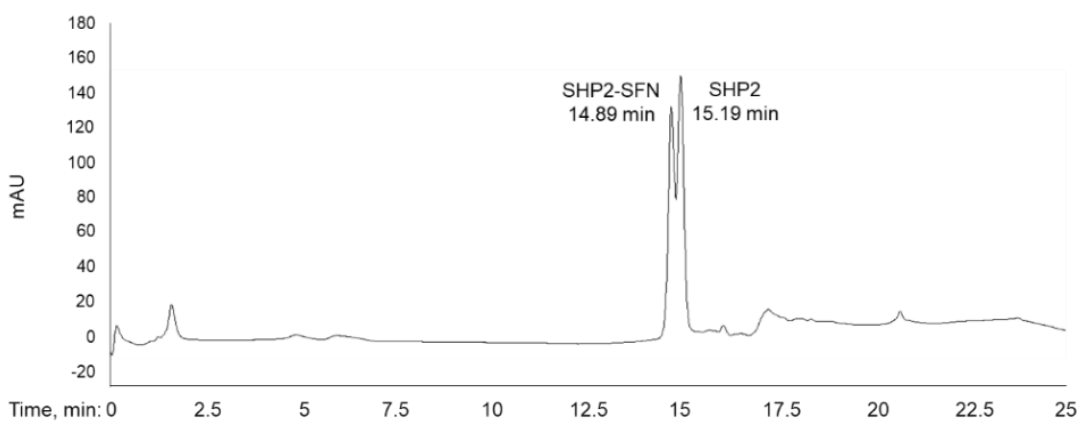

D)
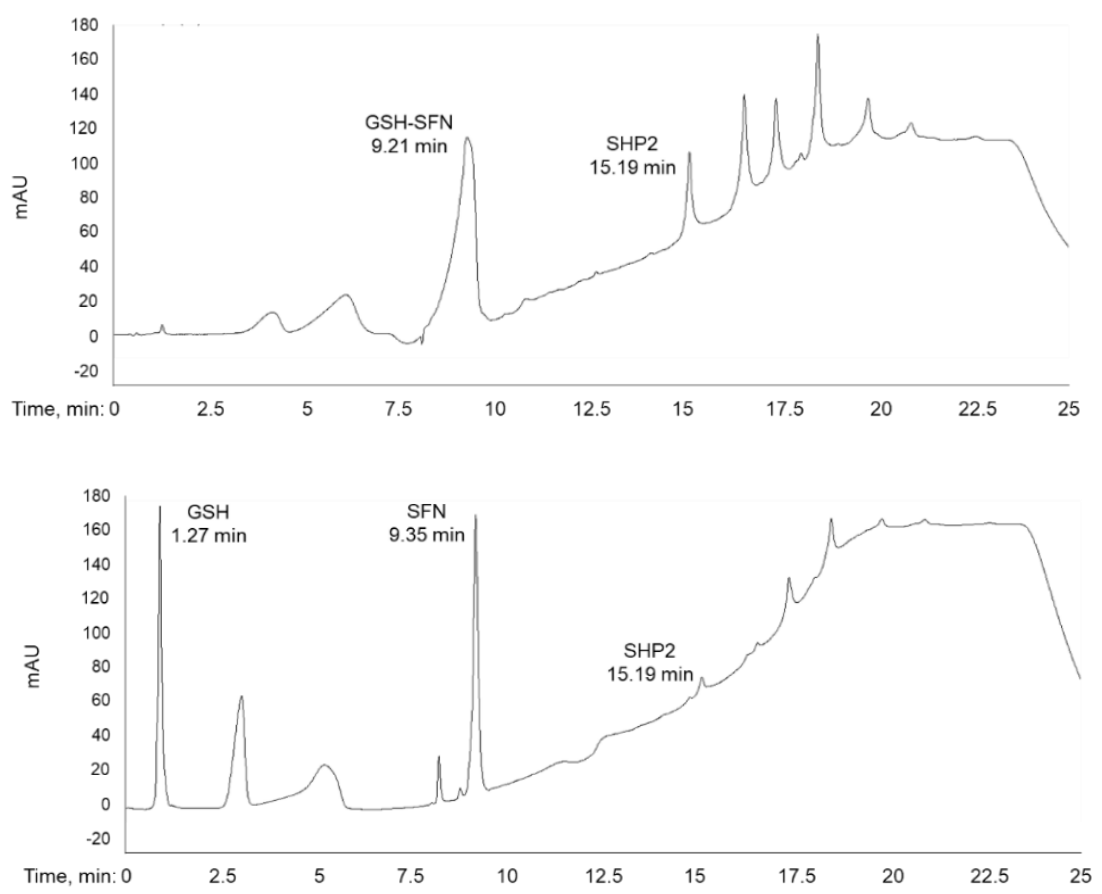

Figure 4.21. HPLC chromatograms showing SFN moving from SHP2 to GSH.

A) A GSH-SFN standard. B) $65 \mu \mathrm{M}$ SFN incubated with $10 \mu \mathrm{M}$ SHP2 for 30 minutes. C) SHP2-SFN incubated with $10 \mathrm{mM}$ GSH for 30 minutes. D) SHP2-SFN incubated with 10 $\mathrm{mM}$ GSH for 30 minutes and subsequent incubation with $10 \mathrm{mM}$ TCEP for 30 minutes. mAU represents absorbance using a UV detector set at a wavelength of $205 \mathrm{~nm}$. 


\subsubsection{SFN can transfer from BSA to a protein present in the blood}

To determine whether an SFN adduct could transfer from BSA to proteins within a biological sample, BSA-SFN was incubated with blood from WT mice for 1-60 minutes. Immunoblotting revealed SFN adducted with a $\sim 15 \mathrm{kDa}$ protein following 40 or 60 minutes of incubation (figure 4.22). A loss in SFN adduction of BSA was also noted over time. A time-matched untreated BSA-SFN control showed no loss of the adduct over time in the absence of blood.

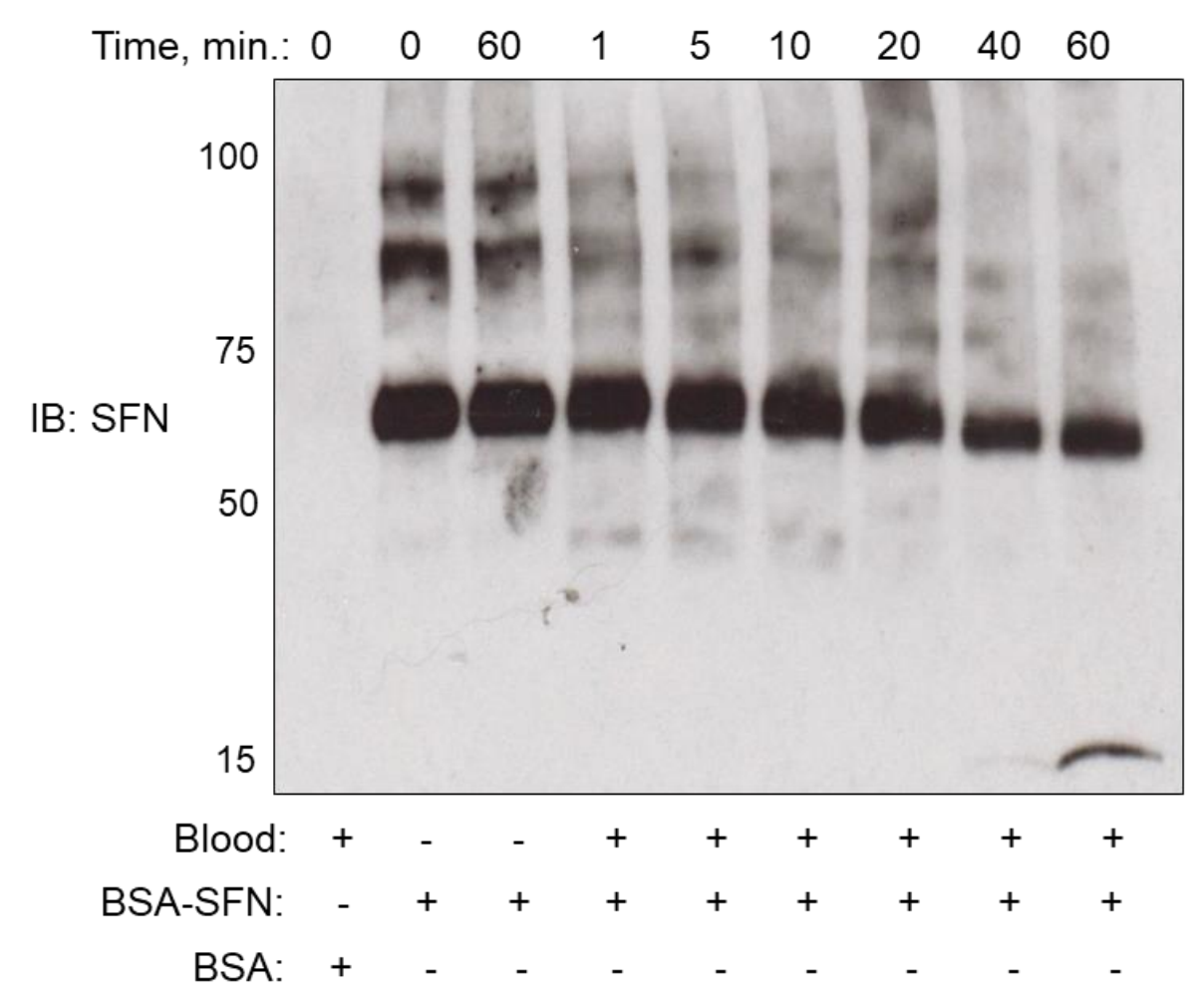

Figure 4.22. SFN can transfer from BSA to blood proteins.

A representative immunoblot showing a loss of an SFN adduct from BSA in conjunction with adduction of the electrophile to a $\sim 15 \mathrm{kDa}$ protein present in blood from WT mice following a 40 - or 60 -minute incubation. 


\subsection{Discussion}

The studies outlined here along with those in chapter 3 showed there is a prominent protein target of SFN, with adduction increasing in a time-dependent manner, following chronic treatment with SFX-01 or ingestion of broccoli sprouts. As well as identifying this protein I analysed the mechanism by which the SFN adduct accumulated on the prominent target protein.

Naturally derived sources of SFN in the form of cruciferous vegetables are frequently consumed by the general public. In this chapter, I first sought to determine if ingestion of the electrophile in this manner would result in its adduction to proteins, as occurred when synthetic SFN or SFX-01 was orally consumed. Increasing levels of chemophobia among the general population also support the need for such studies to identify if biological effects can also be achieved using these natural sources ${ }^{184}$. This aversion to chemical compounds including therapeutics gained momentum with the introduction of dichlorodiphenyltrichloroethane as a routinely-used pesticide as well as the production of genetically-modified organisms. This has led to a sustained increase in sales of both organic foods and natural health supplements. The use of natural therapies either alone or in combination with prescribed drugs is also surprisingly prevalent among patients not only with minor illnesses but also chronic and life-threatening diseases, driven largely by chemophobia 185186187. There has consequently been extensive research into the efficiency of naturally derived compounds as therapeutics, including ITC ${ }^{188} 189$. For example, 1-Methyl1,2,3,4-tetrahydroisoquinoline, which is present in several plant sources as well as the human brain, exhibits neuroprotective and antidepressant effects by inducing the production of brain-derived neurotropic factor and nerve GF as well 
as scavenging free radicals ${ }^{190}{ }^{191}$. Resveratrol, present in the seeds of many plants has shown multiple biological activities, including cardioprotection, in part due to its induction of antioxidant enzymes including glutathione peroxidase, superoxide dismutase and $\mathrm{HO} 1$ via an Nrf2-mediated pathway ${ }^{32}{ }^{33}$. The efficient antioxidant properties of vitamin $\mathrm{C}$, a prominent component in many fruit and vegetables, is also well characterised ${ }^{34} 35$. Biological effects following ingestion of plant/fruit/vegetable sources of the vitamin have been studied with promising outcomes 196197198 . For example, drinking beetroot juice protected against postischemic reperfusion injury, myocardial infarction and ventricular dysfunction in mice by a mechanism in which cyclic guanosine monophosphate and subsequently hydrogen sulfide were increased ${ }^{199}$. I detected SFN adducting to proteins following voluntary ingestion of broccoli sprouts, providing evidence that the ITC is bioactive and that any therapeutic effects of this electrophile may potentially be achievable following administration in this manner. This is consistent with previous studies identifying an increase in antioxidant protein expression in cardiovascular and kidney tissue of stroke-prone hypertensive rats following ingestion of broccoli sprouts 200201 . These studies also observed a correlation to attenuated oxidative stress, increased endothelial-dependent relaxation of the aorta, lowered blood pressure and increased response to NO in vascular smooth muscle cells 200 201. Cardioprotective effects were also seen following ingestion of broccoli sprouts for 30 days in rats whereby increased ventricular function, reduced myocardial infarct size and reduced cardiomyocyte cell death were observed following ischemia and reperfusion ${ }^{202}$. A reduced incidence of prostate cancer in mice via SFN-mediated reduction of histone 
deacetylase enzyme expression and subsequent loss in interaction with their corepressors has also been documented ${ }^{203}$.

Consistent with previous in vivo studies where SFX-01 was administered to mice in the drinking water, SFN adduction of a $\sim 15 \mathrm{kDa}$ protein was detected following ingestion of broccoli sprouts. The amount of SFN adducted to this protein following treatment with $2.5 \mathrm{mg} / \mathrm{ml} \mathrm{SFX}-01$ for 10 days was much greater than after the ingestion of broccoli sprouts. This is somewhat unsurprising as these in vivo studies vary largely in the amount of SFN received. Broccoli sprouts contain $\sim 255 \mathrm{mg}$ glucoraphanin per $100 \mathrm{~g}{ }^{204}$. Throughout this study, the mice ate between $5-10 \mathrm{mg}$ sprouts a day and therefore had a maximum intake of 0.025 mg glucoraphanin. It has previously been calculated that $\sim 75 \%$ of glucoraphanin is cleaved to form active SFN ${ }^{204}$. If I, therefore, assume that the mice received $0.00625 \mathrm{mg}$ SFN/day this is a considerably lower amount than those who received SFX-01 treatment $(0.115 \mathrm{mg}$ SFN/day) for an equivalent time period.

Upregulation of antioxidant proteins by SFN via the Nrf2 pathway is well characterised and outlined in detail in the general introduction. This includes the low molecular weight ubiquitous disulfide reductases Trx and GRX1, which have molecular weights of 11.7 and $12 \mathrm{kDa}$ respectively ${ }^{205}$. Figures 4.23 and 4.24 below show the enzymatic reactions of the Trx or GRX1 disulfide reduction systems respectively. These dithiol proteins each contain reactive cysteines within their active site motifs, Cys-Gly-Pro-Cys and Cys-Pro-Tyr-Cys respectively, and I therefore rationally investigated if the $\sim 15 \mathrm{kDa}$ protein on which SFN accumulated was either of these 206207. 
$\operatorname{Tr} x$

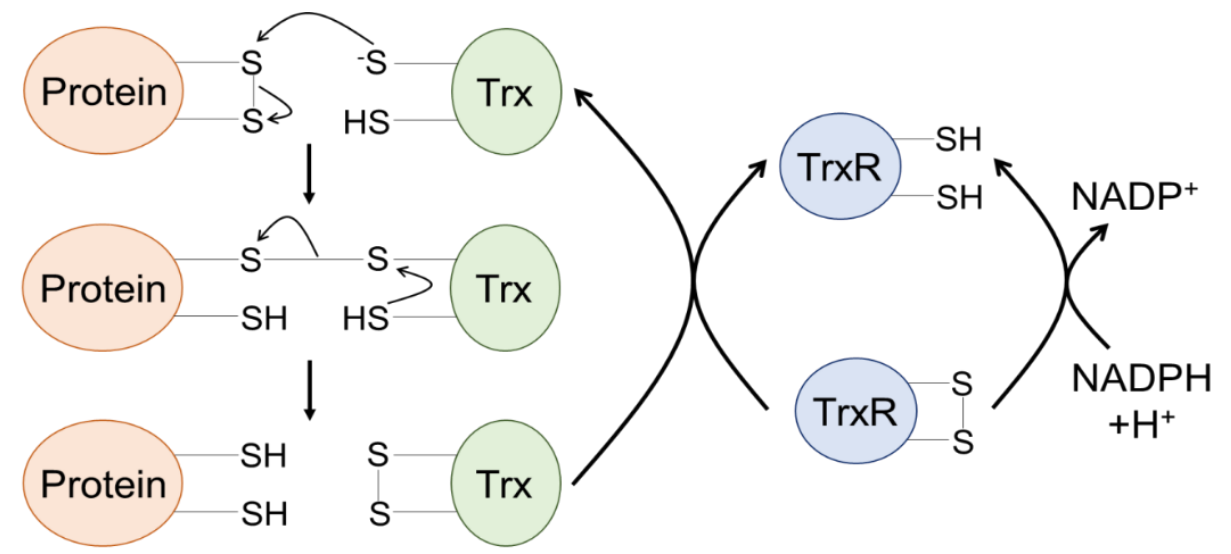

Figure 4.23. A diagram showing the enzymatic reactions involved in a two-step dithiol mechanism for catalytic reduction of protein disulfides by Trx.

GRX1

A)

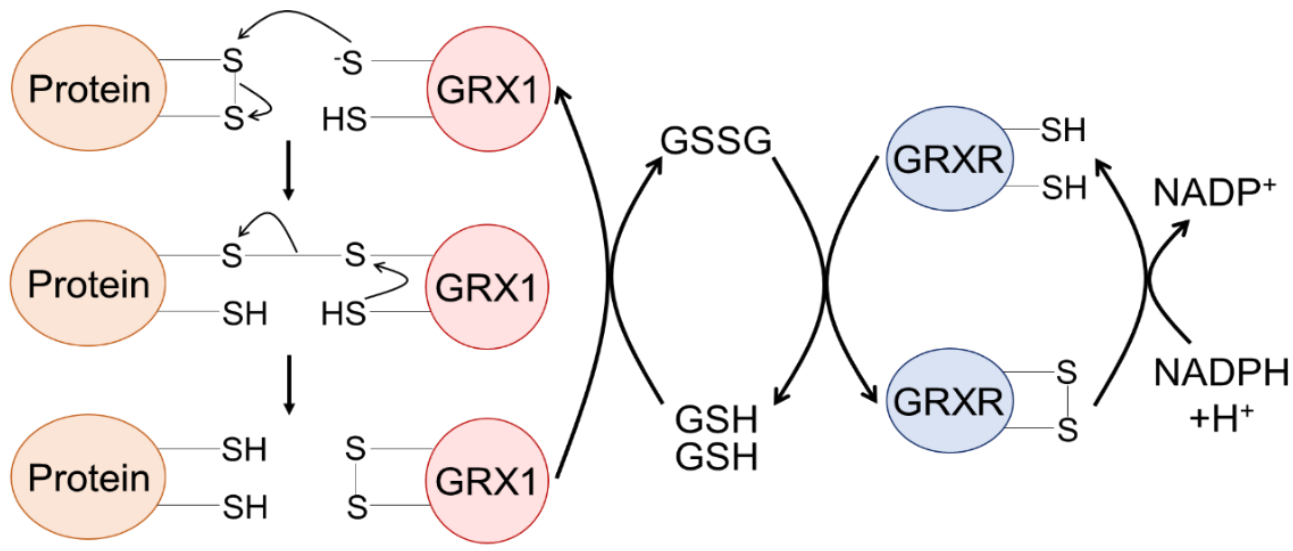

B)

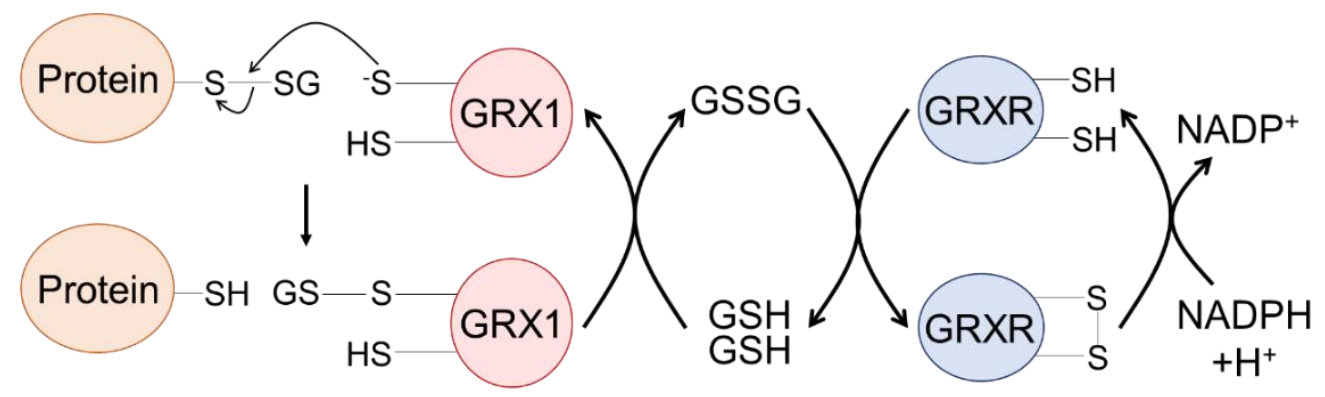

Figure 4.24. A diagram showing enzymatic reactions of GRX1.

A) A two-step dithiol mechanism for catalytic reduction of protein disulfides by GRX1 B) A one-step monothiol mechanism for catalytic reduction of protein-SG disulfides by GRX1. 
As anticipated and consistent with stimulation of an Nrf2-mediated cellular antioxidant gene response upon exposure to electrophiles ${ }^{208} 209$, treatment with SFX-01 for several days increased Trx and GRX1 protein expression. These observations are in alignment with previous studies whereby Trx protein expression was increased in the neural retina of mice following 3 days of oral treatment with SFN ${ }^{210}$. Treatment of a human breast epithelial cell line with SFN for 48 hours was also shown to upregulate GRX1 expression levels ${ }^{211}$. As depicted in figure 4.24 B a GSH adduct can trans-thiolate to GRX1. Therefore, as well as accumulation of TrX- or GRX1-SFN as a result of increased protein expression i.e. there is more reactive protein available for the electrophile to adduct to, perhaps trans-thiolation of the adduct from proteins onto the antioxidant is also occurring (figures 4.25 and 4.26). This concept of transthiolation of an SFN adduct between proteins is discussed in greater detail on page 133. If GSH and the TrxR/glutaredoxin reductase (GRXR) systems were unable to remove the SFN adduct from Trx or GRX1, then it may be anticipated this continual exposure to SFX-01 may cause accumulation of the adduct on either or both of the proteins and so explain the observations made. 

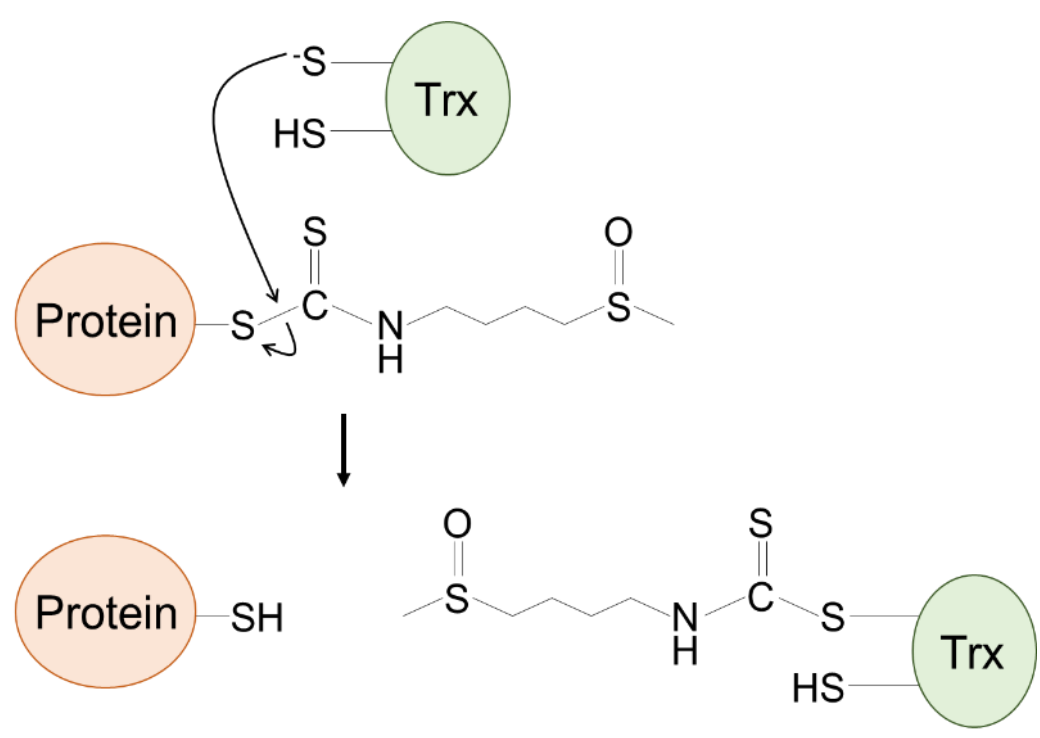

Figure 4.25. Proposed mechanism for transfer of the SFN adduct to Trx.
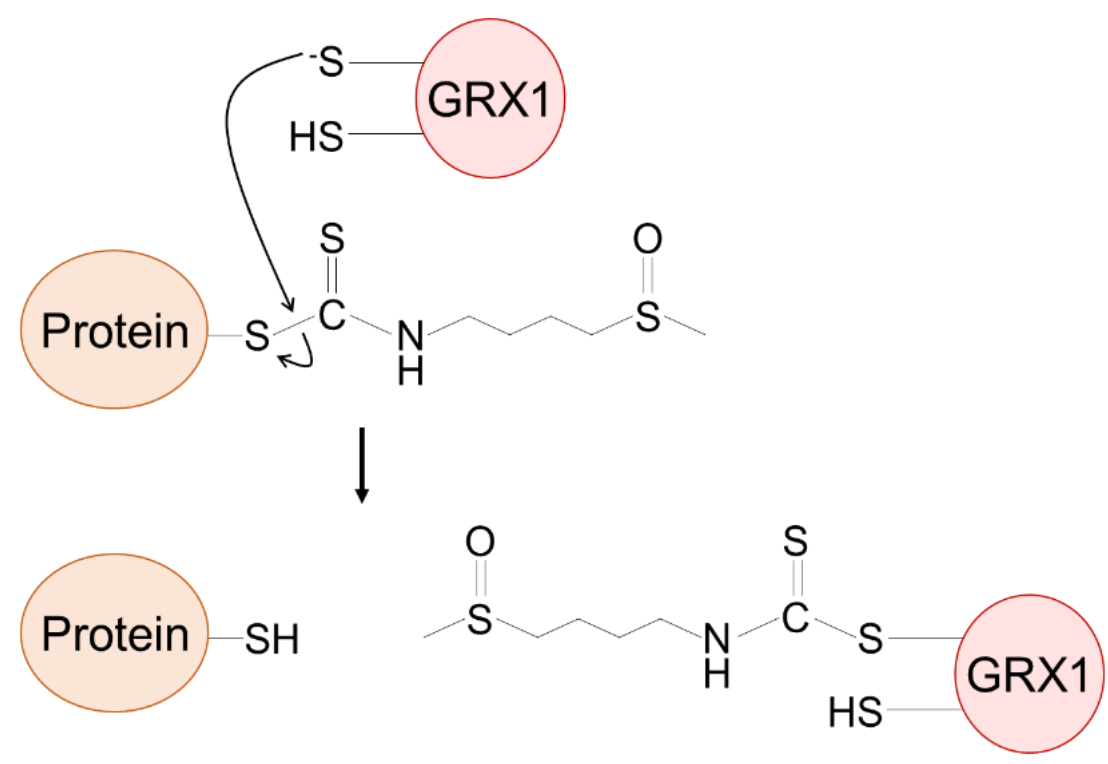

Figure 4.26. Proposed mechanism for transfer of the SFN adduct to GRX1.

Western immunoblotting concluded the $\sim 15 \mathrm{kDa}$ protein was not Trx or GRX1 as neither resolved at the same molecular weight as the protein containing the SFN adduct following gel electrophoresis. Consequently, LC-MS/MS analysis was 
undertaken as a method of identifying proteins present in a gel piece taken from where the modified protein migrated on an SDS-PAGE gel. No silver or Colloidal Coomassie staining was required to locate the $\sim 15 \mathrm{kDa}$ modified protein on the gel as it could be visualised by a yellow colouring, likely due to the high presence of sulphur within the adducted SFN. In addition to identifying proteins present in the gel sample, a mass equivalent to that of SFN was detected covalently adducted to $\mathrm{Cys}^{93}$ of the $\beta 1$ subunit of $\mathrm{Hgb}$.

Mammalian $\mathrm{Hgb}$ is a tetrameric structure consisting of two sets of dimeric polypeptide chains; $\alpha$-globin subunits 1 and 2 and $\beta$-globin subunits 1 and $2{ }^{212}$. Bound by non-covalent forces within each globin subunit is an ironprotoporphyrin IX molecule, which is primarily in the physiological ferrous (Fell) chemical valance state ${ }^{213}$. Two highly conserved amino acids, a proximal histidine and a distal phenylalanine, coordinate the iron moiety and without them, $\mathrm{Hgb}$ cannot perform its primary function as a two-way respiratory carrier ${ }^{214}$. Reversible binding of $\mathrm{O}_{2}$ at the four ferrous iron atoms allows transport of the gas from pulmonary vessels to tissues throughout the body ${ }^{215}$. The return of $\mathrm{CO}_{2}$ is then subsequently facilitated not by the ferrous iron but instead via weak interactions at the amino-terminal of the globins ${ }^{213}$.

Although the mechanism is still under investigation, circulating NO plays a role in the regulation of hypoxic vasodilation with both proposed hypotheses being dependent on red blood cells and deoxy-Hgb. The first involves the highly reactive Cys ${ }^{93}$ within the $\beta$ subunit of $\mathrm{Hgb}$ which is conserved between all vertebrate species possessing advanced cardiovascular systems ${ }^{216}$. Extensive research from many laboratories, predominantly led by the Stamler group, have demonstrated NO can bind at this highly reactive residue, either directly or via 
trans-nitrosylation (the transfer of $\mathrm{NO}$ ) from the heme group to the thiol, generating Hgb-SNO 217218 219. They report significant levels of Hgb-SNO within red blood cells isolated from arterial blood of anesthetised rats and almost undetectable amounts in venous blood ${ }^{220}$. It is proposed the increased rate of $S$ nitrosylation in areas of high $\mathrm{O}_{2}$ concentration is due to an allosteric change induced by $\mathrm{O}_{2}$ binding, which exposes $\mathrm{Cys}^{93}$ which would otherwise be blocked by the $\mathrm{C}$-terminal $\mathrm{His}^{146220}$. It is well characterised that physiological $\mathrm{O}_{2}$ gradients regulate vascular tone and blood flow, whereby lower concentrations of the gas result in hypoxic vasodilation ${ }^{221} 222$. It is now suggested that this endocrine-like transportation of $\mathrm{NO}$ upon $\mathrm{Hgb}$ may have a role in this coupling of metabolic demand with increased delivery of $\mathrm{O}_{2}$. Not only via the induction of allosteric changes which enhance the release of $\mathrm{O}_{2}$ but also the disassociation of $\mathrm{NO}$ itself from the macromolecule in areas of low $\mathrm{O}_{2}$ where it regulates vascular tone. Whereby, this offloading of bioactive $\mathrm{NO}$ from $\mathrm{Cys}^{93}$, via trans-nitrosylation to GSH or the cytoplasmic domain of the anion exchanger AE1 facilitates its excretion from red blood cells, where it subsequently enters the vascular endothelium and elicits its role as a potent vasodilator 223224225226227 . If Cys 93 does indeed play a key role in transportation and bioactivity of NO, it's adduction by SFN may have detrimental effects on the NO-mediated regulation of vascular tone. Perhaps if $\mathrm{Cys}^{93}$ is occupied by SFN, it can no longer scavenge $\mathrm{NO}$ at high $\mathrm{O}_{2}$ concentrations and subsequently cannot deliver it to areas of low saturation. Consequently, this may cause vasoconstriction and reduced blood flow in areas where metabolic demand for $\mathrm{O}_{2}$ is high.

This being said, the relevance of $\mathrm{Hgb}$-SNO formation in erythrocytes to the overall regulation of hypoxic vasodilation is still debated, with some researchers 
in favour of a second ideology named the nitrite-reductase hypothesis 228229230 . Studies have observed a clear arterial to venous plasma nitrate gradient with arterial levels reduced during exercise 231232 . A significant increase in red blood cell NO metabolite accumulation is also detected in veins compared to arteries, with the most dominant species being iron-nitrosylated $\mathrm{Hgb}^{231}{ }^{232}$. This theory, therefore, proposes the endocrine reservoir is in fact nitrite which is converted to $\mathrm{NO}$ by deoxy-Hgb which can then either diffuse through the vessel wall and elicit local effects on vasodilation or adduct vicinal deoxy-heme groups. NO adducted to deoxy-heme produces the six-coordinate species nitrosyl-Hgb, involving iron bound to four nitrogens, a proximal histidine and NO 233234 which may act as a transport mechanism for $\mathrm{NO}$. In areas of low $\mathrm{O}_{2}$ tension, the proximal histidine bond can break producing a five-coordinate species resulting in lowered affinity for and release of $\mathrm{O}_{2}$ from the neighbouring heme groups ${ }^{233}{ }^{234}$. These studies do detect non-significant formation of $\mathrm{Hgb}$-SNO in arterial plasma although to a much lower extent than NO-heme and suggest it's likely not a primary transport mechanism for $\mathrm{NO}$ although may facilitate its release from heme. As well as this, a mouse model with a $\mathrm{Cys}^{93}$ to $\mathrm{Ala}^{93}$ mutation generated by the Townes group identified no deficit in systemic or pulmonary haemodynamics and concluded it is not essential for the physiological coupling of erythrocyte deoxygenation to NO bioactivity ${ }^{235}$. Nevertheless, the effects of prolonged SFN treatment on blood pressure warrant further investigation.

Regulation of $\mathrm{NO}$ bioavailability by $\mathrm{Hgb}$, in particular, $\mathrm{Hgb}$ a, has also been demonstrated in endothelial cells at myoendothelium junctions within the blood vessel wall ${ }^{236}$. The expression of $\mathrm{Hgb}$ a increases as the diameter of the vascular wall decreases, correlating with an increase in microvascular junctions in these 
smaller resistance arteries ${ }^{237}$. Again, Hgb appears to play a role in regulating NO-dependent vasodilation of these arterioles ${ }^{238} 239$. When $\mathrm{O}_{2}$ concentrations are high, NO produced by endothelial NO synthase is scavenged by oxy-Hgb to produce nitrate ions and methaemoglobin with a ferric iron, which prevents diffusion into and vasodilation of vascular smooth muscle cells 236240 . As endothelial cells predominantly express $\mathrm{Hgb} \alpha$ and only minimal $\mathrm{Hgb} \beta$, it is unlikely that adduction of SFN at $\mathrm{Cys}^{93}$ of the $\beta$ subunit is occurring within this cell type. Hgb $\alpha$ and/or $\beta$ expression have been reported in multiple somatic cell types such as alveolar epithelial cells ${ }^{241}$, renal mesangial cells ${ }^{242}$, hepatocytes 243, macrophages ${ }^{244}$ and neurons ${ }^{245}$. However, I did not detect $\mathrm{Hgb}$ in isolated mouse ventricular cardiomyocytes. Further to this, flushing coronary arteries from isolated hearts of mice who had received SFX-01 with increasing amounts of buffer correlated with a loss of $\mathrm{Hgb}$ as well as Hgb-SFN. This is consistent with Hgb-SFN originating from the blood and likely red blood cells. Immunostaining of blood cells from mice who had received SFX-01 treatment with an anti-SFN antibody and subsequent analysis by flow cytometry or fluorescent microscopy should be conducted to confirm this.

As outlined in the introduction to this chapter, it has been shown a protein-SFN adduct can either spontaneously resolve, or trans-thiolate to another thiol containing protein. Following treatment of HEK293 cells with GSH-SFN, the accumulation of SFN adducts was seen upon multiple proteins with no detectable increase in glutathionylation. This corroborates findings that the interaction between the electrophile and GSH is reversible and perhaps the accumulation of SFN upon cellular proteins occurred following its direct trans-thiolation from the tripeptide. 
I next sought to assess if SFN adducts could also be reversed from proteins other than GSH and if so, whether trans-thiolation of the electrophile may account for its time-dependent accumulation on $\mathrm{Hgb} \beta \mathrm{Cys}^{93}$ following chronic in vivo treatment with SFX-01.

It is established that amine groups have nucleophilic properties and so can adduct ITCs including SFN 246247248 249, albeit with reduced affinity compared to thiols as they are a weak base with high $\mathrm{p} K_{\mathrm{a}} 250251$. Transfer of allyl-ITC from $N^{\alpha}$ acetyl-L-cysteine to either $N^{a}$-benzoyl-glycyl-L-lysine or lysine residues on BSA has been reported ${ }^{252}$. Similar transfer reactions of fluorescein-ITC were also shown between thiol and amine groups upon sarcoplasmic vesicles in vitro ${ }^{253}$. Indeed, in the studies reported here in which I purified BSA-SFN in an aminecontaining Tris buffer, this resulted in the loss of the SFN adduct over time which was not observed using a PBS buffer. A loss of the electrophile was also seen following incubation of BSA-SFN with L-cysteine or GSH. In addition, HPLC analysis suggested that SFN can transfer from SHP2 to GSH. Together these data provide evidence that SFN-modification of protein thiols can be reversed, which may be mediated by nucleophilic attack towards the protein-SFN interaction by another thiol or amine group, which results in the transfer of the electrophile to this second nucleophilic moiety. Importantly, I also observed SFN can indeed trans-thiolate from BSA to $\mathrm{Hgb} \beta$, supporting the ideology Cys ${ }^{93}$ may serve as a sink for the electrophile as a result of trans-thiolation from other thiolcontaining proteins.

Whilst published MS data from the Uchida group suggests that trans-thiolation of an SFN adduct occurs by direct transfer to a second thiol without the generation of a free-electrophile ${ }^{182}$, other published studies suggest the transfer of ITCs 
occurs via a two-step process whereby the dithiocarbamate resolves to regenerate the free-electrophile, which can subsequently adduct other thiol or amine groups 176179252253 . Should sporadic reversal be solely responsible for the transfer of the electrophile, we would expect an equal loss of the adduct when BSA-SFN was incubated with either GSH or Hgb $\beta$. However, a greater loss of the adduct was detected following incubation with GSH. This increased loss of the adduct following incubation with GSH was unexpected, as $\mathrm{Hgb} \beta$ was predicted as the stronger nucleophile due to $\mathrm{Cys}^{93}$ being recognised as highly reactive with a much lower $\mathrm{p} K_{\mathrm{a}}$ than the tripeptide. Nevertheless, this data suggests a second and perhaps dominant mechanism for trans-thiolation involving direct nucleophilic attack of the unbound thiol towards the dithiocarbamate, which is outlined in figure 4.27. This paradigm is further supported by a detectable increase in both removal of the SFN adduct from BSA and trans-thiolation to $\mathrm{Hgb} \beta$ at $\mathrm{pH} 9.0$ compared with 7.4. A salt bridge between Asp ${ }^{94}$ and $\mathrm{His}^{146}$ which is responsible for structural auto-inhibition of $\mathrm{Cys}^{93}$ of $\mathrm{Hgb}$ $\beta$ is loosened in alkaline conditions. The higher $\mathrm{pH}$ of 9.0 , therefore, induces a conformational change in $\mathrm{Hgb} \beta$ which would increase access of the thiolate of $\mathrm{Cys}^{93}$ to conduct nucleophilic attack towards the SFN adduct upon BSA ${ }^{220}$. A lowered $\mathrm{H}^{+}$content will also create an equilibrium in favour of the thiol being reduced, lowering its $\mathrm{p} K_{\mathrm{a}}$ and therefore increasing its reactivity. 

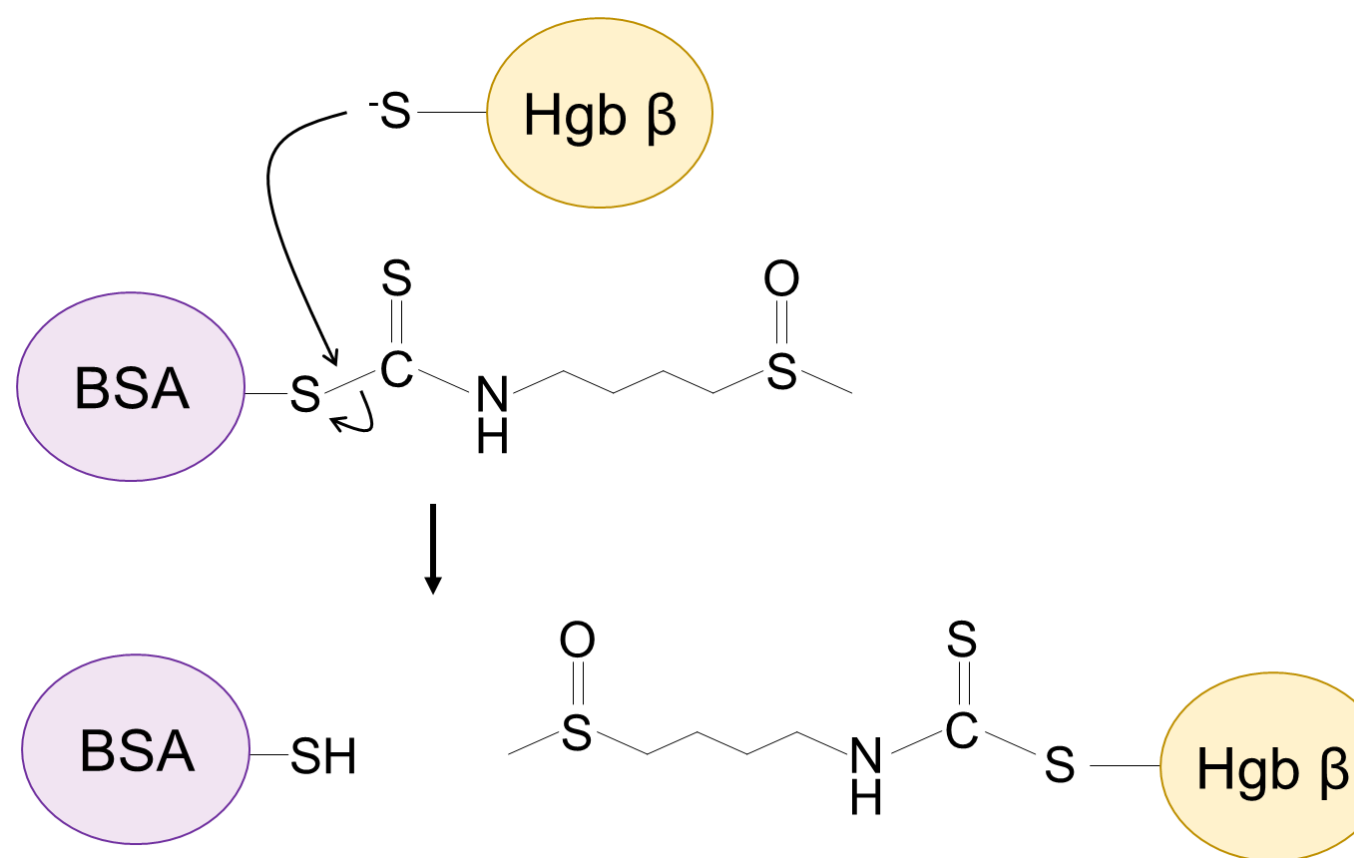

Figure 4.27. Proposed mechanism for trans-thiolation of SFN from BSA to Hgb $\beta$ involving directed nucleophilic attack of the unbound thiol towards to adduct.

Notably, SFN adduction to $\mathrm{Hgb} \beta$ following incubation of the protein with BSASFN occurs rapidly but does not accumulate over time. Perhaps Cys ${ }^{93}$ of $\mathrm{Hgb} \beta$ becomes saturated with SFN within 1-minute of exposure, with the availability of the cysteine increasing at $\mathrm{pH} 9.0$ than 7.4 for reasons explained above. Repeating these studies with a higher concentration of $\mathrm{Hgb} \beta$ may result in timedependent accumulation of the adduct. Indeed, time-dependent accumulation of SFN upon an $\sim 15 \mathrm{kDa}$ protein, presumably Cys ${ }^{93}$ of $\mathrm{Hgb} \beta$, was observed when BSA-SFN was incubated with blood. Also, time-dependent loss of the SFN adduct from BSA did not correlate with the level of accumulation upon $\mathrm{Hgb} \beta$. Transfer of the adduct to amines may also be occurring, either via direct nucleophilic attack or subsequently to sporadic reversal, which could not be detected using the SFN antibody. 
Of course, SFN accumulation upon $\mathrm{Hgb} \beta$ following chronic in vivo treatment with SFX-01 could be due to direct adduction of the protein by a free-form of the electrophile. However, together, these studies suggest Cys ${ }^{93}$ of $\mathrm{Hgb} \beta$ may serve as a sink for SFN as a result of the transfer of the electrophile from other protein thiol or even amine groups. The efficiency of trans-thiolation of SFN from BSA to GSH compared with $\mathrm{Hgb} \beta$ highlights this tripeptide may facilitate this transient movement of the electrophile. Perhaps the adduct first trans-thiolates from a protein thiol to $\mathrm{GSH}$, with a subsequent nucleophilic attack by $\mathrm{Cys}^{93}$ of $\mathrm{Hgb} \beta$. As the $\mathrm{pK}$ a of the thiol of GSH is notably higher than that of the reactive thiol of BSA, this highlights that transfer of the adduct may not depend on the thiol $p K_{a}$ of the receiving protein, although what does control the direction of trans-thiolation is still undetermined. Perhaps thiol accessibility plays a role. The simple structure of GSH may allow the tripeptide to easily access protein-SFN adducts to perform a nucleophilic attack, and also, GSH-SFN would subsequently be easily accessible by other free protein thiols. Why Cys ${ }^{93}$ upon $\mathrm{Hgb} \beta$ is more efficient in this process than other abundant reactive-thiol containing blood proteins such as Cys $^{34}$ upon BSA is unclear ${ }^{254}$. Perhaps it is simply due to $\mathrm{Hgb}$ being $3 \mathrm{X}$ more abundant 255256 . The progressive build-up of Hgb $\beta$-SFN could elude the adduct is relatively stable. However, these studies suggest it is likely GSH will subsequently remove SFN and perhaps due to the large amount of SFX-01 administered to mice the rate of adduct formation is simply greater than the rate by which GSH or other proteins can remove the adduct. Further investigation into trans-thiolation of SFN from $\mathrm{Hgb} \beta$ to other proteins warrants investigation as this may provide a method for transportation of the electrophile between tissues. 


\section{Therapeutic potential of sulforaphane in a NS}

\section{mouse model}

\subsection{Introduction}

\subsubsection{The role of SHP2 in cardiac development}

SHP2 protein expression is distributed throughout the embryo suggesting it holds various functions in embryonic development ${ }^{126}$. Indeed, homozygous $\mathrm{N}$-terminal deletion of SHP2 results in embryonic lethality mid-gestation due to several defects in cell differentiation, mesodermal patterning and body organisation including node, notochord and posterior elongation ${ }^{257}$. These developmental processes are all dependent upon SHP2 for full and sustained activation of the SFK/Ras/ERK signalling pathway following stimulation by fibroblast GF, which stabilises the pro-apoptotic protein Bcl-2-like-protein-22 (BIM) ${ }^{257} 258$. Studies using amphibian and avian embryos showed cardiac cell specification and differentiation occur at the onset of gastrulation 259260 . The fibroblast GF signalling pathway induces expression of key progenitors which regulate cardiac cell differentiation, including homeobox protein NK-2 homolog B, T-box 5 and GATA-binding protein $4{ }^{261}$. A lack of cardiac cell differentiation was seen in Xenopus embryos with knockdown of SHP2 following cardiac explant assays, revealing an essential role of the phosphatase in the specification and maintenance of such cardiac progenitors ${ }^{262}$. Following gastrulation, cardiac development and morphogenesis occur ${ }^{263}$. As homozygous deletion of the phosphatase in mice results in embryonic lethality mid-gestation, elucidation of the role of SHP2 in cardiac development has been challenging ${ }^{257}$. Progress has since been made using Xenopus and zebrafish whereby embryonic lethality pre- 
or mid-gastrulation were overcome. Morpholino-mediated knockdown in zebrafish showed SHP2 regulates convergence and extension of myocardial and endocardial precursor cells during gastrulation via RhoA and SFK signalling 264 61. The same conclusion was made by expressing mRNA encoding gain-offunction or loss-of-function SHP2 mutations associated with NS or NS with multiple lentigines respectively in zebrafish embryos ${ }^{61}$. Interestingly, they suggest both an activating and an inhibitory role of SHP2 in SFK signalling during gastrulation, as the injection of either a hyperactivating or deactivating mutation had the same physiological outcome ${ }^{61}$. Parallel studies injecting mRNA encoding a loss-of-function mutation found in patients with NS with multiple lentigines into SHP2-deficient Zebrafish embryos further identified a role of the phosphatase in neural crest development and migration post-gastrulation in an ERK-dependent manner ${ }^{265}$.

Rightward looping of the linear heart tube initiated by fusion of bilateral heart primordia is the first morphological manifestation of embryonic laterality ${ }^{264}$. This mainly occurs due to left/right asymmetric localisation of transcription factors and signalling molecules such as the transcription GF $\beta$ cytokine, nodal ${ }^{266}$. The leftward movement of nodal occurs by transport within Kupffer's vesicles which are mechanistically transported by motile cilia 267268269 . SHP2 plays multiple roles in this process with randomisation of such left/right markers and impaired leftward heart displacement in zebrafish embryos was seen when expressing SHP2 mutations which altered the phosphatases activity ${ }^{270}$. Expression of Kupffer's vesicles is stimulated by fibroblast GF 8 , and this signalling event is regulated by SHP2 ${ }^{261}{ }^{271}$. Vesicle development is dependent upon the distribution of receptor-mediated calcium release from the endoplasmic reticulum 
272. Calcium signalling via the calcium-permeable cation channel polycystin 2 is also important in both the formation and sensing of cilia ${ }^{273}$. It is suggested SHP2 functions in calcium signalling either indirectly via ERK-mediated phosphorylation of the $\operatorname{cav}(1.2)$ subunit or direct association with inositol triphosphate 3 receptors 274275 . As well as asymmetrical localisation of nodal, actin polymerisation and non-muscle myosin 2 activity are also required for left/right patterning ${ }^{276}$. Gainof-function SHP2 mutations cause reduced formation and polarity of cardiac actin fibres likely via hyperactivation of Rho-associated protein kinase, resulting in smaller hearts with impaired cardiac looping 277.

Segmentation into the atrium, ventricles, atrioventricular canal and outflow tract, as well as the aorta and pulmonary tract, is the next stage of cardiac development 278. Endocardial cushions subsequently form between the atrioventricular canal and outflow tract which later evolve into semilunar and atrioventricular valves. The localisation of multiple signalling molecules and pathways regulate this process, including vascular endothelial GF, ERK, nuclear factor of activating Tcells cytoplasmic 1, notch, $\beta$-catenin and bone morphogenetic protein 279280281 , all of which involve SHP2 and indeed, heterozygous deletion of the phosphatase results in valve enlargement ${ }^{282}$.

Finally, aortic and pulmonary circulation are separated by the development of ventricles, atria and the outflow tract whilst the septum forms due to the expansion of the left and right ventricle 278283284 . Hyperactivation of SHP2 in the myocardium of mouse embryos results in thinner ventricular walls and septal defects ${ }^{285}$. Although, the same is also seen following reduced activation of the phosphatase ${ }^{286}$. Perhaps SHP2 regulates cardiac chamber maturation via a phosphatase-independent mechanism, a hypothesis proposed by Paardekooper 
Overman et al. They propose increased association of kinase c-Src with SHP2 due to the phosphatase being in an open conformation, acting as a scaffold to bring the proteins into proximity 287288 .

\subsubsection{White blood cell production}

Blood cells, including leukocytes, are short-lived and require continual production via differentiation from haematopoietic stem cells (HSCs), a process termed haematopoiesis 289290291292 . A detailed overview of this process is shown in figure 5.1. The main site of haematopoiesis in adult mammals under normal circumstances is the bone marrow, however, can also occur extramedullary in the liver and spleen in response to severe haematopoietic stresses ${ }^{293}$. This process is hierarchical, with a progressive commitment to a certain cell-type coupled with a loss of self-renewal 4547295 . Initially, HSCs are defined as longterm, which self-renew indefinitely and are distinguished by cell surface markers 296297298299 . Molecular regulation of self-renewal and inhibition of differentiation of long-term HSCs is coupled with notch and wingless signalling ${ }^{300}$. The progress of long-term HSCs to multipotent progenitor (MPP) cells is marked by RTK foetal liver kinase-2 (Flk-2) ${ }^{+}$and cluster of differentiation 90 (Thy-1.1)- with an intermediate HSC formed named short-term HSC (Flk-2+ Thy-1.1+), which has limited self-renewal capacity ${ }^{296}$. MPP cells can no longer self-renew but are heterogeneous in their cellular content and retain the potential to differentiate into any white blood cell lineage ${ }^{301}$. Long- and short-term HSCs and MPP cells differ in expression of transcription factors as shown in figure 5.1302 303304 305, which is regulated by discrete changes in the niche microenvironment in which HSCs reside. Long- and short-term HSCs and MPP cells reside in perivascular niches associated with sinusoidal blood vessels in adult bone marrow 306307308 . 
Certain GFs maintain HSCs in these niches, including stem cell factor (SCF), CXC-chemokine ligand 12 and thrombopoietin (TPO), synthesised by endothelial and reticular perivascular stromal cells 309310311312313314 . The niche microenvironment is also manipulated by other cell types including megakaryocytes, monocytes and macrophages, which directly or indirectly alter the fate of residing HSCs 315316317318 . Akashi et al identified MPP cells differentiate to common lymphoid or common myeloid MPP cells, distinguished by different cell surface markers, IL-7Ra ${ }^{+}$Thy $-1.1^{-} \mathrm{Lin}^{-} \mathrm{Sca}-1^{\mathrm{lo}} \mathrm{C}-\mathrm{Kit} \mathrm{it}^{\mathrm{lo}}$ and CD34+FcyRIII-/lo Thy-1.1-IL-7Ra- ${ }^{-}{ }^{+}{ }^{-}$Sca- ${ }^{-}{ }^{-}$C-Kit ${ }^{+}$respectively ${ }^{71} 72$. This cellular differentiation occurs in part due to stimulation by circulating cytokines, which is also true for subsequent differentiation and commitment steps, as depicted in figure $5.1321322{ }^{323}$. As well as differentiation commitment, these cytokines promote cell survival, induction of maturation and functional activation ${ }^{321}$. Common lymphoid cells differentiate into mature B-, T-, or natural killer (NK)-cells where the lymphoid lineage ends ${ }^{324}$. Common myeloid cells on the other hand further differentiate into megakaryocyte-erythroid or granulocyte-myeloid progenitor cells 325 . The megakaryocyte-erythroid lineage ends with differentiation to either platelets or erythrocytes ${ }^{291} 325$. Granulocyte-myeloid cells, however, require an additional differentiation step forming either myeloblasts or monoblasts, with final maturation into basophils, eosinophils and neutrophils or monocytes respectively 319326 .

Although most lymphocytes then enter the circulation, a large pool of neutrophils reside in the bone marrow until mobilised in response to chemoattractant cues 8182327 . Whilst a proportion of monocytes circulate freely in the blood awaiting recruitment and differentiation into macrophages, these are outnumbered by 
monocytes residing in the subcapsular red pulp of the spleen 330331332333 . Genetic and cell-fate mapping identified spleen-resident macrophage populations represent a separate and distinct phagocyte lineage established prior to birth, either from elements present in the yolk-sac or foetal liver precursors $\begin{array}{llll}334 & 335 & 336 & 337\end{array}$. How and why monocyte clusters are recruited and maintained within the spleen remains unclear. Although inflammatory signals mobilize these cells en masse to distant tissues, so it likely serves as a reservoir until required to differentiate to macrophages and fight infection ${ }^{338}$. Perhaps they also contribute to the replenishment of spleen-residing macrophages, although this remains undetermined. It is also suggested the spleen can act as a reservoir for neutrophils within the perifollicular zone, which aid antibody production by marginal zone residing B-cells 339340341 . An immature population of spleen residing neutrophils have also been discovered, although whether they arise via bone marrow-dependent or -independent haematopoiesis could not be established 342343 . 


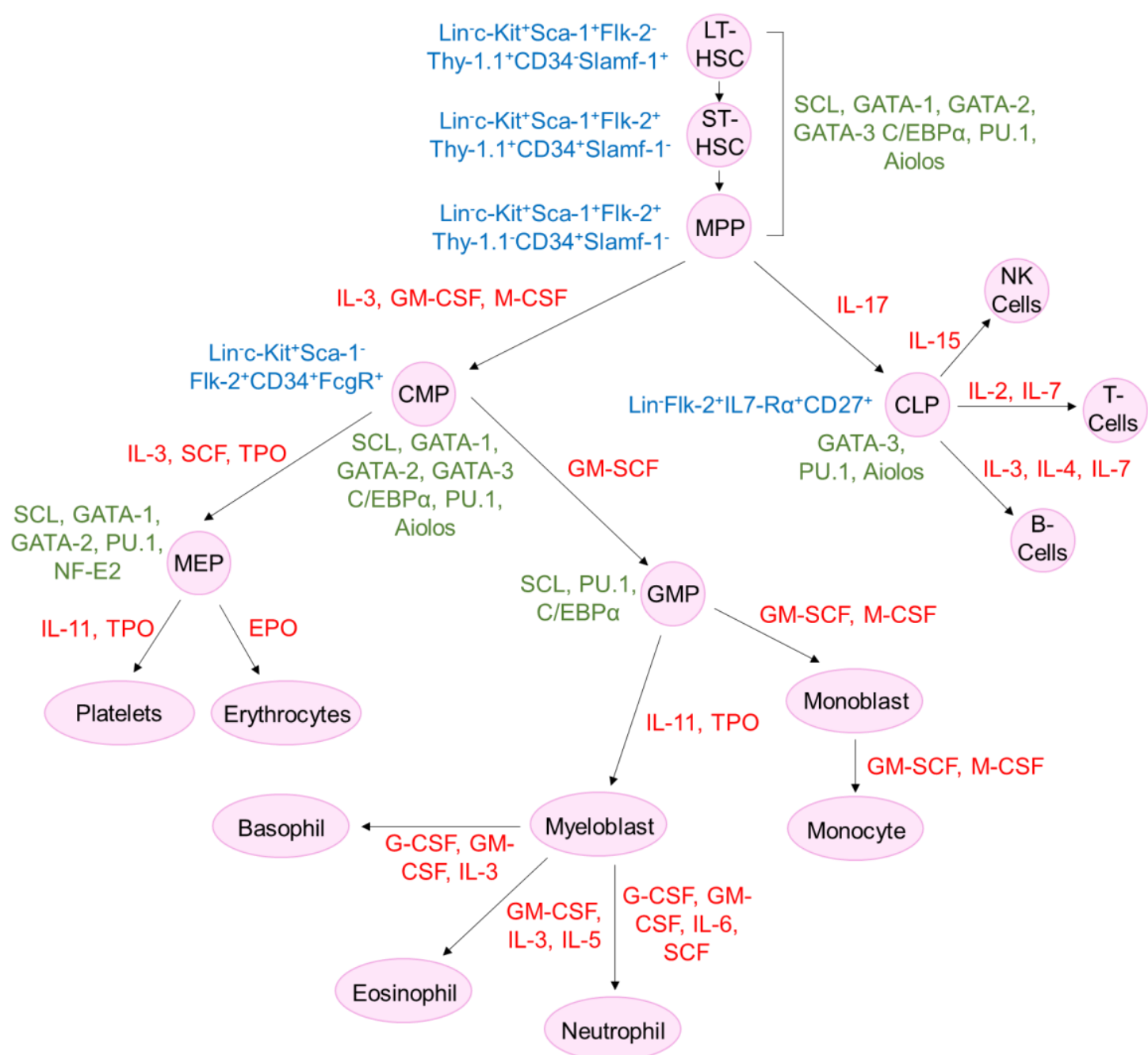

Figure 5.1. White blood cell production in the bone marrow by haematopoiesis.

Blue $=$ cell surface receptors. Lin (Linear), Kit (Mast/stem cell GF receptor), Sca (Stem cell antigen), CD (Cluster of differentiation), Slam (Signalling lymphocyte activation molecule), FcgR (Fragment crystallizable gamma receptor, IL7-R (Interleukin 7 receptor). Green = cellular transcription factors. SCL (Stem cell leukaemia), GATA (Formerly termed Erythroid transcription factor), C/EBP (CCAAT-enhancer binding protein), PU (PU-box binding). Red = cytokines and chemokines which aid each differentiation step. IL (Interleukin), GM-CSF (Granulocyte-macrophage colony stimulating factor), M-CSF (Macrophage colony stimulating factor), G-CSF (Granulocyte colony stimulating factor), TPO, SCF. 
A detailed overview of haematopoiesis was provided above to highlight the important roles of SHP2 in the proliferation and survival of HSCs and progenitors. As indicated in figure 5.1, the cell-surface receptor Kit is highly expressed in HSCs ${ }^{344}$. Following binding of SCF to Kit, SHP2 creates a positive feedback loop, an important process in maintaining adult HSC quiescence and survival ${ }^{345}$ 346347 . The Eder group showed SHP2 acts downstream of the RTK breakpoint cluster region-abelson murine leukaemia (Bcr/Abl), promoting HSC proliferation and survival through activating STAT5 signalling ${ }^{348}$. SHP2 also acts upstream of the cell-proliferation pathways Ras, ERK and Akt, with inducible SHP2-deficient HSC and progenitors undergoing apoptosis in response to SCF and TPO ${ }^{349 .}$ Through overexpressing gain-of-function SHP2 mutants in progenitor cells the Chan group demonstrate a higher number of these cells residing in the synthesis and gap 2 phase of the cell cycle along with increased markers of progression, including cyclin D1, B-cell lymphoma-2 and B-cell lymphoma extra-large ${ }^{350}$, as well as reduced expression of apoptotic markers p27, p21 and B-cell lymphomalike-protein $11^{350}$. Using shRNA-mediated inhibition of SHP2 expression, Li et al identified reduced myeloid differentiation following GF stimulation ${ }^{351}$. This was driven by an initial increase in STAT5 phosphorylation, correlating with my own observations in cardiac tissue as outlined in chapter 3 , with a subsequent decrease that reduced expression of anti-apoptotic genes including myeloid leukaemia cell differentiation protein and B-cell lymphoma extra-large ${ }^{351 .}$ Through the use of a lymphocyte cell line, $\mathrm{Ba} / \mathrm{F} 3$, the Friedman group further suggest stimulation with G-CSF leads to SHP2 phosphorylation with subsequent activation of STAT3 signalling and granulocyte lineage determination ${ }^{352}$. Finally, Xu et al show hyperproliferation of lineage-committed myeloid, T-cell and B-cell 
progenitors following transfection with SHP2 which has a gain-of-function mutation, E76K/+ ${ }^{353}$. They speculate activated SHP2 distributes into centrosomes driving the amplification and genomic instability of these transfected cells ${ }^{353}$.

As highlighted above, SHP2 plays key roles in cardiac development and white blood cell production. It is perhaps unsurprising that NS patients with gain-offunction SHP2 mutations often present with cardiac structural defects and a myeloproliferative phenotype as detailed in the general introduction ${ }^{78} 354.100$ $\%$ of homozygous Ptpn11 1 61G+/+ NS mice and approximately $50 \%$ of heterozygous Ptpn11 1 D61G/+ NS mice, which I will refer to as HOM and HET respectively in this chapter when referencing foetal experiments, die in utero due to several cardiovascular structural defects ${ }^{78}$. Here, I first sought to establish if foetal treatment with SFX-01, by administering the drug to pregnant dams, inhibits SHP2 phosphatase activity to perhaps enhance the birth rate of HET offspring and allow HOM offspring to survive through to birth. Of course, if SFX01 did enhance viability, a rational next question was whether their cardiac function and bone and skeletal muscle defects were also improved. The second aim of this chapter was to establish if prolonged treatment with SFX-01 could reduce white blood cell production in these mice, in particular, the myeloid lineage. As the increase white blood cell production in this mouse model likely contributes to the splenomegaly they present with, the effect of prolonged SFX01 treatment on spleen growth was also assessed. 


\subsection{Materials and methods}

\subsubsection{SDS-PAGE and western blotting}

SDS-PAGE and western immunoblotting were performed as outlined in the general methods. For this chapter the following primary antibodies were used:

\begin{tabular}{|l|l|l|}
\hline Primary antibody & Company & Species \\
\hline Sulforaphane & In-house & Rabbit \\
\hline SHP2 (for immunocapture) & Santa Cruz \#sc-280 & Rabbit \\
\hline Total ERK 1/2 & Cell signalling \#9102 & Rabbit \\
\hline Phosphorylated ERK 1/2 & Cell signalling \#9101 & Rabbit \\
\hline SHP2 (for immunodetection) & R\&D Systems \#AF1894 & Goat \\
\hline
\end{tabular}

Table 5.1. List of primary antibodies used for western immunoblotting in chapter 5.

\subsubsection{Immunoprecipitation}

Immunoprecipitation of proteins from tissue was performed as outlined in the general methods. For this chapter the following antibodies were used:

\begin{tabular}{|l|l|l|l|}
\hline Antibody & Company & Species & Capture beads \\
\hline SHP2 & Santa Cruz \#sc-7384 & Rabbit & Agarose conjugated \\
\hline GRB2 & Santa Cruz \#sc-8034 & Rabbit & Agarose conjugated \\
\hline
\end{tabular}

Table 5.2. List of antibodies used for immunoprecipitation in chapter 5. 


\subsubsection{Foetal treatment with SFN}

Three genotype breeding pairings were used for foetal studies: WTMT, WT/HET, with the male mouse being HET, and HET/HET. In preliminary studies, male and females were housed separately and received $2.5 \mathrm{mg} / \mathrm{ml}$ or $0.8 \mathrm{mg} / \mathrm{ml}$ SFX-01, which is equivalent to $0.385 \mathrm{mg} / \mathrm{ml}$ or $0.123 \mathrm{mg} / \mathrm{ml} \mathrm{SFN}$ respectively, for 3 days in their drinking water prior to breeding. Males were then added to the females' cages, where they remained until pregnancy was confirmed by visual inspection of the female. Dams continued receiving SFX-01 throughout their pregnancy. Neonates were sacrificed and snap frozen by liquid nitrogen within 24 hours of birth.

In additional studies, males and females were mated, and the date of conception was calculated by identification of a vaginal plug and at this point, the males were removed. Treatment with $2.5 \mathrm{mg} / \mathrm{ml} \mathrm{SFX-01} \mathrm{in} \mathrm{the} \mathrm{dams} \mathrm{drinking} \mathrm{water} \mathrm{began} 11$ days post-conception, the time point determined as the completion of gastrulation. Neonates were sacrificed and snap frozen by liquid nitrogen within 24 hours of birth.

\subsubsection{Blood sampling via the tail vein}

10 minutes prior to sampling, mice were placed in a warming chamber set to 37 ${ }^{\circ} \mathrm{C}$ to induce dilation of the blood vessels. Mice were then placed into an induction chamber and anaesthesia was induced for 1 -minute using $3 \%$ isoflurane mixed with $97 \% \mathrm{O}_{2}$ at a flow rate or $1 \mathrm{l} / \mathrm{min}$. Mice were put into a face-down position and held in place using surgical tape. Anaesthesia was maintained using a nose mask with $1.5-2 \%$ isoflurane and $98-98.5 \% \mathrm{O}_{2}$ at a flow rate of $1 \mathrm{l} / \mathrm{min}$. Body temperature was measured using a rectal probe and maintained at $37{ }^{\circ} \mathrm{C} \pm 1.5$ ${ }^{\circ} \mathrm{C}$ via a heat lamp when required. Tails were washed with an antimicrobial 
solution prior to sample collection, and a heparinised $0.3 \mathrm{mI} \mathrm{U}-100$ insulin syringe (BD Benelux) inserted into the lateral vein. $50 \mu \mathrm{l}$ of blood was immediately collected into the syringe and pressure was carefully applied to terminate bleeding at the sampling site. After sampling, animals recovered in a temperature-controlled incubator.

\subsubsection{Wright-Giemsa stain}

$1 \mu \mathrm{l}$ of blood was spread thinly onto a glass slide and allowed to air dry. Once fully dried, blood films were submerged in Wright-Giemsa stain for 30 seconds, after which they were placed directly into deionised water for 10 minutes. Slides were then washed in running deionised water and left to fully air dry before being analysed by light microscopy. The nucleus and cytoplasm of white blood cells were stained dark purple, whilst red blood cells were stained light pink. White blood cells were counted from five fields of view at a 10X magnification for all samples and white blood cell count was represented as an average of these five values.

\subsubsection{Tissue preparation for flow cytometry}

Following the sacrifice of mice, the spleen, femur and tibia were harvested and placed into separate falcon tubes containing $30 \mathrm{ml}$ of PBS supplemented with 5 $\mu \mathrm{l}$ heparin. Blood was also collected via the abdominal aorta into a $1 \mathrm{ml}$ syringe lined with heparin. All samples were placed on ice until needed and prepared as subsequently described. All centrifugation steps were carried out for 10 minutes at $1800 \times \mathrm{g}$ at $4{ }^{\circ} \mathrm{C}$ and all incubation steps were carried out at room temperature. Blood: $50 \mu \mathrm{l}$ of blood was transferred to a $15 \mathrm{ml}$ falcon tube with the remaining blood used to analyse MIP1 $\alpha$ and MIP2 levels as described below. For lysis of red blood cells, $1 \mathrm{ml}$ 10X PBS and $9 \mathrm{ml}$ deionised water was added to the $15 \mathrm{ml}$ 
falcon tube followed by inverting 5 times. $5 \mathrm{ml}$ of $1 \mathrm{X}$ PBS was then added, and samples were centrifuged. Spleen: Spleen tissue was transferred into a petri dish containing $20 \mathrm{ml}$ PBS, any attached fat tissue was removed, and the spleen was dissected into two halves along the long axis. Half of the tissue was snap frozen at $-80{ }^{\circ} \mathrm{C}$ to be used in future experiments, whilst the remaining tissue was placed into a $40 \mu \mathrm{m}$ nylon mesh cell strainer (Thermo Fischer), which was placed into a fresh petri dish containing $5 \mathrm{ml}$ PBS. Using the flat end of a syringe plunger the tissue was forced through the cell strainer. PBS containing the strained spleen cells was transferred into a $50 \mathrm{ml}$ falcon tube containing $25 \mathrm{ml}$ PBS and centrifuged. Cells were resuspended in $10 \mathrm{ml}$ red blood cell lysis buffer (BioLegend), incubated for 10 minutes on a rolling shaker and then centrifuged. Bone marrow: Femur and tibia bones were transferred into a petri dish containing $20 \mathrm{ml}$ PBS. The knee joint and either side of each bone were cut using scissors. A needle attached to a $1 \mathrm{ml}$ syringe filled with PBS was inserted into the cavity of each bone and the marrow was flushed out into a $50 \mathrm{ml}$ falcon tube containing $25 \mathrm{ml} \mathrm{PBS}$ and samples were centrifuged. Cells were resuspended in $10 \mathrm{ml}$ red blood cell lysis buffer, incubated for 10 minutes on a rolling shaker and then centrifuged. After centrifugation of all tissue samples, supernatants were removed, and pellets were resuspended in $1 \mathrm{ml}$ PBS. Myeloid cell staining was then carried out.

\subsubsection{Cell staining for flow cytometry}

Tissue samples prepared as described above were passed through a $35 \mu \mathrm{m}$ nylon mesh cell strainer upon a $5 \mathrm{ml}$ round-bottomed glass test tube (Thermo Fisher). $50 \mu \mathrm{l}$ from each tissue type were combined in a separate $5 \mathrm{ml}$ roundbottomed glass test tube to be used for staining with a dead cell marker. $200 \mu \mathrm{l}$ 
of each sample was also transferred into a fresh $5 \mathrm{ml}$ round-bottomed glass test tube to be used as unstained sample controls, with the remaining sample to be used for myeloid cell staining. $5 \mathrm{ml}$ PBS was added to all tubes and centrifuged, after which $4.8 \mathrm{ml}$ of the supernatant was discarded, and the pellet was resuspended thoroughly by vortexing in the remaining $200 \mu$ l. The remaining protocol was carried out in reduced lighting. $1 \mu \mathrm{l}$ of cell viability dye, Zombie Aqua (BioLegend), was added to each 'staining' sample as well as the dead cell marker only control. Zombie Aqua is an amine-reactive dye which is non-permeant to live cells but permeant to cells with compromised membranes. Samples were incubated in the dark for 20 minutes. During this time, flow cytometry buffer was prepared (2\% FBS, 2 mM EDTA in PBS) and passed through a filter unit (Thermo Fisher). $5 \mathrm{ml}$ of buffer was then added to each sample tube and centrifuged. 4.8 $\mathrm{ml}$ of supernatant was discarded, and the pellet was resuspended thoroughly by vortexing in the remaining $200 \mu \mathrm{l} .1 \mu \mathrm{l}$ of each antibody; cluster of differentiation molecule 11b (CD11b)- allophycocyanin Cy7 (ApcCy7), lymphocyte antigen 6C (Ly6C)-peridinin-Chlorophyll-Protein (PerCP) lymphocyte antigen 6C (Ly6G)fluorescein Isothiocyanate (FITC), all purchased from BD Biosciences, were added to each 'staining' tube and were incubated for 1-hour. During this time, single staining control samples were prepared as follows: $100 \mu \mathrm{l}$ of compensation beads (BioLegend) were added to $400 \mu \mathrm{l}$ buffer and split equally between three $5 \mathrm{ml}$ round-bottomed glass test tubes which were to be used as single staining control. 30 minutes later, $1 \mu$ of either antibody was added to one of the single staining control tubes and incubated for 30 minutes. Following incubation, $5 \mathrm{ml}$ of buffer was added to all tubes and centrifuged. $4.8 \mathrm{ml}$ of supernatant was removed, and the pellets were resuspended thoroughly by vortexing in the 
remaining $200 \mu \mathrm{l}$. Caps were then placed on all tubes and stored in the dark at 4 ${ }^{\circ} \mathrm{C}$ until flow cytometry analysis was carried out.

\subsubsection{Flow cytometry analysis}

Flow cytometry was conducted using a FACSCanto II cell analyser system (BD Biosciences) using a $633 \mathrm{~nm}$ excitation red laser to detect CD11b-ApcCy7, a 488 $\mathrm{nm}$ excitation red laser to detect Ly6C-PerCP and a $488 \mathrm{~nm}$ excitation green laser to detect Ly6G-FITC. 50,000 cells per sample were analysed. Firstly, using an 'unstained' sample, the cell population of interest was gated and the photomultiplier tubes (PMT) voltages for forward scatter (FCS) and side scatter (SSC) as well as fluorescent channels were optimised. Compensation \% values were calculated using each single staining antibody control and values were applied to all experiments conducted on the same day. 'Staining' tubes for each tissue sample were then analysed by the cytometer and the following analysis was conducted for each sample using FlowJo software (BD Biosciences): Elimination of cell debris: SSC-area (SSC-A) (y-axis) was plotted against FSCheight (FSC-H) (x-axis) and a fluorescence intensity threshold of 5,000 was generated to eliminate any cell debris from the analysis. Elimination of cell doublets: FSC-H (y-axis) was plotted against FSC-A ( $\mathrm{x}$-axis) and the main cell population was gated for. Elimination of dead cells: Cell count (y-axis) was plotted against $405 \mathrm{~nm}$ excitation using a violet laser. Two peaks were generated; correlating to live cells (lower fluorescence intensity) and dead cells (higher fluorescence intensity). Dead cells were eliminated by gating only for the lower intensity peak. Identification of inflammatory monocyte population: Ly6C (y-axis) was plotted against CD11b (x-axis) and inflammatory monocytes were analysed by gating only the $\mathrm{CD} 11 \mathrm{~b}+\mathrm{Ly} 6 \mathrm{C}^{\mathrm{Hi}}$ cell population. Identification of neutrophil 
population: Ly6G (y-axis) was plotted against CD11b (x-axis) and neutrophils were analysed by gating only the CD11b+Ly6G $\mathrm{G}^{\mathrm{Hi}}$ cell population.

\subsubsection{Ultrasound}

Ultrasound was performed using the VisualSonics Vevo 770 imagine system fitted with an RMV707B scan head at 15-45 MHz. Mice were placed into an induction chamber and anaesthesia was induced for 1-minute using $3 \%$ isoflurane mixed with $97 \% \mathrm{O}_{2}$ at a flow rate or $1 \mathrm{l} / \mathrm{min}$. Mice were then placed in a supine position on top of a heated pad embedded with electrocardiogram electrodes and fixed in place using surgical tape. Anaesthesia was maintained using a nose mask with $1.5-2 \%$ isoflurane and $98-98.5 \% \mathrm{O}_{2}$ at a flow rate of 1 $1 /$ min. The electrocardiogram was monitored throughout the procedure, as was the body temperature, which was measured using a rectal probe and maintained at $37^{\circ} \mathrm{C} \pm 1.5^{\circ} \mathrm{C}$ via the heated pad as well as a heat lamp when required. Hair was removed from the abdominal area using a razor followed by hair removal cream. A generous layer of preheated ultrasound gel was then added to the abdominal area. The probe was lowered onto the gel and moved into position until the largest area of the long axis of the spleen was identified, which was measured in $\mathrm{mm}^{2}$. After the ultrasound was completed, treatment animals recovered in a temperature-controlled incubator.

\subsubsection{Macrophage inflammatory protein 1 alpha (MIP1a) measurement}

Levels of MIP1a were measured in blood samples using an enzyme-linked immunosorbent assay (ELISA) kit (Abcam \#ab200017). Wash buffers and an antibody cocktail were prepared as advised by the manufacturer's protocol. All incubation steps were carried out at room temperature, with shaking at $400 \mathrm{rpm}$. Plasma samples were then prepared. Firstly, $\sim 1 \mathrm{ml}$ of blood was collected in a 
heparin-coated syringe and transferred to a microcentrifuge tube. Blood was centrifuged at $2000 \mathrm{xg}$ for 10 minutes with separated plasma placed into a fresh microcentrifuge tube. $50 \mu \mathrm{l}$ of plasma sample or standard were added to wells of the provided 96-well plate, which were pre-lined with an immobilized anti-tag MIP1 $\alpha$ antibody. All samples were run in triplicates. $50 \mu$ of an antibody cocktail containing an affinity-tag labelled capture antibody and a reporter conjugated detector antibody was added and incubated for 2 hours followed by $3 \mathrm{X}$ washing with $350 \mu \mathrm{l}$ wash buffer. After the final wash, $100 \mu \mathrm{l}$ of $1: 1 \mathrm{H}_{2} \mathrm{O}_{2}$ and the chromogenic substrate $3,3^{\prime}, 5,5^{\prime}$-tetramethylbenzidine were added, which is catalysed by the addition of HRP which generates a blue colour during incubation in the dark for 10 minutes. The reaction was terminated by adding $100 \mu \mathrm{l}$ of sulfuric acid-containing stop solution, generating a yellow colour during incubation for 1-minute. An endpoint reading of optical density was then measured at $450 \mathrm{~nm}$ using a microplate reader. Values were calculated by using the average of triplicate samples and subtracting the average zero standard from each sample.

\subsubsection{Macrophage inflammatory protein 2 (MIP2) measurement}

Levels of MIP2 were measured in blood samples using an ELISA kit (R\&D Systems \#DY435). Plasma was prepared as described previously. All incubation steps were carried out at room temperature and all washes conducted 3X with $400 \mu \mathrm{l}$ wash buffer $(0.05 \%$ Tween 20 in PBS, $\mathrm{pH}$ 7.4). The assay plate was prepared by first coating required wells of a 96-well plate with $100 \mu$ of MIP2 capture antibody, incubated overnight and the following morning the plate was washed. Wells were blocked using $300 \mu \mathrm{l}$ of $1 \%$ BSA in PBS, pH 7.2-7.4 for 1hour followed by washing. $100 \mu \mathrm{l}$ of sample or standard was added to the wells 
and the plate was incubated for 2 hours and then the wells were washed. $100 \mu \mathrm{l}$ of detection antibody was then added to each well and again incubated for 2 hours and washed. $100 \mu \mathrm{l}$ of Streptavidin-HRP was then added in the dark and incubated for 20 minutes followed by washing. $100 \mu$ of $1: 1 \mathrm{H}_{2} \mathrm{O}_{2}$ and 3,3',5,5'tetramethylbenzidine were then added in the dark and incubated for 20 minutes followed by washing. Finally, $50 \mu \mathrm{l}$ of $2 \mathrm{M} \mathrm{H}_{2} \mathrm{SO}_{4}$ was added. An endpoint reading of optical density was then immediately measured at $450 \mathrm{~nm}$ using a microplate reader. Values were calculated by using the average of triplicate samples and subtracting the average zero standard from each sample. Average values from positive control standards were plotted against their concentration and a standard curve was generated. Unknown MIP2 concentrations were then interpolated from this curve. 


\subsection{Results}

\subsubsection{High amounts of SFX-01 is embryonic lethal in pregnancies carrying}

NS foeti

I first sought to investigate if foetal treatment with SFX-01 would result in HOM offspring being born from HET/HET NS breeding pairs or increase the number of HET offspring born from WT/HET breeding pairs. Treatment with $2.5 \mathrm{mg} / \mathrm{ml}$ SFX01 had no detrimental effects on conception, pregnancy or litter size for WT/WT breeding pairs (figure $5.2 \mathrm{~A}$ ). All females from WT/HET breeding pairs who received SFX-01 conceived and gave birth to litters. However, all females from HET/HET breeding pairs that received SFX-01 before pregnancy, and in which pregnancy had been confirmed, did not deliver litters, with termination estimated by visual inspection within 10 days of conception (figure 5.2 A). Treatment of WT/HET breeding pairs with SFX-01 significantly reduced litter size (figure 5.2 B) with genotyping identifying a trend towards fewer HET offspring born compared to untreated controls (figure $5.2 \mathrm{C}$ ). All females from HET/HET breeding pairs that received SFX-01 post-gastrulation, and in which pregnancy had been confirmed, also did not deliver litters (figure 5.3). 
A)

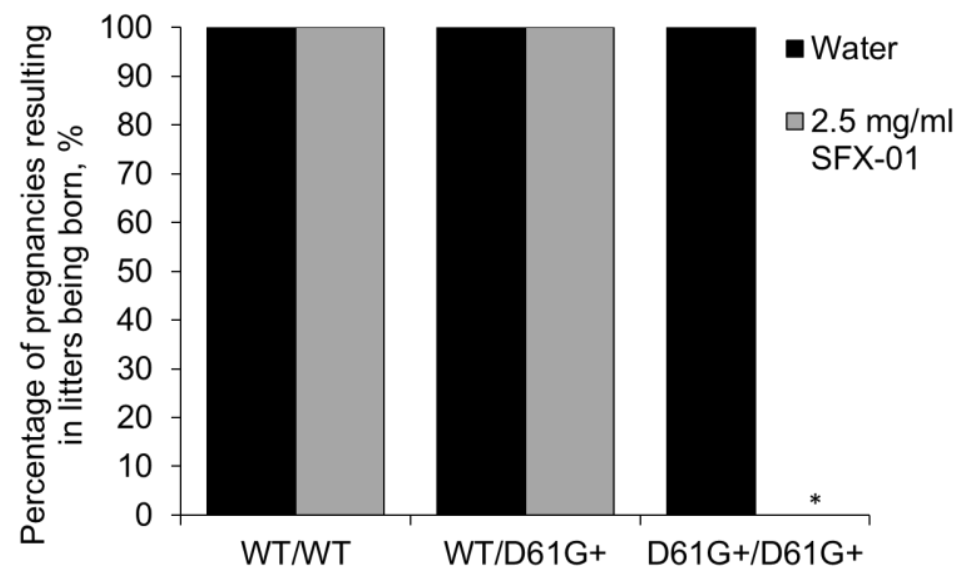

B)

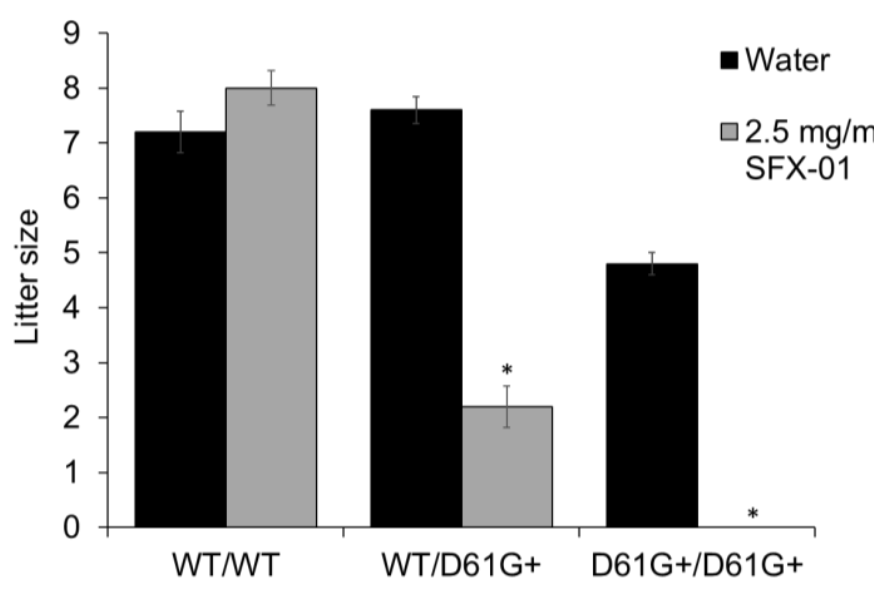

C)

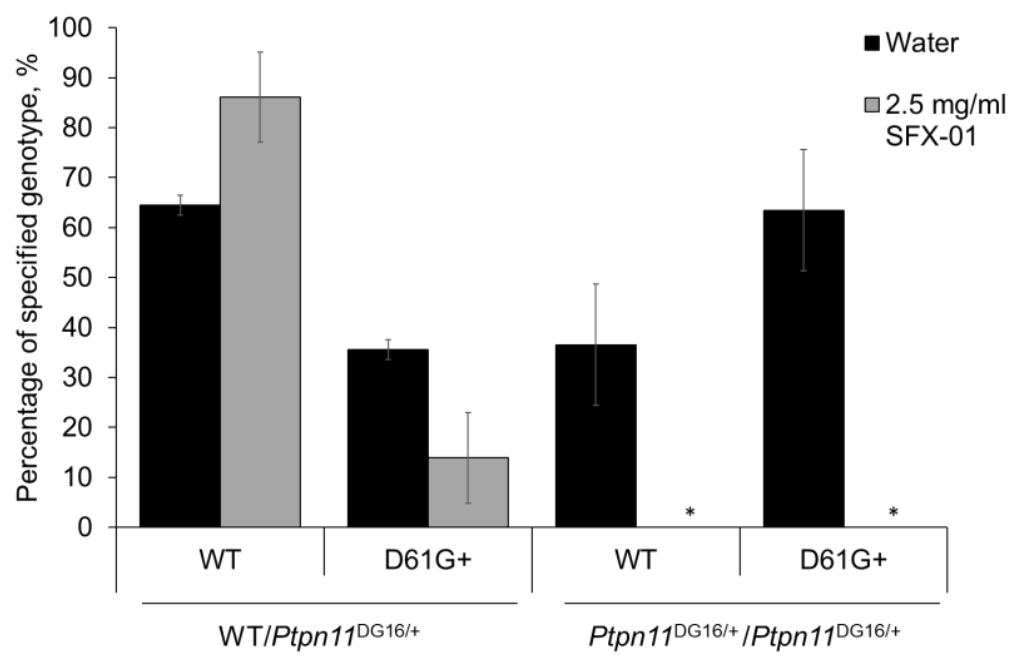

Figure 5.2. Foetal treatment with high amounts of SFX-01 pre-gastrulation is embryonic lethal in pregnancies carrying NS foeti.

A) Treatment of HET/HET breeding pairs with $2.5 \mathrm{mg} / \mathrm{ml} \mathrm{SFX-01} \mathrm{in} \mathrm{their} \mathrm{drinking} \mathrm{water}$ before and during pregnancy resulted in no litters being born. B, C) Treatment of WT/HET breeding pairs with $2.5 \mathrm{mg} / \mathrm{ml} \mathrm{SFX-01} \mathrm{in} \mathrm{their} \mathrm{drinking} \mathrm{water} \mathrm{before} \mathrm{and} \mathrm{during}$ pregnancy significantly reduced litter size with a trend for a lower percentage of HET offspring. ( $n=6,{ }^{*} p<0.05$ versus untreated control). 


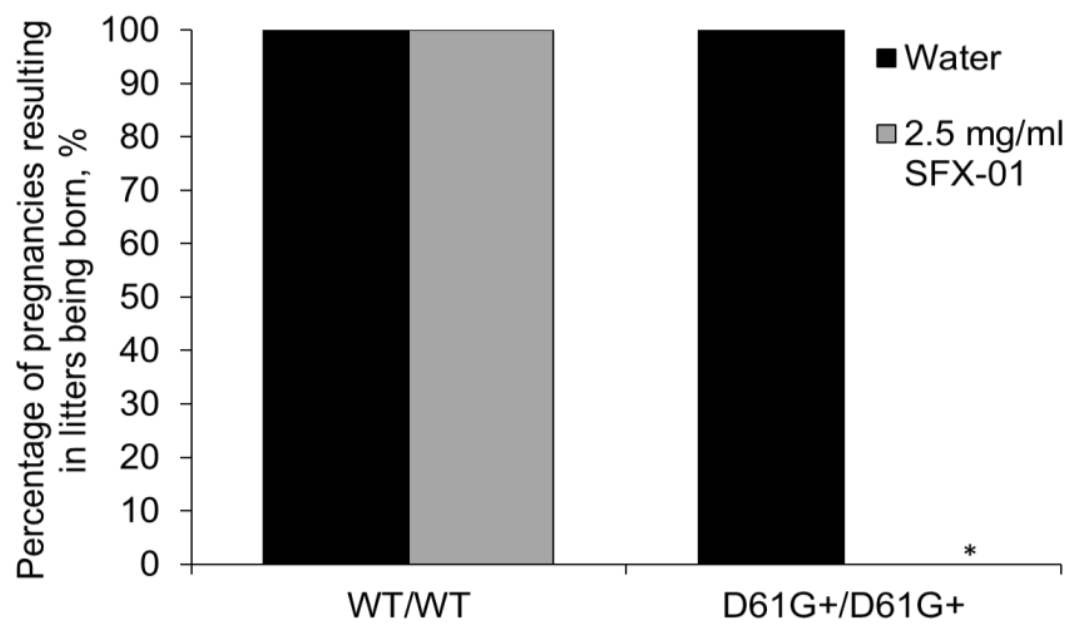

Figure 5.3. Foetal treatment with high amounts of SFX-01 post-gastrulation under these conditions is embryonic lethal in pregnancies carrying NS foeti.

A) Treatment of HET/HET breeding pairs with $2.5 \mathrm{mg} / \mathrm{ml}$ SFX-01 in their drinking water 11 days post-conception resulted in no litters being born. ( $n=3,{ }^{*} p<0.05$ versus untreated control).

\subsubsection{Only WT neonates are born following foetal treatment with low amounts of SFX-01}

As treatment of HET/HET breeding pairs with $2.5 \mathrm{mg} / \mathrm{ml}$ SFX-01 resulted in the termination of pregnancies, foetal studies were repeated using $0.8 \mathrm{mg} / \mathrm{ml} \mathrm{SFX-}$ 01. As above, SFX-01 treatment had no detrimental effects on conception, pregnancy or litter size for WTMT breeding pairs (figure 5.4 A). All females from HET/HET breeding pairs who received SFX-01 conceived and gave birth to litters (figure 5.4 A). However, SFX-01 treatment significantly reduced the litter size of HET/HET breeding pairs and genotyping confirmed all offspring were WT (figure $5.4 \mathrm{~B}, \mathrm{C})$. 
A)

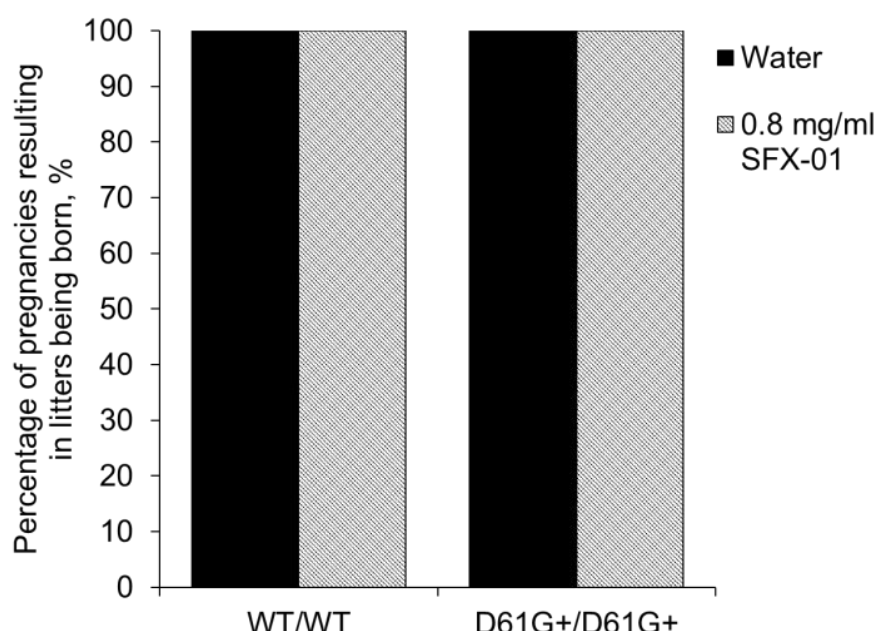

B)

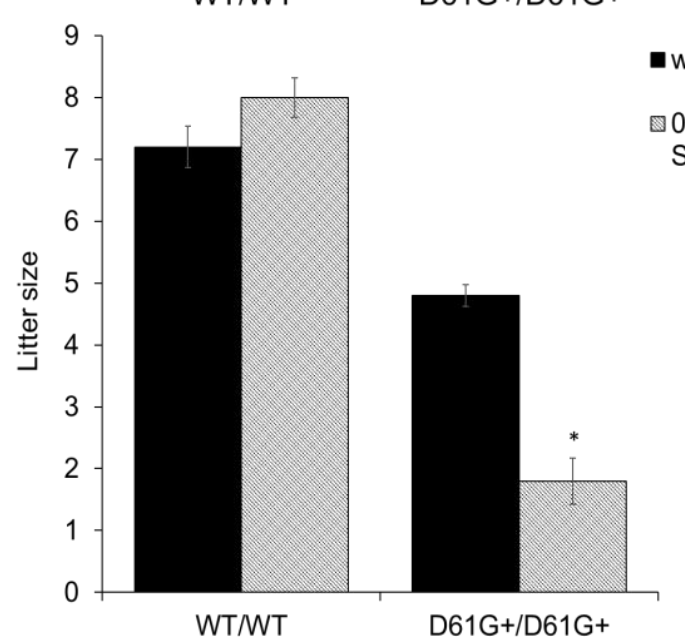

C)

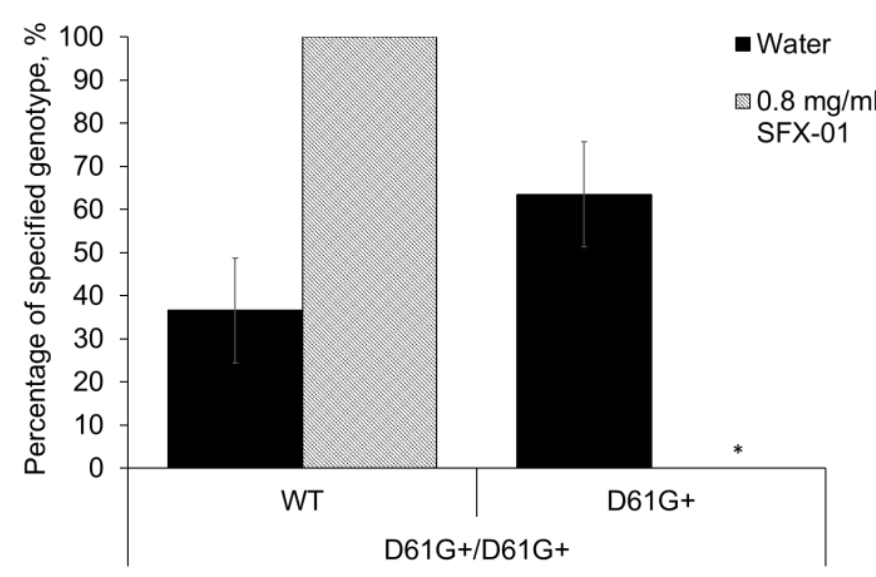

Figure 5.4. Foetal treatment with low amounts of SFX-01 results in the birth of only WT neonates in pregnancies carrying NS foeti.

A) Percentage of pregnancies resulting in the birth of litters was not altered following treatment of WTNT or HET/HET breeding pairs with $0.8 \mathrm{mg} / \mathrm{ml} \mathrm{SFX-01} \mathrm{before} \mathrm{and}$ during pregnancy. B, C) Treatment of HET/HET breeding pairs with $0.8 \mathrm{mg} / \mathrm{ml} \mathrm{SFX-01}$ significantly reduced litter size with only WT offspring born. $\left(n=6,{ }^{*} p<0.05\right.$ versus untreated control). 


\subsubsection{Foetal treatment with SFX-01 only partially reduces neonatal SHP2}

phosphatase activity

A fluorescence-based activity assay identified no inhibition in global SHP2 phosphatase activity in WT neonates from WT/HET or HET/HET breeding pairs following foetal treatment with 2.5 or $0.8 \mathrm{mg} / \mathrm{ml} \mathrm{SFX-01} \mathrm{respectively} \mathrm{(figure} 5.5$ A, B). Partial inhibition of SHP2 phosphatase activity by $\sim 17 \%$ was detected in HET offspring from WT/HET breeding pairs following foetal treatment with 2.5 $\mathrm{mg} / \mathrm{ml} \mathrm{SFX-01} \mathrm{(figure} \mathrm{5.5} \mathrm{A).}$ 
A)

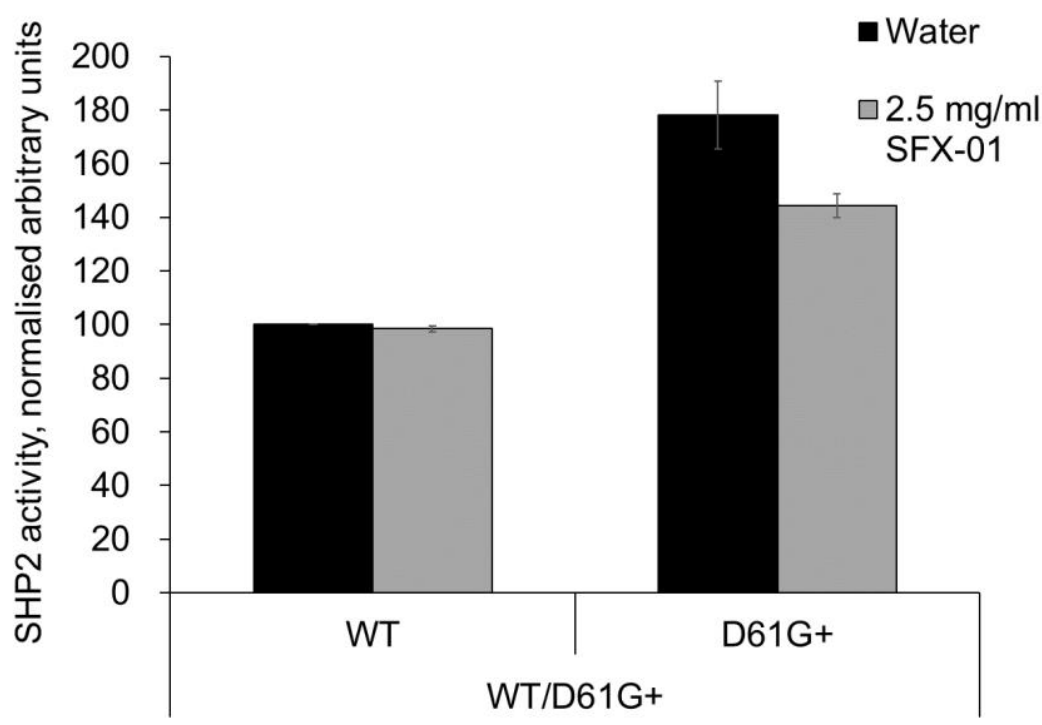

B)

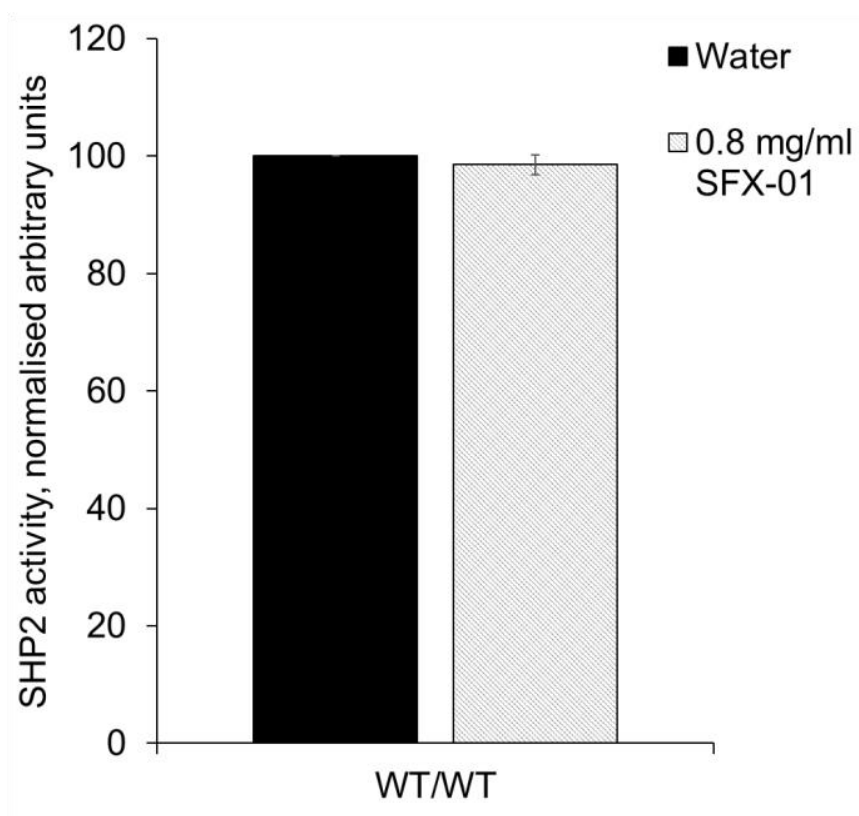

Figure 5.5. Foetal treatment with SFX-01 partially reduces SHP2 phosphatase activity in HET neonates.

A) A fluorescence-based phosphatase activity assay which shows no inhibition of global SHP2 activity in WT neonates and partial inhibition in HET neonates from WT/HET breeding pairs who received $2.5 \mathrm{mg} / \mathrm{ml} \mathrm{SFX-01} \mathrm{in} \mathrm{their} \mathrm{drinking} \mathrm{water} \mathrm{before} \mathrm{and}$ throughout pregnancy. B) A fluorescence-based phosphatase activity assay identified no inhibition of global SHP2 activity in WT neonates from HET/HET breeding pairs who received $0.8 \mathrm{mg} / \mathrm{ml} \mathrm{SFX-01} \mathrm{in} \mathrm{their} \mathrm{drinking} \mathrm{water} \mathrm{before} \mathrm{and} \mathrm{throughout} \mathrm{pregnancy.} \mathrm{(} \mathrm{n}$ $=2-6)$. 
5.3.4 Protein-SFN adducts are detected in neonates following foetal treatment with SFX-01

Western immunoblotting was conducted to determine whether SFN crosses the placenta and labels foetal tissue. Protein-SFN adducts were detected in both WT and HET neonates from WT/HET breeding pairs who had received $2.5 \mathrm{mg} / \mathrm{ml}$ SFX-01 in their drinking water before and during pregnancy as outlined above (figure 5.6).

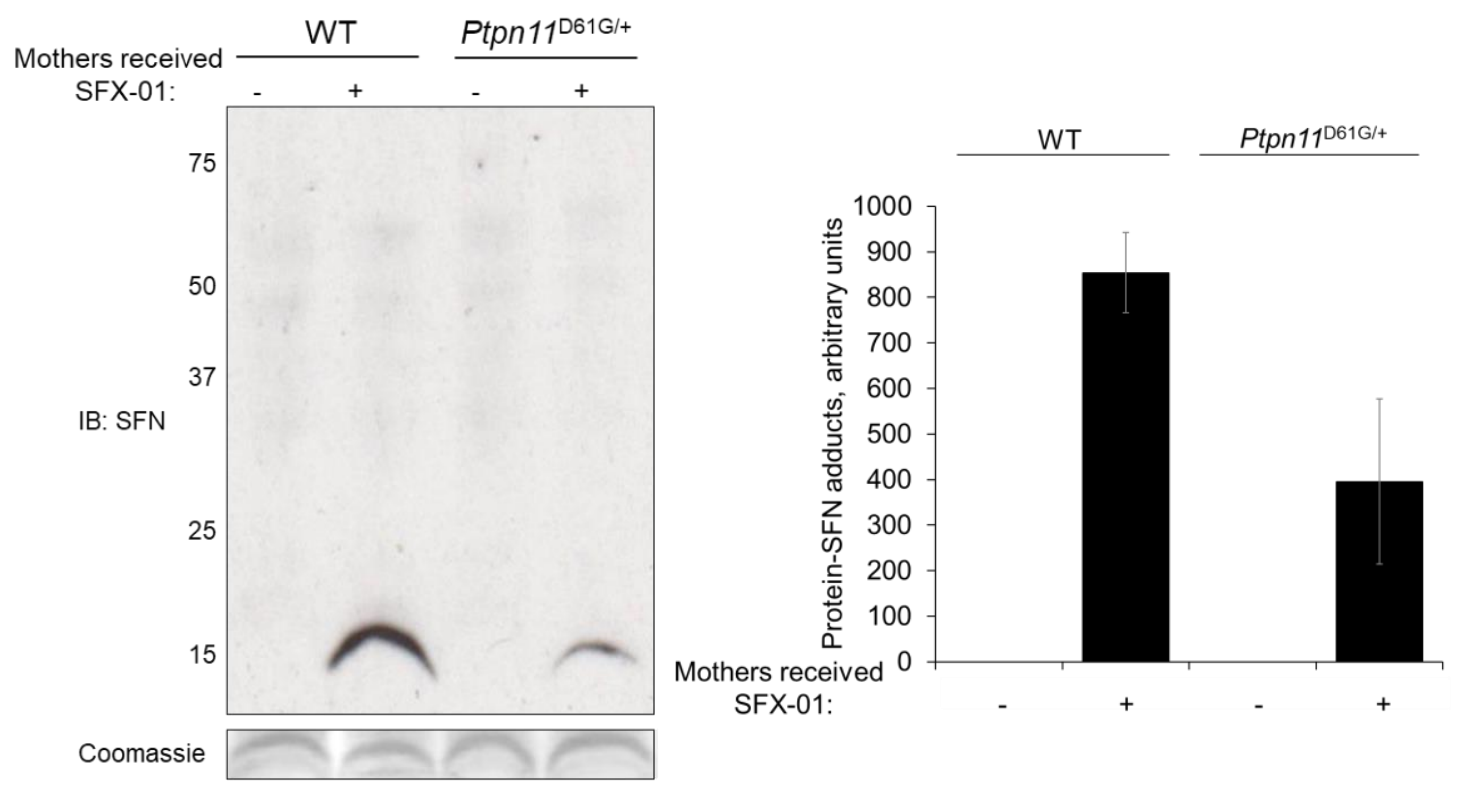

Figure 5.6. SFN adducts proteins in WT or HET neonates following foetal treatment with SFX-01.

An immunoblot showing SFN adduction of a $\sim 15 \mathrm{kDa}$ protein in WT and HET neonates from WT/HET breeding pairs who had received $2.5 \mathrm{mg} / \mathrm{ml} \mathrm{SFX-01} \mathrm{in} \mathrm{their} \mathrm{drinking} \mathrm{water}$ before and during pregnancy. $(n=5)$. 
5.3.5 Foetal treatment with SFX-01 increases neonatal ERK phosphorylation

Western immunoblotting identified increased ERK phosphorylation in WT neonates from WTMT breeding pairs who had received $0.8 \mathrm{mg} / \mathrm{ml}$ SFX-01 in their drinking water before and during pregnancy as outlined above (figure 5.7).
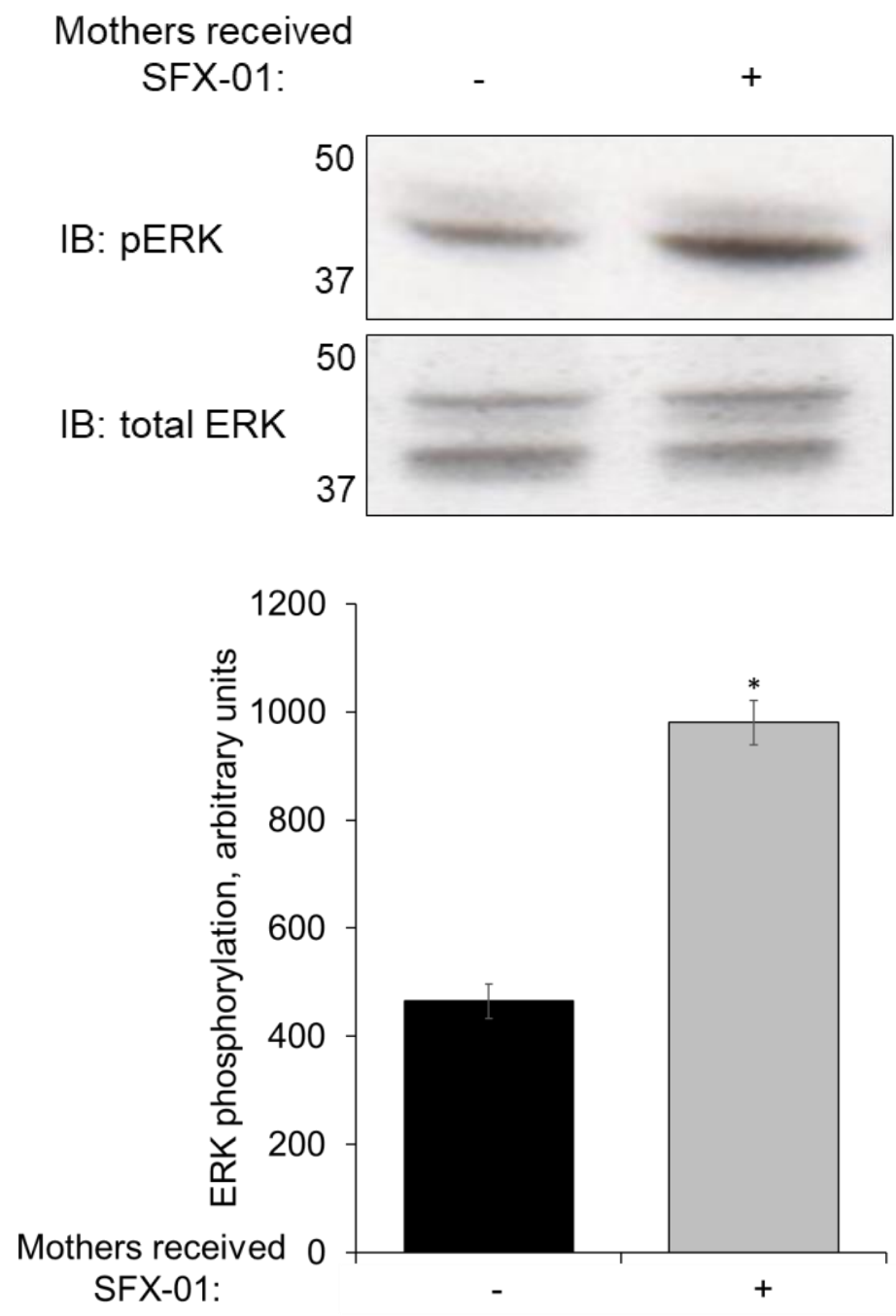

Figure 5.7. Foetal treatment with SFX-01 increases ERK phosphorylation in WT neonates.

An immunoblot showing increased ERK phosphorylation in WT neonates from WTMT breeding pairs who had received $0.8 \mathrm{mg} / \mathrm{ml} \mathrm{SFX-01} \mathrm{in} \mathrm{their} \mathrm{drinking} \mathrm{water} \mathrm{before} \mathrm{and}$ during pregnancy. $\left(n=3,{ }^{*} p<0.05\right.$ versus untreated control). 


\subsubsection{SFX-01 treatment lowers total white blood cell count in WT and NS}

mice

Blood sampling followed by Wright-Giemsa staining, as represented in figure 5.8 A, showed total white blood cell count was elevated in 17- and 22-week-old NS mice compared to their WT littermates (figure 5.8 B) which was in line with previous studies. 5- and 10-week treatment with SFX-01 reduced total white blood cell count in NS mice compared to untreated controls, which was also reduced in WT mice at the later time point (figure $5.8 \mathrm{~B}$ ). 
A)

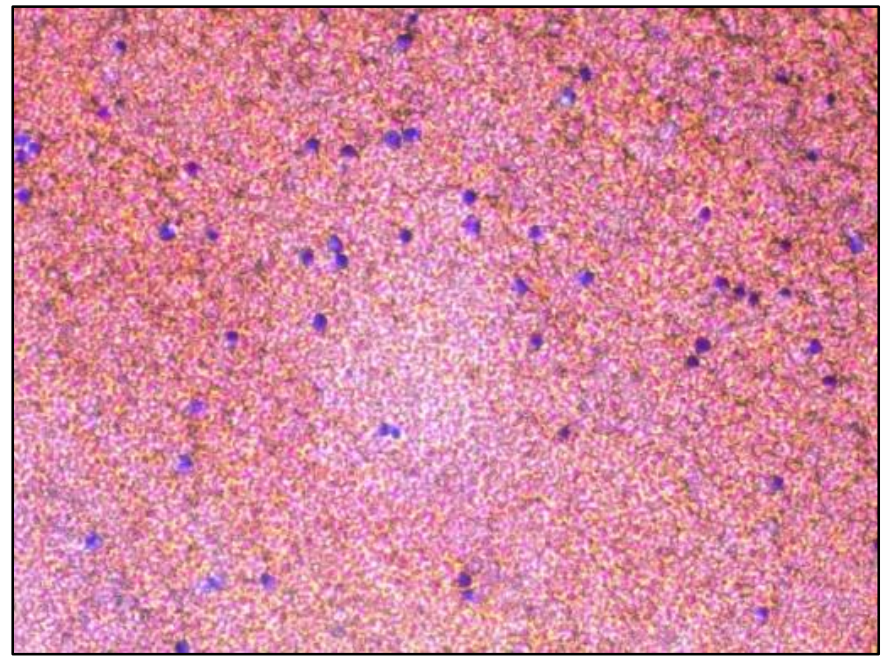

B)

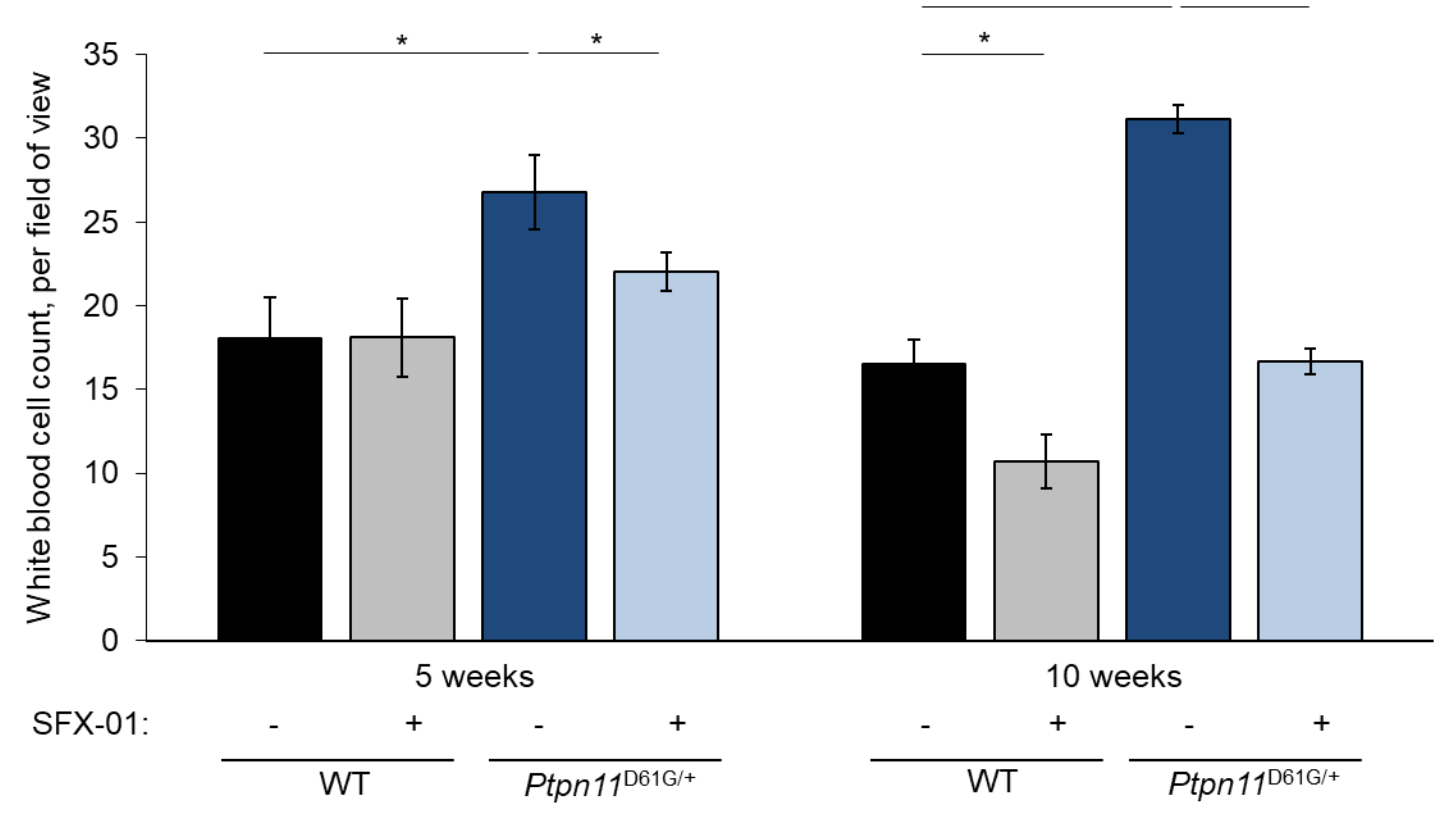

Figure 5.8. SFX-01 treatment reduces white blood cell count in WT and NS mice.

A) A representative light microscopy image of Wright-Giemsa stained blood samples from a 22-week-old WT mouse. B) Total white blood cell count was lowered in NS mice following 5- and 10-weeks treatment with SFX-01. Cell count was also lowered in WT mice at the later time point. $\left(n=13-16,{ }^{*} p<0.05\right.$ versus untreated control). 


\subsubsection{SFX-01 treatment lowers myeloid cell count in the blood and spleen of NS mice}

Inflammatory monocyte and neutrophil cell counts were calculated using flow cytometry as outlined in figure 5.9. Analysis from 17- or 22-week-old NS mice revealed an increase in inflammatory monocytes and neutrophils in the blood compared to their WT littermates (figure 5.10). No difference in myeloid cell count was detected in bone marrow of 22-week-old NS mice compared to their WT littermates (figure 5.11), however, the number of neutrophils was significantly greater in the spleen (figure 5.12). 10-week treatment with $2.5 \mathrm{mg} / \mathrm{ml} \mathrm{SFX-01} \mathrm{in}$ drinking water had no effect on total myeloid cell count in the blood of WT mice (figure 5.10), however, caused a significant decrease in the number of neutrophils and therefore total myeloid count in the blood of NS mice (figure 5.10). No elevation of total myeloid cell count was detected in bone marrow of 22-week-old NS mice compared to WT littermates, although 10-week treatment with SFX-01 caused a small reduction in neutrophil count in this tissue in either genotype (figure 5.11). A significant increase in total myeloid cell count comprising of an increase in neutrophils was detected in the spleen of 22-weekold NS mice compared to their WT littermates (figure 5.12). Treatment with SFX01 for 10 weeks significantly reduced neutrophil and inflammatory monocyte counts in the spleen of NS mice with the latter also decreased in WT littermates (figure 5.12). 

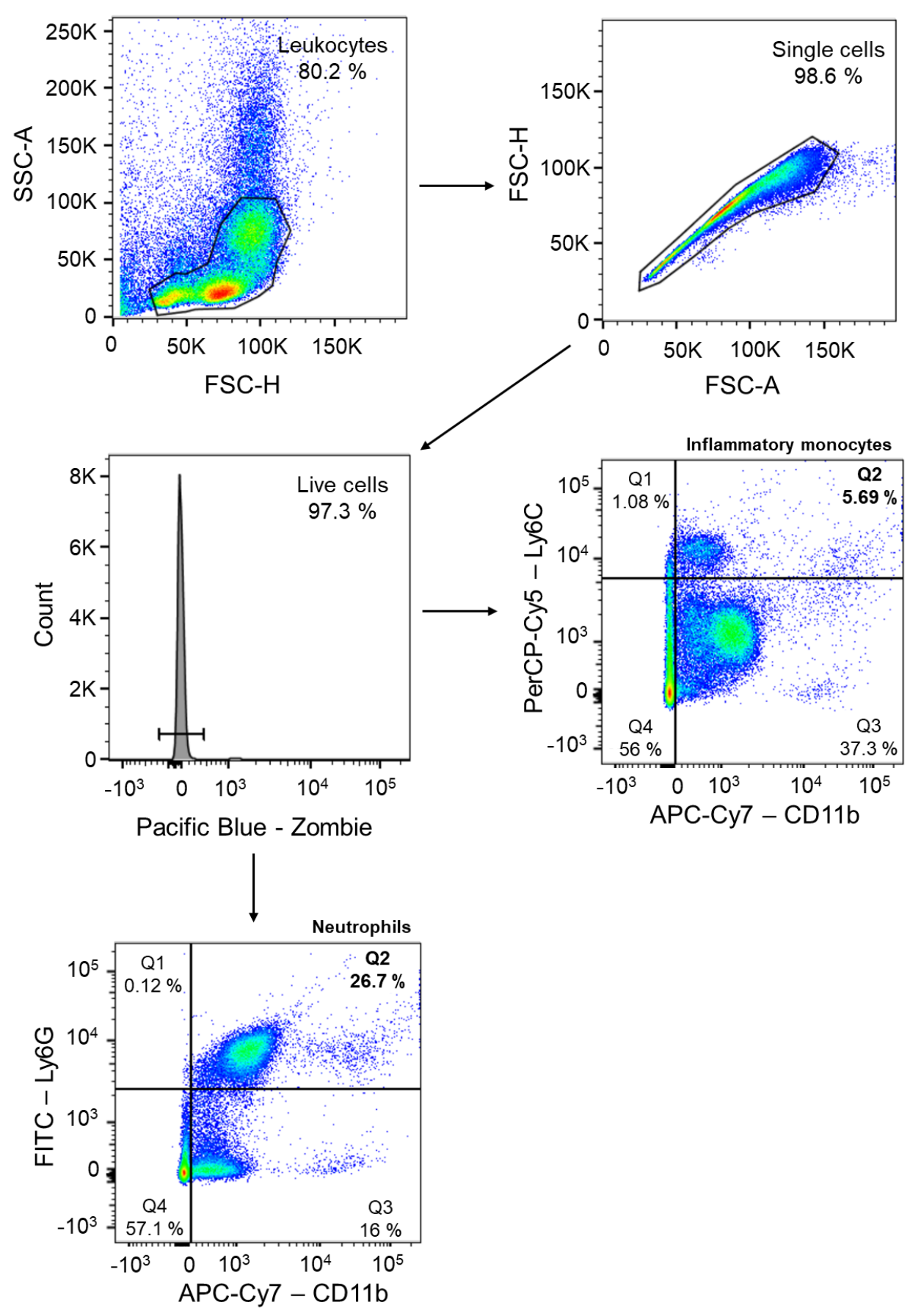

Figure 5.9. Representative flow cytometry analyses of blood from 22-week-old NS mice.

Representative FLOW-JO plots and histograms showing gates used to calculate percentages of each cell population within the blood. Leukocytes (SSC-A vs FSC-H), single cells (FSC-H vs FSC-A), live cells (Cell count vs Pacific Blue), inflammatory monocytes (CD11b $\left.\mathrm{b}^{+} \mathrm{Ly} 6 \mathrm{C}^{\mathrm{Hi}}\right)$ and neutrophils $\left(\mathrm{CD} 11 \mathrm{~b}^{+} \mathrm{Ly} 6 \mathrm{C}^{\mathrm{Hi}}\right)$. Values represent percentage of the previous gate. For example, $5.69 \%$ of live, single-cell leukocytes are inflammatory monocytes. 
A)

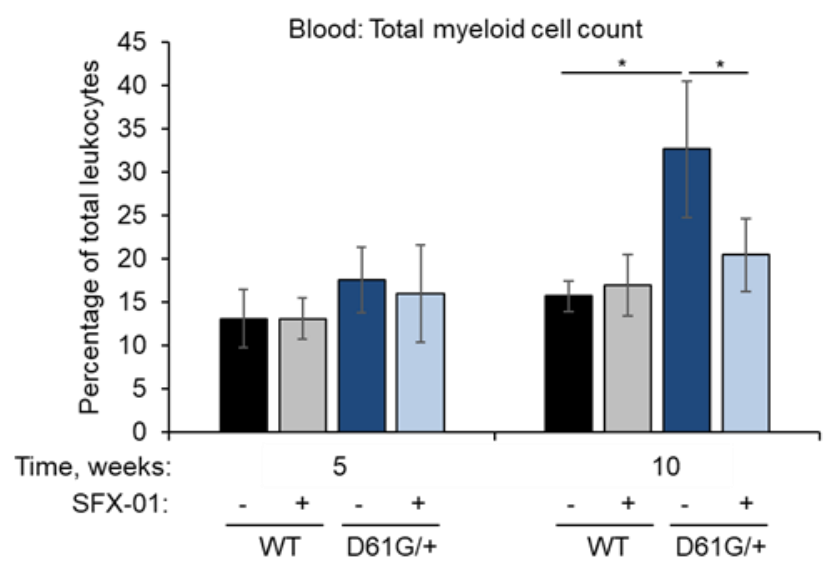

B)

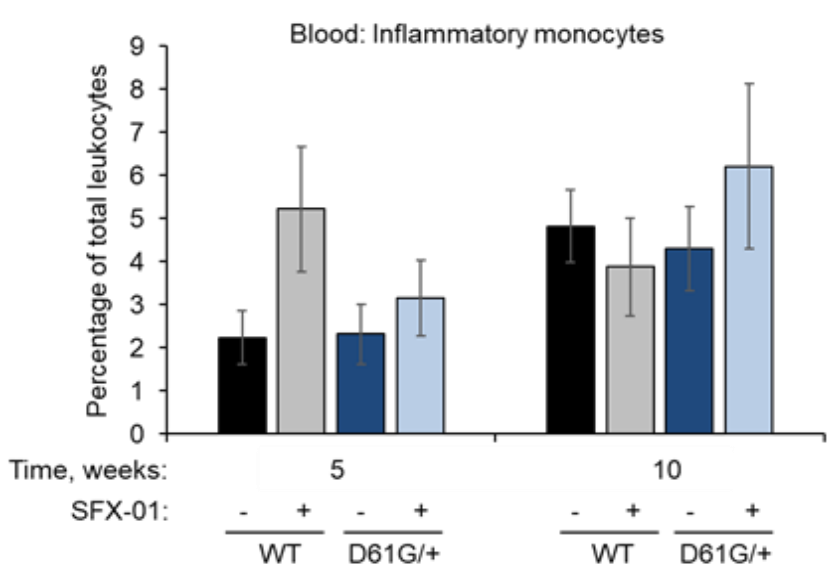

C)

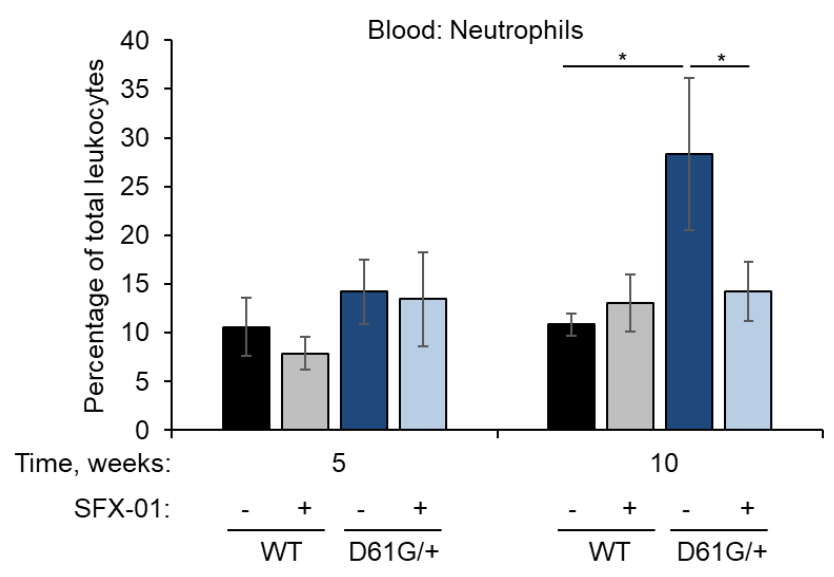

Figure 5.10. SFX-01 treatment reduces neutrophil count in the blood of NS mice.

A-C) Flow cytometry analysis showing reduced myeloid cell count in the blood from 22week-old NS mice following 10-week treatment of $2.5 \mathrm{mg} / \mathrm{ml} \mathrm{SFX-01} \mathrm{in} \mathrm{their} \mathrm{drinking}$ water, which was attributed to a reduction in neutrophils. No effect on total myeloid cell count from the blood of WT mice was detected. $\left(n=13-16,{ }^{*} p<0.05\right.$ versus WT or untreated control as stated). 
A)

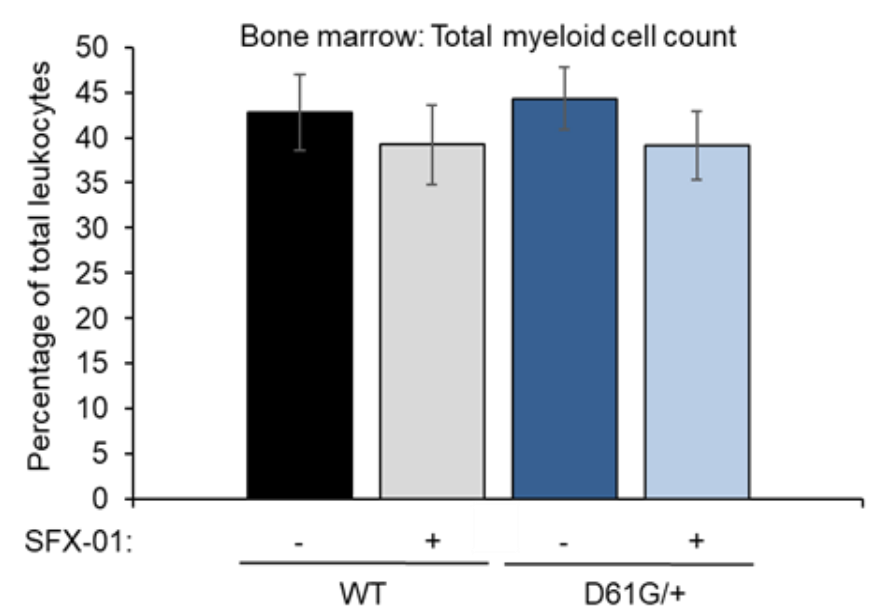

B)

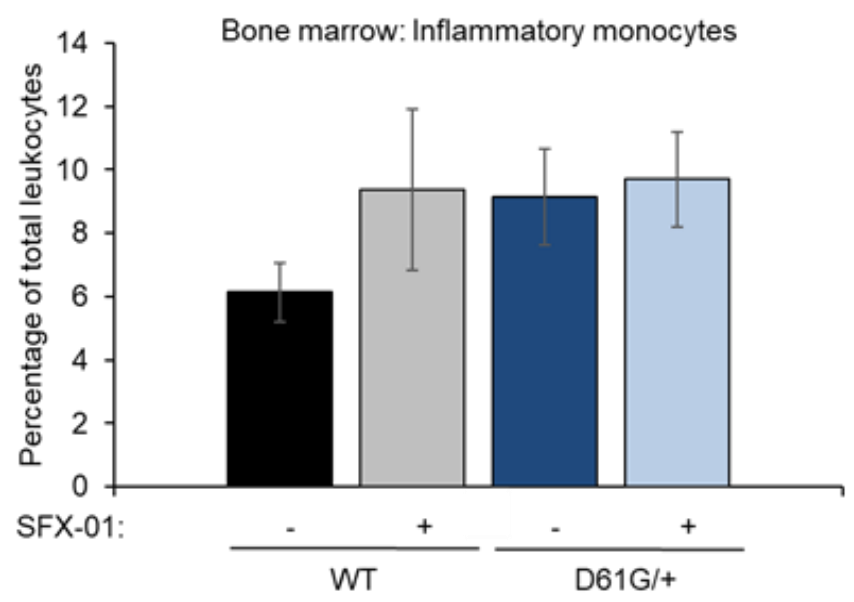

C)

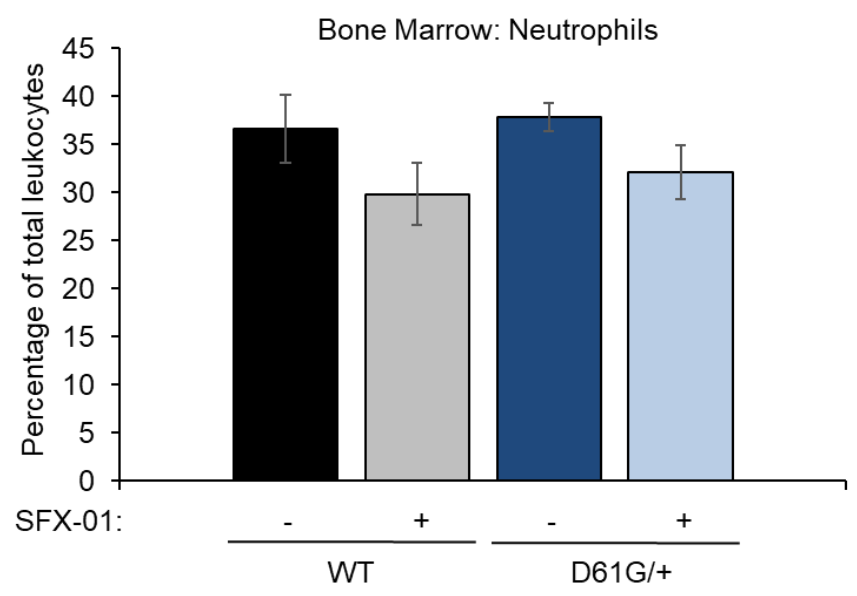

Figure 5.11. SFX-01 treatment causes a small reduction in neutrophil cell count in the bone marrow of NS mice.

A-C) Flow cytometry analysis showing a small reduction in myeloid cell count in the bone marrow of 22-week-old WT or NS mice following 10-week treatment of $2.5 \mathrm{mg} / \mathrm{ml}$ SFX-01 in their drinking water due a reduction in the number of neutrophils. $(n=14-16)$. 
A)

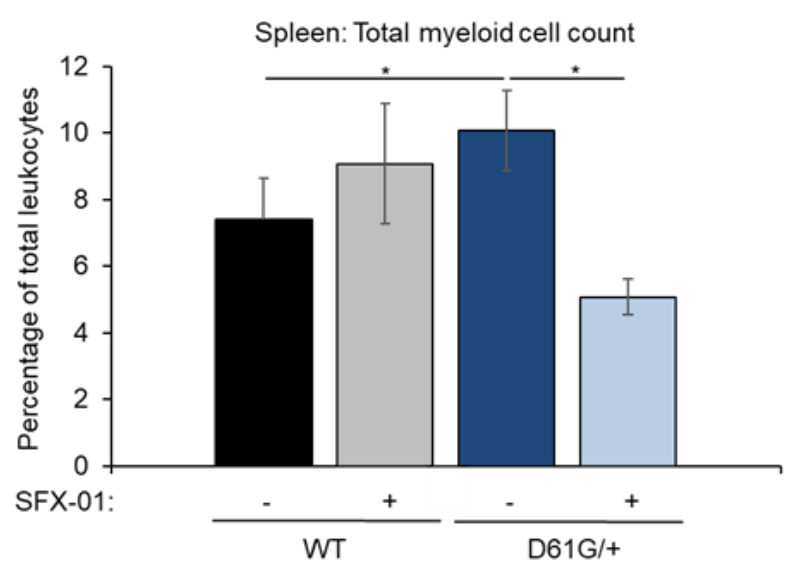

B)

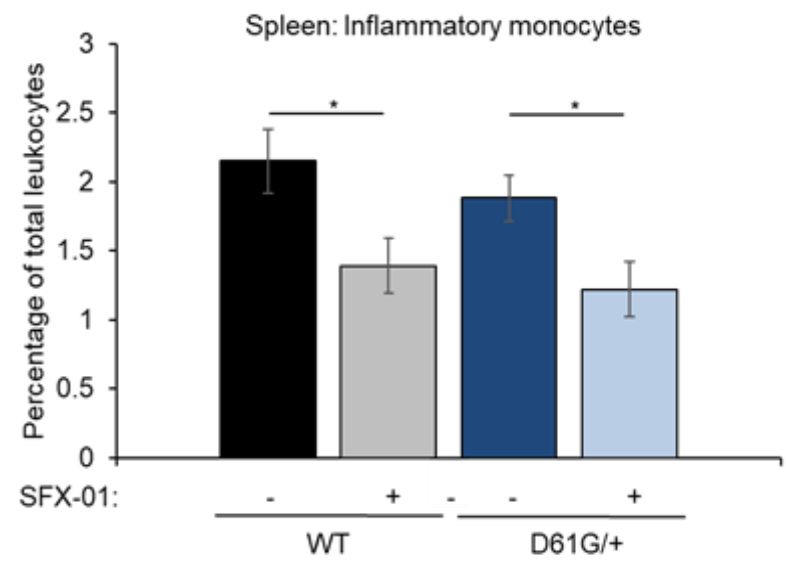

C)

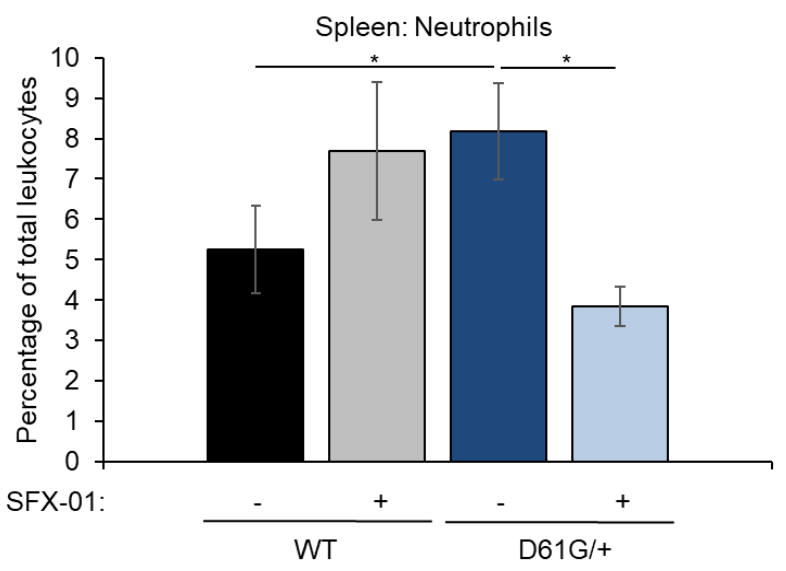

Figure 5.12. SFX-01 treatment reduces total myeloid cell count in the spleen of NS mice.

A-C) Flow cytometry analysis showing reduced myeloid cell count in the spleen of 22week-old NS mice following 10-week treatment of $2.5 \mathrm{mg} / \mathrm{ml} \mathrm{SFX-01} \mathrm{in} \mathrm{their} \mathrm{drinking}$ water, which was attributed to a reduction in both inflammatory monocytes and neutrophils. A reduction in inflammatory monocyte cell count was detected in the spleen of 22-week-old WT mice following treatment with the drug. $\left(n=14-16,{ }^{*} p<0.05\right.$ versus WT or untreated control as stated). 


\subsubsection{SFX-01 treatment reduces the plasma concentration of MIP2 but not}

MIP1a in NS mice

MIP1a was not elevated in the plasma of untreated 22-week-old NS mice compared to their WT littermates and no reduction of the chemokine was seen following 10-week treatment with $2.5 \mathrm{mg} / \mathrm{ml}$ SFX-01 in their drinking water (figure 5.13 A). However, a trend towards an increase in levels of MIP2 was seen in the plasma of 22-week-old NS mice compared to WT, with a trend for a decrease in levels of MIP2 following 10-week treatment with $2.5 \mathrm{mg} / \mathrm{ml}$ SFX-01 treatment in NS compared to untreated controls (figure $5.13 \mathrm{~B}$ ). 
A)

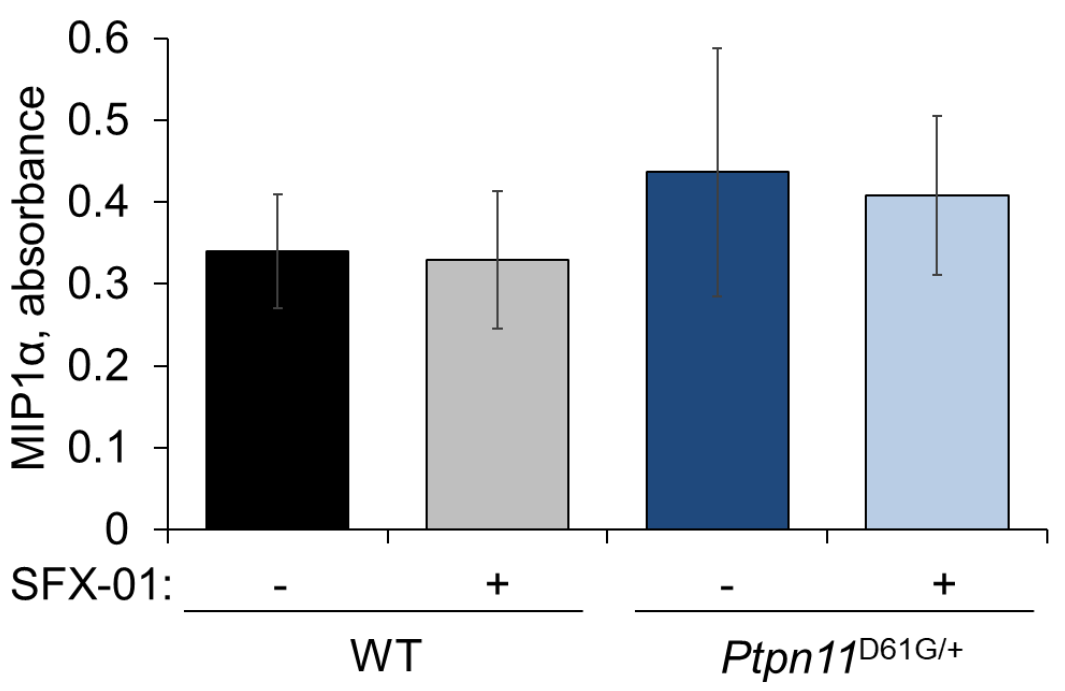

B)

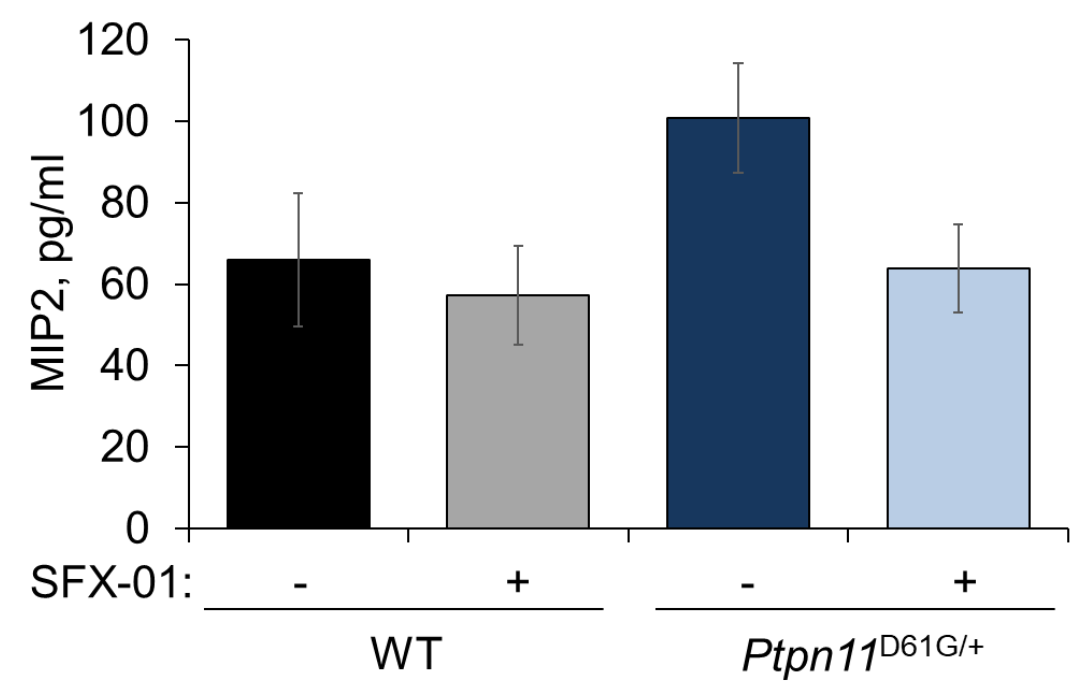

Figure 5.13. The concentration of MIP2 but not MIP1a is reduced in the plasma of NS mice following SFX-01 treatment.

A, B) Analyses of MIP1 $\alpha$ and MIP2 levels in plasma from 22-week-old WT or NS mice following 10-week treatment with $2.5 \mathrm{mg} / \mathrm{ml} \mathrm{SFX-01} \mathrm{in} \mathrm{their} \mathrm{drinking} \mathrm{water.} \mathrm{SFX-01}$ treatment reduced MIP2 levels in NS mice. Neither chemokine was reduced in WT mice. $(n=13-15)$.

\subsubsection{SFX-01 treatment reduces the spleen growth of NS mice}

Ultrasound analysis of the size of the spleen and weighing of this tissue showed 22-week-old NS mice had larger and heavier spleens compared to their WT littermates (figures 5.14 and 5.15). The ultrasound analysis also identified a trend 
for a decrease in the spleen size of NS mice following 10-week treatment with $2.5 \mathrm{mg} / \mathrm{ml} \mathrm{SFX}-01$ in their drinking water compared to untreated controls (figure 5.15 B). A significant reduction in spleen growth was apparent in NS mice when data was analysed as a delta change in the spleen size for each mouse over the duration of the 10-week treatment period with $2.5 \mathrm{mg} / \mathrm{ml} \mathrm{SFX-01} \mathrm{(figure} 5.15 \mathrm{C}$ ). Weighing of the spleens at the end of the experiment corroborated the trend for a reduction in the spleen weight of NS mice following 10-week treatment with SFX-01 (figure 5.15 A). Spleen weight and size were unaffected in WT mice following SFX-01 treatment. 
A)

WT

Average size

$$
=25 \mathrm{~mm}^{2}
$$

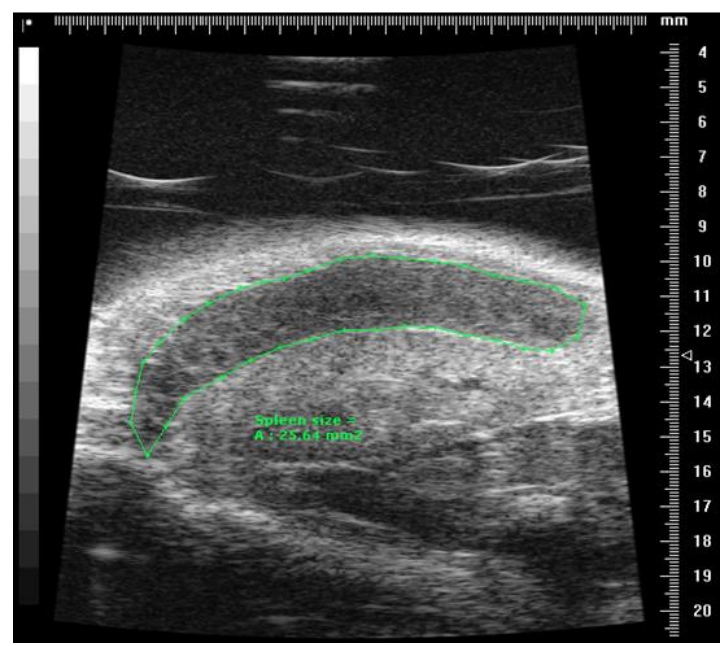

B)

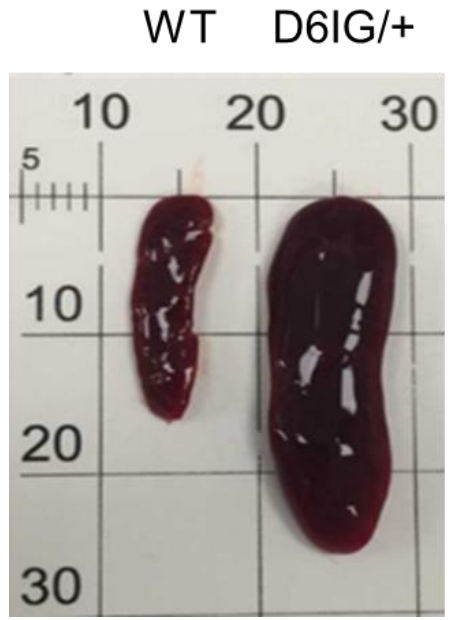

Ptpn11061G/+

Average size

$=42 \mathrm{~mm}^{2}$

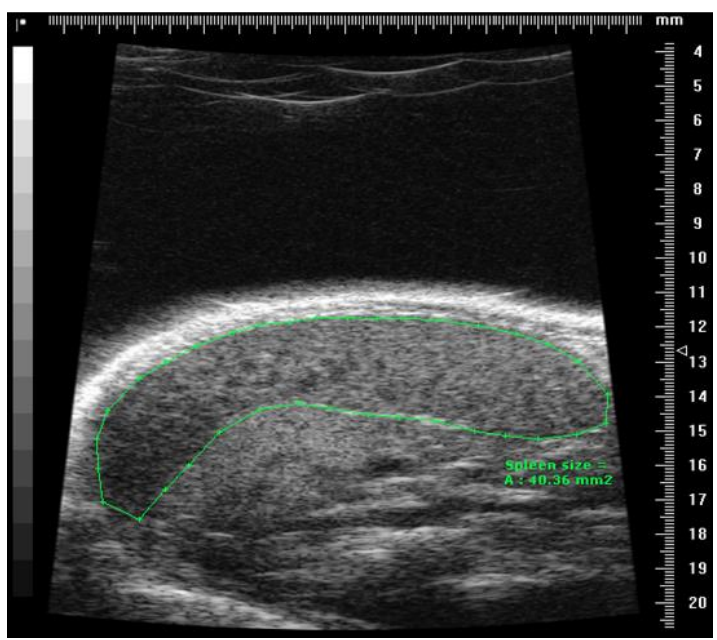

Figure 5.14. Adult NS mice have splenomegaly.

A, B) Representative ultrasound and photographic images of spleens from 22-week-old WT or NS mice. 
A)

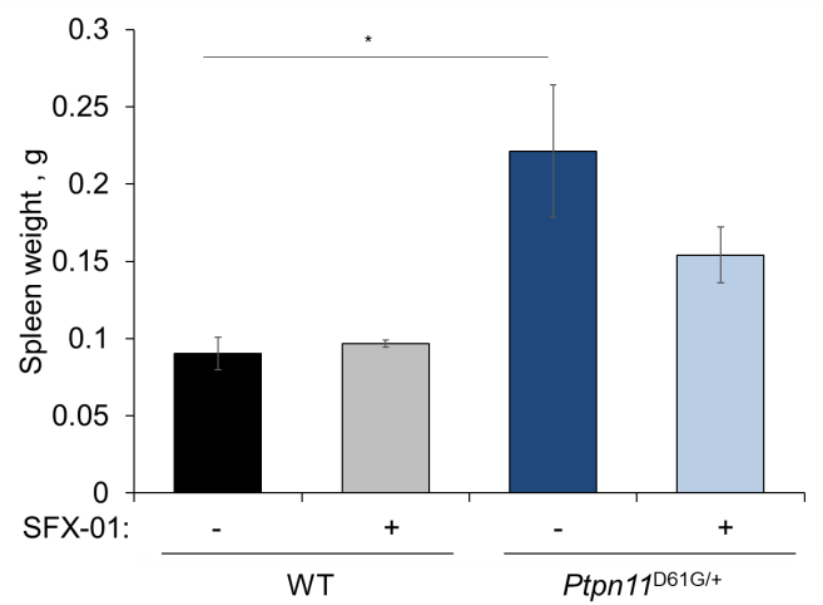

B)

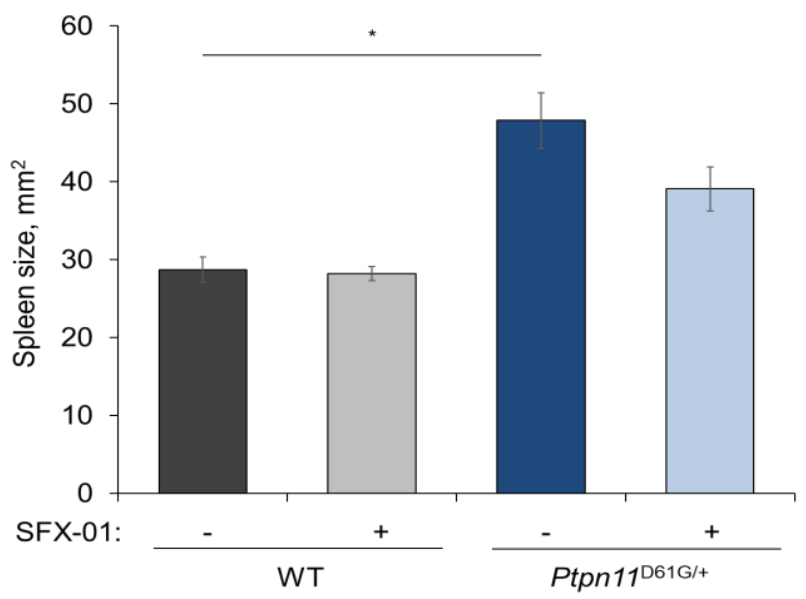

C)

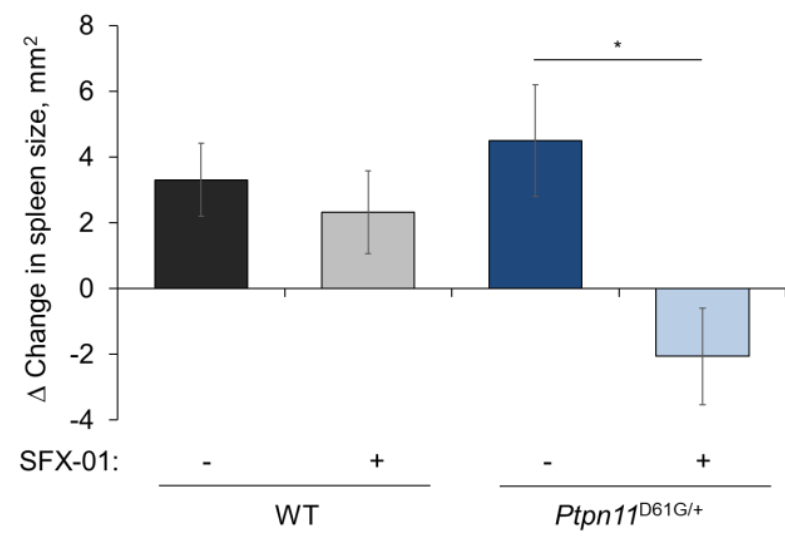

Figure 5.15. The growth of the spleen of NS mice is reduced when they receive a 10-week treatment of SFX-01.

A, B) Weighing of the spleen tissue and analysis by ultrasound identified a trend for a reduction in spleen weight and size from 22 -week-old NS mice following treatment with $2.5 \mathrm{mg} / \mathrm{ml} \mathrm{SFX}-01$ in their drinking water for 10 weeks compared to untreated controls. C) Representation of ultrasound data as $\Delta$ change in the spleen size for each mouse over the duration of the treatment period identified a significant reduction in NS mice who received the drug. ( $n=14-16,{ }^{*} p<0.05$ versus WT or untreated control as stated). 


\subsubsection{SFN adducts proteins in the spleen}

Western immunoblotting revealed SFN-adducted proteins in spleen tissue from 22-week-old WT or NS mice following 10-week treatment with $2.5 \mathrm{mg} / \mathrm{ml} \mathrm{SFX-}$ 01 in their drinking water (figure 5.16).

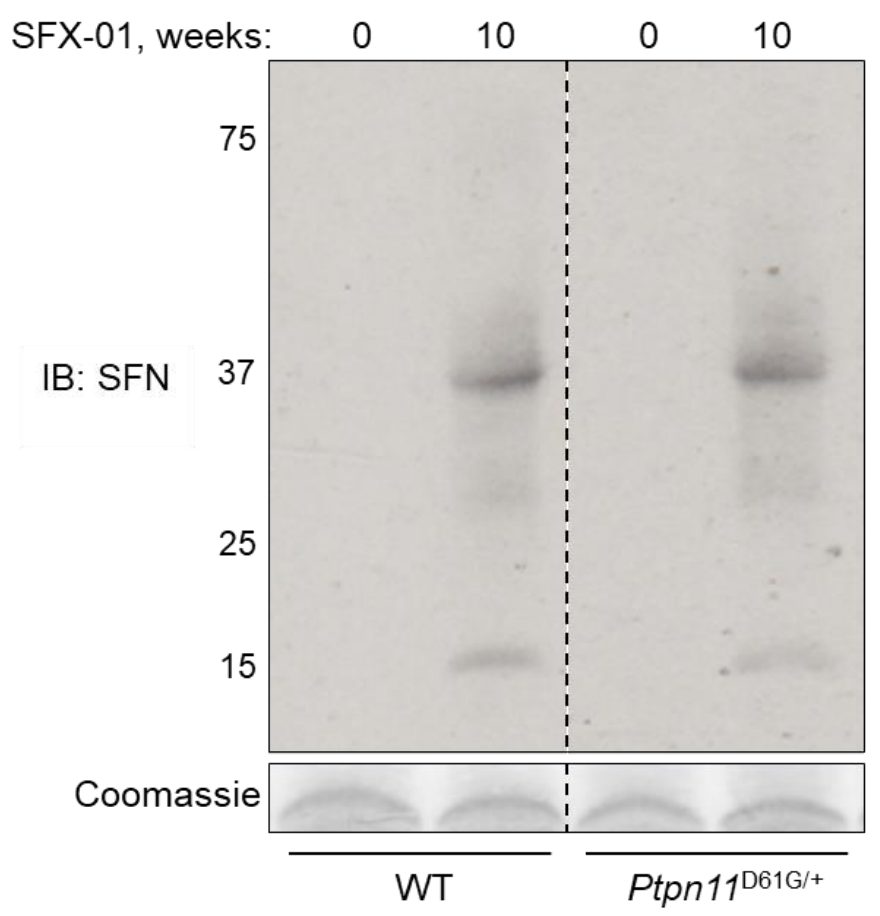

Figure 5.16. Protein-SFN adducts are detected in the spleen following SFX-01 treatment.

A representative immunoblot showing SFN-adducted proteins in spleen tissue from 22week-old WT or NS mice following 10-week SFX-01 treatment with $2.5 \mathrm{mg} / \mathrm{ml} \mathrm{SFX-01} \mathrm{in}$ their drinking water.

5.3.11 SFX-01 treatment reduces SHP2 phosphatase activity in the spleen of NS mice

SHP2 was immunoprecipitated from spleen tissue of 22-week-old WT or NS mice following 10-week treatment with $2.5 \mathrm{mg} / \mathrm{ml} \mathrm{SFX}-01$ in their drinking water (figure 5.17 A). The activity of the phosphatase was subsequently analysed using the 
fluorescence-based assay, which was reduced in NS mice following 10-week SFX-01 treatment compared to untreated controls. The activity of the phosphatase was unaffected following treatment in WT mice (figure 5.17 B).

A)

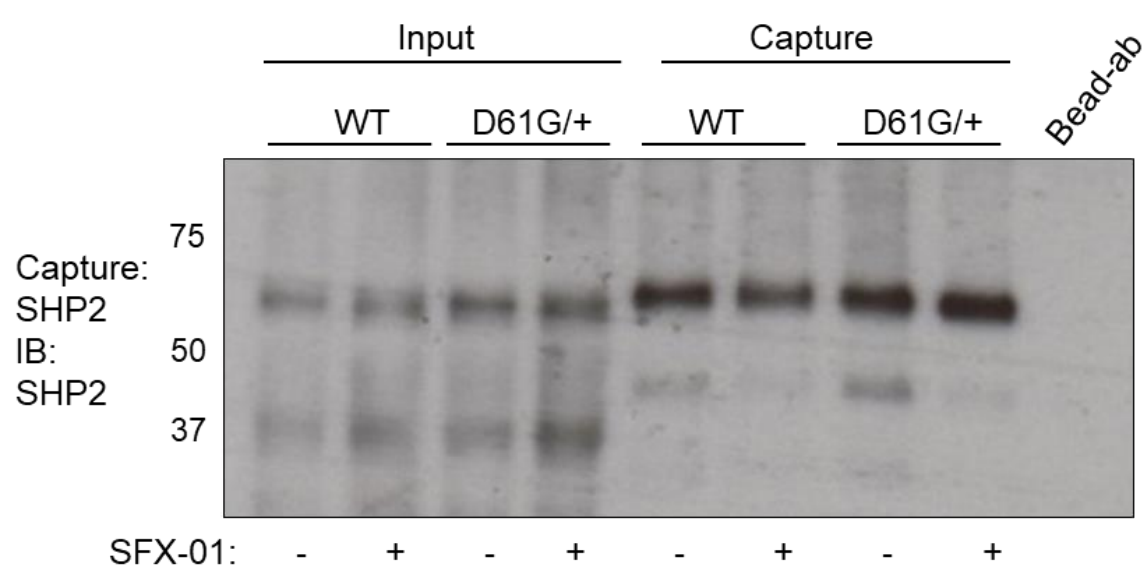

B)

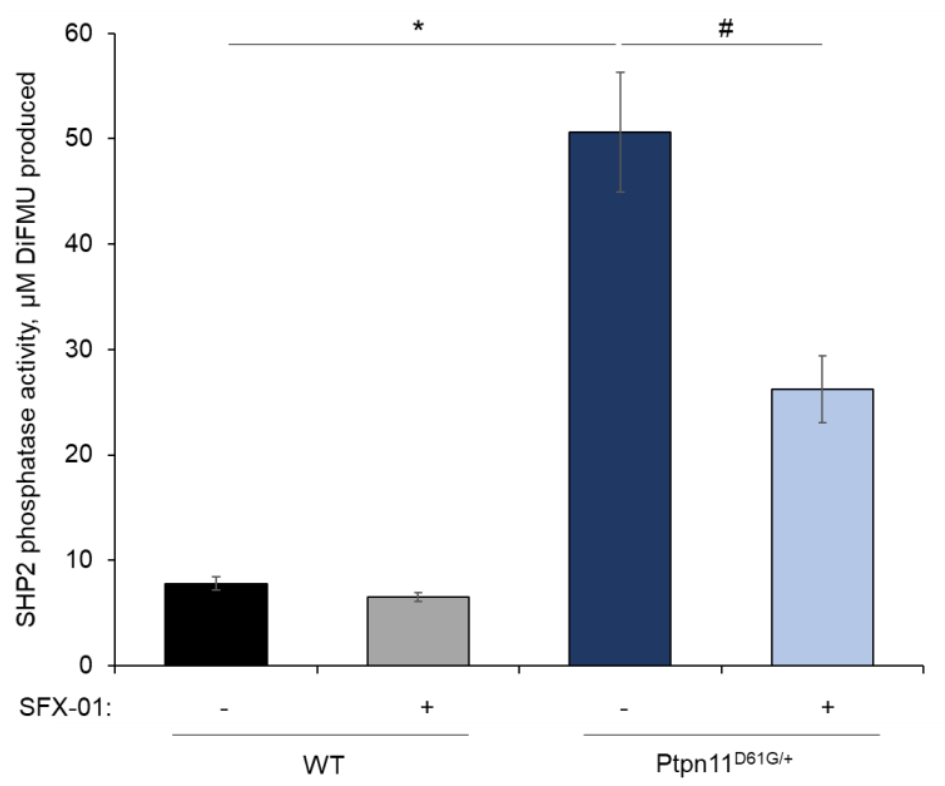

Figure 5.17. SFX-01 treatment reduces the phosphatase activity of SHP2 in the spleen of NS mice.

A) An immunoblot of input and capture samples following immunoprecipitation of SHP2 from spleen tissue of22-week-old WT or NS mice following 10-week treatment with 2.5 $\mathrm{mg} / \mathrm{ml} \mathrm{SFX-01} \mathrm{in} \mathrm{their} \mathrm{drinking} \mathrm{water.} \mathrm{B)} \mathrm{A} \mathrm{fluorescence-based} \mathrm{phosphatase} \mathrm{activity}$ assay using immunoprecipitated SHP2 shows inhibition of SHP2 activity in NS mice following 10-week treatment with $2.5 \mathrm{mg} / \mathrm{ml} \mathrm{SFX-01.} \mathrm{(} n=6{ }^{*} p<0.05$ versus WT or untreated control as stated). 


\subsection{Discussion}

In chapter 3 I demonstrated and characterised the inhibition of SHP2 by SFN both in vitro and in vivo. In this chapter, I sought to determine if this inhibition would prove therapeutic in the NS mouse model, which was considered a rational prospect as it expresses hyperactive SHP2. Thus, perhaps by supplying SFN to pregnant dams carrying NS foeti, their aberrant cardiac and skeletal development during embryogenesis would be corrected. Alternatively, perhaps administering SFN to NS mice after they are born might reduce the incidence of myeloproliferative disease that develops during adulthood.

NS patients with gain-of-function SHP2 mutations often have several congenital defects in cardiac structure ${ }^{354} 355$. The NS mouse model also develop congenital cardiac defects in a gene-dosage dependent manner 78137356357358 and the variable penetrance and severity of phenotypes of this mouse model are discussed in greater detail in the general introduction. HOM NS embryos have severe atrial, atrioventricular or ventricular septal defects, double-outlet right ventricle, enlarged outflow tract and defective atrioventricular valve primordia which all contribute to heart failure and lethality by $\mathrm{E} 13.5^{78}$. Approximately $50 \%$ of HET embryos also die mid-gestation, although with less severe cardiac defects 78. The remaining HET embryos are born with less-severe non-cardiac abnormalities ${ }^{78}$. As outlined in the introduction of this chapter, several studies identified roles for SHP2 in multiple stages of cardiac development. Through inducible knock-in approaches using NS mouse models with either a D61G/+ or D61Y/+ mutation, which cause hyperactivation of SHP2, the Neel group conclude all NS cardiac defects develop in utero and arise from the increased activity of the phosphatase in the endocardium. Detection by ultrasound of excessive 
amniotic fluid is suggestive of NS in the unborn child which can further be diagnosed through genetic testing ${ }^{359}$. If the diagnosis was achieved prior to or in the early stages of cardiac development, perhaps inhibition of hyperactive foetal SHP2 may prove therapeutic in reducing cardiac structural defects along with other congenital phenotypes. Using concentrations previously shown as inhibitory to SHP2 of adult mice $(2.5 \mathrm{mg} / \mathrm{ml})$, foetal treatment with SFX-01 resulted in all mothers from WTNT conceiving and giving birth to healthy litter sizes. Detection of SFN adducted to a $\sim 15 \mathrm{kDa}$ protein in these neonates, which is likely $\mathrm{Hgb} \beta$ for reasons outlined in chapter 4 , is indicative that the electrophile can cross the placenta. Although not statistically significant, this amount of drug did induce partial inhibition of SHP2 phosphatase activity in HET neonates from WT/HET breeding pairs. To achieve greater inhibition of SHP2, it is likely a higher amount of SFX-01 is required. However, unfortunately, treatment with the drug at these amounts resulted in no HOM NS offspring born from HET/HET breeding pairs. Litter size from WT/HET pairs was also reduced, with a lower number of HET neonates born and therefore this amount of SFX-01 exerted harmful effects upon foeti with this genotype. Foetal treatment with a lower amount of SFX-01 $(0.8 \mathrm{mg} / \mathrm{ml})$ again had no effect on rates of conception, the number of births or litter size of WT mice. With this reduced amount of drug, offspring were also born from HET/HET breeding pairs, although again, unfortunately, this treatment was detrimental to NS foeti.

Further analysis showed foetal treatment with this lower amount of SFX-01 increased ERK phosphorylation in WT neonates born from HET/HET breeding pairs. As SHP2 activity was not significantly altered in neonates following foetal treatment with this lower amount of the drug, it is logical to assume the SFN- 
induced increase in ERK phosphorylation occurs independently of SHP2. An SFN-induced increase in the phosphorylation of ERK via SHP2-independent mechanisms was discussed in chapter 3. Basally, NS foeti have increased ERK phosphorylation in endocardial cushion cells as well as their face and limb buds compared to WT mice, which is causative of their developmental abnormalities for reasons outlined below ${ }^{78}$. If SFX-01, therefore, increased ERK phosphorylation even further in NS foeti, perhaps severe cardiac and skeletal developmental defects were causative of their embryonic lethality. As mentioned previously, endocardial cushions evolve into semilunar and atrioventricular valves. During normal early and late stage valve development ERK activation is increased in the endocardial cushion cells overlaying distal tips of the atrioventricular canal and outflow tract valves and its hyperactivation has been shown to increase cushion explant outgrowth ${ }^{74}{ }^{360}$. Constitutive activation of ERK in mouse valve primordia replicates the valve phenotypes observed in embryos which have either the D61G/+ or D61Y/+ gain-of-function SHP2 mutation, whilst deletion of ERK completely rescued the endocardial cushion phenotype ${ }^{361}$. It is therefore accepted that hyperactivation of ERK in SHP2 gain-of-function NS mouse models as well as patients is causative of their valvular septal defects, although the exact mechanism of action is still under investigation. Krenz et al have shown that increased ERK activation in the endocardial cushion cells of embryos who have the gain-of-function SHP2 mutation Q79R/+ causes excessive mesenchymal cell proliferation, with no effect on endocardialmesenchymal transformation ${ }^{361}$. The Neel group, on the other hand, suggest ERK hyperactivation does extend the interval during which cardiac endocardial cells undergo an endocardial-mesenchymal transformation, likely by enhancing 
ErbB RTK signalling, in particular, Erb3, which is a positive regulator of valve development 7378 281. Nakamura et al also report increased ERK activation and enhanced cardiomyocyte proliferation in the trabecular myocardium which inhibits ventricular compaction and closure of the ventricular septum in their cardiomyocyte-specific Q79R/+ NS mouse model ${ }^{362 .}$

These studies suggest SFX-01 could not prevent congenital skeletal and cardiac developmental defects in NS foeti. However, optimising the amount of the drug received by the parents, as well as the stage of embryogenesis which it is administered are worth pursuing. For example, foetal treatment with SFX-01 post-gastrulation may avoid embryonic lethality by allowing initial skeletal and cardiac development to occur, with the electrophile then correcting SHP2 dependent abnormalities associated with later stages of cardiac development ${ }^{263}$. Whilst this would not allow for full correction of the cardiac phenotype, it may improve cardiac function. A pilot study administering SFX-01 post-gastrulation was unsuccessful, although only a higher, perhaps sub-optimal, dose of the drug was tested. The date of conception was also only an estimate, based on the presence of a vaginal plug, and so perhaps the SFX-01 was in fact administered pre- or mid-gastrulation in some mice. In future studies, conception and gastrulation should be confirmed using ultrasound. As ERK phosphorylation is increased in WT offspring from parents who received the drug and increased activity of the kinase is implicated in congenital phenotypes associated with NS, subsequent studies monitoring these offspring for skeletal and cardiac defects should be carried out.

Finally, the phosphorylation and activation of ERK stimulate downstream upregulation of cell growth and proliferation. Therefore, although ERK 
hyperactivation is detrimental in the context of embryonic development, perhaps the increase in the phosphorylation of this kinase which was observed in adult mice following SFX-01 treatment, as seen in chapter 3, may be beneficial regarding the impaired growth that NS patients face.

Some NS patients also present with myeloproliferative disease, which can develop into JMML; a rare myeloproliferative neoplasm associated with excessive monocytic and macrophagic proliferation 363364365366 367. Unlike cardiac complications, haematopoietic defects are completely penetrant in NS mice. As outlined in the introduction of this chapter, multiple studies have implicated SHP2 in HSC survival, proliferation and differentiation and both the Neel group and I have demonstrated elevated total white blood cell counts in adult NS mice ${ }^{96-105}$. Further to this, SHP2 promotes progenitor cell differentiation of the myeloid cell lineage. Indeed, bone marrow haematopoietic progenitors from NS mice have cell-autonomous signalling defects, developing into myeloid colonies independently of factor-stimulation. They are also more sensitive to IL3 and GM-CSF, factors which promote differentiation and proliferation of the myeloid lineage ${ }^{78}$. Flow cytometry analysis conducted by the Neel group showed myeloid count in the bone marrow and spleen of NS mice, which was conducted using a granulocyte receptor 1 antibody with affinity for both Ly6C and Ly6G cell surface markers, was elevated ${ }^{78}$. Separate Ly6C or Ly6G antibodies were used in my analysis reported here, allowing for differentiation between inflammatory monocyte and neutrophil cell populations respectively ${ }^{368}$. Using this method, I too identified adult NS mice had increased myeloid cell count in the spleen and blood but also made the novel observation this was predominantly due to an increased number of neutrophils. Although, there was only a slight increase in 
neutrophil count in the bone marrow of NS mice compared to WT littermates. Using a mouse model with a gain-of-function SHP2 mutation which was panhaematopoietic cell-inducible, E76K/+, Xu et al similarly observed accumulation of neutrophils in the blood. Observations made following my own flow cytometry analysis support studies outlined in the introduction of this chapter which implicates a more specific role for SHP2 in differentiation and proliferation of the granulocyte lineage, which encompasses neutrophils ${ }^{352}$.

I also observed a trend towards an increase in levels of the chemokine MIP2 in the plasma of NS mice compared to WT. MIP2 is a potent chemoattractant for neutrophils, with intravenous injection of the chemokine causing selective and rapid mobilisation of neutrophils from the bone marrow ${ }^{369}{ }^{370}$. Perhaps in NS mice, this higher abundance of circulating MIP2 causes greater attraction of neutrophils from the bone marrow, thus, as soon as they are produced, they are rapidly released into the circulation. This may also explain why the number of neutrophils appears not to be elevated in the bone marrow. There was, however, a trend towards increased inflammatory monocytes in the bone marrow of NS mice compared to WT. This was coupled with no increase in plasma levels of the chemokine MIP1a, a chemoattractant known to drive recruitment and maturation of monocytes 371372 . These mice may, therefore, have increased production of monocytes and neutrophils within the bone marrow, with the former accumulating because of deficient chemoattractant signals that would otherwise trigger their release into the circulation. It would perhaps be illuminating to conduct an analysis of a broader range of monocyte chemoattractants, such as chemokine ligand 2 or protein expression levels of its receptor chemokine ligand receptor 2 373 , as this may further support this speculation. As differentiation between 
inflammatory monocyte and neutrophil populations in the bone marrow or spleen of NS mice was not achievable by the Neel group, perhaps the elevated myeloid cell counts they identified in these mice also represented monocytes in the bone marrow and neutrophils within the spleen.

Together with an increase in the number of neutrophils in the spleen, I have shown adult NS mice develop splenomegaly, a phenotype that characterises some NS patients 374 375. As total white blood cell count, including neutrophils, was increased in the circulation, perhaps this enlargement of the spleen occurs due to their accumulation within this organ. Such an idea is consistent with evidence outlined in the introduction of this chapter whereby the spleen can act

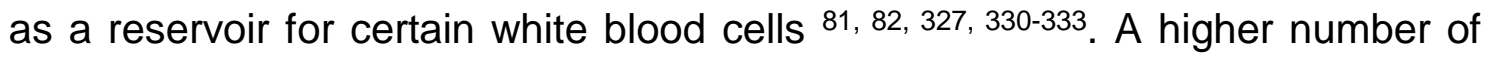
long- and short-term HSCs, as well as MPP cells, have been observed in spleens of mice carrying the gain-of-function SHP2 mutation E76K/+ ${ }^{353}$. With this in mind, perhaps splenomegaly in the $\mathrm{D} 61 \mathrm{G} /+$ mouse model is a result of extramedullary haematopoiesis occurring in this tissue. As my flow cytometry analysis only assessed mature neutrophils, a more in-depth analysis of cell-surface markers of lymphocytes from the spleen may help elucidate if excessive extramedullary haematopoiesis is also occurring simultaneously to white blood cell production in the bone marrow.

Consistent with the in vivo data described in chapter 3 where mice were treated chronically for 10 days with SFX-01, prolonged 10-week treatment with the drug significantly reduced SHP2 phosphatase activity in NS mice. Notably, inhibition of SHP2 occurs to a much greater extent in these mice compared to WT, which may be due to the gain-of-function SHP2 mutation alleviating $\mathrm{N}$-terminal structural inhibition of the phosphatase domain allowing easier access to the 
catalytic cysteine, an idea outlined in the general introduction. Also discussed previously was the logical assumption that SFN adducts multiple proteins in vivo and with this modification potentially altering their biological function. Even so, SHP2 plays essential roles in multiple stages of myeloid cell differentiation and progression, in particular, granulocytes. I therefore strongly suggest the reduction in neutrophil count in the blood, spleen and bone marrow of NS mice following treatment with SFX-01 was substantially mediated by inhibition of the phosphatase. To further confirm this, fluorescence-activated cell sorting could be utilised to isolate HSCs and progenitor cells from bone marrow and spleen following drug treatment. Subsequent culture and analyses of SHP2 inhibition, as well as cell proliferation, differentiation or apoptosis markers may uncover which haemopoietic cell type is most affected by SFN.

Balasubramanian et al discovered SHP2 is a positive regulator of p38 MAPKdependent MIP2 production through the formation of a stable complex with the kinase and GRB2 ${ }^{376}$. Plasma levels of MIP2 are reduced in NS mice following treatment with SFX-01. MIP2 is produced in a range of tissue and blood cells. Perhaps SFN-dependent inhibition of SHP2 is also preventing its recruitment to GRB2 and p38 MAPK, resulting in reduced MIP2 expression levels. In turn, this may reduce the stimulation for neutrophils to exit the bone marrow, potentially explaining the reduced neutrophil counts in the blood and spleen. If this was the case, an accumulation of neutrophils within the bone marrow following SFX-01 treatment may have been anticipated, which I did not observe. Perhaps neutrophils initially accumulate within the bone marrow, which may act as a feedback mechanism to reduce white blood cell production. Although, I anticipate a more rational explanation is SFN-dependent inhibition of SHP2 reduced both 
MIP2-mediated release of neutrophils from the bone marrow as well as myeloid cell proliferation and differentiation.

Studies have also shown that SHP2 can translocate to the mitochondria where it interacts with and dephosphorylates $\mathrm{p} 135$. This stimulates the production of ROS in myeloid progenitor cells and causes hypersensitivity to cytokines ${ }^{377}$. Treatment of these cells with the antioxidant $\mathrm{N}$-acetyl-cysteine prevents the biological effects caused by the modification of proteins by these ROS and in turn, reduces myeloid colony formation. Perhaps SFN is also lowering cytokine sensitivity and differentiation of myeloid progenitor cells in NS mice by reducing the intracellular levels of ROS, either through inhibition of SHP2 or activation of the KEAP1/Nrf2 signalling pathway.

As SHP2 phosphatase activity was not significantly inhibited in WT tissue following 10-week treatment with SFX-01, the observed reduction in total white blood cells, as well as inflammatory monocytes in the spleen of these mice, may be caused by the modification of other proteins by SFN. Of course, biological events which may be stimulated by adduction of the electrophile to proteins other than SHP2 may also contribute towards the reduction in total white blood cell count and inflammatory monocytes in the spleen of NS mice. Multiple studies have demonstrated anti-inflammatory effects of the electrophile regarding the monoblast lineage. Particularly through suppression of tumour necrosis factor-ainduced NF-KB transcriptional activity in monocytes, macrophages and endothelial cells by either adducting and inhibiting the catalytic cysteine within NF-KB $\beta$ subunit or inhibiting the RhoA/ROCK signalling pathway ${ }^{378} 379$. This leads to a subsequent reduction in gene expression of intracellular adhesion molecule 1 and vascular adhesion molecule 1 and reduces the adhesion of 
monocytes to sites of inflammation 380381382 . SFN also reduces the expression and release of macrophage migration inhibitory factor, IL-1 $1 \beta$, IL-6, tumour necrosis factor- $\alpha$ and matrix metallopeptidase 9 , which are pro-inflammatory cytokines that stimulate the release of monocytes from the bone marrow as well as their maturation to macrophages 248379383384 .

As well as decreasing total white blood cell and myeloid cell populations, the prolonged treatment of SFX-01 also prevented excessive growth of the spleen and the incidence of splenomegaly in the NS mouse model. Analysis of the spleen size of mice was achieved using ultrasound. When only end-point ultrasound data was analysed, the spleen size of NS mice did not appear to be significantly reduced by SFX-01 treatment compared to water only controls. However, the ultrasound method enabled calculation of spleen size before, during and after drug treatment, allowing analysis of the amount of spleen growth of each mouse through the duration of the study. When analysed in this way, spleen growth was significantly reduced in NS mice when treated with SFX-01. I outlined in the introduction of this chapter that myeloid cells can reside within the spleen until they are stimulated by an inflammatory response. Perhaps the reduction in the growth of the spleen of NS mice who received the drug was due to a lower number of circulating, and as a result, spleen residing neutrophils, which prevented the expansion of this tissue. The necessity to analyse each mouse as an individual and not as a grouped cohort can be explained by the variability in the severity of the splenomegaly that is present in different NS mice, and the variable penetrance of different phenotypes in this mouse model is explained in greater detail in the general introduction. 


\section{The mechanism of sulforaphane-induced inhibition of SHP2}

\subsection{Introduction}

\subsubsection{Catalytic mechanism of SHP2}

Amino acid sequence alignment of PTPs showed an evolutionarily conserved catalytic domain with a signature motif (I/V)VHCXAGXGR(S/T)G, whereby X can be any amino acid 385386387 . Site-directed mutagenesis and active site-labelling elucidated the invariant cysteine residue as essential for PTP activity 388389390 . Several studies show PTPs, including SHP2, have a conserved catalytic mechanism involving a two-step process in which substrate binding is followed by phosphate monoester hydrolysis as shown in figure 6.1387391392393394395 . The active site within the PTP domain of SHP2 forms a 'pocket' consisting of three loops; P, Q and WDP. The catalytic cysteine of SHP2, Cys ${ }^{459}$, resides within the P-loop of the active site along with a critical arginine ${ }^{43}$. The $p K_{a}$ of this cysteine thiol is low, which facilitates the nucleophilic attack of the thiolate towards protein phosphate groups. This low $\mathrm{pKa}$ is maintained through electrostatic interactions between the negatively charged thiolate and the positively charged side chains of the critical arginine ${ }^{43} \mathrm{H}$ bonding has also been shown to stabilize the proton-dissociated state of the reactive cysteine residue to maintain the low $\mathrm{p} K \mathrm{a}$. This was demonstrated by Chigadze et al, who showed the side chains of $\mathrm{Asp}^{61}$ within the WDP-loop form multiple $\mathrm{H}$ bonds, including with Ser ${ }^{460}$ from the catalytic P-loop, a water-mediated $\mathrm{H}$ bond with the catalytic Cys $^{459}$, two water-mediated $\mathrm{H}$ bonds with $\mathrm{Arg}^{465}$ and another water-mediated $\mathrm{H}$ bond with Asp 425392397 . The orientation of the positively charged critical arginine 
within the P-loop facilitates correct binding of the phosphorylated substrate by coordinating with negatively charged oxygens of the phosphate group 398399400 . The first step of the reaction involves nucleophilic attack of the catalytic cysteine thiolate towards the phosphorus atom 390392401 . Concurrently with cleavage of this ester bond, an adjacent aspartic acid residue residing in the proteins WPDloop donates a proton to the leaving group oxygen upon the substrate 391399402 403. This results in a cysteinyl-phosphorus intermediate covalently bound via a thioester linkage, which is stabilised by the P-loop arginine, and release of the dephosphorylated substrate ${ }^{387}{ }^{403}$. The second rate-limiting step involves positioning of a water molecule within the phosphatase domain by coordination with a glutamate in the Q-loop ${ }^{404}$. The catalytic aspartate now acts as a general base by accepting a proton from the water molecule and facilitating hydrolysis of the scissile phosphorous-sulfur bond 402405406407 . This generates free-phosphate and also restores the reactive thiolate, allowing the PTP to engage in a new reaction cycle. As outlined in chapters 3 and 5, SFN inhibited SHP2 phosphatase activity in vitro and in vivo. Interestingly, the in vitro studies showed SFN adduction to SHP2 was followed by the loss of a detectable adduct, even though inhibition of the phosphatase was maintained. A possible explanation for this was that loss of the adduct was coupled with the formation of a residual modification within the active site that prevents the catalytic mechanism described above. Here I sought to investigate this incompletely understood mechanism of inhibition of SHP2 by SFN which unexpectedly and intriguingly cannot be accounted for by stable adduction by the electrophile. 

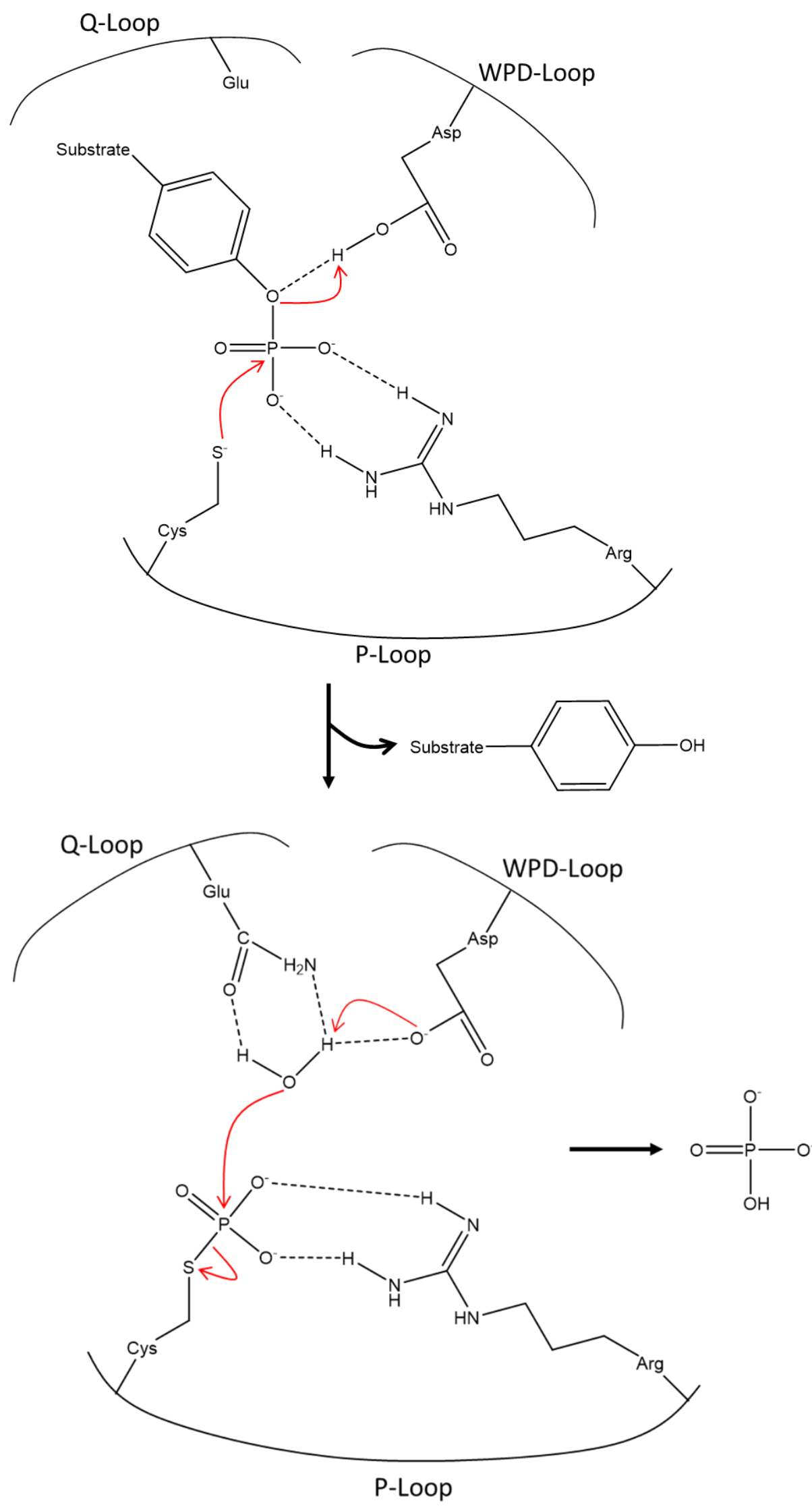

Figure 6.1. Catalytic mechanism of cysteine-based PTPs, including SHP2. 


\subsection{Materials and methods}

\subsubsection{SDS-PAGE and western immunoblotting}

SDS-PAGE and western immunoblotting were performed as outlined in the general methods. For this chapter the following primary antibodies were used:

\begin{tabular}{|l|l|l|}
\hline Primary antibody & Company & Species \\
\hline Sulforaphane & In-house & Rabbit \\
\hline SHP2 (for immunocapture) & Santa Cruz \#sc-280 & Rabbit \\
\hline SHP2 (for immunodetection) & R\&D Systems \#AF1894 & Goat \\
\hline
\end{tabular}

Table 6.1. List of primary antibodies used for western immunoblotting in chapter 6.

\subsubsection{Immunoprecipitation}

Immunoprecipitation of proteins from tissue was performed as outlined in the general methods. For this chapter, the capture antibody was agarose-conjugated anti-SHP2 (Rabbit, Santa Cruz, \#sc-7384 AC).

\subsection{3 $\mathrm{H}_{2} \mathrm{O}_{2}$ treatment of recombinant SHP2}

$10 \mathrm{ng}$ of recombinant SHP2 in $20 \mu \mathrm{l}$ of PBS containing $0-250 \mu \mathrm{M} \mathrm{H}_{2} \mathrm{O}_{2}$ was incubated at room temperature for 15 minutes. $20 \mu \mathrm{l}$ of $2 X$ SDS-PAGE sample buffer was then added and western immunoblotting was performed as described in the general methods.

\subsubsection{Polyethylene glycol (PEG)-switch method}

The PEG-switch method was used to assess potential reversible oxidative modification of SHP2 following treatment with SFN. SHP2 was 
immunoprecipitated from cardiac tissue of control WT or NS mice or following treatment with $2.5 \mathrm{mg} / \mathrm{ml} \mathrm{SFX-01} \mathrm{for} 4$ days in their drinking water and resuspended in $50 \mu \mathrm{l}$ PBS. $50 \mu \mathrm{l}$ of a maleimide containing buffer $(100 \mathrm{mM}$ maleimide, $100 \mathrm{mM}$ Tris- $\mathrm{HCl}, 1 \% \mathrm{SDS}, \mathrm{pH} 7.4$ ) was then added to each SHP2 containing mixture and incubated at $50{ }^{\circ} \mathrm{C}$ for 25 minutes. $200 \mathrm{mM}$ TCEP was then added, and the mixture was incubated at room temperature for 30 minutes. Each reaction mixture was desalted using a $7 \mathrm{kDa}$ cut-off $0.5 \mathrm{ml}$ Zeba Spin desalting column (Thermo Fisher) and $20 \mu \mathrm{l}$ of a PEG-maleimide containing buffer (70 mM PEG-maleimide, 500 mM Tris-HCl, $7 \%$ SDS, pH 7.4) was added followed by incubation at room temperature for 2 hours rotating at $20 \mathrm{rpm}$. An equal volume of 2X SDS-PAGE sample buffer containing $50 \mathrm{mM}$ maleimide was then added to quench the reaction. Samples were analysed under non-reducing conditions by SDS-PAGE and western immunoblotting using an anti-SHP2 antibody. PEG-maleimide specifically reacts with free cysteine thiols and carries a pegylated tail with a molecular weight of $5 \mathrm{kDa}$. This method therefore potentially allows determination of the number of cysteines within SHP2 which had been subjected to reversible oxidative modification by analysis of the proteins banding pattern following western immunoblotting.

\subsubsection{Biotinylated iodoacetamide (BIAM) labelling method}

The BIAM labelling method was used to identify if the catalytic cysteine within SHP2 was post-translationally modified following treatment with SFN. SHP2 was immunoprecipitated from cardiac tissue of control WT or NS mice or following treatment with $2.5 \mathrm{mg} / \mathrm{ml} \mathrm{SFX-01} \mathrm{for} 4$ days in their drinking water and resuspended in $50 \mu \mathrm{l}$ PBS, pH 6.4. $2 \mu$ of a $10 \mathrm{mM}$ BIAM stock (400 
$\mu \mathrm{M}$ final concentration) was then added and the reaction mixture was incubated at room temperature for 30 minutes followed by addition of an equal volume $2 \mathrm{X}$ SDS-PAGE sample buffer containing $100 \mathrm{mM}$ maleimide to quench the reaction. Samples were analysed under non-reducing conditions by SDS-PAGE and western immunoblotting. PVDF membranes were probed using HRP-conjugated streptavidin (1:10,000 in $5 \%$ BSA in PBS-T) which was detected using ECL. If the catalytic cysteine is post-translationally-modified, BIAM is unable to adduct and loss of labelling is seen.

\subsubsection{Phenylarsinic acid (PAA) labelling method}

The PAA labelling method was used to identify if two vicinal thiols within the catalytic domain of SHP2 had been post-translationally-modified following treatment with SFN. SHP2 was immunoprecipitated from cardiac tissue of control WT or NS mice or following treatment with $2.5 \mathrm{mg} / \mathrm{ml} \mathrm{SFX-01} \mathrm{for} 4$ days in their drinking water and resuspended in $50 \mu \mathrm{l}$ PBS followed by an equal volume of $2 \mathrm{X}$ SDS-PAGE sample buffer. Samples were analysed under non-reducing conditions by SDS-PAGE and far-western blotting. PVDF membranes were incubated with $1 \mathrm{mM}$ biotinylated-PAA (Synlnnova) in $40 \mathrm{ml} 5 \%$ BSA in PBS-T for 1 -hour at room temperature. Membranes were then washed for $5 \times 15$ minutes with PBS-T and incubated for a further hour with HRP-conjugated streptavidin (1:10,000 in $5 \%$ BSA in PBS-T) which was detected using ECL. If two vicinal thiols are post-translationally-modified, PAA is unable to adduct and loss of labelling is seen.

\subsubsection{Amplification and purification of WT and Cys ${ }^{459}$ Ser SHP2 plasmid} WT or Cys ${ }^{459}$ Ser SHP2 plasmids were purchased from Addgene (plasmid \#8381 and \#8382 respectively). Both plasmids comprised a cytomegalovirus (pCMV) 
vector backbone with ampicillin resistance and human SHP2 inserted (with or without mutation). The WT plasmid can be visualised in figure 6.2. Plasmids were received in transformed bacteria in an agar stab. $5 \mathrm{ml}$ Luria-Bertani (LB) broth supplemented with $100 \mathrm{mg} / \mathrm{ml}$ ampicillin was inoculated with the transformed bacteria and incubated for 7 hours at $37^{\circ} \mathrm{C}$ shaking at $100 \mathrm{rpm}$. Transformed LB broth was then added to $245 \mathrm{ml}$ fresh LB broth supplemented with $100 \mathrm{mg} / \mathrm{ml}$ ampicillin and incubated overnight at $37{ }^{\circ} \mathrm{C}$ shaking at $100 \mathrm{rpm}$. The following morning, the plasmid was extracted from the bacterial cells using a plasmid maxiprep kit (QIAGEN) following the manufacturer's instructions. DNA was then sequenced by Eurofins Genomics and results were analysed using ApE software. 


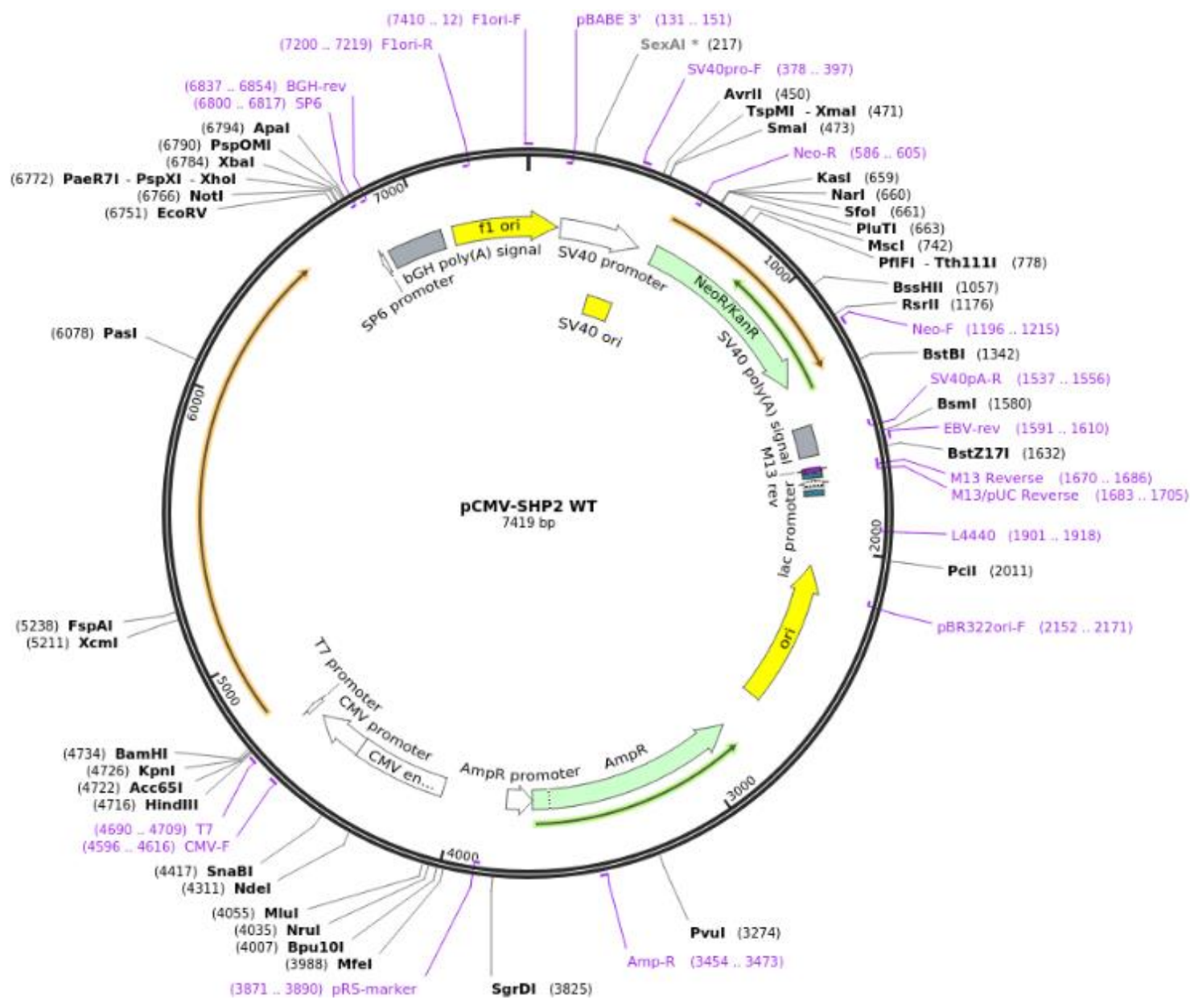

Figure 6.2. WT SHP2 plasmid used for site-directed mutagenesis.

\subsubsection{Site-directed mutagenesis of SHP2 plasmid}

Multiple cysteine to serine single or double SHP2 mutants, as named in table 6.2, were generated via site-directed mutagenesis. 


\begin{tabular}{|c|c|c|c|}
\hline $\begin{array}{l}\text { Mutant } \\
\text { name }\end{array}$ & $\begin{array}{l}\text { Plasmid } \\
\text { used }\end{array}$ & Forward primer for PCR & $\begin{array}{l}\text { Reverse primer for } \\
\text { PCR }\end{array}$ \\
\hline Cys $^{333}$ Ser & $\begin{array}{l}\text { SHP2 } \\
\text { WT }\end{array}$ & $\begin{array}{l}\text { 5' - ACACAAGGCTCCCTG } \\
\text { CAAAAC - 3' }\end{array}$ & $\begin{array}{l}\text { 5' - GGCAATGTAACT } \\
\text { CTTTTTGG - 3' }\end{array}$ \\
\hline Cys $^{367}$ Ser & $\begin{array}{l}\text { SHP2 } \\
\text { WT }\end{array}$ & 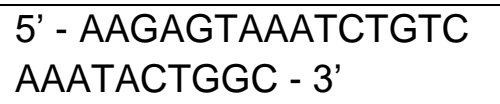 & $\begin{array}{l}\text { 5' - TCCTCTCTCCAC } \\
\text { TTCTTTC- 3' }\end{array}$ \\
\hline Cys ${ }^{333 / 367 S e r}$ & $\begin{array}{l}\text { SHP2 } \\
\text { Cys }^{333} \text { Ser }\end{array}$ & $\begin{array}{l}\text { 5' - AAGAGTAAATCTGTC } \\
\text { AAATACTGGC - 3' }\end{array}$ & $\begin{array}{l}\text { 5' - TCCTCTCTCCAC } \\
\text { TTCTTTC - 3' }\end{array}$ \\
\hline Cys ${ }^{333 / 459}$ Ser & $\begin{array}{l}\text { SHP2 } \\
\text { Cys }{ }^{459} \text { Ser }\end{array}$ & $\begin{array}{l}\text { 5' - ACACAAGGCTCCCTG } \\
\text { CAAAAC - 3' }\end{array}$ & $\begin{array}{l}\text { 5' - GGCAATGTAACT } \\
\text { CTTTTTGG - 3' }\end{array}$ \\
\hline
\end{tabular}

Table 6.2. List of primers used to generate cysteine to serine SHP2 mutants.

Site-directed mutagenesis was achieved via polymerase chain reaction (PCR) using a Q5 High-Fidelity DNA Polymerase kit (New England Biolabs), the oligonucleotide primers stated in table 6.2 and the PCR protocol stated in table 6.3.

\begin{tabular}{|l|l|l|}
\hline Step & Temperature & Time \\
\hline Initial denaturation & $98{ }^{\circ} \mathrm{C}$ & 30 seconds \\
\hline 35 cycles & $98{ }^{\circ} \mathrm{C}$ & 10 seconds \\
& $60{ }^{\circ} \mathrm{C}$ & 30 seconds \\
& $72{ }^{\circ} \mathrm{C}$ & 15 minutes \\
\hline Final extension & $72{ }^{\circ} \mathrm{C}$ & 15 minutes \\
\hline Holding & $4{ }^{\circ} \mathrm{C}$ & Until removed \\
\hline
\end{tabular}

Table 6.3. PCR protocol used for site-directed mutagenesis.

Following PCR, $1 \mu$ l of the restriction enzyme Dpnl (New England Biolabs) was added to the mixture and incubated at $37^{\circ} \mathrm{C}$ for 1 -hour. 


\subsubsection{Transformation of Escherichia coli and plasmid purification following mutagenesis}

Following incubation of the PCR mixture with Dpn1, $5 \mu$ l of PCR mix was added to one vial of 5- $\alpha$ competent Escherichia coli cells (New England Biolabs), incubated on ice for 30 minutes, heat shocked at $42{ }^{\circ} \mathrm{C}$ for 30 seconds followed by a final incubation on ice for 5 minutes. $500 \mu$ of super optimal broth with catabolite repression (SOC) medium was added to the competent cells, incubated at $37^{\circ} \mathrm{C}$ for 1 -hour shaking at $300 \mathrm{rpm} .100 \mu \mathrm{l}$ of the transformed competent cells were then spread evenly onto a LB agar plate supplemented with $100 \mathrm{mg} / \mathrm{ml}$ ampicillin and incubated at $37{ }^{\circ} \mathrm{C}$ overnight. $5 \mathrm{ml}$ of LB broth supplemented with $100 \mathrm{mg} / \mathrm{ml}$ ampicillin was then inoculated with single bacterial colonies which grew on the LB agar plate and incubated at $37{ }^{\circ} \mathrm{C}$ overnight shaking at $100 \mathrm{rpm}$. The following morning, the plasmid was extracted from the bacterial cells using a plasmid mini-prep kit (QIAGEN) following the supplied instructions. DNA was then sequenced by Eurofins Genomics and sequencing results were analysed using ApE software.

\subsubsection{Transfection of HEK293 cells with SHP2 plasmids}

For each well of a 6 -well plate, the following was prepared; $4 \mu$ Lipofectamine 2000 (Thermo Fischer) was added to $100 \mu \mathrm{l}$ opti-MEM reduced serum media in a microcentrifuge tube. In a separate tube, $0-500 \mathrm{ng}$ of SHP2 plasmid was added to $100 \mu \mathrm{l}$ Lipofectamine. Both tubes were incubated separately for 5 minutes at room temperature. The contents of the tubes were then combined and incubated for a further 15 minutes. During this time media was replenished upon HEK293 cells. The DNA-containing transfection solution was then added drop-wise to 
each well. Cells were placed back into the incubator and experiments were carried out 24 hours post-transfection.

\subsubsection{Cell treatment with SFX-01}

Media from HEK293 cells seeded into 6-well plates, either with or without prior transfection with an SHP2 plasmid was replaced with $2 \mathrm{ml}$ warmed, serum-free media (DMEM plus GlutaMAX-I supplemented with penicillin/streptomycin (1

unit $/ \mathrm{ml} ; 1 \mu \mathrm{g} / \mathrm{ml})$ ). Cells were treated with varying amounts of SFX-01, 0-250 $\mu \mathrm{M}$, and placed back into the incubator for $0.5-4$ hours. Following this, if western immunoblotting was required, $200 \mu \mathrm{l}$ of 2 X SDS-PAGE sample buffer was added to each well. When immunoprecipitation of SHP2 was required, $200 \mu \mathrm{l}$ of PBS was added instead. Cells were then detached from the well using a cell scraper, moved into a microcentrifuge tube and lysed via sonication for 7 seconds at 30 $\mathrm{kHz}$ and $40 \%$ amplitude. Western immunoblotting or immunoprecipitation protocols previous outlined were then followed.

\subsubsection{Generation of 5-thio-2-nitrobenzoic acid (TNB)}

To generate TNB, 0.5 g 5, 5-dithio-bis-2-nitrobenzoic acid (dTNB, Ellman's reagent) was dissolved in $25 \mathrm{ml} 0.5 \mathrm{M}$ Tris- $\mathrm{HCl} \mathrm{pH} 8.8$ containing $2.5 \mathrm{ml} \beta$ mercaptoethanol. The $\mathrm{pH}$ of the solution was adjusted to 1.5 with $6 \mathrm{M} \mathrm{HCl}$. The solution was rotated at $4{ }^{\circ} \mathrm{C}$ to aid TNB crystal formation. The following morning, the bright orange TNB crystals were filtered and washed with $2 \mathrm{~L}$ cold $0.1 \mathrm{M} \mathrm{HCl}$ to remove $\beta$-mercaptoethanol. The crystals could be stored indefinitely at room temperature. 


\subsubsection{Treatment of TNB and analysis by HPLC}

$1 \mathrm{mg}$ of TNB crystals were dissolved in $200 \mu \mathrm{l}$ deionised water followed by a further 1:500 dilution in water to make a $50 \mu \mathrm{M}$ TNB solution which was incubated with or without $5 \mu \mathrm{M} \mathrm{SFX-01} \mathrm{for} \mathrm{1-hour} \mathrm{or} \mathrm{overnight.} \mathrm{An} \mathrm{equivalent} \mathrm{amount} \mathrm{of}$ dissolved TNB was also incubated with $100 \mu \mathrm{M} \mathrm{H}_{2} \mathrm{O}_{2}$ for 1-hour. The solutions were analysed by HPLC using the protocol outlined in the general methods but with the UV detector set to $320 \mathrm{~nm}$ to aid detection of TNB. A deionised wateronly sample was used as a negative control followed by TNB, dTNB alone or SFX-01 alone standards to determine their retention time.

\subsubsection{Liquid chromatography-mass spectrometry analysis of GSH following treatment with SFX-01}

LC-MS/MS was carried out by Dr Francesca Mazzacuva (Franklin-Wilkins Building, King's College London). 10 or $100 \mu \mathrm{M}$ of reduced GSH was incubated with $10 \mu \mathrm{M}$ L-SFN (dissolved in DMSO) and incubated for $0.5-24$ hours. Immediately prior to analysis, samples were diluted 1:50 with $1: 1 \mathrm{H}_{2} \mathrm{O} / 1,1^{\prime}$ azobis(cyclohexanecarbonitrile) $+0.1 \%$ formic acid. $5 \mu \mathrm{l}$ of sample was first separated by LC using a reverse-phase column (Luna Omega, C18, $1.6 \mu \mathrm{M}, 100$ $\AA$ A, $100 \mathrm{~mm} \times 2.1 \mathrm{~mm}$ inner diameter, Thermo Fisher). An atomic absorption spectrometer was incorporated into the LC system which monitored the absorption of light (atomic absorbance), which was produced by a hollow cathode lamp, to measure the amount of each reaction product in the solution.

Separated reaction products were then applied to an interfacing linear ion trap mass spectrometer with an electron transfer dissociation source (LTQ Orbitrap $\mathrm{XL}$, Thermo Fisher) and collected from the analyser using full ion scan mode over an $m / z$ range of $100-1000$. MS conditions used were: capillary voltage $14 \mathrm{~V}$, 
capillary temperature $350{ }^{\circ} \mathrm{C}$, tube lens $35 \mathrm{~V}$. During the 15 -minute run time the machine simultaneously performed several experiments:

1) MS scan in positive ion mode, range $100-1000 \mathrm{~m} / \mathrm{z}$

2) $\mathrm{MS}^{2}$ in positive ion mode of $613.3 \mathrm{~m} / \mathrm{z}$, collision energy 17 , range $300-650 \mathrm{~m} / \mathrm{z}$ for GSSG

3) $\mathrm{MS}^{2}$ in positive ion mode of $308.3 \mathrm{~m} / \mathrm{z}$, collision energy 15 , range $150-310 \mathrm{~m} / \mathrm{z}$ for GSH

4) $\mathrm{MS}^{2}$ in positive ion mode of $178.6 \mathrm{~m} / \mathrm{z}$, collision energy 15 , range $50-150 \mathrm{~m} / \mathrm{z}$ for SFN

5) $\mathrm{MS}^{3}$ in positive ion mode of $485.2 \mathrm{~m} / \mathrm{z}$, collision energy 23, and then of 356 $m / z$, collision energy 35 , range $95-1000 \mathrm{~m} / z$ for GSH-SFN 


\subsection{Results}

\subsubsection{An SFN adduct is not detected upon inhibited cardiac SHP2}

As outlined in figure 3.12 of chapter 3, immunoprecipitation of cardiac SHP2 from WT or NS mice revealed inhibition of the protein's phosphatase activity following 4-day treatment with $2.5 \mathrm{mg} / \mathrm{ml} \mathrm{SFX-01} \mathrm{in} \mathrm{their} \mathrm{drinking} \mathrm{water.} \mathrm{Re-analysis} \mathrm{of}$ immunocaptured SHP2 by SDS-PAGE followed by immunoblotting unexpectedly revealed the absence of an SFN adduct upon the inhibited phosphatase from WT or NS mice following treatment (figure 6.3).

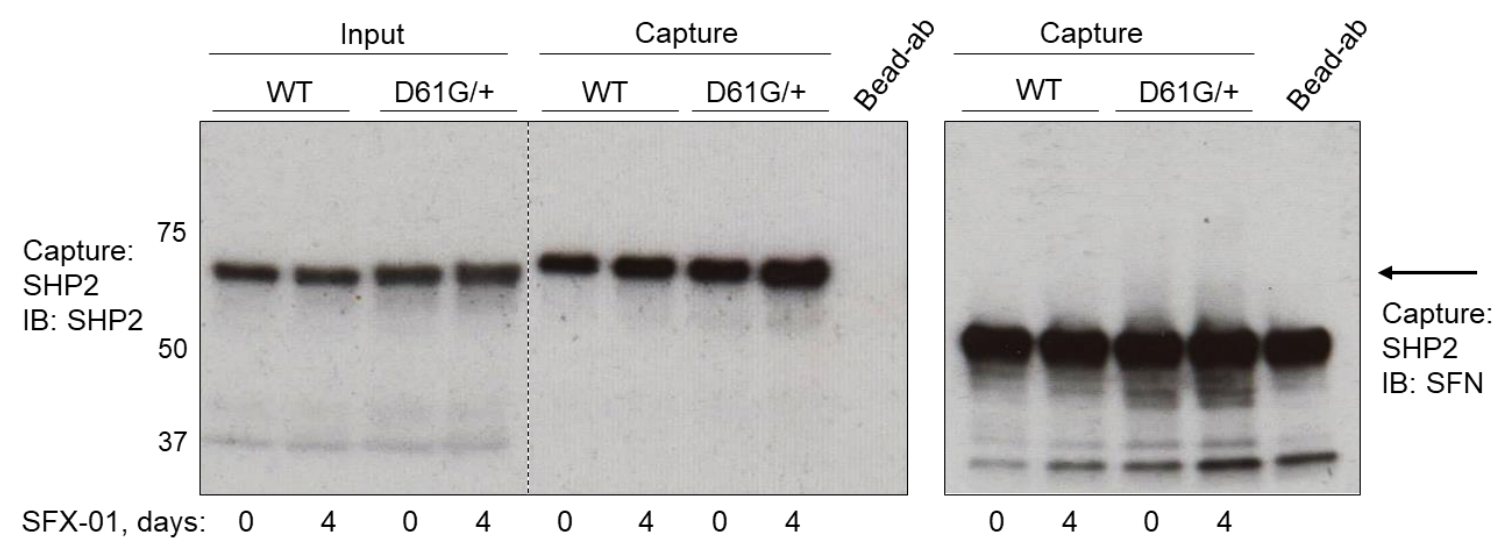

Figure 6.3. An SFN adduct is not detected upon SHP2 following chronic in vivo treatment with SFX-01.

A representative immunoblot showing the absence of an SFN adduct upon immunocaptured cardiac SHP2 from WT or NS mice following 4-day treatment with 2.5 $\mathrm{mg} / \mathrm{ml} \mathrm{SFX-01} \mathrm{in} \mathrm{their} \mathrm{drinking} \mathrm{water.}$

\subsubsection{Recombinant SHP2 migrates faster by SDS-PAGE following $\mathrm{H}_{2} \mathrm{O}_{2}$ treatment}

To investigate if oxidative modification induced an intramolecular disulfide within SHP2, recombinant protein was incubated with 0-500 $\mu \mathrm{M} \mathrm{H}_{2} \mathrm{O}_{2}$ for 15 minutes. Immunoblotting revealed a proportion of the recombinant SHP2 protein migrated 
faster on a polyacrylamide gel following $\mathrm{H}_{2} \mathrm{O}_{2}$ treatment in a concentrationdependent manner, consistent with the formation of an intramolecular disulfide (figure 6.4).

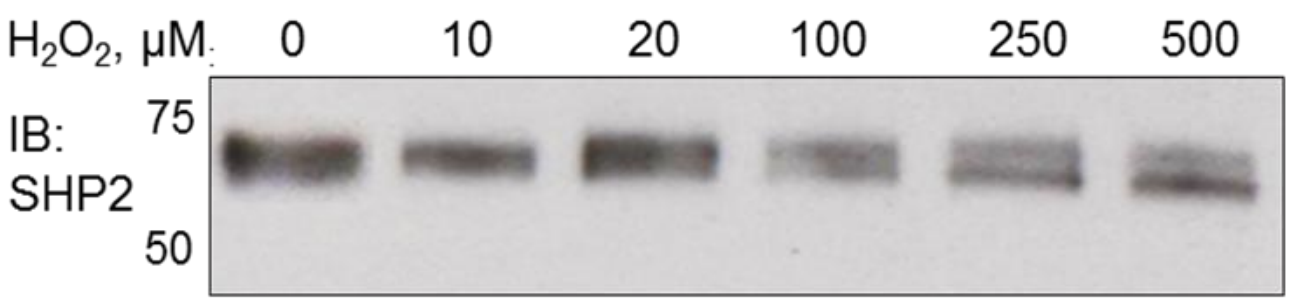

Figure 6.4. $\mathrm{H}_{2} \mathrm{O}_{2}$-treated recombinant SHP2 migrates faster on a polyacrylamide gel.

A representative immunoblot showing a proportion of recombinant SHP2 protein migrated faster on a polyacrylamide gel in a concentration dependent manner following 15-minute treatment with 10-500 $\mathrm{\mu M} \mathrm{H}_{2} \mathrm{O}_{2}$.

\subsubsection{SFN treatment induces a non-reducible molecular weight shift of SHP2 in vivo}

Immunoprecipitation of SHP2 from cardiac tissue of WT mice that received 2.5 $\mathrm{mg} / \mathrm{ml} \mathrm{SFX-01} \mathrm{in} \mathrm{their} \mathrm{drinking} \mathrm{water} \mathrm{for} \mathrm{4-10} \mathrm{days} \mathrm{followed} \mathrm{by} \mathrm{western}$ immunoblotting identified two lower molecular weight protein bands following drug treatment (as shown by the red arrows in figure 6.5). These bands were still present when the protein was incubated with $5 \mathrm{mM}$ of the reducing agent DTT, indicating these molecular weight shifts are unlikely to result from SFN inducing an intramolecular disulfide within the phosphatase (figure 6.5). 


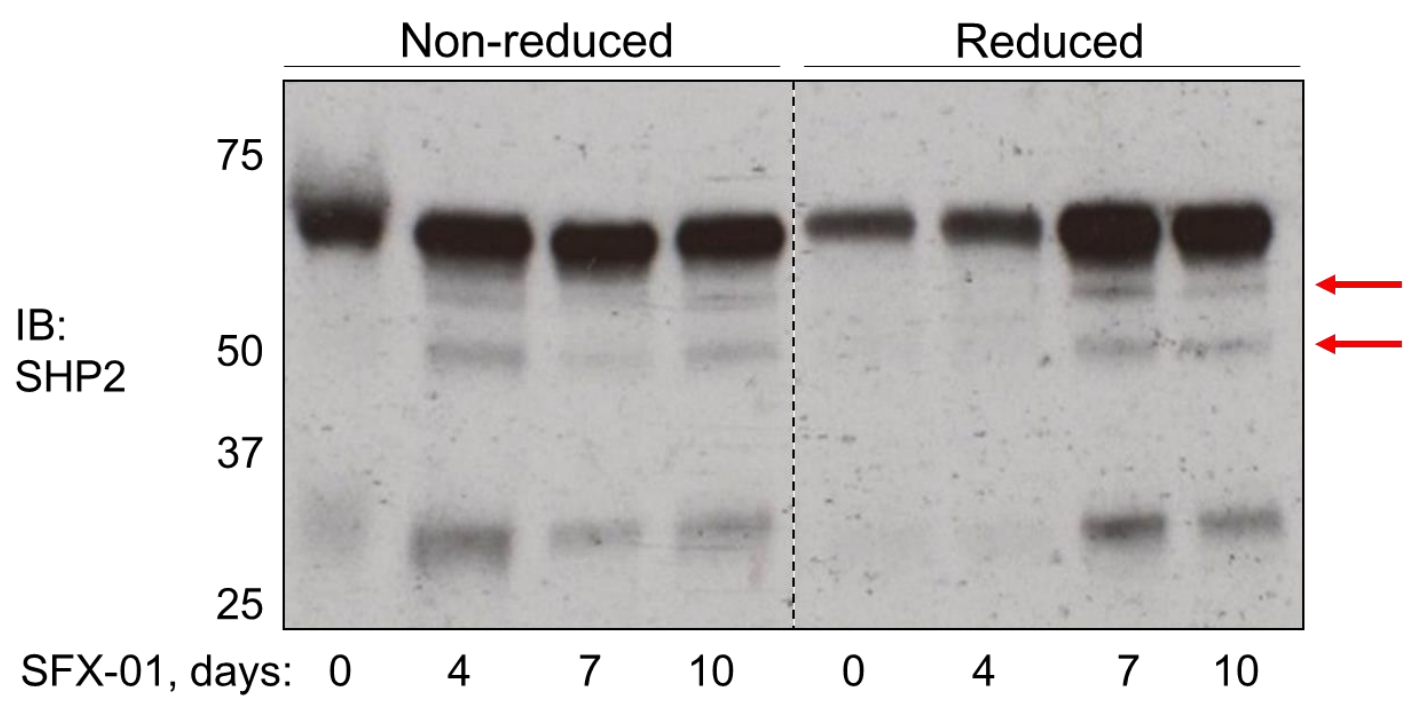

Figure 6.5. A small portion of cardiac SHP2 migrates faster a polyacrylamide gel following SFX-01 treatment.

A representative immunoblot showing SFN induces two molecular weight shifts in a small proportion of cardiac SHP2 (indicated by red arrows) following in vivo treatment of WT mice with $2.5 \mathrm{mg} / \mathrm{ml}$ SFX-01 in their drinking water for 4-10 days. Lower molecular weight protein bands were still present following treatment of immunoprecipitated SHP2 with 5 mM DTT.

\subsubsection{SFN-induced inhibition of cardiac SHP2 is not reversed by treatment}

\section{with DTT}

A fluorescence-based phosphatase activity assay showed cardiac SHP2 immunoprecipitated from WT or NS mice was inhibited following 4-10-day treatment with $2.5 \mathrm{mg} / \mathrm{ml} \mathrm{SFX-01} \mathrm{in} \mathrm{their} \mathrm{drinking} \mathrm{water} \mathrm{(figure} \mathrm{6.6).} \mathrm{Subsequent}$ analyses with this assay revealed treatment of the inhibited SHP2 protein with 5 mM DTT did not significantly rescue the phosphatase activity of SHP2 (figure 6.6). 


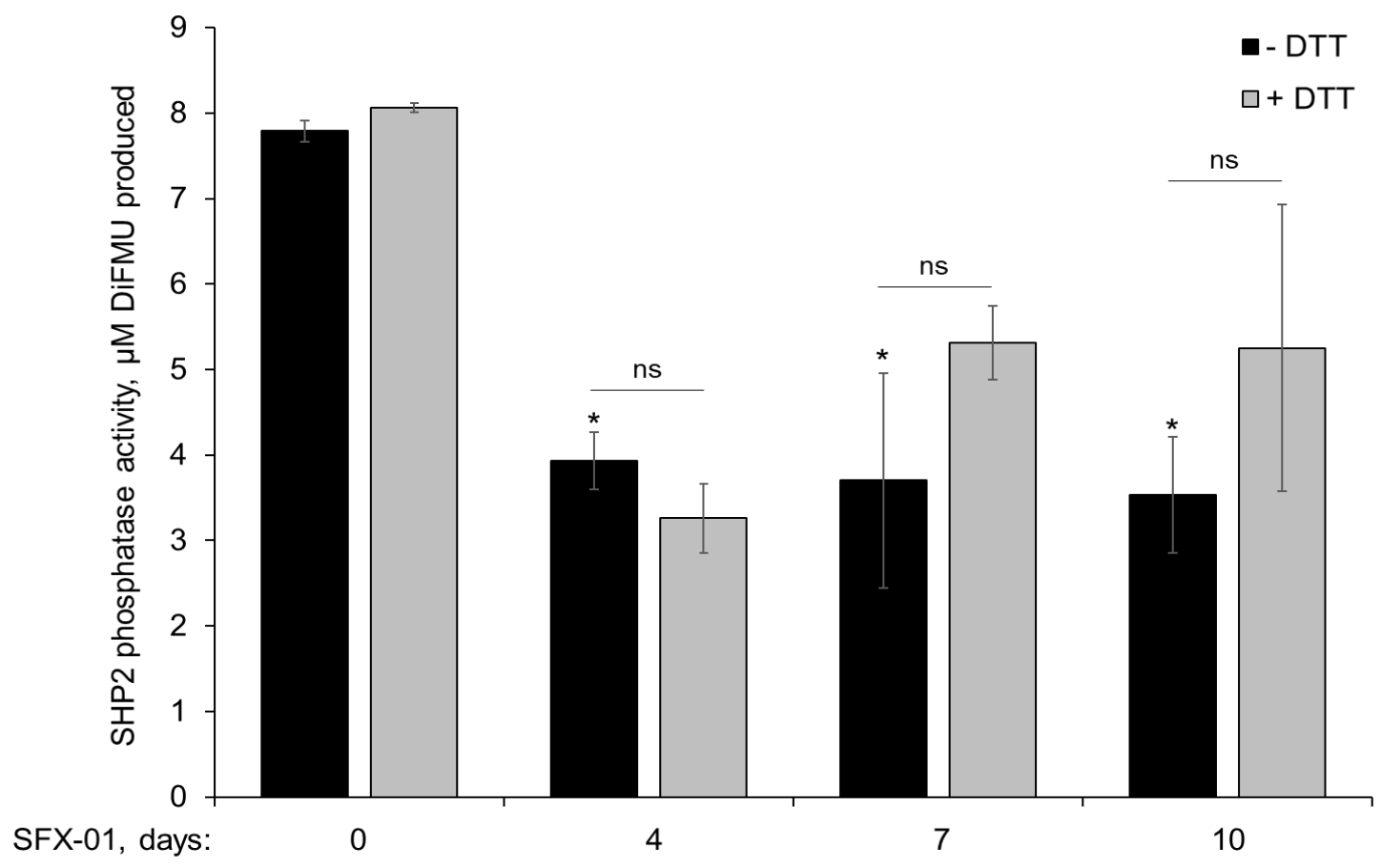

Figure 6.6. SFX-01 treatment inhibits SHP2 phosphatase activity in vivo, which is not recovered by DTT.

A fluorescence-based assay using cardiac SHP2 immunoprecipitated from WT or NS mice following 4-10-day treatment with $2.5 \mathrm{mg} / \mathrm{ml} \mathrm{SFX-01}$ in their drinking water revealed inhibition of the phosphatases activity which was not recovered by treatment with $5 \mathrm{mM}$ DTT.

\subsubsection{SFN treatment induces a small mobility shift of cardiac SHP2 following the PEG-switch assay}

PEG-maleimide treatment of SHP2 immunoprecipitated from cardiac tissue of WT or NS mice that received $2.5 \mathrm{mg} / \mathrm{ml}$ SFX-01 in their drinking water for 4 days induced a mobility shift in a small but detectable proportion of the protein on a polyacrylamide gel, as detected by a $5 \mathrm{kDa}$ increase in molecular weight by western immunoblotting (Figure 6.7). 

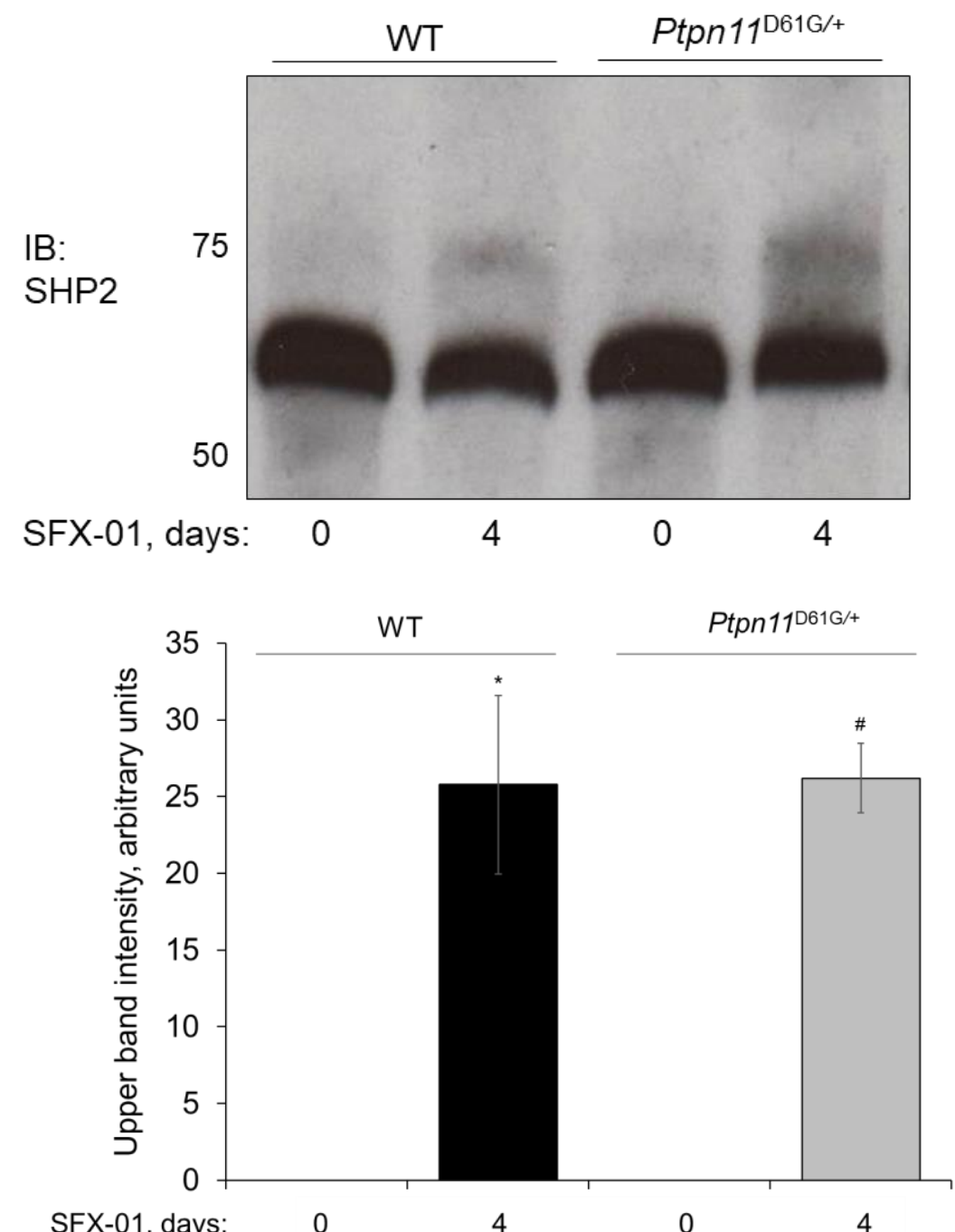

Figure 6.7. Analysis of the oxidative modification of cardiac SHP2 following in vivo treatment with SFX-01 using the PEG-switch method.

An immunoblot showing a small but detectable increase in molecular weight of cardiac SHP2 immunoprecipitated from WT or NS mice who had received $2.5 \mathrm{mg} / \mathrm{ml} \mathrm{SFX-01} \mathrm{in}$ their drinking water for 4 days when incubated with PEG-maleimide. ( $n=4,{ }^{*} p<0.05$ versus untreated control).

\subsubsection{In vivo treatment with SFX-01 reduces BIAM labelling of SHP2}

SHP2 immunoprecipitated from cardiac tissue of WT or NS mice following 4-day treatment with $2.5 \mathrm{mg} / \mathrm{ml} \mathrm{SFX-01} \mathrm{in} \mathrm{their} \mathrm{drinking} \mathrm{water} \mathrm{was} \mathrm{incubated} \mathrm{with} \mathrm{BIAM.}$ 
Immunoblotting revealed reduced binding of BIAM to SHP2 in samples derived from mice exposed to SFX-01 (figure 6.8).
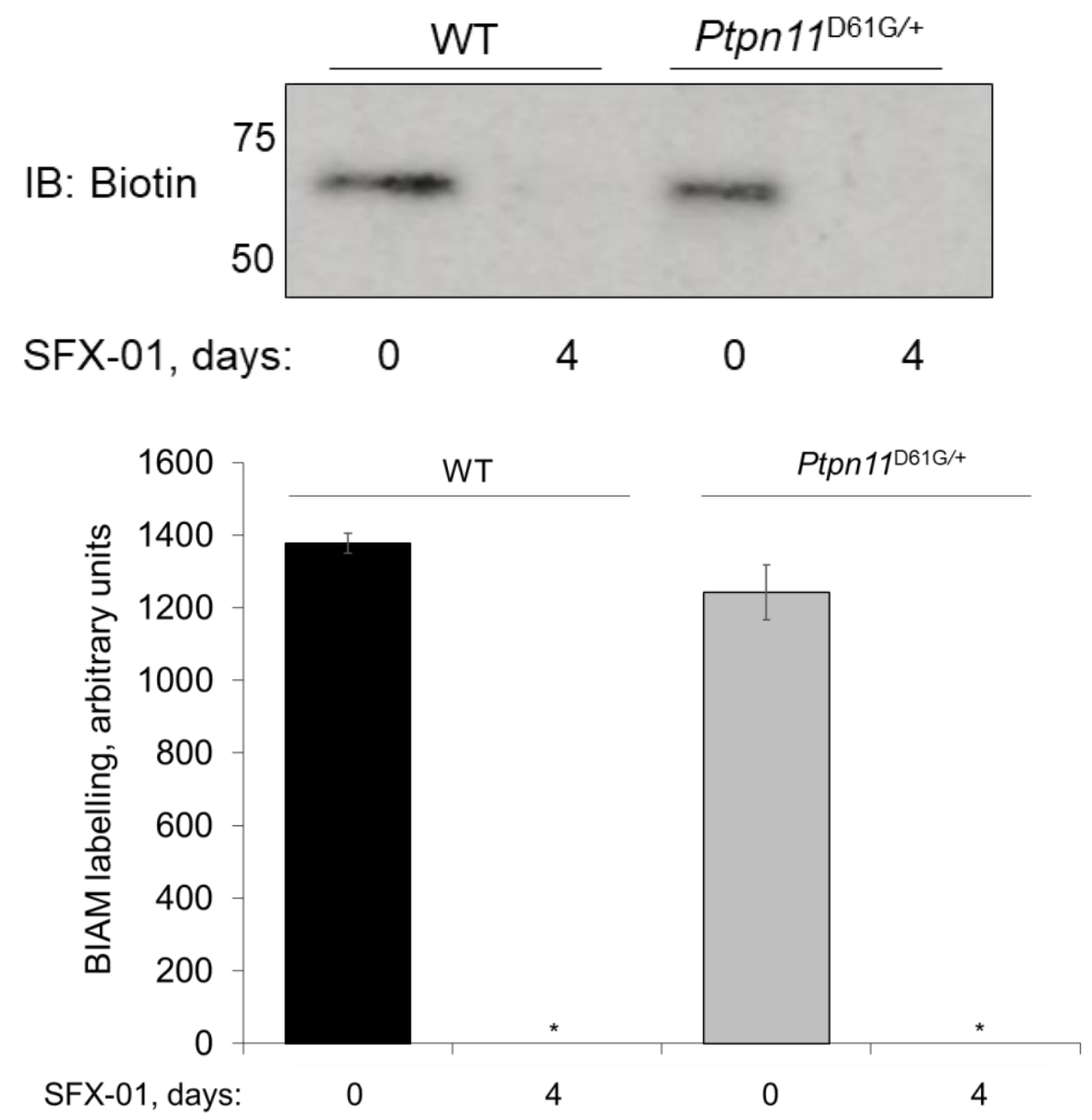

Figure 6.8. BIAM labelling of cardiac SHP2 is reduced following SFN-induced inhibition of the protein.

An immunoblot showing reduced binding of BIAM to SHP2 immunoprecipitated from cardiac tissue of WT or NS mice following 4-day treatment with $2.5 \mathrm{mg} / \mathrm{ml} \mathrm{SFX-01} \mathrm{in}$ their drinking water. $\left(n=4,{ }^{*} p<0.05\right.$ versus water only control).

\subsubsection{In vivo SFX-01 treatment reduces PAA labelling of SHP2}

SHP2 immunoprecipitated from cardiac tissue of WT or NS mice following 4-day treatment with $2.5 \mathrm{mg} / \mathrm{ml} \mathrm{SFX-01} \mathrm{in} \mathrm{their} \mathrm{drinking} \mathrm{water} \mathrm{was} \mathrm{incubated} \mathrm{with}$ 
biotinylated-PAA. Far-western blotting revealed reduced binding of PAA to SHP2 following drug treatment (figure 6.9).

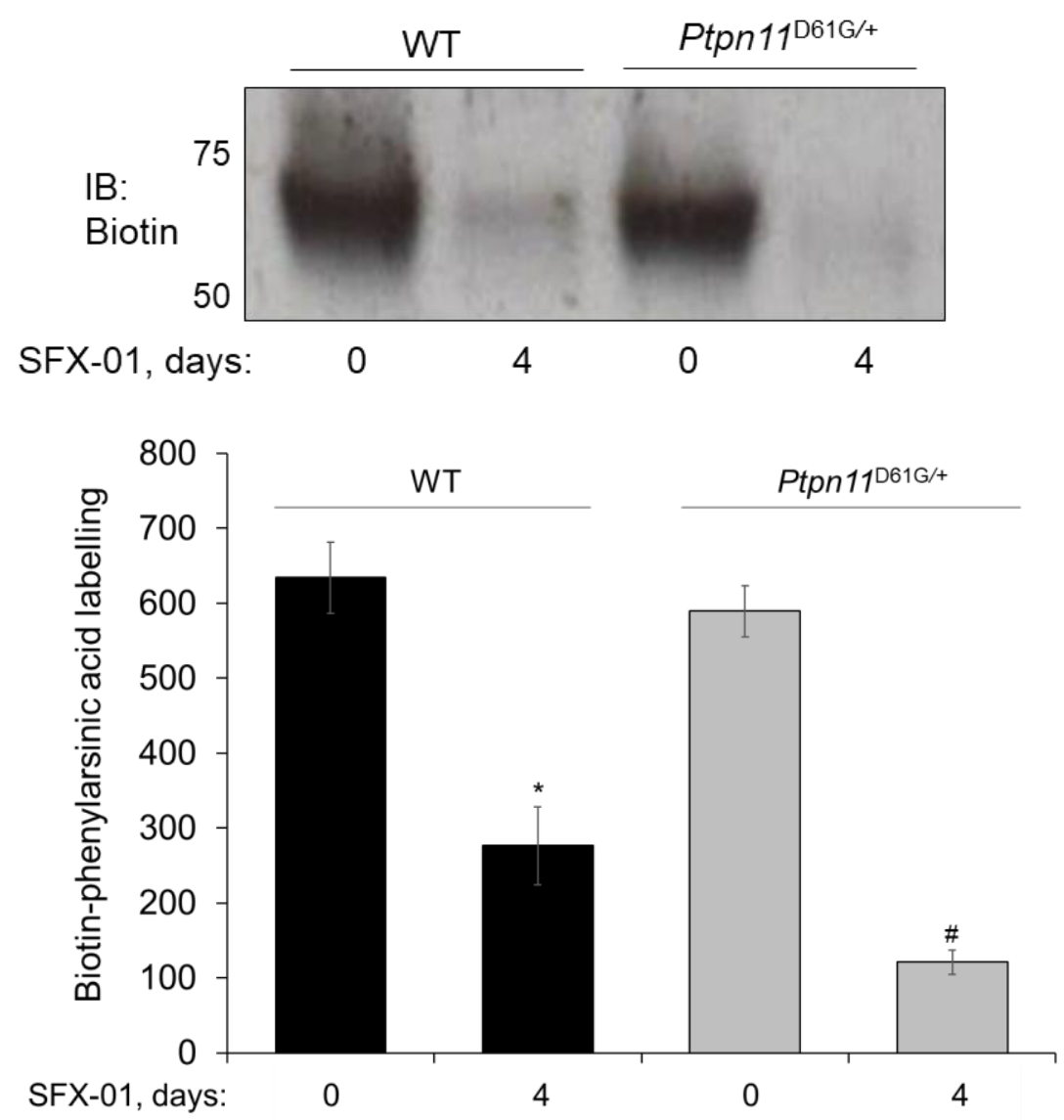

Figure 6.9. PAA labelling of cardiac SHP2 is reduced following SFN-induced inhibition of the protein.

A far-western blot showing reduced binding of PAA to SHP2 immunoprecipitated from cardiac tissue of WT or NS mice following 4-day treatment with $2.5 \mathrm{mg} / \mathrm{ml} \mathrm{SFX-01} \mathrm{in}$ their drinking water. $\left(n=4,{ }^{*} p<0.05\right.$ versus water only control).

\subsubsection{Cysteine to serine SHP2 mutants were successfully generated}

Using site-directed mutagenesis to exchange a guanine for a cytosine, two single mutants and two double mutants in which cysteine (TGC (Cys $\left.{ }^{333}\right)$ or TGT $\left(\right.$ Cys $\left.\left.^{367}\right)\right)$ was changed to serine (TCC $\left(\right.$ Cys $\left.^{333}\right)$ or TCT $\left(\right.$ Cys $\left.^{367}\right)$ ) in an SHP2 
expression plasmid were generated. These included: $\mathrm{Cys}^{333} \mathrm{Ser}$ and $\mathrm{Cys}^{367} \mathrm{Ser}$ (generated from the WT plasmid) Cys ${ }^{333 / 367}$ Ser (made from Cys ${ }^{333}$ Ser plasmid) and $\mathrm{Cys}^{333 / 459} \mathrm{Ser}$ (made from Cys ${ }^{459}$ Ser plasmid). Successful mutagenesis was confirmed using nucleotide sequencing and a subsequent alignment comparison with WT SHP2 cDNA (figures 6.10-6.13). 


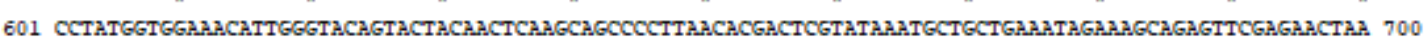
10 111111110111111111111111111111111111 6 -

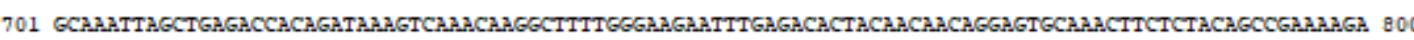
||||||||||||||||||||||||||||||||||||||||||||||||||||||||||||||||||||||||||||||||||||||||||||||||

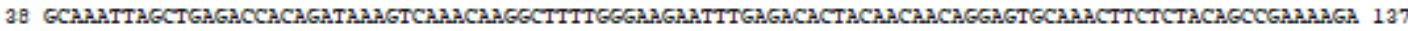
s

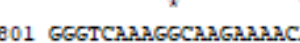
a

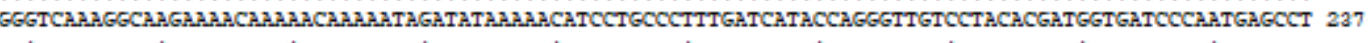

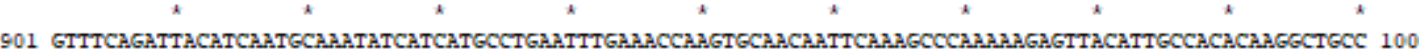
111111111111111111111111111111111111111111111111111111111111111111111111111111111111111111011

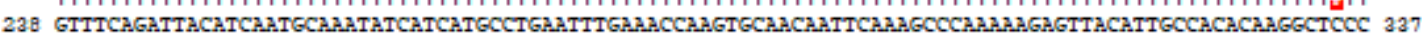
*

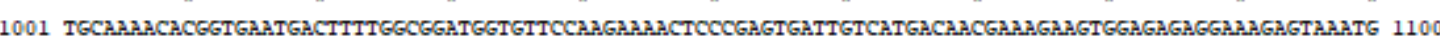
111111111111111111111111111111111111111111111111111111111111111111111111111111111111111111111111111111 338 TGCAAAACACGGTGATGACTTTTGGCGGATGGTGTTCCAAGAAAACTCCCGAGTGATTGTCATGACAACGA.AGAAGTGGAGAGAGGAAAGAGTAAATG 437

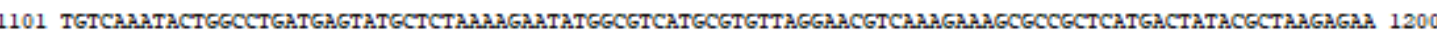
1111111111111111111111111111111111111111111111111111111111111111111111111111111111111111111111 438 TGTCAAATACTGGCCTGATGATATGCTCTAAAAGATATGGCGTCATGCGTGTTAGGACGTCAAAGAAGCGCCGCTCATGACTATACGCTAAGAGA 537 * * * * * * * * * * * * * *

1201 CTTAAACTTTCAAAGGTTGGACAAGCTCTACTCCAGGGGATACGGAGAGAACGGTCTGGCAATACCACTTTCGGACCTG6CCGGACCACG6CGTGCCCA 1300 $1111111111111111111111111-$ 11111111111111111111111111111111111111111111111111111111111111111 538 CTTAAACTTTCAAAGGTGGACAAG--0-0---GGATACGGAGGACGGTCTGGCAATACCACTTTCGGACCT6GCCGGACCACG6CGTGCCCA 625

1301 GCGACCCTGGGGGCGTGCTGGACTTCCTGGAGGAGGTGCACCATAAGCAGGAGAGCATCATGGATGCAGGGCCGGTCGTGGTGCACTGCAGTGCTGGAAT 1400 11111111111111111111111111111111111111111111111111111111111111111111111111111111111111111111111111 626 GCGACCCTG6GGGCGTGCTGGACTTCCTGGAGGAGGTGCACCATAAGCAGGAGAGCATCATGGATGCAGGGCCGGTCGTGGTGCACTGCAGTGCTGGA.AT 725

1401 TGGCCGGACAGGGACGTCATTGTGATGATATTCTTATTGACATCATCAGAGAGAAGGTGTTGACTGCGATATTGACGTTCCCAAAACCATCCAGATG 1500

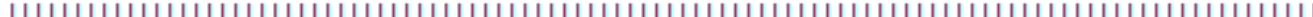
726 TGGCCGGACAGGGACGTTCATTGTGATTGATATTCTTATTGACATCATCAGAGAGAAAGTGTTGACTGCGATATTGACGTTCCCAAAACCATCCAGATG 825
*
A
*
*
t.
* *

1501 GTGCGGTCTCAGAGGTCAGGGATGGTCCAGACAGAaGCACAGTACCGATTTATCTATATG-GCGGTCCAGCATTATATTGAAACACTACAGCGCAGGATT 1599

111111111111111111111111111111111111111111111111111111111111111111111111111111111111111111111111111111111 . 826 GTGCGGTCTCAGAGGTCAGGGTGGTCCAGACAGA.GCACAGTACCGATTTATCTATATG6GCGGTCCAGCATTATATTGAACACTACAGCGCAGG--- 922

\section{Figure 6.10. Nucleotide sequence alignment of Cys ${ }^{333}$ Ser SHP2 mutant with WT} plasmid.

Alignment of nucleotide sequences from WT SHP2 plasmid purchased from Addgene (top line) with $\mathrm{Cys}^{333}$ Ser SHP2 plasmid generated by site-directed mutagenesis. The red highlighted \# at position 998 represents exchange of a guanine for a cytosine, showing successful mutation of this cysteine (TGC) to a serine (TCC). The red sections at the beginning and end of the Cys ${ }^{333}$ Ser sequence represent areas of primer binding. 


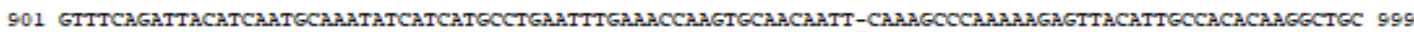
11111111111111111111111111111111111111111111111111111111111111111011111111111111111111111111111111111111111

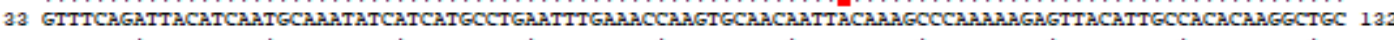

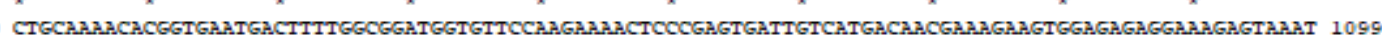
1111111111111111111111111111111111111111111111111111111111111111111111111111111111111111111111111111111 133 CTGCAAACACGGTGATGACTTTTGGCGATGGTGTTCAAGAAACTCCCGAGTGATGTCATGACAACGAAGAAGTGAGAGAGGAAGAGTAAAT 232

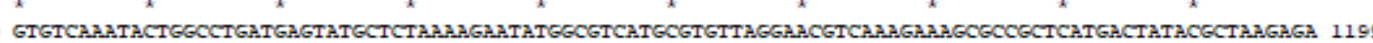

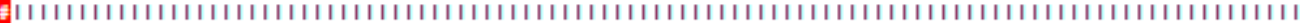
233 CTGTCAAATACTGGCCTGATGATATGCTCTAAAAGATATGGCGTCATGCGTGTTAGGACGTCAAAGAAGCGCCGCTCATGACTATACGCTAAGAGA 332

1200 ACTTAAACTTTCAAAGGTTGGACAAGCTCTACTCCAGGGGATACGGAGAGAACGGTCTGGCAATACCACTTTCGGACCTGGCCGGACCACGGCGTGCCC 1299 $111111111111111111111111111+111111111111111111111111111111111111111111111111111111111111111111$

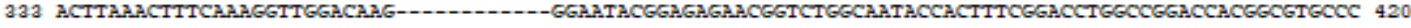

AGCGACCCTGGGGGCGTGCTGGACTTCCTGGAGGAGGTGCACCATAAGCAGGAGAGCATCATGGATGCAGGGCCGGTCGTGGTGCACTGCAGTGCTGGAA 1399 11111111111111111111111111111111111111111111111111111111111111111111111111111111111111111111111111111 421 AGCGACCCTGGGGGCGTGCTGGACTTCCTGGAGGAGGTGCACCATAAGCAGGAGAGCATCATGGATGCAGGGCCGGTCGTGGTGCACTGCAGTGCTGGA 520

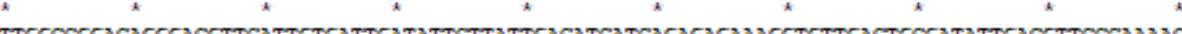

1400 TTGGCCGGACAGGGACGTTCATTGTGATTGATATTCTTATTGACATCATCAGAGAGAAAGGTGTTGACTGCGATATTGACGTTCCCAAAACCATCCAGAT 1499 111111111111111111111111111111111111111111111111111111111111111111111111111111111111111111111111111

521 TTGGCCGGACAGGGACGTTCATTGTGATTGTATTCTTATTGACATCATCAGAGAGAAGGTGTTGACTGCGATATTGACGTTCCCAAAACCATCCAGAT 620

*

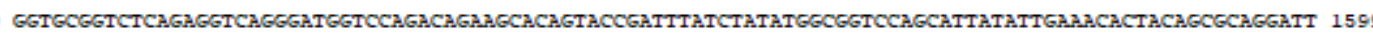

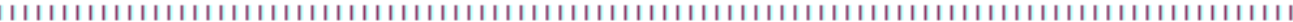

621 GGTGCGGTCTCAGAGGTCAGGGATGGTCCAGACAGAACACAGTACCGATTATCTATATGGCGGTCCAGCATTATATTGAACACTACAGCGCAGGATT 720

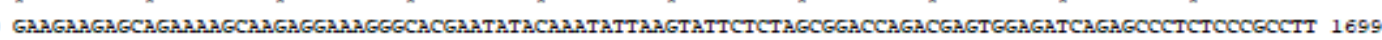
11111111111111111111111111111111111111111111111111111111111111111111111111111111111111111111111111111111111 721 GAAGAAGAGCAGAAAACAAGAGGAAGGGCACGAMTATACAAATATTAATATTCTCTAGCGGACCAGACGAGTGGAGATCAGAGCCCTCTCCCGCCTT 820

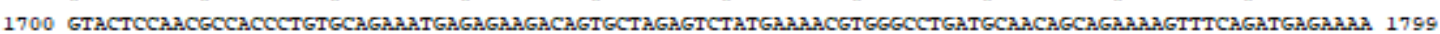
11111111111111111111111111111111111111111111111111111111111111111111111111111111111111111111111111111

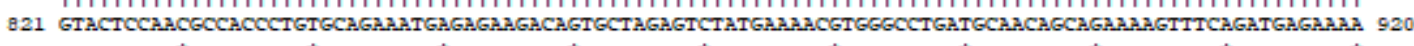
C

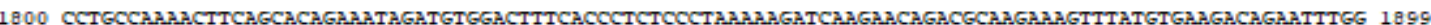
1111111111111111111111111111111111111111111111111110111111111111111111111111111111111111111111

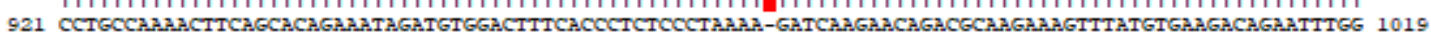

4.

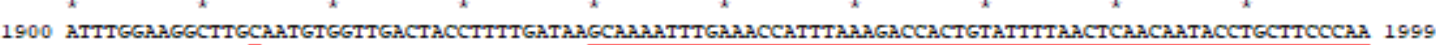
$1111111111111101111111111111111111111111-2$

1020 ATTTGGAAGGCTTG-AATGTGGTTGACTACCTTTTGATAA1058

\section{Figure 6.11. Nucleotide sequence alignment of Cys ${ }^{367}$ Ser SHP2 mutant with WT} plasmid.

Alignment of nucleotide sequences from WT SHP2 plasmid purchased from Addgene (top line) with Cys ${ }^{367}$ Ser SHP2 plasmid generated by site-directed mutagenesis. The red highlighted \# at position 1100 represents exchange of a guanine for a cytosine, showing successful mutation of this cysteine (TGT) to a serine (TCT). The red sections at the beginning and end of the $\mathrm{Cys}^{367}$ Ser sequence represent areas of primer binding. 


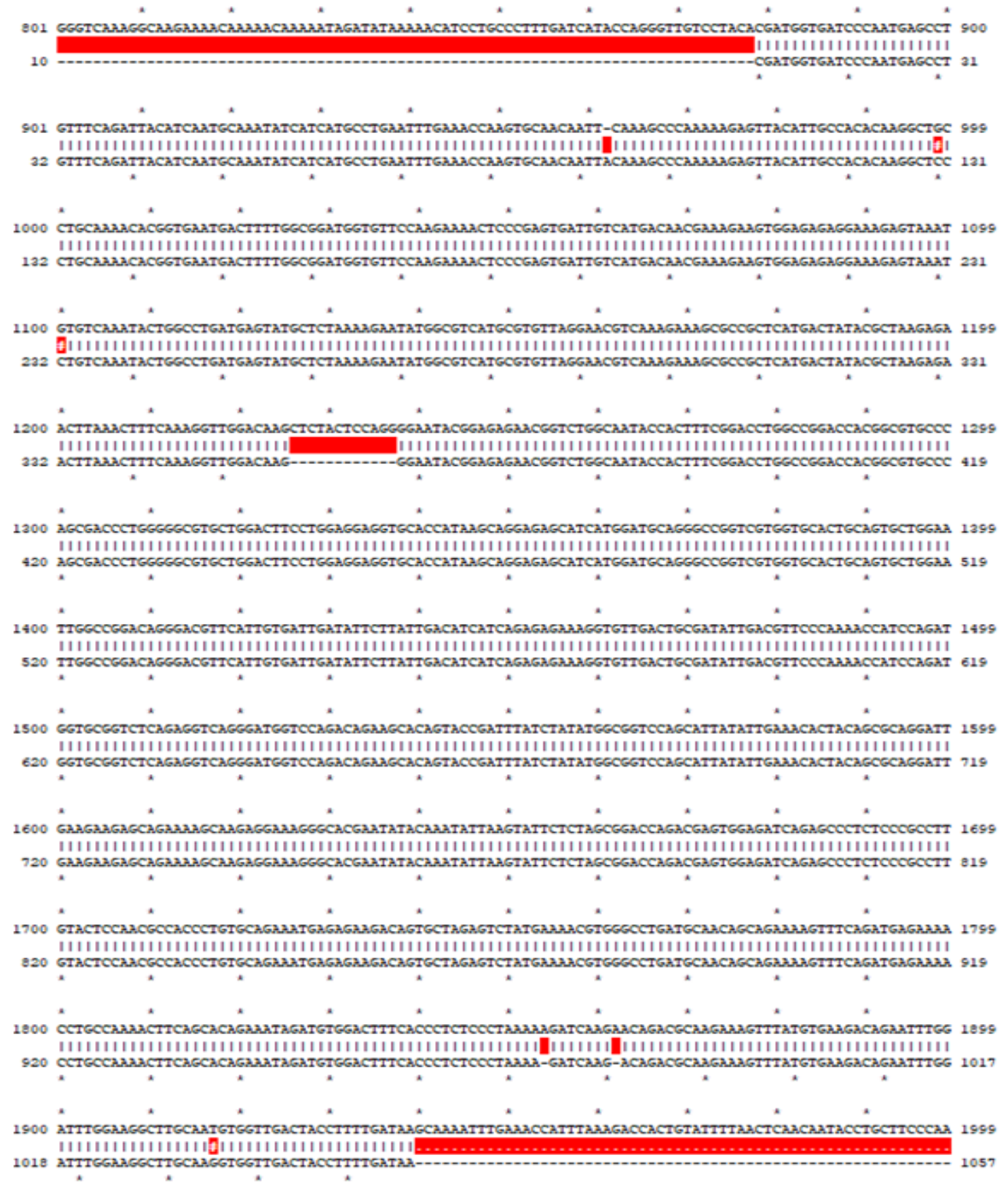

\section{Figure 6.12. Nucleotide sequence alignment of Cys ${ }^{333 / 367}$ Ser SHP2 mutant with WT plasmid.}

Alignment of nucleotide sequences from WT SHP2 plasmid purchased from Addgene (top line) with $\mathrm{Cys}^{333 / 367}$ Ser SHP2 plasmid generated by site-directed mutagenesis. The red highlighted \# at positions 998 and 1100 represents exchanges of guanine for cytosine, showing successful mutation of these cysteines (TGC or TGT) to serines (TCC or TCT). The red sections at the beginning and end of the Cys ${ }^{333 / 367}$ Ser sequence represent areas of primer binding. 


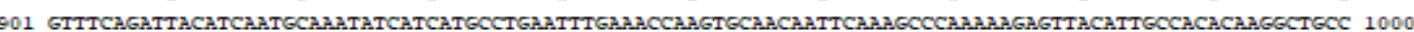

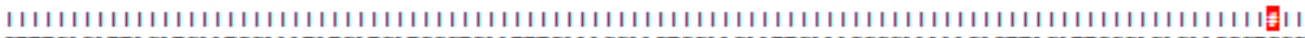

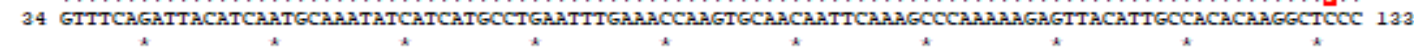

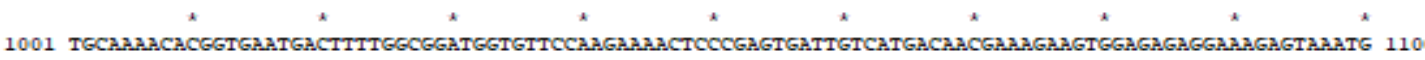
111111111111111111111111111111111111111111111111111111111111111111111111111111111111111111111111111 134 TGCAAAACACGGTGATGACTTTTGGCGGATGGTGTCCAAGAAAACTCCCGAGTGATTGTCATGACAACGAAAGAAGTGGGAGAGGAAGAGTAAATG 233

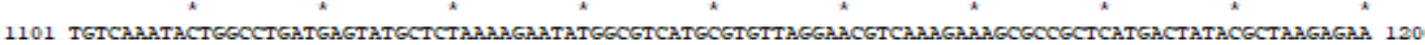
111111111111111111111111111111111111111111111111111111111111111111111111111111111111111111111111111111

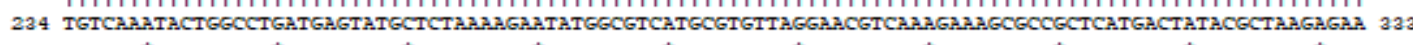

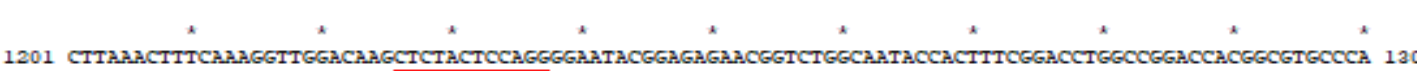
111111111111111111111111

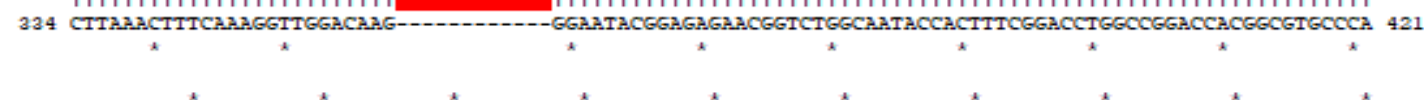

1301 GCGACCCTGGGGGCGTGCTGGACTTCCTGGAGGAGGTGCACCATAAGCAGGAGAGCATCATGGATGCAGGGCCGGTCGTGGTGCACTGCAGTGCTGGAAT 1400

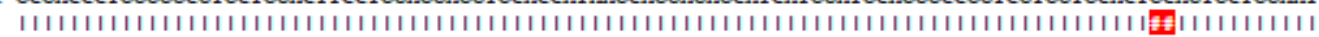
422 GCGACCCTGGGGGCGTGCTGGACTTCCTGGAGGAGGTGCACCATAAGCAGGAGAGCATCATGGATGCAGGGCCGGTCGTGGTGCACTCGAGTGCTGGAAT 521

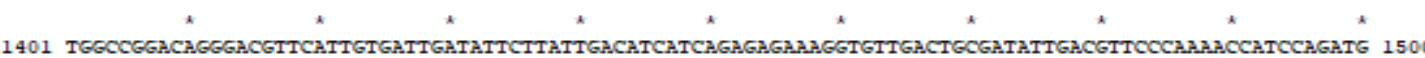
11111111111111111111111111111111111111111111111111111111111111111111111111111111111111111111111111111

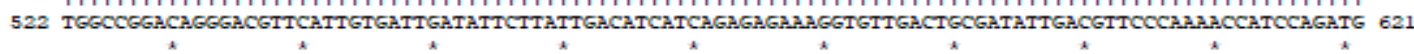
$\star$

150161690

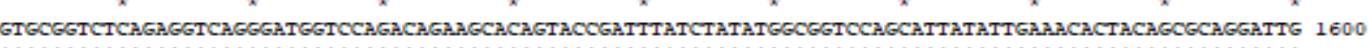
111111111111111111111111111111111111111111111111111111111111111111111111111111111111111111111111111111111111 622 GTGCGGTCTCAGAGGTCAGGGATGGTCCAGACAGAaGCACAGTACCGATTTATCTATATGGCGGTCCAGCATTATATTGAaCACTACAGCGCAGGATT 721

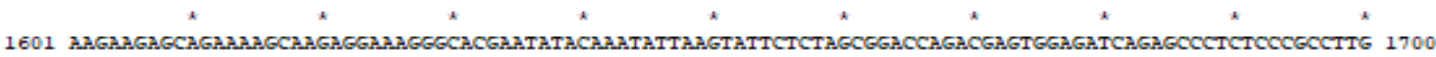
11111111111111111111111111111111111111111111111111111111111111111111111111111111111111111111111111111

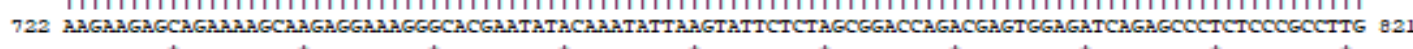

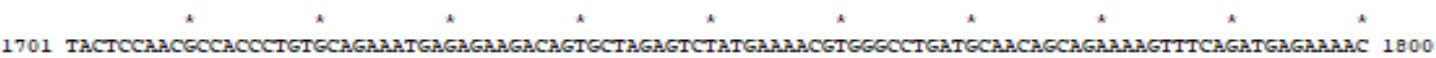
11111111111111111111111111111111111111111111111111111111111111111111111111111111111111111111111111

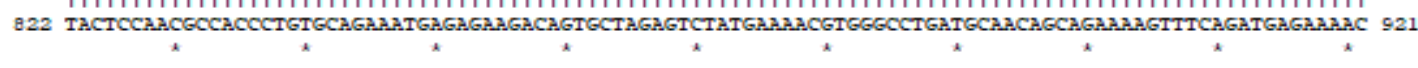

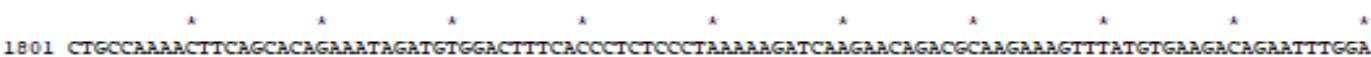
1111111111111111111111111111111111111111111111111111111111111111111111111111111111111111111111111111111111111

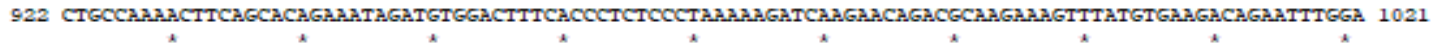
1901 * 111111111111111111111111111111111111111111111 1022 TTTGGäGCTTGCAaTGTGGTTGACTACCTTTTGaTAaGCAaA

\section{Figure 6.13. Nucleotide sequence alignment of Cys ${ }^{333 / 459} \mathrm{Ser}$ SHP2 mutant with WT} plasmid.

Alignment of nucleotide sequences from WT SHP2 plasmid purchased from Addgene (top line) with $\mathrm{Cys}^{333 / 459} \mathrm{Ser}$ SHP2 plasmid generated by site-directed mutagenesis. The red highlighted \# at positions 998 and 1388 \& 1389 represents exchanges of cysteines to serines. The red sections at the beginning and end of the Cys ${ }^{333 / 459}$ Ser sequence represent areas of primer binding. 
6.3.9 SFN adducts proteins in a concentration-dependent manner in HEK293 cells

To establish if SFN could adduct protein targets in a cultured cell line, HEK293 cells were treated with 0-250 $\mu \mathrm{M}$ SFX-01 for 30 minutes. An increase in SFX-01 concentration correlated with an increased abundance of protein-SFN adducts (figure 6.14).

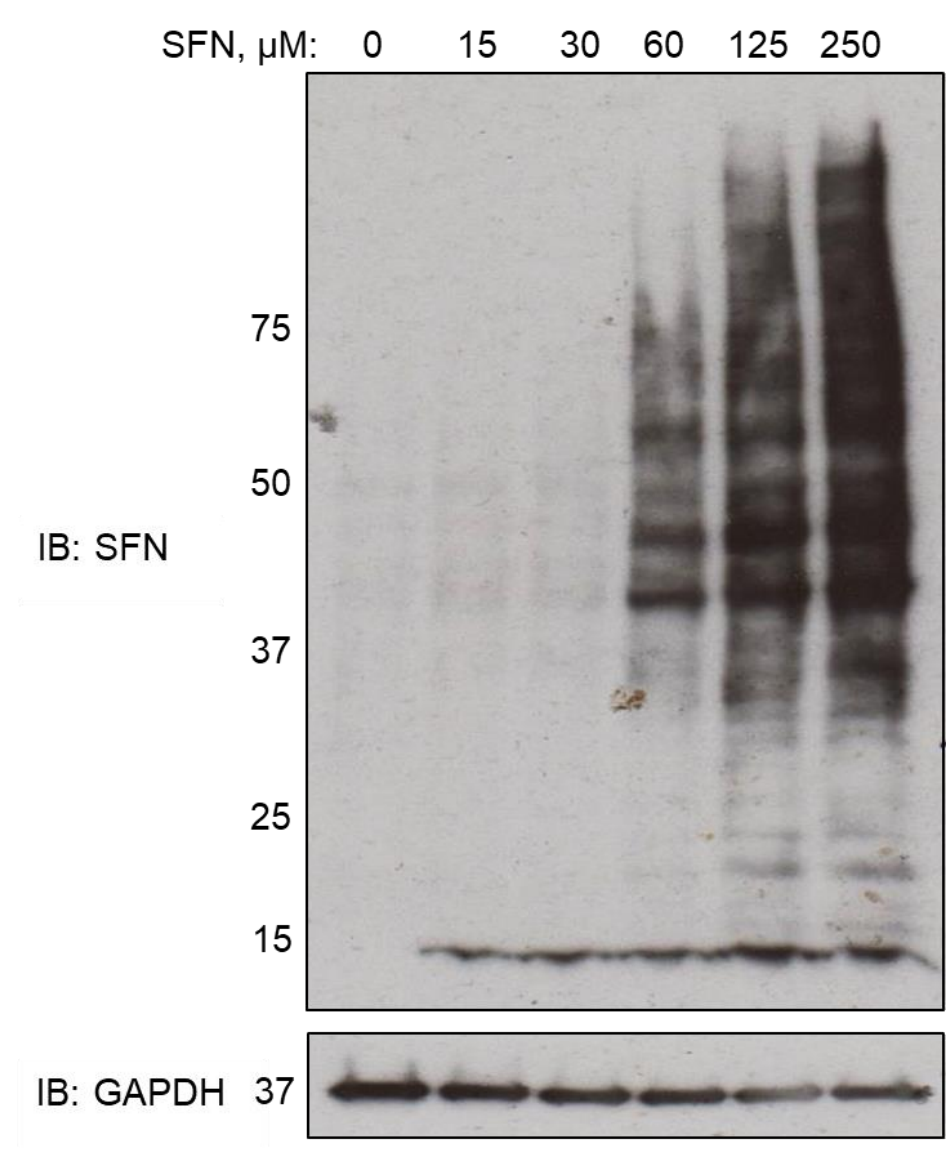

Figure 6.14. SFN adducts proteins in HEK293 cells in a concentration-dependent manner.

A representative immunoblot showing multiple proteins adducted by SFN in a concentration-dependent manner following treatment of HEK293 cells with $0-250 \mu \mathrm{M}$ SFX-01. 


\subsubsection{Cellular transfection with SHP2 plasmid increases protein}

expression in an amount-dependent manner

To determine if cellular transfection with increasing amounts of SHP2 plasmid correlated to an increase in the protein's expression, HEK293 cells were transfected with 0-500 ng WT SHP2 plasmid and protein expression was analysed 24 hours later. Western immunoblotting revealed transfection with increasing amounts of SHP2 plasmid correlated with increased expression of the phosphatase (figure 6.15).

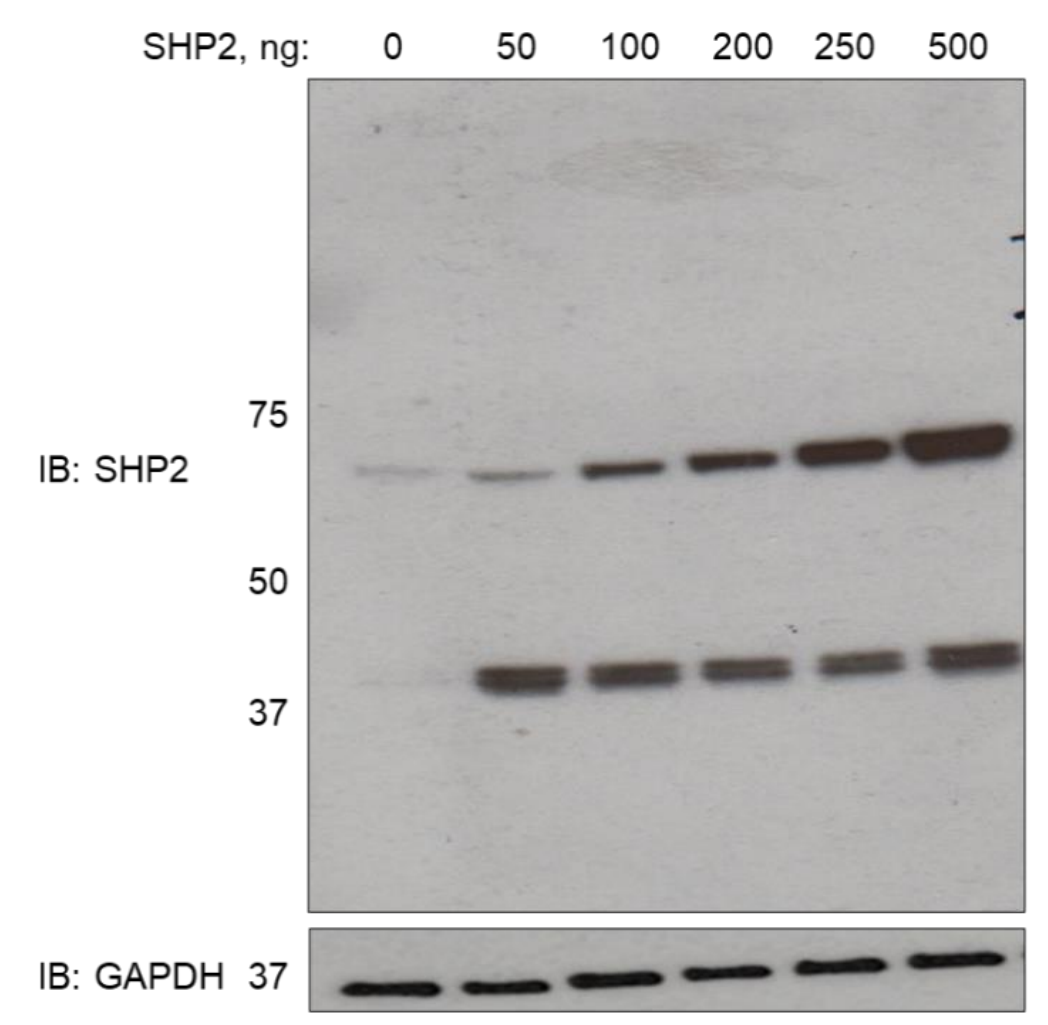

Figure 6.15. Cellular transfection with higher amounts of SHP2 plasmid generates increased protein expression.

A representative immunoblot showing increased expression of SHP2 protein in HEK293 cells 24 hours post-transfection with increasing amounts of WT plasmid. 


\subsubsection{Cys ${ }^{459}$ Ser SHP2 mutation does not affect plasmid transfection efficiency or protein expression}

Western immunoblotting showed SHP2 protein expression was comparable 24 hours following transfection of HEK293 cells with $0-500 \mathrm{ng}$ of plasmid regardless of whether WT or Cys ${ }^{459}$ Ser plasmid was used, suggesting transfection efficiency was unaffected by the mutation (figure 6.16).

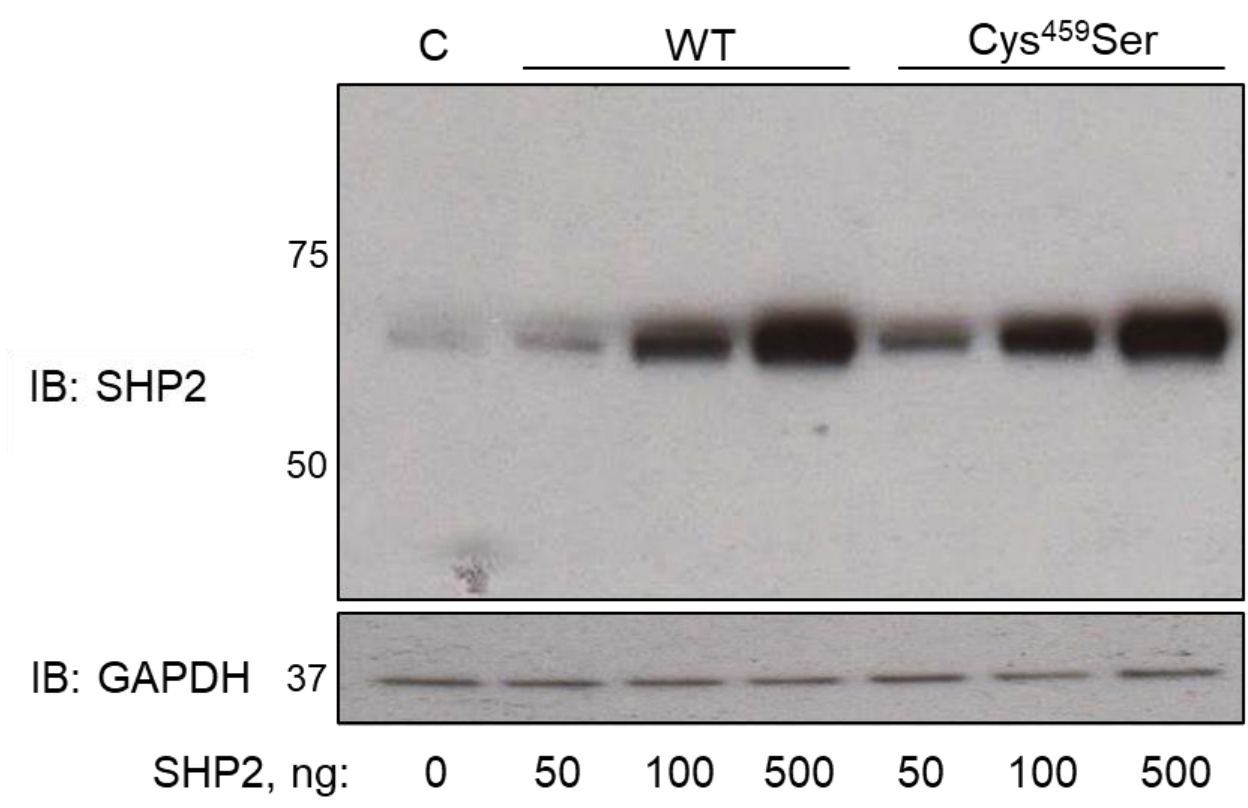

Figure 6.16. SHP2 protein expression is unaffected by a Cys ${ }^{459}$ Ser mutation.

A representative immunoblot showing comparable SHP2 protein expression in HEK293 cells 24 hours after transfection with WT or Cys ${ }^{459}$ Ser SHP2 plasmid.

\subsubsection{The phosphatase activity of SHP2 is lost following mutation of the protein's catalytic cysteine}

I next assessed if site-directed mutagenesis of cysteines within the active site of SHP2 affected the phosphatase's activity. To do so, HEK293 cells were transfected with 500 ng of WT, Cys ${ }^{459}$ Ser, Cys ${ }^{333}$ Ser, Cys ${ }^{367}$ Ser, Cys ${ }^{333 / 367}$ Ser or Cys $^{333 / 459}$ Ser SHP2 plasmid followed by protein immunoprecipitation 24 hours 
later. Western immunoblotting identified comparable protein expression in input samples as well as captured protein between all plasmids (figure 6.17 A). A subsequent fluorescence-based activity assay revealed that mutants which did not contain Cys ${ }^{459}$ Ser, such as Cys $^{333}$ Ser, Cys ${ }^{367}$ Ser or Cys ${ }^{333 / 367}$ Ser, had comparable phosphatase activity to WT SHP2. However, mutation of Cys ${ }^{459}$ to $\mathrm{Cys}^{459} \mathrm{Ser}$ resulted in the loss of the phosphatase's activity (figure $6.17 \mathrm{~B}$ ). 
A)

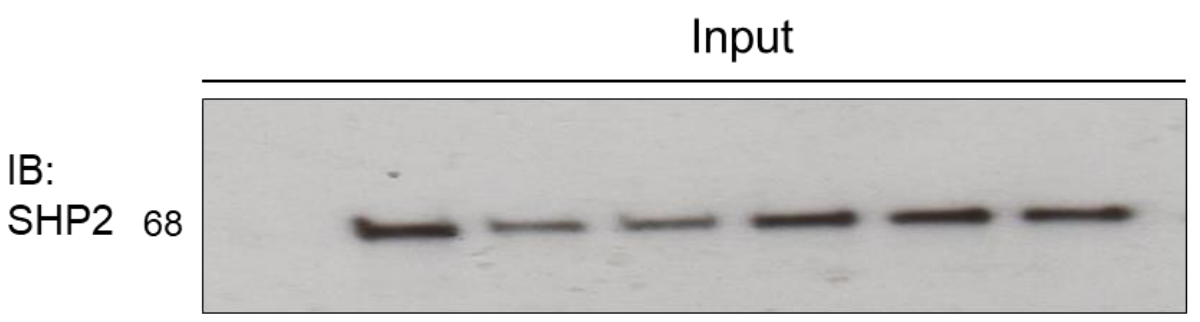

\section{Capture}

IB:

SHP2 68

B)
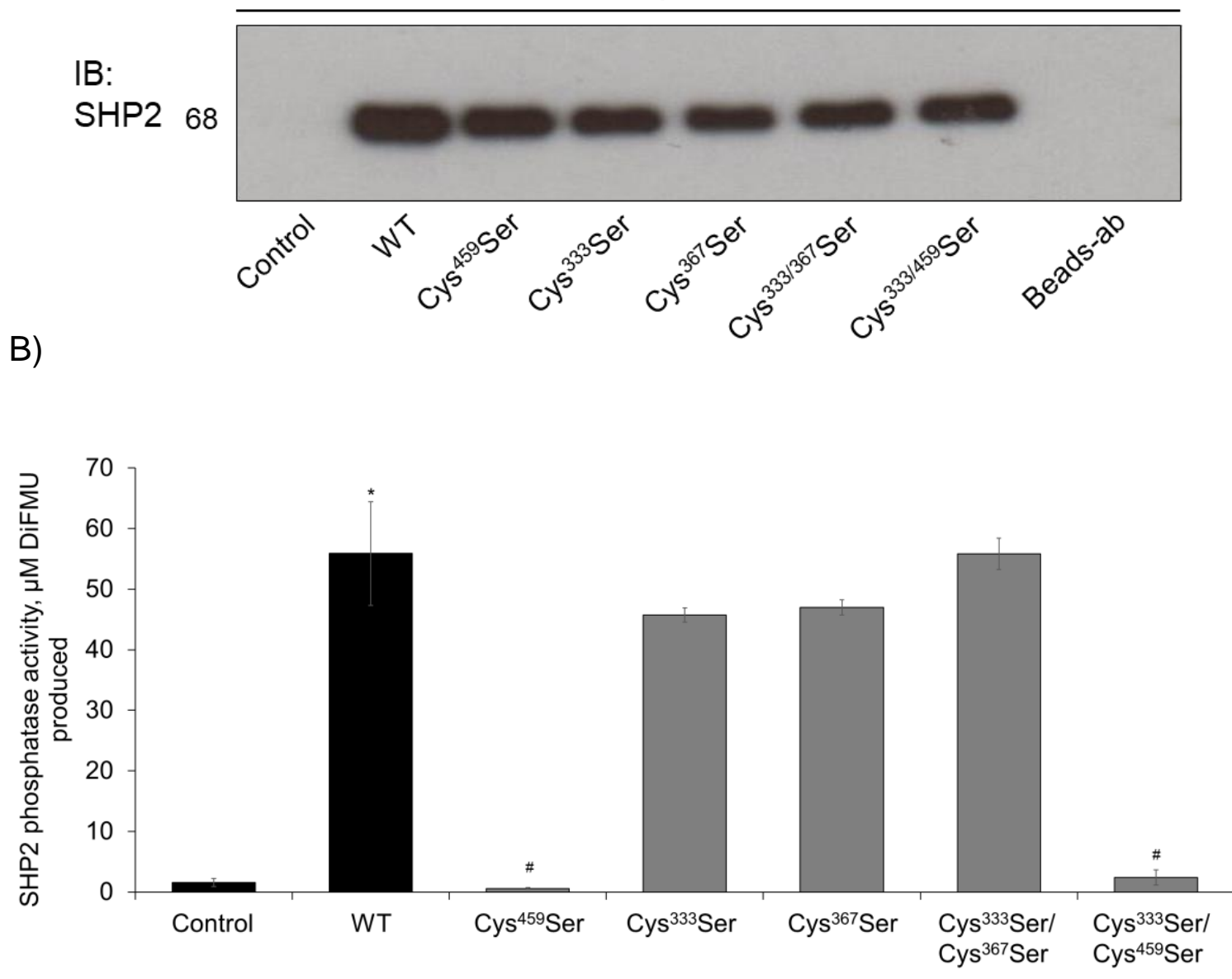

Figure 6.17. Cys ${ }^{459}$ Ser SHP2 protein has no phosphatase activity

A) An immunoblot showing comparable SHP2 protein expression in HEK293 cells 24 hours following transfection with $500 \mathrm{ng}$ of WT, Cys ${ }^{459} \mathrm{Ser}$, Cys ${ }^{33} \mathrm{Ser}$, Cys ${ }^{367} \mathrm{Ser}$, $\mathrm{Cys}^{333 / 367}$ Ser or $\mathrm{Cys}^{333 / 459}$ Ser plasmid. The amount of SHP2 protein immunocaptured from cells was also comparable between all plasmids. B) A fluorescence-based assay showing comparable SHP2 phosphatase activity between WT SHP2 and Cys ${ }^{333}$ Ser, $\mathrm{Cys}^{367} \mathrm{Ser}$ and $\mathrm{Cys}^{333 / 367} \mathrm{Ser}$ mutants. The phosphatase activity of SHP2 was lost following mutation of the catalytic cysteine to a serine. $\left(n=5,{ }^{*} p<0.05\right.$ versus WT control). 


\subsubsection{Mutation of any cysteine pair within the active site of SHP2 results in}

retention of the SFN adduct

I next explored if SFN that adducts to SHP2 in HEK293 cells can be removed from the phosphatase in a similar fashion to that observed in in vitro experiments. I hypothesised that retention of the electrophile upon SHP2 might be achieved if two of the triadic vicinal thiols in the catalytic domain of the phosphatase were mutated to serine. To assess this idea, HEK293 cells were transfected with 500 ng WT, Cys ${ }^{333 / 367}$ Ser or Cys ${ }^{333 / 459}$ Ser SHP2 plasmid and 24 hours later cells were treated with $10 \mu \mathrm{M}$ SFX-01. Immunocapture followed by western immunoblotting revealed SFN adduction to WT, Cys ${ }^{333 / 367}$ Ser or Cys ${ }^{333 / 459}$ Ser SHP2 protein 2 hours post-treatment with SFX-01 (figure 6.18). Whilst an SFN adduct was not detected 4 hours post-treatment on WT SHP2, adduction of the electrophile to SHP2 was observed at this time point on Cys ${ }^{333 / 367}$ Ser or Cys ${ }^{333 / 459}$ Ser protein. Immunoblotting also revealed comparable SHP2 protein expression 28 hours following transfection with each of the plasmids (figure 6.18). 
Hours after

SFX-01 added:

$10 \mu \mathrm{m}$ SFN

Capture: 75

SHP2

IB: SFN

50

Capture: 75

SHP2

IB: SHP2

50

$\begin{array}{llllll}2 & 4 & 2 & 4 & 2 & 4\end{array}$

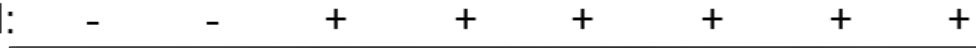
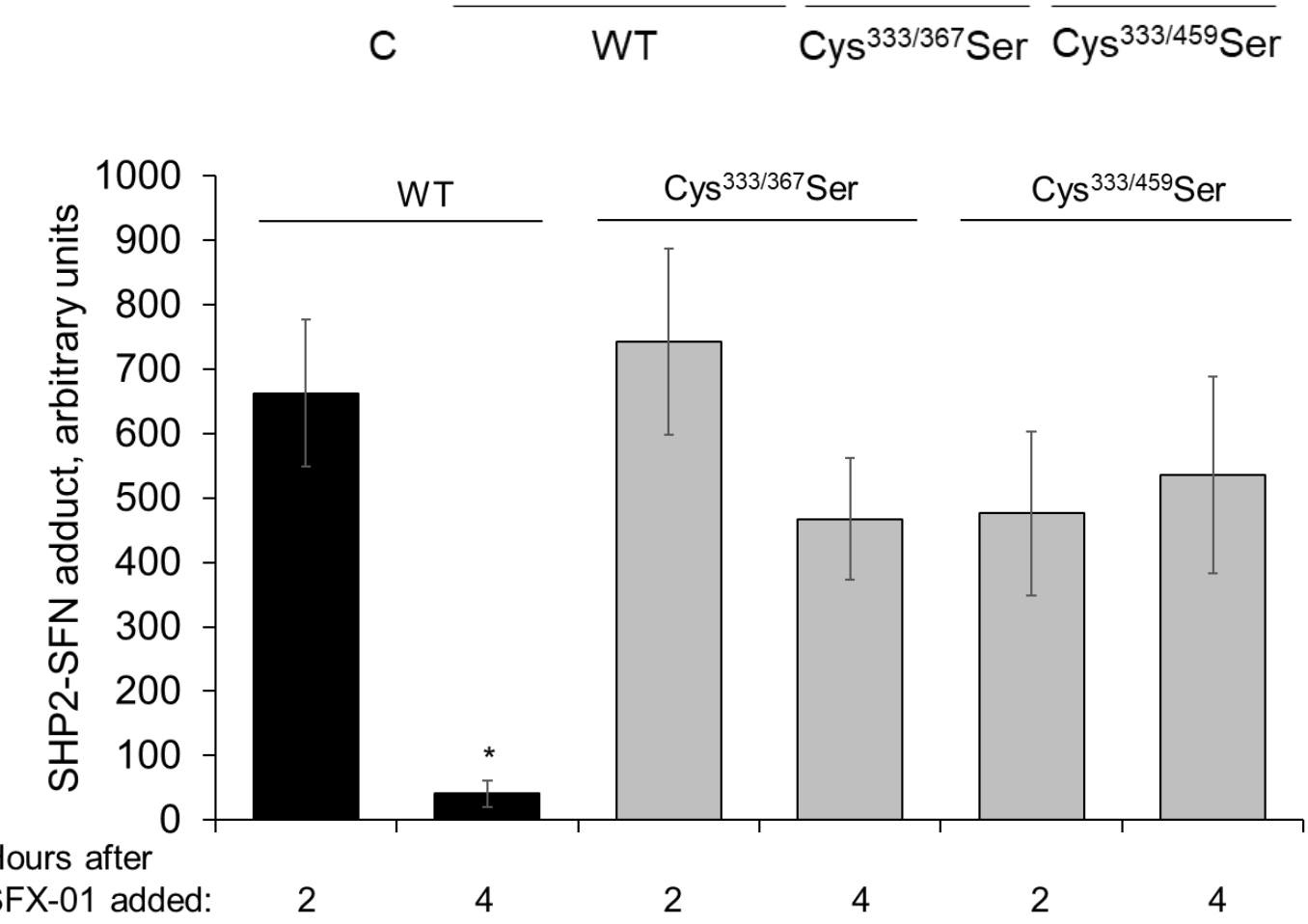

Figure 6.18. SFN adduct upon SHP2 is stabilised following mutation of two active site cysteines.

An immunoblot showing SFN adducted to WT, Cys ${ }^{333 / 367}$ Ser or Cys ${ }^{333 / 459}$ Ser SHP2 immunoprecipitated from HEK293 cells 2 hours post-treatment with $10 \mu \mathrm{M}$ SFX-01. The SFN adduct was not detected on WT SHP2 immunoprecipitated from HEK293 cells 4 hours post-treatment with SFX-01 but was retained at this time point on Cys ${ }^{333 / 367}$ Ser or Cys ${ }^{333 / 459}$ Ser SHP2. ( $n=3,{ }^{*} p<0.05$ versus WT plasmid following 2-hour treatment with SFX-01). 


\subsubsection{4 dTNB is formed following incubation of TNB with SFX-01}

HPLC analysis following incubation of $50 \mu \mathrm{M}$ TNB with $100 \mu \mathrm{M} \mathrm{H}_{2} \mathrm{O}_{2}$ identified a chromatographic peak at 11.27 minutes (figure $6.20 \mathrm{~A}$ ), which was highly likely dTNB as this retention time was very similar to the retention time of an authentic standard, which was 11.21 minutes (figure 6.19 A). This showed oxidative modification of TNB by $\mathrm{H}_{2} \mathrm{O}_{2}$ could induce a disulfide between two TNB molecules. Further HPLC analysis identified a peak on the chromatogram at 11.24 minutes following treatment of $50 \mu \mathrm{M}$ TNB with $5 \mu \mathrm{M}$ SFX-01 for 1-hour (figure $6.20 \mathrm{~B}$ ). Again, this product was highly likely dTNB, as this retention time was very similar to the retention time of an authentic standard. The area under the hypothesised dTNB peak following incubation of SFX-01 with TNB increased following 24-hour incubation, whilst a peak at $\sim 1.08$ minutes, corresponding to TNB decreased (figure $6.20 \mathrm{C}$ ). A peak corresponding to SFN alone was also present on chromatograms from both the 1-hour and 24-hour incubation time points, as well as one at 10.87 minutes, which may represent TNB-SFN, although this could not be confirmed due to the lack of an authentic standard (figure 6.20 B, C). 
A)

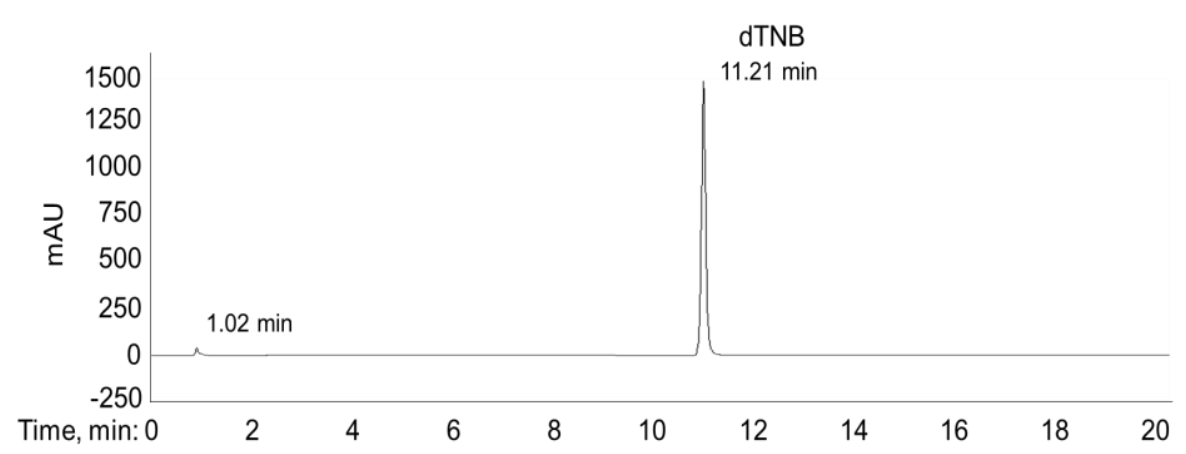

B)

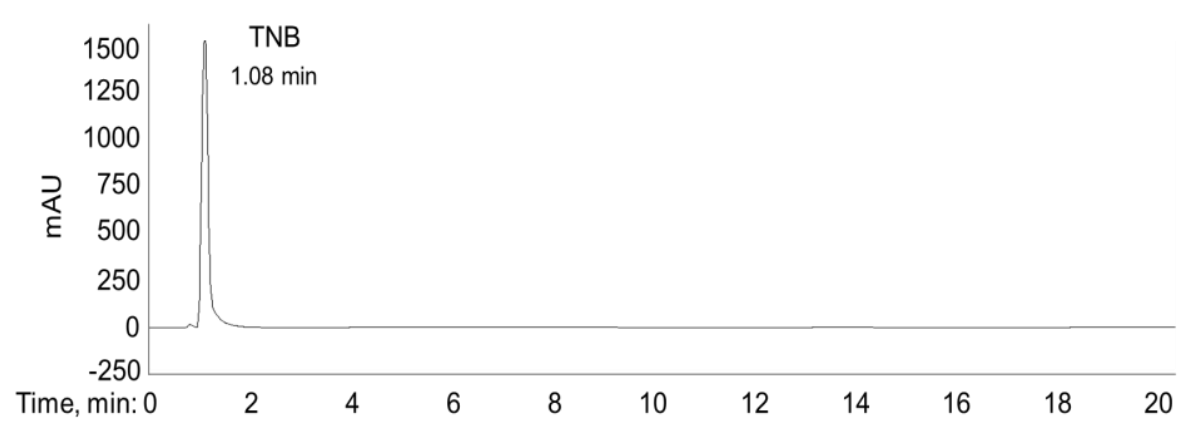

C)

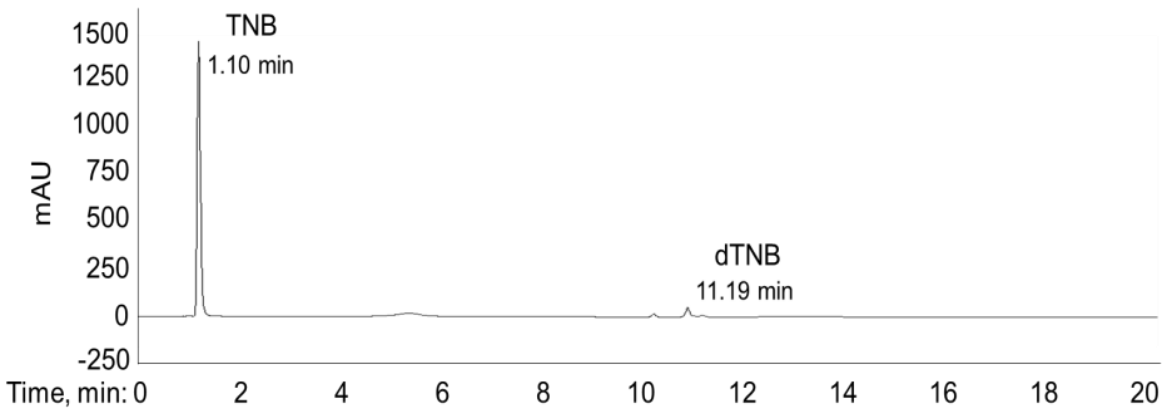

D)

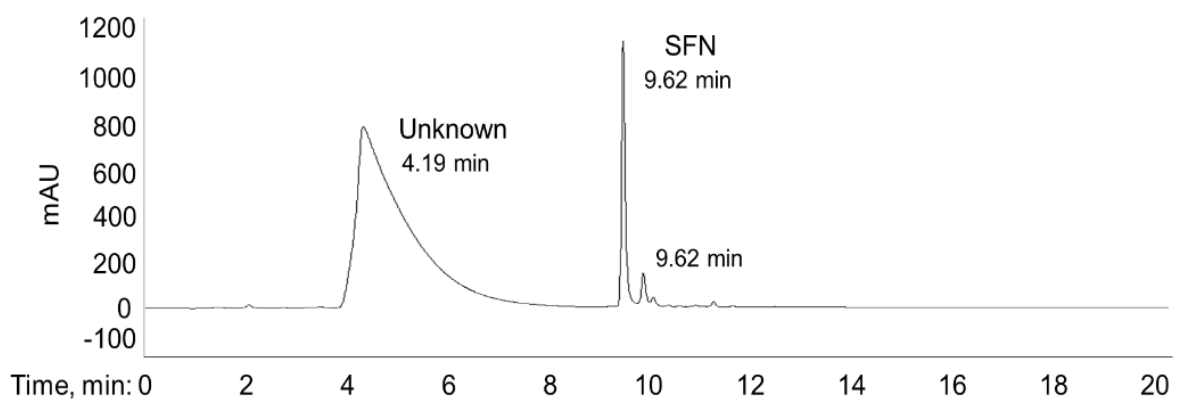

Figure 6.19. HPLC chromatograms of standards used for analysis.

A) A dTNB standard. B) A TNB standard. C) A TNB standard left at room temperature for 24 hours. D) An SFX-01 standard. mAU represents absorbance using a UV detector set at a wavelength of $320 \mathrm{~nm}$. 
A)

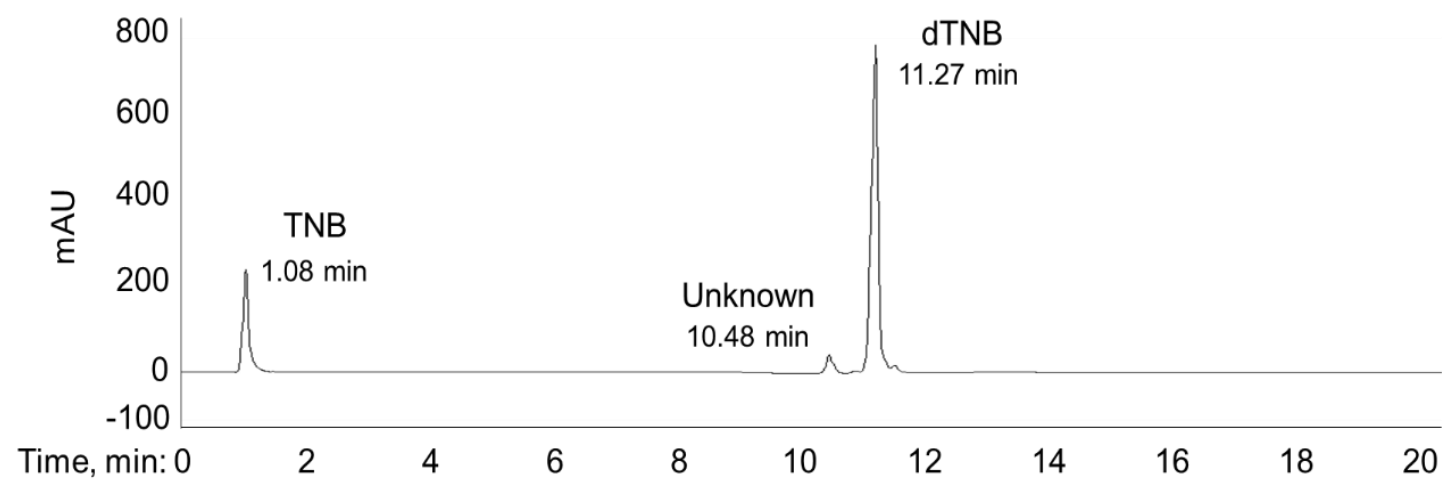

B)

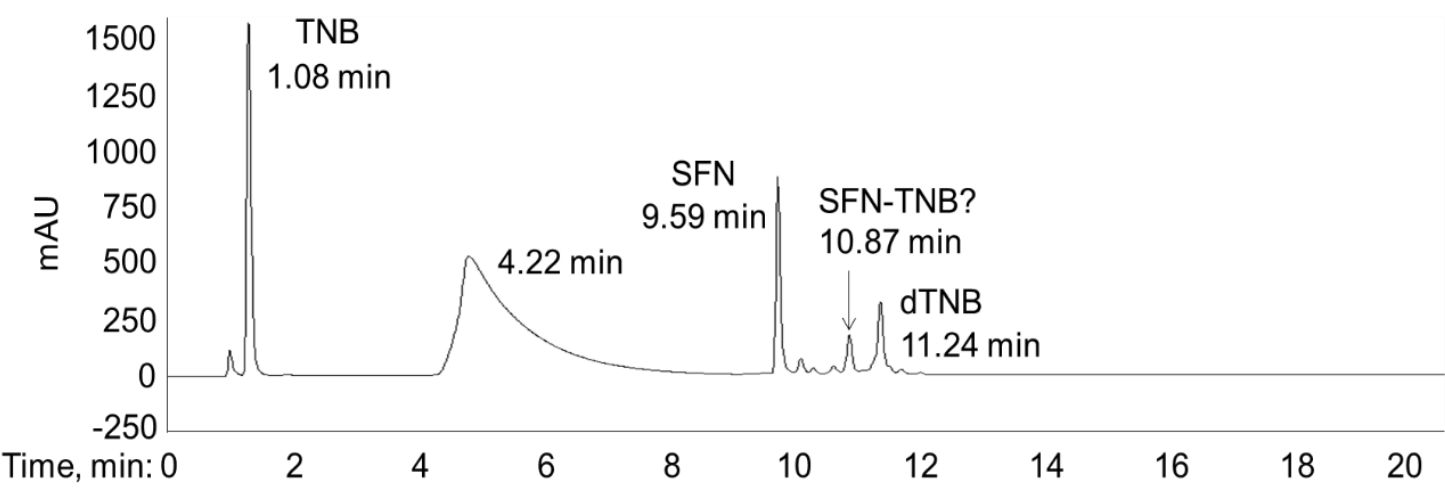

C)

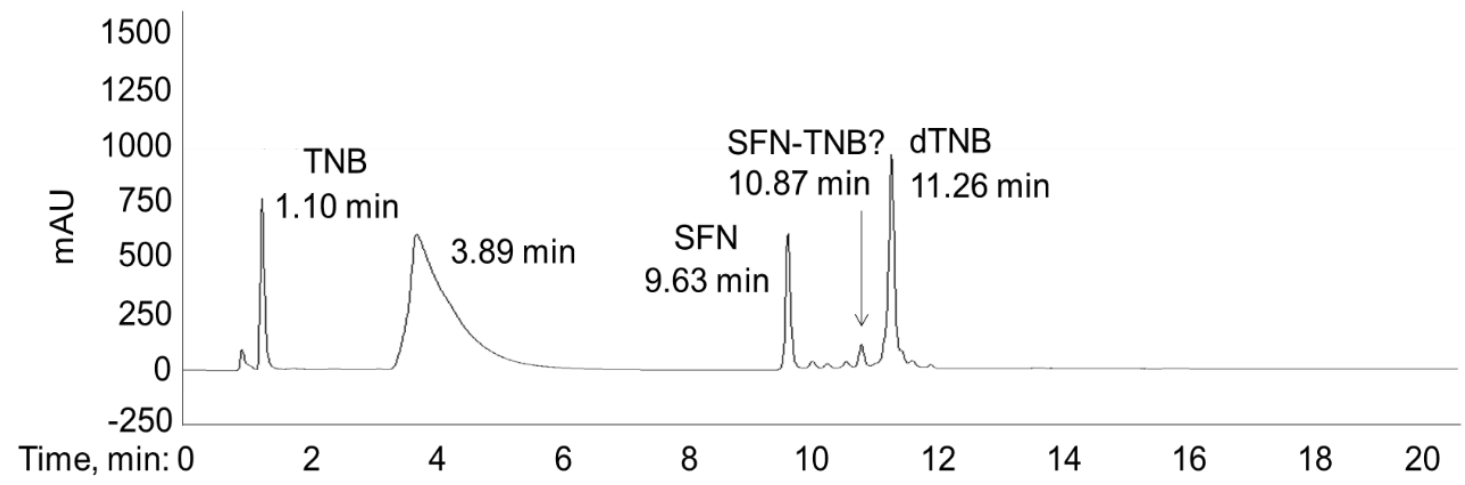

Figure 6.20. HPLC chromatograms showing SFN incubation with TNB inducing dTNB formation.

A) $50 \mu \mathrm{M} \mathrm{TNB}$ incubated with $100 \mu \mathrm{M} \mathrm{H}_{2} \mathrm{O}_{2}$ for 1-hour. B, C) $50 \mu \mathrm{M} \mathrm{TNB}$ incubated with $5 \mu \mathrm{M}$ SFX-01 for 1 - or 24-hours respectively. mAU represents absorbance using a UV detector set at a wavelength of $320 \mathrm{~nm}$. 


\subsubsection{The identification of a dithiolethione was not achieved by mass spectrometry following incubation of GSH with SFN}

To identify if SFN could induce dithiolethione formation between two GSH molecules, $10 \mu \mathrm{M}$ L-SFN was incubated with $10 \mu \mathrm{M}$ GSH for 8 hours or $100 \mu \mathrm{M}$ GSH for $0.5,3,18$ or 24 hours and the resultant reaction products were analysed by LC-MS/MS. Chromatograms from LC of experiments containing $100 \mu \mathrm{M}$ GSH are shown here which identified 4 major products within all reaction mixtures with retention times of $5.94,1.46,1.35$ and 2.86 minutes (figure 6.21). LC chromatograms were the same for experiments using $10 \mu \mathrm{M} \mathrm{GSH}$. Following injection into the linear ion trap mass spectrometer, subsequent $\mathrm{MS}^{2}$ analysis performed on the first three peaks identified base peaks of $114.09,179.03+$ 308.15 and $484.18 \mathrm{~m} / \mathrm{z}$ which correspond to SFN, GSH and GSSH respectively as calculated from standards (figure 6.22 A-C). $\mathrm{MS}^{3}$ was performed on the final peak identifying a base peak of $136.03 \mathrm{~m} / \mathrm{z}$ (figure $6.22 \mathrm{D}$ ). Analysis of the base peaks formed following $\mathrm{MS}^{2}$ and $\mathrm{MS}^{3}$ of this latter peak strongly suggest this reaction product was GSH-SFN (figure 6.23-6.25). Analysis of atomic absorbance (AA) of LC peaks identified a time-dependent increase in the amount of GSH-SFN or GSSG formed (figure 6.26 and 6.27 A). A time-dependent increase in AA of GSSG was also seen following incubation of $10 \mu \mathrm{M} \mathrm{GSH}$ alone at room temperature for 8 hours (figure 6.27 B). 
A)

RT: 1.60

AA: 4084664069

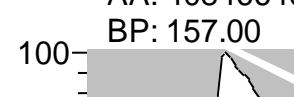

B)

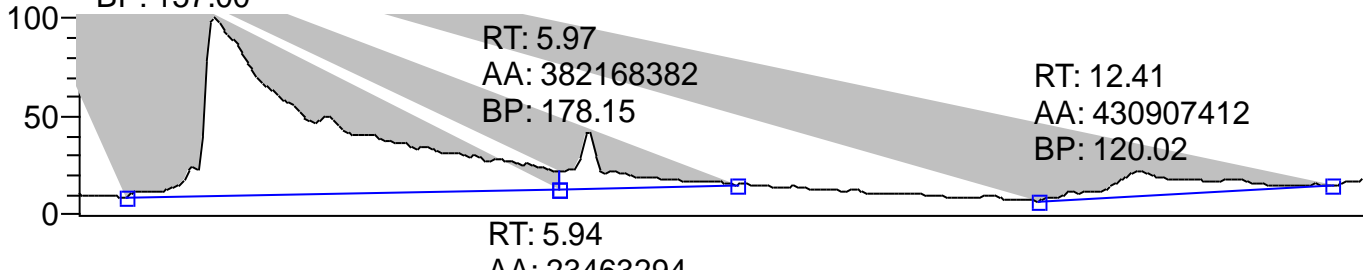

)

AA: 23463294

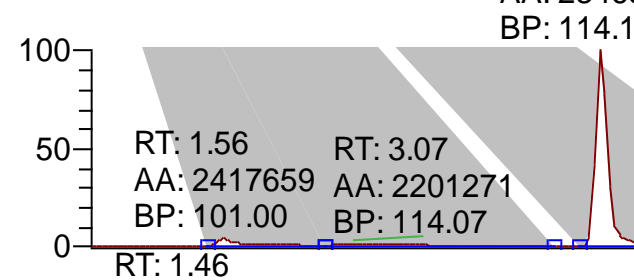

14.10

C)

AA: 32265427

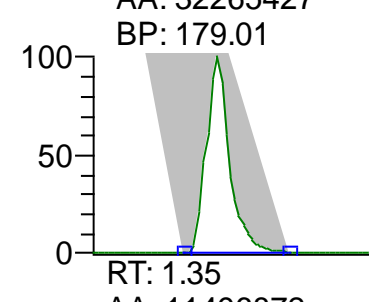

D) $\quad \mathrm{AA}: 11496872$

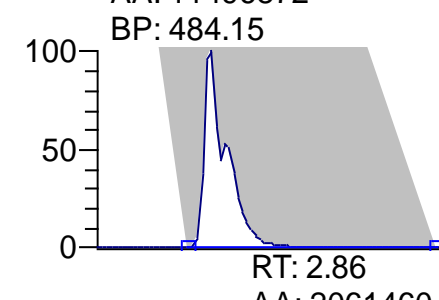

E)

AA: 2061460

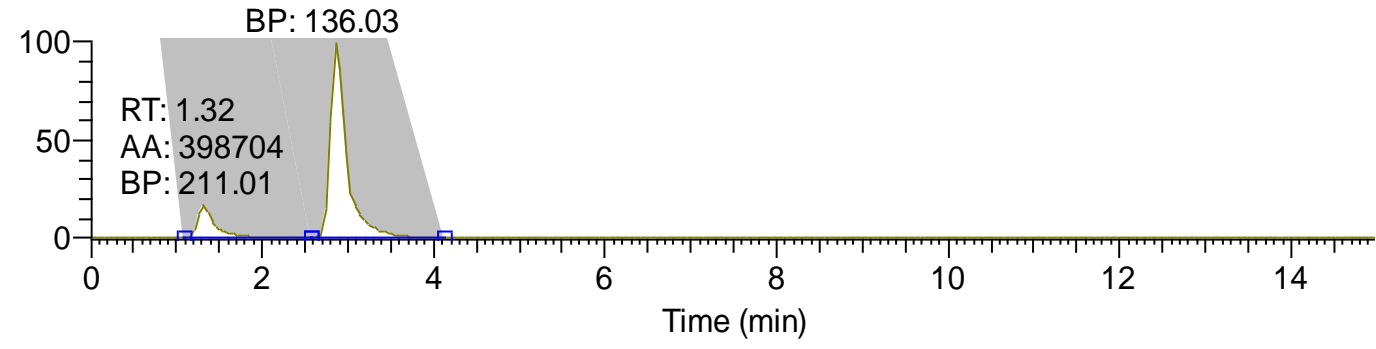

Figure 6.21. Representative chromatograms from LC performed prior to linear ion trap MS.

A) $10 \mu \mathrm{M}$ L-SFN incubated with $100 \mu \mathrm{M}$ GSH for 24 hours. B-E) Optimisation of chromatogram A showing peaks for different reaction products. B) A product with a retention time (RT) of 5.94 minutes. C) A product with a RT of 1.46 minutes. D) A product with a RT of 1.35 minutes. E) A product with a RT of 2.86 minutes. $A A=$ atomic absorbance which represents amount of each product in the reaction. $B P=$ corresponding base peak when analysed by MS which represents the most intense peak in the mass spectra for that product. 


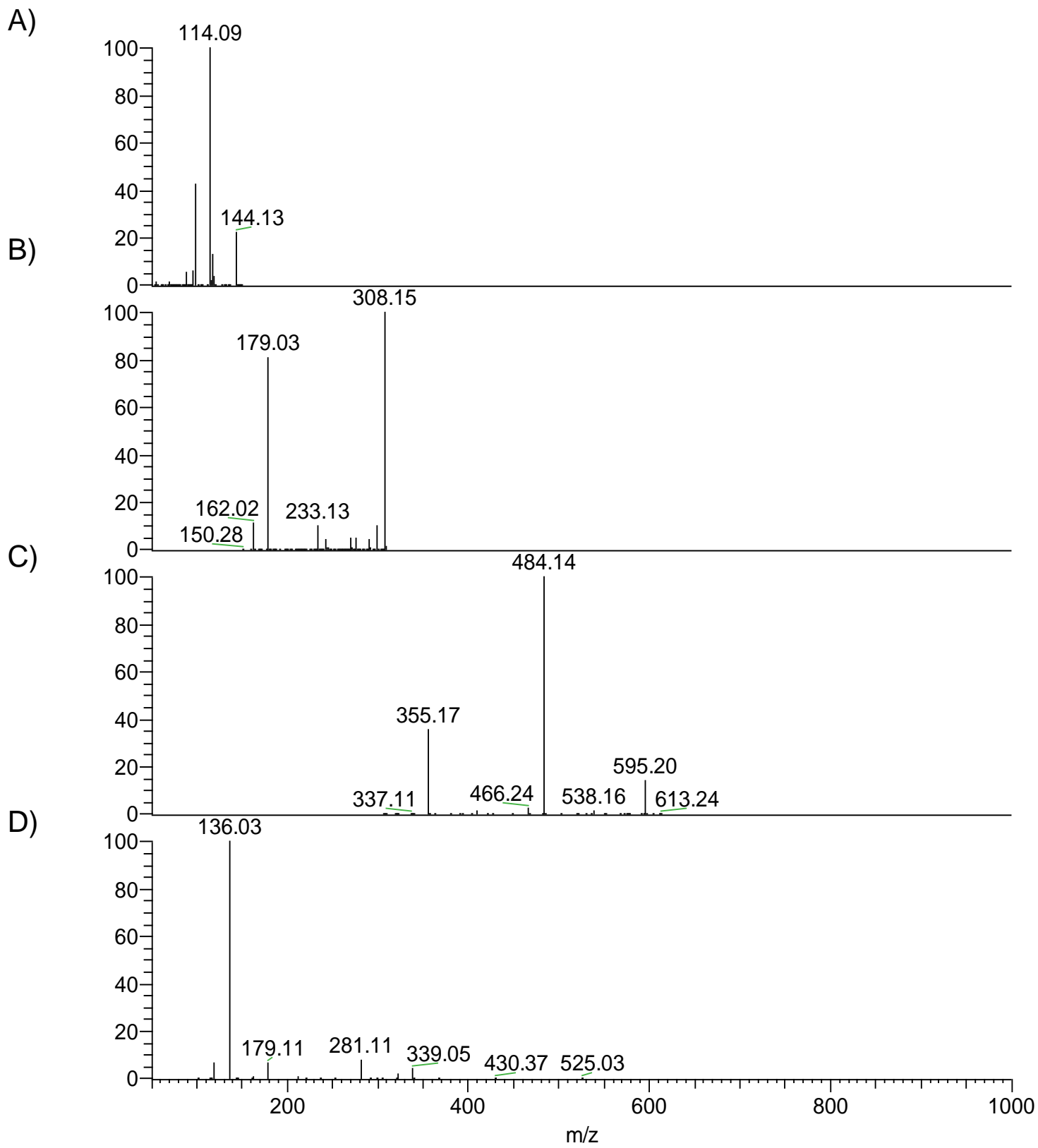

Figure 6.22. Representative mass spectrums from linear ion trap tandem MS performed following the incubation of GSH with L-SFN.

A-D) Mass spectrums from linear ion trap MS performed after LC following 24-hour incubation of $10 \mu \mathrm{m} \mathrm{L-SFN}$ with $100 \mu \mathrm{m} \mathrm{GSH}$. A) $\mathrm{MS}^{2}$ performed at an $\mathrm{m} / z$ range of 50 150 identifying a base peak of $114.09 \mathrm{~m} / z$ which corresponds to SFN. B) MS ${ }^{2}$ performed at an $\mathrm{m} / \mathrm{z}$ range of $150-310$ identifying base peaks of $179.03 \mathrm{~m} / \mathrm{z}$ and $308.15 \mathrm{~m} / \mathrm{z}$ which correspond to GSH. C) $\mathrm{MS}^{2}$ performed at an $\mathrm{m} / \mathrm{z}$ range of 300-650 identifying a base peak of $484.14 \mathrm{~m} / z$ which corresponds to GSSG. D) $M^{3}$ performed at an $\mathrm{m} / z$ range of 95-1000 identifying a base peak of $136.03 \mathrm{~m} / \mathrm{z}$ which corresponds to GSH-SFN. 


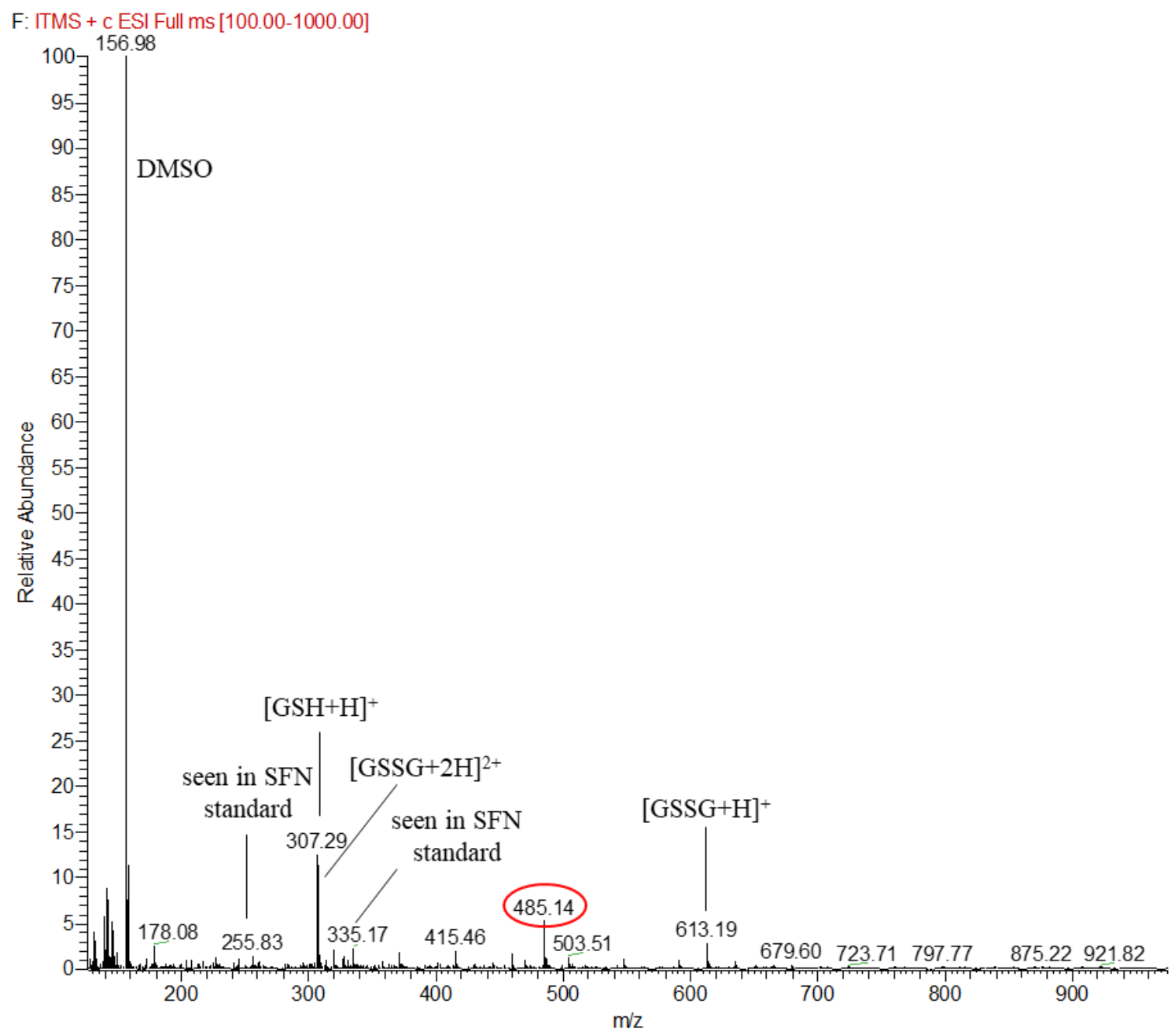

Figure 6.23. Full MS performed following the incubation of GSH with L-SFN.

A representative MS mass spectrum performed after LC following 24-hour incubation of 10 $\mu \mathrm{m}$ L-SFN with $100 \mu \mathrm{m} \mathrm{GSH}$ at an $\mathrm{m} / z$ range of 100-1000. Peaks were identified which corresponded to SFN, GSH and GSSG as well as a new peak, circled in red, formed at an $m / z$ of 485.14 . 

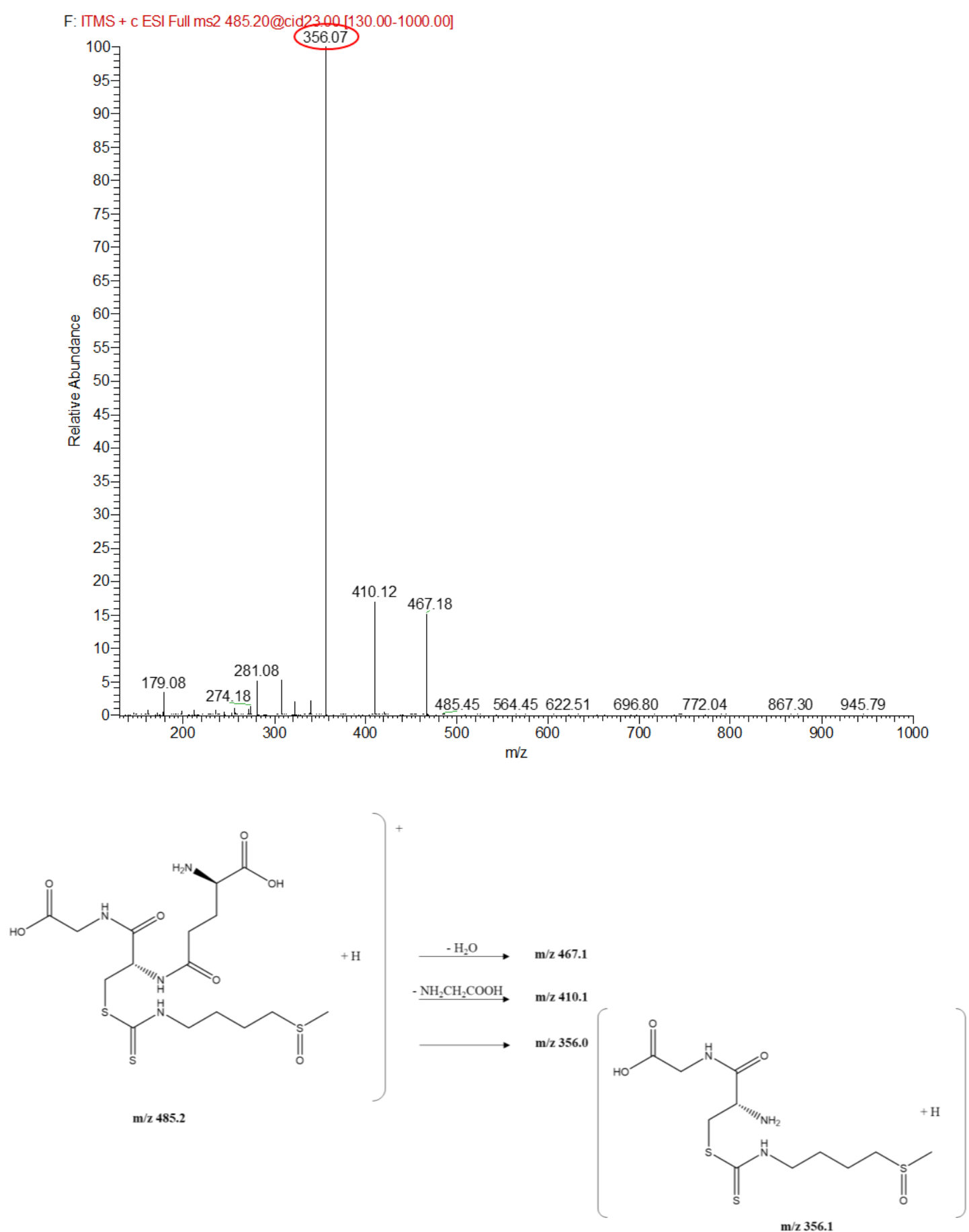

Figure 6.24. $\mathrm{MS}^{2}$ performed following the incubation of GSH with L-SFN.

A representative $\mathrm{MS}^{2}$ mass spectrum performed at an $\mathrm{m} / \mathrm{z}$ range of $130-1000$ following fragmentation of an unidentified MS mass spectrum peak formed at an $m / z$ of 485.14 after LC following 24-hour incubation of $10 \mu \mathrm{m} \mathrm{L-SFN} \mathrm{with} 100 \mu \mathrm{m} \mathrm{GSH}$. A new base peak was formed at an $\mathrm{m} / \mathrm{z}$ of 356.07 , likely SFN adducted to fragmented GSH. Two smaller peaks formed at an $\mathrm{m} / z$ of 410.12 and 467.1 , correspond to $\mathrm{NH}_{2} \mathrm{CH}_{2} \mathrm{COOH}$ and $\mathrm{H}_{2} \mathrm{O}$ respectively. 

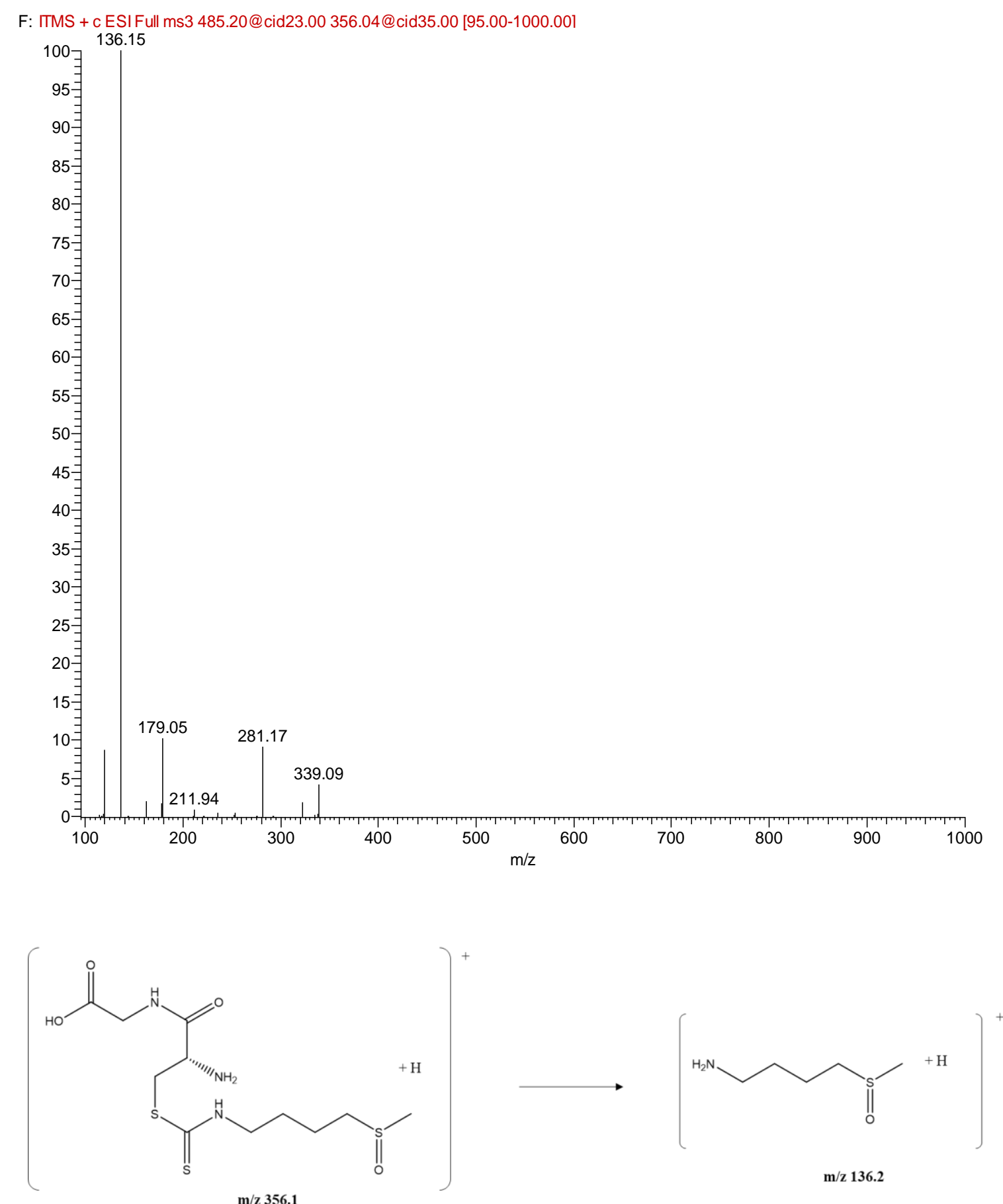

Figure 6.25. $\mathrm{MS}^{3}$ performed following the incubation of GSH with L-SFN.

A representative $\mathrm{MS}^{3}$ mass spectrum performed at an $\mathrm{m} / \mathrm{z}$ range of $95-1000$ following fragmentation of a base peak formed at an $\mathrm{m} / \mathrm{z}$ of 356.07 following $\mathrm{MS}^{2}$ after LC following 24-hour incubation of $10 \mu \mathrm{m} \mathrm{L-SFN}$ with $10 \mu \mathrm{m} \mathrm{GSH}$. A new base peak was formed at an $\mathrm{m} / \mathrm{z}$ of 136.2 , likely truncated SFN whereby the ITC group has been truncated to an amine. 


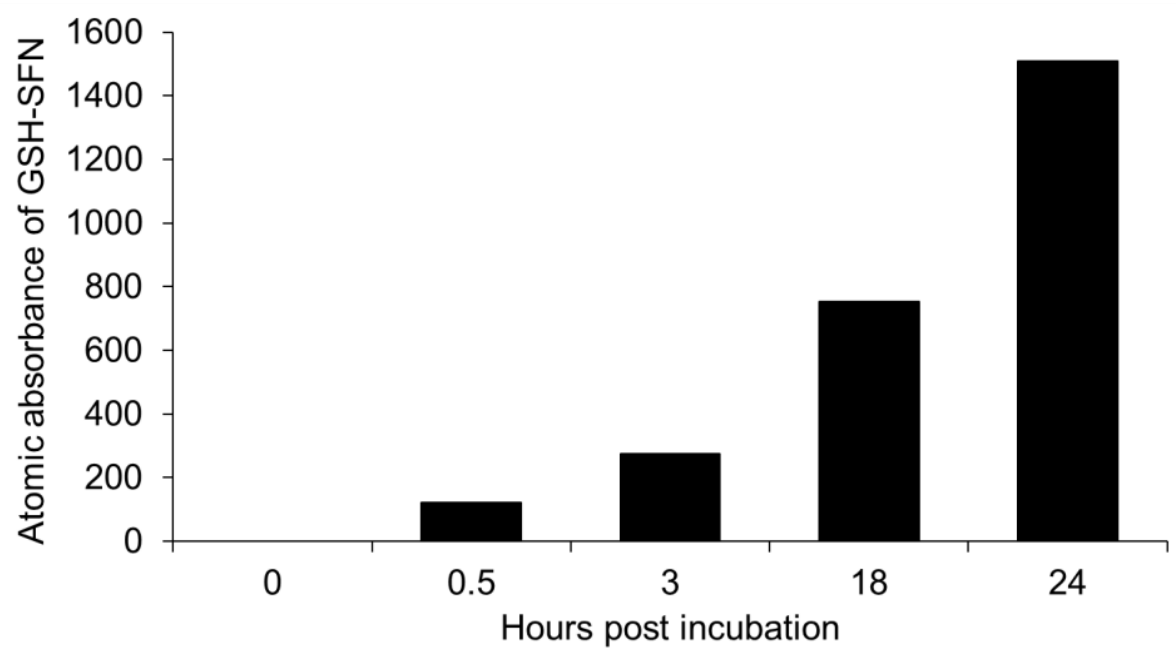

Figure 6.26. The amount GSH-SFN formed following incubation of GSH with L-SFN increases in a time-dependent manner.

AA of GSH-SFN calculated from LC performed following 24-hour incubation of $10 \mu \mathrm{m}$ SFN with $100 \mu \mathrm{m} \mathrm{GSH}$ identified a time-dependent increase in abundance of GSH-SFN.

A)

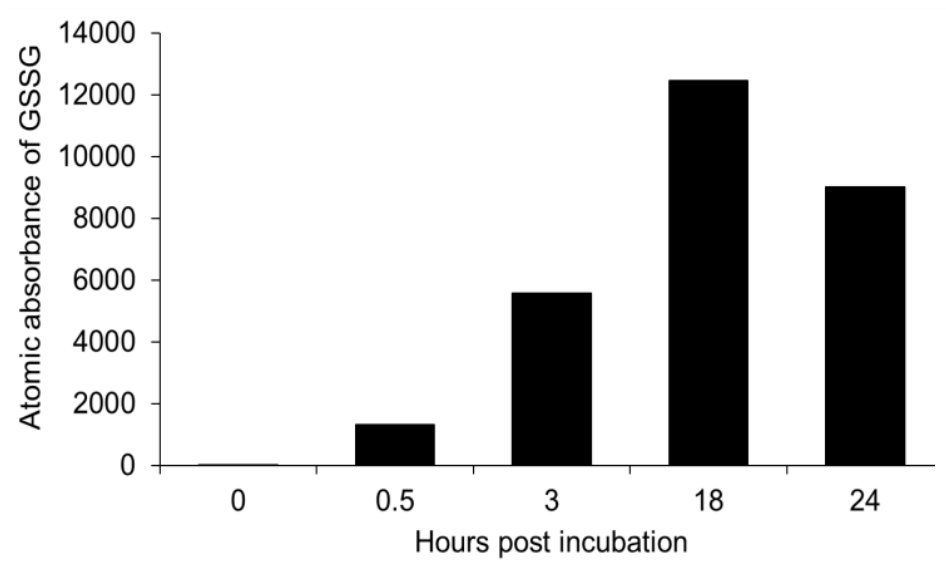

B)

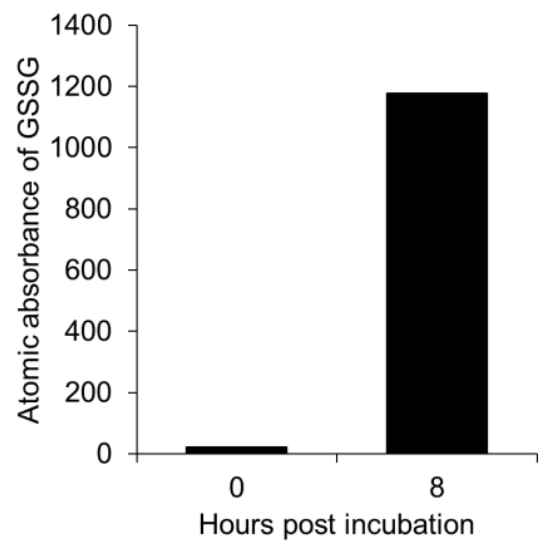

Figure 6.27. The amount of GSSG formed following the incubation of GSH at room temperature increases in a time-dependent manner.

A) AA of GSSG calculated from LC performed following 24-hour incubation of $10 \mu \mathrm{m}$ SFN with $100 \mu \mathrm{m}$ GSH identified a time-dependent increase in abundance of GSSG. B) AA of GSSG calculated from LC performed following 8-hour incubation of $10 \mu \mathrm{m} \mathrm{SFN}$ with $10 \mu \mathrm{m} \mathrm{GSH}$ identified a time-dependent increase in abundance of GSSG. 


\subsection{Discussion}

SFN can react with nucleophilic biomolecules and covalently adduct to them. Typically, covalent interactions are stable and permanent or only slowly reversed.

Chapters 3 and 5 explained and characterised in detail the inhibition of recombinant or cardiac SHP2 phosphatase activity by SFN. Although unexpected, the in vitro studies showed that SFN initially forms an adduct with the recombinant SHP2, but over time the modification was lost. However, despite this loss of the electrophilic adduct, the phosphatase itself remained inhibited. Additional biochemical analyses outlined in this chapter showed that SFX-01 treatment in vivo also induced inhibition of SHP2 phosphatase activity without a detectable SFN adduct. In this chapter, I sought to investigate the molecular mechanism of inhibition of SHP2 by SFN which occurs despite the absence of a stable covalent adduct that is initially observed when the phosphatase encounters this electrophile.

As described in the general introduction, quantitative MS analysis of proteins immunocaptured with a pan-specific anti-SFN-protein antibody identified cardiac SHP2 as a significant target of the electrophile in vivo. For SHP2 to be immunocaptured, prior to its subsequent identification by MS, this required the presence of an SFN adduct to which the capture antibody binds. These initial studies involved an acute, large bolus dose of SFN being administered orally to mice, before isolation of the heart for analysis 3 hours later. The large dose and the relatively short exposure time would account for the presence of an SFNadduct, as it is likely a time point which captures the initial adduction of the electrophile. In the follow-up studies outlined above, this adduct was shown to be prone to removal or alteration such that is cannot be detected on SHP2 by the 
anti-SFN antibody, depending on the conditions assessed. For example, the in vivo studies outlined in this chapter as well as those in chapter 3 were conducted using chronic 4-10-day exposure of mice to SFX-01 in their drinking water, which resulted in SHP2 inhibition, but no SFN adduct was detected. Considering the reversal of the adduct observed in the in vitro studies and the fact that the immunocapture-MS studies identified SHP2 as a target of the electrophile, it is rational to conclude SFN initially adducts, with the electrophile then somehow altered such that it cannot be detected but maintains the inhibition of the phosphatase.

In line with the observations and considerations made in chapter 4 , perhaps the loss of the adduct was achieved via trans-thiolation of the electrophile from SHP2 to another cellular protein. Although this is considered unlikely, as typically this mechanism would result in phosphatase activity being regained as the catalytic thiolate would be reformed, whereas SHP2 remains inhibited following the loss of detectable SFN.

Several studies have shown RTK stimulation is associated with an increase in cellular $\mathrm{H}_{2} \mathrm{O}_{2}$, which is necessary for maximal phosphorylation of the receptor 408 . As $\mathrm{H}_{2} \mathrm{O}_{2}$ inhibits PTP activity, including SHP2, it has been strongly suggested this increase in oxidant levels serve as a regulatory mechanism to inhibit PTPs and optimise receptor phosphorylation $\begin{array}{llllll}409 & 410 & 411 & 412 & 413\end{array}$. Indeed, $\mathrm{H}_{2} \mathrm{O}_{2}$-induced oxidative modification of SHP2 is observed following cellular stimulation with PDGF ${ }^{414}$. PTPs with only one cysteine in their active domain, such as PTP1B, undergo oxidative modification of this residue forming a sulfenic acid intermediate 415416 . To prevent higher irreversible oxidation to a sulfinic or sulfonic acid, the oxygen is rapidly eliminated via formation of a cyclic sulfenamide; a 5-atom ring 
structure whereby a covalent bond is formed between the cysteine sulfur and the main chain nitrogen of an adjacent serine residue ${ }^{417}$. This cysteine-serine link induces structural changes within the active site, exposing the oxidative modification to cellular reducing agents such as GSH and Trx, regenerating the active form of the enzyme ${ }^{418}$. Some PTPs, on the other hand, have a second thiol within their active domain which is vicinal to their catalytic cysteine, including the cell cycle cdc25 phosphatases, low molecular weight-PTP and tensin homologue (PTEN). These are often termed 'backdoor' cysteines, and protect against irreversible oxidation of the sulfenic acid intermediate by facilitating the formation of an intramolecular disulfide 419420421 . Again, the S-S bond can be readily reduced enabling reversibility of this inhibitory oxidative modification and so providing a mechanism of regulation. Crystallographic studies showed the active site of SHP2 contains two of these 'backdoor' cysteines at positions 333 and 367 , which are vicinal not only to the catalytic cysteine, Cys ${ }^{459}$, but also each other 43422 . The proximity of these cysteines was visualised using the computer software PyMol, as shown in figure 6.28. As mentioned, oxidative modification of the catalytic cysteine of SHP2 acts as an inhibitory mechanism to regulate downstream signalling pathways, such as MAPK and endothelin 1423424425 . Through biochemical and structural analysis, Machado et al propose a sulfenic acid intermediate forms upon Cys ${ }^{459}$, inhibiting the phosphatases catalytic activity, which is quickly resolved by $\mathrm{Cys}^{367}$ to form an intramolecular disulfide, maintaining inhibition of the protein as the catalytic cysteine is still unavailable 426. A similar mechanism has been demonstrated for MAP kinase phosphatase 3, whereby oxidative modification of its catalytic cysteine by $\mathrm{H}_{2} \mathrm{O}_{2}$ induces disulfide formation with one of the multiple cysteines distributed within the 
proteins $\mathrm{N}$ - and C-terminal domains ${ }^{427}$. Through kinetic and MS analysis, the Rudolph group demonstrate $\mathrm{H}_{2} \mathrm{O}_{2}$ treatment of SHP2 results in an inhibitory intramolecular disulfide between $\mathrm{Cys}^{333}$ and $\mathrm{Cys}^{367}{ }^{142}$. Further to this, disulfide formation was not achieved following $\mathrm{H}_{2} \mathrm{O}_{2}$ treatment of Cys ${ }^{459}$ Ser mutant protein 142. They conclude a sulfenic acid intermediate is first formed upon Cys ${ }^{459}$ which is then resolved by the formation of an intramolecular disulfide with either Cys ${ }^{333}$ or Cys ${ }^{367}$, with this S-S bond itself resolved by formation of a subsequent intramolecular disulfide between both of the 'backdoor' cysteines. They propose the resultant intramolecular disulfide acts as a protective mechanism to block Cys $^{459}$ from further oxidative modification until itself is resolved by cellular disulfide reductases. Kinetic studies by the Gates group also support that SHP2 phosphatase activity can be inhibited by an oxidant-induced intramolecular disulfide within the active domain of the protein ${ }^{428}$.

As demonstrated in chapter 3 , in vitro treatment of recombinant SHP2 with low amounts of SFX-01 resulted in the formation of SHP2-SFN, followed by a loss in the detection of the electrophile adduct, even though inhibition of the phosphatase was maintained. With this in mind, I hypothesised that an SFN adduct may behave akin to a sulfenic acid intermediate, with this oxidative-like modification chemically transitioning to a secondary modification with resultant loss of the adduct, for example, an intramolecular disulfide. As shown in figure 6.29, perhaps adduction of SFN at $\mathrm{Cys}^{459}$ causes initial inhibition of the phosphatase, with subsequent loss of the adduct due to a resolving intramolecular disulfide between $\mathrm{Cys}^{459}$ and $\mathrm{Cys}^{333}$ or $\mathrm{Cys}^{367}$ and maintaining inhibition as the catalytic cysteine remains unavailable. These 'backdoor' cysteines are also maintained in a reduced state and reside near the opening of 
the active site pocket of SHP2. Perhaps due to its reactive nature, SFN can also adduct here. If so, inhibition of the phosphatase may occur due to reduced access of phosphorylated substrates to the catalytic cysteine of SHP2, firstly by the presence of an SFN adduct and subsequently due to a resolving intradisulfide between the backdoor cysteine (figure 6.29).

Notably, in vitro treatment with high amounts of SFX-01 inhibited SHP2 phosphatase activity with SFN adduction maintained over time. It was likely at such high abundance, SFN adducted the catalytic thiol as well as both 'backdoor' cysteines, making induction of an intramolecular disulfide within the active domain unachievable.

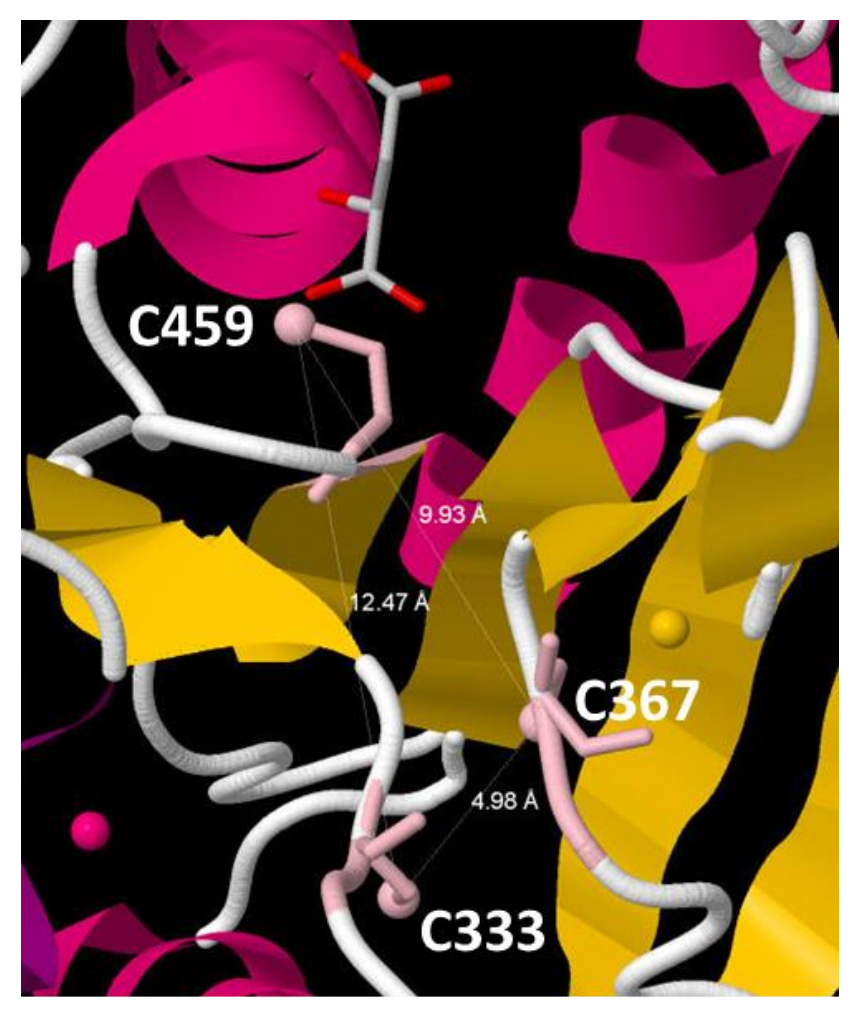

Figure 6.28. A PyMol image of the active site of SHP2.

The catalytic cysteine of SHP2, Cys ${ }^{459}$, can be visualised as vicinal to $\mathrm{Cys}^{333}$ and $\mathrm{Cys}^{367}$ separated by $12.47 \AA$ and $9.93 \AA$ respectively. Cys $^{333}$ and $\mathrm{Cys}^{367}$ are also vicinal to each other, separated by $4.98 \AA$. 
However, SFN-induced intramolecular disulfide formation would require a concomitant reductive modification of the electrophile, which has not been reported and chemically is considered an unlikely reaction. Further to this, $\mathrm{H}_{2} \mathrm{O}_{2}-$ treated recombinant SHP2 migrated faster on an acrylamide gel, consistent with intra-disulfide formation, whilst inhibited cardiac SHP2 immunoprecipitated following in vivo SFX-01 treatment did not. Lower molecular weight protein bands were detected by immunoblotting following in vivo treatment with SFX-01, however, the shift in molecular weight was much greater than would be anticipated for the formation of an intra-disulfide and was perhaps more likely a result of protein degradation. Also, as SFN-induced inhibition of cardiac SHP2 could not be reversed following treatment with DTT, this helps to rule out the formation of a disulfide by the direct reaction of the electrophile with the phosphatase. The PEG-switch method utilises a step in which oxidised thiols are chemically reduced to generate free-thiols which are then labelled with PEGmaleimide ${ }^{429}$. Only minimal amounts of inhibited cardiac SHP2 became labelled with PEG-maleimide following in vivo treatment with SFX-01, indicating further the inhibitory modification induced by SFN was not reducible. These observations together are consistent with SHP2 inhibition by SFN not being mediated by an active site intramolecular disulfide.

However, whilst cardiac SHP2 from control mice was labelled by the thiol-reactive BIAM reagent ${ }^{430}$, it failed to label the protein when mice were administered SFX01 for 4 days. BIAM-labelling was carried out under acidic conditions, driving protonation of thiols with a higher $\mathrm{p} K_{\mathrm{a}}$ whilst maintaining the reduced, deprotonated state of the catalytic cysteine. The loss of BIAM labelling, therefore, indicates the catalytic cysteine was likely covalently modified, although to 
reiterate, this is unlikely to be a disulfide or a simple SFN adduct. Further analysis showed that the cardiac SHP2 that was inhibited following 4-day exposure to SFX-01 was also no longer able to bind PAA, a dithiol cross-linking reagent which can form stable dithioarsine rings with protein vicinal thiols ${ }^{431}$. This suggests SFN-induced inhibition of SHP2 was most probably driven by an oxidative-like modification of two cysteines within its active domain. To explore this further, cysteine to serine double SHP2 mutants were generated, Cys ${ }^{333 / 367}$ Ser and Cys $^{333 / 459}$ Ser, leaving only one available cysteine within the catalytic domain. As expected, catalytic activity was maintained in the former mutant and diminished in the latter. Following a 2-hour treatment of cells with SFX-01, SFN was detected upon WT, Cys ${ }^{333 / 367}$ Ser or Cys $^{333 / 459}$ Ser SHP2. This is consistent with SFN adducting not only to the catalytic cysteine, but also one or both of the 'backdoor' cysteines. In line with in vitro experiments, the WT SHP2-SFN adduct was lost over time. In contrast, a stable adduct was maintained upon either double mutant. Again, this is consistent with SFN adducting and inhibiting SHP2, but with this modification subsequently reacting with an adjacent cysteine to form another oxidative modification that underlies the sustained inhibition of the phosphatase. The chemical nature of this inhibitory modification is an important consideration and the subject of the discussion below.

Zhang et al have demonstrated a cyclocondensation reaction between ITCs and the vicinal dithiol-containing compound 1,2-benzenedithol ${ }^{432}$. This results in the formation of a 1,3-dithiole-2-thione cyclic dithiolethione compound, with this stable and non-reducible modification occurring between the two vicinal thiols of 1,2-benzenedithol and release of $\mathrm{R}$-substituted nitrogen as an amine. In addition, this group showed ITC metabolites, including GSH-SFN, cysteinylglycine-, 
cysteine- as well as $\mathrm{N}$-acetylcysteine-conjugates can also undergo the same cyclocondensation reaction with 1,2-benzenedithol and produce a dithiolethione 433. Perhaps SFN or its metabolites induced dithiolethione formation, which is characterised by two sulfur atoms linked by a $\mathrm{C}=\mathrm{S}$ group, between two of the vicinal thiols in the active domain of SHP2 as shown in figure 6.30 . Such an adduct would likely not be detectable by the anti-SFN antibody developed inhouse and used throughout these studies, as the dithiolethione is markedly different than an SFN adduct from a structural standpoint. This would initially involve adduction of SFN at Cys ${ }^{459}$ or either of the 'backdoor' cysteines, followed by nucleophilic attack by a second vicinal thiol at the same carbon atom of SFN as outlined in figure 6.31. A resulting sulfur-containing dithiolethione condensation product would then be formed between either Cys ${ }^{459}$ and a backdoor cysteine, driving inhibition as the catalytic cysteine is unavailable, or between the backdoor cysteines themselves, likely preventing access of phosphorylated proteins into the active domain.
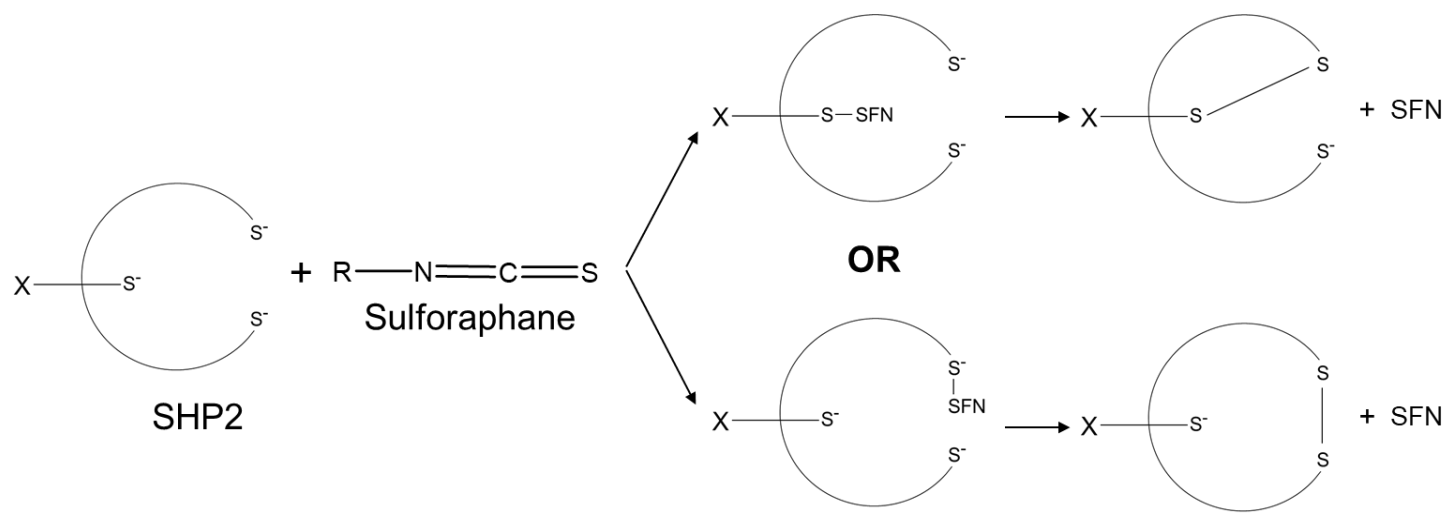

Figure 6.29. A proposed scheme of SFN-induced intra-disulfide formation.

Following adduction of SFN to the catalytic cysteine of SHP2, or either of the proteins 'backdoor' cystines, resolution of the adduct by an intramolecular disulfide would maintain inhibition of the phosphatase. This considered mechanism is unlikely to occur and the analogy with $\mathrm{H}_{2} \mathrm{O}_{2}$-induced disulfide formation, therefore, is not valid. 
The premise that SFN may induce such a dithiolethione 'chemical bridge' between two thiols is supported by the generation of dTNB following incubation of TNB with L-SFN. However, MS analysis is required to confirm this idea, which was underway at the time of writing.

Incubation of SFN with GSH resulted in a time-dependent increase in the formation of a compound which did not correspond to SFN, GSH or GSSG standards when analysed by MS. Subsequent $\mathrm{MS}^{3}$ analysis of this compound and review of resultant base peaks strongly suggested the formation of GSHSFN and not two GSH molecules linked via a dithiolethione. Nevertheless, the formation of GSH-SFN is the first step required for dithiolethione formation. A higher relative abundance of GSH or longer incubation times may increase the probability that a second tripeptide would contact and react with GSH-SFN to facilitate dithiolethione formation. Although perhaps generation of such a chemical structure between two thiol-containing proteins is unlikely and requires two thiols to be in close vicinal proximity, such as those found within the active domain of SHP2.

Enzymatic digestion and analysis by MS of SHP2 exposed to SFN would be a rational way of assessing whether a dithiolethione condensation product does indeed form within the active site of the protein to causatively mediate phosphatase inhibition by this electrophile. The presence of a mass correlating to two peptide chains each containing $\mathrm{Cys}^{459}$, $\mathrm{Cys}^{333}$ or $\mathrm{Cys}^{367}$ linked by the dithiolethione structure would be robustly consistent with such an inhibitory mechanism. 


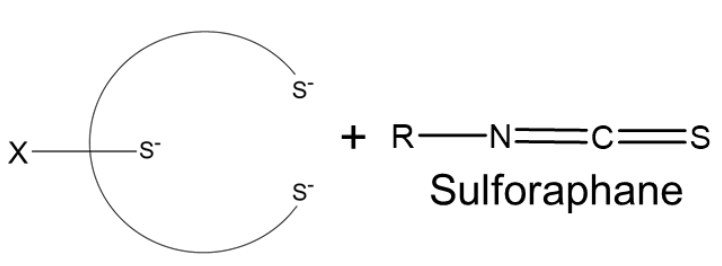

SHP2

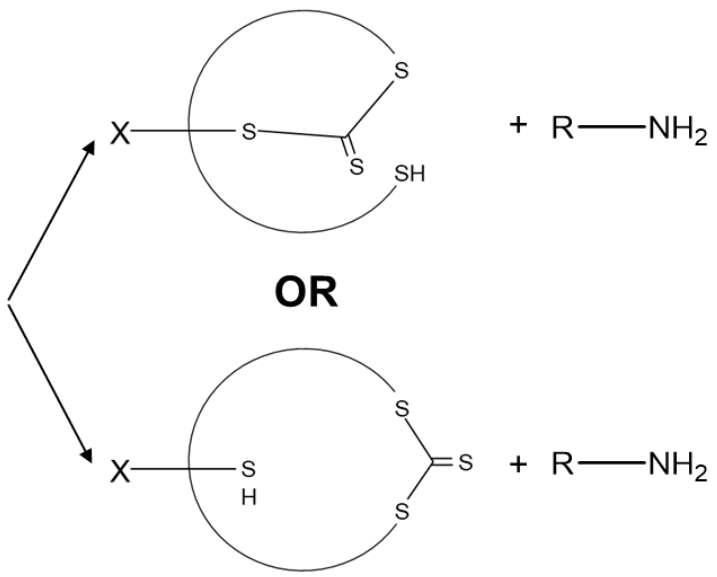

Figure 6.30. A proposed scheme for SFN-induced dithiolethione formation between two vicinal thiols within the active site of SHP2.

Following the adduction of SFN to either the catalytic cysteine of SHP2 or either of the 'backdoor' cysteines, a cyclocondensation reaction may occur which forms a dithiolethione product, which may be responsible for maintaining the SFN-induced inhibition of the phosphatase.

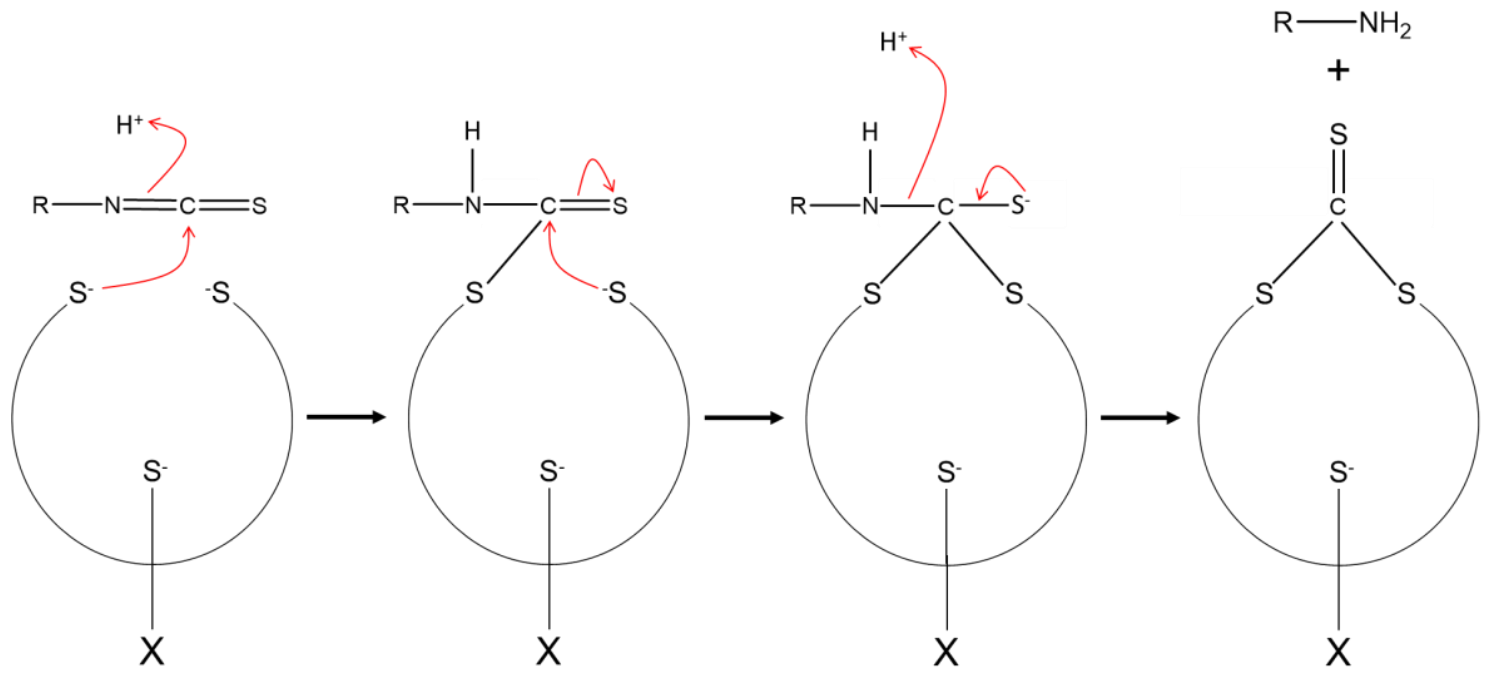

Figure 6.31. A possible cyclocondensation reaction of an SFN adduct with vicinal thiols within the active site of SHP2. 


\section{Summary and Conclusion}

\subsection{Summary}

This thesis reports a comprehensive investigation of the inhibition of the PTP SHP2 by the electrophilic ITC SFN. Specific focus is given to how this inhibition may prevent the development and progression of phenotypes that NS patients present with.

Cysteine thiols with a low $p K_{a}$ value, meaning they can ionise and so become reactive at cellular $\mathrm{pH}$, are often involved in the catalytic activity of a protein, either through regulation of tertiary structure or conducting the catalytic events themselves. These thiolate moieties are susceptible to modification by oxidants, with their negative charge also enabling nucleophilic reactions with electrophilic compounds such as SFN, which mediates covalent adduction of such compounds that potentially modulates the activity and function of the protein.

It is established SFN adducts to cysteines of multiple proteins and the biological effects of these interactions accumulatively contribute to the cancer prevention activity of the electrophile, which has sparked an increase in studies investigating this ITC 2533209434 435. A comprehensive review of these events is not in the scope of this thesis; however, they include the induction of anti-inflammatory and pro-apoptotic responses ${ }^{436}$. Data also suggest that SFN may alter epigenetic changes which occur in some cancers, which reverse aberrant changes in gene transcription through mechanisms of histone deacetylase inhibition, global demethylation, and microRNA modulation ${ }^{437}$.

SFN can also induce an antioxidant response by adducting cysteines within KEAP1 32438439 . Upregulation of antioxidants in non-pathological conditions can 
prevent homeostatic failures caused by an increase in cellular oxidants ${ }^{440} 441$. Cancer cells, on the other hand, can activate the Warburg effect, which increases the conversion of glucose-6-phosphate into ribulose-5-phosphate and generates $\mathrm{NADPH} 442443444$. NADPH is required for $\mathrm{GSH}$ production as well as some disulfide reductase systems and protects tumour cells from apoptosis by counteracting oxidative stress and facilitating DNA damage repair ${ }^{445}$. Therefore, whilst cancer has been considered a result of increased oxidative stress, its treatment with antioxidants would contribute to the already increased reducing conditions of these cells. Indeed, successful cancer therapies such as radiotherapy or doxorubicin induce oxidant production 446447448449 . Although SFN induces an antioxidant response, the electrophile also adducts proteins at moieties which are otherwise modified by oxidants and therefore can mimic them. Further to this, whilst SFN rapidly conjugates with GSH which delineates the drug from modifying other cellular proteins, this interaction also prevents the reductive capabilities of the GSH ${ }^{22}$. Therefore, even though SFN upregulates the production of antioxidants, their own adduction by the electrophile may alleviate this antioxidant response. Indeed, a number of human clinical trials have been performed with rigour using SFN with published study results indicating potential therapeutic benefit 450451 . The electrophile is structurally simple and small which underlies the ability of SFN to interact with and adduct to a large number of protein targets, some of which likely remain unknown, and may induce multiple biological events. Nevertheless, human clinical trials using SFN as well as SFX01 , a chemically stabilised variant of SFN in which it is encapsulated in acyclodextrin ring structure, to our knowledge, have shown little, if any, undesired biological outcomes at the therapeutic concentrations used 9192939495104105 . 
Therefore, despite SFN adducting multiple cellular proteins, as can be observed with the anti-SFN antibody used throughout this study, the electrophile is tolerated and is considered a tractable therapeutic.

Identifying protein targets of SFN may help define the impact of the electrophile on biological systems. For example, if SFN attenuates the development of a specific pathology, its adduction to one of the protein targets identified may mediate, at least in part, its therapeutic actions. Another prospect following target identification is it may highlight proteins which are functionally altered during pathological conditions, and perhaps their adduction by SFN may be therapeutic by modifying their function.

Indeed, as reported herein, immunoprecipitation of SFN adducted to protein cysteines following in vivo treatment with SFX-01 and subsequent proteomic analysis identified cardiac SHP2 as a significant target. This then leads to the rational hypothesis, as considered below, that SFN likely adducts to the catalytic cysteine in SHP2 to inhibit it and so may prove therapeutic in pathologies mediated by hyperactivation of this phosphatase.

Point mutations in Ptpn11, the gene which encodes SHP2, are causative of all phenotypes present in the multisymptomatic developmental disorder NS, including abnormal facial features, short stature and in some cases myeloproliferative disease $\begin{array}{llll}65 & 69 & 137 & 452\end{array}$ chromosomal pathology associated with congenital heart defects, which include pulmonary valve stenosis and atrial and ventricular septal defects, among others 66 67. 12 missense, germline mutations of the Ptpn11 gene have been identified in NS patients, which all cause hyperactivation of SHP2 phosphatase activity. 
Hyperactivation of the phosphatase, either because of a gain-of-function mutation in Ptpn11, or other dysregulated signalling events, have also been characterised in multiple cancers. Hyperactive SHP2 phosphatase activity is therefore associated with the initiation and progression of both pathologies. This has driven significant pharmacological programmes developing inhibitors of SHP2. Whilst some of these compounds are potent inhibitors of the phosphatase, their interaction with other off-target proteins has been observed which may cause undesired biological functions, and studies are still needed to evaluate potential side-effects of these interactions in vivo 798283 .

Due to the electrophilicity of SFN, it was logical to assume its adduction to SHP2 was occurring at the proteins negatively charged thiolate moiety of its catalytic cysteine, Cys ${ }^{459}$. This nucleophilic thiolate is required for the phosphatase activity of SHP2, so it was anticipated that adduction of SFN would inhibit its ability to dephosphorylate substrates. Consistent with this, incubation of recombinant SHP2 with SFX-01 resulted in adduction of SFN and concentration-dependent inhibition of the phosphatase's activity. A rational implication was that SFN may be therapeutic in scenarios in which hyperactive SHP2 activity contributes to pathogenesis, such as NS. A logical next step was, therefore, investigating SFNmediated inhibition of the phosphatase in an NS mouse model engineered to express a heterozygous gain-of-function mutation, $\mathrm{D} 61 \mathrm{G} /+$, found to cause the highest level of SHP2 activation in human patients ${ }^{452}$.

Biochemical analysis showed multiple proteins had been adducted by SFN in a variety of tissue types in WT or NS mice that received an acute (30-minute) bolus dose of high amounts of SFX-01. This data provided evidence of successful absorption of SFN and transport into tissue cells when SFX-01 was used as the 
source of the electrophile. Even so, western immunoblot analysis of SHP2 immunoprecipitated from cardiac tissue of WT or NS mice following this 30minute treatment with SFX-01 did not show an SFN adduct upon the protein. Follow-up assays also revealed no inhibition of the phosphatase's activity. Perhaps 30-minute post-treatment with SFX-01 was too short to allow adduction of the electrophile to cardiac SHP2, as initial identification of SFN as a target of the phosphatase in vivo was achieved using MS analysis 3-hours post-treatment with the drug.

Data from biochemical analysis of SHP2 phosphatase activity following incubation of recombinant protein with varying concentrations of SFX-01 for different lengths of time showed chronic treatment with lower amounts of the electrophile caused the same level of inhibition of the phosphatase as when incubated with higher amounts of the drug for a much shorter duration. This corroborated studies by the Darley-Usmar group which show continual treatment with low amounts of an electrophilic compound over time caused its accumulative adduction to target proteins which was not achieved through short-term treatment regimens ${ }^{144}$. Indeed, immunoprecipitation of cardiac SHP2 and analysis of its activity revealed the phosphatase was inhibited following chronic 4-, 7- or 10-day treatment with low amounts of SFX-01 continually in the drinking water of WT or NS mice, which otherwise was not seen following acute treatment with a high bolus amount of the drug.

An increase in the phosphorylation of STAT5, an essential protein of the GH signalling pathway which acts downstream of SHP2 was also seen, which was logically assumed as a direct effect of SFN-induced inhibition of the phosphatase. The Ras/ERK pathway is another signalling event which occurs downstream of 
SHP2, with activation of the phosphatase leading to increased phosphorylation of the kinase. It was therefore anticipated that inhibition of SHP2 by SFN would decrease phosphorylation of ERK, which has previously been observed with other inhibitors of the phosphatase 798283 . In fact, chronic in vivo SFX-01 treatment caused an increase in ERK phosphorylation in cardiac tissue of WT mice, even though SHP2 was inhibited. This was perhaps a consequence of adduction of SFN to proteins other than SHP2 and this data corroborates previous studies showing an increase in ERK phosphorylation following treatment with the electrophile in a variety of cell types 151152153154 . Therefore, whilst SFN-induced inhibition of the phosphatase may reduce SHP2-mediated stimulation of the Ras/ERK signalling pathway, this might be outweighed by the electrophile adducting and modifying the activity of other proteins also responsible for regulating ERK activity, such as MAPK phosphatase 1, a phosphatase known to dephosphorylate ERK.

As SFN-induced inhibition of SHP2 in vivo had been characterised it was rational to explore if this was therapeutic in the D61G/+ NS mouse model. Although the underlying mechanism remains unclear, NS patients and this mouse model often present with different phenotypes which can also be present with different severities ${ }^{73}$. For example, short stature, cranial defects and myeloproliferative disease are present in $100 \%$ of $\mathrm{D} 61 \mathrm{G} /+$ offspring, however, the severity of these phenotypes differs between mice. On the other hand, $100 \%$ of homozygous foeti and $50 \%$ of those that are heterozygous for this mutation die mid-gestation due to severe cardiac complications, as well as skeletal defects and liver necrosis, whilst the remainder are born with no cardiac phenotype. As these developmental defects are caused by a single gain-of-function SHP2 mutation, it was rational to 
assume a reduction of the phosphatase's activity by SFN could improve cardiac, skeletal and liver development in foeti with NS that would otherwise succumb to embryonic lethality. To address this, foeti were exposed to SFN via treatment of heterozygous parents with SFX-01 in their drinking water before and throughout pregnancy. Whilst foeti from WTMT breeding pairs were unaffected, the number of homozygous or heterozygous neonates born from NS breeding pairs following treatment with SFX-01 was unfortunately decreased compared to water only controls. Analysis of the activity of SHP2 immunoprecipitated from NS neonates which were born from these studies revealed no inhibition of the phosphatase. Further biochemical analysis showed SFX-01 increased foetal ERK phosphorylation. As SHP2 is a positive regulator of the Ras/ERK signalling pathway, hyperactivation of the phosphatase causes an increase in phosphorylation of the kinase. Thorough studies conducted by the Neel group allowed the conclusion that this basal increase in ERK phosphorylation in the $\mathrm{D} 61 \mathrm{G} /+$ NS mouse model is causative of the majority of developmental defects occurring in these mice ${ }^{78}$. It was, therefore, logical to assume that SFN increased ERK phosphorylation even higher in NS foeti which may have exacerbated developmental defects. Therefore, this may be responsible for the decreased number of heterozygous offspring born following foetal treatment with the electrophile. Nevertheless, it remains rational that reduction of SHP2 phosphatase activity would improve the embryonic development of NS foeti and further studies of this nature should be conducted using inhibitors of the protein that do not result in elevated ERK phosphorylation.

Activation of ERK leads to the stimulation of downstream transcription factors which positively regulate cell growth and proliferation. Although SFN-induced 
increases in ERK phospho-activation induces undesired biological events during the embryonic development of NS foeti, perhaps this increase in the activity of this kinase in neonates would be therapeutic towards the growth retardation these patients present with. SFN-induced increase in STAT5 phosphorylation, which positively regulates the GH signalling pathway, may also contribute to an increase in growth of these patients post-birth.

SHP2 regulates multiple stages of white blood cell production 345346347 . It is therefore unsurprising that some children with NS develop JMML, characterised by increased myeloid and monocyte cell count which can sometimes escalate into leukaemia ${ }^{68} 69$. Similarly, adult NS mice presented with elevated white blood cell count, myeloid cell count (myeloproliferative disease) and splenomegaly. As well as regulating the proliferation and survival of white blood cell progenitor cells, SHP2 has more specific roles in granulocyte lineage determination i.e. the differentiation of premature white blood cells into neutrophils, basophils or eosinophils ${ }^{352} 353$. Flow cytometry analysis lead to the novel observation that increased myeloid cell count in the blood, spleen and bone marrow of NS mice was predominantly an increase in the number of neutrophils. As this myeloproliferative phenotype was caused by a single gain-of-function mutation in SHP2, it was logical to determine if SFN-induced inhibition of the phosphatase would prevent increased myeloid cell production and assess if this could be sustained over time. To do so, adult NS mice were treated with SFX-01 for a prolonged 10-week period which indeed reduced the phosphatase activity of the protein and fewer mice developed a myeloproliferative phenotype of the blood, spleen and bone marrow. Further analysis of this data showed that neutrophils were the predominant cell-type that was lower in SFX-01-treated NS mice 
compared to water only controls. A reduction in the growth of the spleen of NS mice, which was monitored using ultrasound, was also observed when mice were treated with the drug. As the splenomegaly observed in NS mice is attributed in part to an increase in spleen-residing myeloid cells, it is logical that the reduced spleen growth observed when treated with SFX-01 was due to fewer circulating and spleen-residing myeloid cells. Perhaps if children with NS were treated with SFN or SFX-01 from birth this may prevent the hyperproliferation of myeloid cells and lower the incidence of JMML and leukaemia in these juvenile patients.

The electrophilicity of SFN allows covalent interaction of the electrophile with nucleophilic moieties. Therefore, it was rationally anticipated that inhibition of SHP2 following 4-day or 10-week treatment with SFX-01 was caused by a stable SFN adduct upon the protein, likely at its negatively charged catalytic cysteine. However, immunoprecipitation of SHP2 and biochemical analysis showed that whilst the electrophile induced inhibition of the phosphatase's activity, an SFN adduct was not seen upon the protein. It was logical to assume SFN did adduct SHP2 which inhibited the protein, due to its identification as a target of the electrophile being achieved by MS following immunoprecipitation of proteins with an SFN adduct. However, perhaps this adduction was followed by loss or chemical modification of SFN, which maintained the inhibition of the phosphatase.

Spontaneous reversal of thiol-SFN adducts has previously been documented ${ }^{176}$ 177. The Uchida group have also shown using MS analysis that as well as the spontaneous reversal of a GSH-SFN interaction, the electrophile can actively transfer directly from GSH to a second thiol-containing protein ${ }^{182}$, a mechanism termed 'trans-thiolation' in this document. Data presented herein also shows 
trans-thiolation of SFN can occur and perhaps this mechanism enables the electrophile to exert biological effects on multiple proteins in vivo.

It is likely spontaneous reversal of a thiol-SFN interaction or trans-thiolation of the adduct regenerates a reactive form of the thiol that the electrophile was initially adducted to and as a result, the protein would regain its activity. As inhibition of SHP2 phosphatase activity was maintained following the loss of the SFN adduct it was perhaps unlikely removal of the electrophile occurs via either of these mechanisms. Indeed, biochemical assays carried out using the alkylating agent iodoacetamide, which readily adducts cysteine thiolates, showed chronic in vivo treatment with SFX-01 inhibited the phosphatase activity of SHP2 and although no SFN adduct was detected, iodoacetamide could no longer adduct at the protein's catalytic cysteine. To our knowledge, this is the first evidence that functional modifications of a protein's activity induced by adduction of SFN can be maintained following the loss of the adduct. To reiterate, these data supported the hypothesis that loss of the SFN adduct from SHP2 was not achieved by spontaneous reversal of the electrophile-protein interaction or transthiolation, as these mechanisms would regenerate a reactive thiolate at the protein's catalytic cysteine.

Analysis of the crystal structure of SHP2 showed the catalytic cysteine within its phosphatase domain is in proximity to two other vicinal cysteines, creating a triad of reactive thiols. Cellular oxidants can modify the catalytic cysteine of SHP2 and inhibit its phosphatase activity. One of the two other cysteines which reside nearby form an intramolecular disulfide with the catalytic cysteine which reduces the oxidative modification and prevents further irreversible oxidation. The catalytic cysteine remains unreactive as its thiolate moiety is participating in the 
intramolecular disulfide and the proteins phosphatase activity remains inhibited until the bond itself is reduced by cellular disulfide reductases. PAA is a dithiol cross-linking agent which can only bind to proteins when two protein vicinal thiols are in a reduced and reactive state. Biochemical analysis utilizing this compound showed it could no longer adduct to cardiac SHP2 from mice following 4-day treatment with SFX-01, suggesting at least two of the three vicinal thiols within the phosphatase domain of SHP2 were impeded, which to reiterate was not by stable SFN adducts. To our knowledge, we have shown the novel observation that SFN can induce an oxidative-like modification between two vicinal thiols when adduction of the electrophile occurs at a cysteine which resides close to at least one other reactive thiol.

Whilst induction of an inhibitory intramolecular disulfide within the active domain of SHP2 following adduction of the electrophile to the proteins catalytic cysteine was a logical assumption, this would require a reductive modification of the electrophile upon its resolution from the catalytic cysteine, which chemically is unlikely. Data produced from biochemical assays carried out following the reduction of protein disulfide bonds, including the PEG-switch, also suggested that SFN-induced inhibitory modification of at least two of the three vicinal thiols within the phosphatase domain of SHP2 was not an intramolecular disulfide.

It has been shown that a dithiolethione can be formed by a series of condensation reactions following adduction of SFN to a dithiol containing compound, which is characterised by two sulfur atoms linked by a $\mathrm{C}=\mathrm{S}$ chemical structure ${ }^{433}$. It is rational to suggest SFN adducts the catalytic cysteine of SHP2, with a second vicinal thiol within the protein's phosphatase domain subsequently attacking the electrophile, which results in the formation of a dithiolethione condensation 
product between two of the vicinal thiols within the proteins active domain. Inhibition of SHP2 would, therefore, be induced by initial adduction of the electrophile at the catalytic cysteine, with the resolution of the adduct to a dithiolethione maintaining the inhibition of the phosphatase as the catalytic cysteine remains unreactive. The resultant dithiolethione epitope would be undetectable by the antibody developed in-house against SFN adducted to protein cysteines and may explain why no SFN adduct is detected upon inhibited SHP2 following chronic in vitro and in vivo treatment with the electrophile.

Biochemical data collected following the treatment of different SHP2 cysteine to serine mutants in HEK293 cells with SFX-01 suggested SFN can adduct at the two thiols vicinal to the catalytic cysteine, as well as Cys ${ }^{459}$ itself. Further to this, when two of the three cysteines within the phosphatase domain of SHP2 were mutated to a serine, a stable SFN adduct was seen following treatment with SFX01, which was otherwise resolved when two reactive thiols were present. This data suggests SFN can also adduct SHP2 at one of the two non-catalytic vicinal thiols within its phosphatase domain, with either the catalytic cysteine or the second vicinal thiol inducing dithiolethione formation. If the latter were to occur, inhibition of SHP2 would be achieved as this chemical structure would prevent access of phosphorylated substrates to the catalytic cysteine of the protein.

\subsection{Conclusion}

Sulforaphane, or a formulation in clinical development called SFX-01, inhibits the catalytic activity of SHP2; a protein-tyrosine phosphatase which when hyperactivate causatively mediates multiple cancers as well as Noonan syndrome. Sulforaphane-mediated inhibition of SHP2 is not achieved by a stable adduct but likely by induction of a chemical modification of two vicinal thiols within 
the protein's catalytic domain, perhaps the formation of a dithiolethione. SFNinduced inhibition of the phosphatase reduced the incidence of myeloproliferative disease and splenomegaly in a mouse model for Noonan syndrome which is genetically engineered with a single gain-of-function SHP2 mutation, D61G/+. Chronic treatment with SFN is well tolerated in adult mice and is a promising candidate to utilize as a therapy regarding not only Noonan syndrome but also cancers which are driven by hyperactivation of SHP2.

\subsection{Future work}

It is evident from published studies and data presented herein that SFN modulates multiple biological effects in vivo, due to the electrophile adducting many cell signalling proteins. An increase in ERK phosphorylation in NS embryos may have contributed to their embryonic lethality following foetal treatment with SFX-01. Therefore, identifying how SFN induced an increase in ERK phosphorylation may be beneficial if the electrophile is to be pursued as a therapy for this pathology. It was rational to speculate the increase in ERK phosphorylation occurred due to adduction of SFN to MAPK phosphatase 1 which would inhibit its ability to dephosphorylate ERK. This could be assessed using the same biochemical techniques utilised to analyse the activity of SHP2 and its adduction by SFN throughout this research project. It has also been shown SFN can increase the levels of cellular ROS, which in turn can promote ERK phosphorylation in an IGF1 dependent manner. Assays such as with dichlorodihydrofluorescein diacetate ${ }^{453}$, dihydroethidium ${ }^{454}$ or the HyPer probe ${ }^{455}$ could be used to analyse cellular levels of ROS following treatment with SFN.

Perhaps treatment of NS foeti post-gastrulation would inhibit SHP2 phosphatase activity and allow correct development of the later stages of embryogenesis, 
whilst the anticipated increase in ERK phosphorylation may not compromise key stages of foetal development as they would have already occurred. Although preliminary data suggested treatment of NS foeti post-gastrulation also decreased the number of NS neonates born, a definitive conclusion should not be taken from these studies. The date of conception was predicted by the presence of a vaginal plug and these studies should be repeated whereby gastrulation is confirmed using ultrasound, as perhaps SFN was administered before gastrulation was fully completed.

During this research project, the pharmaceutical company Novartis documented a potent $\left(\mathrm{IC}_{50}=71 \mathrm{nM}\right)$, highly selective and orally bioavailable small-molecule SHP2 inhibitor named SHP099 ${ }^{456}$. SHP099 stabilises the closed and autoinhibited conformation of SHP2 by concurrently binding the interface of the Nand $\mathrm{C}$-terminal $\mathrm{SH} 2$ domains and the PTP domain, thereby driving inhibition through an allosteric mechanism. Not only does SHP099 suppress downstream Ras/ERK signalling, but the drug has no significant effect on the biological activity of a panel of PTPs or kinases tested. However, subsequent studies by Sun et al identified SHP2 with gain-of-function mutations within the N- or C-SH2 domains, including D61G/+, were resistant to inhibition by SHP099 in cultured GM-CSFdependent TF 1 myeloid cells ${ }^{457}$. Nevertheless, in vivo treatment with the drug has not been documented. Treatment of NS foeti with SHP099 may inhibit SHP2 with no off-target biological effects, which may prevent the defects in embryonic development which are caused by hyperactivation of the phosphatase.

NS patients are born with short stature and this growth retardation continues into adulthood. Studies reported herein showed that combined treatment of SFN and GH potentiated activation of the JAK/STAT GH signalling pathway compared to 
GH alone, which is currently the only therapy available for NS patients. Therefore, as activation of ERK stimulates cell growth and proliferation, perhaps upregulation of this pathway as well as GH signalling using a combined SFN/GH therapy post-birth would be therapeutic in increasing the growth of NS patients and reducing the incidence of short stature.

Proteomic analysis showed SFN adducted to $\mathrm{Cys}^{93}$ of $\mathrm{Hgb} \beta$ during chronic in vivo exposure to SFX-01. Published studies showed NO also readily adducts at $\mathrm{Cys}^{93} 223224$. Although elucidation of the role and mechanism of NO binding at this reactive cysteine is still ongoing, it is suggested this interaction aids regulation of NO-mediated vessel relaxation and therefore blood pressure 225226 227. SFN adduction to Cys ${ }^{93}$ may impede binding and transport of NO to areas where vascular relaxation is required which could prevent NO-mediated regulation of blood pressure, and such physiological changes could be monitored in mice treated with SFX-01 using telemetry probes. However, the stoichiometry of the SFN modification at $\mathrm{Hgb} \beta$ Cys $^{93}$ may be very low that it is functionally insignificant.

Finally, observations from biochemical studies suggest SFN adduction at the catalytic cysteine of SHP2 inhibits its phosphatase activity, which is maintained by the induction of an oxidative-like modification between at least one other vicinal thiol, with resultant loss of a detectable adduct. This SFN-induced modification may be the formation of a dithiolethione condensation product and proteomic analysis using LC-MS/MS could be used to look for this theoretical modification. Following in vivo treatment with SFN, immunoprecipitation of SHP2 and enzymatic digestion of the protein may preserve a dithiolethione between two peptides that each contain one of the discussed vicinal thiols. The 
identification of a product with a mass that corresponds to two of these thiolcontaining peptides plus the mass of the dithiolethione would strongly suggest SFN induced such a chemical structure between two vicinal thiols within the phosphatase domain of SHP2. Such an analysis would also allow the identification of the two protein cysteines involved in this potential posttranslational modification that may mediate inhibition of SHP2 in vivo during chronic exposure to SFX-01. If a dithiolethione is indeed present, the generation of an antibody to detect this specific epitope may aid identification of other vicinal thiol-containing proteins which are also inhibited or activated by this chemical structure following treatment with SFN. 


\section{Bibliography}

1. Stadtman, E. R. \& Levine, R. L. Free radical-mediated oxidation of free amino acids and amino acid residues in proteins. Amino Acids. 25, 207-218 (2003).

2. Murphy, M. P. How mitochondria produce reactive oxygen species. Biochem. J. 417, 1-13 (2009).

3. Bhattacharyya, A., Chattopadhyay, R., Mitra, S. \& Crowe, S. E. Oxidative stress: an essential factor in the pathogenesis of gastrointestinal mucosal diseases. Physiol. Rev. 94, 329-354 (2014).

4. Poole, L. B. The basics of thiols and cysteines in redox biology and chemistry. Free Radic. Biol. Med. 80, 148-157 (2015).

5. Rhodes, G. \& Kyte, J. Structure in protein chemistry, 2nd Edition. Biochem. Mol. Biol. Educ. 36, 90-91 (2008).

6. Sahaf, B., Heydari, K., Herzenberg, L. A. \& Herzenberg, L. A. The extracellular microenvironment plays a key role in regulating the redox status of cell surface proteins in HIV-infected subjects. Arch. Biochem. Biophys. 434, 26-32 (2005).

7. Hanschmann, E.-M., Godoy, J. R., Berndt, C., Hudemann, C. \& Lillig, C. H. Thioredoxins, glutaredoxins, and peroxiredoxins--molecular mechanisms and health significance: from cofactors to antioxidants to redox signaling. Antioxid. Redox Signal. 19, 1539-1605 (2013).

8. Ansley, D. M. \& Wang, B. Oxidative stress and myocardial injury in the diabetic heart. J. Pathol. 229, 232-241 (2013).

9. Kattoor, A. J., Pothineni, N. V. K., Palagiri, D. \& Mehta, J. L. Oxidative Stress in Atherosclerosis. Curr. Atheroscler. Rep. 19, 42 (2017).

10. Gupta, R. K. et al. Oxidative stress and antioxidants in disease and cancer: a review. Asian Pac. J. Cancer Prev. 15, 4405-4409 (2014).

11. Lin, M. T. \& Beal, M. F. Mitochondrial dysfunction and oxidative stress in neurodegenerative diseases. Nature. 443, 787-795 (2006).

12. Paul, B. D. et al. Cystathionine $\mathrm{y}$-lyase deficiency mediates neurodegeneration in Huntington's disease. Nature. 509, 96-100 (2014).

13. Pereira, A. C. \& Martel, F. Oxidative stress in pregnancy and fertility pathologies. Cell Biol. Toxicol. 30, 301-312 (2014).

14. Beetham, K. S. et al. Oxidative stress contributes to muscle atrophy in chronic kidney disease patients. Redox Rep. 20, 126-132 (2015).

15. Holguin, F. Oxidative Stress in Airway Diseases. Ann. Am. Thorac. Soc. 10, S150-S157 (2013).

16. Johnson, I. T. Glucosinolates: Bioavailability and Importance to Health. Int. J. Vitam. Nutr. Res. 72, 26-31 (2002).

17. Holst, B. \& Williamson, G. A critical review of the bioavailability of glucosinolates and related compounds. Nat. Prod. Rep. 21, 425 (2004).

18. Shapiro, T. A., Fahey, J. W., Wade, K. L., Stephenson, K. K. \& Talalay, P. Human metabolism and excretion of cancer chemoprotective glucosinolates and isothiocyanates of cruciferous vegetables. Cancer Epidemiol. Biomarkers Prev. 
7, 1091-1100 (1998).

19. Fahey, J. W., Zalcmann, A. T. \& Talalay, P. The chemical diversity and distribution of glucosinolates and isothiocyanates among plants. Phytochemistry. 56, 5-51 (2001).

20. Fahey, J. W. et al. Sulforaphane Bioavailability from Glucoraphanin-Rich Broccoli: Control by Active Endogenous Myrosinase. PLoS One. 10 (2015).

21. Fahey, J. W., Zhang, Y. \& Talalay, P. Broccoli sprouts: an exceptionally rich source of inducers of enzymes that protect against chemical carcinogens. Proc. Natl. Acad. Sci. 94, 10367-10372 (1997).

22. Forman, H. J., Zhang, H. \& Rinna, A. Glutathione: overview of its protective roles, measurement, and biosynthesis. Mol. Aspects Med. 30, 1-12 (2009).

23. Holst, B. \& Williamson, G. A critical review of the bioavailability of glucosinolates and related compounds. Nat. Prod. Rep. 21, 425 (2004).

24. Egner, P. A. et al. Bioavailability of Sulforaphane from Two Broccoli Sprout Beverages: Results of a Short-term, Cross-over Clinical Trial in Qidong, China. Cancer Prev. Res. 4, 384-395 (2011).

25. Caulfield, J. L., Wishnok, J. S. \& Tannenbaum, S. R. Nitric oxide-induced deamination of cytosine and guanine in deoxynucleosides and oligonucleotides. J. Biol. Chem. 273, 12689-12695 (1998).

26. Wang, D. \& Dubois, R. N. Prostaglandins and cancer. Gut 55, 115-22 (2006).

27. Heiss, E., Herhaus, C., Klimo, K., Bartsch, H. \& Gerhäuser, C. Nuclear factor kappa $\mathrm{B}$ is a molecular target for sulforaphane-mediated anti-inflammatory mechanisms. J. Biol. Chem. 276, 32008-32015 (2001).

28. Zhang, D. D. \& Hannink, M. Distinct Cysteine Residues in Keap1 Are Required for Keap1-Dependent Ubiquitination of Nrf2 and for Stabilization of Nrf2 by Chemopreventive Agents and Oxidative Stress. Mol. Cell. Biol. 23, 8137-8151 (2003).

29. Dinkova-Kostova, A. T. et al. Direct evidence that sulfhydryl groups of Keap1 are the sensors regulating induction of phase 2 enzymes that protect against carcinogens and oxidants. Proc. Natl. Acad. Sci. 99, 11908-11913 (2002).

30. Zhang, D. D. \& Hannink, M. Distinct cysteine residues in Keap1 are required for Keap1-dependent ubiquitination of Nrf2 and for stabilization of Nrf2 by chemopreventive agents and oxidative stress. Mol. Cell. Biol. 23, 8137-51 (2003).

31. Bonnesen, C., Eggleston, I. M. \& Hayes, J. D. Dietary indoles and isothiocyanates that are generated from cruciferous vegetables can both stimulate apoptosis and confer protection against DNA damage in human colon cell lines. Cancer Res. 61, 6120-6130 (2001).

32. Hu, C., Eggler, A. L., Mesecar, A. D. \& van Breemen, R. B. Modification of keap1 cysteine residues by sulforaphane. Chem. Res. Toxicol. 24, 515-521 (2011).

33. Talalay, P., Fahey, J. W., Holtzclaw, W. D., Prestera, T. \& Zhang, Y. Chemoprotection against cancer by phase 2 enzyme induction. Toxicol. Lett. 82, 173-179 (1995).

34. Prochaska, H. J., Santamaria, A. B. \& Talalay, P. Rapid detection of inducers of enzymes that protect against carcinogens. Proc. Natl. Acad. Sci. 89, 2394-2398 (1992). 
35. Zhang, Y., Talalay, P., Cho, C. G. \& Posner, G. H. A major inducer of anticarcinogenic protective enzymes from broccoli: isolation and elucidation of structure. Proc. Natl. Acad. Sci. 89, 2399-2403 (1992).

36. Brown, K. K. \& Hampton, M. B. Biological targets of isothiocyanates. Biochim. Biophys. Acta - Gen. Subj. 1810, 888-894 (2011).

37. Gamet-Payrastre, L. Signaling pathways and intracellular targets of sulforaphane mediating cell cycle arrest and apoptosis. Curr. Cancer Drug Targets. 6, 135-145 (2006).

38. Myzak, M. C. \& Dashwood, R. H. Histone deacetylases as targets for dietary cancer preventive agents: lessons learned with butyrate, diallyl disulfide, and sulforaphane. Curr. Drug Targets. 7, 443-452 (2006).

39. Cheung, K. L. \& Kong, A.-N. Molecular Targets of Dietary Phenethyl Isothiocyanate and Sulforaphane for Cancer Chemoprevention. AAPS J. 12, 8797 (2010).

40. Mi, L. et al. Identification of potential protein targets of isothiocyanates by proteomics. Chem. Res. Toxicol. 24, 1735-1743 (2011).

41. Clulow, J. A. et al. Competition-based, quantitative chemical proteomics in breast cancer cells identifies new target profiles for sulforaphane. Chem. Commun. 53, 5182-5185 (2017).

42. Angeloni, C. et al. Novel Targets of Sulforaphane in Primary Cardiomyocytes Identified by Proteomic Analysis. PLoS One. 8 (2013).

43. Hof, P., Pluskey, S., Dhe-Paganon, S., Eck, M. J. \& Shoelson, S. E. Crystal structure of the tyrosine phosphatase SHP-2. Cell. 92, 441-450 (1998).

44. Barford, D. \& Neel, B. G. Revealing mechanisms for SH2 domain mediated regulation of the protein tyrosine phosphatase SHP-2. Structure. 6, 249-254 (1998).

45. Lauriol, J., Jaffré, F. \& Kontaridis, M. I. The role of the protein tyrosine phosphatase SHP2 in cardiac development and disease. Semin. Cell Dev. Biol. 37, 73-81 (2015).

46. Volker Strack, et al. The Protein-Tyrosine-Phosphatase SHP2 Is Phosphorylated on Serine Residues 576 and 591 by Protein Kinase $C$ Isoforms $\alpha, \beta 1, \beta 2$, and $\eta$. Biochemistry. 41, 603-608 (2001).

47. Cha, Y. \& Park, K.-S. SHP2 is a downstream target of ZAP70 to regulate JAK1/STAT3 and ERK signaling pathways in mouse embryonic stem cells. FEBS Lett. 584, 4241-4246 (2010).

48. Miura, K. et al. Involvement of EphA2-mediated tyrosine phosphorylation of Shp2 in Shp2-regulated activation of extracellular signal-regulated kinase. Oncogene. 32, 5292-5301 (2013).

49. Harburger, D. S. \& Calderwood, D. A. Integrin signalling at a glance. J. Cell Sci. 122, 159-163 (2009).

50. Giancotti, F. G. \& Ruoslahti, E. Integrin signaling. Science. 285, 1028-1032 (1999).

51. Oh, E.-S. et al. Regulation of Early Events in Integrin Signaling by Protein Tyrosine Phosphatase SHP-2. Mol. Cell. Biol. 19, 3205-3215 (1999). 
52. Van Obberghen, E. et al. Surfing the insulin signaling web. Eur. J. Clin. Invest. 31, 966-77 (2001).

53. Boucher, J., Kleinridders, A. \& Kahn, C. R. Insulin Receptor Signaling in Normal and Insulin-Resistant States. Cold Spring Harb. Perspect. Biol. 6 (2014).

54. Tajan, M., de Rocca Serra, A., Valet, P., Edouard, T. \& Yart, A. SHP2 sails from physiology to pathology. Eur. J. Med. Genet. 58, 509-525 (2015).

55. Van Vactor, D., O'Reilly, A. M. \& Neel, B. G. Genetic analysis of protein tyrosine phosphatases. Curr. Opin. Genet. Dev. 8, 112-126 (1998).

56. Zhang, J., Zhang, F. \& Niu, R. Functions of Shp2 in cancer. J. Cell. Mol. Med. 19, 2075-2083 (2015).

57. Chan, G., Kalaitzidis, D. \& Neel, B. G. The tyrosine phosphatase Shp2 (PTPN11) in cancer. Cancer Metastasis Rev. 27, 179-192 (2008).

58. Grossmann, K. S., Rosário, M., Birchmeier, C. \& Birchmeier, W. The Tyrosine Phosphatase Shp2 in Development and Cancer. in Advances in cancer research. 106, 53-89 (2010).

59. Huang, W.-Q. et al. Structure, Function, and Pathogenesis of SHP2 in Developmental Disorders and Tumorigenesis. Curr. Cancer Drug Targets. 14, 567-588 (2014).

60. Bentires-Alj, M. et al. Activating Mutations of the Noonan Syndrome-Associated SHP2/PTPN11 Gene in Human Solid Tumors and Adult Acute Myelogenous Leukemia. Cancer Res. 62, 6997-7000 (2004).

61. Jopling, C., van Geemen, D. \& den Hertog, J. Shp2 Knockdown and Noonan/LEOPARD Mutant Shp2-Induced Gastrulation Defects. PLoS Genet. 3, 225 (2007).

62. Edouard, T. et al. Functional effects of PTPN11 (SHP2) mutations causing LEOPARD syndrome on epidermal growth factor-induced phosphoinositide 3kinase/AKT/glycogen synthase kinase 3beta signaling. Mol. Cell. Biol. 30, 24982507 (2010).

63. Yu, Z.-H. et al. Molecular Basis of Gain-of-Function LEOPARD SyndromeAssociated SHP2 Mutations. Biochemistry. 53, 4136-4151 (2014).

64. Allanson, J. E. Noonan syndrome. Am. J. Med. Genet. Part C Semin. Med. Genet. 145, 274-279 (2007).

65. Noonan, J. A. Noonan Syndrome. Clin. Pediatr. 33, 548-555 (1994).

66. Romano, A. A. et al. Noonan Syndrome: Clinical Features, Diagnosis, and Management Guidelines. Pediatrics. 126, 746-759 (2010).

67. Yoshida, R. et al. Protein-Tyrosine Phosphatase, Nonreceptor Type 11 Mutation Analysis and Clinical Assessment in 45 Patients with Noonan Syndrome. J. Clin. Endocrinol. Metab. 89, 3359-3364 (2004).

68. Bastida, P., García-Miñaúr, S., Ezquieta, B., Dapena, J. L. \& Sanchez de Toledo, J. Myeloproliferative Disorder in Noonan Syndrome. J. Pediatr. Hematol. Oncol. 33, 43-45 (2011).

69. Kratz, C. P. et al. The mutational spectrum of PTPN11 in juvenile myelomonocytic leukemia and Noonan syndrome/myeloproliferative disease. Blood. 106, 21832185 (2005). 
70. Bezniakow, N., Gos, M. \& Obersztyn, E. The RASopathies as an example of RAS/MAPK pathway disturbances - clinical presentation and molecular pathogenesis of selected syndromes. Dev. period Med. 18, 285-296 (2014).

71. Rauen, K. A. The RASopathies. Annu. Rev. Genomics Hum. Genet. 14, 355-369 (2013).

72. Wu, C.-J. et al. The tyrosine phosphatase SHP-2 is required for mediating phosphatidylinositol 3-kinase/Akt activation by growth factors. Oncogene. 20, 6018-6025 (2001).

73. Araki, T. et al. Noonan syndrome cardiac defects are caused by PTPN11 acting in endocardium to enhance endocardial-mesenchymal transformation. Proc. Natl. Acad. Sci. 106, 4736-4741 (2009).

74. Krenz, M., Yutzey, K. E. \& Robbins, J. Noonan Syndrome Mutation Q79R in Shp2 Increases Proliferation of Valve Primordia Mesenchymal Cells via Extracellular Signal-Regulated Kinase 1/2 Signaling. Circ. Res. 97, 813-820 (2005).

75. Yu, W.-M., Daino, H., Chen, J., Bunting, K. D. \& Qu, C.-K. Effects of a leukemiaassociated gain-of-function mutation of SHP-2 phosphatase on interleukin-3 signaling. J. Biol. Chem. 281, 5426-5434 (2006).

76. Montagner, A. et al. A Novel Role for Gab1 and SHP2 in Epidermal Growth Factor-induced Ras Activation. J. Biol. Chem. 280, 5350-5360 (2004).

77. Keilhack, H., David, F. S., McGregor, M., Cantley, L. C. \& Neel, B. G. Diverse Biochemical Properties of Shp2 Mutants. J. Biol. Chem. 280, 30984-30993 (2005).

78. Araki, T. et al. Mouse model of Noonan syndrome reveals cell type- and gene dosage-dependent effects of Ptpn11 mutation. Nat. Med. 10, 849-857 (2004).

79. Hellmuth, K. et al. Specific inhibitors of the protein tyrosine phosphatase Shp2 identified by high-throughput docking. Proc. Natl. Acad. Sci. 105, 7275-7280 (2008).

80. Mauro Sarmiento et al. Structure-Based Discovery of Small Molecule Inhibitors Targeted to Protein Tyrosine Phosphatase 1B. J. Med. Chem. 43, 146-155 (1999).

81. Liang, F. et al. Aurintricarboxylic Acid Blocks in Vitro and in Vivo Activity of YopH, an Essential Virulent Factor of Yersinia pestis, the Agent of Plague. J. Biol. Chem. 278, 41734-41741 (2003).

82. Zhang, X. et al. Salicylic acid based small molecule inhibitor for the oncogenic Src homology-2 domain containing protein tyrosine phosphatase-2 (SHP2). J. Med. Chem. 53, 2482-2493 (2010).

83. Liu, W. et al. Identification of Cryptotanshinone as an Inhibitor of Oncogenic Protein Tyrosine Phosphatase SHP2. J. Med. Chem. 56, 7212-7221 (2013).

84. Singh, J., Petter, R. C., Baillie, T. A. \& Whitty, A. The resurgence of covalent drugs. Nat. Rev. Drug Discov. 10, 307-317 (2011).

85. Uetrecht, J. Immune-Mediated Adverse Drug Reactions. Chem. Res. Toxicol. 22, 24-34 (2009).

86. Hodgson, J. Admet. Turning chemicals into drugs. Nat. Biotechnol. 19, 722-726 (2001). 
87. Long, M. J. C. \& Aye, Y. Privileged Electrophile Sensors: A Resource for Covalent Drug Development. Cell Chem. Biol. 24, 787-800 (2017).

88. Jöst, C., Nitsche, C., Scholz, T., Roux, L. \& Klein, C. D. Promiscuity and Selectivity in Covalent Enzyme Inhibition: A Systematic Study of Electrophilic Fragments. J. Med. Chem. 57, 7590-7599 (2014).

89. Nemani, R. \& Lee, E. Y. C. Reactivity of Sulfhydryl Groups of the Catalytic Subunits of Rabbit Skeletal Muscle Protein Phosphatases 1 and 2A. Arch. Biochem. Biophys. 300, 24-29 (1993).

90. Ahmed, V. F., Bottini, N. \& Barrios, A. M. Covalent Inhibition of the Lymphoid Tyrosine Phosphatase. Chem. Med.Chem. 9, 296-299 (2014).

91. Sulforaphane in Treating Patients With Recurrent Prostate. Cancer Clinical Trials UK. https://clinicaltrials.gov/ct2/show/results/NCT01228084.

92. Study to Evaluate the Effect of Sulforaphane in Broccoli Sprout Extract on Breast Tissue. Clinical Trials UK. https://clinicaltrials.gov/ct2/show/results/NCT00982319.

93. Sulforaphane in Treating Patients With Recurrent Prostate Cancer. Clinical Trials UK. https://www.clinicaltrials.gov/ct2/show/NCT01228084.

94. The Effects of Broccoli Sprout Extract on Obstructive Lung Disease. Clinical Trials UK. https://www.clinicaltrials.gov/ct2/show/NCT00994604.

95. Effects of Sulforaphane on Normal Prostate Tissue. Clinical Trials UK. https://www.clinicaltrials.gov/ct2/show/NCT00946309.

96. Serafimova, I. M. et al. Reversible targeting of noncatalytic cysteines with chemically tuned electrophiles. Nat. Chem. Biol. 8, 471-476 (2012).

97. Forster, M., Gehringer, M. \& Laufer, S. A. Recent advances in JAK3 inhibition: Isoform selectivity by covalent cysteine targeting. Bioorg. Med. Chem. Lett. 27, 4229-4237 (2017).

98. Ahn, Y.-H. et al. Electrophilic tuning of the chemoprotective natural product sulforaphane. Proc. Natl. Acad. Sci. 107, 9590-9595 (2010).

99. Shibata, T. et al. Transthiocarbamoylation of Proteins by Thiolated Isothiocyanates. J. Biol. Chem. 286, 42150-42161 (2011).

100. Xiao, Q., Liang, H. \& Yuan, Q. Effect of temperature, $\mathrm{pH}$ and light on the stability of sulforaphane solution. Eur. PMC. 193-196 (2010).

101. Franklin, S. J., Dickinson, S. E., Karlage, K. L., Bowden, G. T. \& Myrdal, P. B. Stability of sulforaphane for topical formulation. Drug Dev. Ind. Pharm. 40, 494502 (2014).

102. Jin, Y., Wang, M., Rosen, R. T. \& Ho, C. T. Thermal degradation of sulforaphane in aqueous solution. J. Agric. Food Chem. 47, 3121-3123 (1999).

103. Evgen Pharma | Evgen Pharma | Technology. http://evgen.com/technology/.

104. SFX01 After Subarachnoid Haemorrhage. Clinical Trials UK. https://clinicaltrials.gov/ct2/show/NCT02614742?term=sfx-01\&rank=1.

105. SFX-01 in the Treatment and Evaluation of Metastatic Breast Cancer. Clinical Trials UK. https://clinicaltrials.gov/ct2/show/NCT02970682?term=sfx-01\&rank=2.

106. Araki, T., Nawa, H. \& Neel, B. G. Tyrosyl Phosphorylation of Shp2 Is Required for 
Normal ERK Activation in Response to Some, but Not All, Growth Factors. J. Biol. Chem. 278, 41677-41684 (2003).

107. Cunnick, J. M., Dorsey, J. F., Munoz-Antonia, T., Mei, L. \& Wu, J. Requirement of SHP2 Binding to Grb2-associated Binder-1 for Mitogen-activated Protein Kinase Activation in Response to Lysophosphatidic Acid and Epidermal Growth Factor. J. Biol. Chem. 275, 13842-13848 (2000).

108. Bardelli, A., Longati, P., Gramaglia, D., Stella, M. C. \& Comoglio, P. M. Gab1 coupling to the HGF/Met receptor multifunctional docking site requires binding of Grb2 and correlates with the transforming potential. Oncogene. 15, 3101-3111 (1997).

109. Egan, S. E. et al. Association of Sos Ras exchange protein with Grb2 is implicated in tyrosine kinase signal transduction and transformation. Nature. 363, 45-51 (1993).

110. Lavoie, H. \& Therrien, M. Regulation of RAF protein kinases in ERK signalling. Nat. Publ. Gr. 16, 281-298 (2015).

111. Rajakulendran, T., Sahmi, M., Lefrançois, M., Sicheri, F. \& Therrien, M. A dimerization-dependent mechanism drives RAF catalytic activation. Nature. 461, 542-545 (2009).

112. Roskoski, R. RAF protein-serine/threonine kinases: Structure and regulation. Biochem. Biophys. Res. Commun. 399, 313-317 (2010).

113. Johnson, G. L. \& Lapadat, R. Mitogen-Activated Protein Kinase Pathways Mediated by ERK, JNK, and p38 Protein Kinases. Science. 298, 1911-1912 (2002).

114. Roskoski, R. $\Uparrow$. MEK1/2 dual-specificity protein kinases: Structure and regulation. Biochem. Biophys. Res. Commun. 417, 5-10 (2012).

115. Steelman, L. S. et al. JAK/STAT, Raf/MEK/ERK, PI3K/Akt and BCR-ABL in cell cycle progression and leukemogenesis. Leukemia. 18, 189-218 (2004).

116. Nakano, H. et al. Differential regulation of IB kinase and by two upstream kinases, NF-B-inducing kinase and mitogen-activated protein kinaseERK kinase kinase-1. Biochemistry. 95, 3537-3542 (1998).

117. Chang, F. et al. Signal transduction mediated by the Ras/Raf/MEK/ERK pathway from cytokine receptors to transcription factors: potential targeting for therapeutic intervention. Leukemia. 17, 1263-1293.

118. Brennan, P., Babbage, J. W., Thomas, G. \& Cantrell, D. p70(s6k) integrates phosphatidylinositol 3-kinase and rapamycin-regulated signals for E2F regulation in T lymphocytes. Mol. Cell. Biol. 19, 4729-4738 (1999).

119. Leibiger, I. B., Leibiger, B., Moede, T. \& Berggren, P. O. Exocytosis of insulin promotes insulin gene transcription via the insulin receptor/PI-3 kinase/p70 s6 kinase and CaM kinase pathways. Mol. Cell. 1, 933-938 (1998).

120. Bynda, S., Heir, P. \& Ohh, M. Src promotes GTPase activity of Ras via tyrosine 32 phosphorylation. Prot. Nacl. Acad. Sci. 111 (2014).

121. Agazie, Y. M. et al. Molecular Mechanism for a Role of SHP2 in Epidermal Growth Factor Receptor Signaling. Mol. Cell. Biol. 23, 7875-7886 (2003).

122. Bunda, S. et al. Inhibition of SHP2-mediated dephosphorylation of Ras suppresses oncogenesis. Nat. Commun. 6 (2015). 
123. Hanafusa, H., Torii, S., Yasunaga, T. \& Nishida, E. Sprouty1 and Sprouty2 provide a control mechanism for the Ras/MAPK signalling pathway. Nat Cell Biol. 4, 850858 (2002).

124. Hanafusa, H., Torii, S., Yasunaga, T., Matsumoto, K. \& Nishida, E. Shp2, an SH2containing Protein-tyrosine Phosphatase, Positively Regulates Receptor Tyrosine Kinase Signaling by Dephosphorylating and Inactivating the Inhibitor Sprouty. J. Biol. Chem. 279, 22992-22995 (2004).

125. Yang, W. et al. An Shp2/SFK/Ras/Erk Signaling Pathway Controls Trophoblast Stem Cell Survival. Dev. Cell. 10, 317-327 (2006).

126. Zhang, S. Q. et al. Shp2 Regulates Src Family Kinase Activity and Ras/Erk Activation by Controlling Csk Recruitment signaling strategy. Molecular Cell. 13, 341-355 (2004).

127. Rawlings, J. S., Rosler, K. M. \& Harrison, D. A. The JAK/STAT signaling pathway. J. Cell Sci. 117, 1281-1283 (2004).

128. Ju“, J., Gent, J., Van Den Eijnden, M., Van Kerkhof, P. \& Strous, G. J. Dimerization and Signal Transduction of the Growth Hormone Receptor. Mol Endocrinol. 17, 967-975 (2003).

129. Babon, J. J., Lucet, I. S., Murphy, J. M., Nicola, N. A. \& Varghese, L. N. The molecular regulation of Janus kinase (JAK) activation. Biochem J. 462, 1-13 (2014).

130. Waters, M. J. \& Brooks, A. J. JAK2 activation by growth hormone and other cytokines. Biochem. J. 466, 1-11 (2015).

131. Argetsinger, L. S. et al. Identification of JAK2 as a growth hormone receptorassociated tyrosine kinase. Cell. 74, 237-244 (1993).

132. Xu, D. \& Qu, C.-K. Protein tyrosine phosphatases in the JAK/STAT pathway. Front. Biosci. 13, 4925-4932 (2008).

133. Hennighausen, L. \& Robinson, G. W. Interpretation of cytokine signaling through the transcription factors STAT5A and STAT5B. Genes Dev. 22, 711-721 (2008).

134. Basham, B. et al. In vivo identification of novel STAT5 target genes. Nucleic Acids Res. 36, 3802-3818 (2008).

135. Noguchi, T., Matozaki, T., Horita, K., Fujioka, Y. \& Kasuga, M. Role of SH-PTP2, a protein-tyrosine phosphatase with Src homology 2 domains, in insulinstimulated Ras activation. Mol. Cell. Biol. 14, 6674-6682 (1994).

136. Eck, M. J., Pluskey, S., Trüb, T., Harrison, S. C. \& Shoelson, S. E. Spatial constraints on the recognition of phosphoproteins by the tandem SH2 domains of the phosphatase SH-PTP2. Nature. 379, 277-280 (1996).

137. Allanson, J. E. Noonan syndrome. J. Med. Genet. 24, 9-13 (1987).

138. Chacko, E., Graber, E., Regelmann, M. O., Wallach, E. \& Costin, G. Update on Turner and Noonan Syndromes. Endocrinol. Metab. Clin. North Am. 41, 713-734 (2012).

139. Rogol, A. D. Clinical and humanistic aspects of growth hormone deficiency and growth-related disorders. Am. J. Manag. Care. 17, S4-S10 (2011).

140. Limal, J.-M. et al. Noonan Syndrome: Relationships between Genotype, Growth, and Growth Factors. J. Clin. Endocrinol. Metab. 91, 300-306 (2006). 
141. Serra-Nedelec, A. D. R. et al. Noonan syndrome-causing SHP2 mutants inhibit insulin-like growth factor 1 release via growth hormone-induced ERK hyperactivation, which contributes to short stature. Proc. Natl. Acad. Sci. 109, 4257-4262 (2012).

142. Chen, C.-Y., Willard, D. \& Rudolph, J. Redox Regulation of SH2-DomainContaining Protein Tyrosine Phosphatases by Two Backdoor Cysteines. Biochemistry. 48, 1399-1409 (2009).

143. Lewis, S. Investigation of the kinetics and mechanisms of the inactivation of ptpshp2 by peroxycarbonate and phytochemicals. MSc thesis. (2012).

144. Oh, J. Y., Giles, N., Landar, A. \& Darley-Usmar, V. Accumulation of 15-deoxydelta(12,14)-prostaglandin J2 adduct formation with Keap1 over time: effects on potency for intracellular antioxidant defence induction. Biochem. J. 411, 297-306 (2008).

145. Lorenz, J., Glatt, H. R., Fleischmann, R., Ferlinz, R. \& Oesch, F. Drug metabolism in man and its relationship to that in three rodent species: monooxygenase, epoxide hydrolase, and glutathione S-transferase activities in subcellular fractions of lung and liver. Biochem. Med. 32, 43-56 (1984).

146. Nau, H. Species differences in pharmacokinetics and drug teratogenesis. Environ. Health Perspect. 70, 113-129 (1986).

147. Choi, W. Y., Choi, B. T., Lee, W. H. \& Choi, Y. H. Sulforaphane generates reactive oxygen species leading to mitochondrial perturbation for apoptosis in human leukemia U937 cells. Biomed. Pharmacother. 62, 637-644 (2008).

148. Cho, S.-D. et al. Involvement of c-Jun N-terminal Kinase in G2/M Arrest and Caspase-Mediated Apoptosis Induced by Sulforaphane in DU145 Prostate Cancer Cells. Nutr. Cancer. 52, 213-224 (2005).

149. Gamet-Payrastre, L. et al. Sulforaphane Sensitizes Tumor Necrosis FactorRelated Apoptosis-Inducing Ligand (TRAIL)-Resistant Hepatoma Cells to TRAILInduced Apoptosis through Reactive Oxygen Species-Mediated Up-regulation of DR5. Cancer Res. 60, 1426-1433 (2000).

150. Hu, R. et al. Gene expression profiles induced by cancer chemopreventive isothiocyanate sulforaphane in the liver of $\mathrm{C} 57 \mathrm{BL} / 6 \mathrm{~J}$ mice and $\mathrm{C} 57 \mathrm{BL} / 6 \mathrm{~J} / \mathrm{Nrf} 2$ (-/-) mice. Cancer Lett. 243, 170-192 (2006).

151. Keum, Y.-S. et al. Mechanism of Action of Sulforaphane: Inhibition of p38 Mitogen-Activated Protein Kinase Isoforms Contributing to the Induction of Antioxidant Response Element-Mediated Heme Oxygenase-1 in Human Hepatoma HepG2 Cells. Cancer Res. 66, 8804-8817 (2006).

152. Jo, C. et al. Sulforaphane induces autophagy through ERK activation in neuronal cells. FEBS Lett. 588, 3081-3088 (2014).

153. Yeh, C.-T., Chiu, H.-F. \& Yen, G.-C. Protective effect of sulforaphane on indomethacin-induced cytotoxicity via heme oxygenase-1 expression in human intestinal Int 407 cells. Mol. Nutr. Food Res. 53, 1166-1176 (2009).

154. $\mathrm{Li}, \mathrm{C}$. et al. Sulforaphane Inhibits Invasion via Activating ERK1/2 Signaling in Human Glioblastoma U87MG and U373MG Cells. PLoS One. 9 (2014).

155. Hakak, Y., Hsu, Y. S. \& Martin, G. S. Shp-2 mediates v-Src-induced morphological changes and activation of the anti-apoptotic protein kinase Akt. Oncogene. 19, 3164-3171 (2000). 
156. Zhang, S. Q. et al. Receptor-specific regulation of phosphatidylinositol 3'-kinase activation by the protein tyrosine phosphatase Shp2. Mol. Cell. Biol. 22, 40624072 (2002).

157. Chong, H., Lee, J. \& Guan, K. L. Positive and negative regulation of Raf kinase activity and function by phosphorylation. EMBO J. 20, 3716-3727 (2001).

158. Meng, D., Shi, X., Jiang, B.-H. \& Fang, J. Insulin-like growth factor-I (IGF-I) induces epidermal growth factor receptor transactivation and cell proliferation through reactive oxygen species. Free Radic. Biol. Med. 42, 1651-1660 (2007).

159. Guyton, K. Z., Liu, Y., Gorospe, M., Xu, Q. \& Holbrook, N. J. Activation of mitogenactivated protein kinase by $\mathrm{H} 2 \mathrm{O} 2$. Role in cell survival following oxidant injury. $J$. Biol. Chem. 271, 4138-4142 (1996).

160. Kamata, H. et al. Reactive Oxygen Species Promote TNFa-Induced Death and Sustained JNK Activation by Inhibiting MAP Kinase Phosphatases. Cell. 120, 649-661 (2005).

161. Choi, B.-H., Hur, E.-M., Lee, J.-H., Jun, D.-J. \& Kim, K.-T. Protein kinase C deltamediated proteasomal degradation of MAP kinase phosphatase-1 contributes to glutamate-induced neuronal cell death. J. Cell Sci. 119, 1329-1340 (2006).

162. Hsu, Y.-C., Chang, S.-J., Wang, M.-Y., Chen, Y.-L. \& Huang, T.-Y. Growth Inhibition and Apoptosis of Neuroblastoma Cells Through ROS-Independent MEK/ERK Activation by Sulforaphane. Cell Biochem. Biophys. 66, 765-774 (2013).

163. Jansoon, J.-O., Eden, S. \& Isaksson, O. Sexual Dimorphism in the Control of Growth Hormone Secretion. Endocr. Rev. 6, 128-150 (1985).

164. Fernández, L. et al. Desensitization of the Growth Hormone-Induced Janus Kinase 2 (Jak 2)/Signal Transducer and Activator of Transcription 5 (Stat5)Signaling Pathway Requires Protein Synthesis and Phospholipase C ${ }^{1}$. Endocrinology. 139, 1815-1824 (1998).

165. van Kerkhof, P., Govers, R., Alves dos Santos, C. M. \& Strous, G. J. Endocytosis and degradation of the growth hormone receptor are proteasome-dependent. $J$. Biol. Chem. 275, 1575-1580 (2000).

166. Wormald, S. \& Hilton, D. J. Inhibitors of Cytokine Signal Transduction. J. Biol. Chem. 279, 821-824 (2004).

167. Krebs, D. L. \& Hilton, D. J. SOCS Proteins: Negative Regulators of Cytokine Signaling. Stem Cells. 19, 378-387 (2001).

168. Waxman, D. J., Ram, P. A., Park, S. H. \& Choi, H. K. Intermittent plasma growth hormone triggers tyrosine phosphorylation and nuclear translocation of a liverexpressed, Stat 5-related DNA binding protein. Proposed role as an intracellular regulator of male-specific liver gene transcription. J. Biol. Chem. 270, 1326213270 (1995).

169. Li, X. et al. Sulforaphane promotes apoptosis, and inhibits proliferation and selfrenewal of nasopharyngeal cancer cells by targeting STAT signal through miRNA124-3p. Biomed. Pharmacother. 103, 473-481 (2018).

170. Hahm, E.-R. \& Singh, S. V. Sulforaphane Inhibits Constitutive and Interleukin-6Induced Activation of Signal Transducer and Activator of Transcription 3 in Prostate Cancer Cells. Cancer Prev. Res. 3, 484-494 (2010). 
171. Pinz, S., Unser, S. \& Rascle, A. The Natural Chemopreventive Agent Sulforaphane Inhibits STAT5 Activity. PLoS One. 9 (2014).

172. Weiss-Messer, E. et al. Growth hormone $(\mathrm{GH})$ receptors in prostate cancer: gene expression in human tissues and cell lines and characterization, $\mathrm{GH}$ signaling and androgen receptor regulation in LNCaP cells. Mol. Cell. Endocrinol. 220, 109-123 (2004).

173. Conway-Campbell, B. L. et al. Nuclear targeting of the growth hormone receptor results in dysregulation of cell proliferation and tumorigenesis. Proc. Natl. Acad. Sci. 104, 13331-13336 (2007).

174. Conway-Campbell, B. L., Brooks, A. J., Robinson, P. J., Perani, M. \& Waters, M. J. The Extracellular Domain of the Growth Hormone Receptor Interacts with Coactivator Activator to Promote Cell Proliferation. Mol. Endocrinol. 22, 2190 2202 (2008).

175. Bricker, G. V. et al. Isothiocyanate metabolism, distribution, and interconversion in mice following consumption of thermally processed broccoli sprouts or purified sulforaphane. Mol. Nutr. Food Res. 58, 1991-2000 (2014).

176. Anders, M. W., Marion W. \& Dekant, W. Wolfgang. Conjugation-dependent carcinogenicity and toxicity of foreign compounds. Academic Press. (1994).

177. Baillie, T. A. \& Slatter, J. G. Glutathione: a vehicle for the transport of chemically reactive metabolites in vivo. Acc. Chem. Res. 24, 264-270 (1991).

178. Bruggeman, I. M., Temmink, J. H. \& van Bladeren, P. J. Glutathione- and cysteine-mediated cytotoxicity of allyl and benzyl isothiocyanate. Toxicol. Appl. Pharmacol. 83, 349-359 (1986).

179. Meyer, D. J., Crease, D. J. \& Ketterer, B. Forward and reverse catalysis and product sequestration by human glutathione $S$-transferases in the reaction of GSH with dietary aralkyl isothiocyanates. Biochem. J. 306, 565-569 (1995).

180. Zhang, Y. S., Kolm, R. H., Mannervik, B. \& Talalay, P. Reversible Conjugation of Isothiocyanates with Glutathione Catalyzed by Human Glutathione Transferases. Biochem. Biophys. Res. Commun. 206, 748-755 (1995).

181. Zhang, Y. \& Callaway, E. C. High cellular accumulation of sulphoraphane, a dietary anticarcinogen, is followed by rapid transporter-mediated export as a glutathione conjugate. Biochem. J. 364, 301-307 (2002).

182. Shibata, T. et al. Transthiocarbamoylation of proteins by thiolated isothiocyanates. J. Biol. Chem. 286, 42150-42161 (2011).

183. Pau, P. C. F., Berg, J. O. \& McMillan, W. G. Application of Stokes' law to ions in aqueous solution. J. Phys. Chem. 94, 2671-2679 (1990).

184. Ernst, E. Rise in popularity of complementary and alternative medicine: reasons and consequences for vaccination. Vaccine. 20, 90-93 (2001).

185. Ernst, E. Clinical Rheumatology Usage of Complementary Therapies in Rheumatology: A Systematic Review. Clinical Rheumatology. 17, 301-305 (1998).

186. Ernst, E. Complementary Therapies for Asthma: What Patients Use. J. Asthma. 35, 667-671 (1998).

187. Ernst, E. The prevalence of complementary/Alternative medicine in cancer. Cancer. 83, 777-782 (1998). 
188. Wąsik, A. \& Antkiewicz-Michaluk, L. The mechanism of neuroprotective action of natural compounds. Pharmacol. Reports. 69, 851-860 (2017).

189. Ferrucci, V., Boffa, I., De Masi, G. \& Zollo, M. Natural compounds for pediatric cancer treatment. Naunyn. Schmiedebergs. Arch. Pharmacol. 389, 131-149 (2016).

190. Wąsik, A. et al. Neuroprotective Effect of the Endogenous Amine 1MeTIQ in an Animal Model of Parkinson's Disease. Neurotox. Res. 29, 351-363 (2016).

191. Antkiewicz-Michaluk, L. et al. Different action on dopamine catabolic pathways of two endogenous 1,2,3,4-tetrahydroisoquinolines with similar antidopaminergic properties. J. Neurochem. 78, 100-108 (2001).

192. Ren, J., Fan, C., Chen, N., Huang, J. \& Yang, Q. Resveratrol Pretreatment Attenuates Cerebral Ischemic Injury by Upregulating Expression of Transcription Factor Nrf2 and HO-1 in Rats. Neurochem. Res. 36, 2352-2362 (2011).

193. Mahal, H. S. \& Mukherjee, T. Scavenging of reactive oxygen radicals by resveratrol: antioxidant effect. Res. Chem. Intermed. 32, 59-71 (2006).

194. Coşkun, Ş., Gönül, B., Güzel, N. A. \& Balabanlí, B. The effects of vitamin C supplementation on oxidative stress and antioxidant content in the brains of chronically exercised rats. Mol. Cell. Biochem. 280, 135-138 (2005).

195. Goswami, A. R., Dutta, G. \& Ghosh, T. Effects of vitamin C on the hypobaric hypoxia-induced immune changes in male rats. Int. J. Biometeorol. 58, 19611971 (2014).

196. Zhang, Y.-J. et al. Effects of 20 Selected Fruits on Ethanol Metabolism: Potential Health Benefits and Harmful Impacts. Int. J. Environ. Res. Public Health. 13, 399 (2016).

197. Subash, S. et al. Consumption of fig fruits grown in Oman can improve memory, anxiety, and learning skills in a transgenic mice model of Alzheimer's disease. Nutr. Neurosci. 19, 475-483 (2016).

198. Arora, N., Shah, K. \& Pandey-Rai, S. Inhibition of imiquimod-induced psoriasislike dermatitis in mice by herbal extracts from some Indian medicinal plants. Protoplasma. 253, 503-515 (2016).

199. Salloum, F. N. et al. Beetroot juice reduces infarct size and improves cardiac function following ischemia-reperfusion injury: Possible involvement of endogenous H2S. Exp. Biol. Med. 240, 669-681 (2015).

200. Noyan-Ashraf, M. H., Wu, L., Wang, R. \& Juurlink, B. H. J. Dietary approaches to positively influence fetal determinants of adult health. FASEB J. 20, 371-373 (2006).

201. Wu, L. et al. Dietary approach to attenuate oxidative stress, hypertension, and inflammation in the cardiovascular system. Proc. Natl. Acad. Sci. 101, 7094-7099 (2004).

202. Mukherjee, S. et al. Comparison of the protective effects of steamed and cooked broccolis on ischaemia-reperfusion-induced cardiac injury. Br. J. Nutr. 103, 815 (2010).

203. Beaver, L. M. et al. Broccoli Sprouts Delay Prostate Cancer Formation and Decrease Prostate Cancer Severity with a Concurrent Decrease in HDAC3 Protein Expression in Transgenic Adenocarcinoma of the Mouse Prostate 
(TRAMP) Mice. Curr. Dev. Nutr. 2 (2018).

204. Clarke, J. D. et al. Bioavailability and inter-conversion of sulforaphane and erucin in human subjects consuming broccoli sprouts or broccoli supplement in a crossover study design. Pharmacol. Res. 64, 456-463 (2011).

205. Hanschmann, E.-M., Godoy, J. R., Berndt, C., Hudemann, C. \& Lillig, C. H. Thioredoxins, glutaredoxins, and peroxiredoxins-molecular mechanisms and health significance: from cofactors to antioxidants to redox signaling. Antioxid. Redox Signal. 19, 1539-1605 (2013).

206. Holmgren, A. Thioredoxin structure and mechanism: conformational changes on oxidation of the active-site sulfhydryls to a disulfide. Structure. 3, 239-243 (1995).

207. Lillig, C. H., Berndt, C. \& Holmgren, A. Glutaredoxin systems. Biochim. Biophys. Acta - Gen. Subj. 1780, 1304-1317 (2008).

208. Talalay, P. Chemoprotection against cancer by induction of phase 2 enzymes. BioFactors. 12, 5-11 (2000).

209. Zhang, Y., Talalay, P., Cho, C. G. \& Posner, G. H. A major inducer of anticarcinogenic protective enzymes from broccoli: isolation and elucidation of structure. Proc. Natl. Acad. Sci. 89, 2399-2403 (1992).

210. Tanito, M. et al. Sulforaphane Induces Thioredoxin through the AntioxidantResponsive Element and Attenuates Retinal Light Damage in Mice. Investig. Opthalmology Vis. Sci. 46, 979 (2005).

211. Agyeman, A. S. et al. Transcriptomic and proteomic profiling of KEAP1 disrupted and sulforaphane-treated human breast epithelial cells reveals common expression profiles. Breast Cancer Res. Treat. 132, 175-187 (2012).

212. Marengo-Rowe, A. J. Structure-function relations of human hemoglobins. Proc. Bayl. Univ. Med. Cent. 19, 239-245 (2006).

213. Schechter, A. N. Hemoglobin research and the origins of molecular medicine. Blood. 112, 3927-3938 (2008).

214. Steinberg, M. H. Disorders of hemoglobin : genetics, pathophysiology, and clinical management. Cambridge University Press. (2001).

215. Antonini, E. \& Brunori, M. Hemoglobin and myoglobin in their reactions with ligands. North-Holland Pub. Co. (971).

216. Gaston, B. et al. Essential role of hemoglobin beta-93-cysteine in posthypoxia facilitation of breathing in conscious mice. J. Appl. Physiol. 116, 1290-1299 (1985).

217. Doctor, A. et al. Hemoglobin conformation couples erythrocyte S-nitrosothiol content to O2 gradients. Proc. Natl. Acad. Sci. 102, 5709-5714 (2005).

218. Gaston, B., Singel, D., Doctor, A. \& Stamler, J. S. S-Nitrosothiol Signaling in Respiratory Biology. Am. J. Respir. Crit. Care Med. 173, 1186-1193 (2006).

219. Gow, A. J. \& Stamler, J. S. Reactions between nitric oxide and haemoglobin under physiological conditions. Nature. 391, 169-173 (1998).

220. Jia, L., Bonaventura, C., Bonaventura, J. \& Stamler, J. S. S-nitrosohaemoglobin: a dynamic activity of blood involved in vascular control. Nature. 380, 221-226 (1996). 
221. Singel, D. J. \& Stamler, J. S. Chemical physiology of blood flow regulation by red blood cells: Annu. Rev. Physiol. 67, 99-145 (2005).

222. Ross, J. M., Fairchild, H. M., Weldy, J. \& Guyton, A. C. Autoregulation of blood flow by oxygen lack. Am. J. Physiol. Content. 202, 21-24 (1962).

223. McMahon, T. J. et al. Nitric oxide in the human respiratory cycle. Nat. Med. 8, 711-717 (2002).

224. Zhang, R. et al. Hemoglobin $\beta$ Cys93 is essential for cardiovascular function and integrated response to hypoxia. Proc. Natl. Acad. Sci. 112, 6425-6430 (2015).

225. Pawloski, J. R., Hess, D. T. \& Stamler, J. S. Export by red blood cells of nitric oxide bioactivity. Nature. 409, 622-626 (2001).

226. Schechter, A. N. \& Gladwin, M. T. Hemoglobin and the Paracrine and Endocrine Functions of Nitric Oxide. N. Engl. J. Med. 348, 1483-1485 (2003).

227. Schechter, A. N. Hemoglobin research and the origins of molecular medicine. Blood. 112, 3927 (2008).

228. Gladwin, M. T. How Red Blood Cells Process Nitric Oxide: Evidence for the Nitrite Hypothesis. Circulation. 135, 177-179 (2017).

229. Gladwin, M. T. et al. Relative role of heme nitrosylation and beta -cysteine 93 nitrosation in the transport and metabolism of nitric oxide by hemoglobin in the human circulation. Proc. Natl. Acad. Sci. 97, 9943-9948 (2000).

230. Gladwin, M. T. et al. Role of circulating nitrite and S-nitrosohemoglobin in the regulation of regional blood flow in humans. Proc. Natl. Acad. Sci. 97, 1148211487 (2000).

231. Cosby, K. et al. Nitrite reduction to nitric oxide by deoxyhemoglobin vasodilates the human circulation. Nat. Med. 9, 1498-1505 (2003).

232. Bailey, D. M. et al. Nitrite and Nitrosohemoglobin Exchange Across the Human Cerebral and Femoral Circulation: Relationship to Basal and Exercise Blood Flow Responses to Hypoxia. Circulation. 135, 166-176 (2017).

233. Kosaka, H. Nitric oxide and hemoglobin interactions in the vasculature. Biochim. Biophys. Acta. 1411, 370-377 (1999).

234. Yonetani, T., Tsuneshige, A., Zhou, Y. \& Chen, X. Electron paramagnetic resonance and oxygen binding studies of alpha-Nitrosyl hemoglobin. A novel oxygen carrier having no-assisted allosteric functions. J. Biol. Chem. 273, 2032320333 (1998).

235. Isbell, T. S. et al. SNO-hemoglobin is not essential for red blood cell-dependent hypoxic vasodilation. Nat. Med. 14, 773-777 (2008).

236. Straub, A. C. et al. Endothelial cell expression of haemoglobin a regulates nitric oxide signalling. Nature. 491, 473-477 (2012).

237. Burgoyne, J. R., Prysyazhna, O., Rudyk, O. \& Eaton, P. cGMP-Dependent Activation of Protein Kinase G Precludes Disulfide Activation. Hypertension. 60, 1301-1308 (2012).

238. Rahaman, M. M. \& Straub, A. C. The emerging roles of somatic globins in cardiovascular redox biology and beyond. Redox Biol. 1, 405-410 (2013).

239. Butcher, J. T., Johnson, T., Beers, J., Columbus, L. \& Isakson, B. E. Hemoglobin 
$\alpha$ in the blood vessel wall. Free Radic. Biol. Med. 73, 136-142 (2014).

240. Singel, D. J. \& Stamler, J. S. Chemical physiology of blood flow regulation by red blood cells: the role of nitric oxide and S-nitrosohemoglobin. Annu. Rev. Physiol. 67, 99-145 (2005).

241. Grek, C. L., Newton, D. A., Spyropoulos, D. D. \& Baatz, J. E. Hypoxia UpRegulates Expression of Hemoglobin in Alveolar Epithelial Cells. Am. J. Respir. Cell Mol. Biol. 44, 439-447 (2011).

242. Nishi, H. et al. Hemoglobin Is Expressed by Mesangial Cells and Reduces Oxidant Stress. J. Am. Soc. Nephrol. 19, 1500-1508 (2008).

243. Liu, W., Baker, S. S., Baker, R. D., Nowak, N. J. \& Zhu, L. Upregulation of Hemoglobin Expression by Oxidative Stress in Hepatocytes and Its Implication in Nonalcoholic Steatohepatitis. PLoS One. 6 (2011).

244. Liu, L., Zeng, M. \& Stamler, J. S. Hemoglobin induction in mouse macrophages. Proc. Natl. Acad. Sci. 96, 6643-6647 (1999).

245. Richter, F., Meurers, B. H., Zhu, C., Medvedeva, V. P. \& Chesselet, M.-F. Neurons express hemoglobin $\alpha$ - and $\beta$-chains in rat and human brains. J. Comp. Neurol. 515, 538-547 (2009).

246. Mi, L., Di Pasqua, A. J. \& Chung, F.-L. Proteins as binding targets of isothiocyanates in cancer prevention. Carcinogenesis. 32, 1405-1413 (2011).

247. Cross, J. V. et al. Nutrient isothiocyanates covalently modify and inhibit the inflammatory cytokine macrophage migration inhibitory factor (MIF). Biochem. J. 423, 315-321 (2009).

248. Ouertatani-Sakouhi, H. et al. A New Class of Isothiocyanate-Based Irreversible Inhibitors of Macrophage Migration Inhibitory Factor. Biochemistry. 48, 98589870 (2009).

249. Kawakishi, S., Goto, T. \& Namiki, M. Oxidative Scission of the Disulfide Bond of Cystine and Polypeptides by the Action of Allyl Isothiocyanate. Agric. Biol. Chem. 47, 2071-2076 (1983).

250. Yamabe, T., Akagi, K., Hashimoto, T., Nagata, S. \& Fukui, K. Hydrogen Bonding Type Charge Transfer Interaction between Thiols and Amines. J. Chem. Soc. (1977).

251. Levy, D. E. Daniel E. Arrow pushing in organic chemistry : an easy approach to understanding reaction mechanisms. Wiley. (2008).

252. Nakamura, T., Kawai, Y., Kitamoto, N., Osawa, T. \& Kato, Y. Covalent Modification of Lysine Residues by Allyl Isothiocyanate in Physiological Conditions: Plausible Transformation of Isothiocyanate from Thiol to Amine. Chem. Res. Toxicol. 22, 536-542 (2009).

253. Swoboda, G. \& Hasselbach, W. Reaction of fluorescein isothiocyanate with thiol and amino groups of sarcoplasmic ATPase. Zeitschrift fur Naturforschung. Sect. C, Biosci. 40, 863-875.

254. Sugio, S., Kashima, A., Mochizuki, S., Noda, M. \& Kobayashi, K. Crystal structure of human serum albumin at 2.5 A resolution. Protein Eng. 12, 439-446 (1999).

255. Busher, J. T. Serum Albumin and Globulin. Clinical Methods: Chapter 1. 3, (1990).

256. Yang, X. et al. Simple paper-based test for measuring blood hemoglobin 
concentration in resource-limited settings. Clin. Chem. 59, 1506-1513 (2013).

257. Saxton, T. M. et al. Abnormal mesoderm patterning in mouse embryos mutant for the SH2 tyrosine phosphatase Shp-2. EMBO J. 16, 2352-2364 (1997).

258. Yang, W. et al. An Shp2/SFK/Ras/Erk Signaling Pathway Controls Trophoblast Stem Cell Survival. Dev. Cell. 10, 317-327 (2006).

259. Dehan, R. L. Migration patterns of the precardiac mesoderm in the early chick embrvo. Exp. Cell Res. 29, 544-560 (1963).

260. Warkman, A. S. \& Krieg, P. A. Xenopus as a model system for vertebrate heart development. Semin. Cell Dev. Biol. 18, 46-53 (2007).

261. Brade, T., Pane, L. S., Moretti, A., Chien, K. R. \& Laugwitz, K.-L. Embryonic Heart Progenitors and Cardiogenesis. Cold Spring Harb. Perspect. Med. 3 (2013).

262. Langdon, Y. G., Goetz, S. C., Berg, A. E., Swanik, J. T. \& Conlon, F. L. SHP-2 is required for the maintenance of cardiac progenitors. Development. 134, 41194130 (2007).

263. Pampfer, S. \& Donnay, I. Apoptosis at the time of embryo implantation in mouse and rat. Cell Death Differ. 6, 533-545 (1999).

264. Buckingham, M., Meilhac, S. \& Zaffran, S. Building the mammalian heart from two sources of myocardial cells. Nat. Rev. Genet. 6, 826-835 (2005).

265. Stewart, R. A. et al. Phosphatase-Dependent and -Independent Functions of Shp2 in Neural Crest Cells Underlie LEOPARD Syndrome Pathogenesis. Dev. Cell. 18, 750-762 (2010).

266. Hirokawa, N., Tanaka, Y., Okada, Y. \& Takeda, S. Nodal Flow and the Generation of Left-Right Asymmetry. Cell. 125, 33-45 (2006).

267. Sun, X., Meyers, E. N., Lewandoski, M. \& Martin, G. R. Targeted disruption of Fgf8 causes failure of cell migration in the gastrulating mouse embryo. Genes Dev. 13, 1834-1846 (1999).

268. Boettger, T., Wittler, L. \& Kessel, M. FGF8 functions in the specification of the right body side of the chick. Curr. Biol. 9, 277-280 (1999).

269. Smith, D. J., Blake, J. R. \& Gaffney, E. A. Fluid mechanics of nodal flow due to embryonic primary cilia. J. R. Soc. Interface 5, 567-573 (2008).

270. Bonetti, M. et al. Noonan and LEOPARD syndrome Shp2 variants induce heart displacement defects in zebrafish. Development. 141, 1961-1970 (2014).

271. Tanaka, Y., Okada, Y. \& Hirokawa, N. FGF-induced vesicular release of Sonic hedgehog and retinoic acid in leftward nodal flow is critical for left-right determination. Nature. 435, 172-177 (2005).

272. Kreiling, J. A. et al. Suppression of the endoplasmic reticulum calcium pump during zebrafish gastrulation affects left-right asymmetry of the heart and brain. Mech. Dev. 125, 396-410 (2008).

273. Yoshiba, S. et al. Cilia at the node of mouse embryos sense fluid flow for left-right determination via Pkd2. Science. 338, 226-231 (2012).

274. Hagiwara, Y. et al. SHP2-mediated signaling cascade through gp130 is essential for LIF-dependent ICaL, [Ca2+]i transient, and APD increase in cardiomyocytes. J. Mol. Cell. Cardiol. 43, 710-716 (2007). 
275. Wang, Q., Abreu, M. T. H., Siminovitch, K., Downey, G. P. \& McCulloch, C. A. Phosphorylation of SHP-2 Regulates Interactions between the Endoplasmic Reticulum and Focal Adhesions to Restrict Interleukin-1-induced Ca Signaling. J. Biol. Chem. 281, 31093-31105 (2006).

276. Noël, E. S. et al. A Nodal-independent and tissue-intrinsic mechanism controls heart-looping chirality. Nat. Commun. 4, 2754 (2013).

277. Langdon, Y. et al. SHP-2 acts via ROCK to regulate the cardiac actin cytoskeleton. Development. 139, 948-957 (2012).

278. Lin, C.-J., Lin, C.-Y., Chen, C.-H., Zhou, B. \& Chang, C.-P. Partitioning the heart: mechanisms of cardiac septation and valve development. Development. 139, 3277-3299 (2012).

279. Abdelwahid, E., Pelliniemi, L. J. \& Jokinen, E. Cell death and differentiation in the development of the endocardial cushion of the embryonic heart. Microsc. Res. Tech. 58, 395-403 (2002).

280. Combs, M. D. \& Yutzey, K. E. Heart Valve Development: regulatory networks in development and disease. Circ. Res. 105, 408-421 (2009).

281. Armstrong, E. J. \& Bischoff, J. Heart Valve Development: endothelial cell signaling and differentiation. Circ. Res. 95, 459-470 (2004).

282. Chen, B. et al. Mice mutant for Egfr and Shp2 have defective cardiac semilunar valvulogenesis. Nat. Genet. 24, 296-299 (2000).

283. Anderson, R. H., Webb, S., Brown, N. A., Lamers, W. \& Moorman, A. Development of the heart: Septation of the atriums and ventricles. Heart. 89, 949958 (2003).

284. Wagner, M. \& Siddiqui, M. A. Q. Signal transduction in early heart development: ventricular chamber specification, trabeculation, and heart valve formation. Exp. Biol. Med. 232, 866-880 (2007).

285. Nakamura, T. et al. Mediating ERK1/2 signaling rescues congenital heart defects in a mouse model of Noonan syndrome. J. Clin. Invest. 117, 2123-2132 (2007).

286. Sucov, H. M. et al. RXR alpha mutant mice establish a genetic basis for vitamin A signaling in heart morphogenesis. Genes Dev. 8, 1007-1018 (1994).

287. Paardekooper Overman, J. et al. PZR Coordinates Shp2 Noonan and LEOPARD Syndrome Signaling in Zebrafish and Mice. Mol. Cell. Biol. 34, 2874-2889 (2014).

288. Walter, A. O., Peng, Z.-Y. \& Cartwright, C. A. The Shp-2 tyrosine phosphatase activates the Src tyrosine kinase by a non-enzymatic mechanism. Oncogene. 18, 1911-1920 (1999).

289. Orkin, S. H. Diversification of haematopoietic stem cells to specific lineages. Nat. Rev. Genet. 1, 57-64 (2000).

290. Ogawa, M. Differentiation and proliferation of hematopoietic stem cells. Blood. $\mathbf{8 1}$, 2844-2853 (1993).

291. Orkin, S. H. \& Zon, L. I. Hematopoiesis: An Evolving Paradigm for Stem Cell Biology. Cell. 132, 631-644 (2008).

292. Seita, J. \& Weissman, I. L. Hematopoietic stem cell: self-renewal versus differentiation. Wiley Interdiscip. Rev. Syst. Biol. Med. 2, 640-653 (2010). 
293. Kim, C. H. Homeostatic and pathogenic extramedullary hematopoiesis. J. Blood Med. 1, 13-19 (2010).

294. Akashi, K. et al. Transcriptional accessibility for genes of multiple tissues and hematopoietic lineages is hierarchically controlled during early hematopoiesis. Blood. 101, 383-389 (2003).

295. Arai, F. Self-renewal and differentiation of hematopoietic stem cells. Rinsho. Ketsueki. 57, 1845-1851 (2016).

296. Christensen, J. L. \& Weissman, I. L. Flk-2 is a marker in hematopoietic stem cell differentiation: a simple method to isolate long-term stem cells. Proc. Natl. Acad. Sci. 98, 14541-14546 (2001).

297. Orlic, D., Fischer, R., Nishikawa, S., Nienhuis, A. W. \& Bodine, D. M. Purification and characterization of heterogeneous pluripotent hematopoietic stem cell populations expressing high levels of c-kit receptor. Blood. 82, 762-70 (1993).

298. Banu, N., Deng, B., Lyman, S. D. \& Avraham, H. Modulation of haematopoietic progenitor development by FLT-3 ligand. Cytokine. 11, 679-688 (1999).

299. Lyman, S. D. et al. Molecular cloning of a ligand for the flt3/flk-2 tyrosine kinase receptor: a proliferative factor for primitive hematopoietic cells. Cell. 75, 11571167 (1993).

300. Duncan, A. W. et al. Integration of Notch and Wnt signaling in hematopoietic stem cell maintenance. Nat. Immunol. 6, 314-322 (2005).

301. Morrison, S. J. \& Weissman, I. L. The long-term repopulating subset of hematopoietic stem cells is deterministic and isolatable by phenotype. Immunity. 1, 661-673 (1994).

302. Nutt, S. L. \& Kee, B. L. The Transcriptional Regulation of B Cell Lineage Commitment. Immunity. 26, 715-725 (2007).

303. Arinobu, Y. et al. Reciprocal Activation of GATA-1 and PU.1 Marks Initial Specification of Hematopoietic Stem Cells into Myeloerythroid and Myelolymphoid Lineages. Cell Stem Cell. 1, 416-427 (2007).

304. Kim, S.-I. \& Bresnick, E. H. Transcriptional control of erythropoiesis: emerging mechanisms and principles. Oncogene. 26, 6777-6794 (2007).

305. Rothenberg, E. V. Negotiation of the T Lineage Fate Decision by TranscriptionFactor Interplay and Microenvironmental Signals. Immunity. 26, 690-702 (2007).

306. Crane, G. M., Jeffery, E. \& Morrison, S. J. Adult haematopoietic stem cell niches. Nat. Rev. Immunol. 17, 573-590 (2017).

307. Wei, Q. \& Frenette, P. S. Niches for Hematopoietic Stem Cells and Their Progeny. Immunity. 48, 632-648 (2018).

308. Cordeiro Gomes, A. et al. Hematopoietic Stem Cell Niches Produce LineageInstructive Signals to Control Multipotent Progenitor Differentiation. Immunity. 45, 1219-1231 (2016).

309. Barker, J. E. SI/SId hematopoietic progenitors are deficient in situ. Exp. Hematol. 22, 174-177 (1994).

310. Ogawa, M. et al. Expression and function of c-kit in hemopoietic progenitor cells. J. Exp. Med. 174, 63-71 (1991). 
311. Sugiyama, T., Kohara, H., Noda, M. \& Nagasawa, T. Maintenance of the Hematopoietic Stem Cell Pool by CXCL12-CXCR4 Chemokine Signaling in Bone Marrow Stromal Cell Niches. Immunity. 25, 977-988 (2006).

312. Nagasawa, T. et al. Defects of B-cell lymphopoiesis and bone-marrow myelopoiesis in mice lacking the CXC chemokine PBSF/SDF-1. Nature. 382, 635-638 (1996).

313. Kimura, S., Roberts, A. W., Metcalf, D. \& Alexander, W. S. Hematopoietic stem cell deficiencies in mice lacking c-Mpl, the receptor for thrombopoietin. Proc. Natl. Acad. Sci. 95, 1195-1200 (1998).

314. Qian, H. et al. Critical Role of Thrombopoietin in Maintaining Adult Quiescent Hematopoietic Stem Cells. Cell Stem Cell. 1, 671-684 (2007).

315. Zhao, M. et al. Megakaryocytes maintain homeostatic quiescence and promote post-injury regeneration of hematopoietic stem cells. Nat. Med. 20, 1321-1326 (2014).

316. Chow, A. et al. Bone marrow CD169+ macrophages promote the retention of hematopoietic stem and progenitor cells in the mesenchymal stem cell niche. J. Exp. Med. 208, 261-271 (2011).

317. Ludin, A. et al. Monocytes-macrophages that express $\alpha$-smooth muscle actin preserve primitive hematopoietic cells in the bone marrow. Nat. Immunol. 13, 1072-1082 (2012).

318. Adams, G. B. et al. Stem cell engraftment at the endosteal niche is specified by the calcium-sensing receptor. Nature. 439, 599-603 (2006).

319. Akashi, K., Traver, D., Miyamoto, T. \& Weissman, I. L. A clonogenic common myeloid progenitor that gives rise to all myeloid lineages. Nature. 404, 193-197 (2000).

320. Kondo, M., Weissman, I. L. \& Akashi, K. Identification of clonogenic common lymphoid progenitors in mouse bone marrow. Cell. 91, 661-672 (1997).

321. Metcalf, D. Hematopoietic cytokines. Blood. 111, 485-491 (2008).

322. Borzillo, G. V, Ashmun, R. A. \& Sherr, C. J. Macrophage lineage switching of murine early pre-B lymphoid cells expressing transduced fms genes. Mol. Cell. Biol. 10, 2703-2714 (1990).

323. Till, J. E., McCulloch, E. A. \& Siminovich, L. A stochastic model of stem cell proliferation, based on the growth of spleen colony-forming cells. Proc. Natl. Acad. Sci. 51, 29-36 (1964).

324. Lai, A. Y. \& Kondo, M. T and B lymphocyte differentiation from hematopoietic stem cell. Semin. Immunol. 20, 207-212 (2008).

325. Iwasaki, H. \& Akashi, K. Myeloid Lineage Commitment from the Hematopoietic Stem Cell. Immunity. 26, 726-740 (2007).

326. Goasguen, J. E. et al. Morphological evaluation of monocytes and their precursors. Haematologica. 94, 994-997 (2009).

327. Kolaczkowska, E. \& Kubes, P. Neutrophil recruitment and function in health and inflammation. Nat. Rev. Immunol. 13, 159-175 (2013).

328. Athens, J. W. et al. Leukokinetic studies III. The distribution of granulocytes in the blood of normal subjects. J. Clin. Invest. 40, 159-164 (1961). 
329. Athens, J. W. et al. Leukokinetic studies IV. The total blood, circulating and marginal granulocyte pools and the granulocyte turnober rate in normal subjects. J. Clin. Invest. 40, 989-995 (1961).

330. Geissmann, F. et al. Development of Monocytes, Macrophages, and Dendritic Cells. Science. 327, 656-661 (2010).

331. Serbina, N. V, Salazar-Mather, T. P., Biron, C. A., Kuziel, W. A. \& Pamer, E. G. TNF/iNOS-producing dendritic cells mediate innate immune defense against bacterial infection. Immunity. 19, 59-70 (2003).

332. Swirski, F. K. et al. Identification of Splenic Reservoir Monocytes and Their Deployment to Inflammatory Sites. Science. 325, 612-616 (2009).

333. Pittet, M. J. \& Weissleder, R. Intravital Imaging. Cell. 147, 983-991 (2011).

334. Schulz, C. et al. A Lineage of Myeloid Cells Independent of Myb and Hematopoietic Stem Cells. Science. 336, 86-90 (2012).

335. Hashimoto, D. et al. Tissue-Resident Macrophages Self-Maintain Locally throughout Adult Life with Minimal Contribution from Circulating Monocytes. Immunity. 38, 792-804 (2013).

336. Mercier, F. E., Ragu, C. \& Scadden, D. T. The bone marrow at the crossroads of blood and immunity. Nat. Rev. Immunol. 12, 49-60 (2012).

337. Hoeffel, G. et al. Adult Langerhans cells derive predominantly from embryonic fetal liver monocytes with a minor contribution of yolk sac-derived macrophages. J. Exp. Med. 209, 1167-1181 (2012).

338. Leick, M., Azcutia, V., Newton, G. \& Luscinskas, F. W. Leukocyte recruitment in inflammation: basic concepts and new mechanistic insights based on new models and microscopic imaging technologies. Cell Tissue Res. 355, 647-656 (2014).

339. Puga, I. et al. B cell-helper neutrophils stimulate the diversification and production of immunoglobulin in the marginal zone of the spleen. Nat. Immunol. 13, 170-180 (2012).

340. Magri, G. et al. Innate lymphoid cells integrate stromal and immunological signals to enhance antibody production by splenic marginal zone B cells. Nat. Immunol. 15, 354-364 (2014).

341. Chorny, A. et al. The soluble pattern recognition receptor PTX3 links humoral innate and adaptive immune responses by helping marginal zone B cells. J. Exp. Med. 213, 2167-2185 (2016).

342. Zhu, Y. P. et al. Identification of an Early Unipotent Neutrophil Progenitor with Protumoral Activity in Mouse and Human Bone Marrow. Cell Rep. 24, 2329-2341 (2018).

343. Deniset, J. F., Surewaard, B. G., Lee, W.-Y. \& Kubes, P. Splenic Ly6Ghigh mature and Ly6Gint immature neutrophils contribute to eradication of S. pneumoniae. J. Exp. Med. 214, 1333-1350 (2017).

344. Roonnstrand, L. Signal transduction via the stem cell factor receptor/c-Kit. Cell. Mol. Life Sci. 61, 2535-2548 (2004).

345. Li, C. L. \& Johnson, G. R. Stem cell factor enhances the survival but not the selfrenewal of murine hematopoietic long-term repopulating cells. Blood. 84, 408414 (1994). 
346. Sharma, Y., Astle, C. M. \& Harrison, D. E. Heterozygous kit mutants with little or no apparent anemia exhibit large defects in overall hematopoietic stem cell function. Exp. Hematol. 35, 214-220 (2007).

347. Zhu, H. H. et al. Kit-Shp2-Kit signaling acts to maintain a functional hematopoietic stem and progenitor cell pool. Blood. 117, 5350-5361 (2011).

348. Scherr, M. et al. Enhanced sensitivity to inhibition of SHP2, STAT5, and Gab2 expression in chronic myeloid leukemia (CML). Blood. 107, 3279-3287 (2006).

349. Chan, G. et al. Essential role for Ptpn11 in survival of hematopoietic stem and progenitor cells. Blood. 117, 4253-4261 (2011).

350. Yang, Z., Li, Y., Yin, F. \& Chan, R. J. Activating PTPN11 mutants promote hematopoietic progenitor cell-cycle progression and survival. Exp. Hematol. 36, 1285-1296 (2008).

351. Li, L. et al. A critical role for SHP2 in STAT5 activation and growth factor-mediated proliferation, survival, and differentiation of human CD34+ cells. Blood. 118, 1504-1515 (2011).

352. Jack, G. D., Zhang, L. \& Friedman, A. D. M-CSF elevates c-Fos and phosphoC/EBPalpha(S21) via ERK whereas G-CSF stimulates SHP2 phosphorylation in marrow progenitors to contribute to myeloid lineage specification. Blood. 114, 2172-2180 (2009).

353. Xu, D. et al. Non-lineage/stage-restricted effects of a gain-of-function mutation in tyrosine phosphatase Ptpn11 (Shp2) on malignant transformation of hematopoietic cells. J. Exp. Med. 208, 1977-1988 (2011).

354. van der Burgt, I. Noonan syndrome. Orphanet J. Rare Dis. 2, 4 (2007).

355. Roberts, A. E., Allanson, J. E., Tartaglia, M. \& Gelb, B. D. Noonan syndrome. Lancet. 381, 333-342 (2013).

356. Noonan, J. A. Hypertelorism with Turner phenotype. A new syndrome with associated congenital heart disease. Am. J. Dis. Child. 116, 373-380 (1968).

357. Nora, J. J., Nora, A. H., Sinha, A. K., Spangler, R. D. \& Lubs, H. A. The UllrichNoonan syndrome (Turner phenotype). Am. J. Dis. Child. 127, 48-55 (1974).

358. Marino, B., Digilio, M. C., Toscano, A., Giannotti, A. \& Dallapiccola, B. Congenital heart diseases in children with Noonan syndrome: An expanded cardiac spectrum with high prevalence of atrioventricular canal. J. Pediatr. 135, 703-706 (1999).

359. Croonen, E. A. et al. Prenatal diagnostic testing of the Noonan syndrome genes in fetuses with abnormal ultrasound findings. Eur. J. Hum. Genet. 21, 936-942 (2013).

360. Liberatore, C. M. \& Yutzey, K. E. MAP kinase activation in avian cardiovascular development. Dev. Dyn. 230, 773-780 (2004).

361. Krenz, M. et al. Role of ERK1/2 signaling in congenital valve malformations in Noonan syndrome. Proc. Natl. Acad. Sci. 105, 18930-18935 (2008).

362. Nakamura, T. et al. Mediating ERK1/2 signaling rescues congenital heart defects in a mouse model of Noonan syndrome. J. Clin. Invest. 117, 2123-2132 (2007).

363. Strullu, M. et al. Juvenile myelomonocytic leukaemia and Noonan syndrome. J. Med. Genet. 51, 689-697 (2014). 
364. Bader-Meunier, B. et al. Occurrence of myeloproliferative disorder in patients with Noonan syndrome. J. Pediatr. 130, 885-889 (1997).

365. Loh, M. L. Recent advances in the pathogenesis and treatment of juvenile myelomonocytic leukaemia. Br. J. Haematol. 152, 677-687 (2011).

366. Johannes, J. M., Garcia, E. R., De Vaan, G. A. \& Weening, R. S. Noonan's syndrome in association with acute leukemia. Pediatr. Hematol. Oncol. 12, 571575 (1995).

367. Side, L. E. \& Shannon, K. M. Myeloid disorders in infants with Noonan syndrome and a resident's rule recalled. J. Pediatr. 130, 857-859 (1997).

368. Rose, S., Misharin, A. \& Perlman, H. A novel Ly6C/Ly6G-based strategy to analyze the mouse splenic myeloid compartment. Cytometry. 81, 343-350 (2012).

369. Wengner, A. M., Pitchford, S. C., Furze, R. C. \& Rankin, S. M. The coordinated action of G-CSF and ELR + CXC chemokines in neutrophil mobilization during acute inflammation. Blood. 111, 42-49 (2008).

370. Burdon, P. C. E., Martin, C. \& Rankin, S. M. Migration across the sinusoidal endothelium regulates neutrophil mobilization in response to ELR + CXC chemokines. Br. J. Haematol. 142, 100-108 (2008).

371. DiPietro, L. A., Burdick, M., Low, Q. E., Kunkel, S. L. \& Strieter, R. M. MIP-1alpha as a critical macrophage chemoattractant in murine wound repair. J. Clin. Invest. 101, 1693-1698 (1998).

372. Davatelis, G. et al. Cloning and characterization of a cDNA for murine macrophage inflammatory protein (MIP), a novel monokine with inflammatory and chemokinetic properties. J. Exp. Med. 167, 1939-1944 (1988).

373. Rollins, B. J. Monocyte chemoattractant protein 1: a potential regulator of monocyte recruitment in inflammatory disease. Mol. Med. 2, 198-204 (1996).

374. George, C. D., Patton, M. A., Sawi, M., Sharland, M. \& Adam, E. J. Abdominal ultrasound in Noonan syndrome: a study of 44 patients. Pediatr. Radiol. 23, 316318 (1993).

375. Roti, G. et al. Acute lymphoblastic leukaemia in Noonan syndrome. Br. J. Haematol. 133, 448-450 (2006).

376. Balasubramanian, A., Ganju, R. K. \& Groopman, J. E. Hepatitis C virus and HIV envelope proteins collaboratively mediate interleukin-8 secretion through activation of p38 MAP kinase and SHP2 in hepatocytes. J. Biol. Chem. 278, 35755-35766 (2003).

377. Xu, D., Zheng, H., Yu, W.-M. \& Qu, C.-K. Activating Mutations in Protein Tyrosine Phosphatase Ptpn11 (Shp2) Enhance Reactive Oxygen Species Production That Contributes to Myeloproliferative Disorder. PLoS One. 8 (2013).

378. Hung, C.-N., Huang, H.-P., Wang, C.-J., Liu, K.-L. \& Lii, C.-K. Sulforaphane Inhibits TNF- $\alpha$-Induced Adhesion Molecule Expression Through the Rho A/ROCK/NF-kB Signaling Pathway. J. Med. Food. 17, 1095-1102 (2014).

379. Reddy, S. A. et al. Sulforaphane and its methylcarbonyl analogs inhibit the LPSstimulated inflammatory response in human monocytes through modulating cytokine production, suppressing chemotactic migration and phagocytosis in a NF-KB- and MAPK-dependent manner. Int. Immunopharmacol. 24, 440-450 
(2015)

380. Nallasamy, P. et al. Sulforaphane reduces vascular inflammation in mice and prevents TNF- $\alpha$-induced monocyte adhesion to primary endothelial cells through interfering with the NF-kB pathway. J. Nutr. Biochem. 25, 824-833 (2014).

381. Liu, Y.-C. et al. Sulforaphane inhibition of monocyte adhesion via the suppression of ICAM-1 and NF-KB is dependent upon glutathione depletion in endothelial cells. Vascul. Pharmacol. 48, 54-61 (2008).

382. Chen, X.-L., Dodd, G. \& Kunsch, C. Sulforaphane inhibits TNF- $\alpha$-induced activation of p38 MAP kinase and VCAM-1 and MCP-1 expression in endothelial cells. Inflamm. Res. 58, 513-521 (2009).

383. Healy, Z. R., Liu, H., Holtzclaw, W. D. \& Talalay, P. Inactivation of Tautomerase Activity of Macrophage Migration Inhibitory Factor by Sulforaphane: a Potential Biomarker for Anti-inflammatory Intervention. Cancer Epidemiol. Biomarkers Prev. 20, 1516-1523 (2011).

384. Cross, J. V et al. Nutrient isothiocyanates covalently modify and inhibit the inflammatory cytokine macrophage migration inhibitory factor (MIF). Biochem. J. 423, 315-321 (2009).

385. Fischer, E. H., Charbonneau, H. \& Tonks, N. K. Protein tyrosine phosphatases: a diverse family of intracellular and transmembrane enzymes. Science. 253, 401406 (1991).

386. David. A. \& Dixon, J. E. A thousand and two protein tyrosine phosphatases. Biocllimica el Bieptr,yca Acra. 1136, 35-43 (1992).

387. Zhang, Z.-Y., Wang, Y. \& Dixon, J. E. Dissecting the catalytic mechanism of protein-tyrosine phosphatases. Proc. Nati. Acad. Sci. 91, 1624-1627 (1994).

388. Guan, K. L. \& Dixon, J. E. Protein tyrosine phosphatase activity of an essential virulence determinant in Yersinia. Science. 249, 553-556 (1990).

389. Streuli, M., Krueger, N. X., Thai, T., Tang, M. \& Saito, H. Distinct functional roles of the two intracellular phosphatase like domains of the receptor-linked protein tyrosine phosphatases LCA and LAR. EMBO J. 9, 2399-2407 (1990).

390. Pot, D. A. \& Dixon, J. E. Active Site Labeling of a Receptor-like Protein Tyrosine Phosphatase. J Biol Chem. 267, 140-143 (1992).

391. Guan, K. \& Dixon, J. E. Evidence for Protein-tyrosine-phosphatase catalysis proceeding via a cysteine-phosphate intermediate. J. Biol. Chem. 266, 1702617030 (1991).

392. Denu, J. M. \& Dixon, J. E. Protein tyrosine phosphatases: mechanisms of catalysis and regulation. Curr. Opin. Chem. Biol. 2, 633-641 (1998).

393. Tonks, N. K. \& Neel, B. G. Combinatorial control of the specificity of protein tyrosine phosphatases. Curr. Opin. Cell Biol. 13, 182-195 (2001).

394. Rudolph, J. Catalytic Mechanism of Cdc25. Biochemistry. 49, 14613-14623 (2002).

395. Wang, W., Q., Sun, J.-P. \& Zhang, Z.-Y. An Overview of the Protein Tyrosine Phosphatase Superfamily. Curr. Top. Med. Chem. 3, 739-748 (2003).

396. Kolmodin, K. \& Aqvist, J. The catalytic mechanism of protein tyrosine phosphatases revisited. FEBS Lett. 498, 208-213 (2001). 
397. Qiu, W. et al. Structural insights into Noonan/LEOPARD syndrome-related mutants of protein-tyrosine phosphatase SHP2 (PTPN11). BMC Struct. Biol. 14, 10 (2014).

398. Stuckey, J. A. et al. Crystal structure of Yersinia protein tyrosine phosphatase at $2.5 \AA$ and the complex with tungstate. Nature. 370, 571-575 (1994).

399. Jia, Z., Barford, D., Flint, A. J. \& Tonks, N. K. Structural basis for phosphotyrosine peptide recognition by protein tyrosine phosphatase 1B. Science. 268, 17541758 (1995).

400. Barford, D., Das, A. K. \& Egloff, M.-P. The structure and mechanism of protein phosphatases: Insights into Catalysis and Regulation. Annu. Rev. Biophys. Biomol. Struct. 27, 133-164 (1998).

401. Bornadata Evans, Patrick A. Tishmack, Christine Pokalsky, Marie Zhang, A. \& Etten, R. L. Van. Site-Directed Mutagenesis, Kinetic, and Spectroscopic Studies of the P-Loop Residues in a Low Molecular Weight Protein Tyrosine Phosphatase. Biochemistry. 42, 13609-13617 (1996).

402. Denu, J. M., Lohse, D. L., Vijayalakshmi, J., Saper, M. A. \& Dixon, J. E. Visualization of intermediate and transition-state structures in protein-tyrosine phosphatase catalysis. Proc. Natl. Acad. Sci. 93, 2493-2498 (1996).

403. Pannifer, A. D., Flint, A. J., Tonks, N. K. \& Barford, D. Visualization of the cysteinyl-phosphate intermediate of a protein-tyrosine phosphatase by $\mathrm{x}$-ray crystallography. J. Biol. Chem. 273, 10454-10462 (1998).

404. Tautz, L., Critton, D. A. \& Grotegut, S. Protein Tyrosine Phosphatases: Structure, Function, and Implication in Human Disease. in Methods in molecular biology. 1053, 179-221 (2013).

405. Taddei, N. et al. Aspartic-129 is an essential residue in the catalytic mechanism of the low Mr phosphotyrosine protein phosphatase. FEBS Lett. 350, 328-332 (1994).

406. Denu, J. M., Zhou, G., Guo, Y. \& Dixon, J. E. The Catalytic Role of Aspartic Acid92 in the Human Dual-Specific Protein-Tyrosine-Phosphatase Vaccinia H1Related. Biochemistry. 34, 3396-3403 (1995).

407. Flint, A. J., Tiganis, T., Barford, D. \& Tonks, N. K. Development of substratetrapping mutants to identify physiological substrates of protein tyrosine phosphatases. Proc. Natl. Acad. Sci. 94, 1680-1685 (1997).

408. Schlessinger, J. Cell Signaling by Receptor Tyrosine Kinases. Cell. 103, 211-225 (2000).

409. Monteiro, H. P. \& Stern, A. Redox modulation of tyrosine phosphorylationdependent signal transduction pathways. Free Radic. Biol. Med. 21, 323-333 (1996).

410. Leclerc, P., de Lamirande, E. \& Gagnon, C. Regulation of protein-tyrosine phosphorylation and human sperm capacitation by reactive oxygen derivatives. Free Radic. Biol. Med. 22, 643-656 (1997).

411. Flohé, L., Brigelius-Flohé, R., Saliou, C., Traber, M. G. \& Packer, L. Redox regulation of NF-kappa B activation. Free Radic. Biol. Med. 22, 1115-1126 (1997).

412. Rhee, S. G., Chang, T.-S., Bae, Y. S., Lee, S.-R. \& Kang, S. W. Cellular regulation 
by hydrogen peroxide. J. Am. Soc. Nephrol. 14, 211-215 (2003).

413. Weibrecht, I. et al. Oxidation sensitivity of the catalytic cysteine of the proteintyrosine phosphatases SHP-1 and SHP-2. Free Radic. Biol. Med. 43, 100-110 (2007).

414. Meng, T.-C., Fukada, T. \& Tonks, N. K. Reversible Oxidation and Inactivation of Protein Tyrosine Phosphatases In Vivo. Mol. Cell. 9, 387-399 (2002).

415. Van Montfort, R. L. M., Congreve, M., Tisi, D., Carr, R. \& Jhoti, H. Oxidation state of the active-site cysteine in protein tyrosine phosphatase 1B. Nature. 423, 773777 (2003).

416. Denu, J. M. \& Tanner, K. G. Specific and Reversible Inactivation of Protein Tyrosine Phosphatases by Hydrogen Peroxide: Evidence for a Sulfenic Acid Intermediate and Implications for Redox Regulation. Biochemistry. 16, 5633-5642 (1998).

417. Salmeen, A. et al. Redox regulation of protein tyrosine phosphatase 1B involves a sulphenyl-amide intermediate. Nature. 423, 769-773 (2003).

418. Tonks, N. K. Redox redux: revisiting PTPs and the control of cell signaling. Cell. 121, 667-670 (2005).

419. Chiarugi, P. et al. Two Vicinal Cysteines Confer a Peculiar Redox Regulation to Low Molecular Weight Protein Tyrosine Phosphatase in Response to Plateletderived Growth Factor Receptor Stimulation. J. Biol. Chem. 276, 33478-33487 (2001).

420. Fauman, E. B. et al. Crystal Structure of the Catalytic Domain of the Human Cell Cycle Control Phosphatase, Cdc25A. Cell. 93, 617-625 (1998).

421. Kwon, J. et al. Reversible oxidation and inactivation of the tumor suppressor PTEN in cells stimulated with peptide growth factors. Proc. Natl. Acad. Sci. 101, 16419-16424 (2004).

422. Chio, C. M., Lim, C. S. \& Bishop, A. C. Targeting a cryptic allosteric site for selective inhibition of the oncogenic protein tyrosine phosphatase Shp2. Biochemistry. 54, 497-504 (2015).

423. Lee, K. \& Esselman, W. J. Inhibition of PTPs by H2O2 regulates the activation of distinct MAPK pathways. Free Radic. Biol. Med. 33, 1121-1132 (2002).

424. Kwon, J. et al. Receptor-stimulated oxidation of SHP-2 promotes T-cell adhesion through SLP-76-ADAP. EMBO J. 24, 2331-2341 (2005).

425. Chen, C.-H. et al. Reactive Oxygen Species Generation Is Involved in Epidermal Growth Factor Receptor Transactivation through the Transient Oxidization of Src Homology 2-Containing Tyrosine Phosphatase in Endothelin-1 Signaling Pathway in Rat Cardiac Fibroblasts. Mol. Pharmacol. 69, 1347-1355 (2006).

426. Machado, L. E. S. F., Critton, D. A., Page, R. \& Peti, W. Redox Regulation of a Gain-of-Function Mutation (N308D) in SHP2 Noonan Syndrome. ACS. 2, 83138318 (2017).

427. Seth, D. \& Rudolph, J. Redox Regulation of MAP Kinase Phosphatase 3. Biochemistry. 45, 8476-8487 (2006).

428. Parsons, Z. D. \& Gates, K. S. Thiol-dependent recovery of catalytic activity from oxidized protein tyrosine phosphatases. Biochemistry. 52, 6412-6423 (2013). 
429. Burgoyne, J. R., Oviosu, O. \& Eaton, P. The PEG-switch assay: A fast semiquantitative method to determine protein reversible cysteine oxidation. $J$. Pharmacol. Toxicol. Methods. 68, 297-301 (2013).

430. Hill, B. G., Reily, C., Oh, J.-Y., Johnson, M. S. \& Landar, A. Methods for the determination and quantification of the reactive thiol proteome. Free Radic. Biol. Med. 47, 675-683 (2009).

431. Hoffman, R. D. \& Lane, M. D. lodophenylarsine oxide and arsenical affinity chromatography: new probes for dithiol proteins. J. Biol. Chem. 267, 1400514011 (1992).

432. Zhang, Y., Cho, C. G., Posner, G. H. \& Talalay, P. Spectroscopic quantitation of organic isothiocyanates by cyclocondensation with vicinal dithiols. Anal. Biochem. 205, 100-107 (1992).

433. Zhang, Y., Wade, K. L., Prestera, T. \& Talalay, P. Quantitative Determination of Isothiocyanates, Dithiocarbamates, Carbon Disulfide, and Related Thiocarbonyl Compounds by Cyclocondensation with 1,2-Benzenedithiol. Anal. Biochem. 239, 160-167 (1996).

434. Moncada, S., Palmer, R. M. \& Higgs, E. A. Nitric oxide: physiology, pathophysiology, and pharmacology. Pharmacol. Rev. 43, 109-142 (1991).

435. Prochaska, H. J., Santamaria, A. B. \& Talalay, P. Rapid detection of inducers of enzymes that protect against carcinogens. Proc. Natl. Acad. Sci. 89, 2394-2398 (1992).

436. Bayat Mokhtari, R. et al. The role of Sulforaphane in cancer chemoprevention and health benefits: a mini-review. J. Cell Commun. Signal. 12, 91-101 (2018).

437. Tortorella, S. M., Royce, S. G., Licciardi, P. V \& Karagiannis, T. C. Dietary Sulforaphane in Cancer Chemoprevention: The Role of Epigenetic Regulation and HDAC Inhibition. Antioxid. Redox Signal. 22, 1382-1424 (2015).

438. Fahey, J. W. \& Talalay, P. Antioxidant Functions of Sulforaphane: a Potent Inducer of Phase II Detoxication Enzymes. Food Chem. Toxicol. 37, 973-979 (1991).

439. Kubo, E., Chhunchha, B., Singh, P., Sasaki, H. \& Singh, D. P. Sulforaphane reactivates cellular antioxidant defense by inducing Nrf2/ARE/Prdx6 activity during aging and oxidative stress. Sci. Rep. 7, (2017).

440. Carocho, M. \& Ferreira, I. C. F. R. A review on antioxidants, prooxidants and related controversy: Natural and synthetic compounds, screening and analysis methodologies and future perspectives. Food Chem. Toxicol. 51, 15-25 (2013).

441. Lobo, V., Patil, A., Phatak, A. \& Chandra, N. Free radicals, antioxidants and functional foods: Impact on human health. Pharmacogn. Rev. 4, 118-126 (2010).

442. Phan, L. M., Yeung, S.-C. J. \& Lee, M.-H. Cancer metabolic reprogramming: importance, main features, and potentials for precise targeted anti-cancer therapies. Cancer Biol. Med. 11, 1-19 (2014).

443. Jorgenson, T. C., Zhong, W. \& Oberley, T. D. Redox imbalance and biochemical changes in cancer. Cancer Res. 73, 6118-6123 (2013).

444. Liberti, M. V \& Locasale, J. W. The Warburg Effect: How Does it Benefit Cancer Cells? Trends Biochem. Sci. 41, 211-218 (2016).

445. Ying, W. NAD+/NADH and NADP+/NADPH in Cellular Functions and Cell Death: 
Regulation and Biological Consequences. Antioxid. Redox Signal. 10, 179-206 (2008).

446. Khalil Arjmandi, M. et al. Pre and post radiotherapy serum oxidant/antioxidant status in breast cancer patients: Impact of age, BMI and clinical stage of the disease. Reports Pract. Oncol. Radiotherl. 21, 141-148 (2016).

447. Borek, C. Antioxidants and Radiation Therapy. J. Nutr. 134, 3207-3209 (2004).

448. Asensio-López, M. C., Soler, F., Pascual-Figal, D., Fernández-Belda, F. \& Lax, A. Doxorubicin-induced oxidative stress: The protective effect of nicorandil on $\mathrm{HL}-1$ cardiomyocytes. PLoS One. 12 (2017).

449. Gilliam, L. A. A. et al. Doxorubicin acts via mitochondrial ROS to stimulate catabolism in C2C12 myotubes. Am. J. Physiol. Cell Physiol. 302, 195-202 (2012).

450. Singh, K. et al. Sulforaphane treatment of autism spectrum disorder (ASD). Proc. Natl. Acad. Sci. 111, 15550-15555 (2014).

451. Brown, R. H., Reynolds, C., Brooker, A., Talalay, P. \& Fahey, J. W. Sulforaphane improves the bronchoprotective response in asthmatics through Nrf2-mediated gene pathways. Respir. Res. 16, 106 (2015).

452. Araki, T. et al. Noonan syndrome cardiac defects are caused by PTPN11 acting in endocardium to enhance endocardial-mesenchymal transformation. Proc. Natl. Acad. Sci. 106, 4736-4741 (2009).

453. Rastogi, R. P., Singh, S. P., Häder, D.-P. \& Sinha, R. P. Detection of reactive oxygen species (ROS) by the oxidant-sensing probe 2',7'dichlorodihydrofluorescein diacetate in the cyanobacterium Anabaena variabilis PCC 7937. Biochem. Biophys. Res. Commun. 397, 603-607 (2010).

454. Peshavariya, H. M., Dusting, G. J. \& Selemidis, S. Analysis of dihydroethidium fluorescence for the detection of intracellular and extracellular superoxide produced by NADPH oxidase. Free Radic. Res. 41, 699-712 (2007).

455. Bilan, D. S. et al. HyPer-3: A Genetically Encoded H2O2 Probe with Improved Performance for Ratiometric and Fluorescence Lifetime Imaging. ACS Chem. Biol. 8, 535-542 (2013).

456. Chen, Y.-N. P. et al. Allosteric inhibition of SHP2 phosphatase inhibits cancers driven by receptor tyrosine kinases. Nature. 535, 148-152 (2016).

457. Sun, X. et al. Selective inhibition of leukemia-associated SHP2E69K mutant by the allosteric SHP2 inhibitor SHP099. Leukemia. 32, 1246-1249 (2018). 